\title{
Stereoscopic Particle Image Velocimetry Measurements in Scramjet Combustors
}

\author{
A Dissertation \\ Presented to \\ the faculty of the School of Engineering and Applied Science \\ University of Virginia \\ In Partial Fulfillment \\ Of the requirements for the Degree \\ Doctor of Philosophy \\ Mechanical and Aerospace Engineering \\ by
}

Chad T. Smith

August 2012 
This dissertation is submitted in partial fulfillment of the requirements for the degree of Doctor of Philosophy in Mechanical and Aerospace Engineering

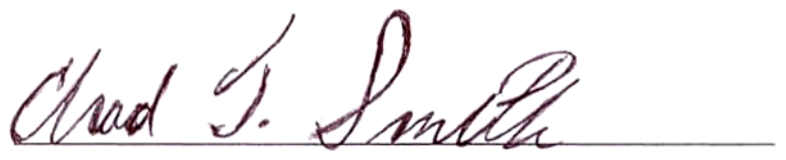

AUTHOR

This dissertation has been read and approved by the examining committee:
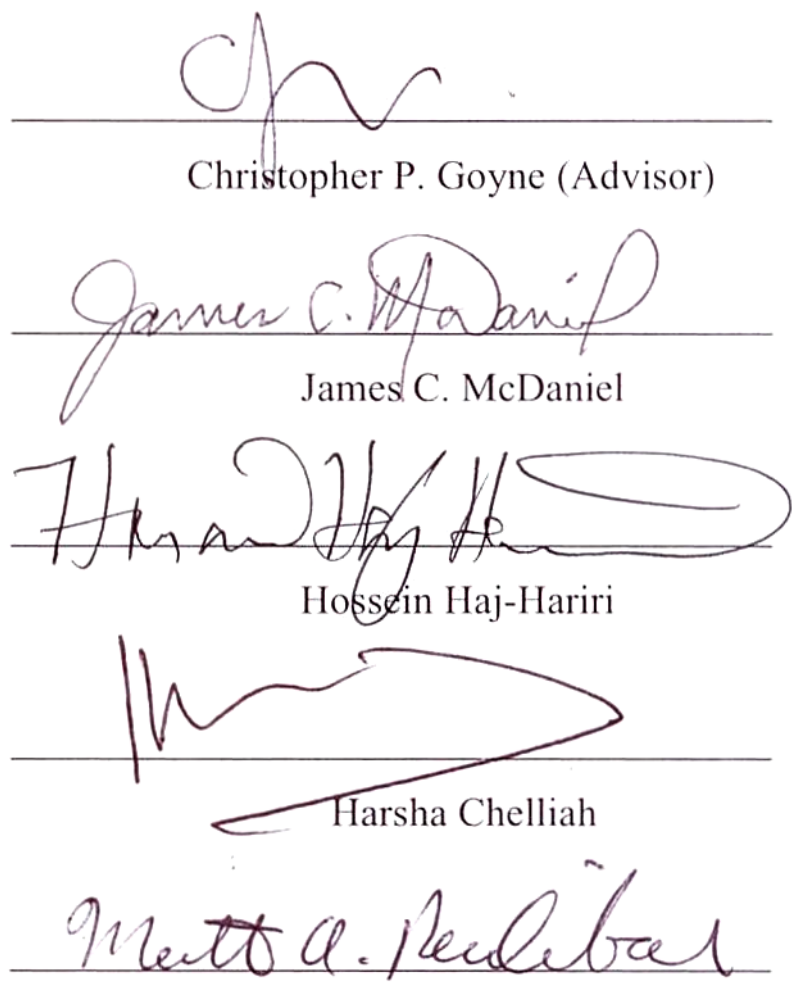

Matthew A. Reidenbach

Accepted for the School of Engineering and Applied Science:
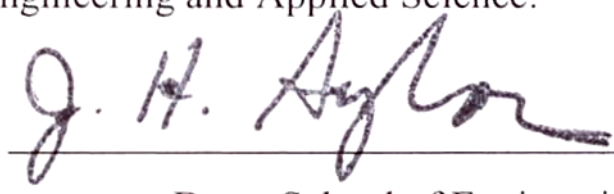

Dean, School of Engineering and

Applied Science

August 2012 


\begin{abstract}
The prospect of atmospheric flight at five times the speed of sound, or greater, has captivated researchers for many years. One type of hypersonic propulsion engine capable of providing such astounding flight-speeds is the Dual-mode scramjet (DMSJ). This engine is especially attractive because a single flowpath is used over a wide range of flight Mach numbers. However, the complex combustion process in a DMSJ is not well understood. The flowfield of a DMSJ combustor is highly turbulent and highly threedimensional due to fuel injection schemes and massive flow separation. These characteristics, along with temperatures in excess of $1200 \mathrm{~K}$, make experimental measurements extremely challenging. Therefore, three-component velocity measurements for the combusting flow of a scramjet are currently lacking in the literature. Velocity measurements in a Dual-Mode Scramjet combustor would further understanding of this complicated flow by providing quantitative in-stream information and a benchmark data set that is needed to facilitate computational model validation.

Classic probe-based measurement techniques are unacceptable for measuring the three-component velocity field of a DMSJ because these techniques alter the flowfield. However, a non-intrusive optical-based technique, such as Stereoscopic Particle Image Velocimetry (SPIV), is a candidate for velocity measurements in scramjet combustors. SPIV experiments have been undertaken at the University of Virginia's Aerospace Research Laboratory in order to measure the flow inside a DMSJ for the case of fuel-air mixing prior to ignition and also for the case of fuel-air combustion. These experiments were conducted at several different measurement locations in two DMSJ combustors with different flowpath geometries, each containing a ramp fuel injector. Because of opposing
\end{abstract}


constraints for SPIV experimental configurations and physical limitations of the supersonic combustion facility, a major accomplishment of this work was the design of appropriate SPIV configurations at each of the desired locations. These SPIV configurations consisted of a laser sheet (between 2.6 and $2.4 \mathrm{~mm}$ thick) placed at crossplanes in the combustor with two cameras to view the flow illuminated by the laser sheet. Each camera was set at angles ranging from 30 to 35-degrees for different experiments and submicron-diameter seed particles (diameter of $0.25 \mu \mathrm{m}$ and $0.3 \mu \mathrm{m}$ ) were added to the flow. These particles track the flow and consecutive images of the particles show displacements over a short time interval (400ns to 600ns) and therefore velocity information is obtained. Experiments conducted using the SPIV configurations produced the first SPIV measurements to provide instantaneous, three-component velocity measurements in a DMSJ during fuel-air combustion.

SPIV measurements generated both instantaneous and averaged velocity fields and quantified for the first time the two counter-rotating vortices induced by the ramp fuel injector. Flow quantities such as fluid rotation and turbulence intensity were then extracted from the velocity measurements. Moreover, because the measurements were obtained for both fuel-air mixing and fuel-air combustion at different combustor locations, these results provide insight into the effect of combustion on the fuel-air mixing process. Turbulent mixing length scales were also found in one measurement plane and are reported along with an estimate of the time scale of turbulence. Qualitative comparisons between SPIV measurements and current Computational Fluid Dynamics (CFD) results have also been made. The SPIV measurements will be available so that more detailed quantitative comparisons can be conducted in the future. While the current 
application of the SPIV technique was quite successful, geometric complications limited the SPIV measurements at certain locations. Using insight gained from the current experiments, recommendations have been made to improve future SPIV measurements. 


\section{Acknowledgements}

My road here at UVA has been a bit longer than I expected and I would not have arrived at the finish line without the support of many people. I would like to thank my advisor, Dr. Chris Goyne for the opportunity to work on such a challenging and interesting project. I would like to thank Dr. Roland Krauss, Dr. Bob Rockwell and Roger Reynolds for their help with running the wind tunnel. I especially appreciate the days they stayed a bit late so I could get my experiments finished. Thank you to Dr. Jim McDaniel for always being excited about seeing my SPIV measurements, even when I thought they needed a great deal of improvement. I want to say thank you to fellow graduate students Brian Rice, Justin Kirik, and Ben Tatman for being my extra sets of hands during my final experiments. These guys may not have thought they were doing much, but their help was very valuable to me during hectic tunnel runs.

I would also like to recognize my fellow graduate students Josh Keely, Jason Howison, and Meghan Snyder for their friendship as we shared the ups and downs of graduate school and working at the ARL. A special thanks to Elliott Bryner. It was great to share my graduate school experience with you and I wouldn't have had it any other way. Thank you to Lisa Stanton and Laura Troutman for their kindness and for always looking out for me. I also had a great deal of support outside of UVA and I must thank my church family at Monticello Wesleyan.

Finally, I cannot forget to thank my parents (and OK, my brother too) for all their love and support. Thank you for the long phone conversations when I was so frustrated by one problem or another. My parents have been my biggest cheering section throughout this process and that has been invaluable. 


\section{Table of Contents}

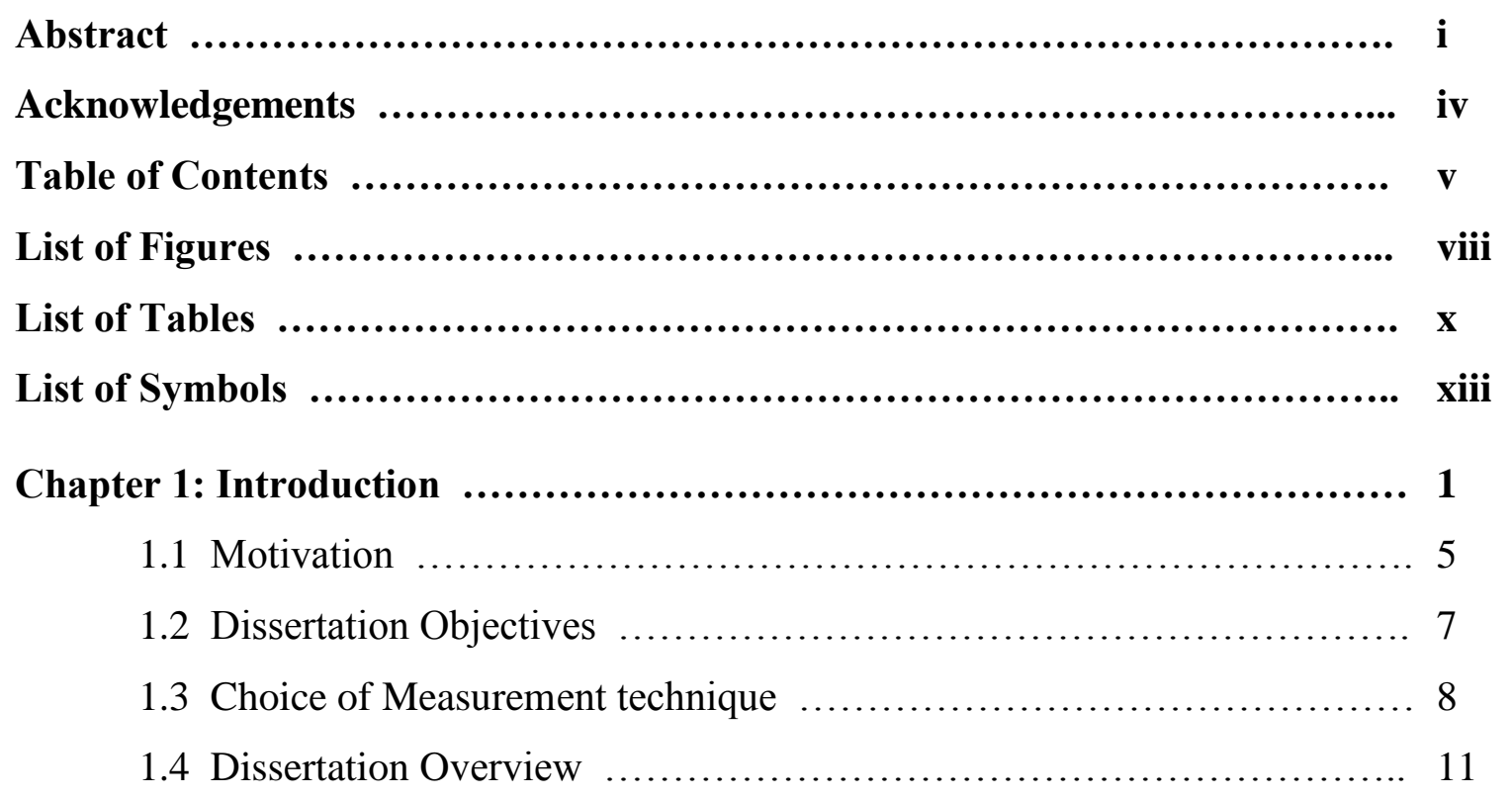

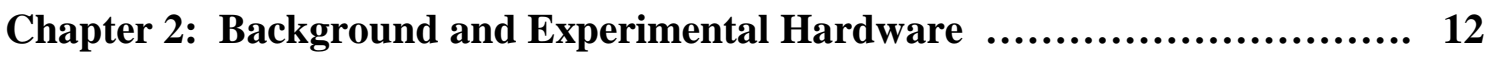

2.1 Particle Image Velocimetry (PIV): Basic Theory ….................. 12

2.1.1 Stereoscopic Particle Image Velocimetry (SPIV) ............. 13

2.1.2 Correlation Methods for PIV ................................... 16

2.2 Relevant PIV and SPIV Applications …............................ 19

2.3 SPIV Experimental Equipment …............................... 21

2.4 Supersonic Combustion Facility and Combustor Flowpaths ............... 31

Chapter 3: SPIV Experiment Design ............................................ 39

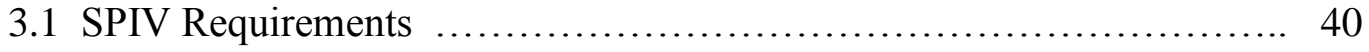

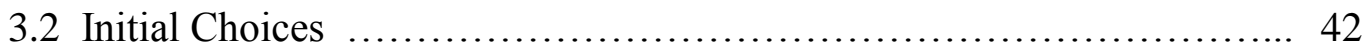

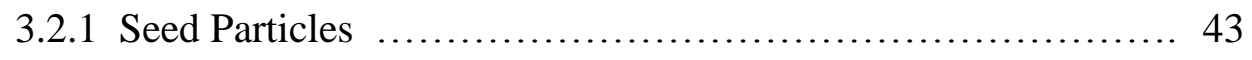

3.2.2 Type of Camera Configuration .............................. 45

3.2.3 The Scheimpflug Condition ................................. 46

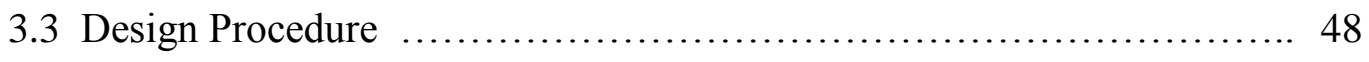

3.4 Example Design Iteration and Configuration at $\mathrm{X} / \mathrm{H}=10 \ldots \ldots \ldots \ldots \ldots .56$

3.5 Initial Results and Success of Experiment Design process ............... 60 
Chapter 4: SPIV Experimental Configurations …............................. 65

4.1 Flowpath 1 Measurement Planes …................................. 66

4.1.1 Experimental Configuration for $\mathrm{X} / \mathrm{H}=10$ plane $\ldots \ldots \ldots \ldots \ldots 68$

4.1.2 Experimental Configuration, $\mathrm{X} / \mathrm{H}=58.6$ plane, $\Phi=0.17 \ldots \ldots .72$

4.1.3 Experimental Configuration, $\mathrm{X} / \mathrm{H}=58.6$ plane, $\Phi=0.34 \ldots \ldots .77$

4.2 Flowpath 2 Measurement Planes ........................................ 81

4.2.1 Experimental Configuration for $\mathrm{X} / \mathrm{H}=12$ plane $\ldots \ldots \ldots \ldots \ldots . . . \quad 82$

4.2.2 Experimental Configuration, $\mathrm{X} / \mathrm{H}=15$ plane $\ldots \ldots \ldots \ldots \ldots \ldots . \ldots . \ldots 7$

Chapter 5: SPIV Experimental Results, Combustor Flowpath 1 ................. 90

$5.1 \mathrm{X} / \mathrm{H}=10$ Measurement Plane Results ............................. 90

5.1.1 Fuel-air Mixing Results ................................ 92

5.1.2 Fuel-air Combustion Results ….......................... 105

5.1.3 Uncertainty Estimation ...................................... 114

$5.2 \mathrm{X} / \mathrm{H}=58.6$ (Exit) Measurement Plane, $\Phi=0.17$ Results $\ldots \ldots \ldots \ldots \ldots \ldots 117$

5.2.1 Fuel-air Mixing Results …............................. 119

5.2.2 Fuel-air Combustion Results ................................. 123

$5.3 \mathrm{X} / \mathrm{H}=58.6$ (Exit) Measurement Plane, $\Phi=0.34$ Results $\ldots \ldots \ldots \ldots \ldots \ldots . . \ldots 131$

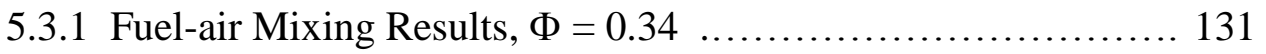

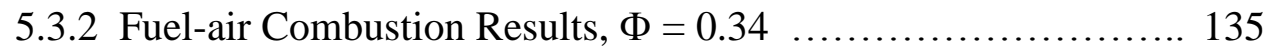

5.4 Qualitative Comparison to CFD results, Flowpath 1 ................. 142

Chapter 6: SPIV Experimental Results, Combustor Flowpath 2 .................. 151

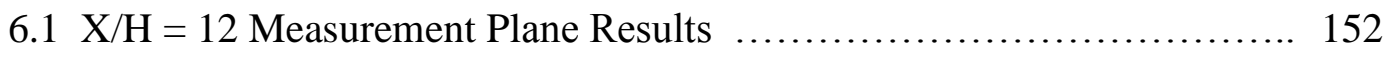

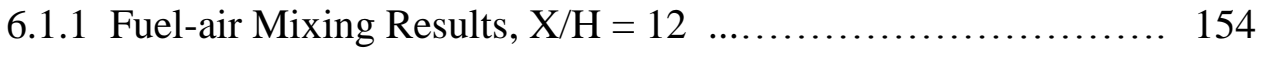

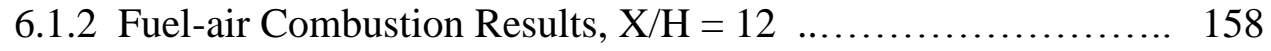

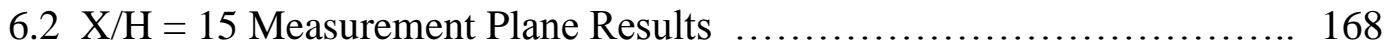

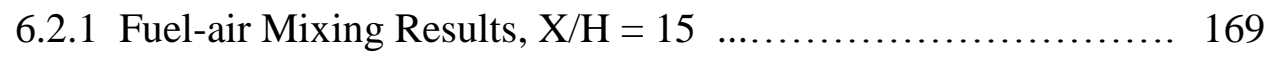

6.2.2 Fuel-air Combustion Results, $\mathrm{X} / \mathrm{H}=15 \quad \ldots \ldots \ldots \ldots \ldots \ldots \ldots \ldots \ldots \ldots \ldots$

6.3 Qualitative Comparison to CFD results, Flowpath $2 \ldots \ldots \ldots \ldots \ldots \ldots \ldots . . . \ldots 178$

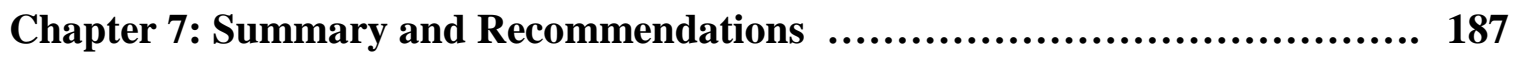


Bibliography

Appendix A: Supplemental figures for $\mathrm{X} / \mathrm{H}=10$ plane, Flowpath $1 \ldots \ldots \ldots \ldots \ldots \ldots 198$

Appendix B: Supplemental figures for $\mathrm{X} / \mathrm{H}=58.6$ plane, $\Phi=0.17 \ldots \ldots \ldots \ldots \ldots \ldots \ldots \ldots 2$

Appendix C: Supplemental figures for $\mathrm{X} / \mathrm{H}=58.6$ plane, $\Phi=0.34 \ldots \ldots \ldots \ldots \ldots \ldots 209$

Appendix D: Supplemental figures for $\mathrm{X} / \mathrm{H}=12$ plane, Flowpath $2 \ldots \ldots \ldots \ldots \ldots \ldots 216$

Appendix E: Supplemental figures for X/H = 15 plane, Flowpath $2 \ldots \ldots \ldots \ldots \ldots \ldots . \ldots 224$

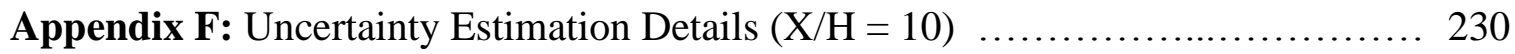




\section{List of Figures}

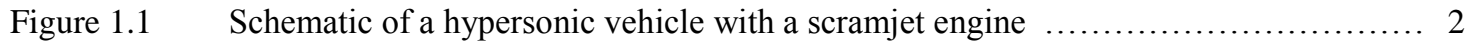

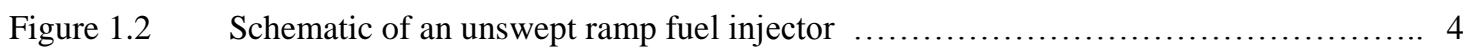

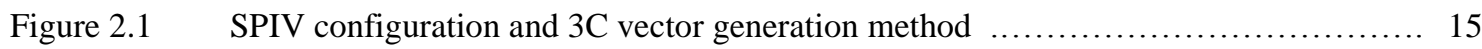

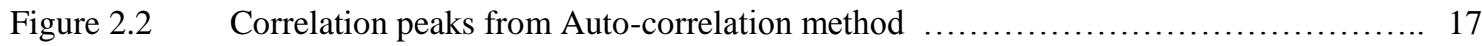

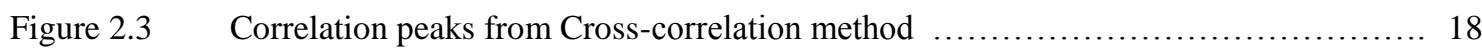

Figure 2.4 SPIV Cameras from TSI system and LaVision System ........................... 24

Figure 2.5 Narrow band pass filter transmittance (Edmund Optics NT43-174) $\ldots \ldots \ldots \ldots \ldots \ldots \ldots . \ldots . \ldots . \ldots 26$

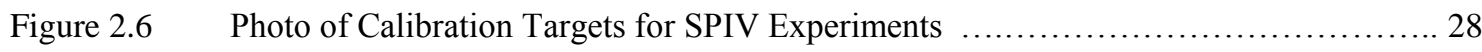

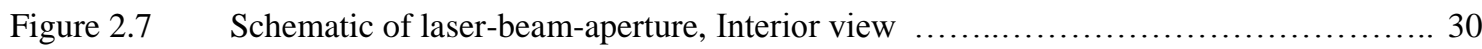

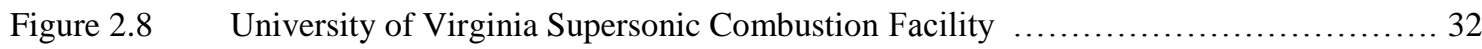

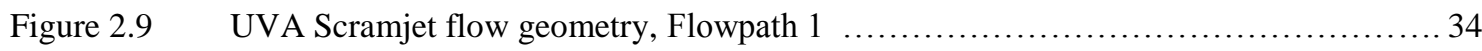

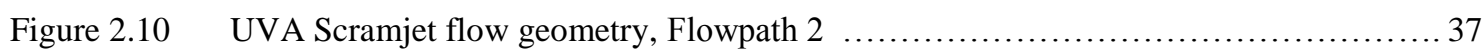

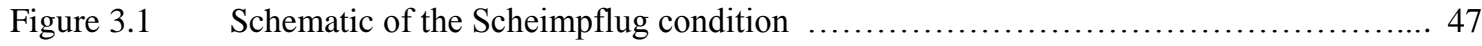

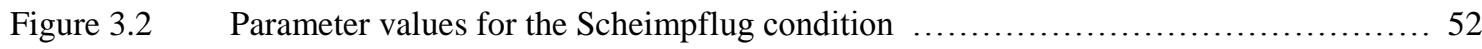

Figure 3.3 Expected particle displacements in image plane of PIV 10-30 cameras .............. 54

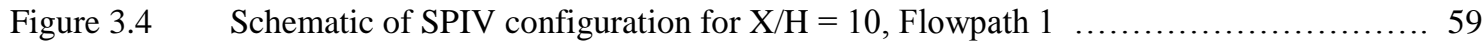

Figure $3.5 \quad$ Velocity results for fuel/air combustion at $\mathrm{X} / \mathrm{H}=10$, Flowpath $1 \ldots \ldots \ldots \ldots \ldots \ldots \ldots \ldots \ldots \ldots \ldots \ldots$

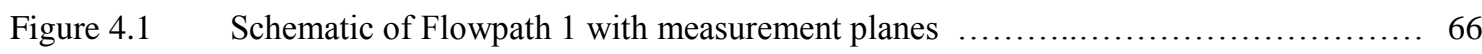

Figure 4.2 Schematic and photo of SPIV configuration for $\mathrm{X} / \mathrm{H}=10$, Flowpath $1 \quad \ldots \ldots \ldots \ldots \ldots . \ldots$

Figure 4.3 Optical train (lens components) for laser sheet at $\mathrm{X} / \mathrm{H}=10$ plane $\ldots \ldots \ldots \ldots \ldots \ldots \ldots \ldots \ldots \ldots \ldots$

Figure 4.4 Schematic and photo of SPIV configuration at X/H $=58.6$ (Exit), Flowpath $1 \ldots \ldots \ldots .75$

Figure 4.5 Optical train (lens components) for laser sheet at Exit plane $(\mathrm{X} / \mathrm{H}=58.6) \ldots \ldots \ldots \ldots .76$

Figure 4.6 Schematic of Flowpath 2 with measurement planes at $\mathrm{X} / \mathrm{H}=12$ and $15 \quad \ldots \ldots \ldots \ldots . \ldots 1$

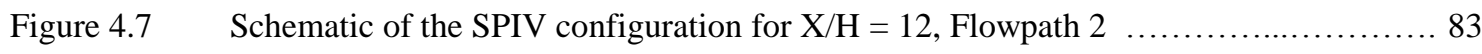

Figure 4.8 Optical train (lens components) for laser sheet at $\mathrm{X} / \mathrm{H}=12$ and 15 planes $\ldots \ldots \ldots \ldots . \ldots 8$ 
Flowpath 1

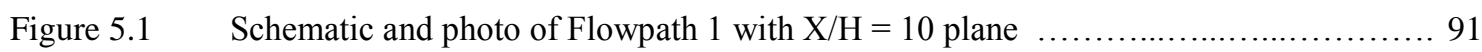

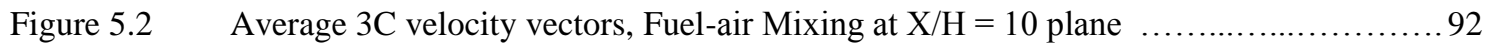

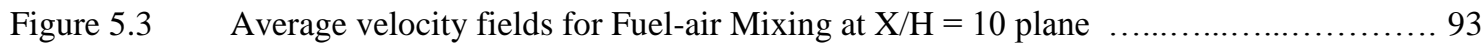

Figure 5.4 Average and Instantaneous velocity fields, Fuel-air Mixing at $\mathrm{X} / \mathrm{H}=10 \quad \ldots \ldots \ldots \ldots \ldots . . \ldots 8$

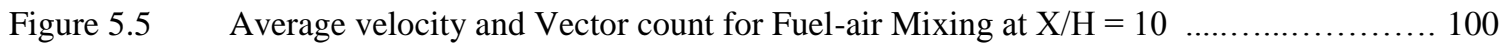

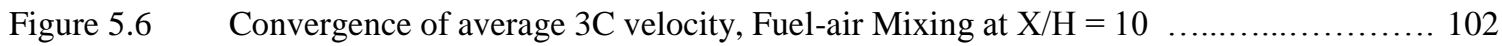

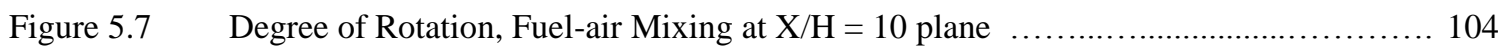

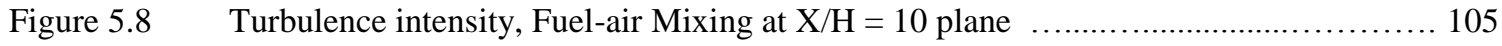

Figure $5.9 \quad$ Average $3 \mathrm{C}$ velocity vectors, Fuel-air Combustion at $\mathrm{X} / \mathrm{H}=10$ plane $\ldots \ldots \ldots \ldots \ldots \ldots \ldots \ldots \ldots$

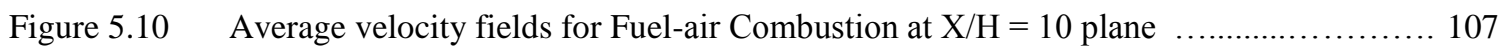

Figure 5.11 Average velocity and Vector count for Fuel-air Combustion at $\mathrm{X} / \mathrm{H}=10 \quad \ldots \ldots \ldots \ldots \ldots . \ldots 109$

Figure 5.12 Convergence of average $3 \mathrm{C}$ velocity, Fuel-air Combustion at $\mathrm{X} / \mathrm{H}=10 \ldots \ldots \ldots \ldots \ldots \ldots$

Figure 5.13 Average and Instantaneous velocity fields, Fuel-air Combustion at $\mathrm{X} / \mathrm{H}=10 \quad \ldots \ldots \ldots \ldots$

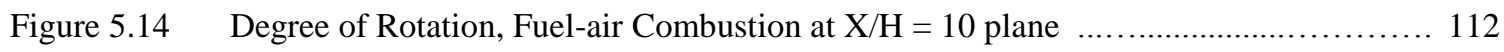

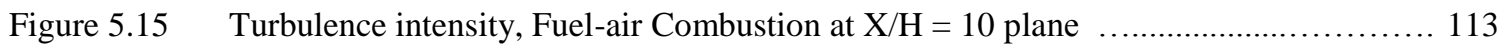

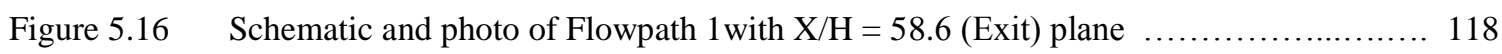

Figure 5.17 Average 3C velocity \& In-plane vectors, Fuel-air Mixing, $\mathrm{X} / \mathrm{H}=58.6, \Phi=0.17 \ldots \ldots .120$

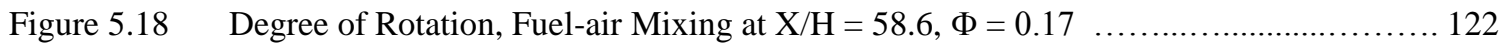

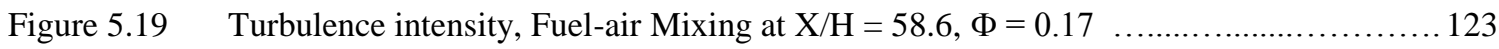

Figure 5.20 3D view of Avg. 3C velocity, Fuel-air Combustion at $\mathrm{X} / \mathrm{H}=58.6, \Phi=0.17 \ldots \ldots \ldots \ldots 124$

Figure 5.21 3C velocity \& In-plane vectors, Fuel-air Combustion, $\mathrm{X} / \mathrm{H}=58.6, \Phi=0.17 \ldots \ldots \ldots \ldots .125$

Figure 5.22 Average and Instantaneous velocity, Fuel-air Combustion, $\mathrm{X} / \mathrm{H}=58.6, \Phi=0.17 \ldots \ldots 127$

Figure 5.23 Average velocity and Vector count, Fuel-air Combustion at $\mathrm{X} / \mathrm{H}=58.6, \Phi=0.17 \ldots \ldots .128$

Figure 5.24 Degree of Rotation, Fuel-air Combustion at $\mathrm{X} / \mathrm{H}=58.6, \Phi=0.17 \quad \ldots \ldots \ldots \ldots \ldots \ldots \ldots \ldots \ldots \ldots \ldots \ldots \ldots \ldots . . . .129$

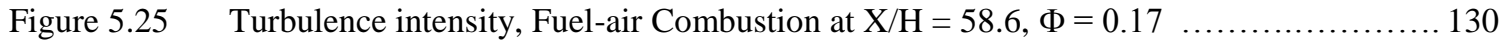

Figure 5.26 Average 3C velocity \& In-plane vectors, Fuel-air Mixing, $\mathrm{X} / \mathrm{H}=58.6, \Phi=0.34 \ldots \ldots .132$

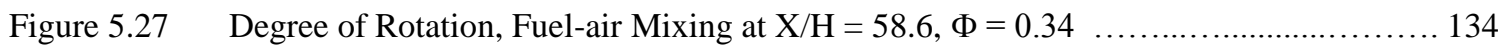




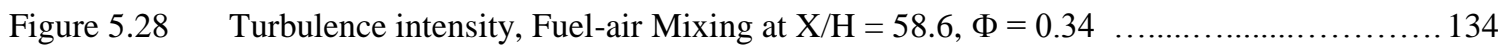

Figure 5.29 3D view of Avg. 3C velocity, Fuel-air Combustion at $\mathrm{X} / \mathrm{H}=58.6, \Phi=0.34 \ldots \ldots \ldots 135$

Figure 5.30 3C velocity \& In-plane vectors, Fuel-air Combustion, $\mathrm{X} / \mathrm{H}=58.6, \Phi=0.34 \ldots \ldots \ldots \ldots 137$

Figure 5.31 Average velocity and Vector count, Fuel-air Combustion at $\mathrm{X} / \mathrm{H}=58.6, \Phi=0.34 \ldots \ldots 138$

Figure 5.32 Average and Instantaneous velocity, Fuel-air Combustion, $\mathrm{X} / \mathrm{H}=58.6, \Phi=0.34 \quad \ldots \ldots 139$

Figure 5.33 Degree of Rotation, Fuel-air Combustion at $\mathrm{X} / \mathrm{H}=58.6, \Phi=0.34 \ldots \ldots \ldots \ldots \ldots \ldots \ldots$

Figure 5.34 Turbulence intensity, Fuel-air Combustion at X/H = 58.6, $\Phi=0.34 \ldots \ldots \ldots \ldots \ldots \ldots 142$

Figure 5.35 CFD and SPIV comparison, Fuel-air Combustion at $\mathrm{X} / \mathrm{H}=10, \Phi=0.26 \ldots \ldots \ldots \ldots 144$

Figure 5.36 CFD and SPIV comparison, Fuel-air Combustion at $\mathrm{X} / \mathrm{H}=58.6, \Phi=0.17 \ldots \ldots \ldots \ldots 146$

Figure 5.37 CFD and SPIV In-plane vectors, Fuel-air Combustion at $\mathrm{X} / \mathrm{H}=58.6, \Phi=0.17 \quad \ldots \ldots \ldots 147$

Figure 5.38 CFD and SPIV comparison, Fuel-air Combustion at $\mathrm{X} / \mathrm{H}=58.6, \Phi=0.34 \ldots \ldots \ldots \ldots 149$

Flowpath 2

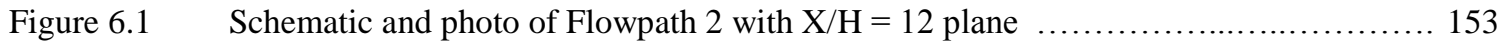

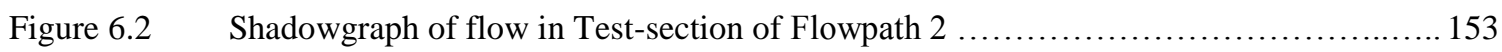

Figure 6.3 Average 3C velocity Magnitude \& In-plane vectors, Fuel-air Mixing, X/H $=12 \quad \ldots \ldots . .155$

Figure $6.4 \quad$ Average velocity and Vector count for Fuel-air Mixing at $\mathrm{X} / \mathrm{H}=12 \quad \ldots \ldots \ldots \ldots \ldots \ldots . \ldots 156$

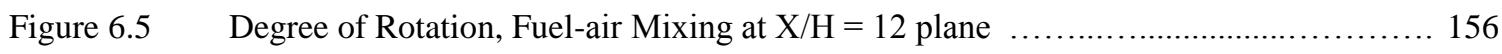

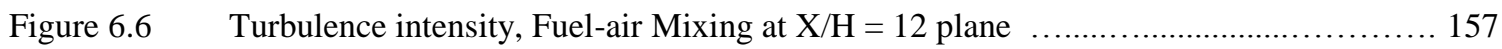

Figure 6.7 3C velocity Magnitude \& In-plane vectors, Fuel-air Combustion, $\mathrm{X} / \mathrm{H}=12 \quad \ldots \ldots \ldots \ldots$

Figure 6.8 3C velocity without incorrect vectors removed, Fuel-air Combustion, $\mathrm{X} / \mathrm{H}=12 \quad \ldots \ldots \ldots$

Figure 6.9 Average and Instantaneous velocity fields, Fuel-air Combustion at X/H $=12 \quad \ldots \ldots \ldots . .161$

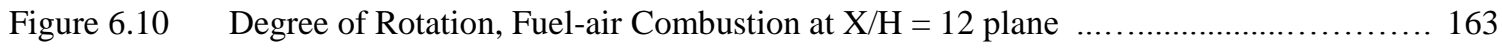

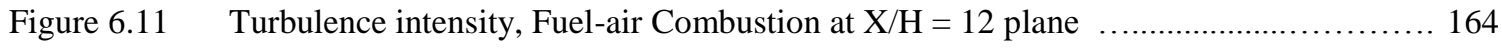

Figure 6.12 Correlation of In-plane velocity components with values at $(Z, Y)=(0,0.8) \quad \ldots \ldots \ldots 166$

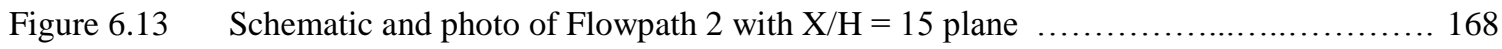

Figure 6.14 Average 3C velocity Magnitude \& In-plane vectors, Fuel-air Mixing, X/H $=15 \quad \ldots \ldots \ldots 169$

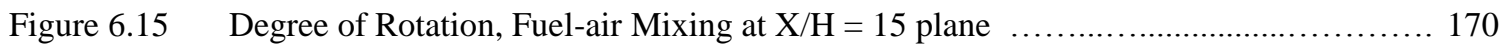




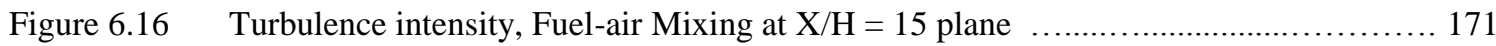

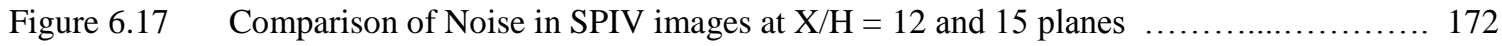

Figure 6.18 $3 \mathrm{C}$ velocity Magnitude \& In-plane vectors, Fuel-air Combustion, $\mathrm{X} / \mathrm{H}=15 \ldots \ldots \ldots \ldots .174$

Figure 6.19 Average velocity and Vector count for Fuel-air Combustion at $\mathrm{X} / \mathrm{H}=15 \ldots \ldots \ldots \ldots \ldots \ldots . . . . .175$

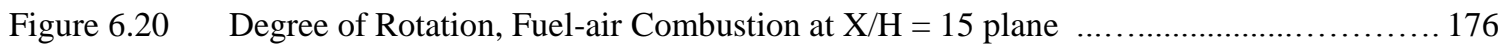

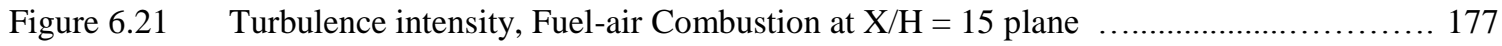

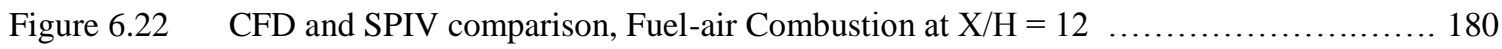

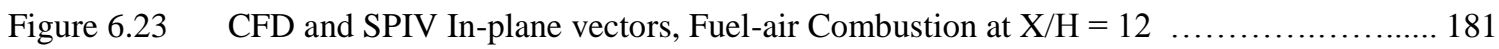

Figure 6.24 CFD and SPIV Turbulent Kinetic Energy Comparison, Combustion at $\mathrm{X} / \mathrm{H}=12 \quad \ldots \ldots \quad 182$

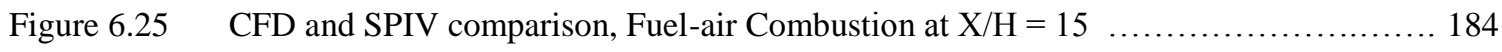




\section{List of Tables}

Table 2.1 Typical University of Virginia Supersonic Combustion facility conditions ........... 34

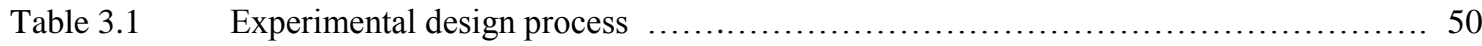

Table 4.1 Experiment conditions and SPIV parameters, $\mathrm{X} / \mathrm{H}=10$ plane $\ldots \ldots \ldots \ldots \ldots \ldots \ldots \ldots$

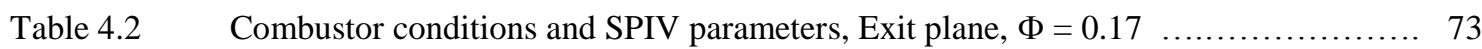

Table 4.3 Combustor conditions and SPIV parameters, Exit plane, $\Phi=0.34 \quad \ldots \ldots \ldots \ldots \ldots \ldots \ldots$

Table 4.4 Experiment conditions and SPIV parameters, $\mathrm{X} / \mathrm{H}=12$ plane, Flowpath $2 \ldots \ldots \ldots \ldots . . \quad 84$

Table 4.5 Experiment conditions and SPIV parameters, $\mathrm{X} / \mathrm{H}=15$ plane, Flowpath $2 \ldots \ldots \ldots \ldots 8$ 


\section{List of Symbols}

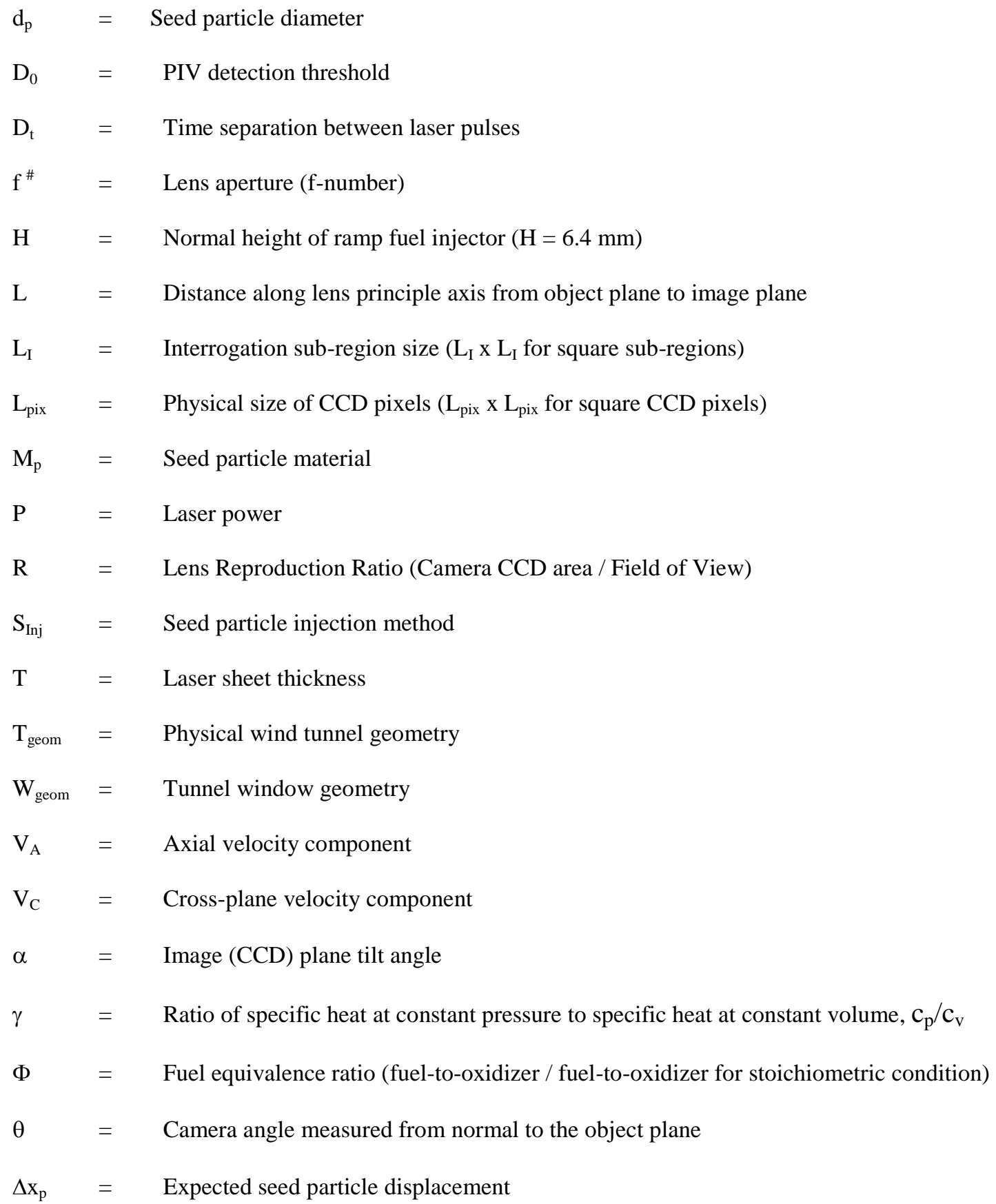




\section{Chapter 1: Introduction}

Recent successful flights of NASA's X-43A hypersonic test vehicle provided concrete evidence of the potential of the Supersonic Combustion Ramjet, or Scramjet, hypersonic airbreathing engine. ${ }^{1}$ Some benefits of atmospheric fight at five times the speed of sound, or more, are quite evident. Scramjet technology could be used for military applications that fundamentally change the way the military deploys unmanned aerial vehicles to acquire ground combat information or the way missile payloads are delivered to their intended targets. Military aircraft outfitted with scramjets could reach their destinations in a relatively short timeframe even if launched from bases or ships at great distances. The potential benefit of scramjet engines over conventional turbinebased engines, for example, can be easily understood by considering that a journey requiring two hours at Mach 0.8 would be completed in just 19 minutes by aircraft using scramjet engines at Mach 5 flight speeds. Furthermore, scramjets will fundamentally change access to outer space, which is currently handled by expensive and only partially reusable rocket-based technology. Since hypersonic air-breathing engines get their oxidizer from the atmosphere, there is no need to carry heavy and expensive oxidizer tanks on board the vehicle. Furthermore, since the vehicle can be flown under power upon reentry, scramjet engine-based vehicles for access to space should therefore ultimately be safer than current technology.

Figure 1.1 shows a schematic of a hypersonic vehicle with a scramjet engine. Tracing the flowpath, incoming air is compressed as it crosses the bow-shock produced at the front of the vehicle and this compressed air enters the inlet of the engine. Further compression then occurs in the isolator section as air passes through an oblique shock 
train. Scramjet engines take advantage of the temperature rise and density increase that occurs across shockwaves created at the front of the hypersonic vehicle and in the isolator section of the engine. ${ }^{2}$ This isolator section is aptly named as it locates the inlet shock train and keeps the oblique shockwaves separated from the combustor section. In the combustor section, injected fuel mixes with incoming air and combustion takes place with the aid of flameholding devices. While the airflow is slowed as it passes through the bow-shock and the isolator's oblique shock train, the flow remains supersonic through the entire engine and therefore combustion occurs at supersonic speeds in a "pure scramjet." After fuel-air combustion takes place in the combustor section, the flow expands through a nozzle integrated into the aft body of the vehicle and here useful thrust is extracted from the flow.

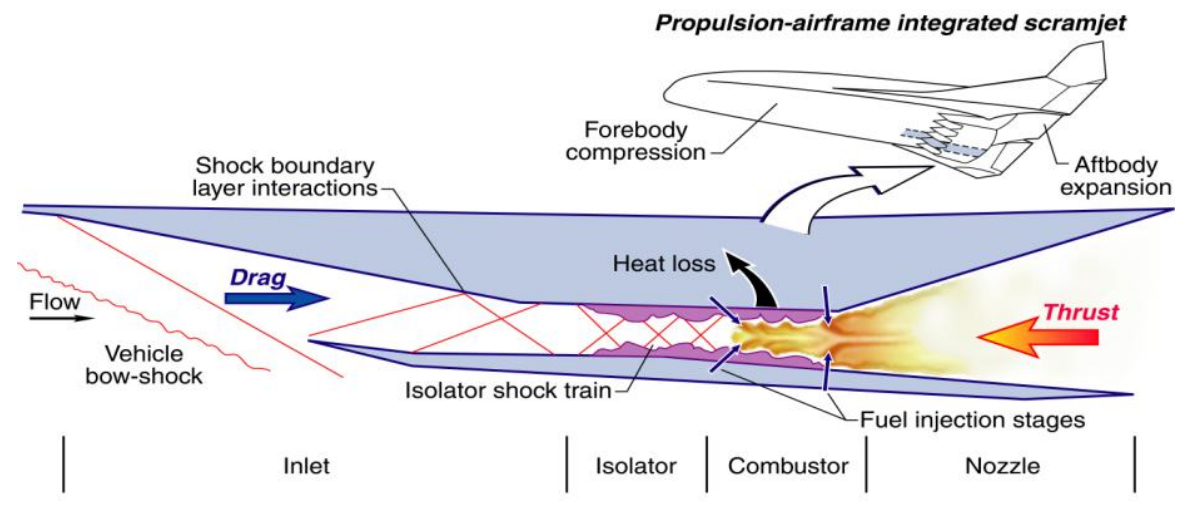

Figure 1.1: Schematic of a hypersonic vehicle with scramjet engine (source: NASA LaRC) ${ }^{3}$

A special class of scramjets is the Dual-Mode Scramjet (DMSJ) which is characterized by the ability to operate in a mode where the combustion process is largely subsonic or in a mode where the flow inside the combustor is predominately supersonic. This type of scramjet is extremely advantageous because both combustion modes use the 
same engine geometry. ${ }^{2}$ Using a single engine geometry allows DMSJs to operate over a wide range of flight Mach numbers. ${ }^{4}$ However, Dual-Mode Scramjets cannot be used as the sole propulsion system from takeoff through supersonic flight. For successful operation of a DMSJ, the vehicle must be traveling at supersonic speeds so that shockwaves are present to compress incoming air before it enters the combustor section of the engine. For this reason, Turbine Based Combined Cycle (TBCC) systems have been proposed where a turbojet is used from takeoff to speeds near Mach 3, at which point a DMSJ takes over and operates in the subsonic combustion mode until the vehicle has been accelerated to speeds near Mach 5. At that point, the engine transitions to the supersonic combustion mode for sustained flight or acceleration past Mach 5. NASA has determined that the Dual-Mode Scramjet is one of the core technologies of combinedcycle systems. ${ }^{5}$ Factors such as boundary layer separation, fuel injection and fuel-air combustion give rise to a complex flowfield inside a DMSJ and this complex combustion process is currently not well understood.

Supersonic flow within the combustor section of a DMSJ causes very short fuelresidence-time and, therefore, efficient fuel-air mixing is essential for fuel-air combustion. Appropriate flameholding mechanisms are also necessary to sustain combustion after ignition. If inefficient fuel-air mixing delays combustion until after fuel has exited the engine, then any useful thrust is lost. Many fuel injection schemes have been proposed to enhance fuel-air mixing and to provide flameholding. One such injector geometry is the physical ramp fuel injector. Figure 1.2 depicts a schematic of a ramp fuel injector in side-view and an end-view of the base of the ramp. For this type of injector, the shockwave emanating from the ramp's leading edge creates an area of high 
pressure on the face of the ramp, while lower pressure regions persist on either side of the ramp fuel injector.

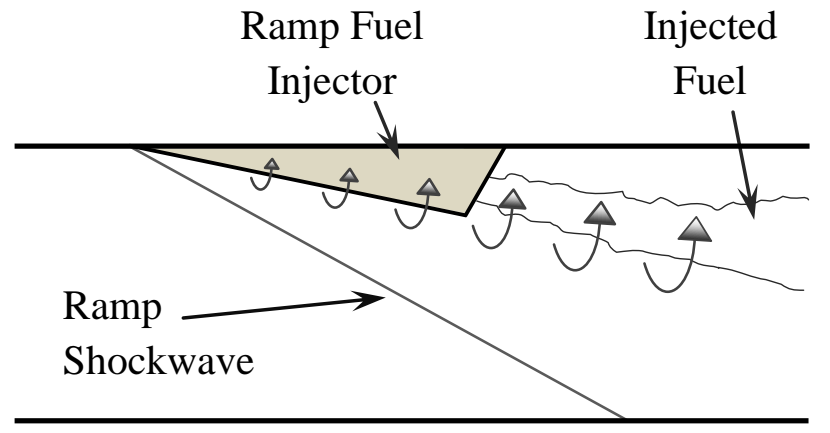

Side View

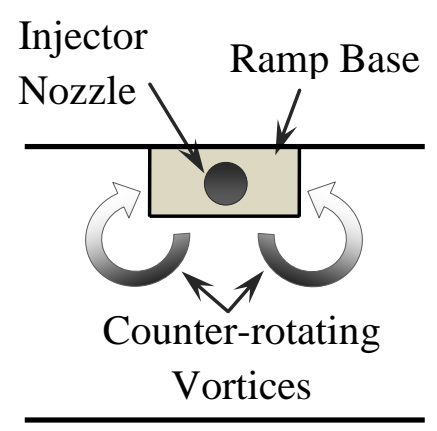

End View

Figure 1.2: Schematic of an unswept ramp fuel injector

Air moves from the high pressure region to the low pressure region causing rotation as the air "spills over" the sides of the ramp. This rotation of flow from the ramp face around to the sides of the ramp creates two counter-rotating, ramp induced vortices. Consequently, the ramp-induced vortices propagate downstream and can be used to enhance fuel-air mixing if fuel is injected, at the base of the ramp, into the region between the two vortices. In addition, the recirculation region at the base of the ramp provides a mechanism for flameholding during combustion. Physical ramps of this type cause the flow to be highly three-dimensional because of the cross-plane velocity components in the vortices created by the ramp. For such an injection scheme, a small wedge-shaped physical ramp is placed in the flow. Ramps are typically designed with an internal fuel passage which is connected to a fuel supply so that fuel can be injected from the ramp base. A supersonic combustor with rectangular cross-section and one wall fitted with an unswept ramp fuel injector is one of the DMSJ combustor geometries currently being investigated at the Aerospace Research Laboratory at the University of Virginia. 


\subsection{Motivation}

Experimental measurements of the turbulent reacting flow inside the combustor section of a Dual-Mode Scramjet and the complex fuel-air mixing process are necessary for gaining a more detailed understanding of the complex flowfield of a DMSJ. It is the fluid flow, fuel-air mixing and ignition in the region downstream of the ramp fuel injector that largely determines the turbulent combustion environment in the scramjet. Efficient combustion of fuel is crucial due to the extremely short fuel residence times within the combustor section of a working scramjet. Visualization of the flow during the fuel-air mixing process without combustion and also during the fuel-air combustion process is useful. Three-component velocity measurements are extremely valuable for understanding the complex flow of a DMSJ. The flowfield of a DMSJ is highly threedimensional, especially in the area downstream of a ramp fuel injector because of the interaction of fuel and air within the ramp-induced vortices as they propagate downstream of the ramp. Ultimately, the rate of fuel-air mixing is the primary factor that determines the time required for combustion (of simple reactants such as hydrogen) and also determines the efficiency of the combustion process. Such mixing information is derived from measurements of three-component velocity inside the combustor during operation and therefore, velocity is a fundamental quantity for understanding the complex motion and mixing of fuel and air. A benchmark data set that includes velocity measurements and key turbulence quantities is needed to facilitate scramjet design and validation of computational fluid dynamics (CFD) models currently being developed at NASA and elsewhere. Such a data set would include spatially resolved instantaneous or time-averaged, three-component (3C) velocity measurements as well as vorticity and 
turbulence intensity values derived from the velocity results. This experimental data would lead to greater understanding of the combustion process in a DMSJ, and such knowledge will ultimately make military and civilian scramjet applications possible.

Unfortunately, no instantaneous, three-component, spatially resolved velocity measurements in scramjets during fuel-air combustion are reported in the literature. This lack of three-dimensional velocity measurements for the combusting flow of a scramjet illustrates the need for new experimental measurements as a means of providing further understanding of the complex flow of a DMSJ and also for CFD model validation, as discussed above. The absence of velocity data in scramjets results because of several characteristics of the flow situation and the limitations of various velocity measurement techniques. First, the flow inside a DMSJ combustor is an extremely challenging environment to experimentally quantify due to high velocity flow and temperatures in excess of 2000K. Second, the flow inside a DMSJ combustor is highly turbulent and highly three-dimensional due to fuel injection schemes and massive flow separation. Additionally, some regions within the combustor contain both subsonic and supersonic flow. Finally, many velocity measurement techniques have at least some impact on, and cause alteration of, the supersonic flow to be measured. Classic probe-based velocity measurement techniques are unacceptable because of their dimensional limitation and intrusion into the flowfield. The flow characteristics and challenging environment of a DMSJ point to classes of optically-based, non-intrusive measurement techniques which are well-suited for velocity measurements of the DMSJ flowfield. 


\subsection{Dissertation Objectives}

The objectives of this research effort center around measuring the velocity field of a DMSJ combustor by means of a non-intrusive optical measurement technique. Therefore, the main goal of this research is to:

Obtain experimental data on the turbulent combustion environment of a dual-mode scramjet and use that data for better understanding of the complex flowfield.

Moreover, the objectives of this work are threefold:

1. Develop an experimental apparatus to apply an optical measurement technique to a DMSJ combustor in order to obtain 3C velocity measurements of the complicated fuel-air mixing and fuel-air combusting flowfields.

2. Use the velocity measurements, and flow characteristics derived from these measurements, to gain a better understanding of the fuel-air mixing and combusting flowfield of a DMSJ.

3. Qualitatively compare time-averaged velocity measurements and other derived flow characteristics to results generated by Computational Fluid Dynamics (CFD) models of a DMSJ that are currently under development. 


\subsection{Choice of Measurement Technique}

Two broad types of optical measurements could be used to measure velocity in a DMSJ combustor. The first is a class based on the measurement of the Doppler shift of light that results from laser-induced fluorescence $(\mathrm{LIF})^{6}$ or from Rayleigh ${ }^{7}$ or $\mathrm{Mie}^{8,9}$ scattered light from molecules or particles, respectively. The second category of optical measurements is based on measuring the displacement of illuminated particles that have been added to fluid flow. Particle Image Velocimetry (PIV) is the most widely used measurement technique of the category based on the measurement of particle displacements. ${ }^{10}$ Since planar measurement techniques are more productive in terms of the number of data points that can be obtained in a given amount of time, only the planar version is addressed here for techniques that have been implemented as both point measurements and in planar form.

Each of the available optical techniques has inherent drawbacks and inherent advantages. The LIF technique is only capable of generating time-averaged velocity measurements and therefore does not give the desired instantaneous data that this study seeks to obtain. ${ }^{11}$ While time-averaged velocity measurements are beneficial, the ability to obtain instantaneous velocity measurements allows for a more complete understanding of the complex flow inside a DMSJ combustor. Such instantaneous velocity information is particularly useful for time resolved simulations of these flows. It is also difficult to identify a fluorescing molecule that is widely present for measurements with and without combustion. LIF measurements that use $\mathrm{OH}$ or $\mathrm{CH}$ molecules cannot obtain velocity measurements for mixing studies without combustion because these molecules are only present during combustion. 
In previous experimental efforts, planar laser-induced fluorescence using the hydroxyl molecule as the fluorescing species (OHPLIF) has been applied to combusting flows in shock tunnels ${ }^{12,13}$ and to a model scramjet combustor ${ }^{14}$. However, in those experiments the OHPLIF technique was used to visualize regions of combustion in the flow, or flow features and flow mixing, and not for velocity measurements. One notable study that used the OHPLIF technique to measure velocity was completed at the University of Virginia with a supersonic combustor fitted with a ramp fuel injector. Gauba $^{15}$ measured two-components of velocity in planes oriented parallel to the injector wall of the supersonic combustion tunnel at the University of Virginia. Two component velocity measurements from those planes are important, in particular because the large axial velocity component was measured, but cross-plane velocities would be more useful for determining the mixing enhancement from the ramp-induced vortices. Additional drawbacks of Gauba's results stem from the limitations mentioned above for the LIF technique. Namely, only time-averaged velocity results could be obtained and velocity could only be measured in areas where the $\mathrm{OH}$ radical was present. The main limitation of employing Doppler shift of Rayleigh or Mie scattered light from molecules or particles to measure velocity is that this technique is hardware intensive; requiring one camera for each velocity component to be measured. ${ }^{16}$ As a result, a test section with three or four large windows would be required for complete optical access and such a supersonic combustor configuration is not practical.

Particle shift techniques have the small disadvantage of slightly impacting the flowfield due to the addition of small tracer particles into the flow. However, the advantages of these particle displacement measurement techniques greatly outweigh this 
drawback. The main advantage of using a particle displacement method to measure velocity is the ability to determine either the instantaneous or time-averaged, spatiallyresolved velocity field. Because particle imaging techniques do not rely on the presence of certain molecules, velocity measurements can be obtained in any area of the flowfield, provided that a sufficient number of particles have been added to the flow. Controlling the application of seed particles to yield a desirable particle concentration can be difficult, but this problem is more desirable than velocity measurements that are subject to an uncontrollable parameter such as the concentration of $\mathrm{OH}$ or $\mathrm{CH}$ molecules. Quantities such as turbulence intensity can also be determined using instantaneous velocity measurements. Unlike measurements based on Doppler shift of scattered light, particle displacement measurements require minimal optical access and camera hardware. Particle Image Velocimetry (PIV) is one of the most popular velocity measurement techniques based on the displacement of tracer particles that have been added to a flow. ${ }^{10}$ For the basic two-dimensional (2D) PIV technique, two components of velocity can be determined from images of particles that have been illuminated by a laser light sheet. For 2DPIV only one camera is necessary, however, if a second camera is used to view the flow from a second perspective, then the resulting Stereoscopic Particle Image Velocimetry (SPIV) technique is capable of determining all three velocity components. Using a particle-based technique such as SPIV, three components of velocity can be determined using only two cameras and one or two windows for optical access. ${ }^{10}$ Because of advantages over other optical measurement techniques, Stereoscopic Particle Image Velocimetry (SPIV) has been identified as the best method for obtaining instantaneous, three-component velocity measurements in a DMSJ combustor. 


\subsection{Dissertation Overview}

This dissertation continues with a brief discussion of the basic theory governing Stereoscopic Particle Image Velocimetry in Chapter 2, along with information about the experimental hardware available for this study, and a description of the Supersonic Combustion Facility at the University of Virginia. Chapter 3 contains a discussion of experimental characteristics necessary for accurate SPIV measurements, along with guidelines for designing a PIV experiment. An experimental design procedure was developed to determine successful SPIV configurations by balancing opposing experimental parameters. The experimental design procedure is presented and Chapter 3 concludes with initial SPIV results for a fuel/air combustion case as evidence of the success of the SPIV configuration determined using the experimental design procedure. SPIV measurements were completed at different locations in two separate scramjet combustor flowpaths. SPIV configurations for each measurement location and discussions of data processing are included in Chapter 4. SPIV results at planes in Flowpath 1 are presented and discussed in Chapter 5. In Chapter 5, SPIV experimental measurements are also qualitatively compared to CFD results produced by other researchers. Chapter 6 contains SPIV measurements, quantities extracted from these measurements, and qualitative CFD comparison for locations in combustor Flowpath 2. SPIV results are analyzed and compared in an effort to gain understanding of the complex flow of a DMSJ combustor. Major results are summarized in Chapter 7 and recommendations for future work are discussed. 


\section{Chapter 2: Background and Experimental Hardware}

In this chapter, the basic theory governing Stereoscopic Particle Image Velocimetry (SPIV) is discussed briefly followed by some examples of PIV and SPIV experiments in the literature that are relevant to application of SPIV to a scramjet combustor. Only a basic description of the SPIV technique is presented here for the sake of brevity and also because details of the technique can be found in the literature. Full descriptions of Particle Image Velocimetry and the stereoscopic version of the technique can be found in a number of excellent books and journal articles on the subject, such as: Raffel, Willert, and Kompenhans ${ }^{10}$; Willert and Gharib ${ }^{17}$; and Prasad ${ }^{18}$; among others. Some details of the SPIV technique are presented in this chapter while others are briefly discussed in Chapter 3 when such details are relevant to choices that had to be made during the experiment design process. After discussion of relevant PIV and SPIV applications, Chapter 2 continues with a presentation of experimental equipment available for SPIV experiments in this study. The chapter concludes with a discussion of the University of Virginia supersonic combustion facility and the two combustor flowpaths of this study.

\subsection{Particle Image Velocimetry (PIV): Basic Theory}

Particle Image Velocimetry (PIV) is based on the simple concept that the velocity of an object can be determined if the change in position of the object and the time required for that displacement are known. ${ }^{10}$ For PIV, particles are added to a particular fluid flow and images of two particle positions are recorded along with the known time between the recordings. The tracer particles are chosen so they accurately follow the flow under investigation and therefore, the velocity field calculated using these particles 
is also the velocity field of the flow. However, the accuracy of flow tracking must be considered, particularly for supersonic flows, to ensure that the particle paths accurately represent the motion of the fluid. PIV is a planar technique where a thin sheet of highintensity laser light is used as the light source for illumination of the tracer particles. For the simple two-dimensional (2D) PIV technique, one camera is positioned to view the portion of the flow illuminated by the laser lightsheet and two particle images are recorded. Using computer software, the recorded image is divided into sub-regions for interrogation. Then statistical correlation is used to determine particle motion and therefore, velocity vectors. For the 2DPIV technique, each sub-region yields only one, two-component velocity vector representing particle motion within the plane of the lightsheet. The sub-region size should be chosen to be small enough so that one velocity vector accurately represents the flow in that location, while at the same time the subregion should be large enough so that it contains enough particles for proper statistical correlation. These competing constraints, among others, have lead to studies to determine appropriate values of experimental parameters in order to maintain a high degree of confidence in the vector results obtained from PIV. One such famous study was completed by Keane and Adrian ${ }^{19}$ in the early 1990's.

\subsubsection{Stereoscopic Particle Image Velocimetry}

An extension of the PIV technique, Stereoscopic Particle Image Velocimetry (SPIV) can be used to obtain three-component velocity fields of the fluid flow under investigation. The same physical principles and statistical correlation methods apply to the SPIV technique just as in the basic (two-component) PIV method; however, a second 
camera is necessary to view the flow from another direction in a SPIV configuration. This second perspective allows determination of the third velocity component. For SPIV, two-component (2C) velocity vectors are first determined using each of the two cameras. These $2 \mathrm{C}$ vectors are then combined using a predetermined image-to-world mapping function to produce three-component (3C) velocity vectors. The SPIV technique introduces additional complications because of the requirement that the flow be viewed from two different perspectives and experimental parameters such as camera angle, camera field of view, and lens depth-of-field all must be taken into account in order to produce well-focused particle images for velocity determination.

Figure 2.1 schematically shows a possible SPIV camera/lightsheet configuration for measuring the velocity field of a dual-mode scramjet combustor test section. Figure 2.1 is discussed here as a way of visually illustrating the theory of the SPIV technique. The top portion of the figure shows two digital cameras positioned to view the flow inside the test section using windows for optical access. As a requirement of the SPIV technique, the cameras are set apart in order to view the flow from two different directions. In this case, the two perspectives are obtained by positioning the cameras at an angle with respect to the lightsheet and this configuration is therefore an "angular-displacement system" for Stereoscopic PIV. ${ }^{18}$ The field of view seen by each camera is divided into small interrogation sub-regions (center of the figure) and correlation algorithms are applied in order to obtain $2 \mathrm{C}$ velocity vectors as viewed by each camera. Furthermore, the images recorded by each camera contain prospective distortion due to the angle of each camera. Once the $2 \mathrm{C}$ velocity vectors have been generated, those in matching 


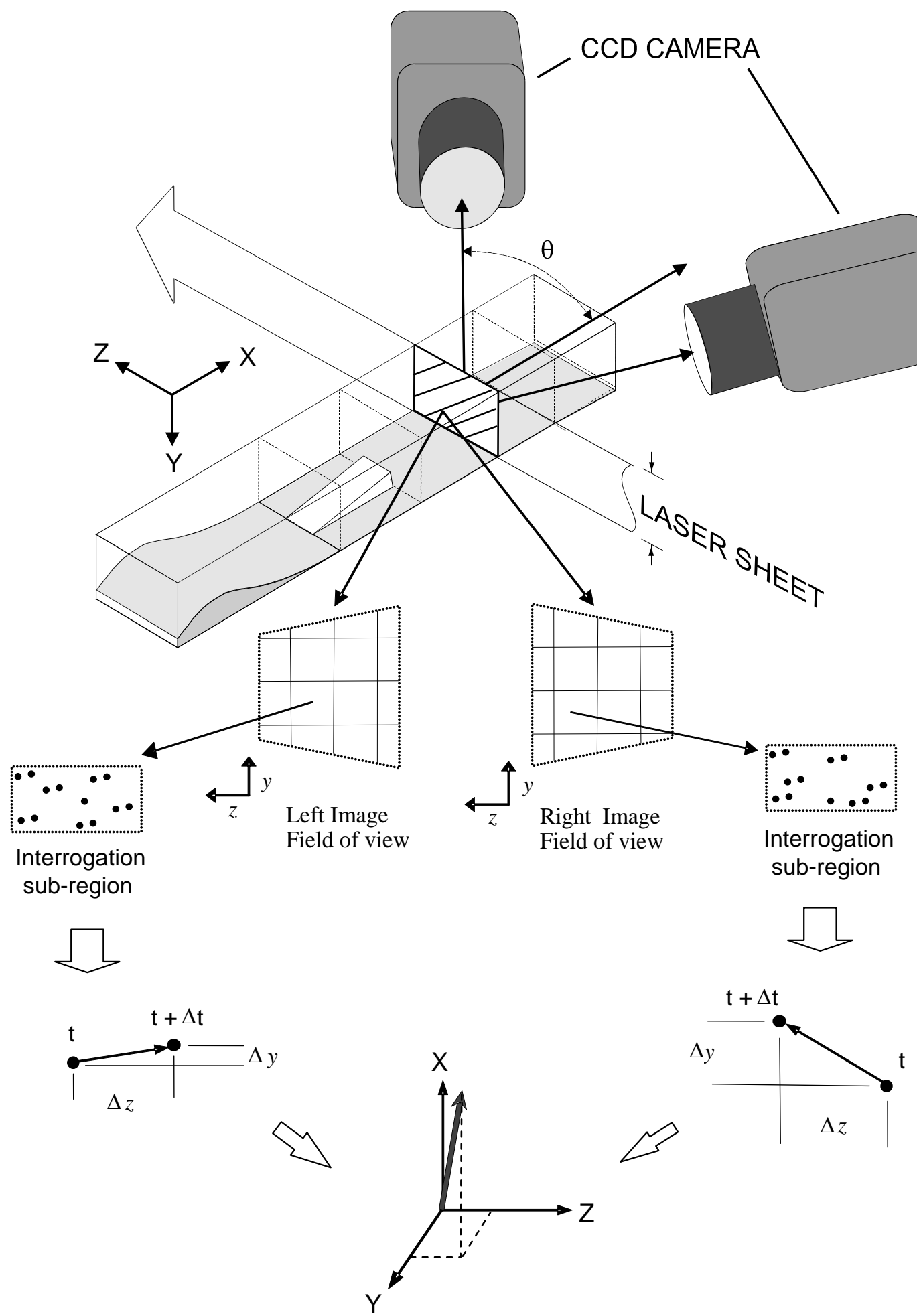

Resulting 3D Vector

Figure 2.1: SPIV configuration and $3 \mathrm{C}$ vector generation method 
physical positions in the flow are combined through a predetermined mapping function into $3 \mathrm{C}$ vectors in real space - shown in the bottom of the figure. The mapping function is determined via a calibration procedure that employs a physical target of markers of known sized and spacing. For calibration, the target is placed into the area where measurements will be obtained and each camera records images of the target which are used to obtain the image-to-world mapping function. Furthermore, the calibration procedure accounts for the perspective distortion induced by setting the cameras at an angle relative to the lightsheet.

\subsubsection{Correlation Methods for PIV}

Statistical correlation of images of particles is at the heart of the PIV measurement technique because it is this correlation that determines the mean particle displacement within a sub-region. The main types of correlation methods available for PIV are briefly discussed here. Two types of correlation methods are available, Autocorrelation and Cross-correlation, ${ }^{10}$ and the choice of correlation method is generally dependent upon the type of flowfield being measured. When using the Autocorrelation method, the positions of the particles as illuminated by both laser pulses are recorded on the same camera image frame. This in general allows for smaller time separations $\left(D_{t}\right)$ between laser pulses because each camera records two exposures on a single image and camera readout time, therefore, does not limit the selection of $D_{t}$. However, as a result of double-exposed recordings, the Autocorrelation method is subject to directional ambiguity because it is unclear which image of the tracer particles was recorded first. ${ }^{10}$ The Autocorrelation method creates three correlation peaks in the correlation domain. A possible correlation 
map arising from the Autocorrelation method is shown in figure 2.2. The three main correlation peaks are: 1$)$ a peak $\left(R_{P}\right)$ showing zero displacement due to particle images perfectly correlating with themselves; 2$)$ one correlation peak $\left(\mathrm{R}_{\mathrm{D}+}\right)$ due to particle images captured at the first laser pulse correlating with images of particles during the second laser pulse - the true displacement peak; and 3) the remaining large peak $\left(\mathrm{R}_{\mathrm{D}-}\right)$ results from the correlation of particle images from the second laser pulse with those of the first - the negative displacement peak. The remaining small peaks $\left(R_{C}+R_{F}\right)$ in the

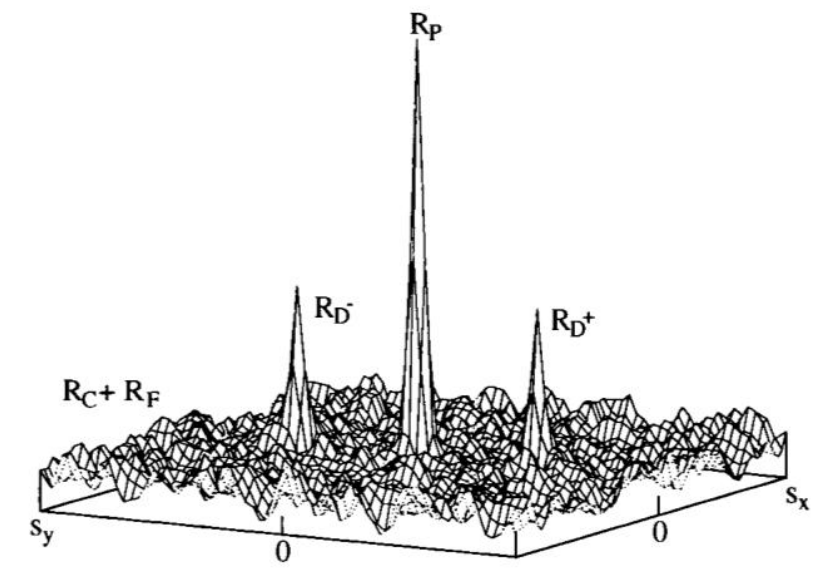

Figure 2.2: Correlation peaks from Auto-correlation method (Reproduced from Ref. 10, p. 71, with kind permission from Springer Science \& Business Media)

correlation domain of Figure 2.2 occur due to random correlations of particle images and constitute background noise. As mentioned above, the Autocorrelation method is subject to directional ambiguity. Therefore, when using Autocorrelation, the main flow direction must be specified so that the correlation peak corresponding to the true displacement is accepted, instead of the negative displacement peak. 
Alternatively, for the Cross-correlation method, the positions of particles are recorded on two different camera frames so it is clear which particle images resulted from the first laser pulse and which images came from the second. As a result, only one main correlation peak $\left(\mathrm{R}_{\mathrm{D}}\right)$ occurs in the correlation domain and no directional ambiguity exists. Figure 2.3 shows a schematic of the correlation domain for a result obtained with cross-correlation. One drawback when using Cross-correlation is that the laser pulse time

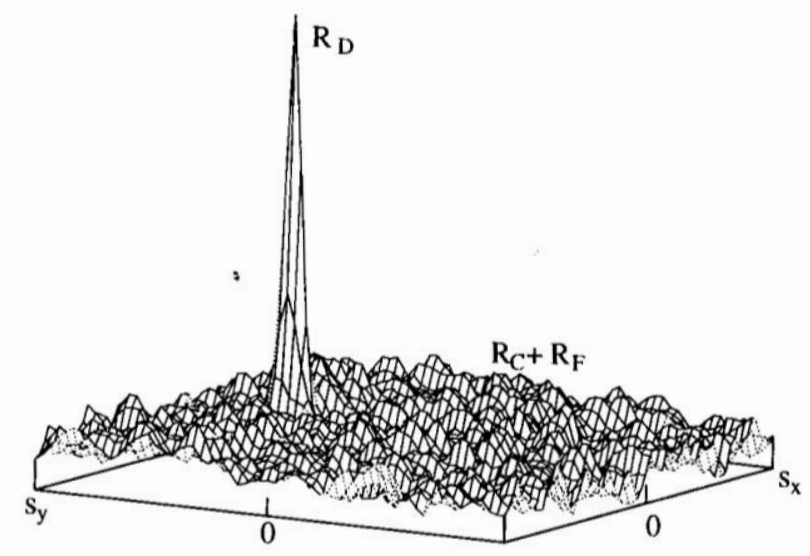

Figure 2.3: Correlation peaks from Cross-correlation method (Reproduced from Ref. 10, p. 69, with kind permission from Springer Science \& Business Media)

separation $\left(D_{t}\right)$ is limited by the time required for cameras to read out the image captured at the first laser pulse to memory locations on the CCD chip before another image can be captured. Furthermore, when Cross-correlation is used, a SPIV measurement consists of a total of four frames; two frames for each camera acquired using illumination from two laser pulses. If a useful particle image is not captured for any one of the four frames, then that SPIV acquisition will not generate $3 \mathrm{C}$ vectors. As mentioned above, some details of the SPIV technique are discussed in Chapter 3 where such information is pertinent to 
experimental design choices. Further details of the SPIV measurement technique can be found in a number of excellent books and papers on the subject.

\subsection{Relevant PIV and SPIV Applications}

The modern digital form of the PIV technique has been in use since the early 1990's and both the two-component and three-component forms of PIV have been applied to many low-speed flow situations. ${ }^{17}$ In order to obtain three-component velocity measurements in a DMSJ combustor, the PIV technique must be capable of velocity measurement in a high-speed flow environment with combustion. Previous studies have successfully applied the SPIV technique in flames, such as the work by Gupta et al. ${ }^{20}$ involving luminous kerosene flames and also the work of Tanahashi et al. ${ }^{21}$ that resulted in $3 \mathrm{C}$ velocity measurements in methane-air turbulent premixing flames. In recent years 2D PIV has been used to measure two velocity components in high-speed flows situations without combustion, such as supersonic flow over a backward facing step, ${ }^{22}$ the flowfield of a compressible wake, ${ }^{23}$ and supersonic flow in a scramjet combustor. ${ }^{24}$ The Stereoscopic PIV technique has been used to measure three velocity components ${ }^{25,26}$ in supersonic jet flows without combustion.

Three notable experimental studies, one by Weisgerber et al., ${ }^{27}$ one by Goyne et al., ${ }^{28}$ and one by Scheel, ${ }^{29}$ have been undertaken to apply PIV to scramjet combustor flows. Weisgerber et al. applied the two-dimensional (2D) PIV technique to a supersonic combustion experiment with the configuration of hydrogen injection into a Mach 2 air flow and compared the PIV results to previous single point Laser Doppler Velocimetry measurements. ${ }^{27}$ Goyne et al. ${ }^{28}$ have completed 2DPIV measurements in the dual-mode 
scramjet facility at the University of Virginia using a commercial PIV system with a light sheet oriented axially in the flow. Those measurements indicated axial velocities ranging from $200 \mathrm{~m} / \mathrm{sec}$ in areas of the flow at locations downstream of the ramp fuel injector, to $1900 \mathrm{~m} / \mathrm{sec}$ for regions near the base of the fuel injector located. Higher velocity was measured where the fuel was injected from the base of the ramp fuel-injector and subsequently mixed into the free-stream. Additionally, Goyne et al. ${ }^{28}$ compared these $2 \mathrm{C}$ streamwise velocity results to CFD results generated by the VULCAN code. Goyne et al. $^{28}$ reported that, in general, velocity magnitudes obtained for areas near the ramp fuel injector agreed well with CFD. However, there were differences between the streamlines calculated from experimental results as compared to the streamlines generated by the VULCAN $\operatorname{code}^{28}$ and it was also determined (using other measurements) that VULCAN results underpredicted the level of turbulent mixing and heat release of combustion.

More recently, Scheel ${ }^{29}$ applied the 2D PIV technique to a scramjet combustor outfitted with two different nozzle geometries and used a laser sheet oriented axially in the flow, similar to the PIV configuration used by Goyne et al. ${ }^{28}$ However, Scheel had to rely on auto ignition of the flow for combusting cases, which did not allow testing of one injector geometry due to delayed ignition that caused combustion near the combustion chamber exit. Furthermore, unlike the work by Goyne et al. ${ }^{28}$, Scheel ${ }^{29}$ completed measurements in a supersonic tunnel that used pre-combustion to heat the freestream air. Therefore all measurements were obtained for a flow containing impurities such as carbon dioxide, water vapor, and other combustion byproducts that resulted from heating tunnel air using pre-combustion. The DMSJ combustor facility at the University of Virginia is electrically heated and provides a freestream air supply that is not 
contaminated by impurities consisting of pre-combustion byproducts. Therefore the UVA facility should better simulate the combustor flowfield of a scramjet vehicle in flight than a tunnel that uses vitiated air.

No instantaneous, three-component, spatially resolved velocity measurements in scramjets during fuel combustion are reported in the literature. Because the governing principles for particle image correlation apply to 2D PIV as well as to SPIV, the work completed by Goyne et al. constitutes an important step toward the application of SPIV to a DMSJ combustor. Moreover, previous Stereoscopic PIV studies of flames and highspeed flows, such as those mentioned above, have shown the capability of the technique in those challenging situations. These studies point to the ability of SPIV to obtain threecomponent velocity measurements in scramjet combustors where both high-speed turbulent flow and fuel-air combustion are present simultaneously.

\subsection{Equipment}

Over the course of this study, two commercial SPIV systems were employed for three-component velocity measurements. The first velocity measurements (measurement plane at $\mathrm{X} / \mathrm{H}=10$ ) were obtained using a commercial PIV system developed by TSI Incorporated. SPIV recordings at the remaining measurements planes were obtained using a commercial SPIV system from LaVision Inc. A dual-cavity Nd:YAG laser was shared by both systems and other equipment such as camera lenses, mirrors, bandpass filters, and laser optical elements were necessary for SPIV measurements in the DMSJ combustor flowpaths of this study. Equipment is briefly described here. 
Laser

Both SPIV systems shared a Spectra Physics (Newport) Quanta-Ray PIV 400-10 dual-cavity Nd:YAG laser capable of delivering laser pulses of wavelength $\lambda=532 \mathrm{~nm}$ and 8 nsec pulse widths at time separations $\left(D_{t}\right)$ between laser pulses on the order of 100 nsec. For the experiments of this study, the shortest time separation between laser pulses used was $D_{t}=350$ nsec, but this Nd:YAG laser is capable of smaller $D_{t}$ values. Documentation of the laser shows that the beam profile of each cavity is approximately "Top-hat" in shape and the beam spots have diameters of approximately $10 \mathrm{~mm}$ at the laser exit, with a slight oval cross-sectional shape along the vertical beam axis. Laser power levels were checked throughout the course of the experiments using a thermopiletype laser calorimeter. Service of the Nd:YAG laser was most recently completed in January 2009 by a laser technician from the Newport Corporation. After service of the laser was complete, power measurements using an Astral $^{\mathrm{TM}}$ series $\mathrm{AC} 2501$ volume absorbing (thermopile-type) laser calorimeter showed that the Nd:YAG laser was capable of a maximum available energy of approximately $445 \mathrm{~mJ} /$ pulse for one cavity and 380 $\mathrm{mJ} /$ pulse for the second cavity. Typically, the best laser beam quality is produced when the laser is operated near the maximum power condition; however, such high laser energies are not necessary for PIV measurements and can be potentially harmful for CCD cameras. Therefore, methods for reducing the laser energy were employed for each SPIV experiment and are discussed below along with the experimental apparatus for each measurement plane. 
Commercial SPIV System by TSI Inc.

The SPIV commercial system by TSI Inc. consists of the Spectra Physics PIV 40010 Nd:YAG laser (shared by both SPIV systems), a pair of TSI PIV 10-30 (Model 630046) 8-Bit digital CCD cameras, a laser-camera synchronizer, and a computer with control software. Each digital camera of the TSI system has a CCD sensor of total size 9 $\mathrm{mm} \times 9.14 \mathrm{~mm}$, containing an array of 1000 x 1016 pixels, of size $9 \mu \mathrm{m} \times 9 \mu \mathrm{m}$ each. The cameras have a moveable back portion that is capable of image-plane tilt angle, $\alpha$, from zero degrees (CCD parallel to lens plane) to near 11 degrees of CCD tilt relative to the camera optical axis. The synchronizer is a TSI model 610032 Laserpulse Synchronizer and is capable of $200 \mathrm{nsec}$ time increments.

As mentioned, this TSI system also included Insight 3 software for data acquisition and analysis of the SPIV recordings for vector generation. However, through the course of the experiments, it was found that the Insight 3 analysis software from TSI Inc. was not capable of proper vector generation from the SPIV data obtained at the first measurement plane in combustor Flowpath 1. For that reason, new DaVis 7.1 software by LaVision Inc. was purchased for analysis of the particle images and the tools of this software package allowed generation of $3 \mathrm{C}$ velocity vectors from the particle images recorded using the TSI system.

LaVision Inc. SPIV system

In November 2009, a commercial SPIV system from LaVision Inc. was purchased and this system consists of the Spectra Physics PIV 400-10 dual-cavity Nd:YAG laser, two Imager Pro X, 14 bit, CCD cameras, Scheimpflug camera mounts, a Programmable 
Timing Unit (PTU), and a personal computer with LaVision's DaVis 7.2 software package for data acquisition and analysis. The CCD sensor of the Imager Pro X cameras contains an array of $1200 \times 1600$ pixels of size $7.4 \times 7.4 \mu \mathrm{m}$, meaning the CCD has physical dimensions of $8.88 \mathrm{~mm}$ x $11.84 \mathrm{~mm}$. These Imager Pro X cameras have lower background noise and better dynamic range (14 bit vs. 8 bit) than the TSI PIV 10-30 cameras. Each of the Scheimpflug mounts consists of an outer frame, which connects to the camera body, and a plate inside the outer frame which is mounted to the frame on a

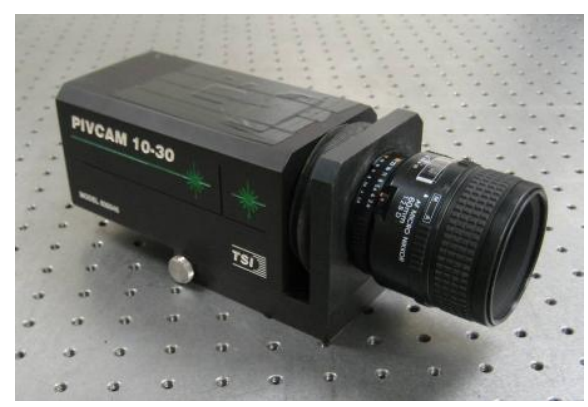

(a) TSI PIVCAM 10-30, 8 bit Camera with $60 \mathrm{~mm}$ Nikkor Lens

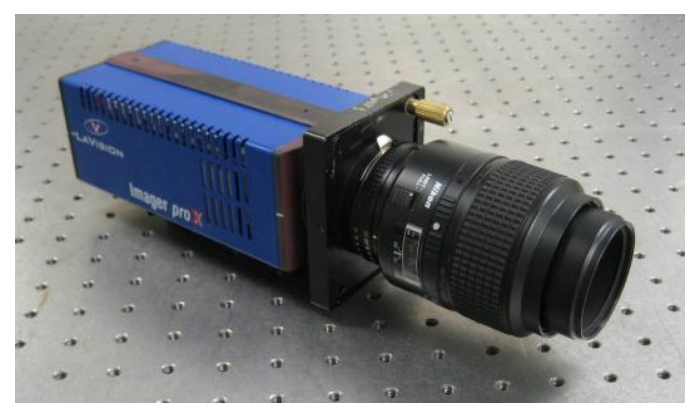

(b) LaVision Imager ProX, 14 bit Camera with Scheimpflug mount and 105mm Nikkor Lens

Figure 2.4: PIV cameras from: (a) SPIV system by TSI Inc. and (b) SPIV system by LaVision Inc.

pivot. Camera lenses attach to the movable inner plate and the pivoting plate allows for tilting of the camera CCD plane to an angle, $\alpha$, similar to the cameras of the TSI system. These LaVision Scheimpflug mounts have been modified to allow for maximum image plane tilt angles near 13 degrees. Figure 2.0 shows pictures of the cameras from the TSI and LaVision systems, respectively. The PTU of the LaVision system is capable of 10 nsec time increments and the DaVis 7.2 software has a vast array of tools for image preprocessing and vector generation and validation. Because of the characteristics of the equipment, the SPIV system from LaVision Inc. is a substantial upgrade over the TSI system that was used to obtain the first set of SPIV measurements in the DMSJ combustor. 


\section{Camera Lenses, filters, and mirrors}

Two sets of Nikon camera lenses were used with the PIV cameras throughout this study. Both PIV cameras could be fitted with either Nikon AF Micro-Nikkor $60 \mathrm{~mm}$ cameras lenses or Nikon AF Micro-Nikkor $105 \mathrm{~mm}$ lenses. Both types of lenses are designed for close-up and general-use photography with continuous focusing from infinity to $1 / 1$ (lifesize) reproduction ratio. These lenses have F-Bayonet type mounts and have minimum and maximum apertures of $\mathrm{f} / 32$ and $\mathrm{f} / 2.8$, respectively. Both types of lenses provide the same reproduction ratios and therefore the same image Field-of-view, but the distance from the object to the CCD is larger for the AF Micro-Nikkor $105 \mathrm{~mm}$ lenses than for the AF Micro-Nikkor $60 \mathrm{~mm}$ lenses, for the same reproduction ratio. For example, focused images are achieved at a distance (L) of $219 \mathrm{~mm}$ from the object to the camera $\mathrm{CCD}$ for the AF Micro-Nikkor $60 \mathrm{~mm}$ lenses at a reproduction ratio of $\mathrm{R}=1 / 1$, while the AF Micro-Nikkor $105 \mathrm{~mm}$ lenses are focused for the same reproduction ratio $(\mathrm{R}=1 / 1)$ at $\mathrm{L}=314 \mathrm{~mm}$. These two types of camera lenses were necessary for SPIV experiments because, for some measurements, it was desirable to have the cameras physically closer to the measurement plane, while for other experiments it was necessary to position the cameras further away from the measurement plane.

Narrow band pass filters were also attached to the camera lenses to allow only light from the Nd:YAG laser to reach the cameras in order to limit background noise in the PIV images. The filters eliminated ambient light from the flame during combustion and light from glowing tunnel wall surfaces. Edmund Optics $532 \mathrm{~nm}$ narrow band pass filters 
(stock number NT43-174) were chosen for this task and the transmittance plot (percent transmittance vs. wavelength) for these filters is shown in figure 2.5. The filters have a

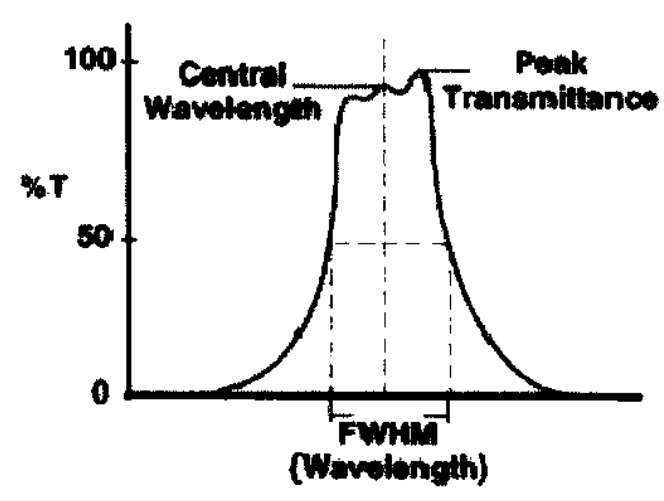

Figure 2.5: Narrow band pass filter transmittance (Source: Edmund Optics Catalog)

central wavelength of $\lambda=532 \mathrm{~nm} \pm 2 \mathrm{~nm}$ and the plot shows nearly $93 \%$ transmittance of $\lambda=532 \mathrm{~nm}$ light through the filters. The full width-half maximum (FWHM) of the transmittance curve is $10 \mathrm{~nm}$ and therefore, these filters effectively blocked ambient light (outside $\lambda=532 \pm 5 \mathrm{~nm}$ ) from reaching the cameras. Unfortunately, this model of filter is no longer available from Edmund Optics, so the set of two filters available for experiments had to be traded between mounts that fit the AF Micro-Nikkor $105 \mathrm{~mm}$ lenses and other mounts for the AF Micro-Nikkor $60 \mathrm{~mm}$ lenses.

Because of space limitations imposed by the geometry of the supersonic tunnel, the PIV cameras could not always be positioned to view the flow directly. In such situations, mirrors were placed near the tunnel to allow the cameras to view the flow. High accuracy first-surface mirrors from Edmund Optics (No. U32-201) were employed to turn the optical paths of the cameras. The circular mirrors each have a diameter of 2 inches and were made of an enhanced Aluminum coating on a Zerodur substrate. Details 
regarding the positions of cameras and mirrors will be discussed with SPIV experimental configurations presented for each measurement plane in Chapters 4 and 5 .

\section{Camera positioning system}

The two cameras for SPIV measurements were mounted on rotating bases that were part of the TSI system and these bases were in turn attached to one of several mounting rails that allowed for positioning of the cameras by sliding along the rail. The mounting rail was held in a vertical position by a Velmex, Inc. BiSlide Model MN10-0100-M02-31 motorized traverse with 10 inches of travel. This heavy-duty traverse is rated for a load of $300 \mathrm{lb}$ normal to the carriage, or $500 \mathrm{inch}-\mathrm{lb}$ for a cantilever load. The Velmex motorized traverse uses a stepper motor for position control with increments of 0.005 $\mathrm{mm} / \mathrm{step}$. Furthermore, the traverse can be operated locally with a joystick, or remotely with a RS232 cable that connects the traverse to computer software.

This traverse was not only useful for fine camera positioning during camera set-up, but also for calibration of the SPIV apparatus. Calibration of a Stereoscopic PIV experiment involves acquisition of images of a calibration grid of known size at several vertical positions through the thickness of the laser sheet. ${ }^{10}$ Calibration can be completed in one of two ways. The calibration grid (target) can be translated while the cameras remain stationary, or the target can be held fixed while the cameras are translated by known distances. The positioning precision of the Velmex translation stage was very useful for calibration because the stage could be used to precisely translate the cameras during the calibration process while the calibration target remained stationary. 


\section{Calibration targets}

Three separate calibration targets where used with SPIV experiments throughout this study. One target was custom-made with dimensions of $1.25 \times 0.95$ inches so that the target would fit inside the combustor test section duct. This target will be referred to as "Calibration Target 1" in subsequent chapters and has 21 columns and 13 rows of 0.5 mm-diameter dots at $1.5 \mathrm{~mm}$ grid spacing. The grid covered $76 \%$ of the area of the target while two holes were drilled into the remaining area so that the target could be attached to rods and held in place inside the combustor test section. All three calibration targets are shown in Figure 2.6.

(a)

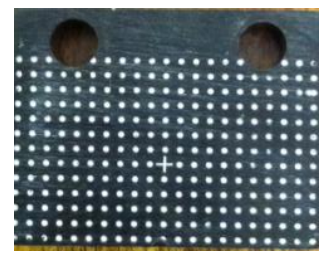

(c)

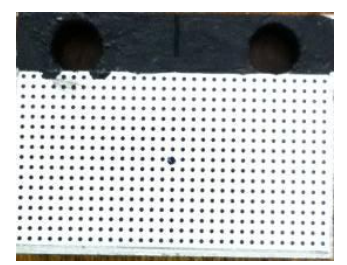

(b)

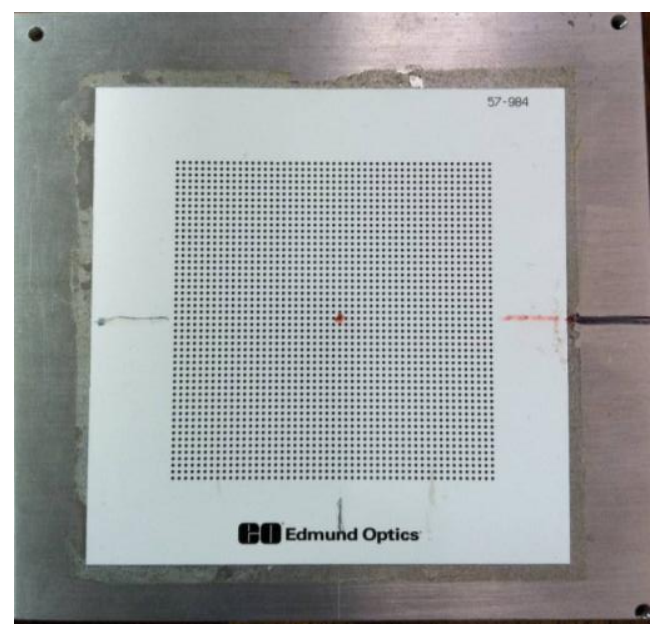

Figure 2.6: Calibration targets for use with SPIV experiments (a) Target 1, (b) Target 2, and (c) Target 3

Calibration Target 2 was constructed by attaching a commercial film grid (Edmund Optics Part number 57-984) to an aluminum plate. This grid is 2 inches $x 2$ inches and consists of $0.5-\mathrm{mm}$-diameter dots at $1 \mathrm{~mm}$ spacing. The commercial film grid was certified for accuracy by Max Levy Autograph, Inc. Calibration Target 2 is too large to 
fit inside the combustor test section and instead was used for calibration of SPIV experiments at the exit plane of the combustor.

The third target (Calibration Target 3) was made to fit within the combustor test section and had the same outer dimensions as Calibration Target 1 . However the dot grid of this target was not custom machined, but instead was constructed in a similar manner to Calibration Target 2. A duplicate of the grid used for Calibration Target 2 (Edmund Optics part number 57-984) was purchased and cut to size so that it could be attached to the 1.25 X 0.95 inch metal plate. This grid was also certified for accuracy by Max Levy Autograph, Inc. The grid of Calibration Target 3 only covered $70 \%$ of the area of the target plate with the remaining area reserved for attachment of rods to hold the target in position inside the combustor test section. The grid consisted of 31 columns and 17 rows of 0.5 -mm-diameter dots at $1 \mathrm{~mm}$ grid spacing.

\section{Laser-beam-aperture and sheet-forming optics}

A commercial beam dump (Edmund Optics number NT55-528) was modified to create and aperture capable of reducing the energy of the Nd:YAG laser pulses. The exterior of the commercial beam dump consists of a hollow anodized aluminum cylinder with a $10 \mathrm{~mm}$ entrance hole and this cylinder creates a chamber for trapping laser beams that pass through the entrance hole. An anodized aluminum cone is located inside the chamber such that laser light entering the beam dump impacts the cone and is reflected to the interior walls of the chamber where laser energy is dissipated. This beam dump was modified by drilling a $6 \mathrm{~mm}$ diameter hole through the cone so that the center portion of the laser beam would pass though the beam dump while the remainder of the laser beam 
impacted the cone and was trapped inside the chamber. In this way, the laser energy could be reduced using this "laser-beam-aperture." Figure 2.7 shows a schematic of the interior of this laser-beam-aperture. The laser-beam-aperture also reduced the laser beam spot diameter from approximately $10 \mathrm{~mm}$ to $6 \mathrm{~mm}$.

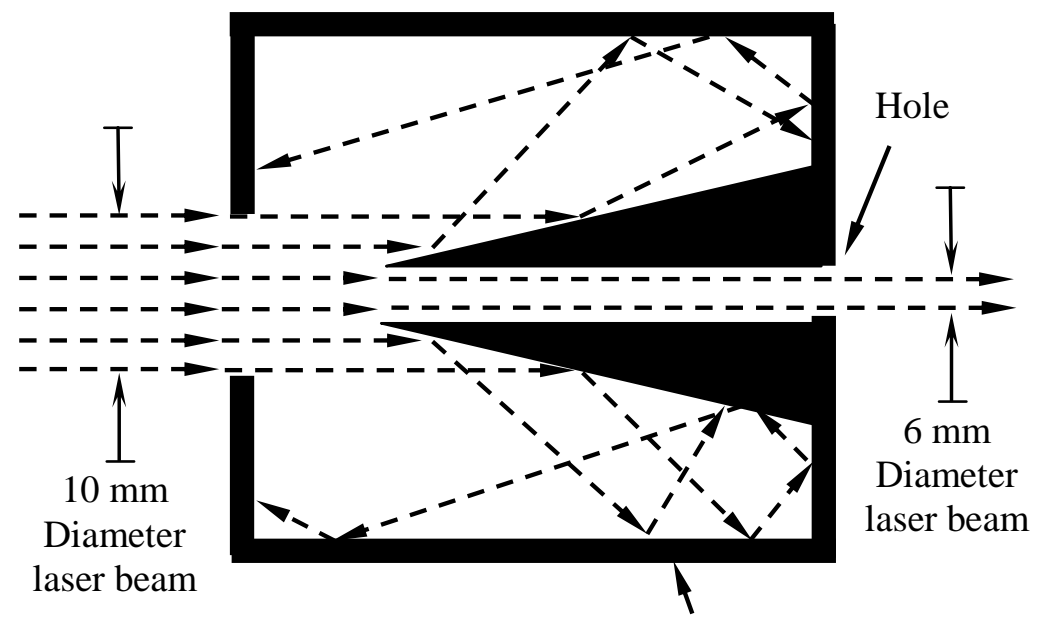

Beam dump section

Figure 2.7: Laser-beam-aperture, Interior view.

For all experiments, the beam produced by the Nd:YAG laser was directed to the supersonic tunnel using commercial mirrors designed for use with Nd:YAG lasers. For each experiment, an appropriate laser sheet for SPIV was produced from the circular laser beam using a set of 3 or 4 cylindrical or spherical lenses placed in sequence to form an "optical train." The combination of sheet-forming optics in the optical train was different for each experiment and each laser optical train is discussed with SPIV experimental configurations presented in Chapters 4 and 5 . 


\subsection{Supersonic Combustion Facility and Tunnel Flowpaths}

The University of Virginia Supersonic Combustion Facility is an electricallyheated, clean-air supersonic wind tunnel that is capable of simulating flight Mach numbers near 5 and has continuous flow capability which allows unlimited duration scramjet combustor testing. The Mach 2 tunnel simulates the combustor conditions that occur for a hypersonic vehicle flying at Mach 5, by heating the free-stream air to match the stagnation temperature. Furthermore, a flight Mach number of 5 falls within the range for which mode-transition is expected to occur for actual flight applications of a dual-mode scramjet. The facility is mounted vertically and is schematically presented in Figure 2.8. Compressed air is supplied by an oil-free compressor and desiccant air-dryer system. This compressed air passes vertically down an annular section between the tunnel's heater tank and heater core. At the bottom of the heater tank, the air turns and passes vertically up through a $300 \mathrm{~kW}, 14$-stage electrical resistance heater where it is heated to a temperature approaching $1200 \mathrm{~K}$. An important feature of the facility comes from the electric heaters that raise the free-stream air temperature without adding contaminates. Unlike supersonic tunnels that heat air through pre-combustion, the UVA combustion facility creates a freestream that is not vitiated with water, carbon dioxide or other contaminants. References 30 and 31 provide further details on the supersonic combustion facility.

Two separate scramjet combustor flowpath geometries were tested and these different geometries are referred to here as "Flowpath 1" and "Flowpath 2." Both flowpaths of the scramjet combustor at the University of Virginia are direct-connect type and are shown in Figures 2.9 and 2.10. Flowpath 1 has been available for experiments since September 


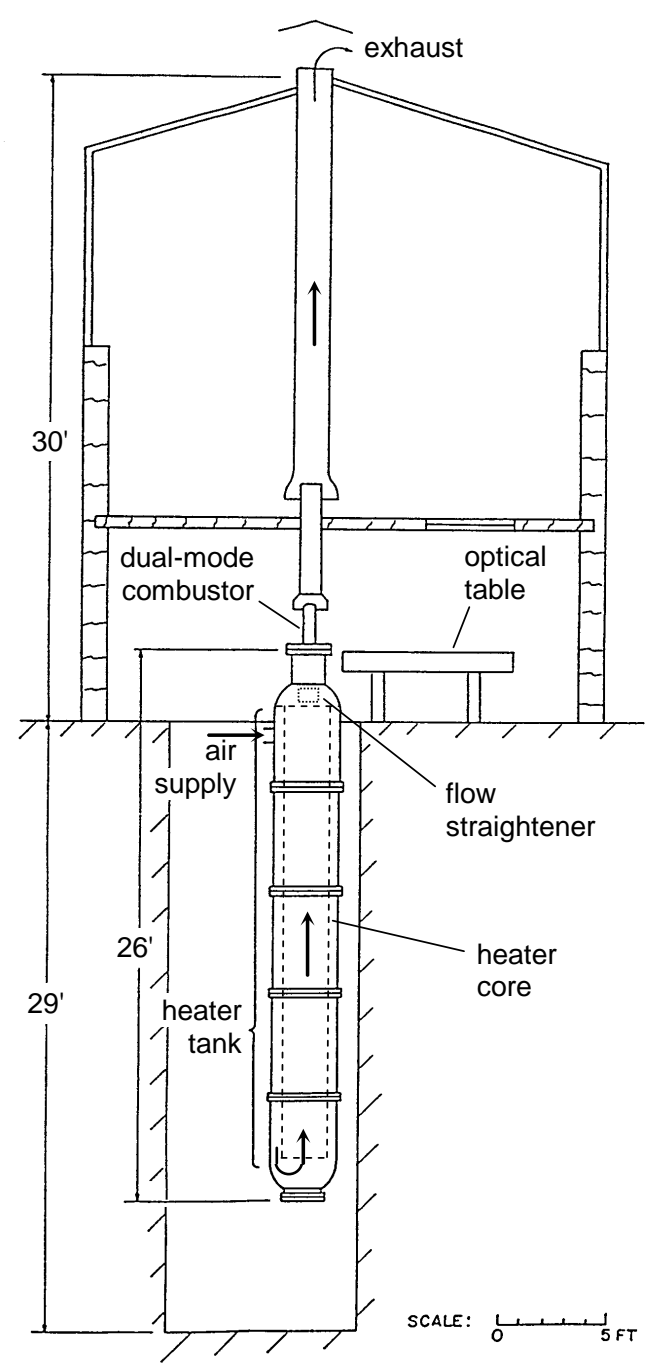

Figure 2.8: Schematic of University of Virginia Supersonic Combustion Facility

2003 and is designed to include a ramp fuel injector. In 2011, installation of flowpath 2 was completed and this flowpath was designed as an improvement over Flowpath 1 in order to allow for a determination of a comprehensive data set. Flowpath 2 has greater optical access than Flowpath 1 due to larger windows, can be fitted with either a ramp fuel injector or a cavity fuel injector, and hydrocarbon fuel can be used with the cavity fuel injector configuration of Flowpath 2. A more detailed discussion of the commonalities and difference between the two flowpaths is presented below. In general, air that has been heated by the facility heater first passes through the facility mach 2 
nozzle and then enters a rectangular duct that contains either two or three separate sections, depending on which flowpath is being tested. Flowpath 1 contains a constant area isolator section just downstream of the Mach 2 nozzle and this section serves to locate the oblique shock train created by the backpressure from fuel-air combustion. An isolator section was not present for Flowpath 2. Both Flowpath 1 and Flowpath 2 have a test section where fuel is injected and combustion takes place. Furthermore, the test sections for both flowpaths contain an unswept ramp fuel injector on one wall and this wall also begins to diverge (at 2.9 degrees) within the test section, but the divergence begins at a different axial location for the two flowpaths.

For both flowpaths, the wall containing the ramp fuel injector and the 2.9 degree divergence is referred to here as the "injector wall" and the wall opposite to the injector wall is referred to as the "observation wall" because this wall can be outfitted with a widow for visual observation of the ramp fuel injector and hydrogen flame. The two walls adjacent to the injector wall are referred to as the tunnel "sidewalls." Furthermore, the divergence of the injector wall, which begins in the test section, continues in the extender section just downstream of the test section. Taken together, the test section and the extender make up the scramjet combustor. The combustion facility does not, however, contain a nozzle for thrust production downstream of the combustor and therefore, each of the tunnel flowpaths must be defined as a direct-connect Dual-mode Scramjet combustor and not as a "full" Dual-mode Scramjet. The DMSJ combustor flowpaths are sufficient for simulating the desired fuel-air mixing and fuel-air combusting flowfields present in the combustion chamber of a scramjet in flight. Details 
of the two flowpaths are discussed below. Typical operating conditions for Flowpath 1 are listed in Table 2.1.

Table 2.1: Typical Test Conditions for University of Virginia Supersonic Combustion Facility, Flowpath 1

\begin{tabular}{|c|c|c|c|}
\hline Parameter & \multicolumn{2}{|c|}{ DMSJ combustor } & Error \\
\hline Gas & Air & $\mathrm{H}_{2}$ & \\
\hline Stag. press. (kPa) & $330-390$ & $220-1600$ & $\pm 3 \%$ \\
\hline Stag. temp. (K) & $900-1200$ & 300 & $\pm 3 \%$ \\
\hline Mach number ${ }^{a}$ & 2.07 & 1.7 & \\
\hline Static press. ${ }^{\mathrm{a}}(\mathrm{kPa})$ & $39-47$ & $50-315$ & \\
\hline Static temp. ${ }^{\mathrm{a}}(\mathrm{K})$ & $480-640$ & 190 & \\
\hline Equivalence ratio & & $0-0.5$ & $\pm 5 \%$ \\
\hline
\end{tabular}

\section{Flowpath 1}

Flowpath 1 has three main sections downstream of the facility nozzle. For

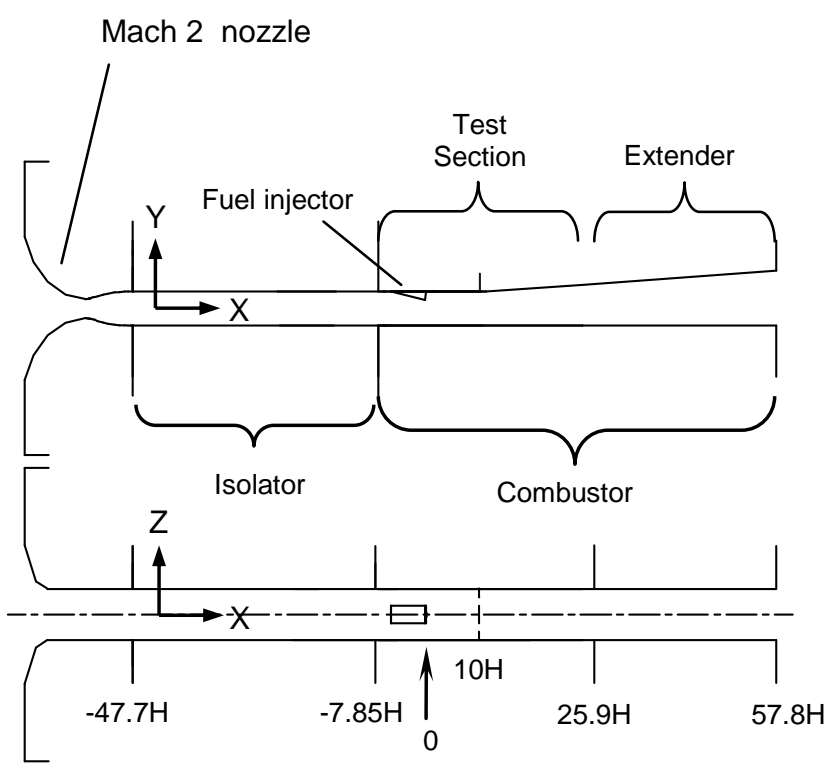

a)

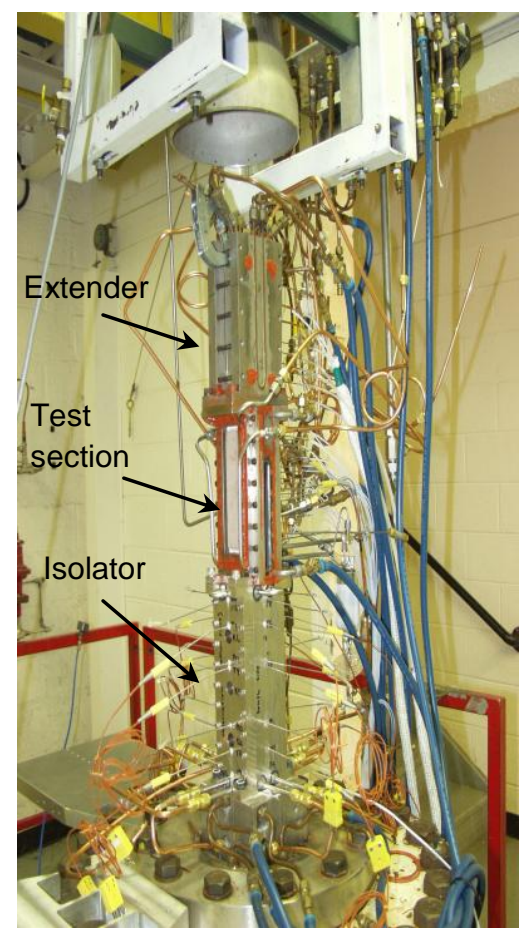

b)

Figure 2.9: University of Virginia Scramjet Combustor, Flowpath 1;

a) Schematic, and b) Photograph of hardware 
experiments using Flowpath 1, air exits the facility's two-dimensional Mach 2 nozzle and passes through a constant area rectangular isolator section. The test section is connected to the downstream end of the isolator, and the final component of the DMSJ combustor is the extender section, located downstream of the test section, where the flow returns to atmospheric pressure. In the test section, the injector wall of the scramjet combustor includes an unswept $10^{\circ}$ compression ramp that is $36.85 \mathrm{~mm}$ long by $6.35 \mathrm{~mm}$ high at the base. Hydrogen fuel is introduced from the base of the ramp by a Mach 1.7 conical nozzle. Flowpath 1 is schematically and photographically depicted in Figure 2.9.

Using the normal height of the ramp $(\mathrm{H}=6.35 \mathrm{~mm}, 0.25$ inches $)$ to normalize linear dimensions, the isolator and combustion duct inlet dimensions are $4 \mathrm{H} \times 6 \mathrm{H}$ and the total length of the isolator is approximately $40 \mathrm{H}$. Distances along the axial direction of the flowpath are also normalized by the height of the ramp fuel injector and the origin for axial dimensions is located at the base of the ramp fuel injector. The isolator section connects directly to the exit of the Mach 2 nozzle at $-47.7 \mathrm{H}$ and ends at $-7.85 \mathrm{H}$, where it connects to the test section. The leading edge of the ramp fuel injector is located at $-5.8 \mathrm{H}$ with the ramp base at zero, and the test section connects to the extender at $25.9 \mathrm{H}$. The test section and the extender have lengths of $33.75 \mathrm{H}$ and $31.9 \mathrm{H}$, respectively. The injector wall starts to diverge by 2.9 degrees at $10 \mathrm{H}$ downstream of the base of the ramp fuel injector and flow exits the combustor to atmosphere at $57.8 \mathrm{H}$. The walls of the isolator, test section and extender are instrumented with low frequency pressure taps, high frequency pressure gauges and thermocouples. The isolator section does not contain windows, but optical access is available on three walls of the test section. In the test section of the combustor, the observation wall (opposite to the injection wall) is fitted 
with an observation window that is $6 \mathrm{H}$ wide and extends from $-2 \mathrm{H}$ to $+22 \mathrm{H}$, while each of the sidewalls adjacent to the injector wall contain side-windows that are $2.7 \mathrm{H}$ wide by $20 \mathrm{H}$ long. The upstream and downstream edges of the side windows are located at $-5.5 \mathrm{H}$ and $+14.5 \mathrm{H}$, respectively. Prior to the start of the divergence of the injector wall, the dimensions of the flowpath cross-section are $4 \mathrm{H}$ from the injector wall to the observation wall, and $6 \mathrm{H}$ from sidewall. Because the injector wall divergence continues from the test section through the extender to the exit of the combustor, the dimensions at the combustor exit are $6.45 \mathrm{H}$ x $6 \mathrm{H}$. Typical hydrogen fuelling conditions are listed in Table 1. The fuel is ignited using a hydrogen-oxygen detonation driven ignition system and combustion is self sustaining following ignition.

\section{Flowpath 2}

Collaboration is currently underway between researchers conducting measurements of the DMSJ combustor at the University of Virginia and others interested in numerically simulating the DMSJ combustor flowfield. Researchers working to generate Computational Fluid Dynamics (CFD) simulations of the DMSJ combustor have requested data for a configuration without an isolator section because they believe such a flow geometry can be simulated more easily than if the isolator were present. Therefore, Flowpath 2 was assembled without an isolator for the experiments presented herein, but the option of including an isolator section for future work is possible. Flowpath 2 was constructed using newly fabricated hardware and no existing hardware from Flowpath 1 was reused for Flowpath 2. A schematic of Flowpath 2 is presented in Figure 2.10. Similar to Flowpath 1, the test section of Flowpath 2 contains a ramp fuel injector on one wall and this injector wall also diverges by 2.9 degrees. However, the ramp fuel injector 


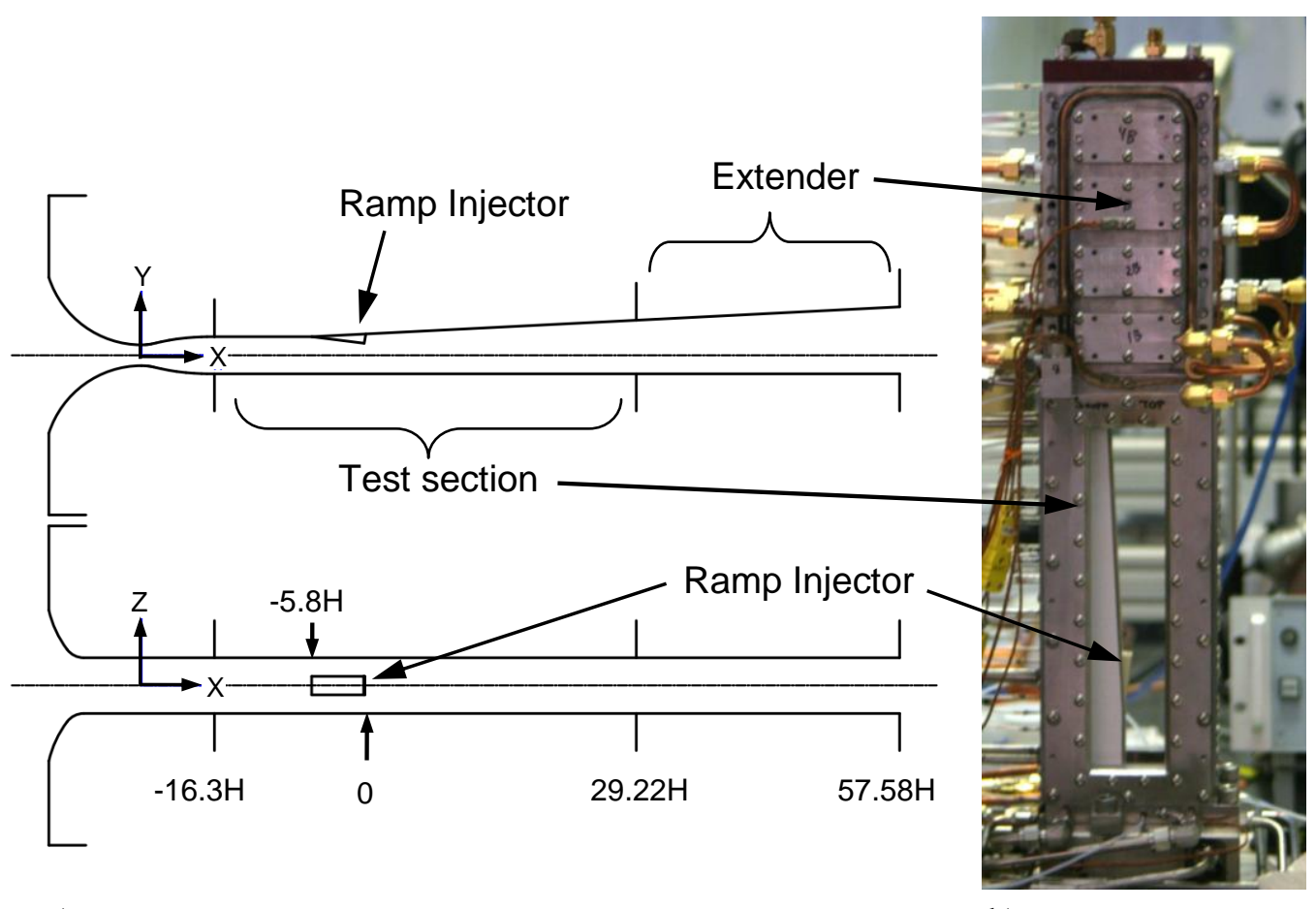

a)

b)

Figure 2.10: University of Virginia Scramjet Combustor, Flowpath 2

a) Schematic, and b) Photograph of hardware

and the starting point of wall divergence in Flowpath 2 are not at the same axial locations as in Flowpath 1. Again, the height of the ramp fuel injector ( $\mathrm{H}=0.25$ inches, $6.35 \mathrm{~mm})$ is used to normalize linear dimensions and the base of the ramp fuel injector is used as the origin for the axial direction. The test section of Flowpath 2 is approximately $45.5 \mathrm{H}$ long and connects directly to the facility mach 2 nozzle at $-16.35 \mathrm{H}$. This test section contains a ramp fuel injector that is of the same dimensions $(5.8 \mathrm{H}$ long by $1 \mathrm{H}$ high at the base) as the injector of Flowpath 1 and hydrogen fuel is also injected from the base of the ramp by means of a Mach 1.7 nozzle. The extender section connects to the test section at $+29.21 \mathrm{H}$ and ends at $57.58 \mathrm{H}$. For Flowpath 2, the divergence of the injector wall begins at the leading edge of the ramp fuel injector and, therefore, the ramp injector is located entirely on the diverging portion of the injector wall. The leading edge of the ramp and start of divergence are located at $-5.8 \mathrm{H}$. 
Optical access is available through the test section sidewalls via two large windows that are $7.6 \mathrm{H}$ wide by $34.55 \mathrm{H}$ long. The upstream end of each side-window is located at $10.85 \mathrm{H}$, while each window's downstream end is located at $23.7 \mathrm{H}$. The side-windows of Flowpath 2 are much larger (in both dimensions) than those of Flowpath 1 and this fact allows for much greater optical access for SPIV measurements. It should also be noted that these side-windows are larger than the cross-section of the flowpath between the injector and observation walls, over the entire length of the windows. The side-windows were oversized, with respect to the ramp fuel injector configuration, so that the windows could also be used for future experiments with a cavity fuel injector.

The cross-section of the duct upstream of the point of divergence is $4 \mathrm{H}$, from injector wall to observation wall, by $6 \mathrm{H}$ between the side-walls. As in Flowpath 1 , the injector wall divergence of Flowpath 2 continues from the test section through the extender to the exit of the combustor and therefore, the cross-section of the combustor exit is $7.21 \mathrm{H} \times 6 \mathrm{H}$. Also similar to Flowpath 1, the test section and extender of Flowpath 2 are both instrumented with pressure taps and thermocouples for wall pressure and wall temperature measurements. Fuel is ignited by the same hydrogen-oxygen detonation driven ignition system used for Flowpath 1. 


\section{Chapter 3: SPIV Experiment Design}

A general experimental design procedure was created in order to determine appropriate SPIV experimental configurations for each of the measurement planes of this study. Creation of such a design procedure was necessary because a straightforward method for determining an appropriate SPIV experimental configuration is not present in the literature. Guidance is given in the literature regarding individual parameters of a SPIV configuration. For example, information can be found about the use of an appropriate laser sheet thickness, or choosing a camera angle to keep the error of out-ofplane and in-plane vectors comparable (such as in references 19 and 32) but no unified method seems to be present in the literature for determining tradeoffs when two parameters cannot both be optimized at the same time. Therefore, an original iterative design procedure was created to aid in determining tradeoffs required to create a SPIV experimental configuration for velocity measurements of the DMSJ combustor flowfield.

Each parameter of a SPIV experimental configuration affects all others so determining a proper SPIV configuration by choosing one parameter at a time, without checking the effect on other parameters, proved to be far too complicated and ultimately futile. Therefore, it was determined that a systematic method for analyzing the effect of parameter choices on all others was necessary so that SPIV experimental configurations could be found. In this chapter, requirements for a successful SPIV configuration are discussed first, along with guidelines for determining values of experimental parameters necessary for meeting these SPIV requirements. Next, initial choices about the seed particles, the type of camera configuration, and correlation method are discussed. The design method is then described and the procedure, as applied for determination of the 
SPIV configuration for the $\mathrm{X} / \mathrm{H}=10$ measurement plane, is presented as an example. The measurement plane at $10 \mathrm{H}$ downstream (x-direction) of the ramp fuel injector is used as the example because this was the location of the first SPIV measurements in the DMSJ combustor at the University of Virginia.

\subsection{SPIV Requirements and Design Guidelines}

Based on the work of others (Ref. 10 and Ref. 17) and that of the author, the following six characteristics need to be present for useful PIV measurements:

1. Seed particles added to the flow that accurately track the flowfield,

2. Camera Field of View (FOV) that is large enough to encompass the area of interest within the flowfield,

3. Good focus across the Field of View (FOV) of each camera,

4. Appropriate time-separation between laser pulses to display the particles at two separate locations,

5. Adequate illumination of the seed particles for photographic recording with good signal-to-noise, and

6. Proper seed particle density across the area of interest for legitimate statistical correlation. 
To aid in meeting the above PIV characteristics, Keane and Adrian ${ }^{19}$ determined a set of general PIV experimental guidelines. Because PIV images are divided into smaller sub-regions and vector generation is based upon statistical correlation of particles in these regions, it is critically important that an adequate number of particles be present within any given sub-region. The design guidelines put forth by Keane and Adrian address particle density, laser sheet thickness, sub-region size, tolerance to velocity gradients within a sub-region, and detection of the correct particle correlation.

a) At least 15 (or more) particle pairs should be present inside an interrogation subregion,

b) Particle motion through the laser sheet should not exceed $25 \%$ of the laser sheet thickness,

c) Mean particle motion within the interrogation sub-region should not exceed $25 \%$ of the sub-region size,

d) Within an interrogation sub-region, the variation of particle displacements due to velocity gradients should be less than $5 \%$ of the sub-region size so that one velocity vector will accurately describe the flow in that region,

e) The detection threshold $\left(\mathrm{D}_{0}\right)$ for detecting a valid correlation peak in the correlation domain should be set between $1.2<\mathrm{D}_{0}<1.5$, where $\mathrm{D}_{0}$ is the ratio between the 
correlation peak and the next-largest noise peak. The choice of $\mathrm{D}_{0}$ defines an acceptable signal-to-noise ratio.

The numeric limits in the above design guidelines were chosen by Keane and Adrian $^{19}$ so that the probability of detecting a valid velocity vector was $90 \%$ or greater. As discussed below, these requirements and guidelines result in many design tradeoffs that must be made in any PIV application. However, the application of SPIV to a DMSJ is particularly challenging due to experimental constraints such as a small tunnel crosssection, limited optical access, and high-speed flow, among others. These experimental constraints, along with limitations of the cameras and lenses, serve to limit the possible range of values for each parameter of the SPIV experimental configuration. This resulted in the necessity for an iterative process for designing the experimental configuration.

\subsection{Initial Choices}

Previous experimental studies using Planar Laser-Induced Iodine Fluorescence (PLIIF) techniques ${ }^{33}$ were completed for an unheated supersonic tunnel with a physical tunnel configuration similar to the DMSJ combustor of the current work. Results from that previous study were used as a priori information to aid in implementing the SPIV technique. Based on PLIIF measurements, the DMSJ combustor flow is expected to contain two stream-wise counter-rotating vortices generated by the ramp fuel injector and the magnitude of the axial velocity component is expected to be approximately 10 times that of the cross-plane components. ${ }^{33,34}$ Computational analysis of a similarly configured DMSJ combustor has been completed previously ${ }^{15}$ and the results indicate that the axial and cross-plane velocity components in the test section of the combustor of the current 
study are expected to be approximately $1000 \mathrm{~m} / \mathrm{sec}$ and $100 \mathrm{~m} / \mathrm{sec}$, respectively, for the case of fuel-air mixing.

Choices regarding correlation method, seed particles, and type of SPIV camera configuration had to be made at the beginning of the SPIV experimental design process because these parameters inform all other design choices. As discussed above, the Autocorrelation method involves directional ambiguity and a mean flow direction must be specified when using Autocorrelation, while the cross-correlation method is not subject to this problem. The DMSJ combustor flow of interest is expected to contain direction changes so cross-correlation was chosen as the analysis method for PIV measurements in this study. The choices of seed particles and the type of camera configuration are discussed below.

\subsubsection{Seed Particles}

General PIV requirement 1 (discussed above) was addressed by choosing seed particles of an appropriate material to withstand the DMSJ combustor flow conditions and an appropriate diameter to track the flow. Prior to the current SPIV experimental study, alumina particles were tested to determine if they were appropriate for use as tracer particles in a DMSJ combustor. That work indicated that 0.3 micron diameter

alumina $\left(\mathrm{Al}_{2} \mathrm{O}_{3}\right)$ particles track velocity gradients in the flow of this DMSJ combustor, ${ }^{35}$ except in the area directly behind shockwaves because the momentum of particles keeps their velocity higher, over the course of a few millimeters, than the lower velocity in the area behind a shock. Moreover, due to a high melting point, the $\mathrm{Al}_{2} \mathrm{O}_{3}$ particles tolerate the high temperatures in the flowfield during the combustion process. For these reasons, 
0.3 micron $\mathrm{Al}_{2} \mathrm{O}_{3}$ particles were chosen for the first SPIV experiments, which were completed at the $\mathrm{X} / \mathrm{H}=10$ measurement plane of Flowpath 1. However, the $\mathrm{Al}_{2} \mathrm{O}_{3}$ particles have been shown to form agglomerates and therefore a method for breaking apart those agglomerates must be employed when using alumina as tracer particles.

During the course of the SPIV experimental study of the DMSJ combustor, silicon dioxide $\left(\mathrm{SiO}_{2}\right)$ was identified as another possible candidate for tracer particles. Based on previous work, ${ }^{35,36} \mathrm{SiO}_{2}$ spherical particles with diameter of $0.25 \mu \mathrm{m}$ accurately track the flow of the DMSJ combustor. Silicon dioxide also has a high melting point $(\sim 1900 \mathrm{~K})$ and very high boiling point $(\sim 2500 \mathrm{~K})$ which is higher than temperatures throughout the combustor. Previous work shows temperatures in the flame of the combustor at between $2000 \mathrm{~K}$ and $2100 \mathrm{~K}$ so $\mathrm{SiO}_{2}$ particles begin to melt in that region, but the particles remain intact in the harsh environment of the DMJS combustor. In addition, testing of the $\mathrm{SiO}_{2}$ spherical particles ${ }^{35}$ showed that the $\mathrm{SiO}_{2}$ particles do not tend to agglomerate, and a method for breaking agglomerates is therefore not necessary. Because of these characteristics, $\mathrm{SiO}_{2}$ particles were chosen as the tracer particles for all SPIV experiments that were undertaken after completion of experiments at the first measurement plane of Flowpath 1.

Two separate particle seeders are available to deliver tracer particles to the DMSJ combustor flowfield. One seeder adds particles to the hydrogen fuel stream and is referred to as the "fuel seeder." The purpose of the other seeder is to deliver particles to the airflow of the DMSJ combustor and that seeder is called the "free-stream seeder." Both seeders are fluidized-bed type and either hydrogen fuel or compressed air levitates particles to create a fluidized bed in a large tube of the seeder. Both seeders have a small 
tube connected to the fluidized bed tube and this "pickup tube" pulls particles from the fluidized bed. Seeded flow from the fuel seeder is routed to the ramp fuel injector, while seeded flow from the free-stream seeder is injected into the DMSJ combustor flowfield at a location that is approximately $0.5 \mathrm{~m}$ upstream of the facility nozzle. Furthermore, the fuel seeder is fitted with a shearing nozzle to aid in breaking particle agglomerates, while the free-stream seeder does not have a shearing nozzle. The shearing nozzle consists of two separate fuel streams, one clean and one containing particles, which are normal to each other. As particles exit the particle chamber of the seeder, they encounter the clean fuel stream flowing perpendicular to the particle laden stream. Particles experience a tangential force due to the clean fuel stream and this effectively shears agglomerates apart into individual particles. With this shearing nozzle, either $\mathrm{Al}_{2} \mathrm{O}_{3}$ particles or $\mathrm{SiO}_{2}$ particles can be used with the fuel seeder, but only $\mathrm{SiO}_{2}$ particles are possible for the free-stream seeder because no shearing nozzle is present in that seeder to break apart particle agglomerates. Further details about both seeders can be found in reference 35 .

\subsubsection{Type of Camera Configuration}

The type of SPIV camera configuration must also be chosen at the onset of a SPIV experiment. For Stereoscopic PIV, two types of camera configurations can be employed - the lens translation method or the angular lens displacement method. ${ }^{10}$ The translation method involves keeping the lenses of each camera parallel to the object plane (the laser sheet) while the image plane (CCD chip) of each camera is translated in order to capture light emanating from a common area of the laser sheet. This method has the disadvantage of being subject to large aberrations due to off-axis viewing. In most 
situations, it is critical to avoid such aberrations in order to obtain high-quality particle images that SPIV requires. ${ }^{37}$ The angular lens displacement method does not require the camera lenses to be placed parallel to the object plane and instead, cameras are set at an angle to view a common area in the laser sheet. A major advantage of this angular arrangement is fine resolution of smaller particles because light rays emanating from across the field of view can pass through the lens near its principle axis. The angular lens configuration was the natural choice because small particle must be used in this study. However, a focusing problem arises when employing the angular method where only a small portion of the field of view is in good focus due to a limited depth-of-field at moderate lens apertures. ${ }^{37}$ This focusing problem can be addressed by enforcing the Scheimpflug condition, discussed below.

\subsubsection{The Scheimpflug Condition}

When the image plane of the camera is parallel to the lens plane, the band of good focus (depth-of-field) is parallel to the lens plane and only intersects part of the object plane. Therefore, only a small portion of the field-of-view (FOV) is in good focus due to angular viewing with limited depth-of-field. ${ }^{37}$ The entire FOV can be brought into focus by tilting the camera's image plane (CCD plane) until the image plane, lens plane and object plane all intersect in a common line. ${ }^{10,18}$ This is known as the Scheimpflug condition and is illustrated in Figure 3.1. 
The geometry shown in Figure 3.1 indicates that larger distances (L) from the Object plane to the Image plane, along the camera optical axis, require smaller image plane tilt angles, $\alpha$, to meet the Scheimpflug condition. Furthermore, for fixed focal length camera lenses, a larger value of $\mathrm{L}$ results in a larger camera field-of-view and therefore, larger camera FOV's requires smaller values of angle $\alpha$ to meet the

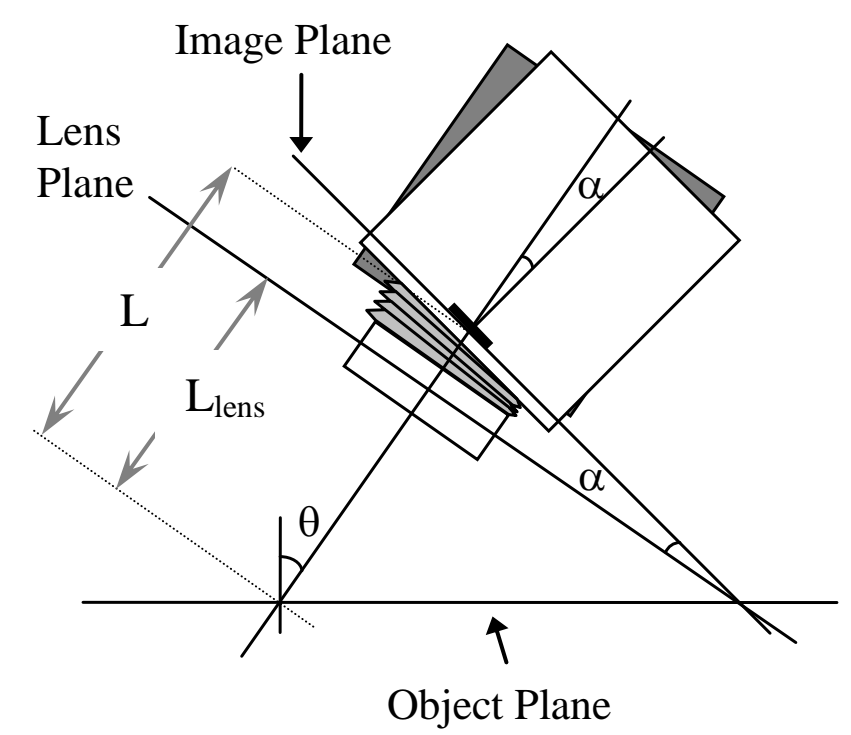

Figure 3.1: Schematic of the Scheimpflug condition

Scheimpflug condition. This trend for required magnitude of Image plane tilt angle also occurs for smaller values of camera angle, $\theta$, which is measured from perpendicular to the object plane. In practice, the maximum magnitude of Image plane tilt angle, $\alpha$, is limited due to camera hardware and this limitation must be considered when choosing a camera FOV and camera angle, $\theta$, so that the Scheimpflug condition can be met, resulting in adequate focus across the entire camera field-of-view. 


\subsection{Design Procedure}

The design procedure for determining an SPIV configuration consists of a set of decisions followed by a check to determine if resulting parameters can both be implemented within physical constraints and are adequate for experimental goals. At any point during the procedure, if the result of previous decisions cannot be tolerated, then a trade-off is accepted for one or more design choices and a new iteration begins. Interactions of some experimental parameters were plotted in order to aid in making tradeoffs. The general design procedure is presented in Table 3.1 and is followed by a discussion of the steps for determining the SPIV configuration, using TSI Cameras and equipment, for the measurement plane at $\mathrm{X} / \mathrm{H}=10$ of Flowpath 1. The plots presented are specifically for the TSI cameras because that equipment was used for the first successful SPIV experiments. The design procedure was also employed for determining SPIV configurations, using the LaVision equipment, for the remaining measurement planes. However, determination of those configurations is not discussed in detail for the sake of brevity and only the final SPIV configurations, which resulted from the design procedure, are presented in the following chapters along with the experimental results for each measurement plane.

When designing a PIV experiment, the following basic parameters can be altered by the experimenter.

- L, Distance (along lens principle axis) from object plane to image plane

- R, Lens Reproduction ratio (Area of CCD sensor / FOV) 
- $\theta$, Camera angle measured from the normal of the object plane

- $\alpha$, Image plane tilt angle

- $\mathrm{f}^{\#}$, Lens f-number ( $\mathrm{f} / \#$ ) - indicates lens aperture diameter

- $\mathrm{T}$, Laser sheet thickness

- $\mathrm{D}_{\mathrm{t}}$, Time separation between laser pulses

- P, Laser power - energy per laser pulse

- $\mathrm{L}_{\mathrm{I}}$, Interrogation sub-region size $\left(\mathrm{L}_{\mathrm{I}} \times \mathrm{L}_{\mathrm{I}}\right.$ for square sub-regions $)$

- $\mathrm{M}_{\mathrm{p}}$, Seed particle material

- $\mathrm{d}_{\mathrm{p}}$, Seed particle diameter

- $\mathrm{S}_{\text {Inj }}$, Seed particle injection method

Furthermore, experimental parameters that often cannot be altered by the experimenter include: the physical geometry $\left(\mathrm{T}_{\text {geom }}\right)$ of the wind tunnel, geometry $\left(\mathrm{W}_{\text {geom }}\right)$ of tunnel windows, physical size $\left(\mathrm{L}_{\mathrm{pix}}\right)$ of the camera's $\mathrm{CCD}$ pixels, and the axial $\left(\mathrm{V}_{\mathrm{A}}\right)$ and cross-plane $\left(\mathrm{V}_{\mathrm{C}}\right)$ velocity components of the flow under investigation. Table 3.1 shows the steps of the iterative design process along with PIV requirements, experimental parameters, and design guidelines related to each step (all discussed above).

Summarizing the sequence, Step 1 is included in the design process because it may be necessary to trade-off using smaller particles that accurately track high frequency 
Table 3.1: Design proceedure for determination of a SPIV experimental configuration

\begin{tabular}{|c|c|c|c|c|}
\hline Step & $\begin{array}{l}\text { Design Step Task } \\
\text { (Decision, Calculation, or Check) }\end{array}$ & $\begin{array}{l}\text { General PIV } \\
\text { Requirement(s) } \\
\text { Addressed }\end{array}$ & $\begin{array}{l}\text { Experiment } \\
\text { Parameters } \\
\text { Involved }\end{array}$ & $\begin{array}{l}\text { Design } \\
\text { Guidelines } \\
\text { Used }\end{array}$ \\
\hline 1 & $\begin{array}{l}\text { Choose seed particles. } \\
\text { Choose particle injection method. }\end{array}$ & 1 & $\mathrm{M}_{\mathrm{p}}, \mathrm{d}_{\mathrm{p}}, \mathrm{S}_{\mathrm{Inj}}$ & \\
\hline 2 & $\begin{array}{l}\text { Identify desired measurement location. } \\
\text { Determine laser sheet orientation. } \\
\text { Choose desired field of view (FOV) and } \\
\text { determine R and L for that FOV. }\end{array}$ & 2 & $\begin{array}{l}\mathrm{L}, \mathrm{R}, \mathrm{V}_{\mathrm{A}}, \mathrm{V}_{\mathrm{C}} \\
\mathrm{T}_{\text {geom }}, \mathrm{W}_{\text {geom }}\end{array}$ & \\
\hline 3 & $\begin{array}{l}\text { Choose camera angle } \theta \text { based on expected } \\
\text { velocity components and experiment goals. }\end{array}$ & $\begin{array}{l}\text { Stereoscopic PIV } \\
\text { Requirement }\end{array}$ & $\begin{array}{l}\mathrm{V}_{\mathrm{A}}, \mathrm{V}_{\mathrm{C}}, \theta \\
\quad \mathrm{W}_{\text {geom }}\end{array}$ & \\
\hline 4 & $\begin{array}{l}\text { Check physical constraints on camera position } \\
\text { to determine if camera location is possible. } \\
\text { Check optical access for chosen FOV. }\end{array}$ & & $\begin{array}{c}\mathrm{L}, \mathrm{R}, \theta \\
\mathrm{T}_{\text {geom }}, \mathrm{W}_{\text {geom }}\end{array}$ & \\
\hline 5 & $\begin{array}{l}\text { Determine image plane tilt angle needed to } \\
\text { meet Scheimpflug condition. (See Figure. 2) } \\
\text { Check if this angle is physically possible. }\end{array}$ & 3 & $\mathrm{R}, \theta, \alpha$ & \\
\hline 6 & $\begin{array}{l}\text { Choose laser separation time, } \mathrm{D}_{\mathrm{t}} \text { based on } \\
\text { approximate expected velocity. } \\
\text { Determine sheet dimensions based on sheet } \\
\text { orientation and design guideline (b). }\end{array}$ & 4 & $\begin{array}{l}\mathrm{D}_{\mathrm{t}}, \mathrm{V}_{\mathrm{A}}, \mathrm{V}_{\mathrm{C}} \\
\quad \mathrm{d}_{\mathrm{p}}, \mathrm{T}\end{array}$ & $\mathrm{b}$ \\
\hline 7 & $\begin{array}{l}\text { Determine: expected particle displacement, } \\
\text { sub-region size based on design guideline (c), } \\
\text { and spatial resolution. (see Figure. } 3 \text { ) }\end{array}$ & 4,6 & $\begin{array}{c}\mathrm{V}_{\mathrm{A}}, \mathrm{V}_{\mathrm{C}}, \mathrm{D}_{\mathrm{t}}, \mathrm{L} \\
\mathrm{R}, \theta, \mathrm{L}_{\mathrm{pix}} \\
\Delta \mathrm{x}_{\mathrm{p}}, \mathrm{L}_{\mathrm{I}}\end{array}$ & $\mathrm{c}, \mathrm{d}$, (a) \\
\hline 8 & $\begin{array}{l}\text { Compare particle displacement to minimum } \\
\text { required displacement ( } \sim 2 \text { pixels). } \\
\text { Check if spatial resolution is adequate. }\end{array}$ & & $\begin{array}{l}\Delta \mathrm{x}_{\mathrm{p}}, \mathrm{L}_{\mathrm{I}} \\
\text { CCD size } \\
\text { (in pixels) }\end{array}$ & \\
\hline 9 & Choose lens aperture and laser power. & 5 & $f^{\#}, P, M_{p}, d_{p}$ & e \\
\hline 10 & $\begin{array}{l}\text { Perform a test experiment and use recorded } \\
\text { PIV images to check: Particle density across } \\
\text { image, signal-to-noise ratio, and particle } \\
\text { displacement variation with-in sub-regions. }\end{array}$ & 6 & $\begin{array}{l}\mathrm{S}_{\text {Inj }}, \mathrm{L}_{\mathrm{I}}, \mathrm{P}, \mathrm{f}^{\#} \\
\mathrm{R}, \mathrm{V}_{\mathrm{A}}, \mathrm{V}_{\mathrm{C}} \\
\mathrm{L}_{\mathrm{pix}}, \mathrm{D}_{\mathrm{t}}\end{array}$ & $\mathrm{a}, \mathrm{d}, \mathrm{e}$ \\
\hline
\end{tabular}

changes in the flow, for larger particles with greater light scattering properties, thus improving signal-to-noise. The laser sheet is typically oriented parallel to the largest expected velocity component to minimize loss of particles through the sheet. However, it 
is sometimes desirable to choose a different sheet orientation so that an area of interest can be captured in each of the instantaneous velocity measurements. The lens reproduction ratio $\mathrm{R}$ is directly related to the Field-of-view (FOV) so $\mathrm{R}$ is also set when the FOV is chosen in step 2. The lens reproduction ratio, $\mathrm{R}$, is defined as the Area of the negative to the Area of coverage ( $\mathrm{R}=$ Area of $\mathrm{CCD} /$ Field-of-view) ${ }^{38}$ and $\mathrm{R}$ is often reported as normalized by the area of the CCD. For example, if a CCD chip is approximately $9 \mathrm{~mm} \times 9 \mathrm{~mm}$, then a reproduction ratio of $\mathrm{R}=1 / 1.6$ results in (undistorted) FOV of $14.4 \mathrm{~mm} \times 14.4 \mathrm{~mm}$. Moreover, choosing the FOV for a (fixed focal length) photographic lens also sets $\mathrm{L}$, and therefore, the location of the camera from the object plane. Corresponding values of $\mathrm{L}$ and $\mathrm{R}$ are found in documentation provided with camera lenses.

The camera angle $\theta$, measured from the normal to the laser sheet, is chosen in step 3 and is typically (but not necessarily) the same for both cameras of an angular SPIV configuration. The movement of particles across the image is due to the geometric projection of the velocity components on to the image plane via the camera angle $\theta$. After making the decisions of design steps $1-3$, the resulting camera position and angle are compared to physical constraints during step 4 of the design procedure. If the camera position cannot be implemented, then another iteration of the design procedure begins after a trade-off is made. If no trade-off is required, then the design process proceeds to step 5 where the image plane tilt angle $\alpha$ is determined in order to meet the Scheimpflug condition. Figure 3.2 depicts the geometric relationship between the camera angle $\theta$ and the image-plane-tilt angle $\alpha$ needed to meet the Scheimpflug condition (Figure 3.1) for several values of $\mathrm{R}$. It is convenient to plot lines of constant reproduction ratio because $\mathrm{R}$ 
indicates both the FOV and the location of the lens plane between the object and image planes. Similar triangles intersecting at the lens plane and having bases of length of the

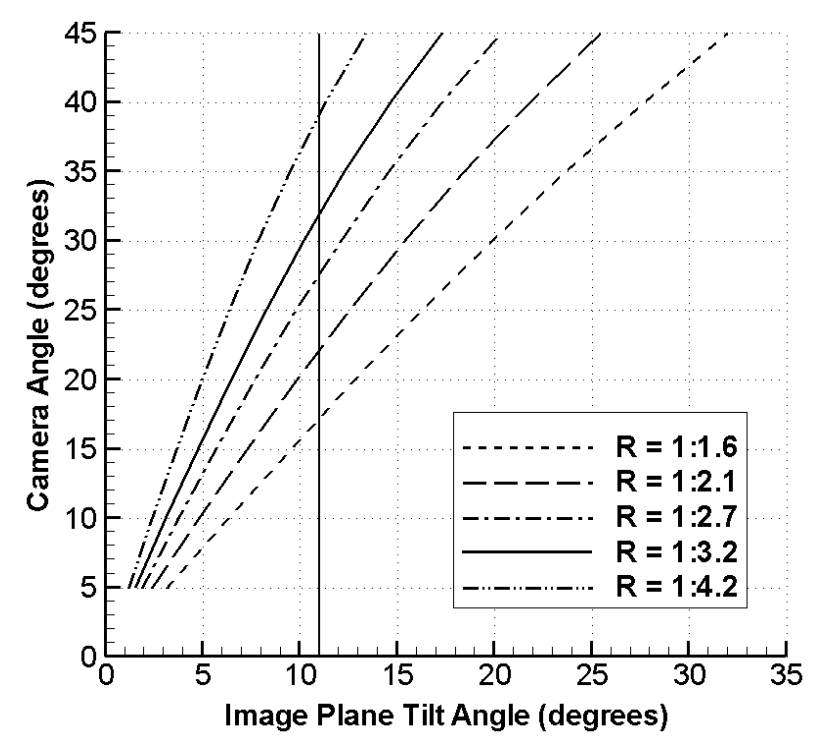

Figure 3.2: Parameter values for the Scheimpflug condition

$\mathrm{CCD}$ and FOV indicate that $\mathrm{R}=\left(\mathrm{L}-\mathrm{L}_{\text {lens }}\right) / \mathrm{L}_{\text {lens }}$ as shown in Figure 3.1 and this allows for determination of the location of the effective lens plane. The general trend of Figure 3.2 shows that both smaller values of the denominator of $\mathrm{R}$ (that is, a smaller FOV) and larger values of $\theta$ require larger values of $\alpha$ to meet the Scheimpflug condition. However, in practice the available image-plane-tilt-angle is limited. The solid vertical line in Figure 3.2 indicates the maximum value of $\alpha$ possible for the PIV 10-30 cameras that were used for the first SPIV measurements in this study. If the calculated value of $\alpha$ cannot be physically achieved, then the entire FOV will not be in good focus.

In step 6, the time separation $D_{t}$, between laser pulses is chosen. The value of the time separation $D_{t}$ is initially chosen based on the expected flow velocity components so that particles travel only a short distance in the through-sheet direction, typically 0.25 $\mathrm{mm}$ to $0.75 \mathrm{~mm}$ for most experiments. The sheet thickness is set to four times the 
through-sheet displacement according to design guideline (b) and sub-millimeter through-sheet displacement keeps the laser sheet reasonably thin. However, $D_{t}$ also affects particle motion across the image plane and this is considered in a subsequent design step.

Once $D_{t}$ has been chosen in step 6, the expected particle displacement across the particle images can be estimated for step 7. To that end, a simple equation was formulated to predict particle displacement in the image plane based on geometric projections of the expected velocity components of the flow. Equation 1 predicts the particle displacement (in pixels) in the image plane based on the physical size ( $\left.\mathrm{L}_{\mathrm{pix}}\right)$ of CCD pixels, the expected velocity components $\mathrm{V}_{\mathrm{A}}$ and $\mathrm{V}_{\mathrm{C}}$, and the previously chosen values for $\mathrm{R}, \theta$ and $\mathrm{D}_{\mathrm{t}}$.

Equation 1 represents an average expected particle displacement $\left(\Delta \mathrm{x}_{\mathrm{p}}\right)$ that results when the geometric projections of the axial and cross-plane velocity components align (and add together) in the image plane. Moreover, equation 1 really predicts an approximate estimate for $\Delta \mathrm{x}_{\mathrm{p}}$ because the equation does not account for varying

$$
\text { Equation 1: } \quad \Delta \mathrm{x}_{\mathrm{p}} \cong\left(\mathrm{V}_{\mathrm{A}} \sin \theta+\mathrm{V}_{\mathrm{C}} \cos \theta\right) * \mathrm{Dt} * \mathrm{R} *\left(\frac{1}{\mathrm{~L}_{\mathrm{pix}}}\right)
$$

magnification across the field-of-view. However, this estimate for $\Delta \mathrm{x}_{\mathrm{p}}$ is useful during the design process. It should be noted for equation $1, \mathrm{~V}_{\mathrm{A}}$ is the (Axial) velocity component normal to the sheet, that is, the velocity in the through-sheet direction. The variable $\mathrm{V}_{\mathrm{C}}$ (cross-plane velocity) in the equation is the velocity component oriented in the plane of the laser sheet, that is, in the object plane. The camera angle $\theta$ has been defined as the angle measured from normal to the object plane (Figure 3.1) and Equation 
1 is applicable regardless of orientation of the laser sheet (object plane) in the flow so long as estimates of the velocity components and camera angle are associated with the appropriate variables $\left(\mathrm{V}_{\mathrm{A}}, \mathrm{V}_{\mathrm{C}}\right.$ and $\left.\theta\right)$ as defined for the equation. After calculating expected particle displacement, proper sub-region size can be determined using design guideline (c) which states that sub-regions should be four times the expected value of $\Delta \mathrm{x}_{\mathrm{p}}$. Using equation 1, Figure 3.3 was created to show expected particle displacements for values of camera angle $\theta$ and lens reproduction ratio $\mathrm{R}$ for the application of SPIV to DMSJ combustor Flowpath 1 using the equipment of the TSI commercial SPIV system.

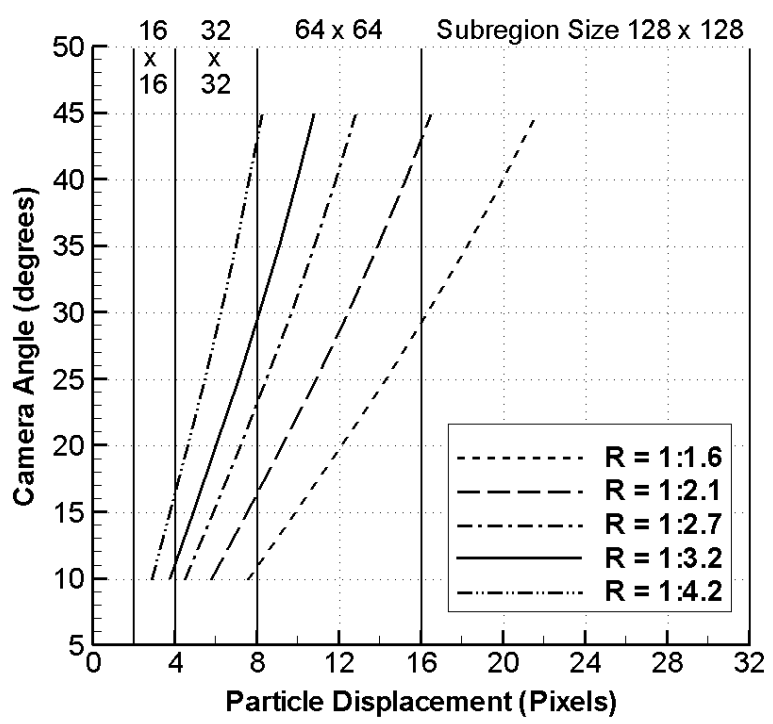

Figure 3.3: Expected particle displacements in PIV image for PIV 10-30 Cameras

Curves were calculated for values of $D_{t}=400 \mathrm{nsec}, V_{A} \cong 1000 \mathrm{~m} / \mathrm{sec}, V_{C} \cong 100 \mathrm{~m} / \mathrm{sec}$ and $L_{\text {pix }} \cong 9 \mu \mathrm{m}$. These values for $\mathrm{D}_{\mathrm{t}}, \mathrm{V}_{\mathrm{A}}$ and $\mathrm{V}_{\mathrm{C}}$ were chosen because the SPIV configuration needed to be designed for velocity measurements for both fuel-air mixing and fuel-air combustion cases. Figure 3.3 was created using values for $\mathrm{V}_{\mathrm{A}}$ and $\mathrm{V}_{\mathrm{C}}(1000$ $\mathrm{m} / \mathrm{sec}$ and $100 \mathrm{~m} / \mathrm{sec}$, respectively) for the fuel-air mixing case because that condition 
contains the largest expected velocities. Furthermore, the smallest value of $D_{t}$ possible using the available PIV equipment from TSI Inc. was $D_{t}=400 \mathrm{nsec}$ and this was chosen to minimize particle displacement across the image plane. The CCD chip of the TSI PIV 10-30 cameras has pixels of physical size $\mathrm{L}_{\text {pix }}=9 \mu \mathrm{m} \times 9 \mu \mathrm{m}$. A plot similar to Figure 3.3 could be created for any flow situation so long as reasonable estimates exist for $\mathrm{V}_{\mathrm{A}}$ and $\mathrm{V}_{\mathrm{C}}$, and the values for $\mathrm{L}_{\mathrm{pix}}$ and $\mathrm{D}_{\mathrm{t}}$ have been set. Indeed, plots similar to Figure 3.3 were created for the pixel size of the Imager ProX cameras $\left(\mathrm{L}_{\mathrm{pix}}=7.4 \mu \mathrm{m} \times 7.4 \mu \mathrm{m}\right)$ of the LaVision SPIV system for several Dt values and these plots aided in the design of SPIV configurations for the remaining measurement planes of Flowpaths 1 and 2.

In Figure 3.3, the vertical axis is the camera angle $\theta$, while the bottom horizontal axis indicates the expected particle displacement across the image plane. A scale for proper sub-region size according to design guideline (c) has been included in Figure 3.3 on the upper horizontal axis. This scale is for square sub-regions, in increments of $2^{\mathrm{n}} \times 2^{\mathrm{n}}$ pixels. For example, for $\theta=40^{\circ}$ and $\mathrm{R}=1 / 2.7$, particles are expected to move approximately 12 pixels during time separation $D t=400 \mathrm{nsec}$. Therefore, $64 \times 64$ pixel sub-regions would be appropriate. It should also be noted that the smaller sub-region size can be used when an expected particle displacement is near the boundary of two subregions sizes, so long as seed particle density remains relatively high as indicated by design guideline (a).

Particles must be recorded at two separate image locations so $\Delta \mathrm{x}_{\mathrm{p}}=2$ pixels is an appropriate minimum displacement for PIV measurements and this is checked in step 8 . Theoretically, a displacement of 1 pixel will yield a velocity measurement, but larger displacements are desired because larger displacements should yield smaller relative 
uncertainties for a fixed sub-pixel accuracy of the measured displacement. One velocity vector results for each sub-region so the spatial resolution is determined by the number of sub-regions in the measurement area. It is also generally accepted to use 50\% overlap of sub-regions ${ }^{17}$ so the spatial resolution is really half the length $\mathrm{L}_{\mathrm{I}}$ of the sub-regions determined in step 7. The lens aperture and laser power are chosen in step 9. Depth-offield increases as the lens aperture diameter decreases (increasing $f^{\#}$ ) but less light reaches the CCD for smaller aperture diameter which can result in poor contrast in the particle images. Therefore, the aperture f-number $\left(\mathrm{f}^{\#}\right)$ should be chosen for a depth-offield that encompasses the laser sheet but also allows the most light to reach the CCD.

Step 10 of the design procedure requires particle images from a PIV experiment and is really a final check of the SPIV configuration. In practice, the requirement of particle density in the image can be achieved during an experiment by adjusting the seeding rate and visually inspecting particle images as they are captured. The upper limit of particle density is exceeded when no space exists between images of particles within an area of the recordings and this must be avoided. Finally, the variation of displacement within a sub-region should be checked using the particle images, but design guideline (d) should already have been met if an appropriately small sub-region size was chosen for good spatial resolution.

\subsection{Example Design Iteration and Configuration at $\mathrm{X} / \mathrm{H}=\mathbf{1 0}$}

As an example of how the design procedure was use to determine the SPIV configuration for the $\mathrm{X} / \mathrm{H}=10$ measurement plane of Flowpath 1, the first iteration of the procedure is discussed here. As discussed above, 0.3 micron alumina particles were 
chosen based on flow-tracking characteristics and a fluidizer bed seeder added particles to the fuel. For step 2 of the procedure, the laser sheet was oriented perpendicular to the flow direction of the combustor so that instantaneous velocity field results could be obtained at planes across the combustor duct downstream of the ramp injector. Previous CFD modeling completed for this supersonic tunnel indicates that an undistorted (crossplane) camera field-of-view of $19 \mathrm{~mm} \times 19 \mathrm{~mm}$ is sufficient to capture the two counterrotating vortices. ${ }^{15}$ The CCD of each PIV 10-30 camera is $9 \mathrm{~mm} \times 9 \mathrm{~mm}$ so $\mathrm{R}=1 / 2.1$ results in a FOV of approximately $19 \mathrm{~mm} \times 19 \mathrm{~mm}$. The largest axial velocity component magnitude occurs for the fuel-air mixing case and is expected to be approximately 1000 $\mathrm{m} / \mathrm{sec}$. Again, the SPIV configuration had to be designed for measurements of both the fuel-air mixing and fuel-air combustion cases. Because of the large axial velocity component, $\theta$ needs to be relatively small to minimize the influence this axial velocity component has on particle displacement in the image plane. For this reason, $\theta$ of less than $45^{\circ}$ is appropriate and $\theta=35^{\circ}$ was a reasonable first choice in step 3 of the design process. The physical constraints were then checked according to step 4 of the design procedure. These parameters resulted in camera locations that could be used with the geometry of the tunnel. The calculation of step 5 showed that an image-plane tilt angle of $\alpha=18.4^{0}$ was required to meet the Scheimpflug condition. However, this value of $\alpha$ is not possible for the PIV $10-30$ cameras ( $\max$ available $\alpha=11^{0}$ ) and trade-offs clearly needed to be made.

Even though a second iteration of the procedure was necessary at this point, it was useful to carry the first iteration through to step 7 in order to determine if a trade-off was needed for camera field-of-view (Reproduction ratio $R$ ) or camera angle $\theta$, or both. $D_{t}=$ 
400 nsec was chosen in step 6 to keep particle displacements small in the through-sheet direction. For the chosen parameters, step 7 indicated that $\Delta \mathrm{x}_{\mathrm{p}}=13.9$ pixels which corresponds to sub-regions of size $\mathrm{L}_{\mathrm{I}}=64 \times 64$ pixel. By consulting the plot (Figure 3.3) of expected particle displacements, it was clear that trade-offs were required for both $\mathrm{R}$ and $\theta$ so that the spatial resolution could be maximized (smaller sub-regions) and so that the Scheimpflug condition could be met. Therefore, trade-offs in field-of-view and camera angle were required. The iterative design process was then repeated numerous times while accepting tradeoffs until an acceptable configuration was determined so that the PIV requirements could be met within physical constraints of the experiment and the DMSJ combustor geometry.

The following values of the experimental parameters yielded an acceptable SPIV configuration using the TSI system for Flowpath 1 location of $\mathrm{X} / \mathrm{H}=10$. The laser sheet was oriented perpendicularly to the axial flow direction and delivered through the tunnel "side-windows." Cameras were also positioned to view the flow through the tunnel sidewindows and therefore one camera received forward scatter signal from tracer particles while the other camera received the weaker backscatter from the particles. Reproduction ratio $R=1 / 3.2$ was chosen, which corresponds to $L=500 \mathrm{~mm}$ for the Nikon AF MircroNikkor 105mm camera lenses and a (undistorted) field-of-view of $29 \mathrm{~mm}$ x $29 \mathrm{~mm}$. Each camera was positioned at $\theta=30$ degrees which required $\alpha=10.2^{0}$ to meet the Scheimpflug condition. The window geometry required the cameras to face downstream and mirrors were employed to turn the optical path due to limitations imposed by the tunnel geometry. Figure 3.4 presents a schematic of the final camera configuration at $\mathrm{X} / \mathrm{H}=10 . \mathrm{D}_{\mathrm{t}}=400 \mathrm{nsec}$ was chosen, but the sheet thickness $(\mathrm{T}=2.6 \mathrm{~mm})$ was set using 
design guideline (b) for $D_{t}=600 \mathrm{nsec}$ so that measurements could be completed using $D_{t}$ $=600 \mathrm{nsec}$ in the event that the cameras were unable to acquire images for $D_{t}=400 \mathrm{nsec}$.

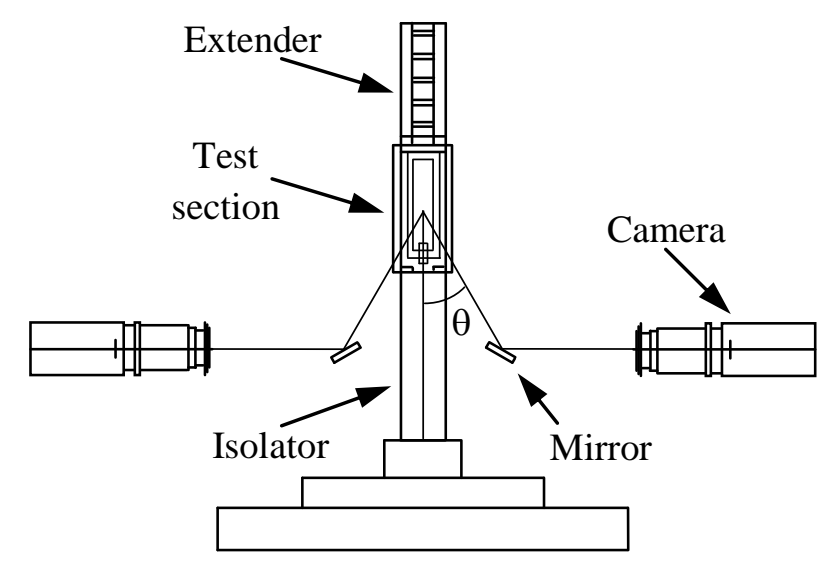

Figure 3.4: Schematic of SPIV configuration for $\mathrm{X} / \mathrm{H}=10$ location of Flowpath 1

During the experiments, it was found that the cameras could indeed record images with $D_{t}=400 \mathrm{nsec}$, however, some images had to be rejected because of one or more missing frames. Each SPIV image consists of four frames, one for each camera for each laser pulse. A missing frame in a SPIV image results when the laser fires before a camera has finished reading $\mathrm{CCD}$ information to memory and therefore the CCD is not ready to acquire a new image of particles. It was decided that acquiring data using $D_{t}=400 \mathrm{nsec}$, with some image rejection due to missing frames, was more beneficial than using $D_{t}=$ $600 \mathrm{nsec}$ because smaller particle displacement for $\mathrm{D}_{t}=400 \mathrm{nsec}$ made it possible to use smaller sub-regions. Using smaller sub-regions allows for a greater number of subregions in a given area and therefore a greater number of velocity vectors. Particles move approximately 8.2 pixels across the Field-of-view between laser pulses for $D_{t}=400 \mathrm{nsec}$ and this displacement required sub-regions of $L_{I}=32 \times 32$ pixels. The camera lens apertures were set to either $\mathrm{f}$-stop of $\mathrm{f} / 8$ or $\mathrm{f} / 11$, with the larger aperture (f/8) used for the camera receiving the weaker backscatter signal. The laser power was adjusted to produce 
good particle illumination. After reducing the sheet width to fit through the tunnel side windows, the available laser power was approximately $23 \mathrm{~mJ} /$ pulse over the $14 \mathrm{~mm}$ wide, $2.6 \mathrm{~mm}$ thick laser sheet. The parameters of step 10 of the design procedure were checked using particle images acquired during the first experiment with this SPIV configuration.

\subsection{Initial Results and Success of Design Procedure}

The SPIV experimental configuration that resulted from the design procedure, presented in section 3.4 , was used to obtain $3 \mathrm{C}$ velocity measurements at the $\mathrm{X} / \mathrm{H}=10$ measurement plane of scramjet combustor Flowpath 1. Measurements were obtained using the commercial SPIV system by TSI Inc. and Figure 3.4 shows the cameras and mirrors position adjacent to the scramjet combustor. Example instantaneous and timeaveraged velocity field measurements using the SPIV configuration are presented in Figure 3.5. These measurements were obtained for the fuel/air combustion case using an equivalence ratio of $\Phi=0.25$ with hydrogen as the fuel. For these SPIV measurements, only the fuel stream was seeded in order to prevent particles from coating the tunnel sidewindows which would obscure optical access. These SPIV measurements are presented here as evidence that the design process was able to identify a successful SPIV experimental configuration. These results are only discussed briefly here. Results at X/H $=10$ for fuel/air mixing and fuel are combustion cases are presented in chapter 5 .

In each part of Figure 3.5, the injector wall of the combustor is located along the top portion of each plot, at a distance of roughly $7 \mathrm{~mm}$ from the origin. The vertical axis of each plot extends beyond the injector wall because the design procedure showed that it 


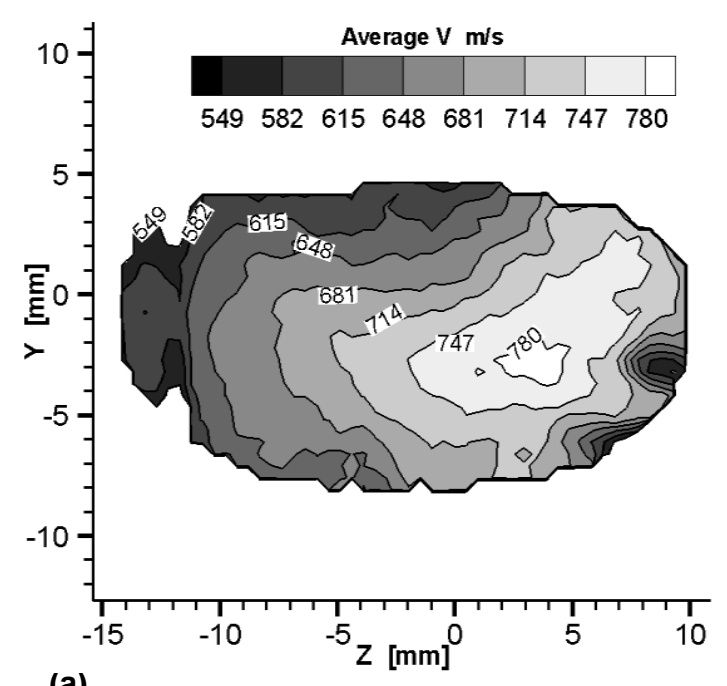

(a)

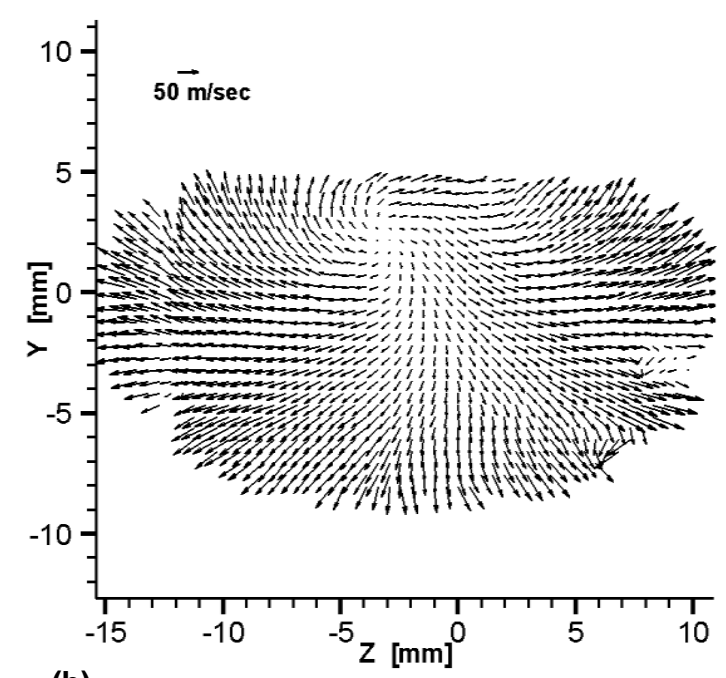

(b)

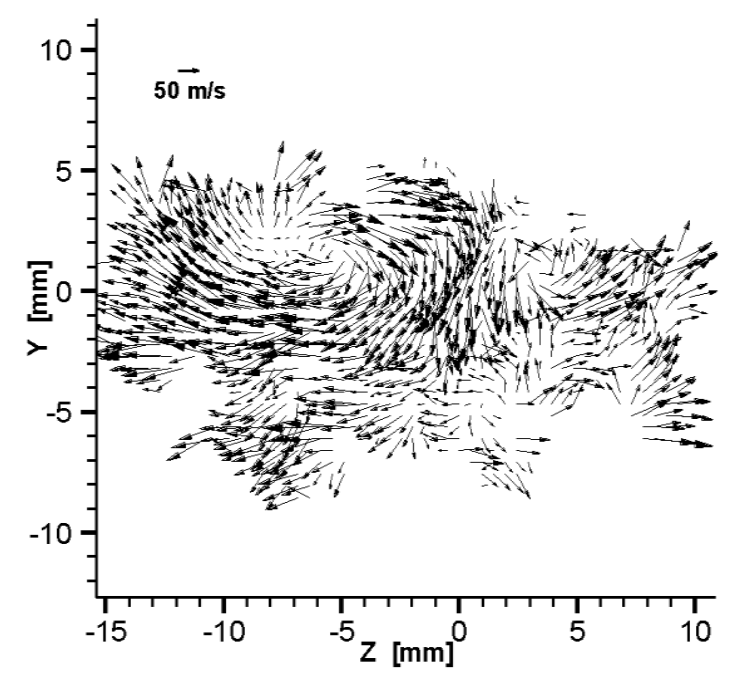

(c)

Figure 3.5: Velocity field at $\mathrm{X} / \mathrm{H}=10$ for fuel/air combustion; (a) Time-averaged 3C velocity magnitude, (b) Time-averaged In-plane vectors, (c) Instantaneous velocity vector result. 
was necessary to choose a camera field-of-view (undistorted FOV $=29 \times 29 \mathrm{~mm}$ and R $=$ 1/3.2) that was slightly larger than combustor cross-section. Figure 3.5(a) shows the magnitude of the three-component (3C) time-averaged velocity vectors for the fuel/air combustion case. For clarity in the figure, velocity contours indicating the magnitude of the $3 \mathrm{C}$ vectors are presented rather than the $3 \mathrm{C}$ vectors themselves. This time-averaged vector field was obtained by averaging 775 instantaneous velocity field results. Average velocity vectors for sub-regions located along the edge of the fuel plume resulted by averaging 50 or more instantaneous vector results, while vector counts near the total number of instantaneous results (775) contributed to the calculation of average vectors for sub-regions located near the center of the measurement area. It should be noted that the two circular areas (colored black in Figure 3.5(a)) at the lower right of the average velocity field, as well as the vertical line at the left of the field, contain incorrect velocity vectors which resulted from correlations of particles with window reflections.

A more interesting representation of the flow can be seen from the in-plane velocity vectors of the time-averaged vector field. Figure 3.5(b) shows a $2 \mathrm{D}$ view of the crossplane of the time-averaged velocity field. In this cross-plane view of the vector field, the influence of the two counter-rotating vortices (induced by the ramp fuel injector) can be seen in the upper center portion of the velocity field. The vortex in the left part of the velocity field can be more easily observed than the one on the right, but the influence of both counter-rotating vortices is evident. Figure 3.5(c) shows a cross-plane view of one of the 775 instantaneous velocity field results that make up the averaged velocity field of Figure 3.5(a) and 3.5(b). Each of the instantaneous velocity field measurements may not contain data for the entire flow field because areas of low seed particle density do not 
yield reliable velocity measurements. However, when averaging a set of instantaneous velocity measurements, the resulting velocity field contains vectors throughout the seeded fuel plume area.

These SPIV results for fuel/air combustion in a scramjet combustor with a ramp fuel injector contain many of the characteristics expected for such a flow situation. First, the effect of the counter-rotating vortices, induced by the ramp injector, can clearly be seen in the cross-plane velocity vectors of Figure $3.5 \mathrm{~b}$, as discussed above. Next, the size and shape of the vector field (the fuel plume) qualitative matches that of previous visualization of the fuel plume. ${ }^{28}$ The measured velocity magnitude, shown in Figure 3.5(a), is in the range expected for this flow situation and the velocity is lower for the fuel/air combustion than the average velocity expected for the fuel/air mixing case. This lower velocity magnitude during fuel/air combustion qualitatively matches the change in velocity due to the effect of heat addition, predicted by theory. Goyne et al. ${ }^{28}$ previously applied a 2D PIV technique to measure the axial flow velocity of a similar DMSJ combustor using one PIV 10-30 camera and a lightsheet oriented parallel to the tunnel xaxis. Goyne et al. ${ }^{28}$ reported axial velocities between $320 \mathrm{~m} / \mathrm{sec}$ and $810 \mathrm{~m} / \mathrm{sec}$ along the tunnel $\mathrm{y}$-axis at $\mathrm{X} / \mathrm{H}=10$ for hydrogen-air combustion. Along the tunnel centerline $(\mathrm{z}=$ 0 ), the average velocity field measured using SPIV in this study indicates axial velocities from $570 \pm 13 \mathrm{~m} / \mathrm{sec}$ to $765 \pm 18 \mathrm{~m} / \mathrm{sec}$. This is in fairly good agreement with the axial velocity results of Goyne et al., however, only an order of magnitude comparison can be made between the two sets of velocity measurements because an isolator section was not present in the DMSJ configuration used by Goyne et al. 
The measurements obtained using the SPIV technique are the first experimentally obtained three-component velocity fields for a scramjet during fuel-air combustion and the cross-plane views extracted from these $3 \mathrm{C}$ measurements show, for the first time, the influence of the two counter-rotating vortices that are induced by the ramp fuel injector. The velocity measurements for the case of fuel-air combustion show that the design procedure successfully identified an appropriate experimental SPIV configuration for measurements in a scramjet combustor and this procedure was used to determine the experimental SPIV apparatus for each of the remaining measurement locations. 


\section{Chapter 4: SPIV Experimental Configurations}

In this study, SPIV experiments were conducted at a total of four measurement planes downstream of the ramp fuel injector - two in Flowpath 1 and two in Flowpath 2. Experiments were not conducted at planes upstream of the injector because such measurements require seed particles in the free-stream air. Particles in the free-stream would have coated the windows of the test-section in either Flowpath 1 or 2, thus obscuring optical access and preventing SPIV measurements. SPIV experiments at planes upstream of the injector may be completed in the future using cameras that obtain images at higher rates, thus allowing data collection before particles coat the windows.

For the measurement planes presented herein, one fuel condition was used at each measurement plane except for one plane in Flowpath 1, where measurements were conducted for two fuel conditions. Therefore, this study contains five separate sets of experiments. A different SPIV experimental configuration was used for each of these five sets of experiments and each configuration was determined using the design procedure of Chapter 3. These final SPIV experimental configurations are presented here. For each of the five sets of experiments, conditions for the DMSJ combustor and the SPIV configuration parameters are summarized in a table. A schematic of the SPIV configuration used for that experiment is also presented. The set of lenses (optical train) that converted the laser beam to a collimated sheet is then shown, followed by a discussion of SPIV data processing and the filtering scheme used to eliminate incorrect velocity vectors. Velocity results obtained using these SPIV experimental configurations are presented in Chapter 5 and Chapter 6 for locations in Flowpath 1 and Flowpath 2, respectively. 


\subsection{Flowpath 1 Measurement Planes}

SPIV measurements were conducted at two different measurement planes for combustor Flowpath 1. One measurement plane was located in the test section at $\mathrm{X} / \mathrm{H}=$ 10, and the second plane at $5 \mathrm{~mm}$ downstream of the exit of the Extender, which corresponds to $\mathrm{X} / \mathrm{H}=58.6$. Figure 4.1 is a schematic of Flowpath 1 with the two measurement planes indicated at their relative locations. The first measurement plane at $\mathrm{X} / \mathrm{H}=10$ corresponds to a location in the test section at $6.35 \mathrm{~cm}$ (2.5 inches) downstream

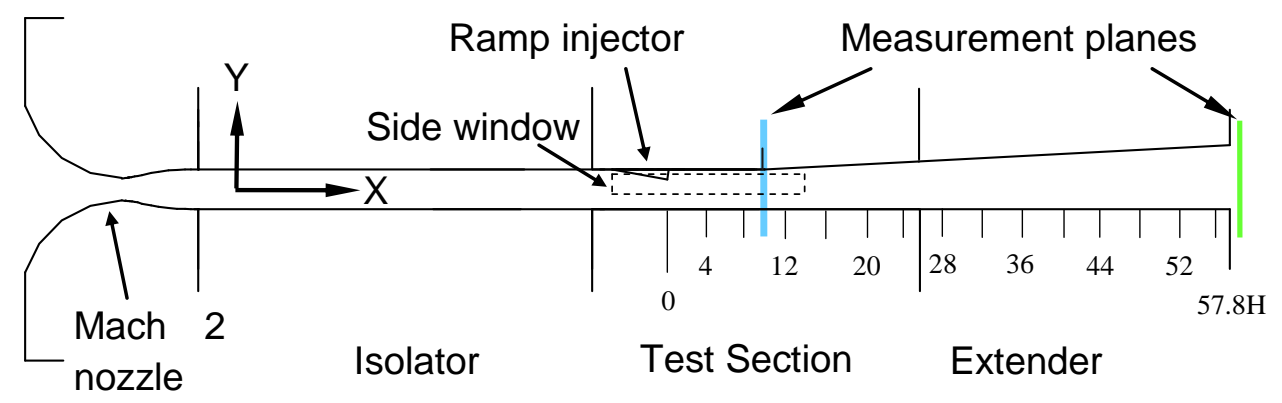

Figure 4.1: Flowpath 1 with SPIV measurement planes at $\mathrm{X} / \mathrm{H}=10$ and $\mathrm{X} / \mathrm{H}=58.6$

of the ramp fuel injector and this location is also the start of the $2.9^{0}$ divergence of the injector wall. This location was chosen based on the position and length of the side windows which provide optical access for the two cameras. After considering several camera arrangements and the physical limitations of the windows, it was decided that cameras would be placed upstream of the fuel injector, adjacent to the Isolator section, and facing downstream. For that arrangement, the most interesting measurement plane was chosen as $\mathrm{X} / \mathrm{H}=10$ because of the proximity to the start of injector wall divergence.

The second measurement plane for Flowpath 1 was located at $5 \mathrm{~mm}$ downstream of the exit of the extender section, at $\mathrm{X} / \mathrm{H}=58.6$. For this plane, cameras were positioned 
downstream of the plane and faced upstream to view the flow. The windows of the test section presented considerable viewing restrictions for the previous plane at $\mathrm{X} / \mathrm{H}=10$, but such limitations do not exist downstream of the Extender so the entire cross-section of the flow could be viewed at $\mathrm{X} / \mathrm{H}=58.6$. Another advantage of the absence of windows at the exit plane is that both the fuel and free-stream can be seeded without the problem of particles coating windows and obscuring optical access. Schematics of the experimental configurations are presented below.

Three separate fuel conditions were used for the experiments conducted with Flowpath 1 , namely the equivalence ratios of $\Phi=0.17,0.26$ and 0.34 . The equivalence ratio, $\Phi$, is defined as the ratio of the fuel-to-oxidizer ratio to the fuel-to-oxidizer ratio at Stoichiometric condition.

$$
\text { Equation 2: } \quad \Phi \equiv \frac{\text { Fuel }- \text { to }- \text { Oxidizer }}{(\text { Fuel }- \text { to }- \text { Oxidizer })_{\text {stoichiomeric }}}
$$

An equivalence ratio of $\Phi=0.26$ was used for experiments at the $\mathrm{X} / \mathrm{H}=10$ measurement plane because this amount of fuel was expected to be in the range of mode transition between supersonic and subsonic combustion for the dual-mode scramjet combustor used in this study. At the $\mathrm{X} / \mathrm{H}=58.6$ plane, measurements were conducted with equivalence ratios of both $\Phi=0.17$ and $\Phi=0.34$ to investigate the difference in the velocity field at the exit plane for a supersonic combustion case $(\Phi=0.17)$ and for a condition where the combusting flow is largely subsonic $(\Phi=0.34)$ in the DSMJ combustor. 


\subsubsection{SPIV Experimental Configuration for $\mathrm{X} / \mathrm{H}=10$}

Both fuel-air mixing and fuel-air combustion experiments for the $\mathrm{X} / \mathrm{H}=10$ plane of Flowpath 1 were conducted with a single fuel equivalence ratio of $\Phi=0.26$. Combustor conditions, equipment, and SPIV experimental parameters at $\mathrm{X} / \mathrm{H}=10$ are summarized in Table 4.1. The experimental configuration for the $\mathrm{X} / \mathrm{H}=10$ measurement plane was previously discussed in Chapter 3 as an example of the experimental design procedure and is only briefly summarized here.

The laser sheet was delivered through the tunnel side-windows and oriented perpendicularly to the axial flow direction so that instantaneous velocity results could be obtained for a cross-section of the combustor duct. The CCD of the PIV 10-30 cameras is $9 \mathrm{~mm} \times 9.14 \mathrm{~mm}$ so the reproduction ratio of $\mathrm{R}=1 / 3.2$ resulted in an (undistorted) fieldof-view of $28.8 \mathrm{~mm}$ x $29.25 \mathrm{~mm}$. Mirrors were employed to turn the optical path due to limitations imposed by the tunnel geometry. Figure 4.2 presents a schematic and photograph of the SPIV camera configuration. A series of lenses and a physical gate baffle were used to transform the laser beam from the Quanta Ray PIV 400-10 laser into a $13 \mathrm{~mm}$ wide laser sheet with sheet thickness (T) of 2.6. The set of lenses (optical train) used to produce this laser sheet is presented in Figure 4.3. During experiments, the laser power was adjusted to create adequate illumination of the alumina particles in PIV images and the energy of the laser was measured using Astral ${ }^{\mathrm{TM}}$ series AC2501 laser calorimeter at the start of the optical train. At that location, the laser energy was found to be $28 \mathrm{~mJ} /$ pulse. After the edges of the laser sheet were removed by the gate of the optical train, the laser power was approximately $23 \mathrm{~mJ} /$ pulse over the $13 \mathrm{~mm}$ wide, $2.6 \mathrm{~mm}$ thick laser sheet. 
Table 4.1: Combustor conditions and experimental parameters for $\mathrm{X} / \mathrm{H}=10$ measurement plane.

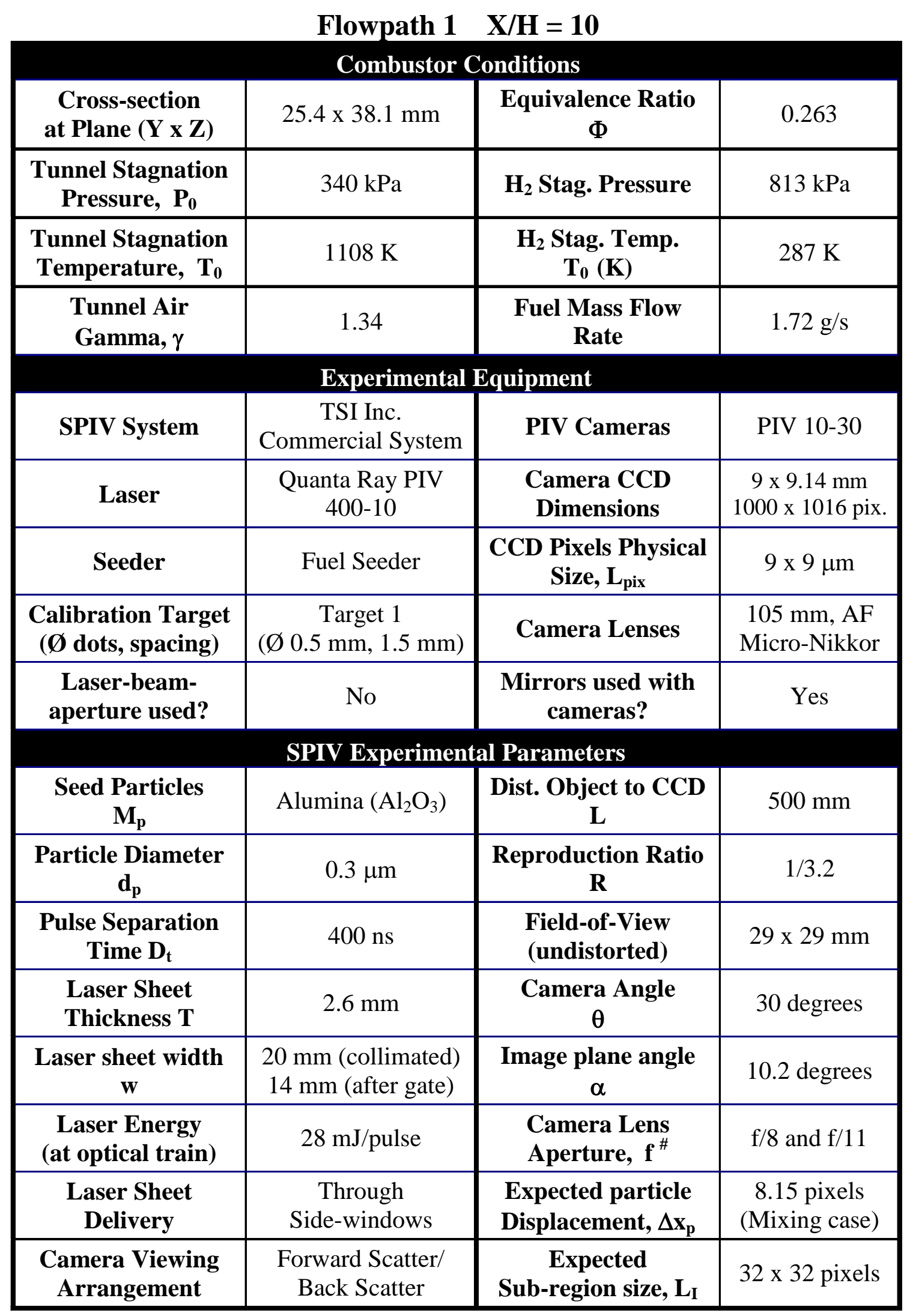


SPIV data processing for measurements at $\mathrm{X} / \mathrm{H}=10$

SPIV images were processed using DaVis 7.1 software by LaVision Inc. The first step of processing SPIV images was to subtract background noise from the images. Laser reflections or light scattered from walls of the combustor produces non-zero areas

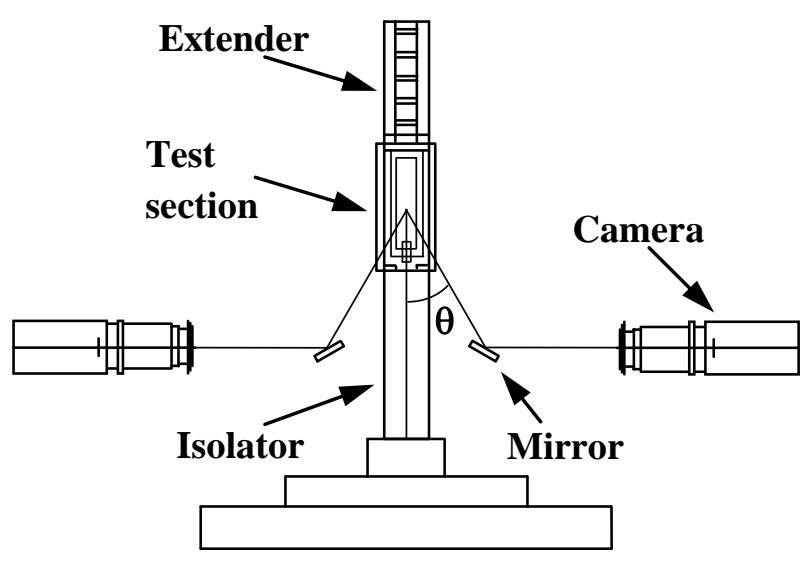

a)

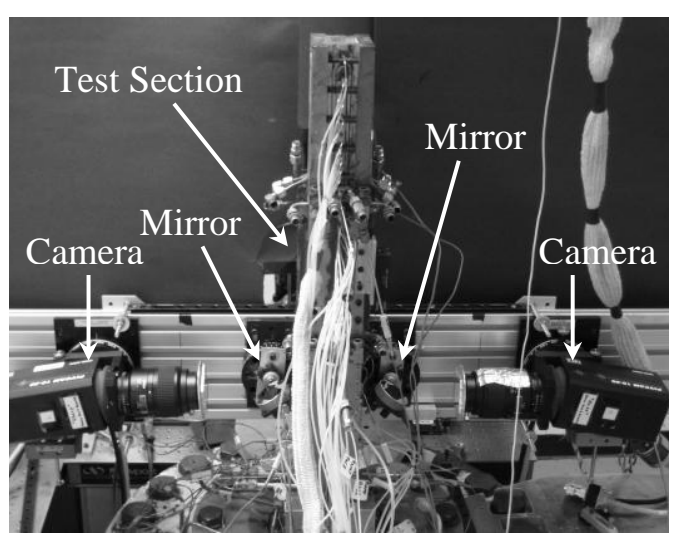

b)

Figure 4.2: SPIV configuration for $\mathrm{X} / \mathrm{H}=10$ location of Flowpath 1

a) Schematic, b) Photograph

in PIV image that can cause incorrect particle correlations and incorrect velocity vectors. During experiments, background images were capture without particles present. The background images were averaged and the average background image was subtracted from all particle images. After background subtraction, a multi-pass processing scheme was employed for vector generation, where a coarse velocity field was initially determined using $64 \times 64$ pixel sub-regions and this information was used to offset smaller 32 x 32 pixel sub-regions for subsequent calculation steps. Results from PIV simulations have shown that properly offsetting interrogation sub-regions greatly reduces the measurement uncertainty. ${ }^{10}$ A standard sub-region overlap of $50 \%$ was used, which yielded spacing of approximately $0.5 \mathrm{~mm}$ between vectors in the measurement area. 
In addition to the multi-pass scheme and the validation filters contained therein, post-processing filters were used to validate the final vectors in each of the instantaneous velocity field results. The first post-processing filter used was a range filter set to accept
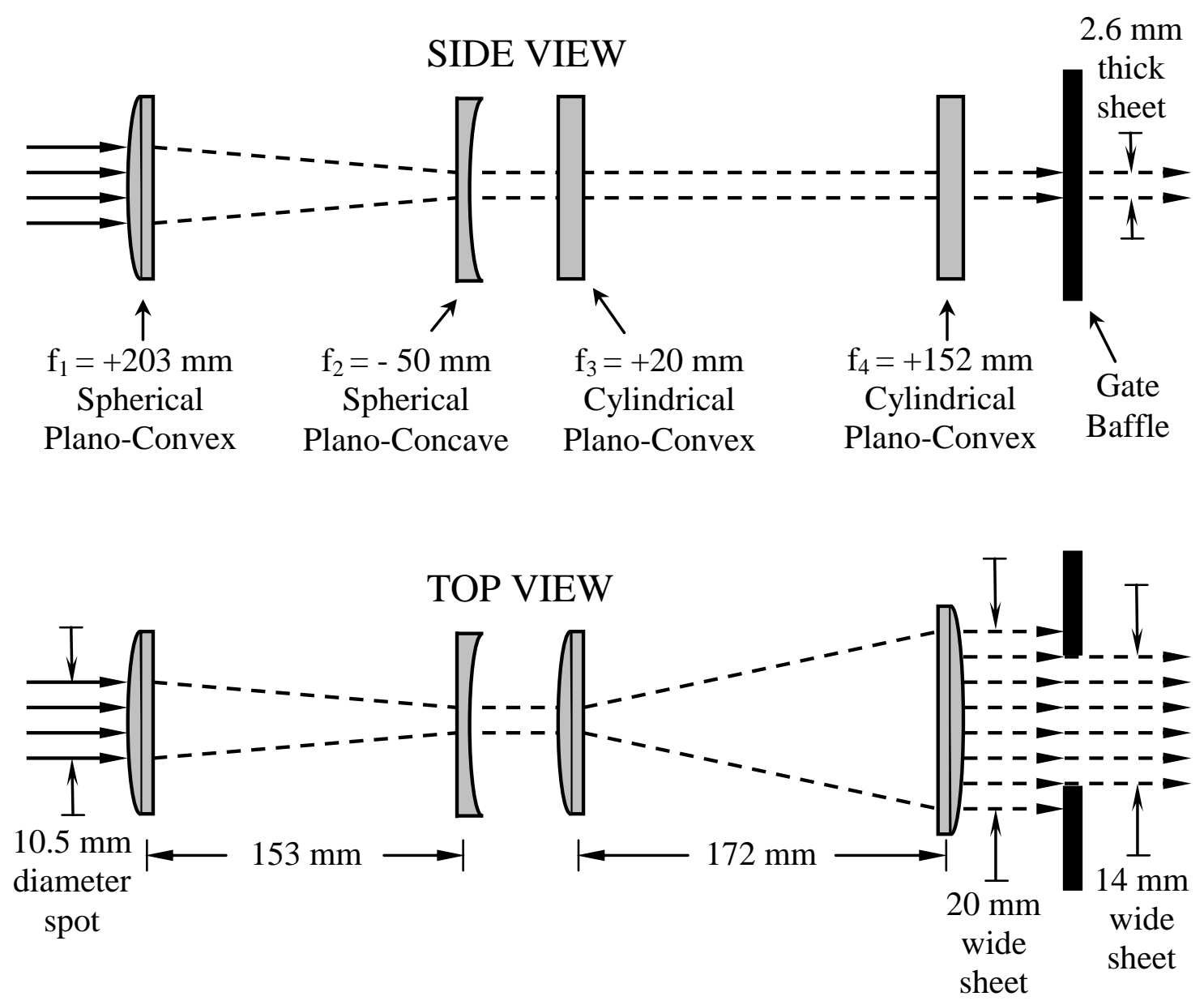

Figure 4.3: Lens elements used to produce the laser sheet for SPIV measurements at $\mathrm{X} / \mathrm{H}=10$

only velocity vectors with axial velocity component in the range of $1000+/-600 \mathrm{~m} / \mathrm{sec}$ and cross-plane components in the range of $0+/-200 \mathrm{~m} / \mathrm{sec}$. Initially, several rounds of test processing were conducted with the data to help optimize the filters. This range filter was initially set wider for both axial and cross-plane velocity components, but that only served to allow clearly incorrect vectors. Vectors allowed by the wider filter stretched across large portions of the measurement region and did not form any discernible flow 
pattern. Therefore, the range filter values stated above were used without loss of correct velocity information. The second post-processing filter was a median filter to compare neighboring velocity vectors. If the difference between a vector being tested and the average of the neighbors was greater than 2 RMS of the neighbors, then the vector was removed. The vector was then tested again and if the difference was less than 3 RMS of the remaining neighbors, the vector was reinserted. All instantaneous vectors that passed the post-processing filters were allowed to contribute to the average velocity results when an average velocity field was created.

\subsubsection{SPIV Experimental Configuration at $\mathrm{X} / \mathrm{H}=58.6, \Phi=0.17$}

For measurements at $5 \mathrm{~mm}$ downstream of the exit, cameras had to be positioned above the exit of the extender section. The exit plane dimensions at the exit are $40.8 \mathrm{~mm}$ $\mathrm{x} 38.1 \mathrm{~mm}(\mathrm{Y} \times \mathrm{Z})$ and $\mathrm{Y}$ dimension is larger than at $\mathrm{X} / \mathrm{H}=10$ due to the divergence of the injector wall. The exit dimensions meant that a larger field-of-view (and therefore a larger lens reproduction ratio) was required for the $\mathrm{X} / \mathrm{H}=58.6$ plane than for plane at $\mathrm{X} / \mathrm{H}=10$. Table 4.2 contains information about the combustor flow conditions, equipment used, and the SPIV experimental configuration at the $\mathrm{X} / \mathrm{H}=58.6$ plane with equivalence ratio $\Phi=0.17$. A shorter focal length lens (AF Micro $60 \mathrm{~mm}$ ) was used for these measurements than for the $\mathrm{X} / \mathrm{H}=10$ plane because space restrictions made it desirable to place the cameras closer to the measurement plane while still obtaining a relatively large field-of-view. For the measurements at $\mathrm{X} / \mathrm{H}=58.6$, both the free-stream and the fuel were seeded with particles using the two seeders discussed previously. It was possible to seed both the fuel and the free-stream because no windows were present 
Table 4.2: Combustor conditions and experimental parameters for $\mathrm{X} / \mathrm{H}=\mathbf{5 8 . 6}$ (Exit plane) $\Phi=0.17$

\begin{tabular}{|c|c|c|c|}
\hline \multicolumn{4}{|c|}{ Combustor Conditions } \\
\hline $\begin{array}{c}\text { Cross-section } \\
\text { at Plane }(Y \times Z)\end{array}$ & $40.8 \times 38.1 \mathrm{~mm}$ & $\begin{array}{c}\text { Equivalence Ratio } \\
\Phi\end{array}$ & 0.17 \\
\hline $\begin{array}{c}\text { Tunnel Stagnation } \\
\text { Pressure, } P_{0}\end{array}$ & $327 \mathrm{kPa}$ & $\mathrm{H}_{2}$ Stag. Pressure & $510 \mathrm{kPa}$ \\
\hline $\begin{array}{l}\text { Tunnel Stagnation } \\
\text { Temperature, } \mathbf{T}_{0}\end{array}$ & $1188-1200 \mathrm{~K}$ & $\mathrm{H}_{2}$ Stag. Temp. & $290 \mathrm{~K}$ \\
\hline $\begin{array}{l}\text { Tunnel Air } \\
\text { Gamma, } \gamma\end{array}$ & 1.34 & $\begin{array}{c}\text { Fuel Mass Flow } \\
\text { Rate }\end{array}$ & $1.01 \mathrm{~g} / \mathrm{s}$ \\
\hline \multicolumn{4}{|c|}{ Experimental Equipment } \\
\hline SPIV System & $\begin{array}{c}\text { LaVision Inc. } \\
\text { Commercial System }\end{array}$ & PIV Cameras & Imager ProX \\
\hline Laser & $\begin{array}{c}\text { Quanta Ray PIV } \\
400-10 \\
\end{array}$ & $\begin{array}{c}\text { Camera CCD } \\
\text { Dimensions } \\
\end{array}$ & $\begin{array}{l}8.88 \times 11.84 \mathrm{~mm} \\
1200 \times 1600 \text { pix. }\end{array}$ \\
\hline Seeder & $\begin{array}{c}\text { Fuel Seeder and } \\
\text { Free-stream Seeder }\end{array}$ & $\begin{array}{c}\text { CCD Pixels Physical } \\
\text { Size, } L_{\text {pix }} \\
\end{array}$ & $7.4 \times 7.4 \mu \mathrm{m}$ \\
\hline $\begin{array}{c}\text { Calibration Target } \\
\text { ( } \varnothing \text { dots, spacing) }\end{array}$ & $\begin{array}{c}\text { Target } 2 \\
(\varnothing 0.5 \mathrm{~mm}, 1.0 \mathrm{~mm})\end{array}$ & Camera Lenses & $\begin{array}{c}60 \mathrm{~mm}, \mathrm{AF} \\
\text { Micro-Nikkor }\end{array}$ \\
\hline $\begin{array}{l}\text { Laser-beam- } \\
\text { aperture used? }\end{array}$ & Yes & $\begin{array}{l}\text { Mirrors used with } \\
\text { cameras? }\end{array}$ & No \\
\hline \multicolumn{4}{|c|}{ SPIV Experimental Parameters } \\
\hline $\begin{array}{c}\text { Seed Particles } \\
\mathbf{M}_{\mathbf{p}} \\
\end{array}$ & $\begin{array}{l}\text { Silicon Dioxide } \\
\left(\mathrm{SiO}_{2}\right)\end{array}$ & $\begin{array}{c}\text { Dist. Object to CCD } \\
\mathrm{L}\end{array}$ & $400 \mathrm{~mm}$ \\
\hline $\begin{array}{c}\text { Particle Diameter } \\
\mathbf{d}_{\mathbf{p}} \\
\end{array}$ & $0.25 \mu \mathrm{m}$ & $\begin{array}{c}\text { Reproduction Ratio } \\
\text { R } \\
\end{array}$ & $1 / 4.6$ \\
\hline $\begin{array}{c}\text { Pulse Separation } \\
\text { Time } D_{t} \\
\end{array}$ & $400 \mathrm{~ns}$ & $\begin{array}{c}\text { Field-of-View } \\
\text { Y x Z (undistorted) }\end{array}$ & $40.8 \times 54.5 \mathrm{~mm}$ \\
\hline $\begin{array}{l}\text { Laser Sheet } \\
\text { Thickness T }\end{array}$ & $2.4 \mathrm{~mm}$ & $\begin{array}{c}\text { Camera Angle } \\
\theta\end{array}$ & 35 degrees \\
\hline $\begin{array}{c}\text { Laser sheet width } \\
\text { w }\end{array}$ & $\begin{array}{c}\text { Spreading, } \\
75 \mathrm{~mm} \text { at combustor }\end{array}$ & $\begin{array}{c}\text { Image plane angle } \\
\alpha \\
\end{array}$ & 8.66 degrees \\
\hline $\begin{array}{c}\text { Laser Energy } \\
\text { (at optical train) }\end{array}$ & $140 \mathrm{~mJ} /$ pulse & $\begin{array}{l}\text { Camera Lens } \\
\text { Aperture, } f^{\#}\end{array}$ & $f / 5.6$ \\
\hline $\begin{array}{c}\text { Laser Sheet } \\
\text { Delivery } \\
\end{array}$ & $\begin{array}{c}\text { Observation Wall to } \\
\text { Injector wall }\end{array}$ & $\begin{array}{l}\text { Expected particle } \\
\text { Displacement, } \Delta x_{p}\end{array}$ & $\begin{array}{c}7.7 \text { pixels } \\
\text { (Mixing case) }\end{array}$ \\
\hline $\begin{array}{l}\text { Camera Viewing } \\
\text { Arrangement }\end{array}$ & $\begin{array}{l}\text { Side Scatter / } \\
\text { Side Scatter }\end{array}$ & $\begin{array}{c}\text { Expected } \\
\text { Sub-region size, } \mathrm{L}_{\mathbf{I}}\end{array}$ & $32 \times 32$ pixels \\
\hline
\end{tabular}


at the exit and the problem of particles coating windows was not a concern. The value for expected particle motion in the table $\left(\Delta x_{p}=7.7\right.$ pixels $)$ was computed using Equation 1 with $\mathrm{D}_{\mathrm{t}}=400 \mathrm{~ns}, \mathrm{R}=1 / 4.6, \mathrm{~L}_{\mathrm{pix}}=7.4$ micrometers and a guess for axial velocity and Inplane velocities of $\mathrm{V}_{\mathrm{A}}=1000 \mathrm{~m} / \mathrm{s}$ and $\mathrm{V}_{\mathrm{C}}=100 \mathrm{~m} / \mathrm{s}$, respectively. No axial velocity data at the extender exit was available prior to these experiments so a guess of $1000 \mathrm{~m} / \mathrm{s}$ was used because it is the approximate freestream velocity from the facility nozzle. A reasonable value for $\Delta \mathrm{x}_{\mathrm{p}}$ was necessary for use with the experimental design procedure.

It should be noted that different cameras were used for measurements at $\mathrm{X} / \mathrm{H}=58.6$ than for previous measurements at $\mathrm{X} / \mathrm{H}=10$. Between the time when measurements at $\mathrm{X} / \mathrm{H}=10$ were completed and the measurements at $\mathrm{X} / \mathrm{H}=58.6$, the SPIV system was upgraded. The new PIV cameras, used for measurements at $\mathrm{X} / \mathrm{H}=58.6$, were 14-Bit, Imager Pro X cameras from LaVision Inc. These cameras were previously discussed in section 2.3 of Chapter 2. A software upgrade from DaVis software 7.1 to DaVis 7.2 was also part of the SPIV system upgrade. The Imager Pro X cameras have a rectangular CCD chip which is why the field of view value listed in Table 4.2 is rectangular. A camera angle of $\theta=35$ degrees was chosen and the value of $\theta$ and $\mathrm{R}$ required an image plane angle of $\alpha=8.66$ degrees to meet the Scheimpflug condition. For this measurement plane, the laser sheet was delivered across the exit plane from the observation wall toward the injector wall along the positive $y$-axis direction, while the cameras were positioned adjacent to the two side-walls. Figure 4.4 shows the SPIV camera configuration for measurements at $\mathrm{X} / \mathrm{H}=58.6$. In the photograph of Fig. 4.4, the second SPIV camera is hidden behind the facility exhaust chimney. 


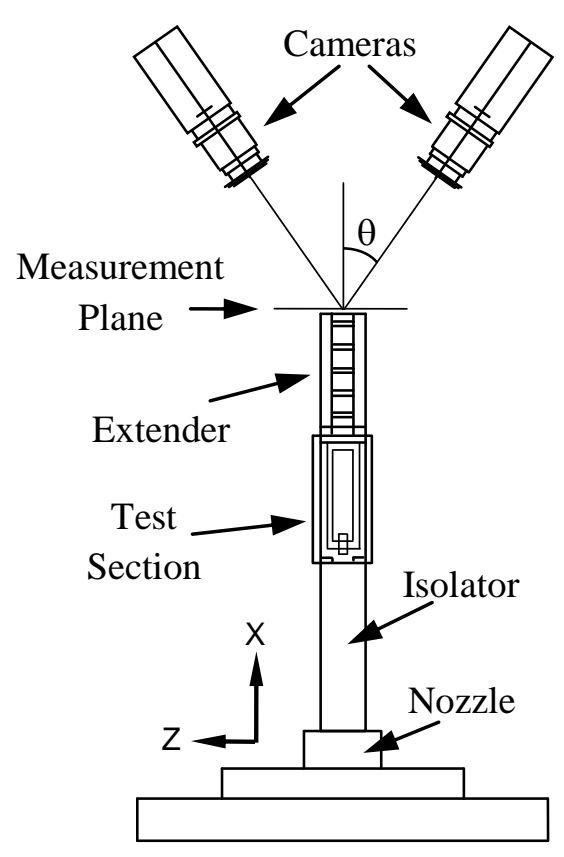

a)

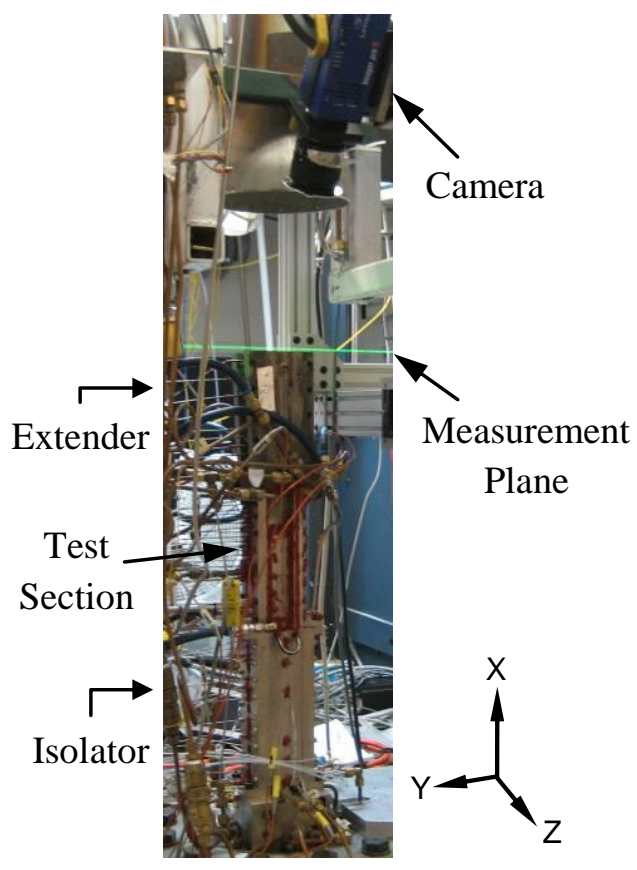

b)

Figure 4.4: $\mathrm{SPIV}$ configuration for $\mathrm{X} / \mathrm{H}=58.6$ (Exit) location of Flowpath 1 a) Schematic, b) Photograph

The set of optical lenses used to produce the laser sheet is presented in Figure 4.5. The sheet was collimated in thickness to produce a $2.4 \mathrm{~mm}$ thick laser sheet for SPIV measurements. However, the width of the sheet was allowed to continuously spread as the sheet propagated in the positive y-axis direction, and the sheet was approximately $75 \mathrm{~mm}$ wide as it crossed the exit of the extender. With the cameras facing perpendicularly to the propagation direction of the laser sheet, a "side-scatter" from the particles is the signal that the cameras record. For Mie scattering, the strongest scattering signal from particles is in the forward scatter direction (scattering in the laser propagation direction) and in this case that direction is toward the injector wall. Backscatter signal from particles is the weakest scattering signal, while the "side-scatter" has strength (intensity) between that of the forward scatter and back scatter signals. Therefore, the signal strength was the same for both cameras in the arrangement shown in Fig. 4.4. 
However, during experiments, light that scattered from the particles in the forward scattering direction (the strongest signal) impacted the injector wall at the combustor exit and caused a very intense reflection off of that wall. The reflection from the injector wall

\section{SIDE VIEW}
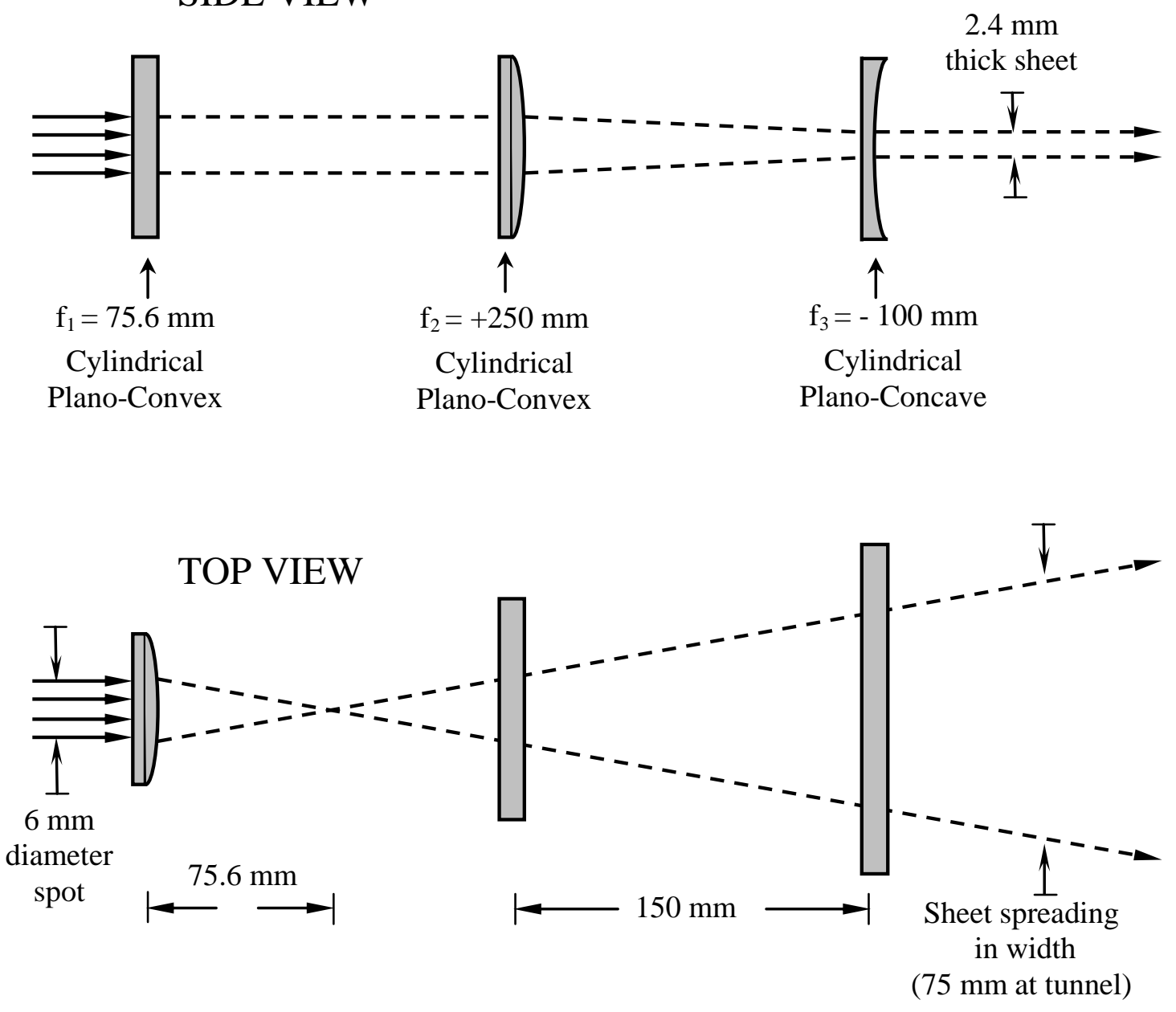

Figure 4.5: Lens elements for production of the laser sheet at $\mathrm{X} / \mathrm{H}=58.6$ (Exit plane)

was so intense that it caused saturation of the camera CCDs which could have permanently damaged the cameras. To fix this problem, the cameras had to be positioned so that the injector wall was outside the field of view of the cameras and that is why there is no data near the injector wall in the results presented below. Reflections off the remaining combustor walls also produced noise in the PIV images, but the reflections 
were not of sufficient intensity to harm the CCD chip of the cameras. However, the noise from wall reflections did cause problems for vector generation in those areas. After presentation of the SPIV configuration for measurements at $\mathrm{X} / \mathrm{H}=58.6, \Phi=0.34$; data processing methods are discussed for both sets of measurements at the $\mathrm{X} / \mathrm{H}=58.6$ plane. Calibration of the SPIV system was completed by acquiring several pictures of Calibration Target 2 and using the calibration software included in the LaVision Davis 7.2 software package.

\subsubsection{Experimental Conditions $\mathrm{X} / \mathrm{H}=58.6, \Phi=0.34$}

Experimental conditions for the $\mathrm{X} / \mathrm{H}=58.6$ measurement plane with $\Phi=0.34$ are listed in Table 4.3. These experiments were conducted on a different day than the tunnel run with $\Phi=0.17$ so the supersonic combustion tunnel conditions were slightly different between the two sets of experiments. However, the real difference for these experiments can be seen in the fuel conditions summarized in Table 4.3. The values for hydrogen $\left(\mathrm{H}_{2}\right)$ stagnation pressure and fuel mass flow rate in Table 4.3 are roughly twice the values of Table 4.2, and therefore, the equivalence ratio was doubled from $\Phi=0.17$ to $\Phi=0.34$. The same experimental configuration was used for measurements at $\mathrm{X} / \mathrm{H}=58.6$ for both equivalence ratios $\Phi=0.17$ and $\Phi=0.34$ and the SPIV configuration is depicted in Figure 4.4. Just like for the measurements at the exit plane for $\Phi=0.17$, the laser aperture was used to reduce the beam diameter to $6 \mathrm{~mm}$ for these measurements with $\Phi=$ 0.34 and the same laser optical train (Fig. 4.5) transformed the $6 \mathrm{~mm}$-diameter beam into a $2.4 \mathrm{~mm}$ thick laser sheet. Again, 0.25 micron $\mathrm{SiO}_{2}$ particles were added to both the fuel and to the free-stream air. 
Table 4.3: Combustor conditions and experimental parameters for $\mathrm{X} / \mathrm{H}=\mathbf{5 8 . 6}$ (Exit) $\Phi=0.34$

\begin{tabular}{|c|c|c|c|}
\hline \multicolumn{4}{|c|}{ Combustor Conditions } \\
\hline $\begin{array}{c}\text { Cross-section } \\
\text { at Plane }(Y \times \text { X) }\end{array}$ & $40.8 \times 38.1 \mathrm{~mm}$ & $\begin{array}{c}\text { Equivalence Ratio } \\
\Phi\end{array}$ & 0.34 \\
\hline $\begin{array}{c}\text { Tunnel Stagnation } \\
\text { Pressure, } \mathbf{P}_{0}\end{array}$ & $330 \mathrm{kPa}$ & $\mathrm{H}_{2}$ Stag. Pressure & $1030 \mathrm{kPa}$ \\
\hline $\begin{array}{l}\text { Tunnel Stagnation } \\
\text { Temperature, } \mathbf{T}_{0}\end{array}$ & $1189 \mathrm{~K}$ & $\mathrm{H}_{2}$ Stag. Temp. & $294 \mathrm{~K}$ \\
\hline $\begin{array}{l}\text { Tunnel Air } \\
\text { Gamma, } \gamma\end{array}$ & 1.34 & $\begin{array}{c}\text { Fuel Mass Flow } \\
\text { Rate }\end{array}$ & $2.025 \mathrm{~g} / \mathrm{s}$ \\
\hline \multicolumn{4}{|c|}{ Experimental Equipment } \\
\hline SPIV System & $\begin{array}{c}\text { LaVision Inc. } \\
\text { Commercial System }\end{array}$ & PIV Cameras & Imager ProX \\
\hline Laser & $\begin{array}{c}\text { Quanta Ray PIV } \\
400-10\end{array}$ & $\begin{array}{c}\text { Camera CCD } \\
\text { Dimensions }\end{array}$ & $\begin{array}{l}8.88 \times 11.84 \mathrm{~mm} \\
1200 \times 1600 \text { pix. }\end{array}$ \\
\hline Seeder & $\begin{array}{c}\text { Fuel Seeder and } \\
\text { Free-stream Seeder }\end{array}$ & $\begin{array}{c}\text { CCD Pixels Physical } \\
\text { Size, } L_{\text {pix }} \\
\end{array}$ & $7.4 \times 7.4 \mu \mathrm{m}$ \\
\hline $\begin{array}{c}\text { Calibration Target } \\
\text { ( } \varnothing \text { dots, spacing) }\end{array}$ & $\begin{array}{c}\text { Target } 2 \\
(\varnothing 0.5 \mathrm{~mm}, 1.0 \mathrm{~mm})\end{array}$ & Camera Lenses & $\begin{array}{c}60 \mathrm{~mm}, \mathrm{AF} \\
\text { Micro-Nikkor }\end{array}$ \\
\hline $\begin{array}{c}\text { Laser-beam- } \\
\text { aperture used? }\end{array}$ & Yes & $\begin{array}{l}\text { Mirrors used with } \\
\text { cameras? }\end{array}$ & No \\
\hline \multicolumn{4}{|c|}{ SPIV Experimental Parameters } \\
\hline $\begin{array}{c}\text { Seed Particles } \\
\mathbf{M}_{\mathbf{p}} \\
\end{array}$ & $\begin{array}{l}\text { Silicon Dioxide } \\
\left(\mathrm{SiO}_{2}\right)\end{array}$ & $\begin{array}{c}\text { Dist. Object to CCD } \\
\mathrm{L}\end{array}$ & $400 \mathrm{~mm}$ \\
\hline $\begin{array}{c}\text { Particle Diameter } \\
\mathbf{d}_{\mathbf{p}}\end{array}$ & $0.25 \mu \mathrm{m}$ & $\begin{array}{c}\text { Reproduction Ratio } \\
\text { R } \\
\end{array}$ & $1 / 4.6$ \\
\hline $\begin{array}{c}\text { Pulse Separation } \\
\text { Time } D_{t} \\
\end{array}$ & $\begin{array}{r}400 \text { ns (Mixing Case) } \\
600 \text { ns (Combustion) }\end{array}$ & $\begin{array}{c}\text { Field-of-View } \\
\text { Y } x \text { Z (undistorted) }\end{array}$ & $40.8 \times 54.5 \mathrm{~mm}$ \\
\hline $\begin{array}{l}\text { Laser Sheet } \\
\text { Thickness T } \\
\end{array}$ & $2.4 \mathrm{~mm}$ & $\begin{array}{c}\text { Camera Angle } \\
\theta \\
\end{array}$ & 35 degrees \\
\hline $\begin{array}{c}\text { Laser sheet width } \\
\text { w }\end{array}$ & $\begin{array}{c}\text { Spreading, } \\
75 \mathrm{~mm} \text { at combustor }\end{array}$ & $\begin{array}{c}\text { Image plane angle } \\
\alpha\end{array}$ & 8.66 degrees \\
\hline $\begin{array}{c}\text { Laser Energy } \\
\text { (at optical train) }\end{array}$ & $151 \mathrm{~mJ} / \mathrm{pulse}$ & $\begin{array}{l}\text { Camera Lens } \\
\text { Aperture, f }\end{array}$ & $\mathrm{f} / 5.6$ \\
\hline $\begin{array}{c}\text { Laser Sheet } \\
\text { Delivery } \\
\end{array}$ & $\begin{array}{c}\text { Observation Wall to } \\
\text { Injector wall }\end{array}$ & $\begin{array}{l}\text { Expected particle } \\
\text { Displacement, } \Delta \mathbf{x}_{p}\end{array}$ & $\begin{array}{c}9.03 \text { pixels } \\
\text { (for combustion) } \\
\end{array}$ \\
\hline $\begin{array}{c}\text { Camera Viewing } \\
\text { Arrangement }\end{array}$ & $\begin{array}{l}\text { Side Scatter / } \\
\text { Side Scatter }\end{array}$ & $\begin{array}{c}\text { Expected } \\
\text { Sub-region size, } \mathrm{L}_{\mathbf{I}}\end{array}$ & $32 \times 32$ pixels \\
\hline
\end{tabular}


Most of the values in Table 4.3 are the same as those of Table 4.2 for the $\Phi=0.17$ measurements, however, two differences should be noted. First, the time separation between laser pulses for the fuel-air combustion case was chosen as $D_{t}=600 \mathrm{~ns}$, while $D_{t}$ remained at $400 \mathrm{~ns}$ for the mixing case. Second, the expected particle displacement $\left(\Delta \mathrm{x}_{\mathrm{p}}\right)$ was slightly larger than that of the previous measurements. After completion of the exit plane measurements with $\Phi=0.17$, a more reliable estimate for axial velocity at the exit plane was available. The maximum value of axial velocity $(750 \mathrm{~m} / \mathrm{s})$ from the previous exit plane measurements was used in Equation 1 for calculation of the expected particle displacement. The experimental configuration summarized in Table 4.3 was used for SPIV measurements of the flowfield at $\mathrm{X} / \mathrm{H}=58.6$ for both fuel-air mixing and fuel-air combustion with an equivalence ratio of $\Phi=0.34$.

\section{SPIV data processing for measurements at $X / H=58.6$}

As mentioned above, the particle images at $5 \mathrm{~mm}$ downstream of the exit plane contained random background noise and also noise from laser reflections off of the interior extender walls. Background images with no particles present (seeders off) were acquired during the experiment and these images were used to create an average background result, which was in turn subtracted from particle images to remove background noise. The intensity of the reflections from tunnel walls was higher when particles were present and, therefore, subtraction of the average background did not entirely remove reflections of tunnel wall from the SPIV images. Attempts were made to separate images of particles (good data) from the wall reflections. Unfortunately, this separation could not be accomplished because both the data and noise were of 
approximately the same intensity. Incorrect velocity vectors due to reflections had to be removed during post-processing. Several post-processing filtering options were attempted, but none removed all incorrect vectors in instantaneous results and enough wrong vectors were present to spoil the average velocity in those areas. Ultimately, separate user-defined masks were created for each fuel condition at $\mathrm{X} / \mathrm{H}=58.6$ in order to blank out bad vectors that resulted from tunnel wall reflections.

After image pre-processing to remove background noise and reflections, a multipass processing scheme was employed to generate velocity vectors. Initially, 64 x 64 pixel sub-regions were used to calculate a coarse velocity field and that information was used to offset smaller $32 \times 32$ vector sub-regions for the final passes. A sub-region overlap of $50 \%$ was also used so that a vector result occurred at approximately every 0.55 mm. In addition to the multi-pass scheme, two main post-processing filters were used to validate the final vectors of the instantaneous fields - a median filter and a velocity range filter. The median filter relies on the fact that a fluid is a continuum and the motion of a small fluid element cannot be drastically different from the surrounding elements. For the measurements here, two separate sets of vector post-processing were used - one for the experiments with $\Phi=0.17$ and one for those with $\Phi=0.34$.

For experiments with $\Phi=0.17$, the median filter was used twice and then the range filter was applied. A vector was removed if its magnitude was greater than 2 RMS of the neighbors and then tested again during a second pass where a vector was reinserted if the difference was less than 2.5 RMS of the remaining neighbors. The filter was applied again and vectors were removed if the magnitude was greater than 1.2 RMS of the neighbors. After the median filter, a range filter was used to remove any groups of very 
large velocity vectors that happened to get past the median filter. The filter was set to accept velocity vectors with axial velocity component ( $\mathrm{u}, \mathrm{x}$-direction) in the range of 0 to $1200 \mathrm{~m} / \mathrm{sec}$ and cross-plane components of $\mathrm{v}=0+/-200 \mathrm{~m} / \mathrm{s}$ and $\mathrm{w}=0+/-125 \mathrm{~m} / \mathrm{s}$.

For measurements with $\Phi=0.34$, the median filter was applied twice - first where vectors were removed if greater than 1.5 RMS of the neighbors and reinserted on the second pass if the magnitude of the vector being tested was less than 2 RMS of the new neighbors. The median filter was applied again with limits of greater than 1 RMS for removal and reinsertion if less than 1.5 RMS of the new neighbors. The range filter was not necessary. As mentioned above, the last post-processing operation was the application of a separate mask was for each set of results at $\mathrm{X} / \mathrm{H}=58.6$.

\subsection{Flowpath 2 Measurement Planes}

SPIV measurements were conducted at two different measurement planes in the Test-section of combustor Flowpath 2. Figure 4.6 shows a schematic of Flowpath 2 with the two measurement planes indicated. Leaders of the National Center for Hypersonic Combined Cycle Propulsion have identified several planes of interest in Flowpath 2 and these include measurement planes at $\mathrm{X} / \mathrm{H}=6, \mathrm{X} / \mathrm{H}=12$ and $\mathrm{X} / \mathrm{H}=18$, among others.

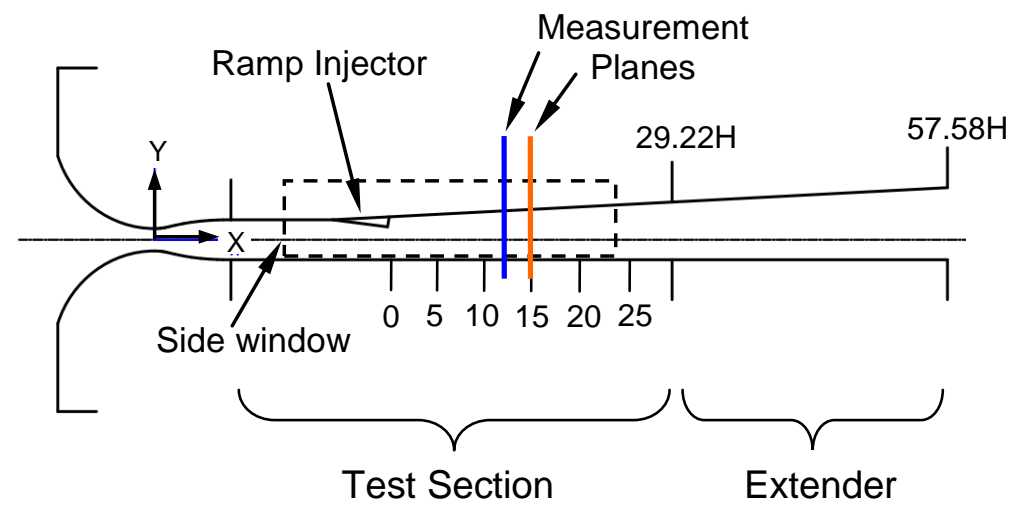

Figure 4.6: Flowpath 2 with SPIV measurement planes at $\mathrm{X} / \mathrm{H}=12$ and $\mathrm{X} / \mathrm{H}=15$ 
The plane located at $\mathrm{X} / \mathrm{H}=12$ in the Test-section of Flowpath 2 was chosen as one of the planes for SPIV measurements. Identifying a second measurement plane downstream of $\mathrm{X} / \mathrm{H}=12$ proved to be difficult. Attempts were made to obtain SPIV measurements at $\mathrm{X} / \mathrm{H}=18$ because that location is also a plane of interest for the work of the National Center for Hypersonic Combined Cycle Propulsion. However, during initial experiments at $\mathrm{X} / \mathrm{H}=18$, SPIV images were found to contain a large area of high background noise which prevented vector generation. It was determined that this large background noise came from viewing the downstream end of the side-windows of the Test-section, so measurements at $\mathrm{X} / \mathrm{H}=18$ could not be conducted. Instead, $\mathrm{X} / \mathrm{H}=15$ was chosen as the location of the second measurement plane in the Test-section of Flowpath 2.

\subsubsection{SPIV Experimental Configuration at $\mathrm{X} / \mathrm{H}=12$}

SPIV measurements at $\mathrm{X} / \mathrm{H}=12$ in Flowpath 2 were conducted using an experimental configuration similar to the configuration at $\mathrm{X} / \mathrm{H}=10$ in Flowpath 1 . Mirrors were placed upstream of the measurement plane to turn the optical path and allowed the cameras to view the flow "facing" downstream. Mirrors were necessary because space upstream of the Test-section is limited by the end of the facility heater. A schematic of the SPIV configuration at $\mathrm{X} / \mathrm{H}=12$ is shown in Figure 4.7. Experimental conditions and characteristics of the SPIV configuration can be found in Table 4.4. During experimental set-up and testing at $\mathrm{X} / \mathrm{H}=12$ it was found that high intensity laser reflections occurred on the side-windows which could have damaged the CCD of the Imager ProX cameras. The solution to this problem was to rotate the cameras 90-degrees so that the long dimension of the CCD was along the y-axis of Flowpath 2, as seen in 
Table 4.4. With the short axis of the CCD parallel to the z-axis of Flowpath, primary laser reflections were outside of the field-of-view of the cameras. Secondary laser reflections were still present, but these were not intense enough to harm the CCD chips.

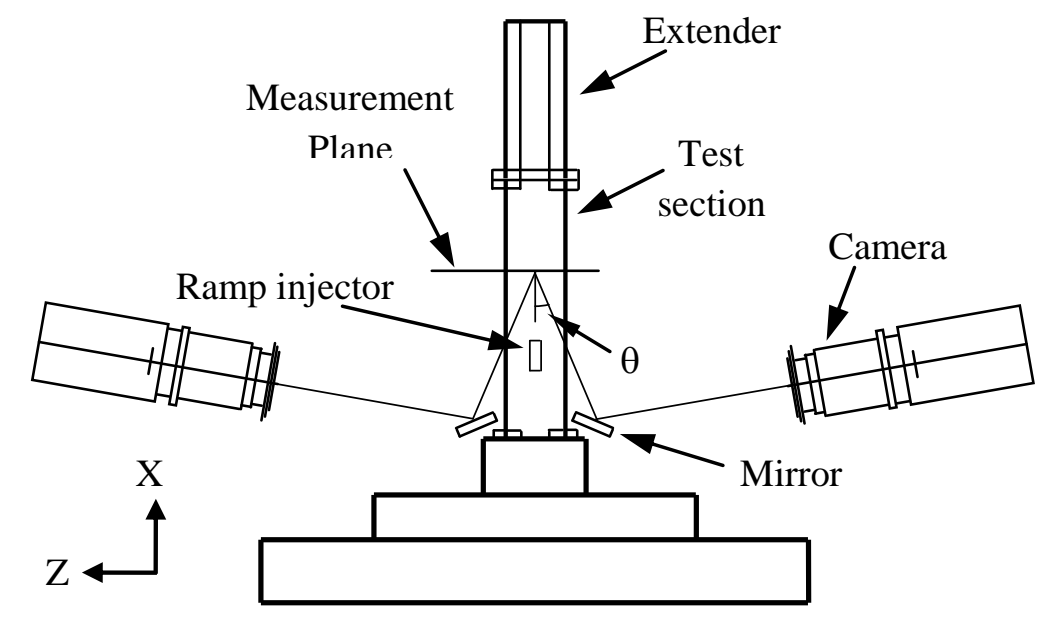

Figure 4.7: Schematic of the SPIV configuration for $\mathrm{X} / \mathrm{H}=12$ location of Flowpath 2

The expected particle displacement value of 9.33 pixels was found using Equation 1 and a guess of $1000 \mathrm{~m} / \mathrm{s}$ for axial and a cross-plane velocity of $100 \mathrm{~m} / \mathrm{s}$, along with the values of $R, \theta, D_{t}$ and $L_{\text {pix }}$ reported in the table. An angle $\alpha=9.6^{0}$ was necessary to meet the Scheimpflug condition for well-focused images. The laser aperture was again used to reduce the laser beam diameter (and power) before the beam reached the optical train. The set of lenses (optical train) used to transform the laser beam into a collimated sheet is presented in Figure 4.8. The laser exited the optical train as a collimated sheet $(28 \mathrm{~mm}$ wide $\mathrm{x} 2.5 \mathrm{~mm}$ thick) and was then directed into the Test-section of Flowpath 2 through the tunnel side-windows. The propagation of the sheet was in the negative z-direction and therefore, one camera viewed the forward scatter signal from the particles (Mie scattering) while the second camera recorded the backscatter signal. 
Table 4.4: Combustor conditions and experimental parameters for $\mathrm{X} / \mathrm{H}=12$ measurement plane.

\begin{tabular}{|c|c|c|c|}
\hline \multicolumn{4}{|c|}{ Combustor Conditions } \\
\hline $\begin{array}{c}\text { Cross-section } \\
\text { at Plane }(Y \times Z)\end{array}$ & $31.1 \times 38.1 \mathrm{~mm}$ & $\begin{array}{c}\text { Equivalence Ratio } \\
\Phi\end{array}$ & 0.172 \\
\hline $\begin{array}{c}\text { Tunnel Stagnation } \\
\text { Pressure, } \mathbf{P}_{\mathbf{0}}\end{array}$ & $297 \mathrm{kPa}$ & $\mathrm{H}_{2}$ Stag. Pressure & $490 \mathrm{kPa}$ \\
\hline $\begin{array}{l}\text { Tunnel Stagnation } \\
\text { Temperature, } \mathbf{T}_{0}\end{array}$ & $1201 \mathrm{~K}$ & $\mathrm{H}_{2}$ Stag. Temp. & $306 \mathrm{~K}$ \\
\hline $\begin{array}{l}\text { Tunnel Air } \\
\text { Gamma, } \gamma\end{array}$ & 1.34 & $\begin{array}{c}\text { Fuel Mass Flow } \\
\text { Rate }\end{array}$ & $0.918 \mathrm{~g} / \mathrm{s}$ \\
\hline \multicolumn{4}{|c|}{ Experimental Equipment } \\
\hline SPIV System & $\begin{array}{c}\text { LaVision Inc. } \\
\text { Commercial System }\end{array}$ & PIV Cameras & Imager ProX \\
\hline Laser & $\begin{array}{c}\text { Quanta Ray PIV } \\
400-10\end{array}$ & $\begin{array}{c}\text { Camera CCD } \\
\text { Dimensions }\end{array}$ & $\begin{array}{l}8.88 \times 11.84 \mathrm{~mm} \\
1200 \times 1600 \text { pix. }\end{array}$ \\
\hline Seeder & Fuel Seeder & $\begin{array}{c}\text { CCD Pixels Physical } \\
\text { Size, } L_{\text {pix }} \\
\end{array}$ & $7.4 \times 7.4 \mu \mathrm{m}$ \\
\hline $\begin{array}{c}\text { Calibration Target } \\
\text { ( } \varnothing \text { dots, spacing) }\end{array}$ & $\begin{array}{c}\text { Target } 3 \\
(\varnothing 0.5 \mathrm{~mm}, 1.0 \mathrm{~mm})\end{array}$ & Camera Lenses & $\begin{array}{l}105 \mathrm{~mm}, \mathrm{AF} \\
\text { Micro-Nikkor }\end{array}$ \\
\hline $\begin{array}{l}\text { Laser-beam- } \\
\text { aperture used? }\end{array}$ & Yes & $\begin{array}{c}\text { Mirrors used with } \\
\text { cameras? }\end{array}$ & Yes \\
\hline \multicolumn{4}{|c|}{ SPIV Experimental Parameters } \\
\hline $\begin{array}{c}\text { Seed Particles } \\
\mathbf{M}_{\mathbf{p}} \\
\end{array}$ & $\begin{array}{c}\text { Silicon Dioxide } \\
\left(\mathrm{SiO}_{2}\right)\end{array}$ & $\begin{array}{c}\text { Dist. Object to CCD } \\
\mathrm{L}\end{array}$ & $519 \mathrm{~mm}$ \\
\hline $\begin{array}{c}\text { Particle Diameter } \\
\mathbf{d}_{\mathbf{p}} \\
\end{array}$ & $0.25 \mu \mathrm{m}$ & $\begin{array}{c}\text { Reproduction Ratio } \\
\text { R } \\
\end{array}$ & $1 / 3.4$ \\
\hline $\begin{array}{c}\text { Pulse Separation } \\
\text { Time } D_{t} \\
\end{array}$ & $400 \mathrm{~ns}$ & $\begin{array}{c}\text { Field-of-View } \\
\text { Y } x \text { Z (undistorted) } \\
\end{array}$ & $40.2 \times 30.2 \mathrm{~mm}$ \\
\hline $\begin{array}{l}\text { Laser Sheet } \\
\text { Thickness T }\end{array}$ & $2.5 \mathrm{~mm}$ & $\begin{array}{c}\text { Camera Angle } \\
\theta\end{array}$ & 30 degrees \\
\hline $\begin{array}{c}\text { Laser sheet width } \\
\text { w }\end{array}$ & $\begin{array}{l}47 \mathrm{~mm} \text { (collimated) } \\
\sim 28 \mathrm{~mm} \text { (after gate) }\end{array}$ & $\begin{array}{c}\text { Image plane angle } \\
\alpha\end{array}$ & 9.6 degrees \\
\hline $\begin{array}{c}\text { Laser Energy } \\
\text { (at optical train) }\end{array}$ & $106 \mathrm{~mJ} /$ pulse & $\begin{array}{l}\text { Camera Lens } \\
\text { Aperture, f }\end{array}$ & $\mathrm{f} / 8$ and $\mathrm{f} / 11$ \\
\hline $\begin{array}{c}\text { Laser Sheet } \\
\text { Delivery } \\
\end{array}$ & $\begin{array}{c}\text { Through } \\
\text { Side-windows } \\
\end{array}$ & $\begin{array}{l}\text { Expected particle } \\
\text { Displacement, } \Delta \mathbf{x}_{\mathbf{p}} \\
\end{array}$ & $\begin{array}{c}9.33 \text { pixels } \\
\text { (Mixing case) }\end{array}$ \\
\hline $\begin{array}{c}\text { Camera Viewing } \\
\text { Arrangement }\end{array}$ & $\begin{array}{c}\text { Forward Scatter / } \\
\text { Back Scatter }\end{array}$ & $\begin{array}{c}\text { Expected } \\
\text { Sub-region size, } \mathrm{L}_{\mathrm{I}}\end{array}$ & $32 \times 32$ pixels \\
\hline
\end{tabular}


Processing of $X / H=12$ data

SPIV data was processed for this measurement location in a similar manner as the data for other planes, discussed previously. Images of background noise were acquired with the seeder off (no particles present) and an average background was subtracted from all SPIV image frames. A multi-pass processing scheme, in the DaVis 7.2 software, was again used where $64 \times 64$ pixel sub-regions calculated coarse velocity field that was then used to off-set smaller $32 \times 32$ pixel sub-regions for the final calculation pass.
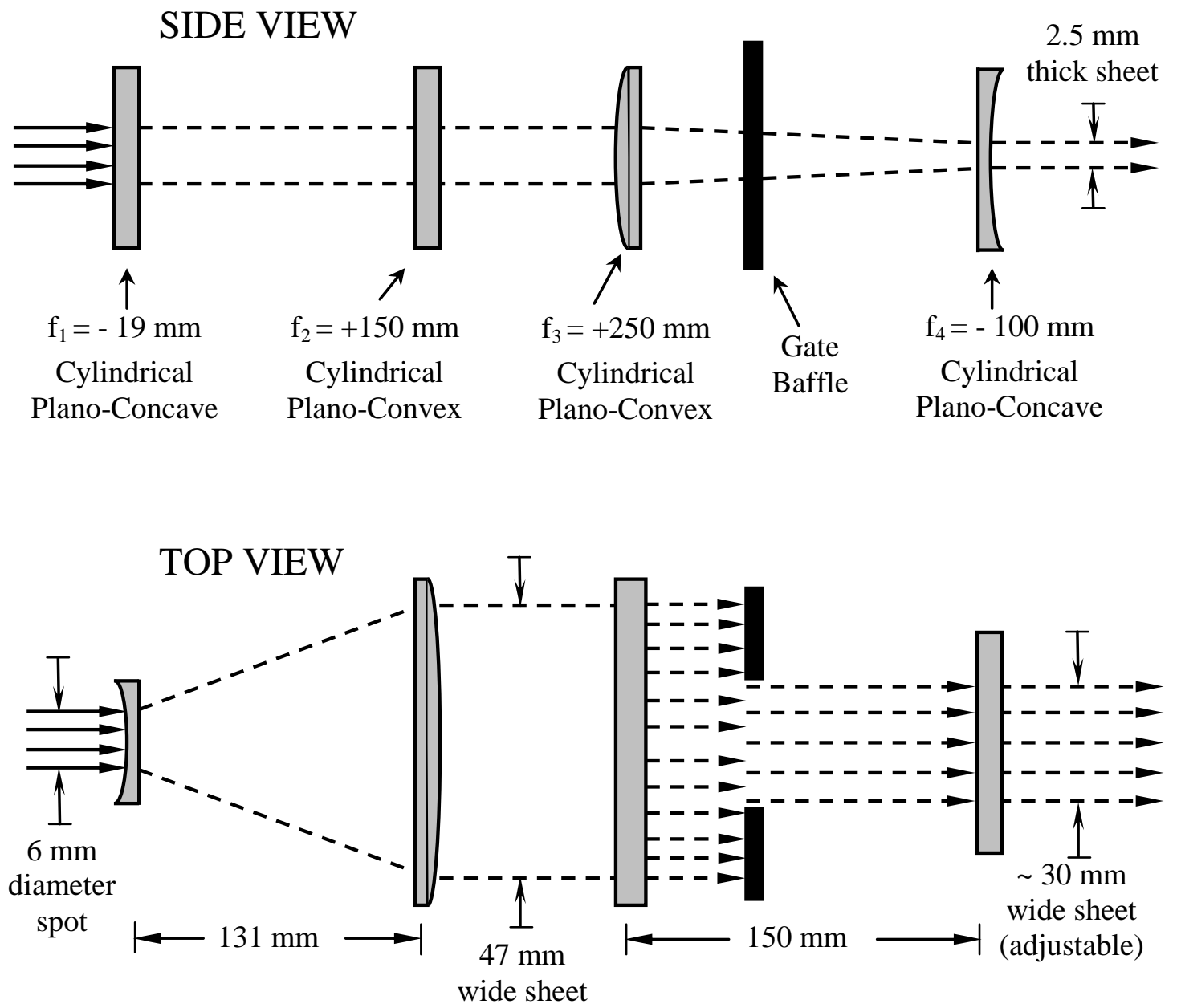

Figure 4.8: Lens elements for production of the laser sheet at $\mathrm{X} / \mathrm{H}=12$ and 15 , Flowpath 2 
Several post-processing schemes were attempted, but the best result came from the pair of filters discussed above. For fuel air mixing measurements, the median filter was optimized and set to remove a vector if its magnitude was greater than 1.5 RMS of the neighbors and then to reinsert if the difference was less than 2 RMS of the remaining neighbors. The range filter was then applied to remove vectors when either in-plane component (y or z-comp) was greater than $+/-200 \mathrm{~m} / \mathrm{s}$ and the axial velocity fell outside the range of 700 to $1300 \mathrm{~m} / \mathrm{s}$. Masking of wrong vectors was not necessary the fuel-air mixing data.

SPIV images for the case of fuel-air combustion at $\mathrm{X} / \mathrm{H}=12$ contained background noise in the area of the fuel plume so additional processing was necessary. Background subtraction was again used as the first step for data processing. The same multi-pass scheme was used for vector generation with the combustion data. For post-processing, the range filter was again set to remove vectors >1.5 RMS of the neighbors and a reinsertion criteria of $<2$ RMS of the remaining neighbors. The range filter was applied and had to be set to allow vectors with axial velocities from 0 to $1200 \mathrm{~m} / \mathrm{s}$ because of the large range in velocity for the combustion case at $\mathrm{X} / \mathrm{H}=12$. Unfortunately, large incorrect velocity vectors on the sides of the fuel plume (in the z-direction) still remained after applying these filters. These incorrect vectors resulted from correlation of particles with laser reflections. Several filtering schemes were attempted, but ultimately a masking filter was created to delete vectors in those areas. In chapter 6 , the average result from one set of SPIV measurements, without masking, is show and the incorrect vectors are evident. 


\subsubsection{SPIV Experimental Configuration at $\mathrm{X} / \mathrm{H}=15$}

The SPIV configuration used for measurements at $\mathrm{X} / \mathrm{H}=15$ was very similar to that of the $\mathrm{X} / \mathrm{H}=12$ measurements. Indeed, the original plan was to use exactly the same experimental configuration for measurements at both $\mathrm{X} / \mathrm{H}=12$ and 15 . However a change in camera angle $(\theta)$ was required to keep reflection noise, from the downstream ends of the side-windows, out of the SPIV images at $\mathrm{X} / \mathrm{H}=15$. The set of experimental conditions and SPIV configuration parameters is summarize in Table 4.5. The same optical train (Fig. 4.8) was used to create the laser sheet for measurements at $\mathrm{X} / \mathrm{H}=15$. For the combustion case, the maximum expected particle displacement $\left(\Delta \mathrm{x}_{\mathrm{p}}\right)$ value in Table 4.5 was calculated using $\mathrm{D}_{\mathrm{t}}=700 \mathrm{nsec}$ and the maximum axial velocity from measurements at $\mathrm{X} / \mathrm{H}=12 . \Delta \mathrm{x}_{\mathrm{p}}$ is large because of the choice of $\mathrm{D}_{\mathrm{t}}$.

\section{Processing of $X / H=15$ data}

The same processing scheme that was employed for vector generation and postprocessing of the data for fuel-air mixing at $\mathrm{X} / \mathrm{H}=12$ was also used for the mixing data at $\mathrm{X} / \mathrm{H}=15$ and is presented above. Images for fuel-air combustion were subject to sever noise in the area of the injector wall and diffuse noise across the remainder of each image. Even with careful vector processing, it was unclear if any useful data would be obtained from these measurements. However, after background subtraction, tests with several different multi-pass processing schemes, and several trials to optimize postprocessing filters; a processing scheme was found that generated some useful measurements. The filters were again a median filter, this time with slightly less restrictive limits, ( > 2 RMS for removal and $<2.5$ RMS for reinsertion) and a range filter 
Table 4.5: Combustor conditions and experimental parameters for $\mathrm{X} / \mathrm{H}=15$ measurement plane.

\begin{tabular}{|c|c|c|c|}
\hline \multicolumn{4}{|c|}{ Combustor Conditions } \\
\hline $\begin{array}{c}\text { Cross-section } \\
\text { at Plane }(Y \times Z)\end{array}$ & $32.1 \times 38.1 \mathrm{~mm}$ & $\begin{array}{c}\text { Equivalence Ratio } \\
\Phi\end{array}$ & 0.172 \\
\hline $\begin{array}{c}\text { Tunnel Stagnation } \\
\text { Pressure, } \mathbf{P}_{\mathbf{0}}\end{array}$ & $297 \mathrm{kPa}$ & $\mathrm{H}_{2}$ Stag. Pressure & $470 \mathrm{kPa}$ \\
\hline $\begin{array}{l}\text { Tunnel Stagnation } \\
\text { Temperature, } \mathbf{T}_{0}\end{array}$ & $1201 \mathrm{~K}$ & $\mathrm{H}_{2}$ Stag. Temp. & $310 \mathrm{~K}$ \\
\hline $\begin{array}{l}\text { Tunnel Air } \\
\text { Gamma, } \gamma\end{array}$ & 1.34 & $\begin{array}{c}\text { Fuel Mass Flow } \\
\text { Rate (grams/s) }\end{array}$ & 0.924 \\
\hline \multicolumn{4}{|c|}{ Experimental Equipment } \\
\hline SPIV System & $\begin{array}{c}\text { LaVision Inc. } \\
\text { Commercial System }\end{array}$ & PIV Cameras & Imager ProX \\
\hline Laser & $\begin{array}{c}\text { Quanta Ray PIV } \\
400-10\end{array}$ & $\begin{array}{c}\text { Camera CCD } \\
\text { Dimensions } \\
\end{array}$ & $\begin{array}{l}8.88 \times 11.84 \mathrm{~mm} \\
1200 \times 1600 \text { pix. }\end{array}$ \\
\hline Seeder & Fuel Seeder & $\begin{array}{c}\text { CCD Pixels Physical } \\
\text { Size, } \mathbf{L}_{\text {pix }}\end{array}$ & $7.4 \times 7.4 \mu \mathrm{m}$ \\
\hline $\begin{array}{c}\text { Calibration Target } \\
\text { (Ø dots, spacing) }\end{array}$ & $\begin{array}{c}\text { Target } 3 \\
(\varnothing 0.5 \mathrm{~mm}, 1.0 \mathrm{~mm}) \\
\end{array}$ & Camera Lenses & $\begin{array}{l}105 \mathrm{~mm}, \mathrm{AF} \\
\text { Micro-Nikkor }\end{array}$ \\
\hline $\begin{array}{c}\text { Laser-beam- } \\
\text { aperture used? }\end{array}$ & Yes & $\begin{array}{l}\text { Mirrors used with } \\
\text { cameras? }\end{array}$ & Yes \\
\hline \multicolumn{4}{|c|}{ SPIV Experimental Parameters } \\
\hline $\begin{array}{c}\text { Seed Particles } \\
\mathbf{M}_{\mathbf{p}} \\
\end{array}$ & $\begin{array}{l}\text { Silicon Dioxide } \\
\left(\mathrm{SiO}_{2}\right)\end{array}$ & $\begin{array}{c}\text { Dist. Object to CCD } \\
\mathrm{L}\end{array}$ & $500 \mathrm{~mm}$ \\
\hline $\begin{array}{c}\text { Particle Diameter } \\
\mathbf{d}_{\mathbf{p}}\end{array}$ & $0.25 \mu \mathrm{m}$ & $\begin{array}{c}\text { Reproduction Ratio } \\
\text { R } \\
\end{array}$ & $1 / 3.2$ \\
\hline $\begin{array}{c}\text { Pulse Separation } \\
\text { Time } D_{t} \\
\end{array}$ & $\begin{array}{l}300 \text { ns (Mixing case) } \\
700 \text { ns (Combustion) }\end{array}$ & $\begin{array}{c}\text { Field-of-View } \\
\text { Y } x \text { Z (undistorted) } \\
\end{array}$ & $37.9 \times 28.4 \mathrm{~mm}$ \\
\hline $\begin{array}{l}\text { Laser Sheet } \\
\text { Thickness T }\end{array}$ & $2.5 \mathrm{~mm}$ & $\begin{array}{c}\text { Camera Angle } \\
\theta \\
\end{array}$ & 35 degrees \\
\hline $\begin{array}{c}\text { Laser sheet width } \\
\text { w }\end{array}$ & $\begin{array}{l}47 \mathrm{~mm} \text { (collimated) } \\
\sim 28 \mathrm{~mm} \text { (after gate) }\end{array}$ & $\begin{array}{c}\text { Image plane angle } \\
\alpha\end{array}$ & 12.3 degrees \\
\hline $\begin{array}{c}\text { Laser Energy } \\
\text { (at optical train) }\end{array}$ & $106-111 \mathrm{~mJ} / \mathrm{pulse}$ & $\begin{array}{l}\text { Camera Lens } \\
\text { Aperture, f }\end{array}$ & $\mathrm{f} / 8$ and $\mathrm{f} / 11$ \\
\hline $\begin{array}{c}\text { Laser Sheet } \\
\text { Delivery } \\
\end{array}$ & $\begin{array}{c}\text { Through } \\
\text { Side-windows }\end{array}$ & $\begin{array}{l}\text { Max Expected } \Delta x_{p} \\
\text { (Particle Displace.) }\end{array}$ & $\begin{array}{l}8.05 \text { pixels (Mix) } \\
17.68 \text { (Combust) } \\
\end{array}$ \\
\hline $\begin{array}{c}\text { Camera Viewing } \\
\text { Arrangement }\end{array}$ & $\begin{array}{c}\text { Forward Scatter / } \\
\text { Back Scatter }\end{array}$ & $\begin{array}{c}\text { Expected } \\
\text { Sub-region size, } \mathrm{L}_{\mathbf{I}}\end{array}$ & $32 \times 32$ pixels \\
\hline
\end{tabular}


set to allow axial velocities between 0 and $1200 \mathrm{~m} / \mathrm{s}$. A larger area of the flowfield had to be masked to eliminate incorrect vectors and this resulted in a smaller area of useful data than combustion at $\mathrm{X} / \mathrm{H}=12$. However, considering the large background noise of the images at $\mathrm{X} / \mathrm{H}=15$, useful results over that small area were considered a success. 


\section{Chapter 5: SPIV Experimental Results, Combustor Flowpath 1}

The Stereoscopic Particle Image Velocimetry (SPIV) technique was implemented at two separate locations in DMSJ combustor Flowpath 1. One of these measurement planes was located at $\mathrm{X} / \mathrm{H}=10$ in the Test-section of Flowpath 1, that is, at $6.35 \mathrm{~cm}$ downstream of the base of the ramp fuel injector. The second plane was located downstream of the exit of the Extender. Chapter 5 contains SPIV measurements at these planes in Flowpath 1, as well as quantities derived from the velocity measurements. This chapter begins with a presentation of SPIV velocity measurements at $\mathrm{X} / \mathrm{H}=10$. Results for the case of fuel-air mixing are shown first, followed by measurements for the fuel-air combustion case. Uncertainty estimation is then discussed. Chapter 5 continues with SPIV measurements for two different fuel conditions at $\mathrm{X} / \mathrm{H}=58.6$, which is $5 \mathrm{~mm}$ downstream of the exit of the Extender. SPIV measurements at the $\mathrm{X} / \mathrm{H}=58.6$ plane are presented in a similar manner to those at the $\mathrm{X} / \mathrm{H}=10$ plane; fuel-air mixing results are shown, followed by fuel-air combustion measurements. Chapter 5 concludes with a qualitative comparison of SPIV velocity measurements to computational fluid dynamics (CFD) results.

\section{1 $\mathrm{X} / \mathrm{H}=10$ Measurement Plane Results}

SPIV results for the $\mathrm{X} / \mathrm{H}=10$ measurement plane are presented here along with quantities calculated from the three-component velocity measurements. Chronologically, the measurements at $\mathrm{X} / \mathrm{H}=10$ were the first SPIV measurements undertaken using DMSJ combustor Flowpath 1. Figure 5.1 shows a schematic and photograph of Flowpath 1 with the $\mathrm{X} / \mathrm{H}=10$ plane indicated. Physically, the DMSJ combustor is oriented 
vertically to allow easy access to the isolator, test section and extender for experiments. It should be noted for Figure 5.1 that the axial direction is the $\mathrm{x}$-direction just as in

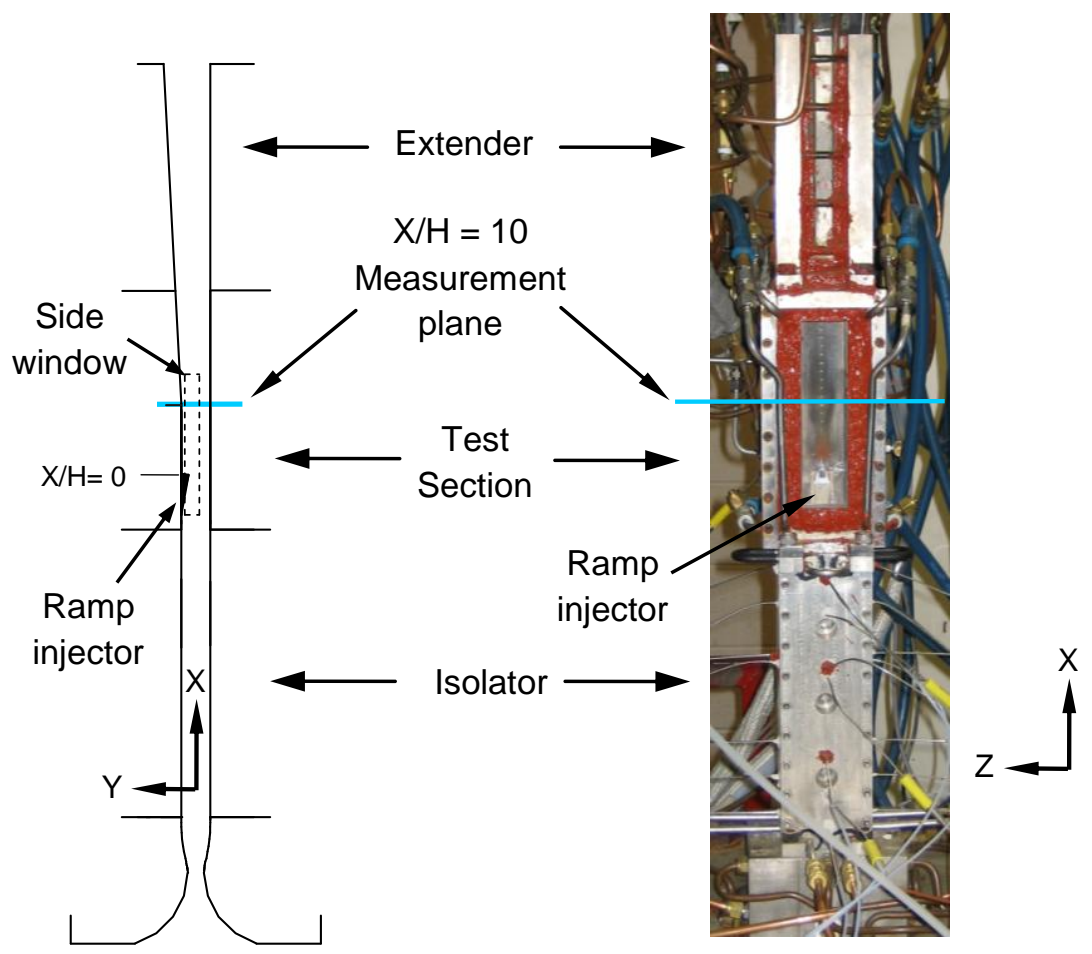

Figure 5.1: Flowpath 1 with SPIV measurement plane at $X / H=10$. a) Schematic, b) Photograph with injector visible through observation window

previous figures, but physically, the axial direction of the DMSJ combustor is vertical and therefore the $\mathrm{x}$-direction is oriented vertically in Figure 5.1. The positive y-direction is pointed toward the injector wall (the wall with the ramp fuel injector) and the positive z-direction points to the left to constitute a correct right-handed coordinate system. This coordinate system is consistent for all figures throughout this dissertation. Consequently, the schematic in Figure 5.1 is a view of the sidewall with the positive z-axis out of the page, while the picture in the figure is a view looking down the positive y-axis and the ramp injector can be viewed through the observation window of the test section. SPIV configuration parameters and experimental details for the $\mathrm{X} / \mathrm{H}=10$ plane measurements 
are presented first along with a summary of data processing, followed by results for fuelair mixing and then results for the fuel-air combustion case.

\subsubsection{Fuel-air Mixing Results, plane at $\mathrm{X} / \mathrm{H}=10$}

The SPIV technique was applied to DMSJ combustor Flowpath 1 for mixing of hydrogen and air at an equivalence ratio of $\Phi=0.26$ without combustion, i.e. measurements obtained prior to igniting the flow. Three-component (3C) velocity measurements were obtained and Figure 5.2 shows an average velocity field of 3C velocity vectors obtained by averaging 708 instantaneous results. The vectors of Figure

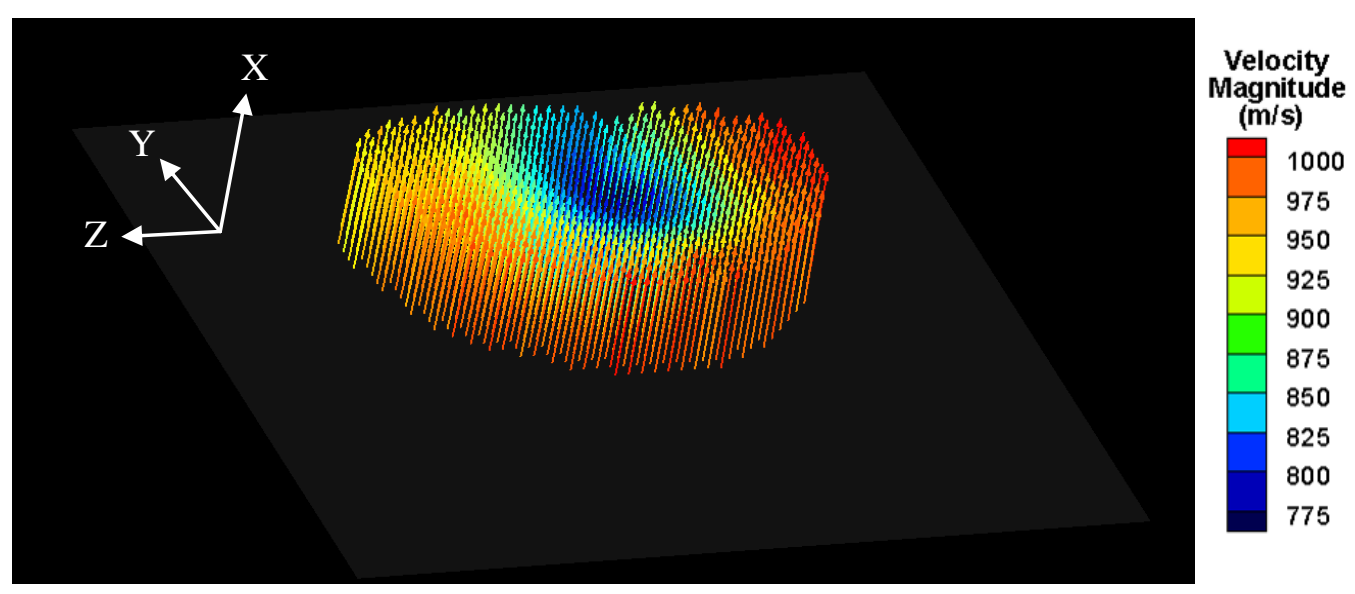

Figure 5.2: Average three-component (3C) velocity vectors from SPIV measurements for Fuel-air Mixing case at $\mathrm{X} / \mathrm{H}=10$

5.2 are colored to show the $3 \mathrm{C}$ velocity magnitude and the vector arrows mostly point along the $\mathrm{x}$-axis indicates the large axial component of the flow. However, some tilt of the vectors away from the $\mathrm{x}$-axis means that a significant in-plane velocity component is present. Figure 5.3 shows four different plots of the time-average velocity field for the fuel-air mixing case at $\mathrm{X} / \mathrm{H}=10$. Parts (a) and (d) of Figure 5.3 indicate $3 \mathrm{C}$ velocity magnitude and in-plane vectors, respectively. The axial velocity component is shown in part (b) of the figure and the in-plane velocity magnitude of the time-average velocity 
field is shown as color contours in part (c). For clarity in the figure, color velocity contours illustrating the magnitude of the $3 \mathrm{C}$ vectors are presented in part (a) rather than the $3 \mathrm{C}$ vectors themselves. In each $2 \mathrm{D}$ plot of SPIV results for $\mathrm{X} / \mathrm{H}=10$, the injector

a) Average $3 \mathrm{C}$ Velocity Field

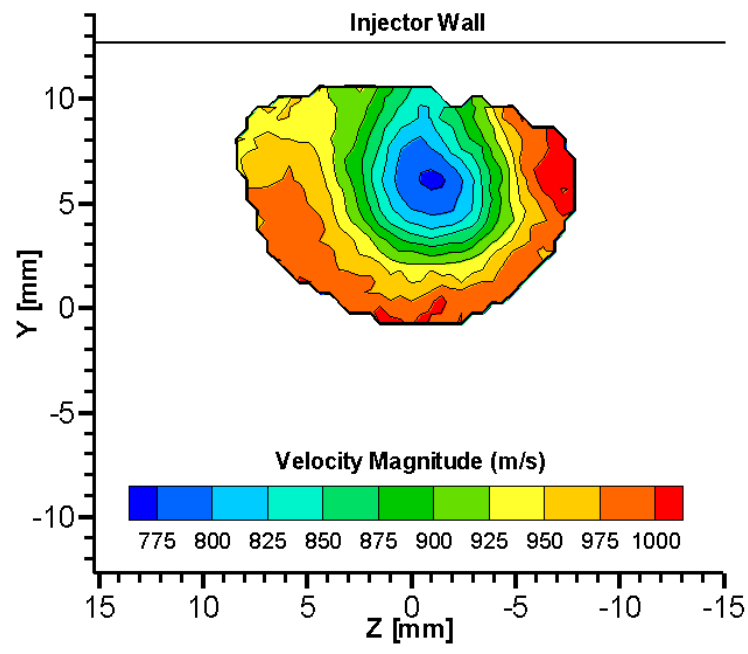

c) Average In-plane Velocity Magnitude

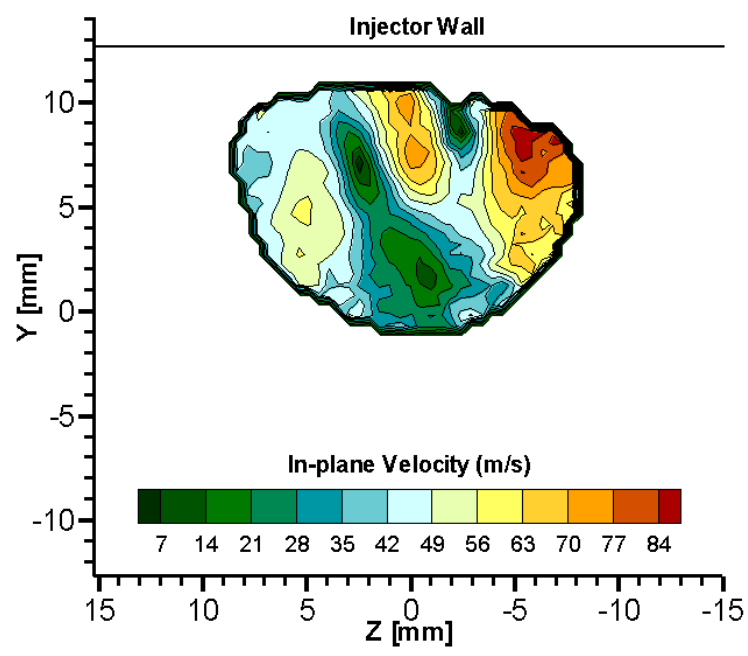

b) Average Axial Velocity

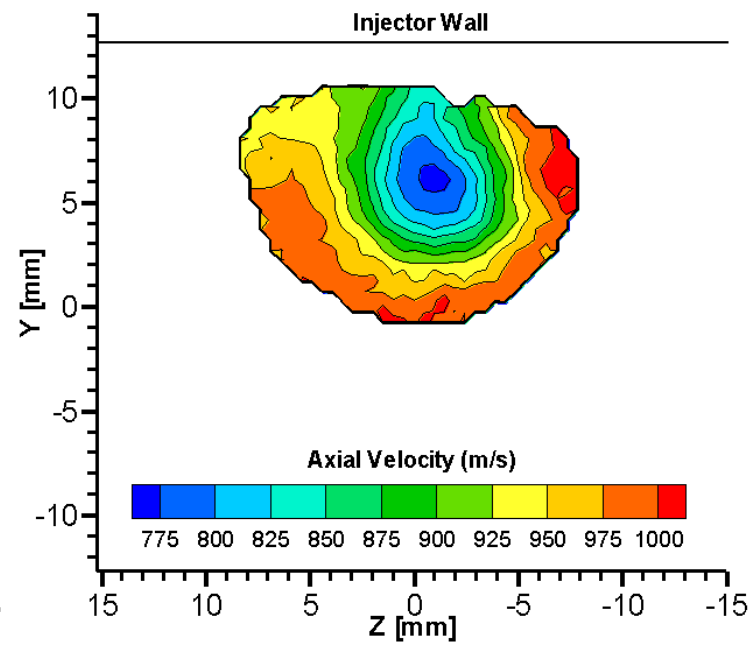

d) Average In-plane Velocity Vectors

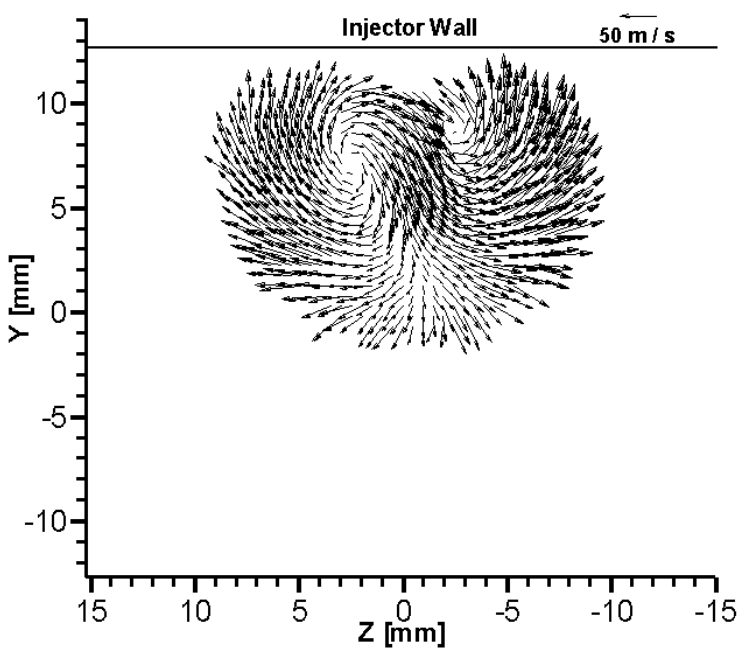

Figure 5.3: $\Phi=0.26$, Fuel-air Mixing case at $\mathrm{X} / \mathrm{H}=10$, Fuel-seeding only Average Velocity fields: a) 3C Velocity Magnitude $\left(u^{2}+v^{2}+w^{2}\right)^{1 / 2}$, b) Axial Velocity, c) In-plane Velocity Magnitude $\left(v^{2}+w^{2}\right)^{1 / 2}$, d) In-plane Velocity Vectors

wall of the tunnel is located along the top portion at a distance of $12.7 \mathrm{~mm}$ from the origin. Moreover, all of these 2D plots show the measurement plane as seen from above, that is, with the positive $\mathrm{x}$-axis out of the page. It should be noted that the y-axis of each 
plot extends beyond the injector wall because the camera filed-of-view was slightly larger than the cross-section of the tunnel in the y-direction. The physical width (z-direction) of the combustor duct is actually $38.1 \mathrm{~mm}$, extending from $-19.05 \mathrm{~mm}$ to $+19.05 \mathrm{~mm}$ along the z-axis. However, only the fuel plume was seeded in these experiments and it did not extend beyond $+/-15 \mathrm{~mm}$ in the $\mathrm{z}$-direction in any instantaneous results for either fuel-air mixing or combustion cases. It was therefore decided to plot these results with a smaller $\mathrm{z}$-axis so that the data would be larger in the figures and more easily viewed, but it must be remembered that the duct width is slightly larger than shown in the plot.

Part (a) of Figure 5.3 illustrates the measured 3C velocity magnitude using color contours just as the color of vectors showed the $3 \mathrm{C}$ velocity magnitude in Figure 5.2. At the exit of the facility nozzle, the velocity is predicted as approximately $1035 \mathrm{~m} / \mathrm{s}$ based on isentropic calculations for a Mach 2 nozzle for the case of fuel-air mixing, namely $\gamma=$ 1.4 and $\mathrm{T}_{0}=1200 \mathrm{~K}$. Some decrease in velocity between the nozzle exit and the testsection is expected due to frictional effects in the isolator section. Figure 5.3 clearly shows that the measured velocity magnitude at $\mathrm{X} / \mathrm{H}=10$ (in the test section) for the fuelair mixing case ranges from $770 \mathrm{~m} / \mathrm{s}$ to $1020 \mathrm{~m} / \mathrm{s}$, which is consistent with the velocity predicted from theory. A more interesting picture of the flow results when examining the cross-plane velocity vectors of the time-averaged vector field as illustrated in Figure 5.3 part (d). A reference vector has been included to indicate the length of the vector arrow corresponding to a velocity of $50 \mathrm{~m} / \mathrm{s}$ in the cross-plane. The shape of the seeded fuel plume is clearly evident and this is consistent with that expected from the work of others. $^{28,15}$ The predominant features in this cross-plane are the two counter-rotating vortices, induced by the ramp fuel injector. Examination of Fig. 5.3 (d) shows that the 
two counter-rotating vortices are contained within an area that is approximately $10 \mathrm{~mm} \mathrm{x}$ $14 \mathrm{~mm}$ in the $\mathrm{y}$ and $\mathrm{z}$ directions, respectively. However, the SPIV results indicate the two vortices are not symmetric about the tunnel y-axis (centerline perpendicular to the injector wall) and are instead symmetric about a line that is rotated by approximately 20 degrees from perpendicular to the injector wall.

The asymmetry of the two measured ramp-induced vortices is an interesting result and should be discussed briefly. The flowfield of the DMSJ combustor used for these experiments is quite complicated due to characteristics such as boundary layer separation, fuel injection and reflected shocks. Factors inherent to the flowfield itself could explain the noted vortex asymmetry. Moreover, the facility fabrication and assembly could have contributed to the asymmetric nature of the ramp-induced vortices. Therefore, some reasons for the measured asymmetry could be: asymmetric boundary layer separation, slight asymmetry in the facility nozzle profile, misalignment between the nozzle and combustor duct, misalignment of the ramp fuel injector, and asymmetry in the temperature profile provided by the facility heater. It is not clear which of these possible factors caused the asymmetry of the vortices, but such asymmetry is common in supersonic tunnels with ramp injectors. Previous velocity measurements of a cold supersonic mixing flow with a ramp fuel-injector by Donohue and $\mathrm{McDaniel}^{33}$ using Planar Laser-Induced Iodine Fluorescence (PLIIF) showed similar asymmetry of the two counter-rotating vortices. However, only qualitative comparison of the SPIV and PLIIF measurement results is possible because the PLIIF results were obtained for a ramp injector with significantly different aspect ratio than the injector used in the current DMSJ combustor study. Furthermore, other measurement techniques have provided 
evidence of the asymmetric nature of the vortices of the DMSJ combustor used in this study. ${ }^{28}$ There is also nothing inherent to the SPIV technique that would cause such an asymmetry in the measurements. For these reasons, it is believe that there is actually some asymmetry in the flowfield and this type of asymmetry is common to such experimental facilities.

Comparison of parts (a) and (b) of Fig. 5.3 clearly shows that nearly the entire 3C velocity magnitude is due to the large axial component that dominates this flow. This was also indicated by the direction of $3 \mathrm{C}$ velocity arrows of Fig. 5.2. Figure 5.3 (c) presents in-plane velocity magnitude as color contours and the in-plane velocity can be more clearly understood with this plot than with the plot of in-plane vectors.

Along the tunnel centerline (y-direction at $\mathrm{z}=0$ ), the SPIV results indicate axial velocities ranging from $780 \pm 18 \mathrm{~m} / \mathrm{sec}$ to $995 \pm 23 \mathrm{~m} / \mathrm{sec}$. Goyne et al. previously applied the 2D PIV technique to measure the axial flow velocity of a similar DMSJ combustor using one PIV 10-30 camera and a laser sheet oriented parallel to the tunnel xaxis. ${ }^{28}$ For the case of hydrogen/air mixing at $10 \mathrm{H}$ downstream of the fuel injector, Goyne et al. reported axial velocities between approximately $800 \mathrm{~m} / \mathrm{sec}$ and $1050 \mathrm{~m} / \mathrm{sec}$ along the tunnel y-axis. ${ }^{28}$ This is in fairly good agreement with the axial velocity results obtained using the SPIV configuration discussed above. However, only a general comparison can be made between the results of Goyne et al. and the SPIV results obtained in the current study because an isolator section was not present in the DMSJ used by Goyne et al. Comparing the axial velocity contours of Fig. 5.3(b) with the crossplane velocity magnitude of Fig. 5.3(c) gives evidence of the large axial velocity component that dominates this flow. Furthermore, previous work ${ }^{15}$ indicates that the 
cross-plane vectors of this flow are expected to be approximately $10 \%$ of magnitude of the axial velocity components. Examination of the in-plane velocity magnitude of Fig. 5.3(c) and the axial velocity of part (b) shows the in-plane magnitude is approximately $6 \%$ to $9 \%$ of the axial velocity at locations throughout most of the fuel plume. The only exceptions to this are at the center of each vortex and in the area near $(z, y)=(-1,2)$ where the in-plane velocity is nearly zero. As mentioned, previous computational work ${ }^{15}$ indicates that the in-plane velocity magnitude is expected to be approximately $1 / 10$ of the axial magnitude and the SPIV velocity measurements show that the in-plane magnitude is indeed on the order of $1 / 10$ of the axial velocity magnitude.

Figure 5.4 shows the average velocity field for the fuel-air mixing case at $\mathrm{X} / \mathrm{H}=10$ and two of the 708 instantaneous velocity field results that make up the averaged velocity field. In-plane vectors are shown in the plots of the left column with the corresponding $3 \mathrm{C}$ velocity magnitude in the plots of the right column. Each of the instantaneous velocity field measurements may not contain data for the entire flow field because areas of low seed particle density do not yield reliable velocity measurements, particularly along the edge of the fuel plume. However, when averaging a set of instantaneous velocity measurements, the resulting velocity field contains vectors throughout the seeded fuel plume area. The instantaneous fields in Fig. 5.4 clearly show the presence of the two counter-rotating vortices and $3 \mathrm{C}$ velocity magnitudes near those of the average field. It was found that the vortices moved (or walked) toward and away from the centerline of the tunnel in each of the instantaneous results and also walked slightly toward and away from the injector wall due to the highly turbulent nature of this flow. This vortex motion is illustrated in the two instantaneous results of Fig. 
Average In-plane Velocity Vectors

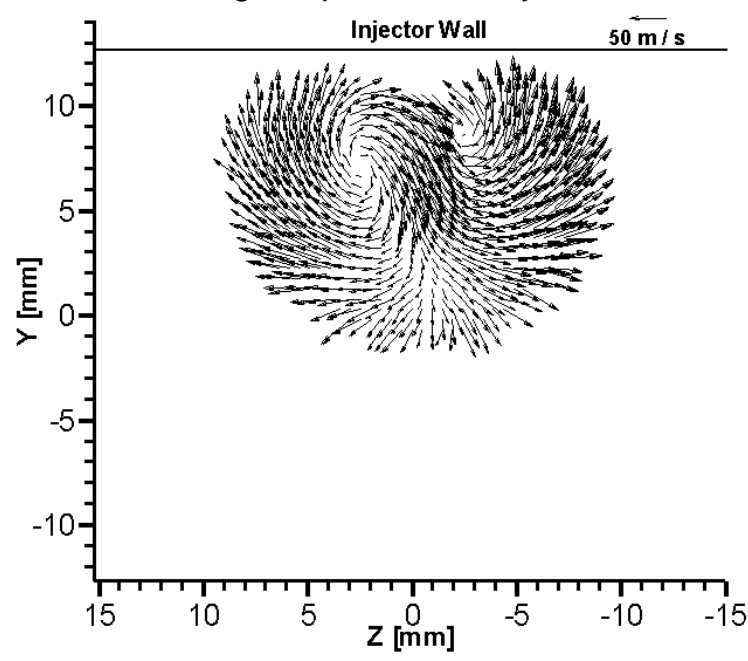

Instantaneous In-plane Velocity Vectors

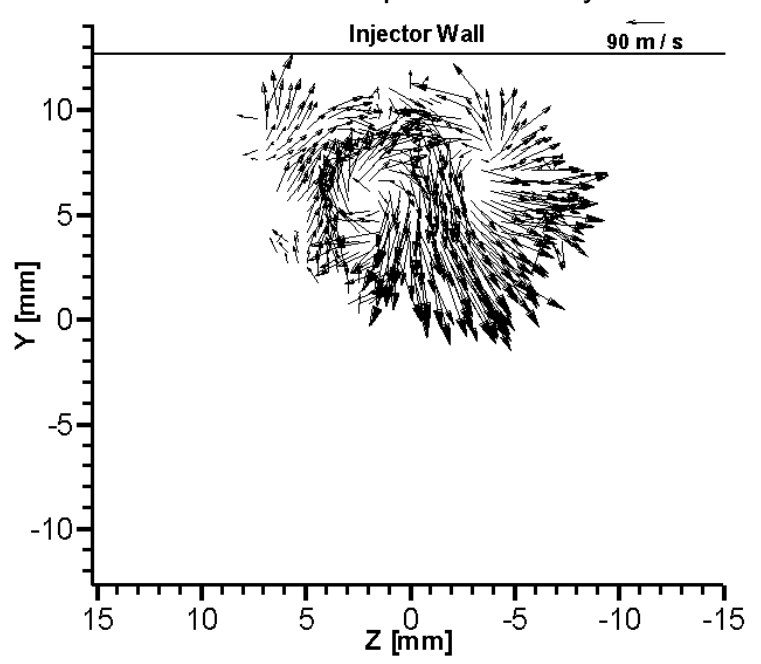

Instantaneous In-plane Velocity Vectors

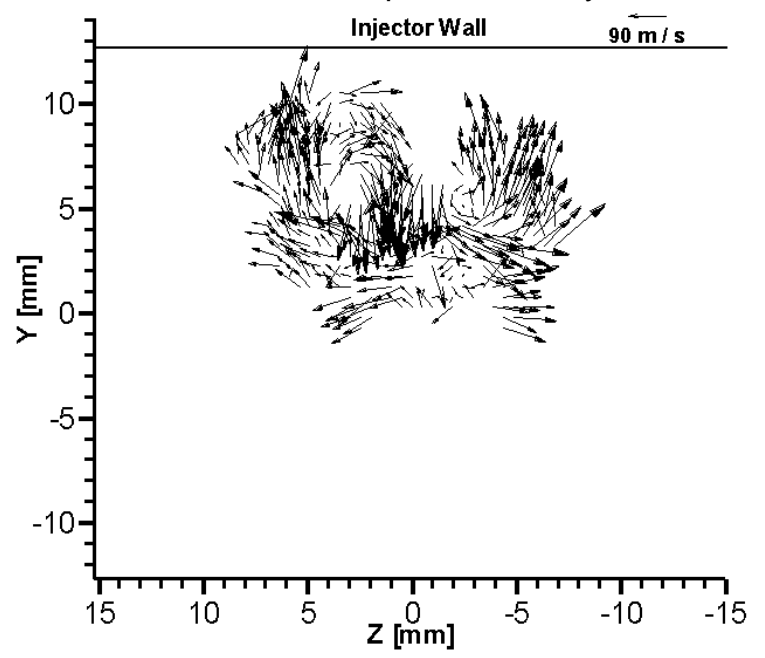

Average $3 C$ Velocity Field

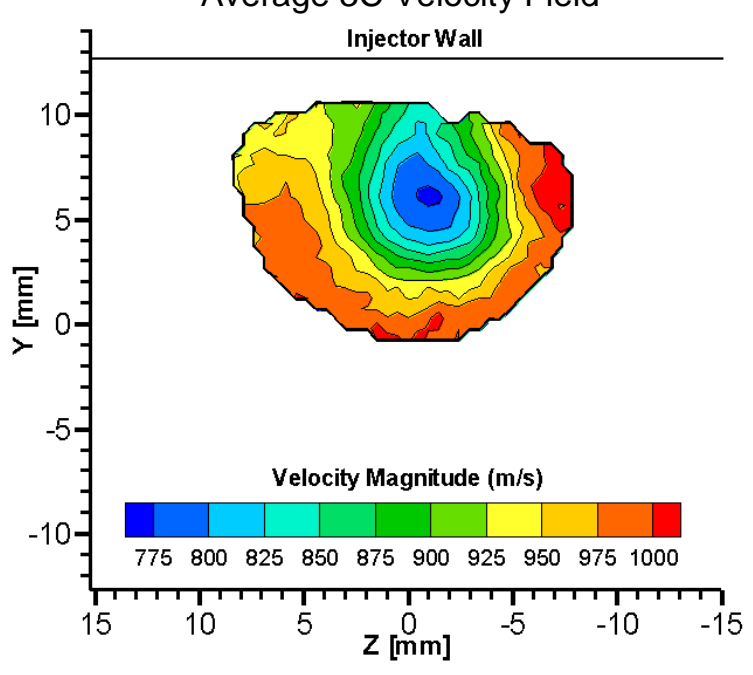

Instantaneous 3C Velocity

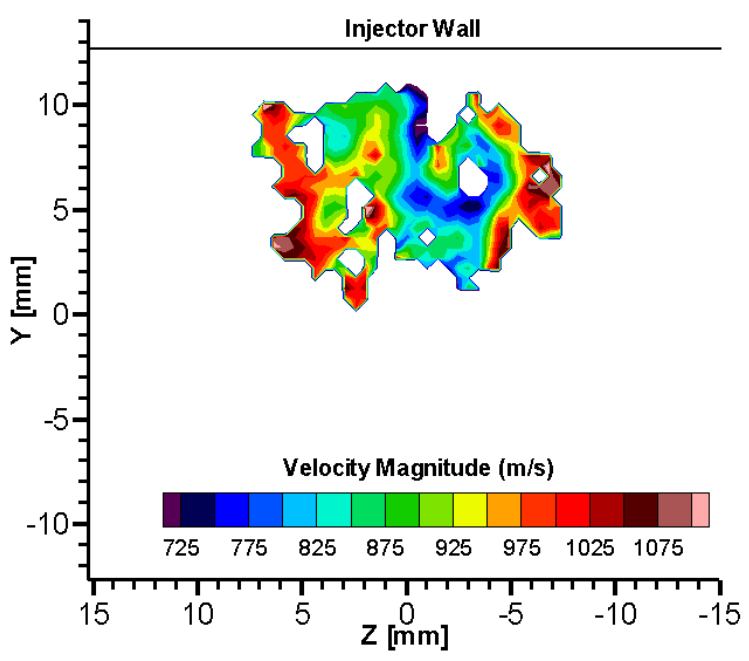

Instantaneous 3C Velocity

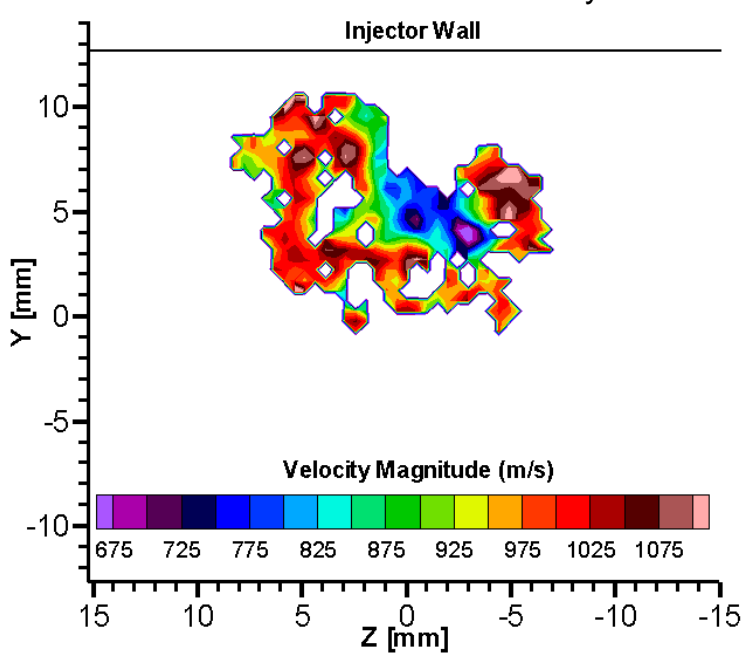

Figure 5.4: Average and Instantaneous velocity fields, Fuel-air Mixing case at $\mathrm{X} / \mathrm{H}=10, \Phi=0.26$ 
5.4. However the vortices were located in one orientation more often than any other and this lead to the predominate asymmetry seen in the average velocity field. Furthermore, the magnitude of the in-plane velocity is larger in some areas of the instantaneous results than in the average result. However, due to the walk of the vortices and also the variation of in-plane velocity magnitude in the instantaneous results, the largest magnitude of the in-plane velocity in the average result is lower than the maximum magnitude in the instantaneous results.

The time-averaged results were obtained by averaging 708 instantaneous velocity fields. However, as seen in the instantaneous velocity fields of Fig. 5.4, each instantaneous result does not contain vectors at every location and therefore, 708 velocity fields does not mean that 708 velocity vectors were averaged to obtain the average velocity at each sub-region across the field. Average velocity vectors for sub-regions located along the edge of the fuel plume resulted by averaging 50 or more instantaneous vector results, while vector counts near $80 \%$ of the total number of instantaneous results (708) contributed to the calculation of average vectors for sub-regions located near the center of the measurement region. Figure 5.5 depicts both the average velocity field and a plot of the number of vectors (vector count) that contributed to the average at each location. As discussed above, filters were used to validate velocity vectors generated during processing of the SPIV images and therefore high confidence exists for the instantaneous velocity measurements. Furthermore, because approximately 550 instantaneous velocity vector results contributed to the average velocity vectors near the core of the measurement area, there is high confidence for the average velocity vectors in the core region. More than 50 instantaneous vectors contributed to average vectors at the 
far edge of the measurement region and therefore, lower confidence exists for the average vectors at the periphery as opposed to average vectors in the core region. However, the magnitude of the average velocity vectors at far edge of the measurement area for the mixing case is near the velocity magnitude expected for the freestream and that fact

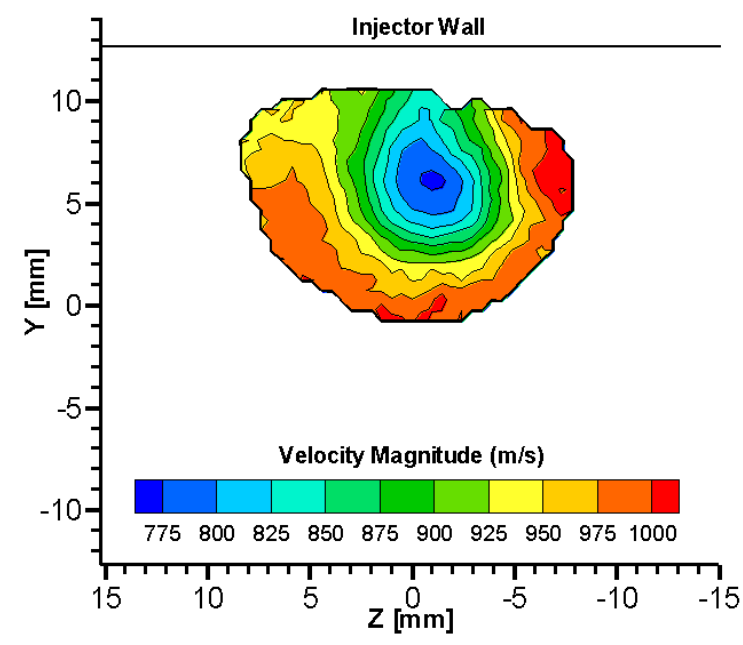

(a) Average of All (708) Vector Fields [3C Velocity magnitude]

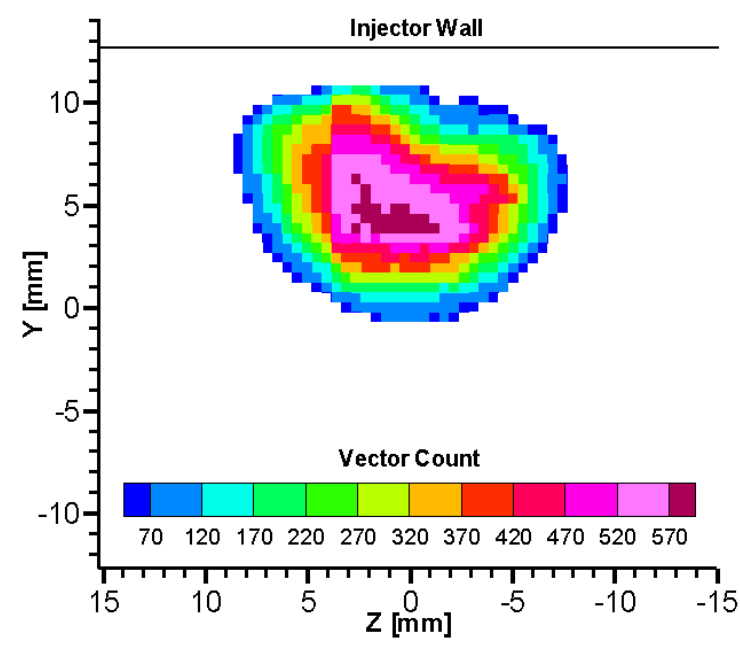

(b) Number of Vectors Averaged for Each Sub-region

Figure 5.5: $\Phi=0.26$, Fuel-air Mixing case at $\mathrm{X} / \mathrm{H}=10$;

(a) Average $3 \mathrm{C}$ velocity field, and (b) Number of vectors averaged at each location

considerably boosts the confidence in those average vector results. A similar result is expected for the fuel-air combustion case.

The question of how many instantaneous velocity fields are needed for a correct average should be addressed here. A reasonable method for determining the minimum number of instantaneous results needed for a good average (statistical convergence) is to use the measurement set itself and plot averages that have been determined from increasing numbers of instantaneous results. This can be done either by plotting entire average fields that have been determined using more and more instant results, or by choosing a single location of the field and constructing a line graph of average values at 
that location versus number of samples used to compute the average. The plots are then visually examined to qualitatively see at what point the average stops changing. Giuni et al. ${ }^{39}$ used this method for determining statistical convergence by plotting velocity fluctuations (RMS) contours for the entire field and also by constructing a line graph of average tangential velocity profiles as a function of number of samples. Son et al. ${ }^{40}$ chose a single location (location of highest fluctuations) and plotted the average value for streamwise velocity and turbulence intensity at that location as functions of number of PIV results. In the work of Giuni et al. and the work of Son et al., visual inspection of the contour plots or line graphs clearly showed the number of samples needed for statistical convergence.

Figure 5.6 presents $3 \mathrm{C}$ velocity contours determined for increasing numbers of SPIV samples (vector fields) at $\mathrm{X} / \mathrm{H}=10$ for fuel-air mixing. Plotting contours for the entire field was the method chosen to determine statistical convergence for the current work. The vector count for each plot in Fig. 5.6 has approximately the same distribution as shown in Fig. 5.5 for the field resulting from averaging the entire data set. Vector count at the center of the plume in each plot of Fig. 5.6 is $\sim 80 \%$ of the number of fields contributing to the average, while the vector count is at a minimum at the far edge of the plume. A threshold of $10 \%$ was used when calculating each average plot so that an average vector is only included, at a give location, if the number of vectors contributing to the average was at least $10 \%$ of the number of instantaneous vector fields. For example, for the field resulting from averaging 100 vector fields, at least 10 vectors at a given location were necessary in order for an average vector to be included. The only exception to the $10 \%$ threshold criteria was the field obtained by averaging the 

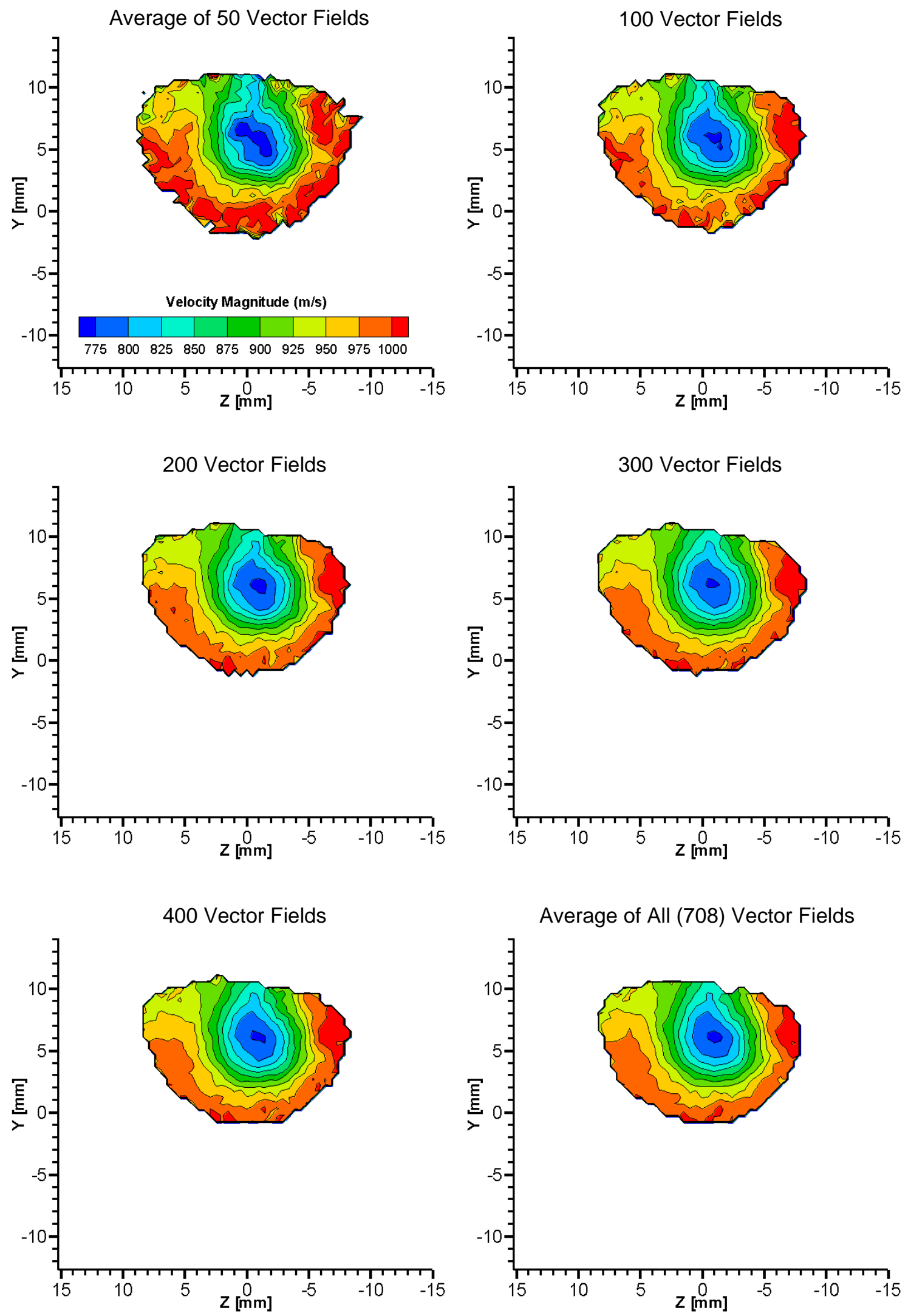

Figure 5.6: Statistical convergence of $3 \mathrm{C}$ velocity, Fuel-air Mixing, $\mathrm{X} / \mathrm{H}=10, \Phi=0.26$ 
entire set of 708 vector fields for fuel-air mixing. For the average of the full set, a threshold of 50 vectors was used. Examining Fig. 5.6, the expected trend for statistical convergence is evident in that plots become more and more self-similar with increasing numbers of instantaneous results contributing to the average. Qualitatively, the plots appear to be nearly the same for an average of 300 instantaneous fields or more, indicating that 300 instantaneous vector fields is sufficient for statistical convergence of $3 \mathrm{C}$ velocity for the case of fuel-air mixing in the test section of Flowpath 1 . However, larger sample sizes are necessary for statistical convergence of flow quantities involving the fluctuation of the velocity, such as turbulence intensity or Reynolds stresses. Based on this result for statistical convergence, the average velocity result from 708 vector fields is clearly converged and the 708 velocity fields of the set were used to two determine other flow quantities.

Flow quantities of rotation and turbulence intensity have been calculated using the SPIV velocity measurements and Fig. 5.7 presents fluid rotation for the case of fuel-air mixing at $\mathrm{X} / \mathrm{H}=10$, with counter-clockwise rotation represented using positive values. Rotation was calculated using a preprogrammed subroutine that was part of the LaVision DaVis 7.1 software package. Fluid rotation for each sub-region is determined using the magnitude of the tangential velocity component of the four neighbors that surround the sub-region. In essence, this is the circulation around a closed loop where the loop is the perimeter of one sub-region. In Fig. 5.7, the rotation due to the two counter-rotating vortices is clearly evident in the upper portion of the plume, near the injector wall and the rotation of the vortices represents the largest magnitude of fluid rotation across the field. It is also clear that the vortices are small in diameter and well-organized. These 
characteristics of the vortices can also be seen in the velocity vector plots, such as Fig. 5.3 (d), however, the rotation plot of Fig. 5.7 more clearly shows the strength and size of the vortices. Rotation of approximately 50,000 radians/sec near the core of the vortices corresponds to roughly 477,000RPM. Such a high rate of rotation is possible because the

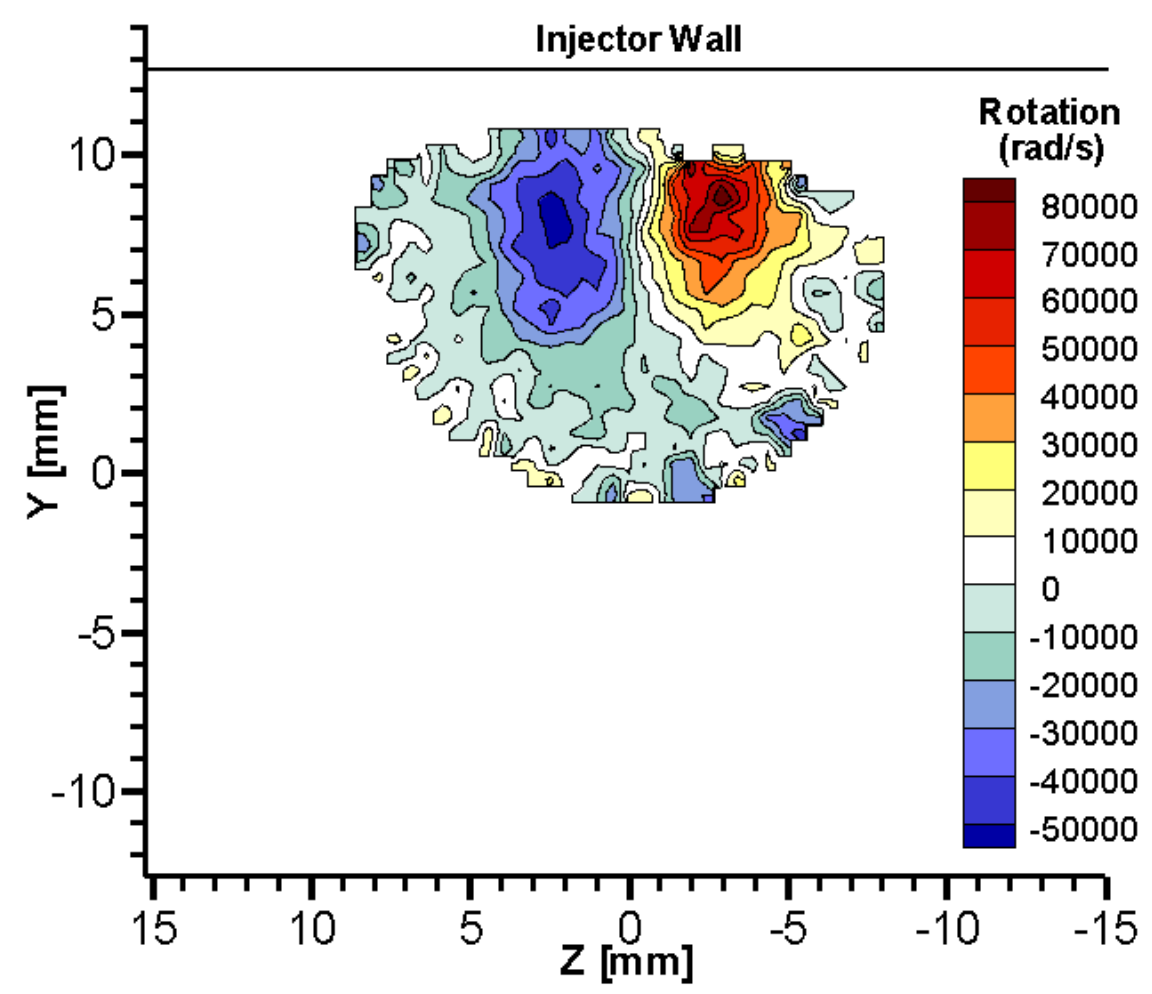

Figure 5.7: Degree of Fluid Rotation for Average SPIV result, $\Phi=0.26$, Fuel-air Mixing case at $\mathrm{X} / \mathrm{H}=10$.

core of each vortex is approximately $1 \mathrm{~mm}$ in diameter. At a radius of $0.5 \mathrm{~mm}$ and rotation of 50,000 radians/sec, the tangential velocity is $25 \mathrm{~m} / \mathrm{s}$, which is the velocity at that location as seen in Fig. 5.3(c). The vortices have also been visually observed during experiments with fuel off and were described as appearing like two ropes emanating from the sides of the ramp injector and having a high rate of rotating.

Figure 5.8 presents turbulence intensity information calculated from the 708 instantaneous velocity results for the fuel-air mixing case. Turbulence intensity was 
calculated by determining the RMS of the 3C velocity for each sub-region and normalizing by the magnitude of the average velocity of that sub-region. Turbulence

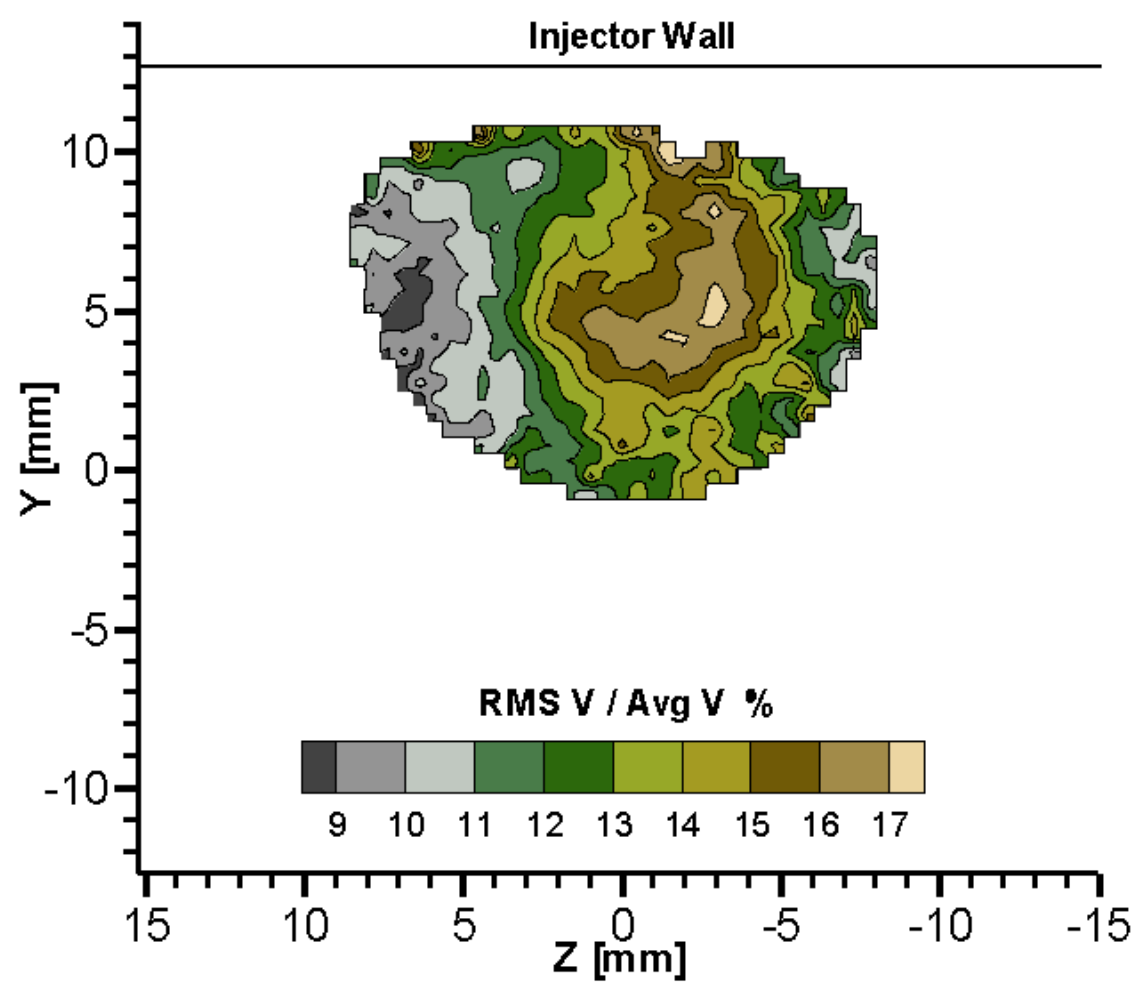

Figure 5.8: Turbulence Intensity (RMS of $3 \mathrm{C}$ Velocity normalized by Average velocity) of Fuel-air Mixing case at $\mathrm{X} / \mathrm{H}=10, \Phi=0.26$

intensity in the fuel-air mixing plume was found to be on the order of $14 \%$ across most of the plume and approached $17 \%$ near the center of the fuel-plume, adjacent to the two counter-rotating vortices.

\subsubsection{Fuel-air Combustion Results}

SPIV measurements of the flowfield at $\mathrm{X} / \mathrm{H}=10$ were also conducted for the case of fuel-air combustion, $\Phi=0.26$. The figures of section 5.2.3 contain the same type of plots for the fuel-air combustion case as the figures of section 5.2.2 did for the fuel-air mixing case. Figure 5.9 shows a three-dimensional view of average velocity vectors for 
the case of fuel-air combustion at $\mathrm{X} / \mathrm{H}=10$ colored for three-component (3C) velocity. Again, the vectors point nearly in line with the axial direction (x-axis) indicating the large

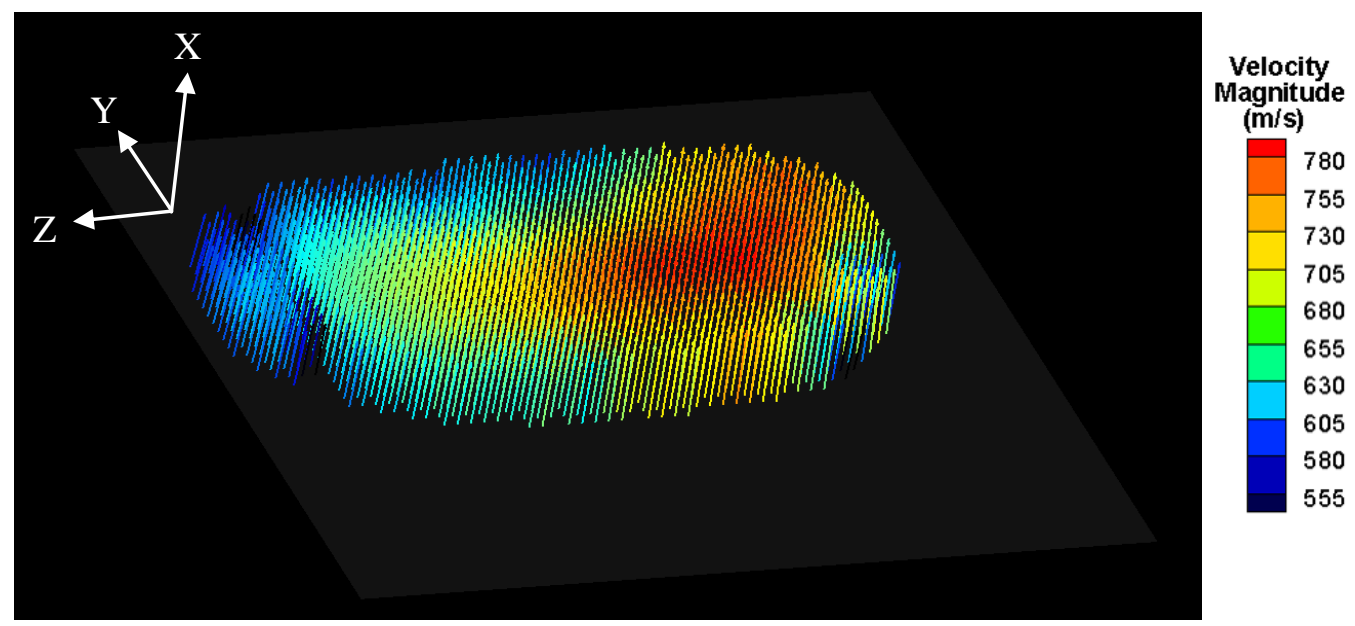

Figure 5.9: Average three-component (3C) velocity vectors from SPIV measurements for Fuel-air Combustion case at $\mathrm{X} / \mathrm{H}=10$

axial component of this flow. The tilt of vectors away from the $\mathrm{x}$-axis implies some inplane velocity component, but the magnitude of in-plane velocity is difficult to determine from the $3 \mathrm{D}$ vector plot. Figure 5.10 shows $3 \mathrm{C}$ velocity magnitude, axial velocity, inplane velocity magnitude and in-plane vectors. Figure 5.10 part (a) presents average $3 \mathrm{C}$ velocity magnitude as color contours in the $\mathrm{X} / \mathrm{H}=10$ plane instead of colored vectors as in Fig.5.9. These time-averaged velocity fields were obtained by averaging 775 instantaneous velocity field results. In the fuel-air combustion situation, Fig. 5.10(a) shows that the average measured $3 \mathrm{C}$ velocity is lower than in the case of fuel-air mixing without combustion and that result qualitatively matches the change in velocity due to heat addition that is predicted by theory. It should be noted that the two circular areas at the lower right of the field are not really areas of local low velocity. These circular areas, along with the vertical break in the flowfield at $\mathrm{z}=+12 \mathrm{~mm}$ are areas of incorrect velocity 
a) Average 3C Velocity Field

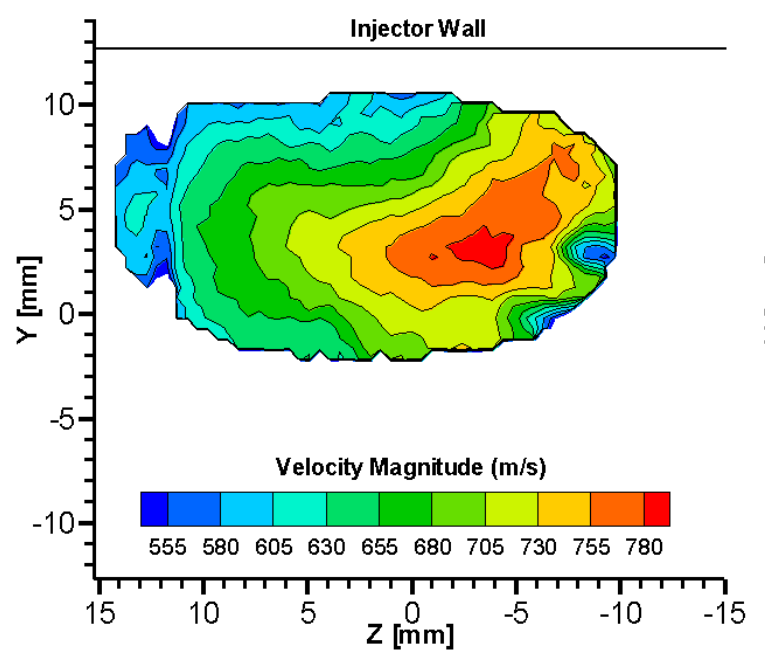

c) Average In-plane Velocity Magnitude

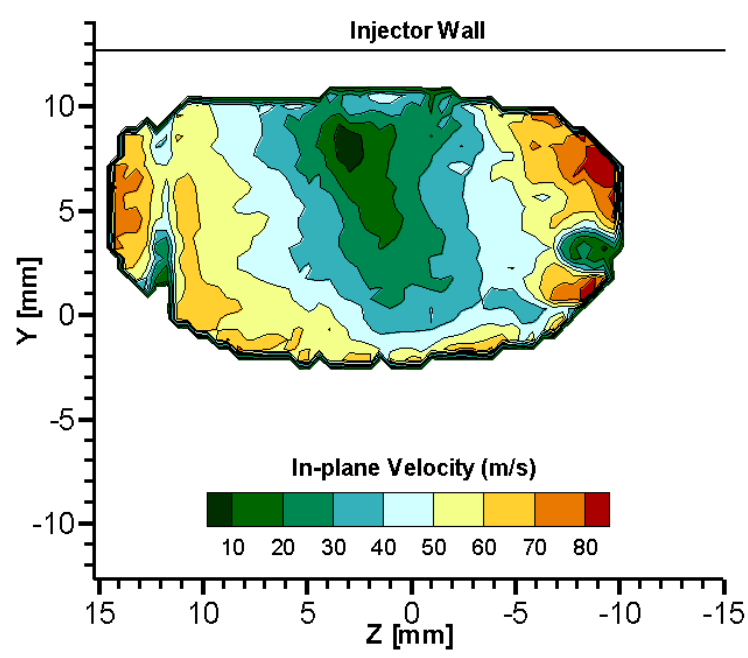

b) Average Axial Velocity

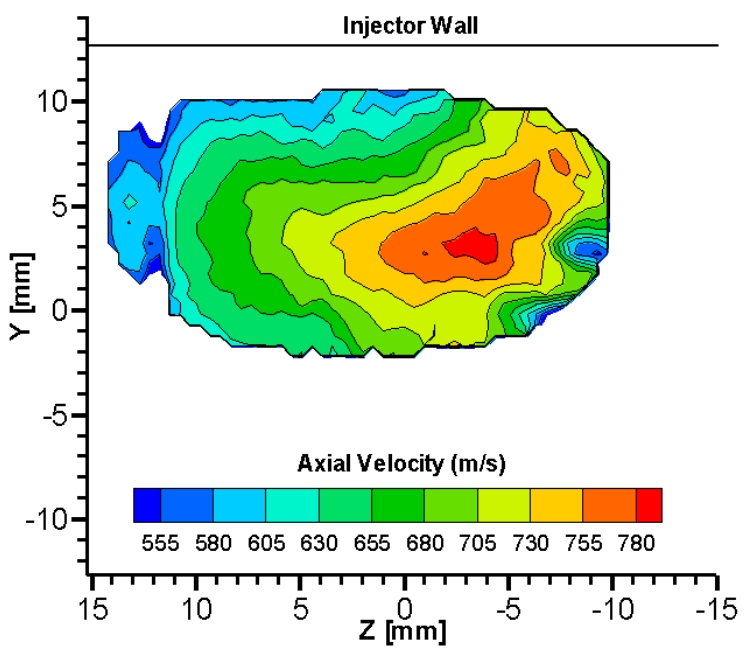

d) Average In-plane Velocity Vectors

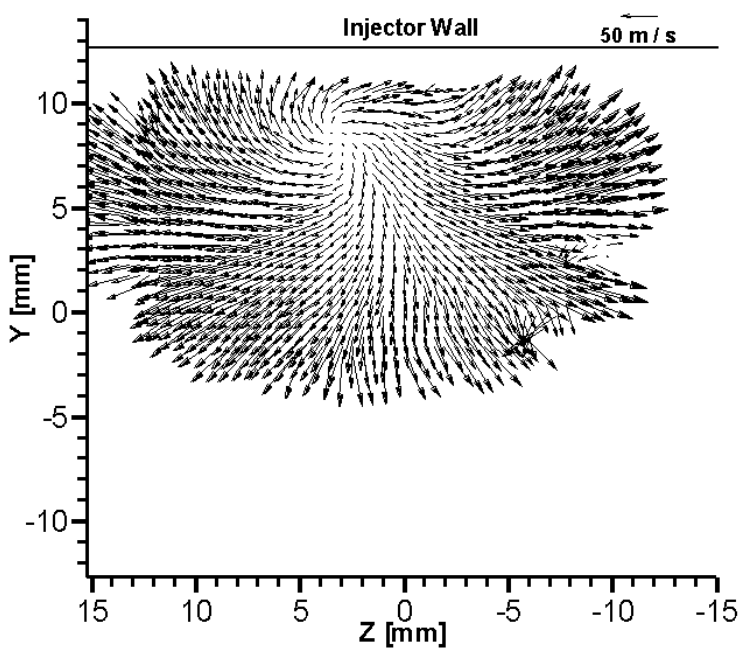

Figure 5.10: $\Phi=0.26$, Fuel-air Combustion at $\mathrm{X} / \mathrm{H}=10$, Fuel-seeding only Average Velocity fields: a) 3C Velocity Magnitude $\left(u^{2}+v^{2}+w^{2}\right)^{1 / 2}$, b) Axial Velocity, c) In-plane Velocity Magnitude $\left(v^{2}+w^{2}\right)^{1 / 2}$, d) In-plane Velocity Vectors

that arose from correlations of particles with laser reflections on the windows of the combustor. Figure 5.10 part (d) shows the in-plane velocity vectors of the average field for fuel-air combustion and the magnitude of the average vectors in the combustion case is lower than the in-plane magnitude for fuel-air mixing. Moreover, the two counterrotating vortices can again be viewed in Fig. 5.10(d) and again show a degree of asymmetry. In the figure, the left vortex is clearly visible and the influence of the right 
vortex is visible, but the center of the right vortex cannot be viewed. It appears that the right vortex has moved closer to the injector wall than in the fuel-air mixing case and the center of the right vortex could be missing in the figure because it is outside the measurement are defined by laser sheet. The vortices also appear to be less organized and weaker than the vortices in the fuel-air mixing case.

Along the tunnel centerline at $\mathrm{z}=0$, the SPIV measurements show axial velocities from $570 \pm 13 \mathrm{~m} / \mathrm{sec}$ to $765 \pm 18 \mathrm{~m} / \mathrm{sec}$ for the fuel-air combustion case. For the case of hydrogen/air combustion at $\mathrm{X} / \mathrm{H}=10$ downstream of the fuel injector, Goyne et al. reported axial velocities between approximately $320 \mathrm{~m} / \mathrm{sec}$ and $810 \mathrm{~m} / \mathrm{sec}$ along the tunnel centerline. ${ }^{28}$ Those previous measurements are also in fairly good agreement with the axial velocity results obtained in the current study using SPIV. Again, because of the absence of an isolator section in the work of Goyne et al., only a general comparison can be made between those previous results and the measurements from the current work. Comparing parts (b) and (c) of Fig. 5.10, the in-plane velocity magnitude again is shown to be approximately $10 \%$ of the axial velocity magnitude across the measurement area except near the vortices where the in-plane velocity is nearly zero.

Similar to the fuel-air mixing case, a minimum of 50 instantaneous velocity vectors contributed to the average vectors for sub-regions located along the edge of the fuel plume, while average vectors near the center of the fuel plume were determined by averaging approximately 700 instantaneous vector results, that is, approximately $90 \%$ of the 775 total instantaneous vector fields contributed a measurement to the average velocity at sub-regions located near the center of the measurement area. Figure 5.11 shows the average $3 \mathrm{C}$ velocity field and the number of instantaneous results that 
contributed to the average at each location. Confidence in vectors at the core of the measurement area and at the edge of the seeded region is similar to the confidence stated above for the fuel-air mixing case based on similar reasoning.

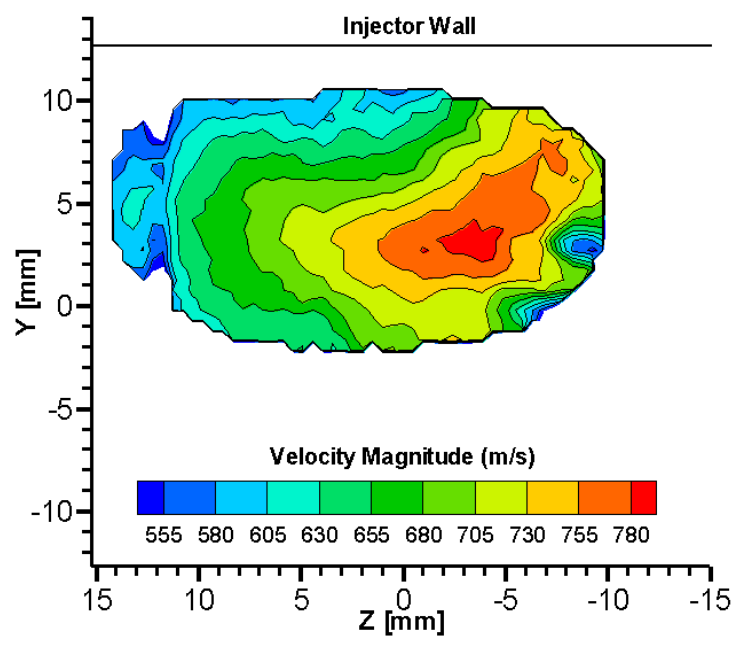

(a) Average of All (775) Vector Fields [3C Velocity magnitude]

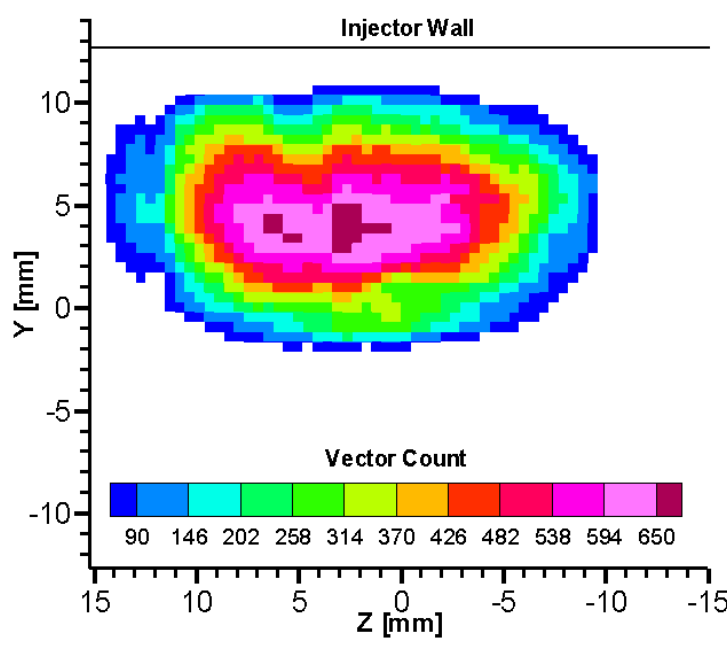

(b) Number of Vectors Averaged for Each Sub-region

Figure 5.11: $\Phi=0.26$, Fuel-air Combustion at $\mathrm{X} / \mathrm{H}=10$;

(a) Average $3 \mathrm{C}$ velocity field, and (b) Number of vectors averaged at each location

Plots to investigate statistical convergence were also created for the fuel-air combustion case and are shown in Figure 5.12. For the fuel-air combustion case, Fig. 5.12 indicates that statistical convergence occurs for most of the field with an average of 400 or more instantaneous. This means that slightly more instantaneous fields are required for statistical convergence of the fuel-air combustion case than for fuel-air mixing. Figure 5.13 shows the average velocity field and two of the 775 instantaneous velocity field results that make up the averaged velocity field. Again, vectors do not occur in all areas of each instantaneous velocity field result and only the fuel stream was seeded for this measurement plane. The vortices can also be viewed in the instantaneous results and appear to "walk" back and forth across the plane for the fuel-air combustion 

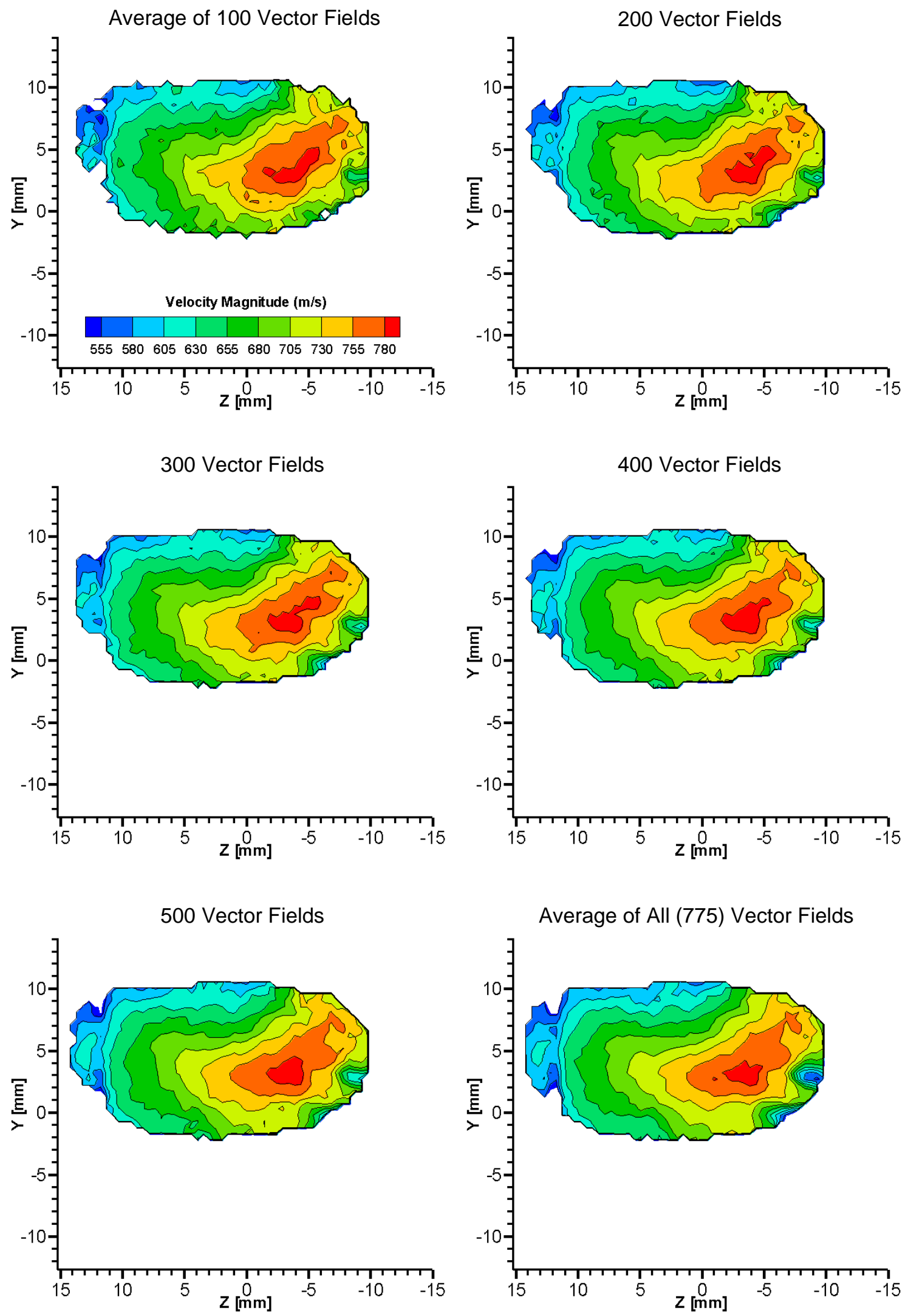

Figure 5.12: Statistical convergence of $3 C$ velocity, Fuel-air Combustion at $X / H=10, \Phi=0.26$ 

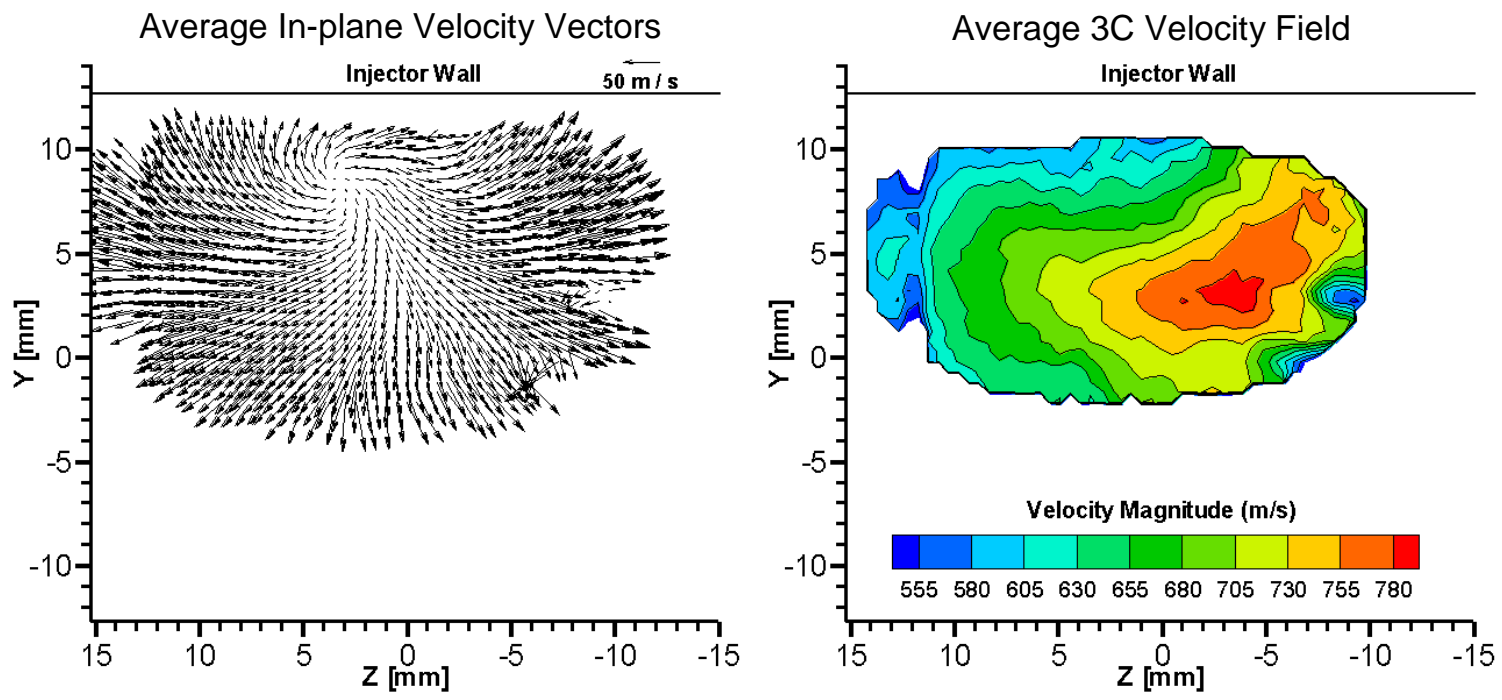

Instantaneous In-plane Velocity Vectors
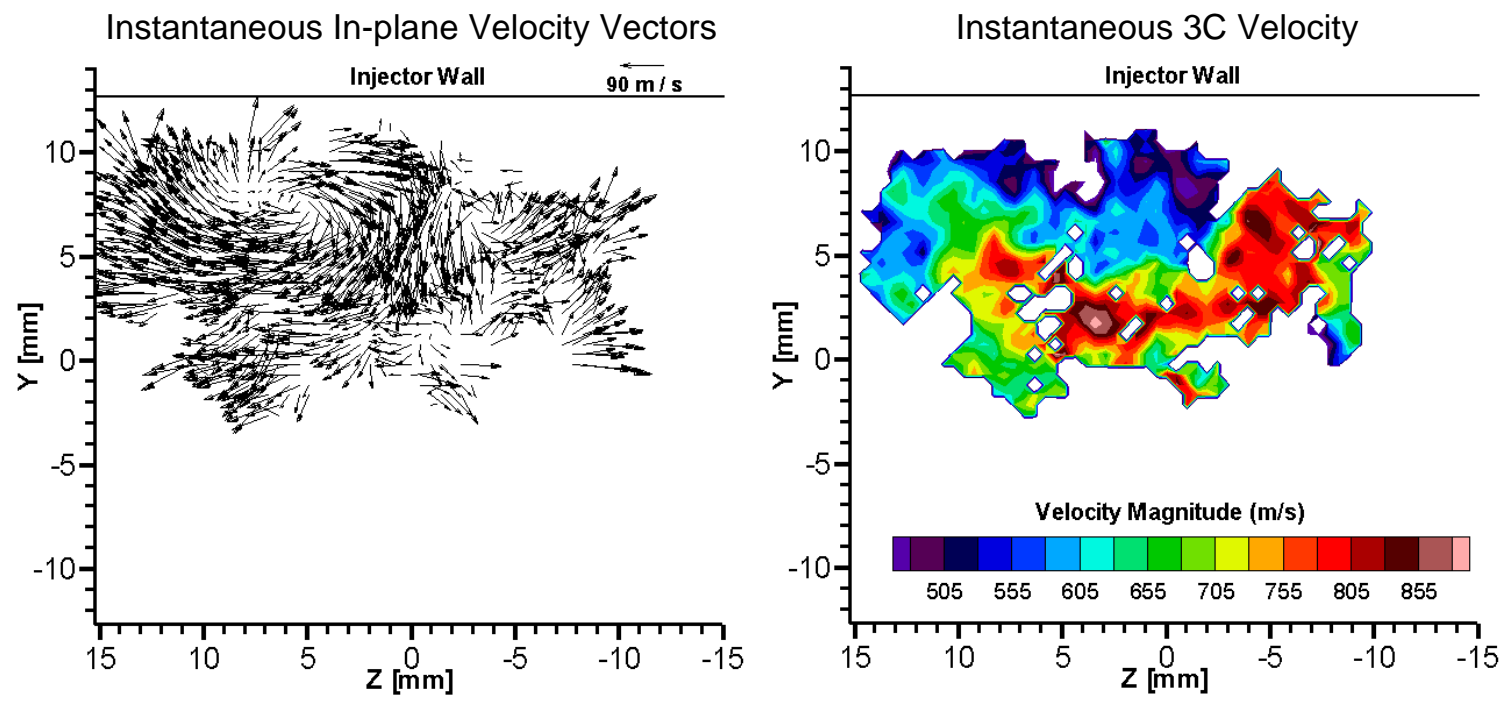

Instantaneous In-plane Velocity Vectors
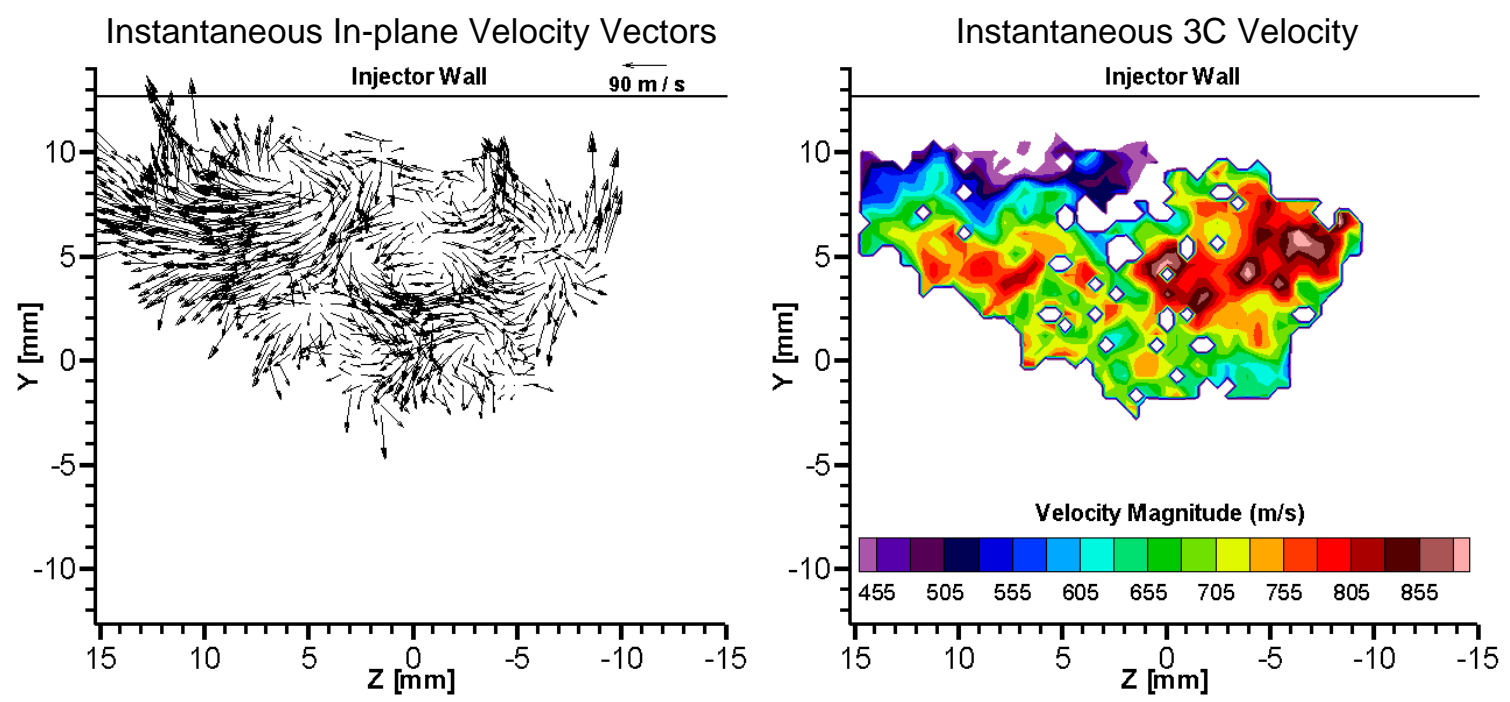

Figure 5.13: Average and Instantaneous velocity fields, Fuel-air Combustion at $X / H=10, \Phi=0.26$ 
case more than the in the fuel-air mixing case presented above. This motion of the vortices again causes the average in-plane velocity magnitude to be lower, in most areas, than the in-plane magnitude of the instantaneous fields as seen in Fig. 5.13.

Rotation for the fuel-air combustion case is presented in Fig. 5.14. The rotation plot allows the two vortices to be seen clearly and the magnitude of the rotation is lower for the fuel-air combustion case than for fuel-air mixing. The vortices are also spread over more of the plume and are weaker than the vortices of the fuel-air mixing.

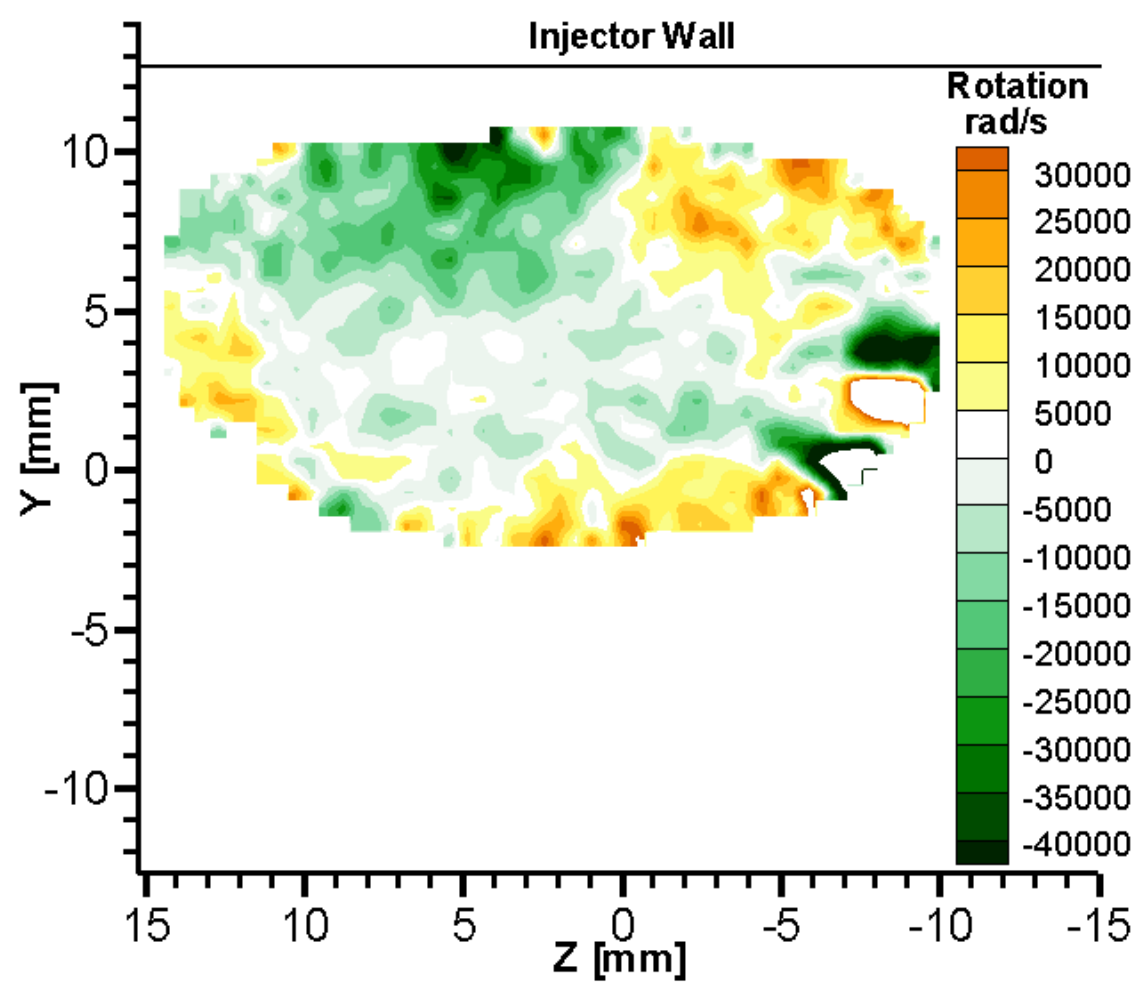

Figure 5.14: Degree of Fluid Rotation for Average SPIV result, $\Phi=0.26$, Fuel-air Combustion case at $\mathrm{X} / \mathrm{H}=10$.

Figure 5.15 presents turbulence intensity information for the fuel-air combustion case at $\mathrm{X} / \mathrm{H}=10$. Turbulence intensity was again calculated by normalizing the $3 \mathrm{C}$ velocity fluctuations by the average velocity at each sub-region across the measurement area. During combustion, turbulence intensity levels are roughly between $15 \%$ and $20 \%$ 
across most of the fuel plume and approach $25 \%$ near the vortices. Again, it should be noted that the two circular areas at the lower right of the turbulence intensity plot, as well

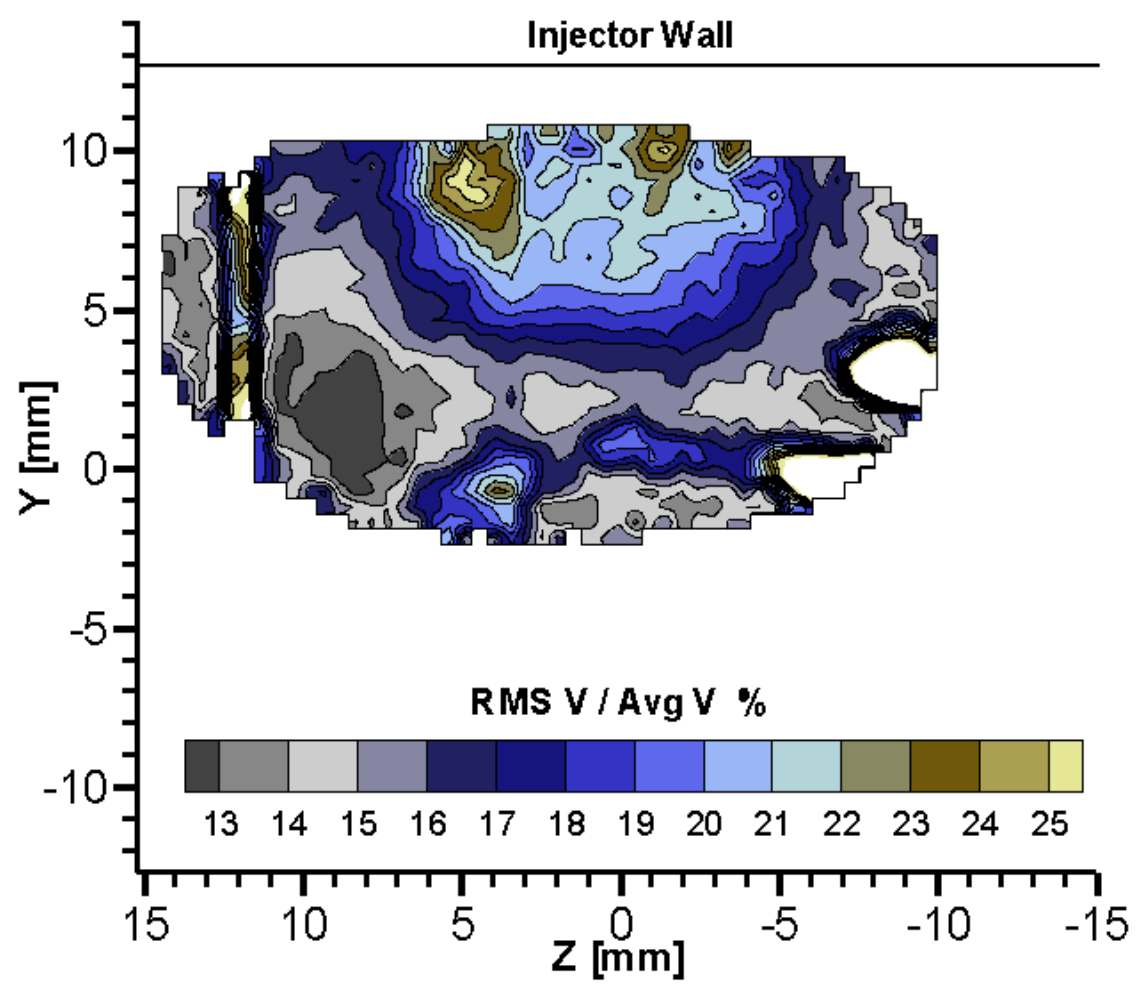

Figure 5.15: Turbulence Intensity (RMS of $3 \mathrm{C}$ Velocity normalized by Average velocity) of Fuel-air Combustion case at $\mathrm{X} / \mathrm{H}=10, \Phi=0.26$

as the vertical line at the left of the plot are not areas of local high turbulence intensity. These areas contain images of laser reflections from the windows that allowed the cameras to view the flow and velocity vectors in these areas were not valid due to the reflections.

Comparison of the velocity fields for the case of fuel-air mixing without combustion and those for the case of fuel-air combustion show some very interesting characteristics of the flow of the DMSJ combustor. The magnitude of the velocity for the case of fuel-air combustion is approximately $20 \%$ less than the average velocity for the 
case of fuel-air mixing and this effect on velocity magnitude is due to the heat addition resulting from the combustion process. Moreover, the area containing velocity vectors in the fuel-air combustion case is larger than that of the non-combusting, mixing case indicating a larger fuel plume during combustion. This result matches fuel plume images previously obtained for this DMSJ combustor which show a larger fuel plume during combustion. $^{41}$ In Fig. 5.14, the presence of the two counter-rotating vortices can again be seen, however the strength of the two vortices appears to be diminished during the fuel/air combustion case. Careful examination of in-plane vector fields for fuel-air combustion show that the vortices are once again not symmetric about the tunnel centerline. It is likely that this asymmetry was caused by one or more of the same factors that caused asymmetry in the fuel-air mixing case discussed above. Furthermore, comparison of Figures 5.8 and 5.15 yields differences in the turbulence intensity distribution induced by the heat release of combustion. The presence of combustion increased turbulence intensity levels by approximately $35 \%$ over the fuel/air mixing case. Some rise in turbulence intensity for the case of fuel-air mixing is expected, however, data for comparison are lacking in the literature at present.

\subsubsection{Uncertainty Estimation}

A brief discussion of uncertainty is presented here along with values for the total estimated uncertainty. A more detailed discussion of the uncertainty estimation for $\mathrm{X} / \mathrm{H}$ $=10$ measurements is presented in Appendix F. The experimental uncertainty of a stereoscopic PIV system is due to four major sources of error: 1) registration error, or when a $2 \mathrm{C}$ vector from one camera is not combined with the correct corresponding $2 \mathrm{C}$ 
vector viewed by the other camera, 2) particle flow tracking error in regions of high flow acceleration or deceleration, 3) uncertainty of measuring the displacement of the particles in the image plane of each camera (uncertainty in determining the two-component (2C) vectors), 4) uncertainty of the time between laser pulses.

Registration error can be introduced if there is misalignment of the calibration target with the laser sheet plane and is described in the literature. ${ }^{18,42}$ A "self-calibration" feature in the LaVision Davis 7.1 software was used to correct misalignment of the target with the laser sheet and registration error was neglected. This "self-calibration" process is fully described by Wieneke. ${ }^{43}$ Particle lag due to shocks or expansion fans was assumed to be negligible because previous CFD and wall pressures ${ }^{28}$ indicate that there are no shocks or expansion fans near the measurement plane of the current work. Furthermore, particle tracking error due to radial motion of a particle in a rotating field was investigated using an equation by Durst et al. ${ }^{44}$ Calculations showed that radial motion of particles due to the rotating field of the ramp-induced vortices produced negligible tracking error at this measurement plane.

Due to the uncertainty in measuring particle displacements in the PIV images, the uncertainty for the axial velocity component was found to be $\pm 14.4 \mathrm{~m} / \mathrm{sec}$, while the error for the cross-plane velocity component was $\pm 8.5 \mathrm{~m} / \mathrm{sec}$. These errors result from a particle displacement measurement accuracy of 0.1 pixels that has been previously reported for PIV cross-correlation algorithms. ${ }^{45}$ Instantaneous velocity fields for the fuelair combustion case were used to determine what fraction the displacement measurement errors were of the mean velocity components. Uncertainty in the time separation between laser pulses $\left(D_{t}\right)$ has been previously estimated to be $1 \%$ and is mostly attributed to laser 
pulse jitter. ${ }^{11}$ Assuming the uncertainties for displacement measurement and time separation $\left(D_{t}\right)$ are independent and normally distributed, they can be combined as a standard root sum square. This yields total uncertainties for the fuel-air combustion case near $2.3 \%$ and $12 \%$ for the axial and cross-plane velocity components, respectively. The uncertainty for the fuel-air combustion case is a worst-case estimate and the uncertainty is less for the fuel-air mixing case.

Because only the fuel was seeded for these measurements, velocity biasing is the final type of error that could have occurred. For velocity biasing to occur, two fluid streams with different velocities need to be present next to one another with one stream containing seeding particles, while the other stream does not. ${ }^{46}$ Biasing can occur because velocity vectors can only be determined based on the displacement of seed particles and therefore the measured velocity can be biased toward the velocity of the seeded stream. In the situation of two fluid streams with nearly the same velocity, biasing should be negligible and the only drawback of seeding only one stream would be that velocity vectors could only be obtained across part of the measurement area.

Based on isentropic calculations, the seeded fuel stream (at the point of injection) is expected to be approximately twice the velocity of the free-stream air flow for the experimental fuel-air mixing case. However, measurements in this study were not conducted at the point of fuel injection, but were acquired at 2.5 inches downstream of the fuel injector (at $\mathrm{X} / \mathrm{H}=10$ ) thus allowing mixing of the fuel and free-stream before reaching the measurement plane. Therefore, the fuel stream had some time to mix with the freestream and to decrease in velocity before reaching the measurement plane at 2.5 inches downstream of the fuel injector. If the velocity of the fuel at the $\mathrm{X} / \mathrm{H}=10$ 
location was significantly different than the velocity of the freestream, then the potential for velocity biasing would still exist. However, the measurements show that this was not the case - the velocity in the core of the fuel plume was $20 \%$ lower than then expected freestream velocity from isentropic calculations. In the core of the fuel jet the number of instantaneous vectors contributing to average vectors was very high (nearly $90 \%$ of the instantaneous vector fields contributed to create average velocity vectors in the core) so very high confidence exists for the average velocity vectors in the core of the fuel jet. Because both the fuel jet and the freestream appear to have attained similar velocities prior to reaching the $10 \mathrm{H}$ measurement plane, velocity biasing is most likely minimal throughout the measurement region for the measurement plane at $\mathrm{X} / \mathrm{H}=10$. This argument is substantiated by the fact that, for the mixing case, the velocities measured at the edges of the fuel plume are actually very close to the expected freestream velocity. Therefore the edges of the plume appear not to have been biased towards the slower seeded fuel plume core. A similar argument is expected to hold for the fuel-air combustion case; however, calculating an expected one-dimensional freestream velocity for the combusting flow is much more complicated.

\section{2 $\mathrm{X} / \mathrm{H}=58.6($ Exit) Measurement Plane, $\Phi=0.17$ Results}

SPIV measurements were conducted at $5 \mathrm{~mm}$ downstream of the exit of Flowpath 1 $(\mathrm{X} / \mathrm{H}=58.6)$ for both fuel-air mixing and fuel-air combustion cases for two different equivalence ratios, $\Phi=0.17$ and $\Phi=0.34$. Figure 5.16 schematically and photographically shows combustor Flowpath 1 with the $\mathrm{X} / \mathrm{H}=58.6$ measurement plane indicated. The measurement plane in the photograph is actually the laser sheet used for 


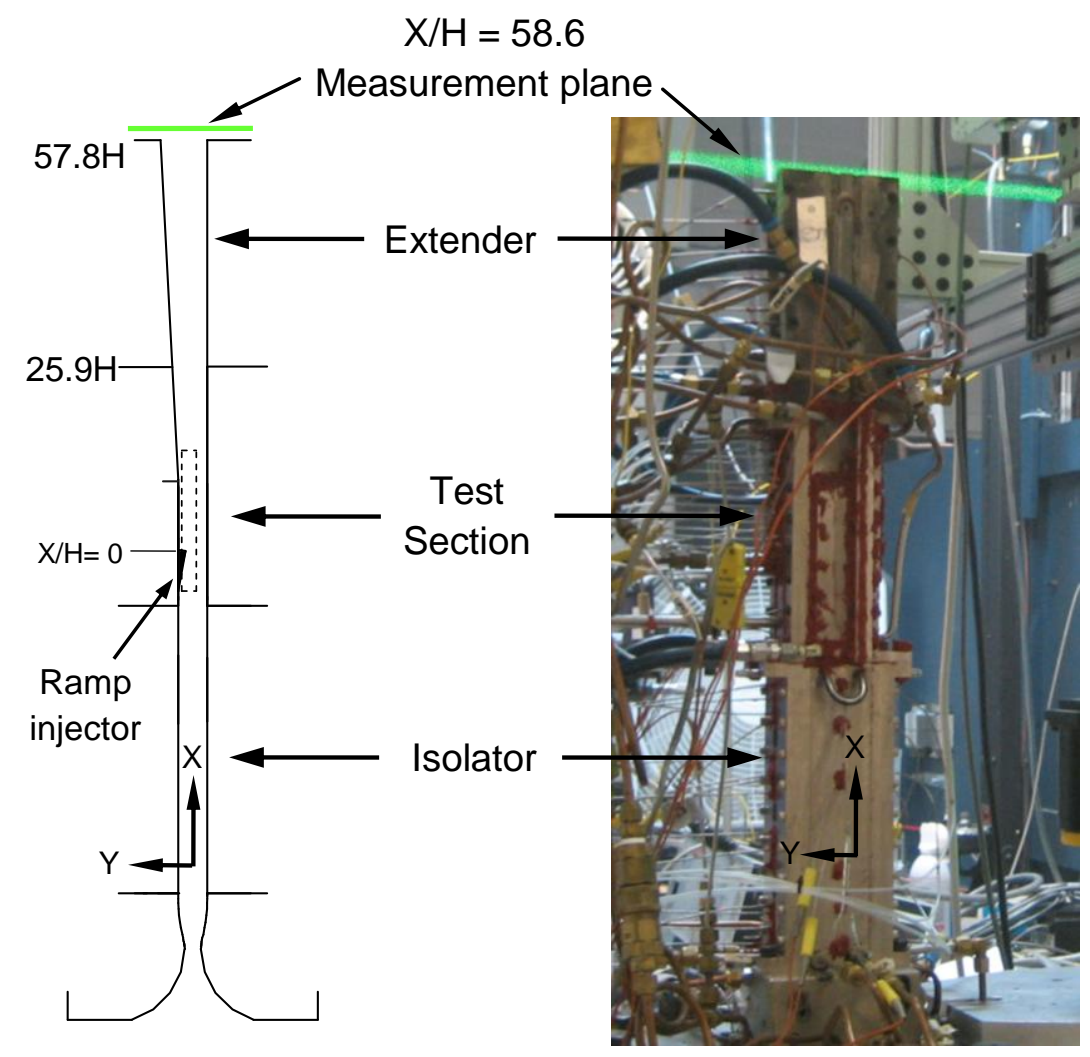

a)

b)

Figure 5.16: Flowpath 1 with SPIV measurement plane at $\mathrm{X} / \mathrm{H}=58.6$

a) Schematic and b) Photograph with laser sheet at the measurement plane.

these measurements and the photograph was taken with artificial fog in the room to make the laser sheet visible. Compressible flow theory predicts that heat addition to a supersonic flow will drive the flow toward the sonic condition. ${ }^{47}$ With greater heat addition than that required to reach sonic condition, the upstream conditions of the flow will change by inclusion of shockwaves so that it is possible to drive the flow to subsonic condition. The equivalence ratio of $\Phi=0.17$ is expected to produce largely supersonic flow during fuel-air combustion because the heat release from combustion is not sufficiently high enough to drive the flow to the sonic condition. Based on previous work $^{28}$ with combustor Flowpath 1 , the heat release from combustion of fuel at $\Phi=0.34$ was sufficient to cause predominantly subsonic flow. These equivalence ratios were 
chosen so that results of the exit flowfield could be compared for the case of supersonic flow to that of predominantly subsonic flow. The results for equivalence ratio of $\Phi=$ 0.17 are presented here, while results for $\Phi=0.34$ are presented in section 5.4. For the remainder of section 5.3, combustor conditions and the SPIV configuration are presented first, followed by a discussion of processing of SPIV data. Fuel-air mixing results for $\Phi$ $=0.17$ are presented briefly and then fuel-air combustion measurements are presented in more detail.

\subsubsection{Fuel-air Mixing Results}

Some fuel-air mixing results for $\Phi=0.17$ at $\mathrm{X} / \mathrm{H}=58.6$ are presented here. However, the SPIV results for the case of fuel-air combustion at the exit were much more interesting than the fuel-air mixing case. Furthermore, the fuel-air mixing results did not give as much useful information about the flow as the fuel-air combustion case. For these reasons, only a brief discussion is presented here for the $\Phi=0.17$ fuel-air mixing results. Three figures for the fuel-air mixing case are shown below and are sufficient to illustrate the characteristics of the average fuel-air mixing flowfield. Other plots for the fuel-air mixing case for $\Phi=0.17$ at $\mathrm{X} / \mathrm{H}=58.6$ can be viewed in Appendix $\mathrm{B}$, but will not be discussed here in detail.

Figure 5.17 shows 3C velocity contours and in-plane velocity vectors for the average velocity field for fuel-air mixing $(\Phi=0.17)$ at the exit of the extender. The average velocity result was obtained by averaging 992 instantaneous velocity fields. During the experiment, 1000 SPIV images were acquired, but 8 of these had one or more camera frames which were blank due to a misfire of the laser. These blank SPIV images 


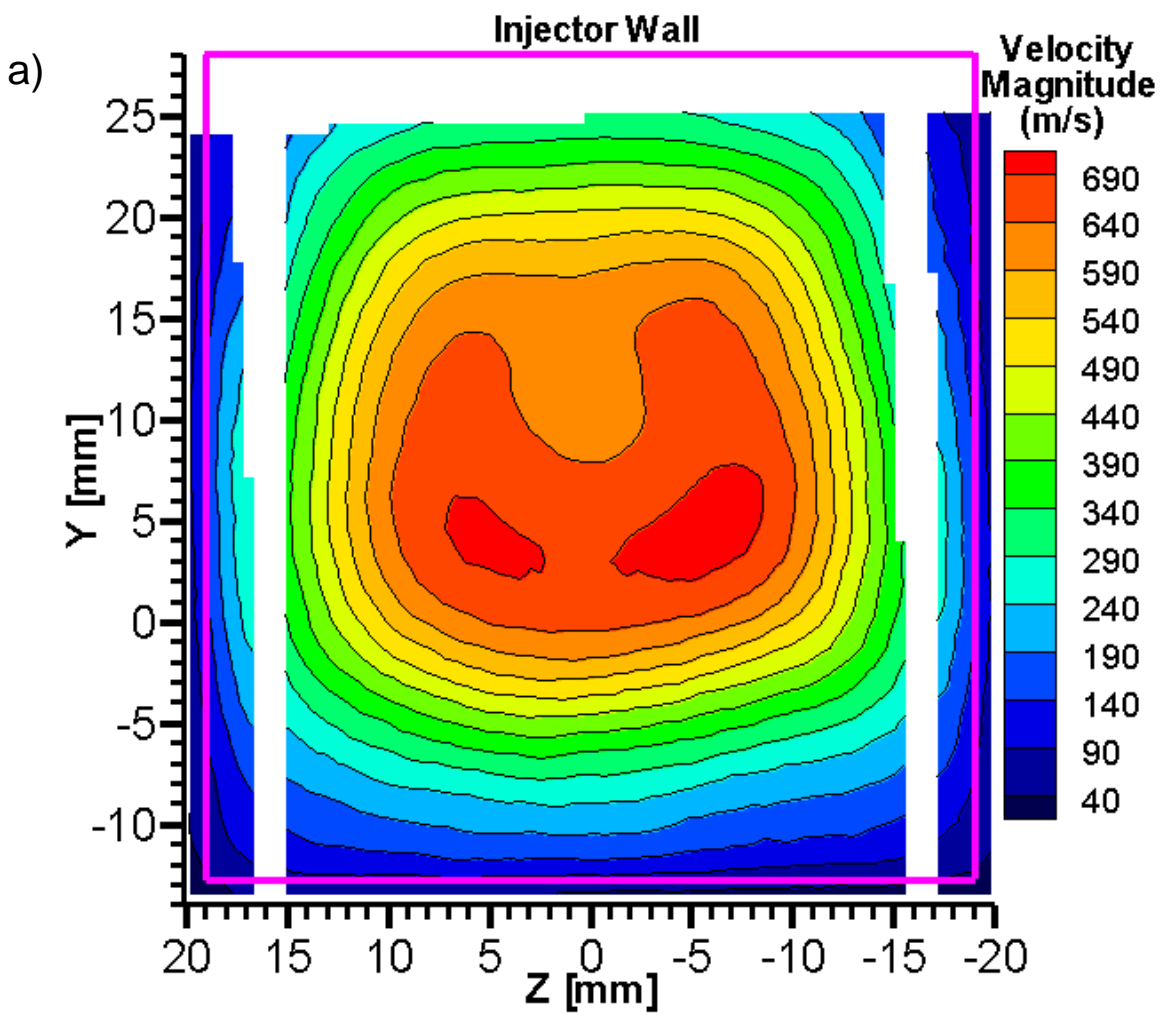

b)

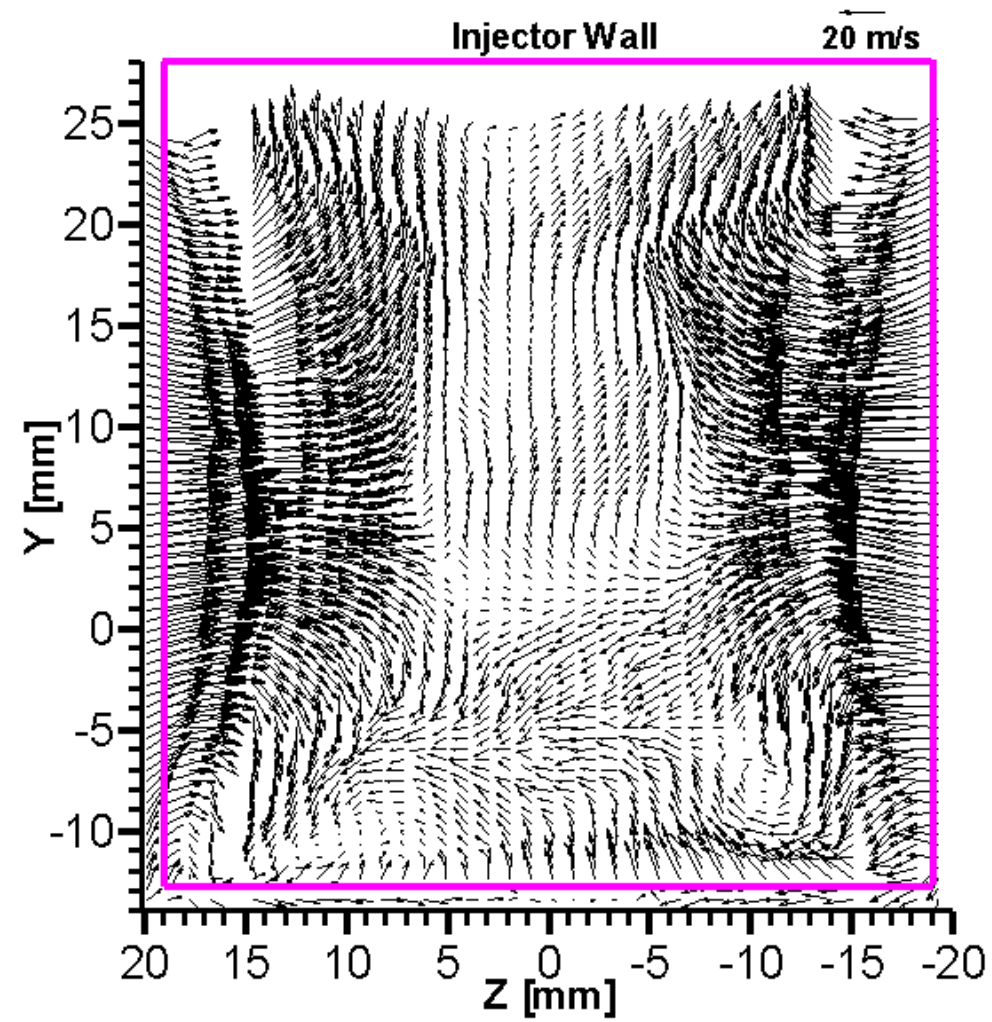

Figure 5.17: Average velocity field for Fuel-air Mixing case $(\Phi=0.17)$ at $X / H=58.6$ a)Three-component $(3 \mathrm{C})$ velocity magnitude, and b) In-plane velocity vectors. 
were removed so that the incorrect vectors from these acquisitions would not contribute do the average field. Good velocity vectors (after filtering) occurred across nearly the entire field-of-view for each instantaneous result and this lead to very high numbers of vectors contributing to the average for each sub-region in the measurement area. In fact, average velocity was calculated using 800 or more contributing vectors for over $92 \%$ of the sub-regions in the measurement area. A plot of vector count can be viewed in Appendix B. In the plots of Fig. 5.17, the lack of data at the top of the plot is because that area was outside of the camera field of view. As mentioned above, this arrangement was necessary because of intense reflections off of the injector wall which could have damaged the cameras if the cameras viewed the injector wall. Furthermore, the vertical areas of missing data occurred because incorrect vectors had to be masked out. Those incorrect vectors resulted because of incorrect correlation of particles with side-wall reflections, as mentioned above. It should be noted that the origin $(0,0)$ in the all plots is the center of the combustor before the start of divergence of the injector wall, that is, the center of the combustor cross-section for locations upstream of $\mathrm{X} / \mathrm{H}=10$. This form of the coordinate axis is consistent for all plots of SPIV measurements and also for CFD results presented in section 5.5. Also, the colored rectangle in each of the plots corresponds to the location of the interior sidewalls of the combustor at the exit plane. The velocity profile in the figure is roughly what would be expected for the mixing case the flow has highest velocity at the center and decreases when moving toward each wall. The influence of the ramp injector can be seen in In-plane vector plot in the area of the injector wall from $\mathrm{Y}=21 \mathrm{~mm}$ to $25 \mathrm{~mm}$. The shape of the $3 \mathrm{C}$ velocity field near the center of the tunnel is also influenced by the injector and this effect is seen by a lower 
velocity of the interior of the "U" shaped contour. In Fig. 5.17(b), long vectors at the left and right of the field have large horizontal (w) velocity components and seem to indicate entrainment of room air into the flow. It should also be noted that, while the influence of the ramp injector can be seen, the ramp-induced vortices are not visible in the In-plane vector plot. Well-organized vortices do not seem to persist this far downstream for this fuel-air mixing case.

Figure 5.18 shows fluid rotation for the $\Phi=0.17$ mixing case. No clear pattern of rotation seems to be present and this plot also indicates that the vortices do not persist this

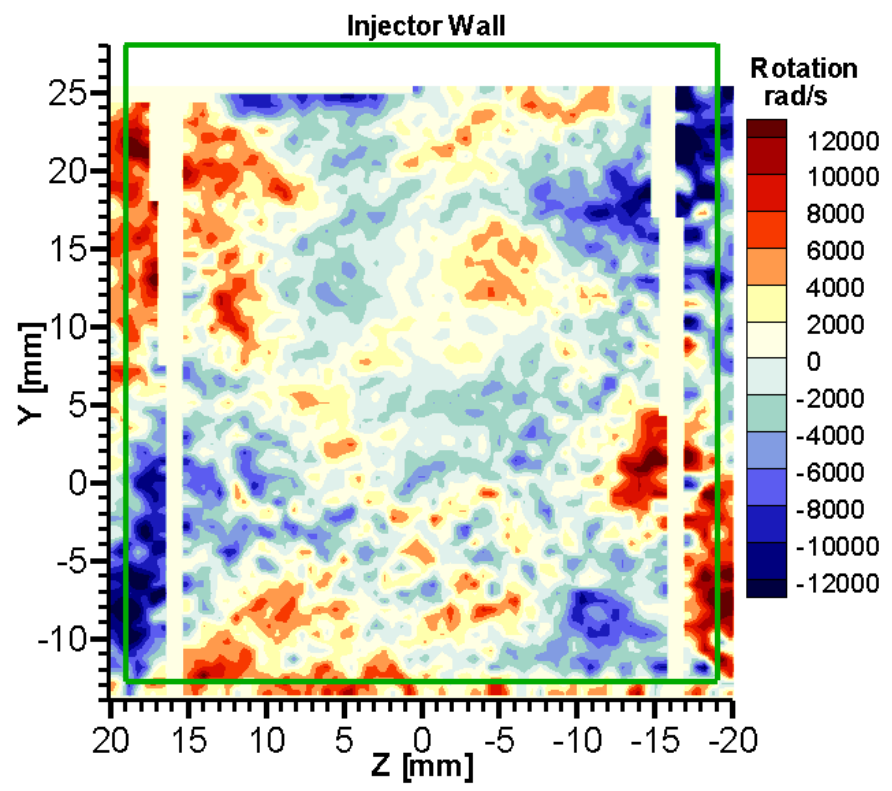

Figure 5.18: Degree of Fluid Rotation for Average SPIV result, $\Phi=0.17$, Fuel-air Mixing case at $\mathrm{X} / \mathrm{H}=58.6$ (Exit plane).

far downstream. If weak vortices were present, they would most easily be viewed in the rotation plot, but that does not seem to be the case. Figure 5.19 shows turbulence intensity for the fuel-air mixing $(\Phi=0.17)$ and turbulence intensity was again calculated by normalizing the RMS of $3 \mathrm{C}$ velocity at each sub-region by the average velocity at each location. Figure 5.19 shows an expected plot of degree of turbulence. The 
turbulence intensity is lowest at the center of the flow where the influence of the walls is minimal and turbulence increases to very large values near the walls and in the

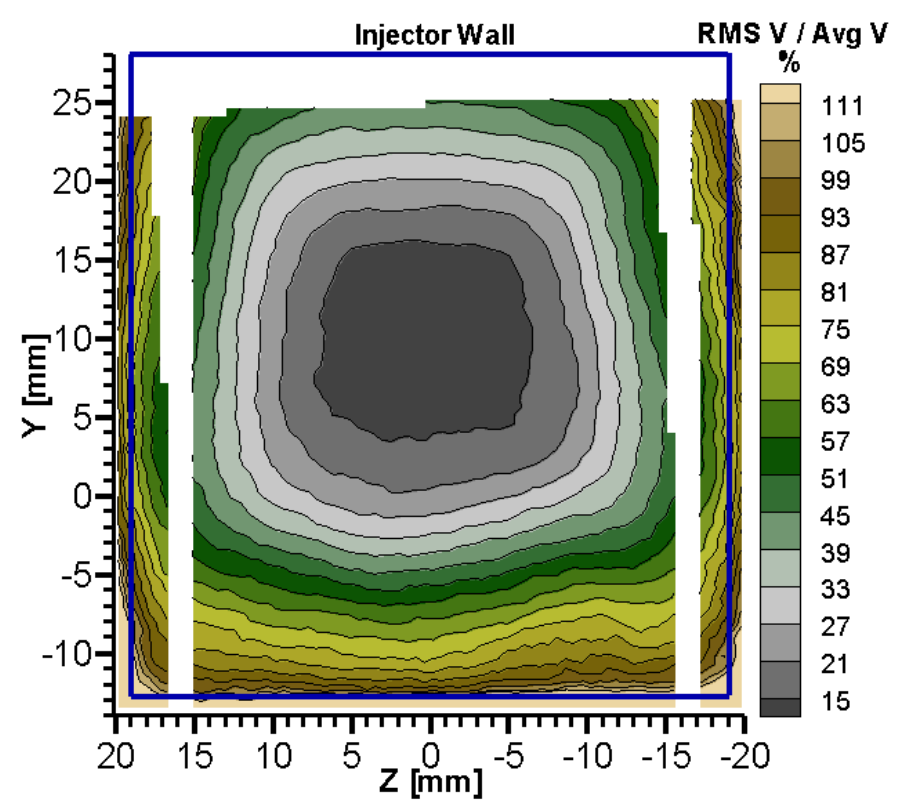

Figure 5.19: Turbulence Intensity (RMS of $3 \mathrm{C}$ Velocity normalized by Average velocity) of Fuel-air Mixing case $(\Phi=0.17)$ at $\mathrm{X} / \mathrm{H}=58.6$ (Exit plane).

entrainment region. Plots which show the in-plane velocity magnitude, examples of instantaneous velocity fields, and the three measured velocity components can be viewed in Appendix B.

\subsubsection{Fuel-air Combustion Results, $\Phi=0.17$}

Fuel-air combustion results for $\Phi=0.17$ at $\mathrm{X} / \mathrm{H}=58.6$ are presented below. The combustion results are discussed in detail here because they are more useful for understanding the flow. As discussed above, the flow in the combustor is expected to be predominantly supersonic for the case of fuel-air combustion with equivalence ratio of $\Phi$ $=0.17$ SPIV measurements were completed for this situation and 1000 instantaneous velocity fields contributed to the average velocity field. Figure 5.20 contains 3D 
perspectives for plots which show the average 3C velocity magnitude. Part (a) of Fig. 5.20 shows resultant velocity vectors, where every $2^{\text {nd }}$ vector is plotted for clarity. Part (b) of the figure shows the $3 \mathrm{C}$ velocity as a three-dimensional surface. The three-

a)
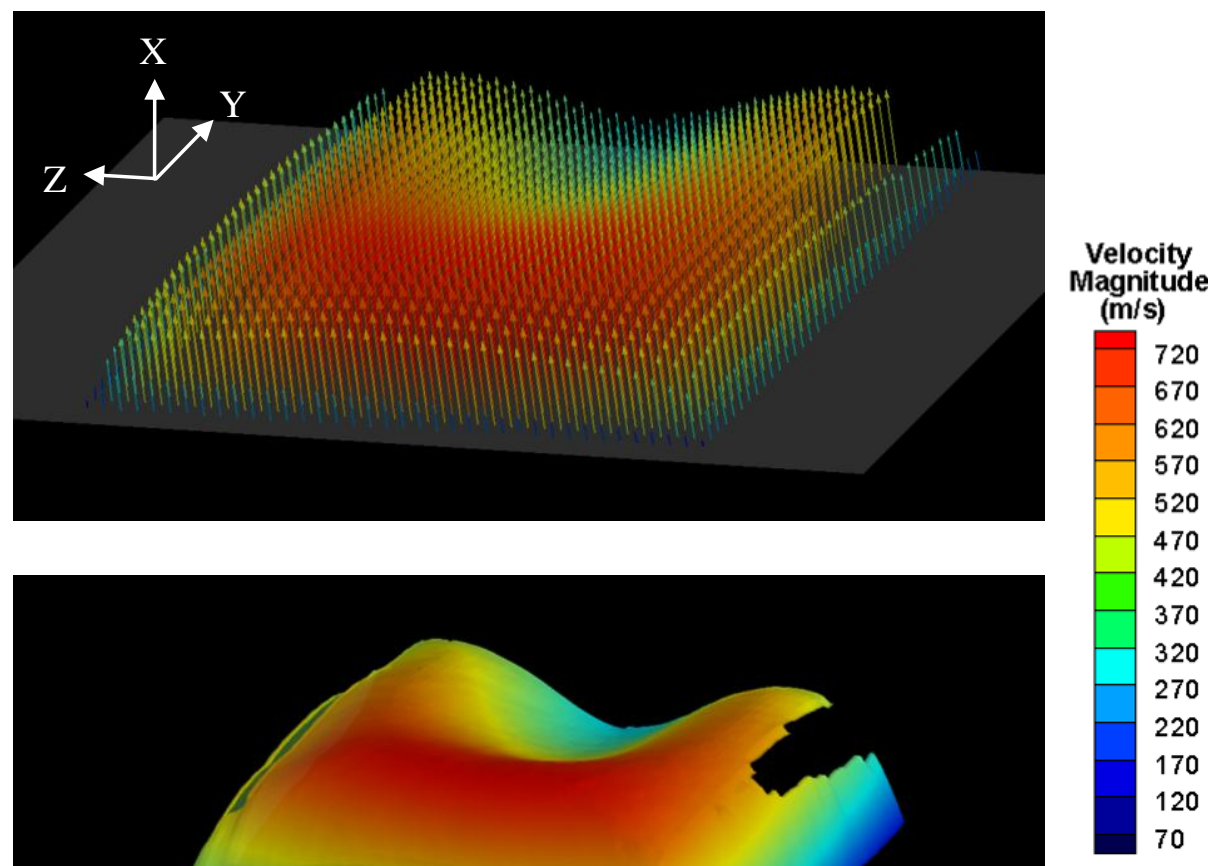

b)

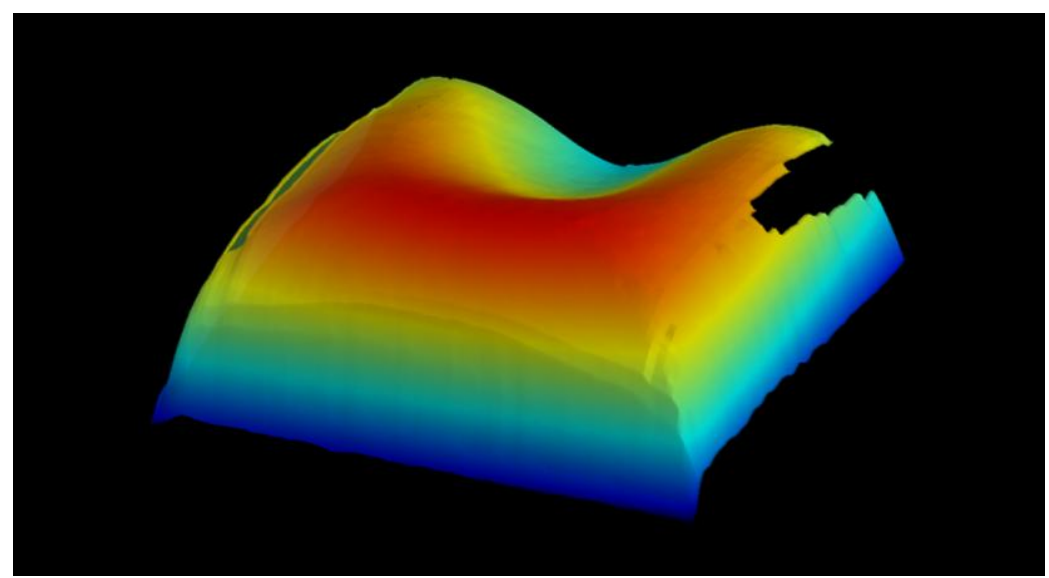

720
20
70
20
70
20
70
20
70
220
70
20
70

Figure 5.20: Average three-component (3C) velocity result from SPIV measurements for Fuel-air Combustion case $(\Phi=0.17)$ at $\mathrm{X} / \mathrm{H}=58.6$ a) Vector representation, and b) Surface representation.

component velocity for this fuel-air combustion case ranges from near $40 \mathrm{~m} / \mathrm{s}$ in the entrainment region, to a maximum of approximately $740 \mathrm{~m} / \mathrm{s}$ in an area of the main flow near the observation wall, that is, the wall opposite to the injector wall. The influence of the ramp injector can be seen in the flow as a lower $3 \mathrm{C}$ velocity on the side of the injector wall and this influence is present from the wall to near the center of the measurement plane. Figure 5.21 shows two-dimensional plots of the average velocity field and part (a) is colored to show 3C velocity magnitude, while part (b) depicts In-plane velocity 
a)

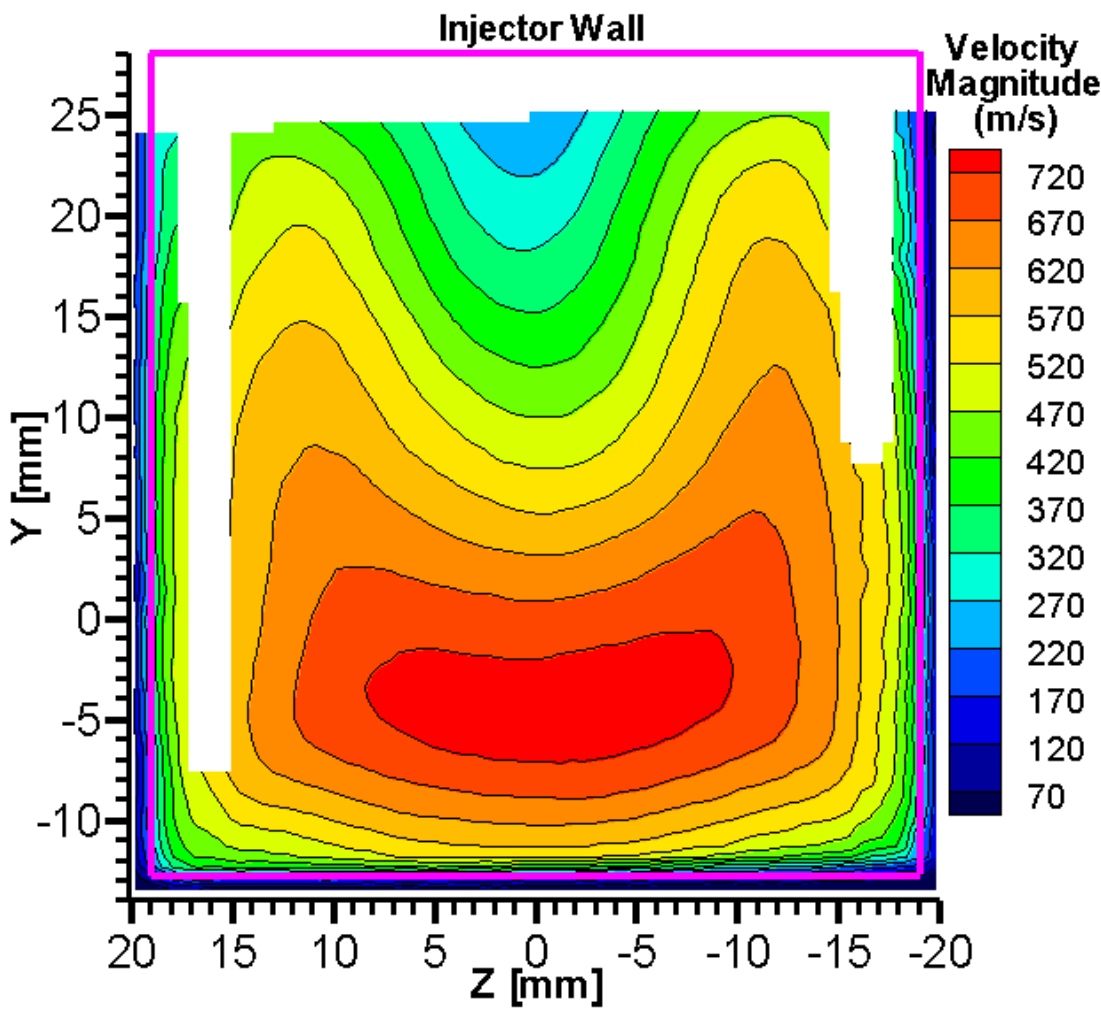

b)

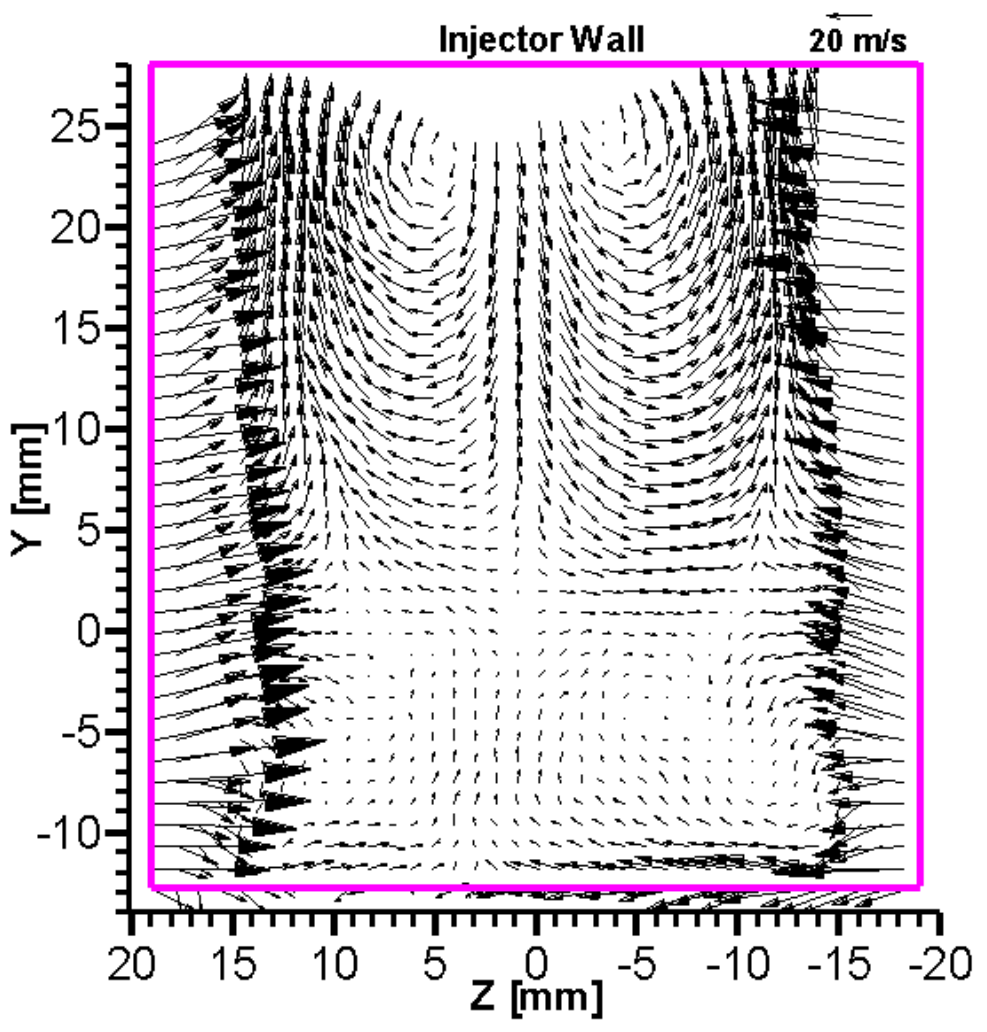

Figure 5.21: Average velocity field for Fuel-air Combustion case $(\Phi=0.17)$ at $\mathrm{X} / \mathrm{H}=58.6$ a)Three-component (3C) velocity magnitude, and b) In-plane velocity vectors. 
vectors. The colored rectangle in the plots shows the edges of the interior combustor walls at the exit plane. The distribution of velocity is clearly visible in part (a) of Fig. 5.21. The In-plane vector plot of Fig. 5.21(b) shows some very interesting results for this situation. The large, horizontal vectors at the left and right of the field indicate entrainment of room air into the flow as it exits the combustor. Even more interesting in the vector plot are the vectors that indicate the presence of the two counter-rotating vortices from the ramp fuel-injector. The vortices seem quite well-defined and are large enough to extend from the injector wall to near a horizontal line through $y=3 \mathrm{~mm}$. The presence of such well-organized vortices this far downstream is somewhat surprising in light of the lack of vortices in the fuel-air mixing case mentioned above. Although the vortices are well-organized, they appear to be rather weak as indicated by the low Inplane velocity magnitude (order of $20 \mathrm{~m} / \mathrm{s}$ ) at a radius of approximately $10 \mathrm{~mm}$ from vortex center.

Figure 5.22 shows the average field and two example instantaneous fields that made up the average. In-plane vectors are shown in the fields of the left column, with the magnitude of the vectors indicated by vector length. A color contour plot of average Inplane velocity magnitude is also include as part of Figure B.7 of Appendix B. The large horizontal vectors of the entrainment area have been omitted in the plots of Fig. 5.22 so that the vectors of the main flow can be viewed more easily. The ramp-induced vortices can clearly be seen near the injector wall in the instantaneous fields and instantaneous vortices contribute to those of the average field. In-plane velocity values near zero in areas around the origin of the average field result because of combination of vectors in opposing directions from the instantaneous fields. At any given instant, the velocity is 
Average In-plane Velocity Vectors

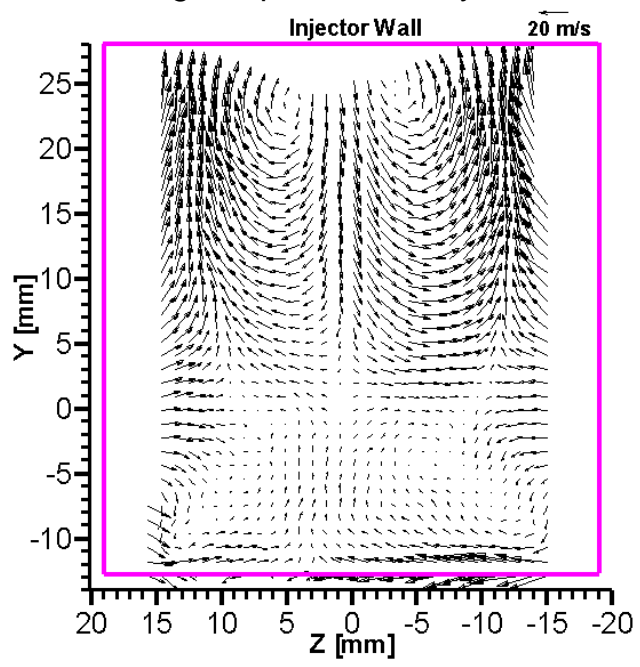

Instantaneous In-plane Velocity Vectors

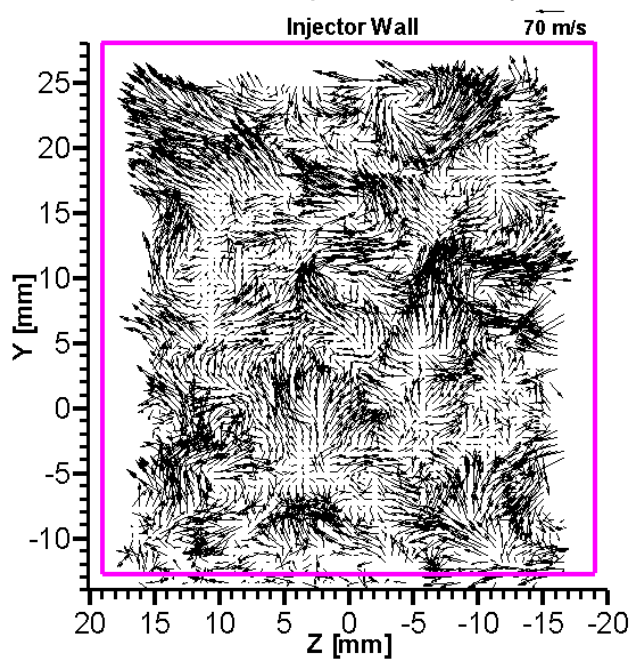

Instantaneous In-plane Velocity Vectors

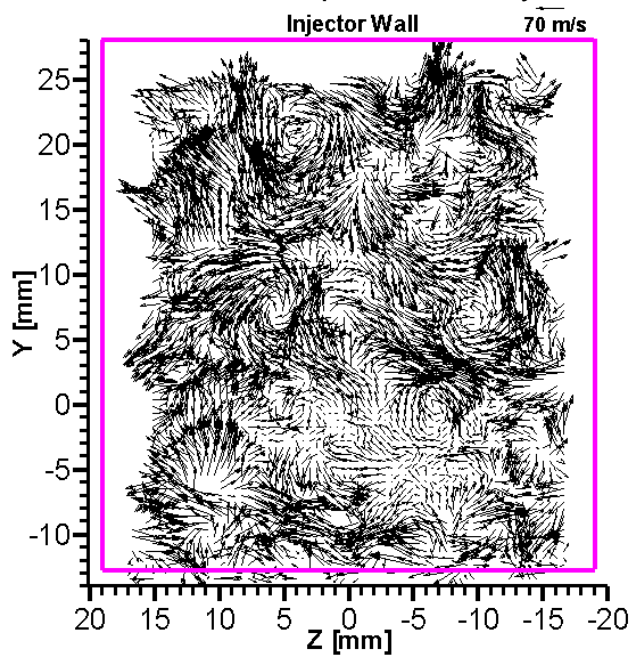

Average 3C Velocity Field

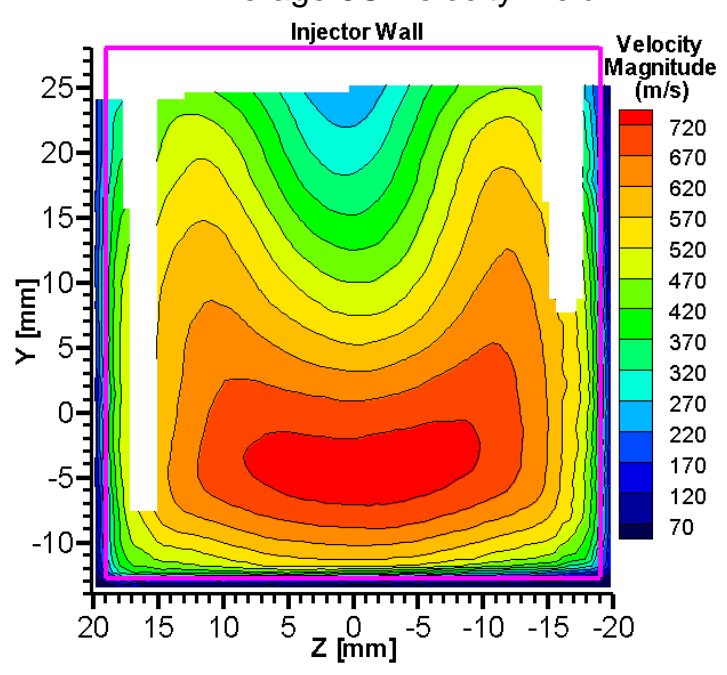

Instantaneous 3C Velocity

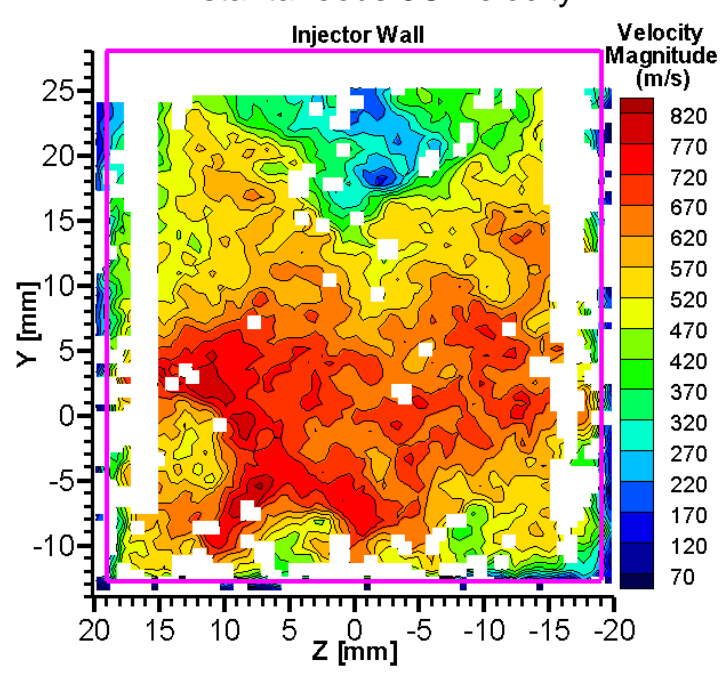

Instantaneous 3C Velocity

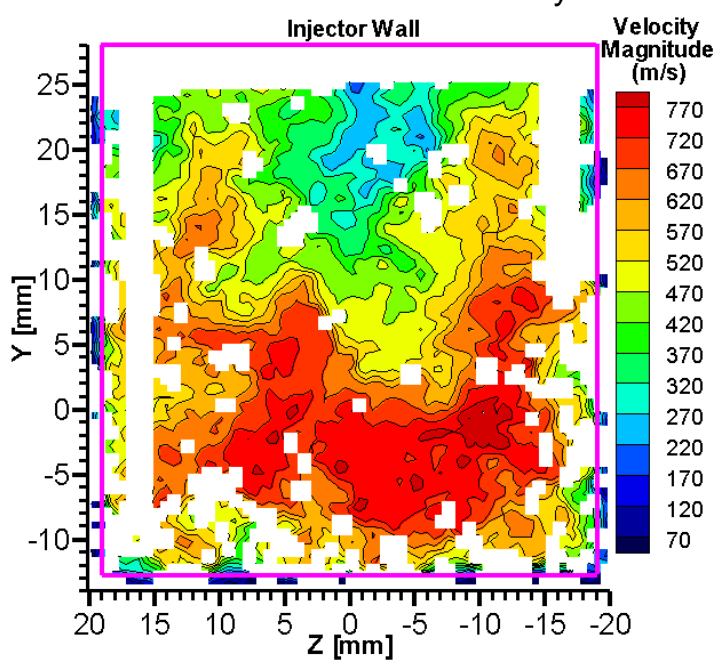

Figure 5.22: Average and Instantaneous velocity fields, Fuel-air Combustion at $\mathrm{X} / \mathrm{H}=58.6, \Phi=0.17$ 
not zero in these areas, but time-average is nearly zero. Furthermore, the general distribution of velocity of the average field can be seen in the instantaneous plots of Fig. 5.22 colored to show $3 \mathrm{C}$ velocity magnitude.

Figure 5.23 shows the number of instant vectors that contributed to the average at each location. The vector count plot clearly shows that the more than 900 instantaneous vectors contributed to the average for the vast majority of the measurement area.

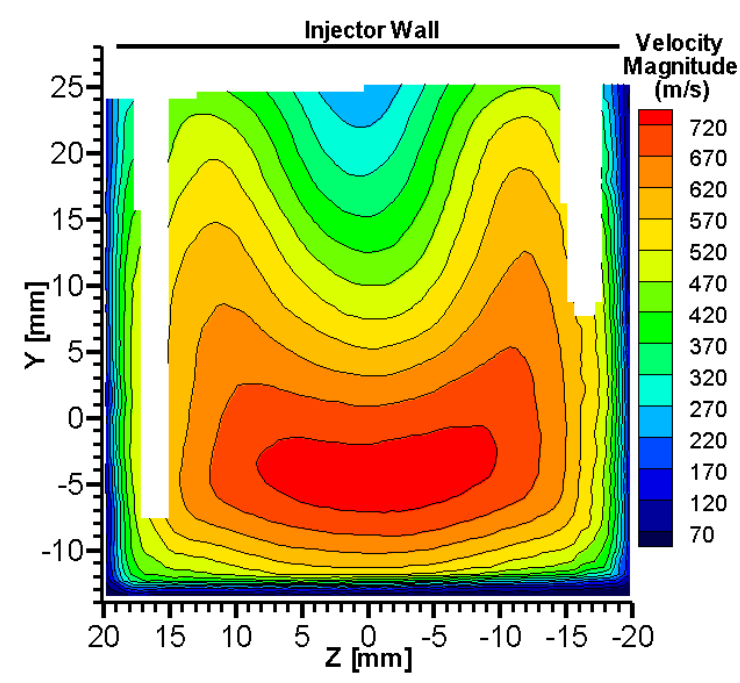

(a) Average of All (1000) Vector Fields [3C Velocity magnitude]

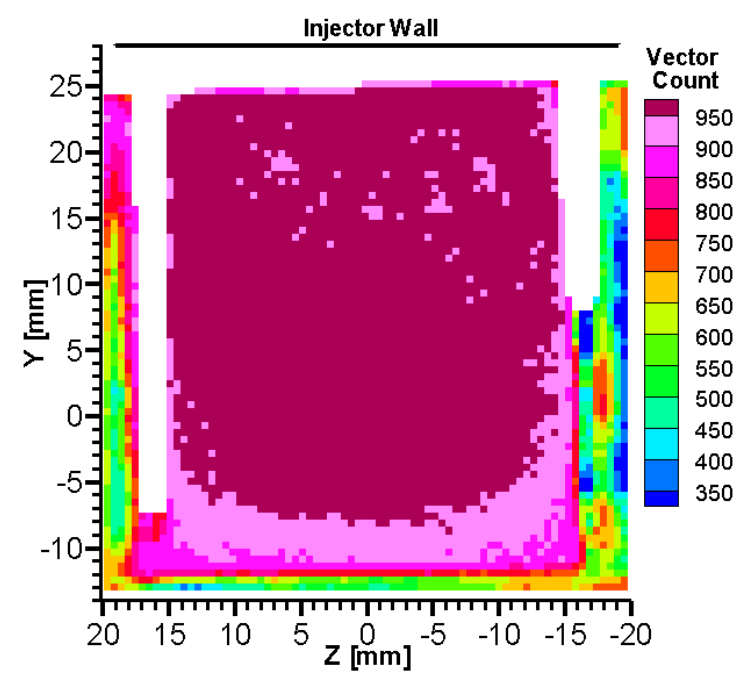

(b) Number of Vectors Averaged for Each Sub-region

Figure 5.23: $\Phi=0.17$, Fuel-air Combustion at $\mathrm{X} / \mathrm{H}=58.6$ (Exit plane);

(a) Average $3 \mathrm{C}$ velocity field, and (b) Number of vectors averaged at each location

Moreover, high confidence exists from the average result in the area of the vortices due to the high vector count in that region. It is unlikely that the average result would be incorrect, considering that it was computed from so many instantaneous velocity vectors that have passed post-processing filters. Therefore, it is likely that the vortices are actually present at this measurement plane and combustion condition. Statistical convergence was again investigated using the qualitative method discussed in section 5.2.2 and it was found that $3 \mathrm{C}$ velocity converged when approximately 300 instantaneous 
results, or more, are averaged. Plots for investigation of statistical convergence for this combustion case $(\Phi=0.17$ at $\mathrm{X} / \mathrm{H}=58.6)$ can be found in Appendix B.

The degree of fluid rotation can be seen in Figure 5.24. In this rotation plot, the two

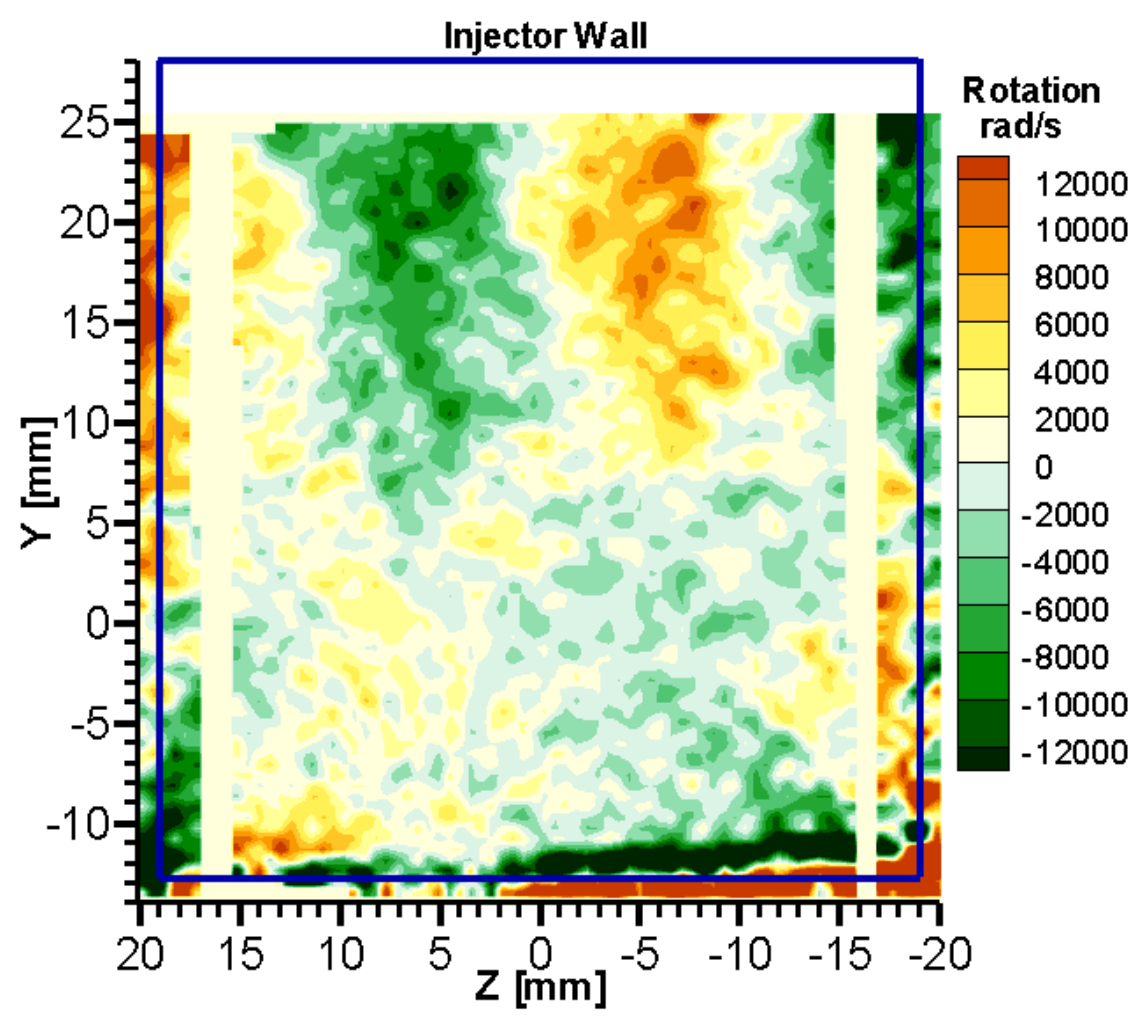

Figure 5.24: Degree of Fluid Rotation for Average SPIV result, $\Phi=0.17$, Fuel-air Combustion case at $\mathrm{X} / \mathrm{H}=58.6$ (Exit plane).

vortices can again clearly be viewed but they are rather weak, as mentioned above. Regardless of strength, the vortices are still clearly present for combustion at this fuel condition. Areas outside the vortices do not seem to have any discernible structure and the rotation in those areas is at most $1 / 5$ of the rotation magnitude of the vortices. Most of the main flow away from the vortices has approximately zero rotation in the average.

Figure 5.25 presents turbulence intensity values for fuel-air combustion $(\Phi=0.17)$ at $\mathrm{X} / \mathrm{H}=58.6$ and turbulence intensity was calculated in the same manner as in other cases discussed above. The scale of the figure was chosen to clearly show turbulence 
intensity values in the main flow and turbulence is only very high in the entrainment region at the outer edges of the measurement area. In that entrainment region (shown with a tan color) turbulence intensity values range from $36 \%$ to over $100 \%$, but such

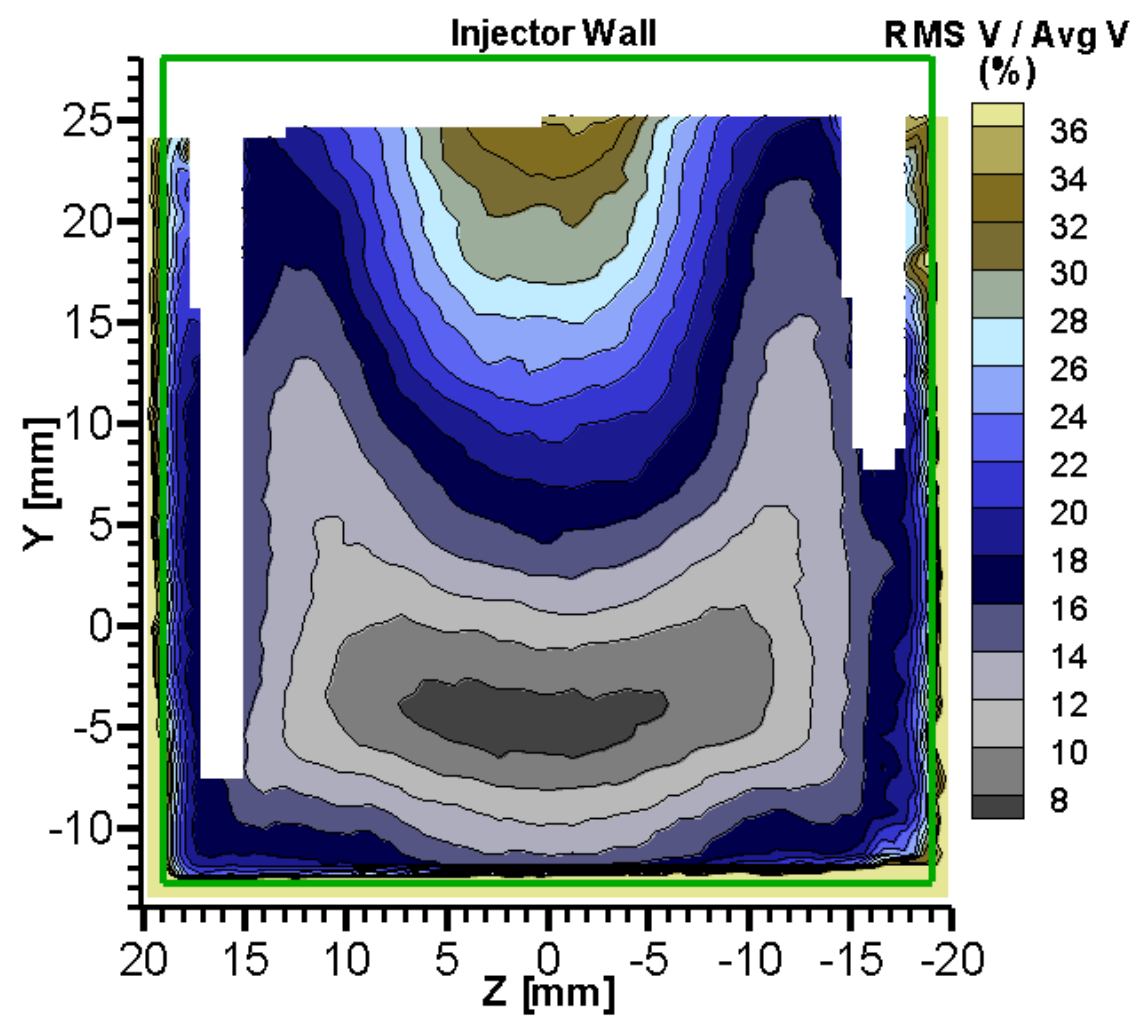

Figure 5.25: Turbulence Intensity (RMS of $3 \mathrm{C}$ Velocity normalized by Average velocity) of Fuel-air Combustion case $(\Phi=0.17)$ at $\mathrm{X} / \mathrm{H}=58.6$ (Exit plane).

large turbulence is not unreasonable in that low-speed region where outside flow is drawn in and mixed with the flow exiting the combustor. The very small area where turbulence intensity is greater than $100 \%$ occurs at the very outer edge of the entrainment region and is not shown as its own contour in the figure. The combination of rotating flow in the area of the vortices and the movement of the vortices between instantaneous fields, gives rise to higher turbulence intensity in the area of the vortices than in the rest of the main flow. Outside of the area of the vortices (and outside the entrainment region) turbulence values are fairly low and are comparable to the magnitude of turbulence intensity in the 
core flow of the $\Phi=0.17$ fuel-air combustion case, presented above. Supplemental figures of the SPIV data are presented in Appendix B for both mixing and combustion cases with $\Phi=0.17$ at $\mathrm{X} / \mathrm{H}=58.6$. The SPIV measurements at $\mathrm{X} / \mathrm{H}=58.6$ were repeated for experiments with an equivalence ratio of $\Phi=0.34$ and results from those experiments are presented in section 5.4.

\subsection{X/H $=58.6$ (Exit) Measurement Plane, $\Phi=0.34$ Results}

SPIV measurements at the exit of the combustor were also completed for an equivalence ratio of $\Phi=0.34$ for cases of both fuel-air mixing and fuel-air combustion. Two equivalence ratios were used at the exit plane to investigate the effect of more fuel on the fuel-air mixing and also the effect of greater heat release during combustion.

\subsubsection{Fuel-air Mixing Results, $\Phi=0.34$}

Results for fuel-air mixing at $\mathrm{X} / \mathrm{H}=58.6$ with and equivalence ratio of $\Phi=0.34$ are briefly presented here. Like the cases with $\Phi=0.17$, the results with $\Phi=0.34$ for fuelair mixing are not as interesting or useful for understanding the flow as are the fuel-air combustion measurements. Therefore, only three figures for the fuel-air mixing case are shown here, with the remaining results located in Appendix C. Figure 5.26 depicts 3C velocity contours and In-plane velocity vectors of the average result for fuel-air mixing, $\Phi=0.34$. The average results for fuel-air mixing were produced by averaging 988 instantaneous velocity fields measured using SPIV. During the experiments, 1000 instantaneous measurements were acquired, but blank frames due to laser misfires or oversaturated frames due to clumps of particles exiting the seeder, required 12 
a)
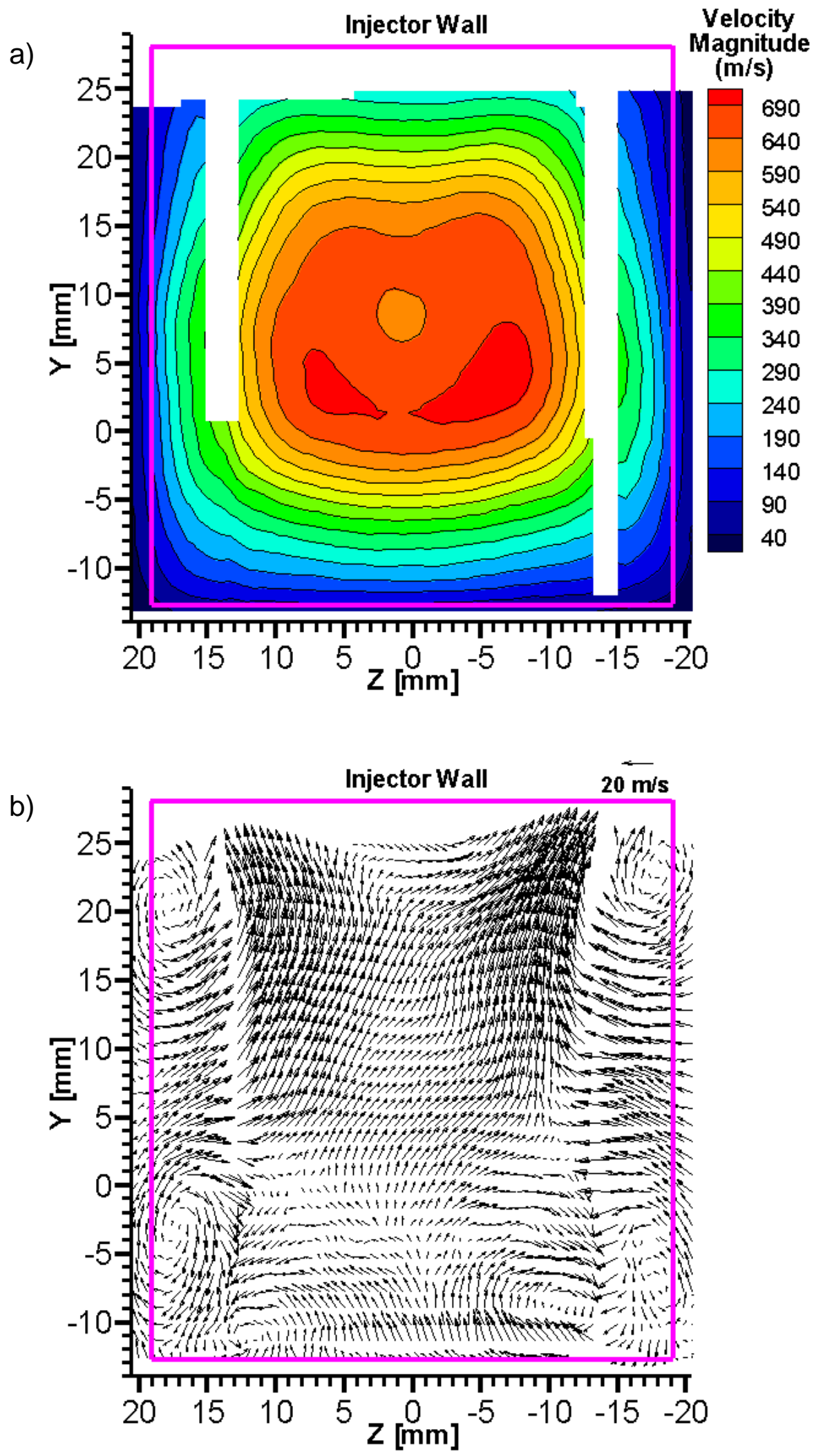

Figure 5.26: Average velocity field for Fuel-air Mixing case $(\Phi=0.34)$ at $X / H=58.6$ a)Three-component (3C) velocity magnitude, and b) In-plane velocity vectors. 
instantaneous results to be removed. In the figure, the colored box indicates the interior walls of combustor Flowpath 1 at the exit plane. Again, data are missing for locations close to the injector wall because the cameras had to be positioned so that the intense reflection from the top edge of the injector wall was outside of the field-of-view of the cameras. The average fuel-air mixing field of Fig. 5.26 (a) is very similar to the mixing results of Fig. 5.17 for fuel-air mixing with $\Phi=0.17$. In fact, the contour patterns and velocity magnitude are almost identical for fuel-air mixing cases with equivalence ratios of 0.17 and 0.34 . The In-plane velocity vectors for mixing with equivalence ratios of $\Phi=$ 0.34 and $\Phi=0.17$ are very similar, specifically, they both have entrainment regions near the edges of the flowfield, almost zero In-plane velocity magnitude in the center core flow, and a noticeable influence of the fuel injector on the In-plane vectors near the injector wall. An interesting result in the vector pattern of Fig. 5.26 indicates the ability of the SPIV technique to resolve a complicated flowfield. In the average vector pattern of Fig. 5.26 part (b) several vortices near the corners of the duct can be seen and such vortices are characteristics of flows through rectangular ducts. Looking back at the mixing case with $\Phi=0.17$, some influence of corner-vortices can also be seen in Fig. 5.17, but to a lesser extent than in the $\Phi=0.34$ mixing case.

Figure 5.27 shows the degree of fluid rotation for the fuel-air mixing case with fuel condition $\Phi=0.34$. Again, there is almost no discernible pattern in the fluid rotation in the core of the flow, although some rotational influence of the ramp-induced vortices can be seen near the injector wall. The rotation near the interior corners of the duct can clearly be viewed as areas of dark blue or dark red in this plot. The turbulence intensity of the average field for the case of fuel-air mixing, $\Phi=0.34$ is shown in Figure 5.28. 


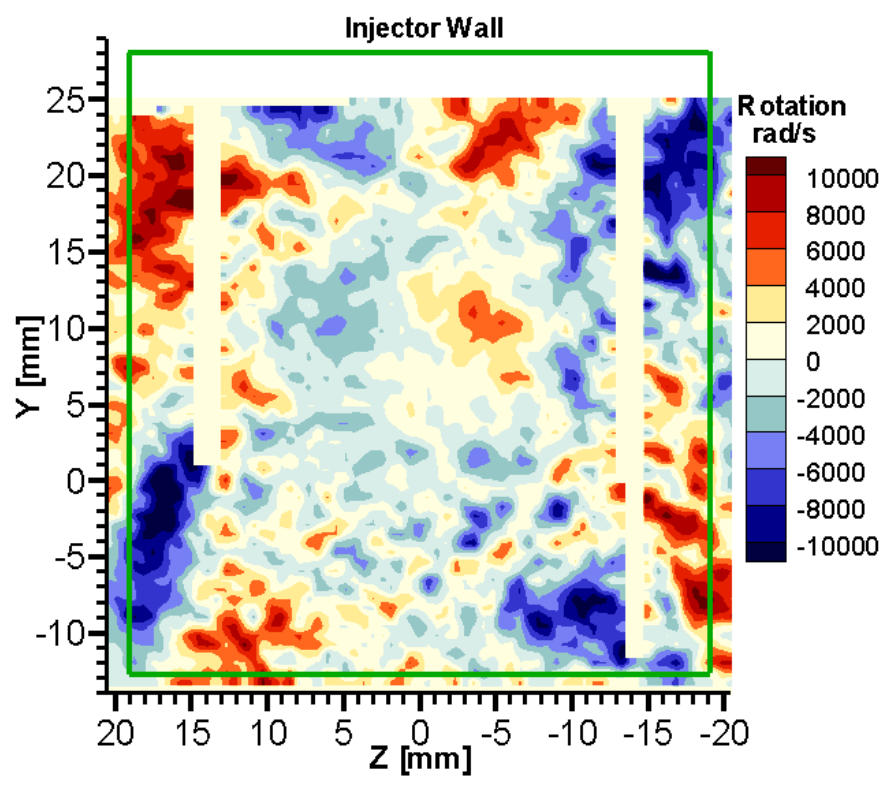

Figure 5.27: Degree of Fluid Rotation for Average SPIV result, $\Phi=0.34$, Fuel-air Mixing case at $\mathrm{X} / \mathrm{H}=58.6$ (Exit plane).

The pattern and magnitude of turbulence intensity is nearly identical for the mixing case with $\Phi=0.34$ to the turbulence intensity for mixing with $\Phi=0.17$. Turbulence is lowest (between $11 \%$ and $15 \%$ ) at the center area of the flow, rises to a much larger degree in

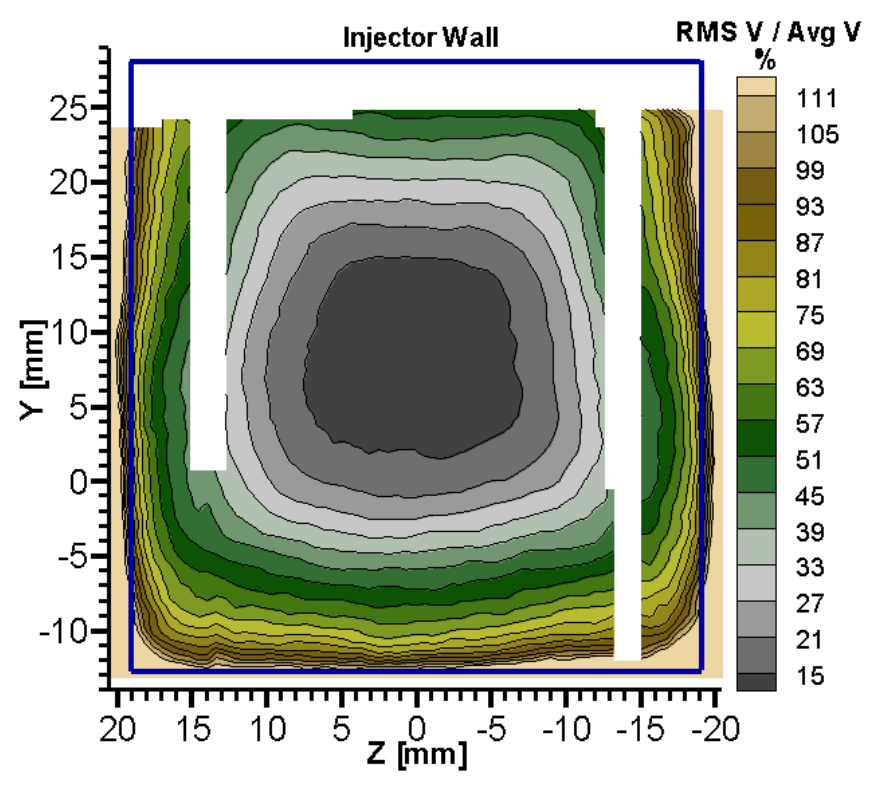

Figure 5.28: Turbulence Intensity (RMS of $3 \mathrm{C}$ Velocity normalized by Average velocity) of Fuel-air Mixing case $(\Phi=0.34)$ at $\mathrm{X} / \mathrm{H}=58.6$ (Exit plane). 
areas near the walls, and is at a maximum in the entrainment regions. Areas near the walls with high turbulence intensities are relatively low-speed regions (approximately 50 $\mathrm{m} / \mathrm{s}$ to $300 \mathrm{~m} / \mathrm{s}$ in the entrainment regions) and high turbulence levels likely result due to the influences of the walls and entraining room air.

\subsubsection{Fuel-air Combustion Results, $\Phi=0.34$}

Combustion results for $\mathrm{X} / \mathrm{H}=58.6, \Phi=0.34$ are presented here. Comparisons are made between the combustion results with $\Phi=0.34$ and the results for $\Phi=0.17$ (presented above) to investigate the effect of greater heat addition due to higher fuel equivalence ratio. Figure 5.29 shows a 3D view of the velocity field for the fuel-air

a)
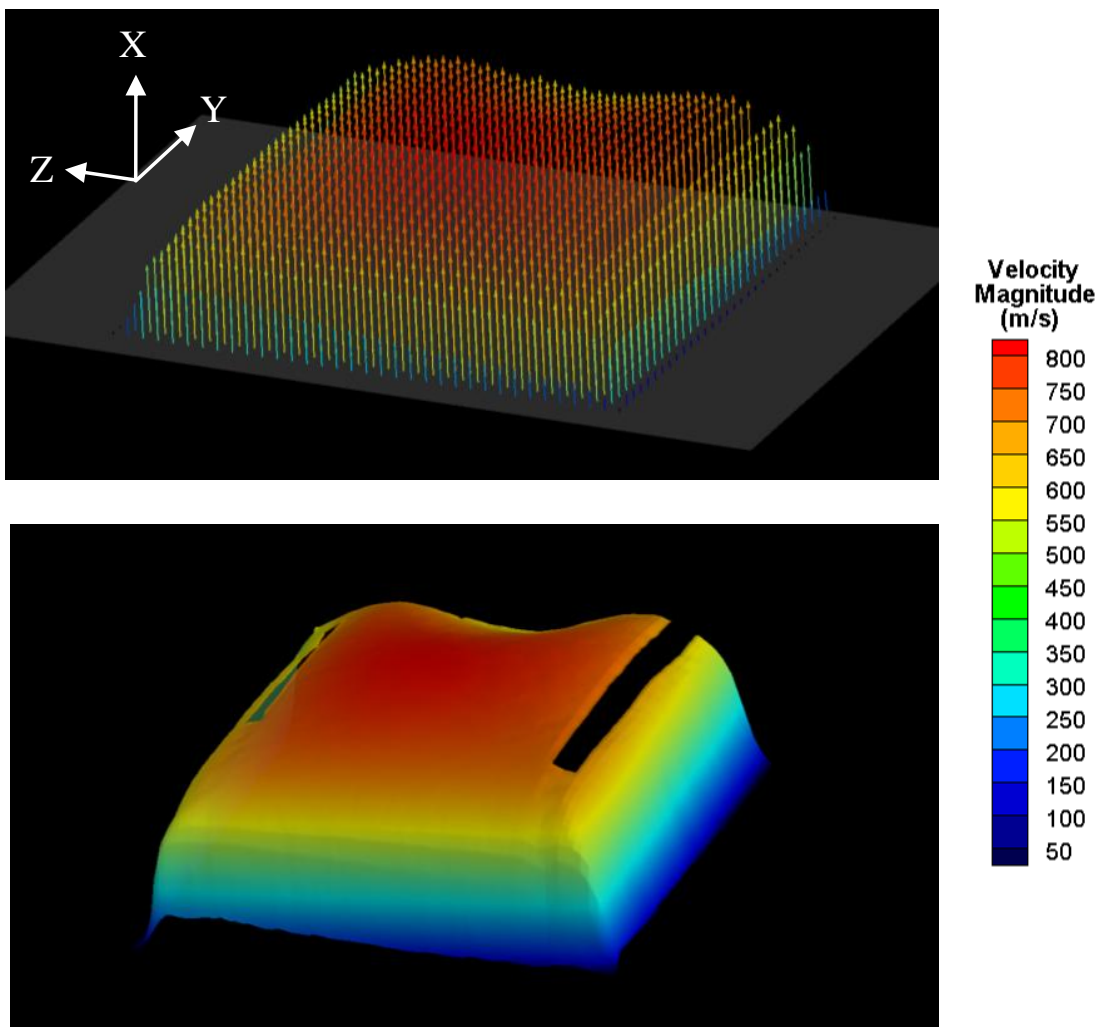

Figure 5.29: Average three-component (3C) velocity result from SPIV measurements for Fuel-air Combustion case $(\Phi=0.34)$ at $\mathrm{X} / \mathrm{H}=58.6$ a) Vector representation, and b) Surface representation. 
combustion at $\mathrm{X} / \mathrm{H}=58.6, \Phi=0.34$, while $3 \mathrm{C}$ velocity contours and In-plane velocity vectors can be viewed in Figure 5.30. Clear differences can be seen in the velocity field for combustion with $\Phi=0.34$ as compared to the combustion results for $\Phi=0.17$. In Fig. 5.29, the velocity is nearly uniform (between $\sim 600$ and $800 \mathrm{~m} / \mathrm{s}$ ) over most of the measurement area, with a maximum velocity of approximately $815 \mathrm{~m} / \mathrm{s}$. This maximum velocity for $\Phi=0.34$ is approximately $70 \mathrm{~m} / \mathrm{s}$ above the maximum for combustion with $\Phi=0.17$. For combustion with $\Phi=0.34$, Fig. 5.29 shows some influence of the rampinduced vortices near the injector wall, but the influence of the vortices does not protrude into the flow nearly to the extent as in the combustion case with $\Phi=0.17$. The influence of the ramp-induced vortices can again be seen in Fig. 5.30 on the injector wall side of the flow, but in contrast to the $\Phi=0.17$ case at the exit plane, well-organized rampinduced vortices do not appear to be present for combustion with equivalence ratio of $\Phi$ $=0.34$. The average velocity field shown in the figures was produced by averaging 979 instantaneous velocity fields and the vector count distribution can be seen if Figure 5.31. It should be noted that counts of over 800 instantaneous vectors contributed to average vectors for over $80 \%$ of the sub-regions in the measurement area. Comparison plots for statistical convergence were also constructed for this case and can be viewed in Appendix C. The $3 \mathrm{C}$ velocity appears to be converged for an average of 300 or more instantaneous velocity fields. Therefore, very high confidence exists in the average velocity field that was calculated using 979 instant results, especially across the center $80 \%$ of the measurement area.

Figure 5.32 shows the average velocity field and two of the 979 instantaneous results that produced the average field for fuel-air combustion with $\Phi=0.34$. The 

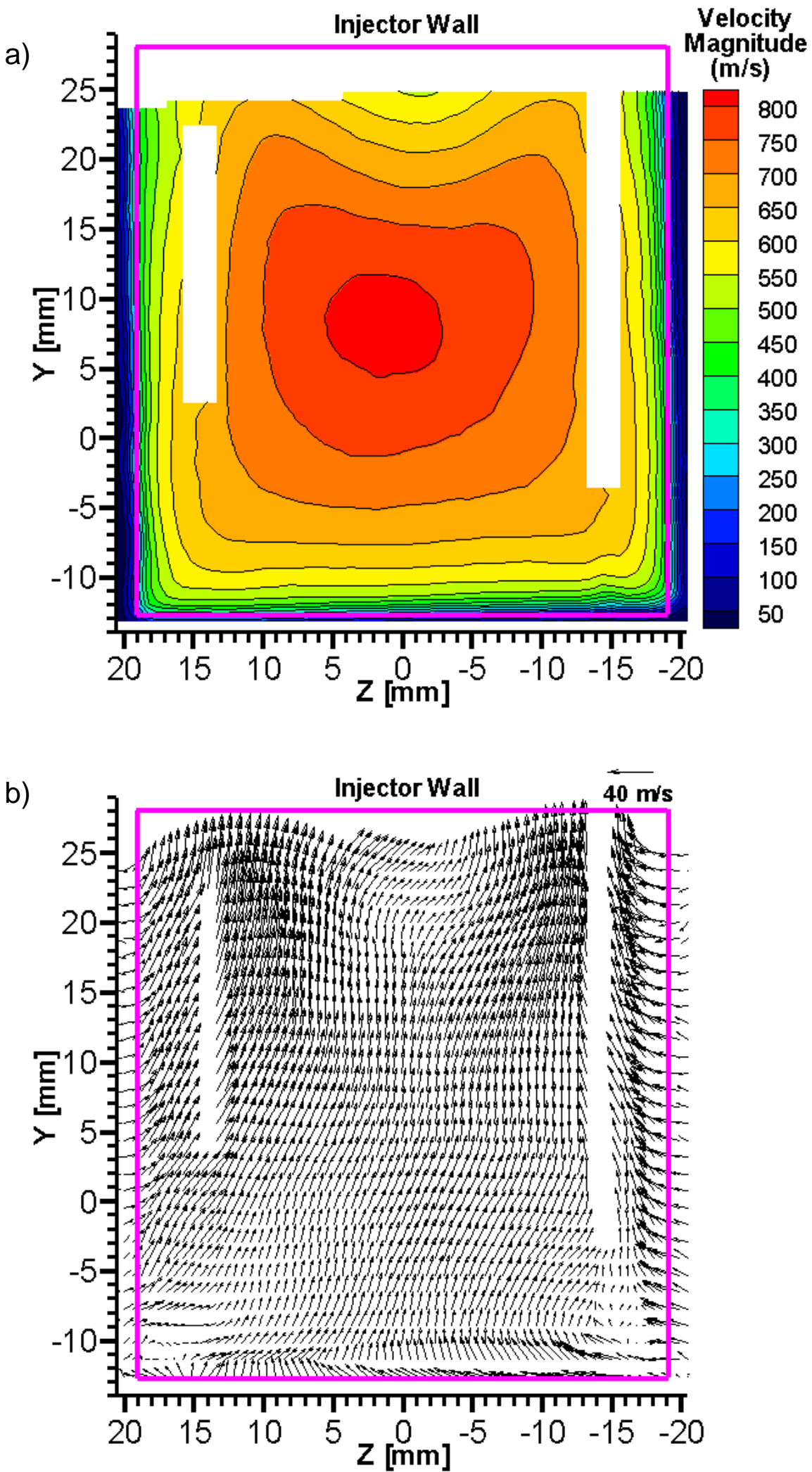

Figure 5.30: Average velocity field for Fuel-air Combustion case $(\Phi=0.34)$ at $X / H=58.6$ a)Three-component (3C) velocity magnitude, and b) In-plane velocity vectors. 
instantaneous results colored to show $3 \mathrm{C}$ velocity clearly show the contour shape seen in the average result. The high-velocity area over most of the flowfield and the sharp drop to low velocity in the entrainment region is clear in the color contours of each of the instantaneous plots. Furthermore, the influence of the ramp-induced vortices is seen as an area of lower velocity flow near the injector wall and this area protrudes into the middle of the field to a different extent in each instantaneous velocity field result.

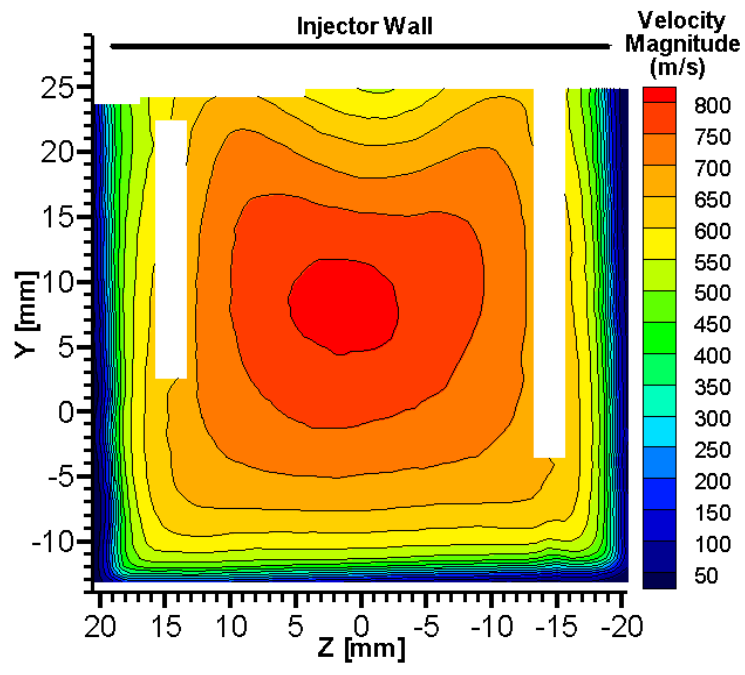

(a) Average of All (979) Vector Fields [3C Velocity magnitude]

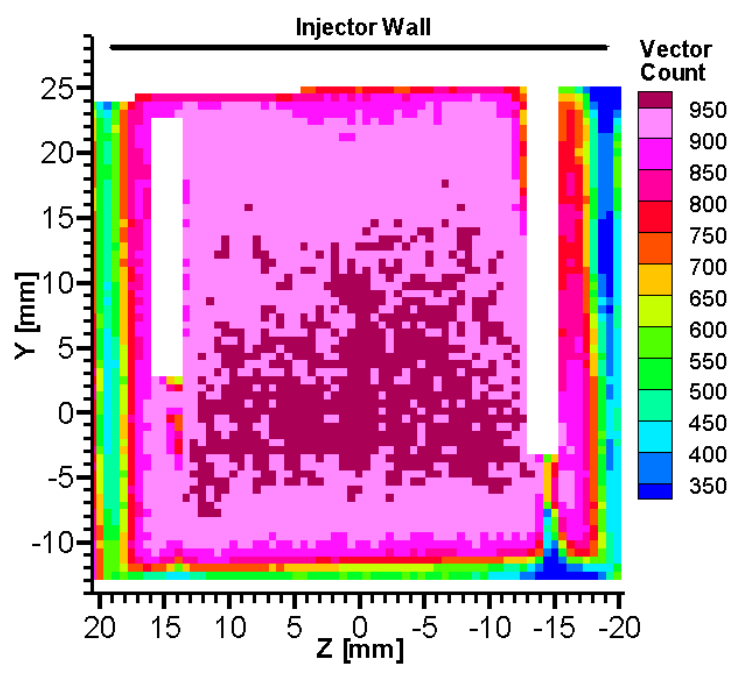

(b) Number of Vectors Averaged for Each Sub-region

Figure 5.31: $\Phi=0.34$ Fuel-air Combustion at $\mathrm{X} / \mathrm{H}=58.6$ (Exit plane);

(a) Average $3 \mathrm{C}$ velocity field, and (b) Number of vectors averaged at each location

Looking at the In-plane vector arrows, the turbulent nature of the flow is noted in the variation of In-plane velocity vectors across the measurement are and also as areas of random rotation which are not found in the average velocity field. Because the In-plane vectors in the average results have low velocity and no distinct pattern, the average result shows that rotation in the instantaneous fields is indeed random in nature. The motion in an area of one instant result is offset by equal and opposite velocity in a different instant result, thus causing nearly zero In-plane velocity for most of the field. The only exception to this random rotation is in the area near the injector wall. In the 
Average In-plane Velocity Vectors

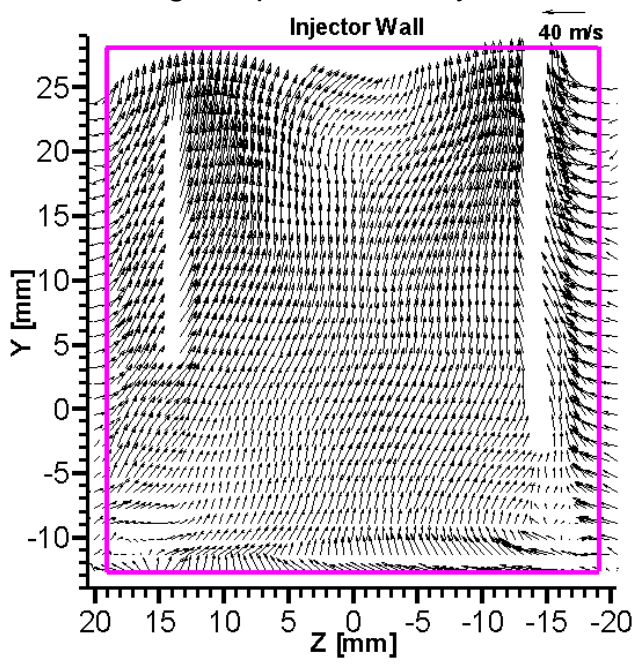

Instantaneous In-plane Velocity Vectors

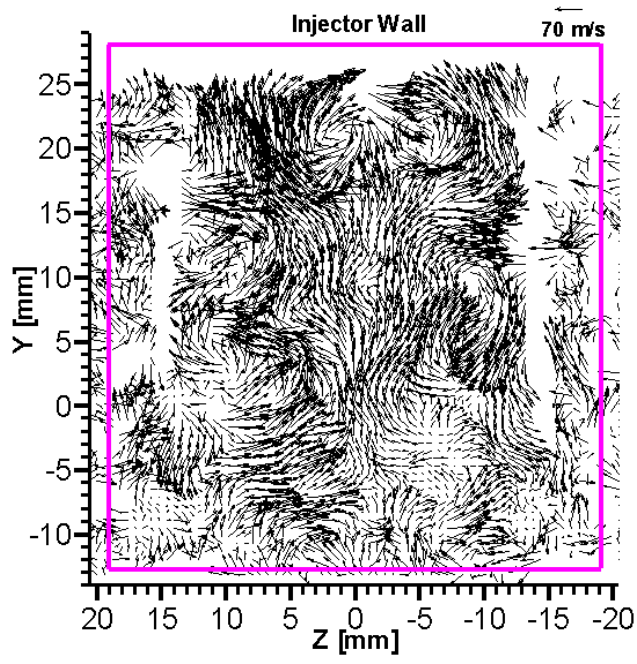

Instantaneous In-plane Velocity Vectors

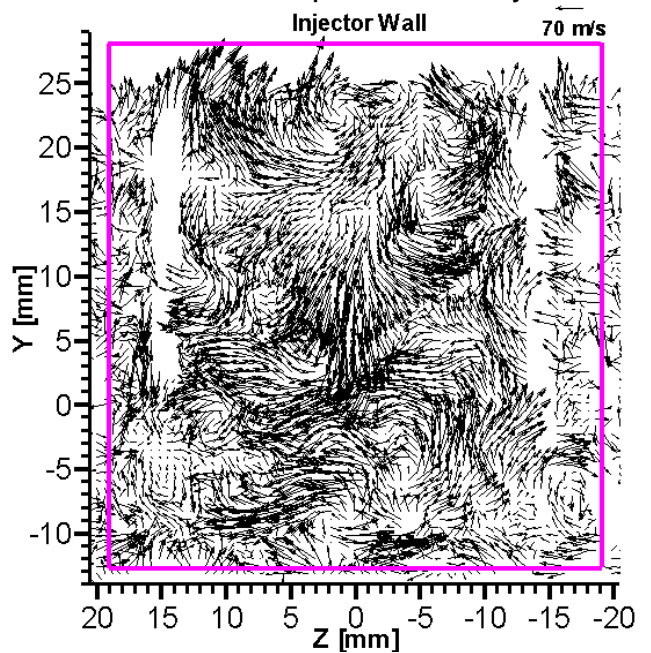

Average 3C Velocity Field

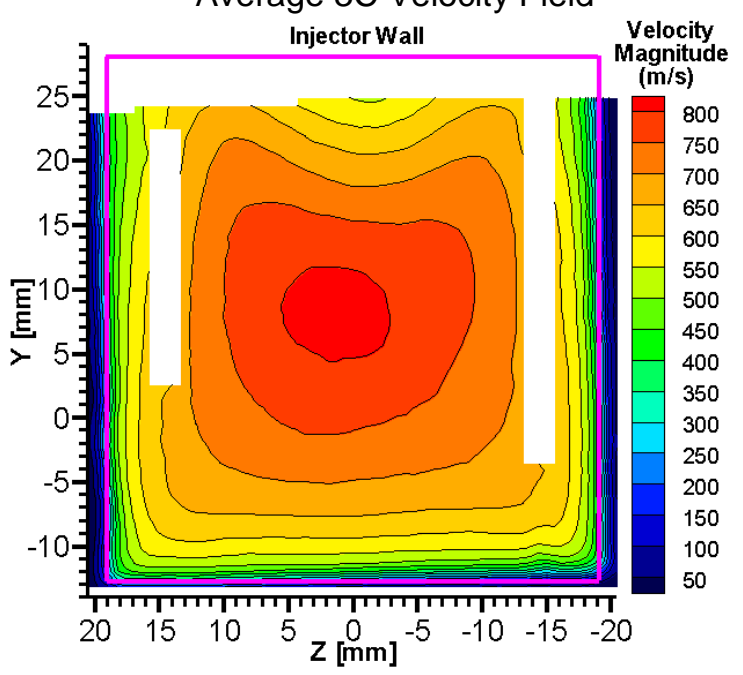

Instantaneous 3C Velocity

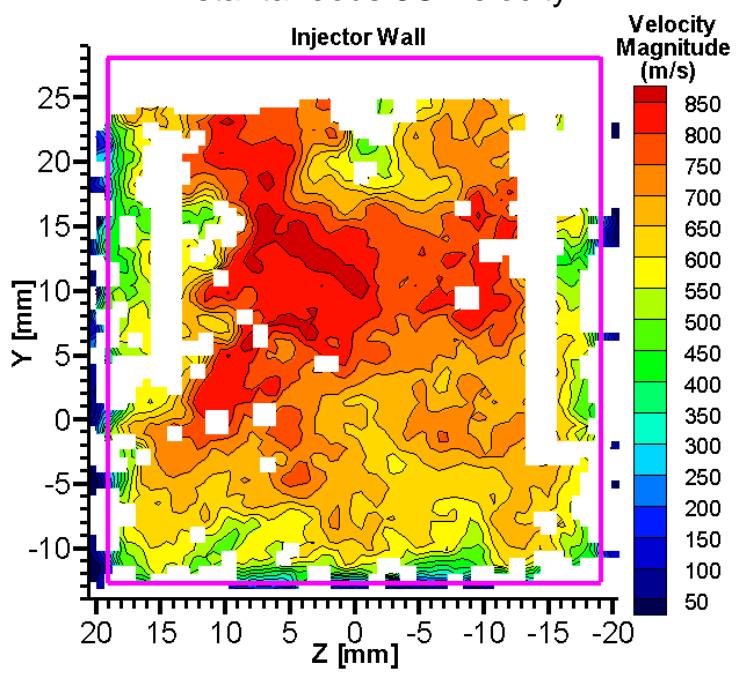

Instantaneous 3C Velocity

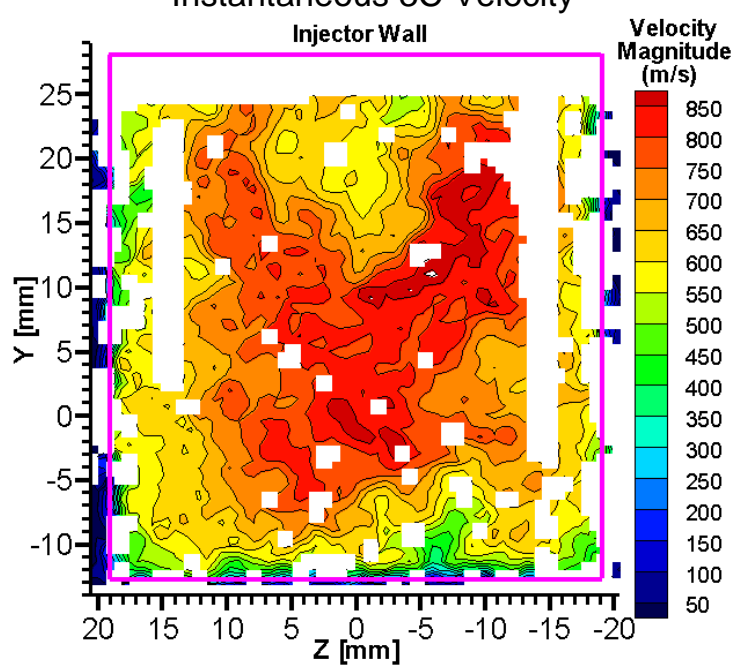

Figure 5.32: Average and Instantaneous velocity fields, Fuel-air Combustion at $X / H=58.6 \Phi=0.34$ 
instantaneous results, some small vortices can be seen near the injector wall and they are in slightly different locations for the two instant fields shown here. These vortices are not present in every instantaneous result and they are not as organized as in the case for $\Phi=$ 0.17 discussed above. Consequently, in the average result for fuel-air combustion with $\Phi$ $=0.34$, the influence of the vortices can clearly be seen in the flow pattern near the injector wall, but not in the form of two well-organized counter-rotating vortices and the influence certainly does not extend out into the main flow to the degree that was present for combustion with $\Phi=0.17$. Figure 5.33 shows the rotation of the average flowfield more clearly than the In-plane vector plots from above. In Fig. 5.33 the rotation in the

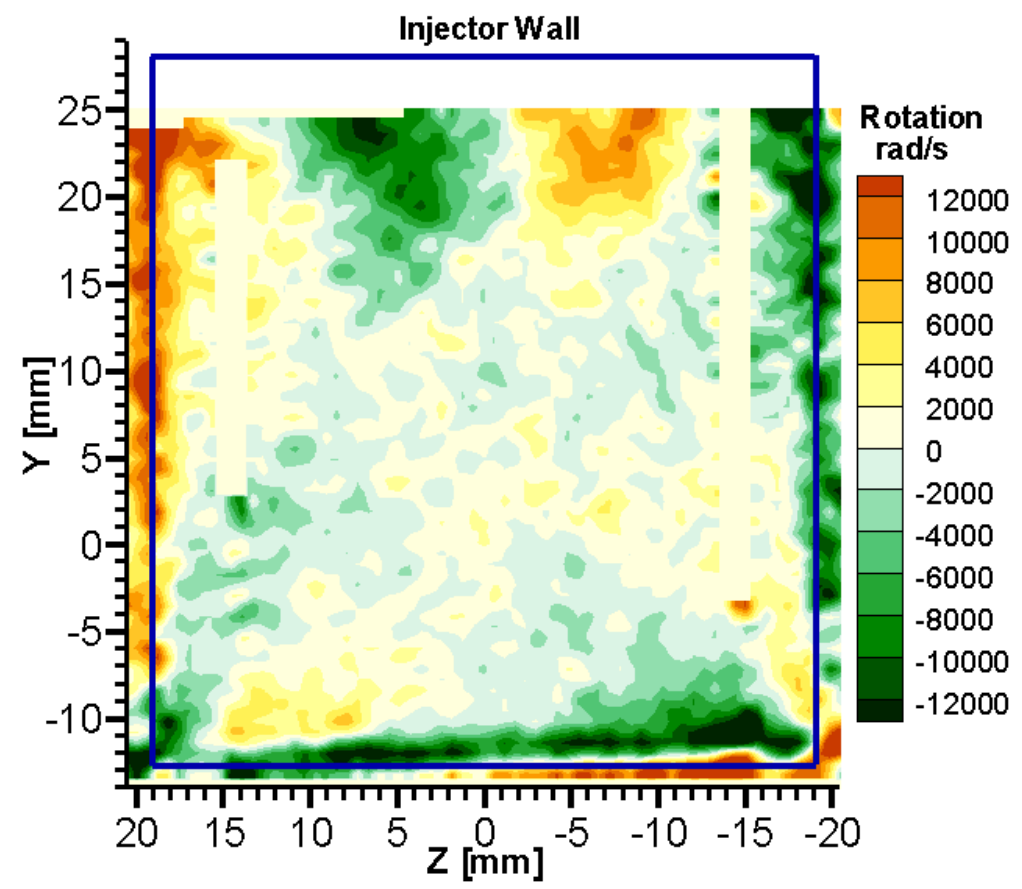

Figure 5.33: Degree of Fluid Rotation for Average SPIV result, $\Phi=0.34$, Fuel-air Combustion case at $\mathrm{X} / \mathrm{H}=58.6$ (Exit plane).

top portion of the plot is due to the two counter-rotating vortices from the ramp fuel injector. Therefore, even though these vortices cannot be clearly seen in the average Inplane vector field, their presence and strength can be inferred from the rotation plot. The 
remaining core-flow area of the field has almost no rotation (values near $0 \mathrm{rad} / \mathrm{s}$ ) and no discernible pattern of rotation. Some non-zero rotation does exist in the entrainment areas, which is not surprising considering the turbulent nature of the entrainment of external air into a supersonic flow. As mentioned above, the larger heat release from combustion at an equivalence ration of $\Phi=0.34$ creates a "thermal choke" and a shock train that causes the flow to be predominantly subsonic in the combustor for the larger equivalence ratio, while the flow remains mostly supersonic for $\Phi=0.17$. It is somewhat surprising that the ramp-induced vortices cannot be viewed for the (predominantly) subsonic combustion case with $\Phi=0.34$, but are clearly seen during supersonic combustion for $\Phi=0.17$. This is likely due to the more chaotic flowfield that exists due to the shock train for the subsonic case and well-organized, ramp-vortices apparently do not remain intact as far downstream as the exit plane for $\Phi=0.34$.

Figure 5.34 shows the turbulence intensity for this combustion case with $\Phi=0.34$. The pattern of turbulence is similar to the combustion case with $\Phi=0.17$ in that the degree of turbulence is at a minimum in the fastest part of the flowfield and turbulence increases in the area near the injector wall due to the influence of the ramp injector. However, for $\Phi=0.34$ combustion, the degree of turbulence is not as high in the area near the injector wall as it was in the case for combustion with $\Phi=0.17$. Furthermore, for $\Phi=0.34$ combustion, the degree of turbulence is low for most of the flowfield (away from the injector wall) in the area of highest velocity. Finally, very large degree of turbulence (ranging from $25 \%$ to $150 \%$ ) can be seen in the entrainment region.

After completion of the SPIV measurements with Flowpath1, a qualitative comparison was completed for the SPIV measurements and Computational Fluid 
Dynamics (CFD) results completed elsewhere. This qualitative comparison is presented in the next section.

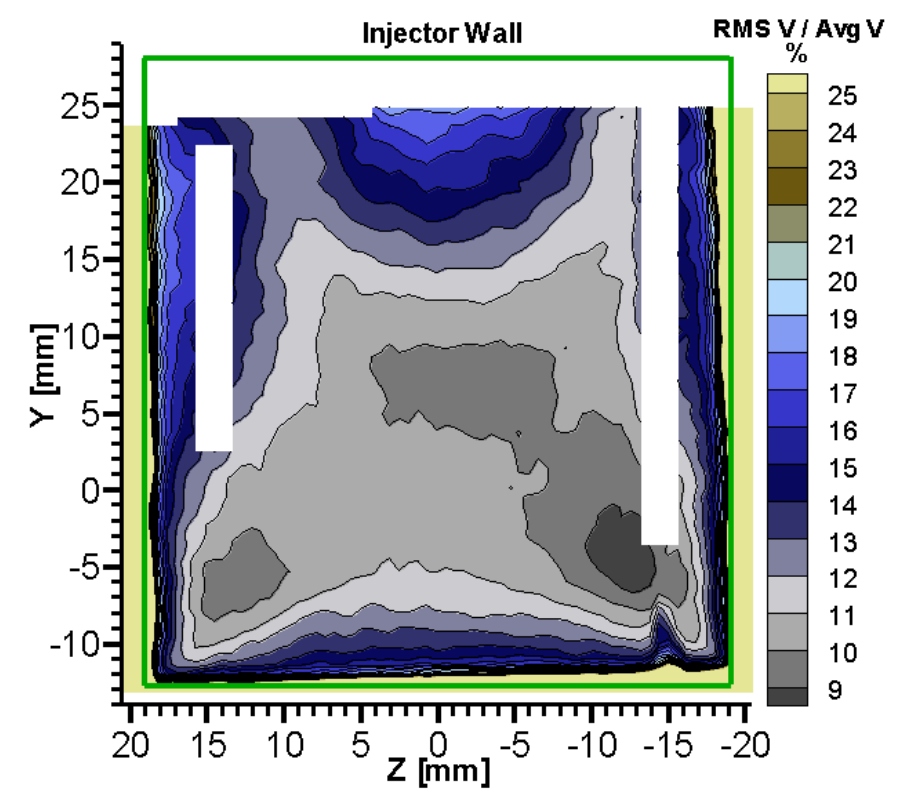

Figure 5.34: Turbulence Intensity (RMS of $3 \mathrm{C}$ Velocity normalized by Average velocity) of Fuel-air Combustion case $(\Phi=0.34)$ at $\mathrm{X} / \mathrm{H}=58.6$ (Exit plane).

\subsection{Qualitative Comparison to CFD results, Flowpath 1}

The Wind-US Computational Fluid Dynamics (CFD) flow solver was used to simulate the flowfield of the direct-connect combustor (Flowpath 1) in the University of Virginia supersonic combustion facility. This CFD work was completed by Dr. William Engblom and his group at Embry-Riddle Aeronautical University. Wind-US is a Reynolds-averaged Navier-Stokes (RANS) flow solver which can be used for simulation of chemically-reacting, compressible flows. Additional information about the Wind-US CFD solver, and application to chemically reacting flows, can be found in references 48 and 49. One half of the flowpath was simulated with the symmetry plane at the centerline at $\mathrm{z}=0$ and a mesh of 5.82 million structured grid points was used for the CFD 
simulations. A denser grid was used to simulate areas near the injector wall, with a coarser grid across the remainder of the flowpath. Thermal boundary conditions were supplied from wall temperature measurements, where available, from experiments with Flowpath 1 at the University of Virginia. Further information about these CFD simulations, including the numerical schemes and turbulence models used, can be found in reference 50 .

Results from CFD simulations were not examined prior to completion of SPIV measurements so that the CFD results would not influence processing of the SPIV data. CFD modelers report reasonably good agreement between these simulations and wall pressure data from experiments. ${ }^{50}$ However, CFD results can match wall pressure data even when the flowfield is not correctly simulated, so the real test of the simulation is a comparison of CFD predictions to experimental measurements for velocity and other quantities in the core of the flow. All three components of velocity from CFD results are compared here to corresponding velocity components from SPIV measurements.

Figure 5.35 shows plots for axial $(\mathrm{u}), \mathrm{v}$, and w velocity components from CFD and SPIV results for fuel-air combustion at the $\mathrm{X} / \mathrm{H}=10$ cross-plane, with fuel equivalence ratio of $\Phi=0.26$. Plots on the left side of the figure are averaged fields from SPIV measurements, while the CFD results are shown in the plots on the right side of the figure. For SPIV measurements, only the fuel was seeded so that velocity measurements occurred for only part of the plane. In order to facilitate comparison of SPIV and CFD results, a dashed box is included in each CFD plot to indicate the location of the corresponding SPIV measurements. Identical color scales have been used to plot SPIV measurements and CFD results for each velocity component. The dominant component 
Experiment (SPIV) Axial (u) Velocity

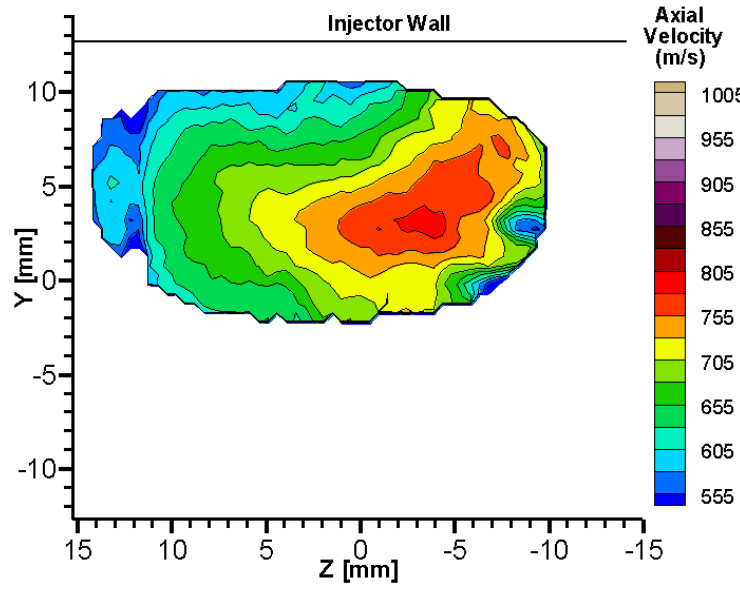

Experiment y-component (v) Velocity

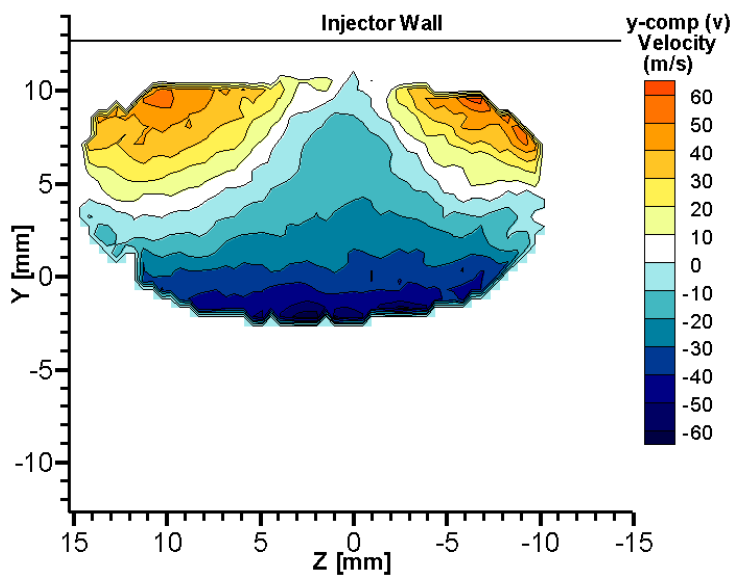

Experiment z-component (w) Velocity

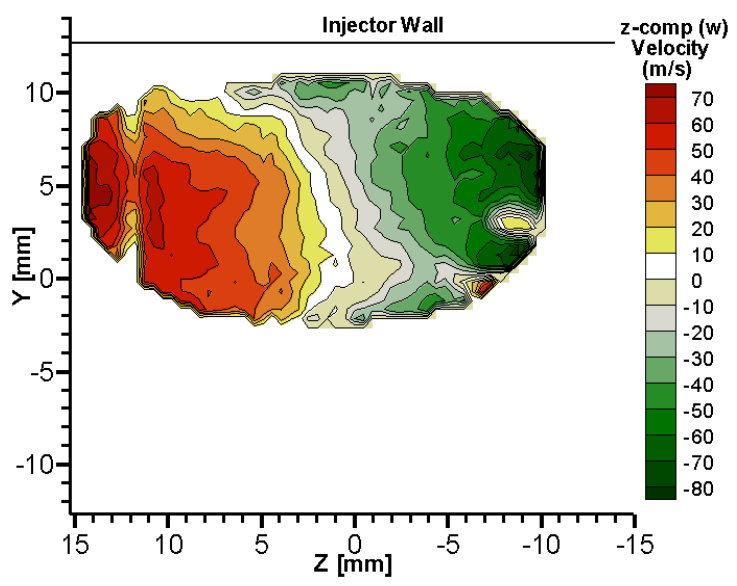

CFD Result, Axial (u) Velocity

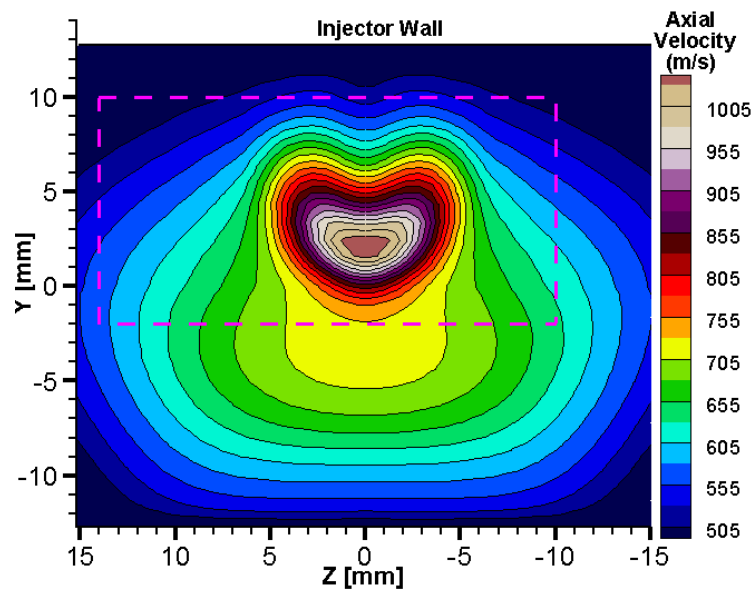

CFD y-component (v) Velocity

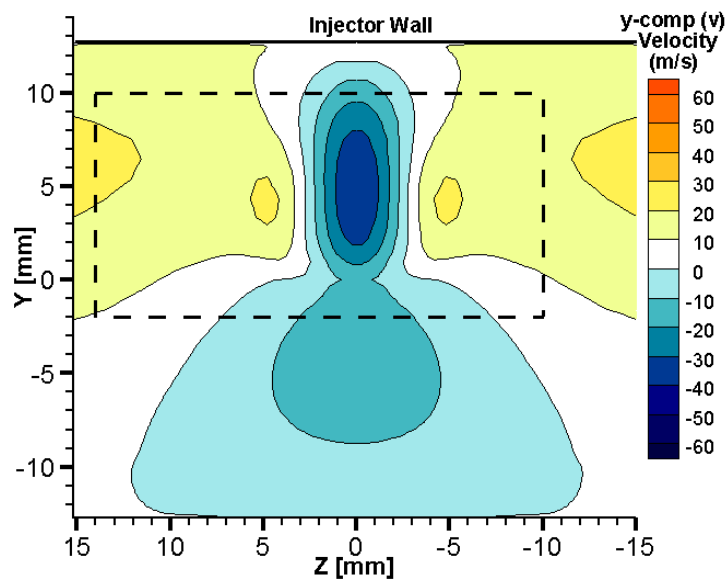

CFD z-component (w) Velocity

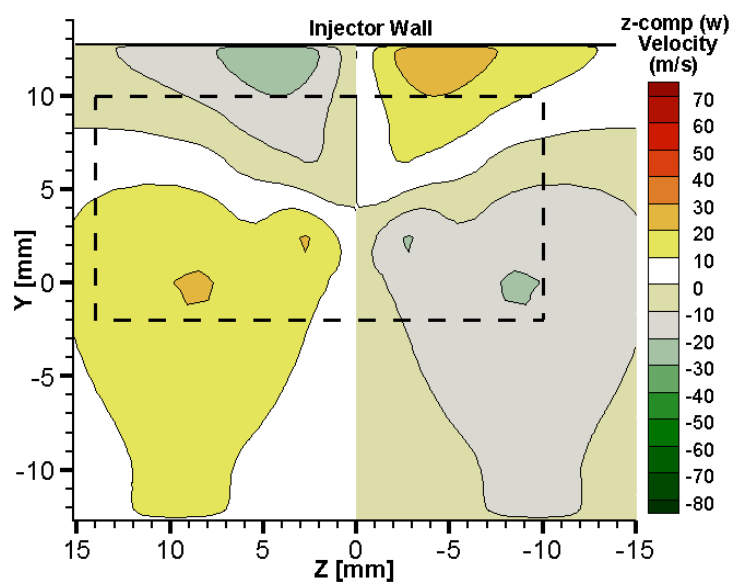

Figure 5.35: Experiment (SPIV) and CFD comparison, Fuel-air Combustion, $\mathrm{X} / \mathrm{H}=10, \Phi=0.26$ 
of this flow is the axial (x-direction) velocity and large differences are observed when comparing SPIV measurements to the CFD result at the $\mathrm{X} / \mathrm{H}=10$ plane. The RANS CFD simulation greatly over-predicts the velocity at the center of the fuel plume. SPIV measurements show velocity ranging from approximately $540 \mathrm{~m} / \mathrm{s}$ to $790 \mathrm{~m} / \mathrm{s}$ across the area of the fuel plume, while the CFD results show axial velocities as high as $1050 \mathrm{~m} / \mathrm{s}$ at the fuel plume core. In the CFD result for axial velocity, the high-speed fuel core with lower velocity around the edge indicates a pure hydrogen core in the plume and the lower velocity outside the core results from combustion in that area. This pure hydrogen core is also indicated by lower temperature $(\sim 630 \mathrm{~K})$ in the core region as opposed to higher temperatures in the remainder of the cross-plane. It seems that the CFD simulation under-predicts the degree of mixing of free-stream air into the hydrogen fuel plume, thus resulting in a pure hydrogen core where no combustion occurs. SPIV measurements indicate a high degree of fuel-air mixing which causes efficient combustion, heat release, and lower velocity across the entire fuel plume area.

Examining the In-plane velocity components, CFD simulations greatly underpredict the magnitude of both the $\mathrm{v}$ and $\mathrm{w}$ velocity components. SPIV measurements show that the y-component (v) velocity ranges from $-65 \mathrm{~m} / \mathrm{s}$ to $+64 \mathrm{~m} / \mathrm{s}$ and z-component (w) velocity from -85 to $+78 \mathrm{~m} / \mathrm{s}$. However, CFD results indicate $\mathrm{v}$ and $\mathrm{w}$ velocity components ranging from $-38 \mathrm{~m} / \mathrm{s}$ to $+22 \mathrm{~m} / \mathrm{s}$ and $-26 \mathrm{~m} / \mathrm{s}$ to $+26 \mathrm{~m} / \mathrm{s}$, respectively. The CFD result under-predicts the y-component magnitude, shows a contour pattern that only slightly resembles the average SPIV measurement, and has in-plane vectors of the wrong direction along the line $\mathrm{Z}=0$. Similar disparity exists for the $\mathrm{z}$-component velocity. Figure 5.36 also shows velocity components from SPIV measurements and CFD results, 
Experiment (SPIV) Axial (u) Velocity

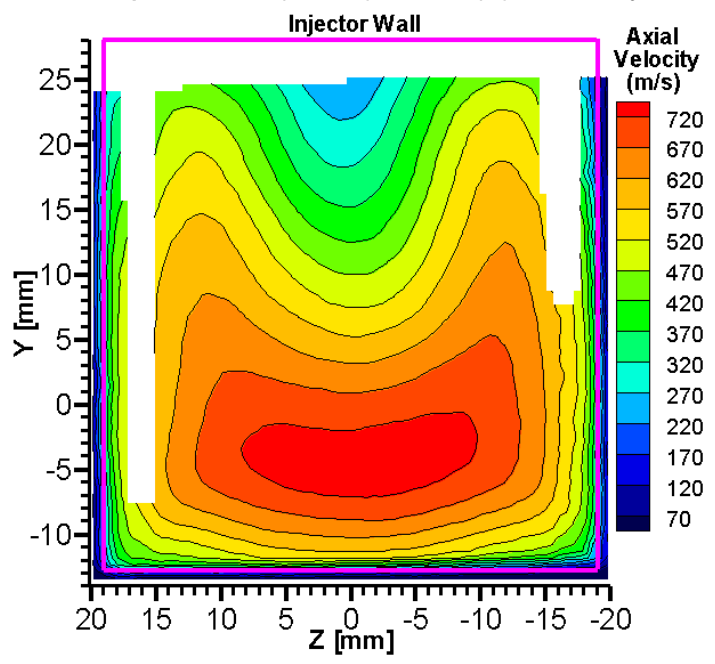

Experiment y-component Velocity

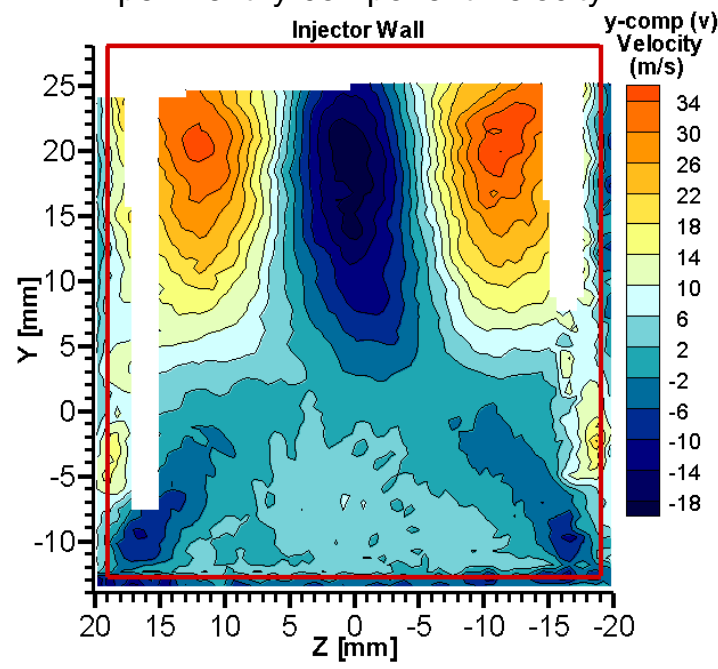

Experiment z-component Velocity

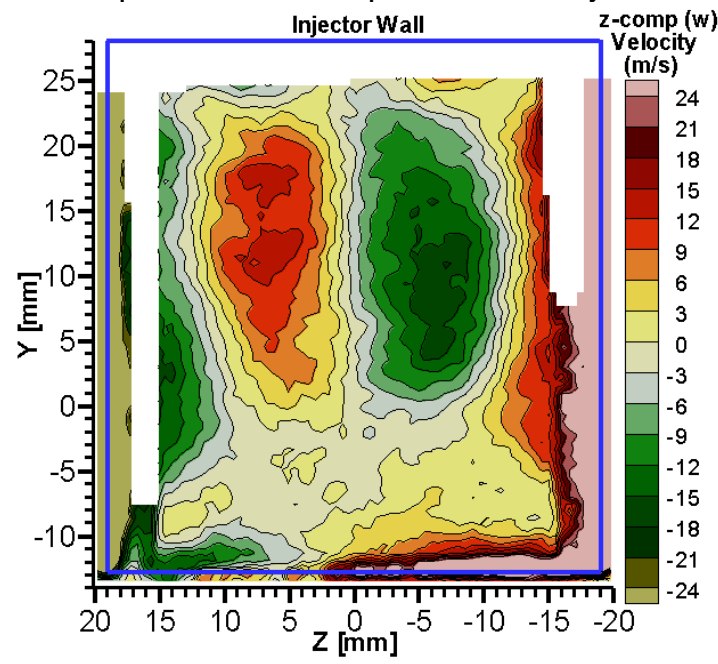

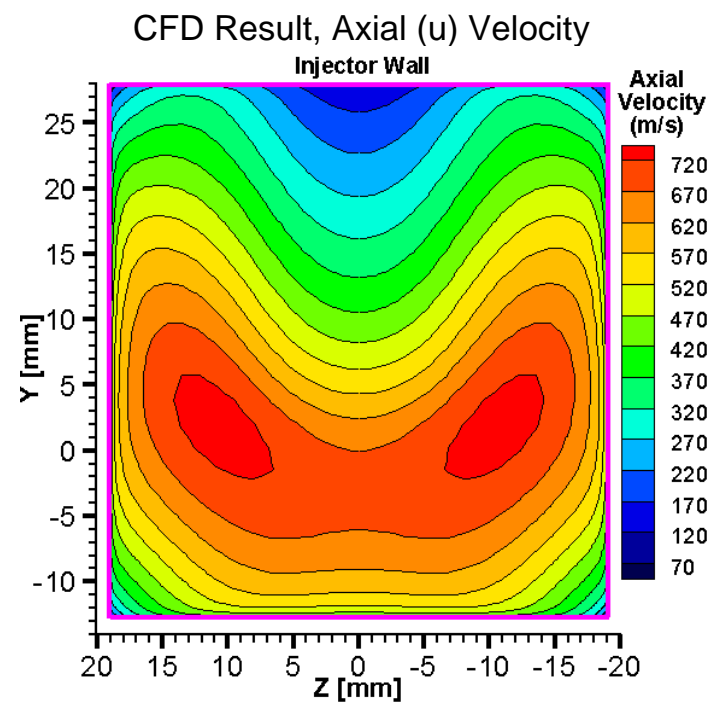
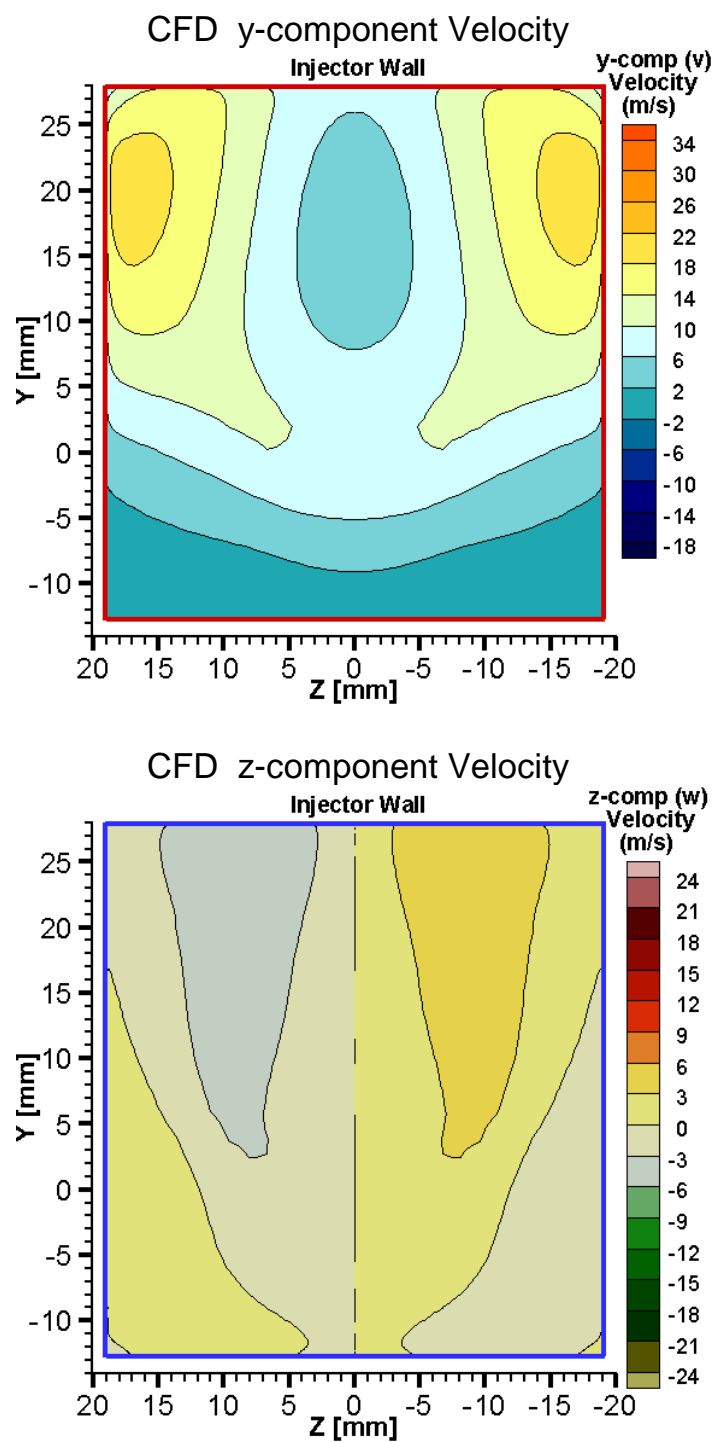

Figure 5.36: Experiment (SPIV) and CFD comparison, Fuel-air Combustion, $\mathrm{X} / \mathrm{H}=58.6, \Phi=0.17$ 
but this time for fuel-air combustion with $\Phi=0.17$ at the exit plane. The RANS CFD simulation matches SPIV results relatively well. For the axial velocity component, the magnitude and contour pattern in the CFD simulation matches the average SPIV result quite well. The maximum velocity region near the observation wall can be seen in both the CFD and SPIV results, albeit the maximum velocity contour is somewhat different in shape. Moreover, the lower velocity region toward the injector wall also matches in the CFD and average SPIV results. The y-component in the CFD result is somewhat similar in shape to the SPIV measurement, except that the CFD under-predicts the magnitude of the y-component. CFD simulation greatly under-predicts the magnitude of the $\mathrm{z}$ component and also incorrectly predicts the direction. This is because the CFD simulation does not accurately capture the ramp-induced vortices in the exit cross-plane for $\Phi=0.17$. Figure 5.37 shows a comparison of In-plane velocity vectors from the average SPIV measurement and the CFD result. CFD simulation was able to accurately

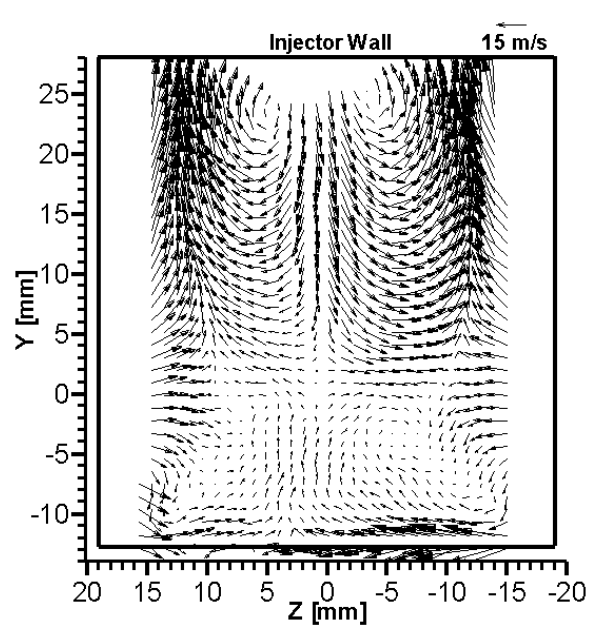

(a) Average Experimental (SPIV) Result

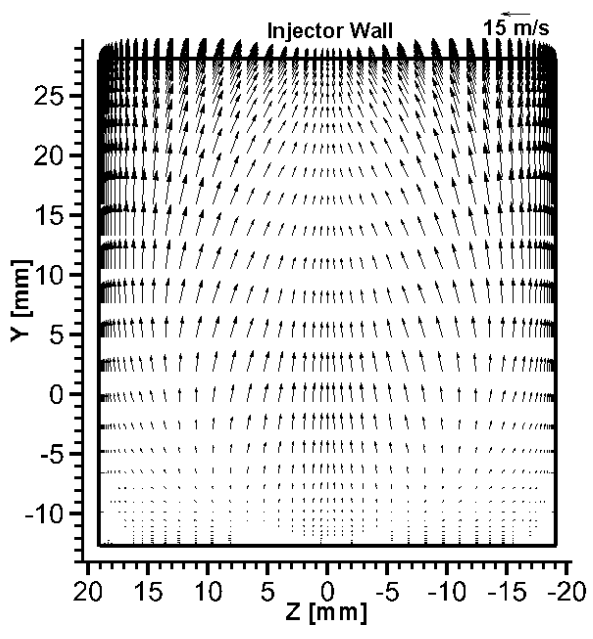

(b) In-plane vectors of CFD Result

Figure 5.37: $\Phi=0.17$, Fuel-air Combustion at $\mathrm{X} / \mathrm{H}=58.6$ (Exit)

(a) Average SPIV velocity field showing In-plane vectors, and (b) CFD Result 
predict the largest velocity component (axial velocity) for combustion with $\Phi=0.17$ at the exit plane, but could not correctly predict the In-plane velocity components.

Figure 5.38 compares CFD and SPIV results for combustion with $\Phi=0.34$ at the exit plane. For this case, the CFD simulation captures the axial velocity component fairly well, with only a slight over-prediction $(\sim 50 \mathrm{~m} / \mathrm{s})$ of the maximum axial velocity for this combustion case $(\Phi=0.34)$ at the exit plane of the Extender. However, near the injector wall, the influence of the ramp-induced vortices on the axial velocity component, as seen in SPIV measurements, is not predicted by the CFD simulation. The shape of the contours predicted by CFD for the $\mathrm{y}$ and z-components is similar to the SPIV result. However, as in the CFD result for $\Phi=0.17$, CFD simulation for combustion with $\Phi=$ 0.34 again greatly under-predicts the magnitude of the $\mathrm{y}$ and z-components and shows the incorrect sign (direction) of the z-component velocities. Comparison of the RANS CFD results to SPIV velocity measurements, presented here, shows clear deficiencies in the CFD simulation for fuel-air combustion, especially for the In-plane velocity components at the exit plane and all components at $\mathrm{X} / \mathrm{H}=10$ with equivalence ratio of $\Phi=0.26$.

\section{Summary}

The measurements obtained for Flowpath 1 show some expected characteristics. The ramp-induced vortices were observed in the test section and the decrease in velocity due to heat release from combustion was also measured. Furthermore, both fuel-air mixing cases at the exit plane showed an expected turbulent flowfield with large velocity at the center and the structured vortices were not observed. However, some surprising results should also be noted. At the $\mathrm{X} / \mathrm{H}=10$ plane, even though they were less- 
Experiment (SPIV) Axial (u) Velocity

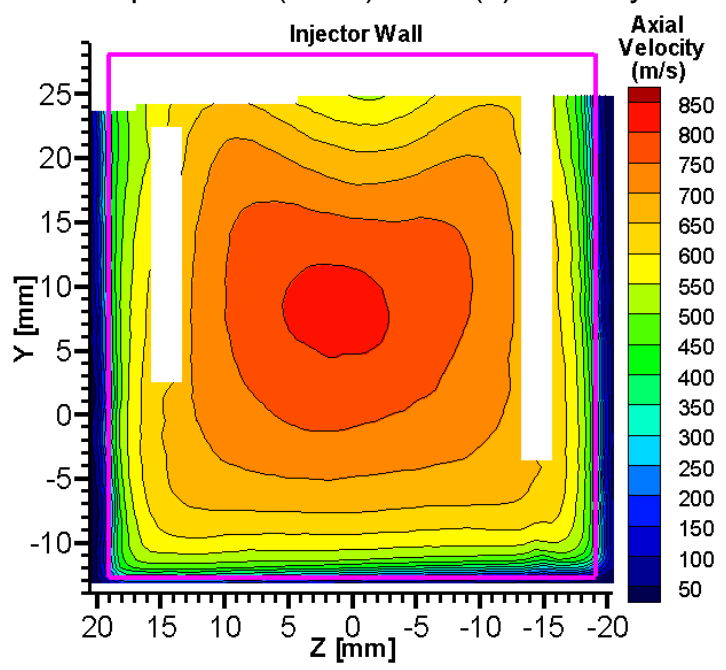

Experiment y-component (v) Velocity

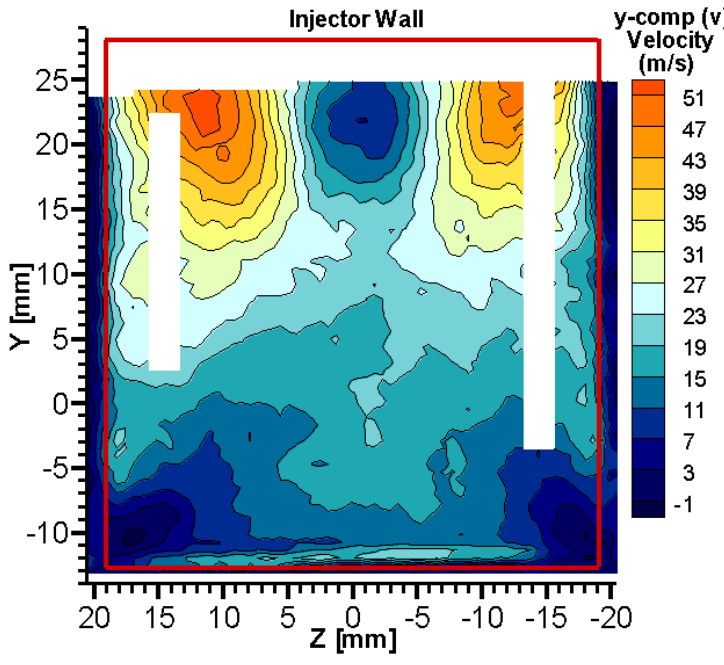

Experiment z-component (w) Velocity

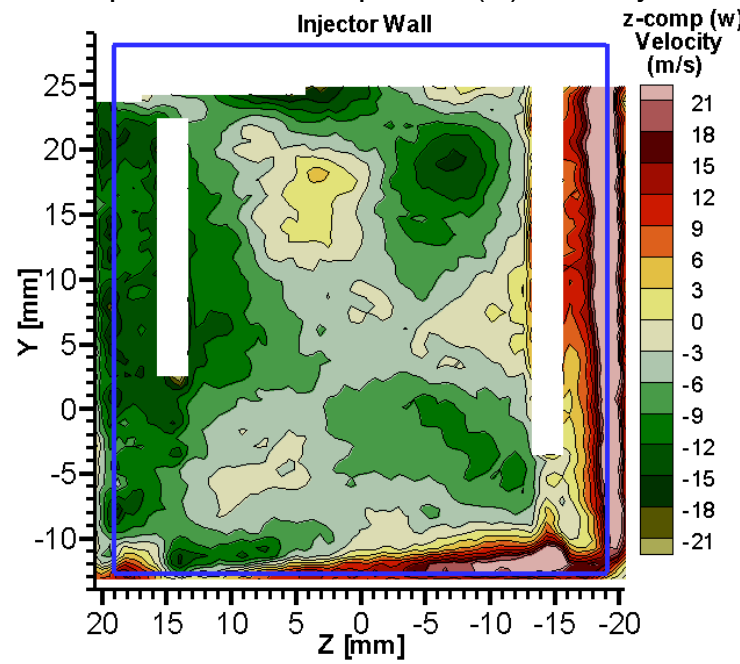

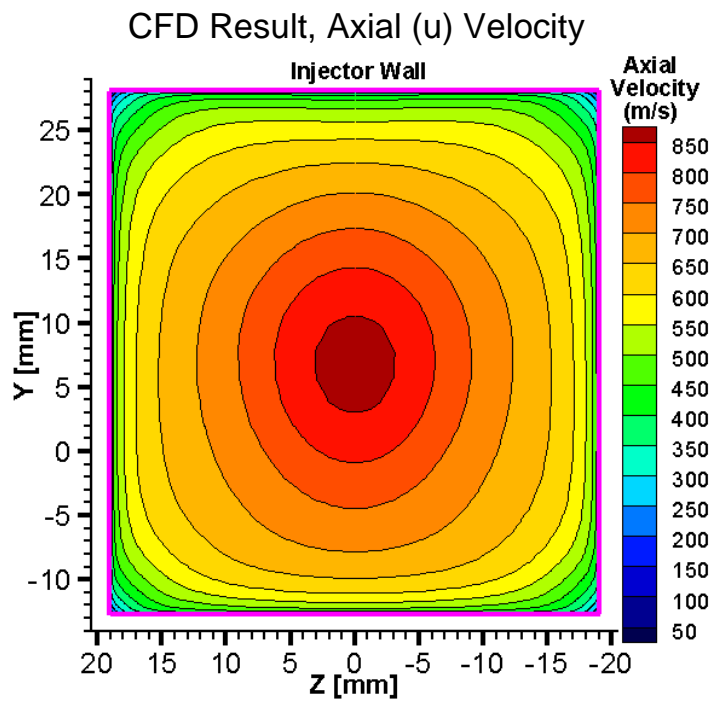

CFD y-component (v) Velocity

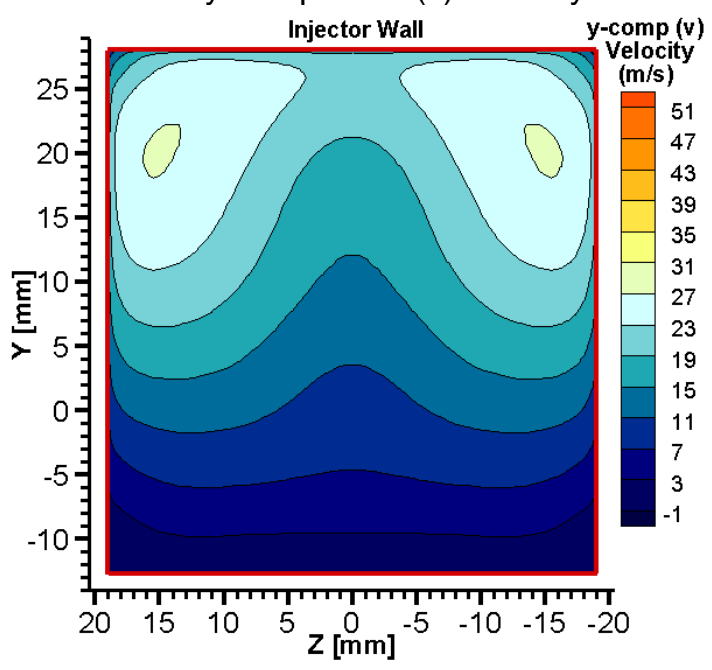

CFD z-component (w) Velocity

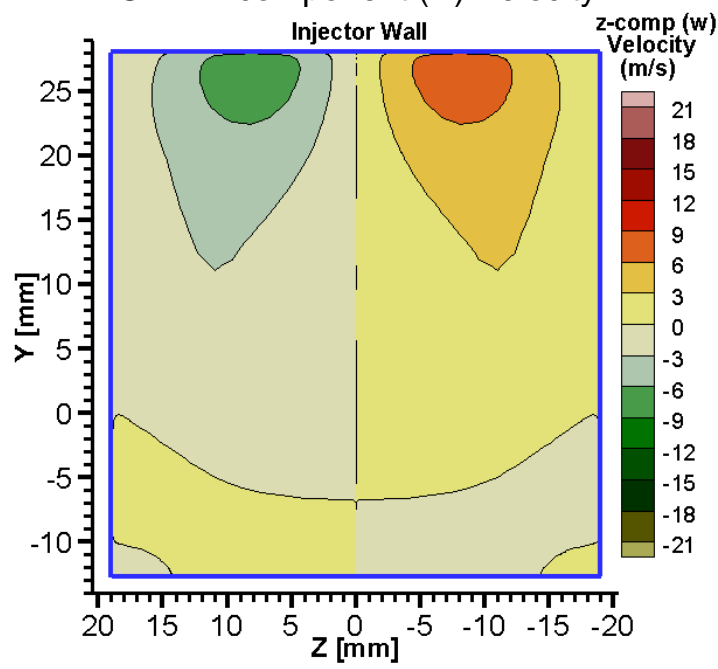

Figure 5.38: Experiment (SPIV) and CFD comparison, Fuel-air Combustion, $X / \mathrm{H}=58.6, \Phi=0.34$ 
organized, the vortices were still observed during fuel-air combustion rather than breaking apart completely. The influence of the vortices was even seen as far downstream as the exit plane, and dominated the flowfield of the exit plane combustion with $\Phi=0.17$. Rotation plots show that, in general, the vortices remain organized as they propagate downstream and that is good for efficient fuel-air mixing required for combustion in such a high-velocity flow. In general, turbulence was more pronounced in the area of the vortices during combustion and turbulence was higher for the combustion case than for mixing in the Test-section. The opposite trend was noted for turbulence intensity between mixing and combustion cases at the exit plane. RANS CFD results seem to under-predict the degree of fuel-air mixing. This explains why agreement is so poor between SPIV and CFD results in the test section $(\mathrm{X} / \mathrm{H}=10)$ but matches better at the exit plane - the fuel has had time to mix by the time the exit plane is reached, while some fuel remains unmixed at $\mathrm{X} / \mathrm{H}=10$ due to inadequate turbulence models used with the simulations. RANS results only matched SPIV measurements well for the axial velocity component and the magnitude of each in-plane component was under-predicted in all cases. Clearly the RANS model used to simulate combustor Flowpath 1 needs to be improved in order for CFD predictions to more accurately match SPIV experimental measurements of this flowfield. 


\section{Chapter 6: SPIV Experimental Results, Combustor Flowpath 2}

Stereoscopic Particle Image Velocimetry (SPIV) was used for velocity measurements at two separate locations in DMSJ combustor Flowpath 2. As mentioned above, Flowpath 2 does not contain an Isolator section. The absence of an Isolator allowed for a well-characterized inflow boundary condition and this combustor configuration was requested by CFD modelers. The absence of an Isolator section also required experiments to only be conducted with fuel equivalence ratio of $\Phi=0.17$ so that the DMSJ combustor would operate in a mode where the flow is predominantly supersonic during combustion. Equivalence ratios much higher than $\Phi=0.17$ would cause predominantly subsonic combustion and, without a long Isolator section to isolate the shock train of the subsonic combustion case, shockwaves could move far upstream and cause damage to the facility nozzle. Injector wall divergence in Flowpath 2 begins upstream of the ramp injector, so the flowfield was expected to be very different than that of Flowpath 1. Further information about Flowpath 2 was presented in Chapter 2.

SPIV measurements were conducted at two planes in the Test-section of Flowpath 2. The measurement plane at $\mathrm{X} / \mathrm{H}=12$ was chosen because of the importance of that location to the work of the National Center for Hypersonic Combined Cycle Propulsion. As mention above, attempts were made to conduct SPIV experiments at $\mathrm{X} / \mathrm{H}=18$, but large background noise from the ends of the side-windows prevented adequate particle images. The plane located at $\mathrm{X} / \mathrm{H}=15$ in the Test-section of Flowpath 2 was chosen as an alternative to $\mathrm{X} / \mathrm{H}=18$. Chapter 4 contains details about the experimental configurations and flow conditions for both the $\mathrm{X} / \mathrm{H}=12$ and 15 planes. SPIV measurements at these planes in Flowpath 2 are presented here (Chapter 6) as well as 
quantities derived from the velocity measurements. Chapter 6 begins with SPIV velocity measurements at $\mathrm{X} / \mathrm{H}=12$ for the case of fuel-air mixing, followed by measurements for the fuel-air combustion case at $\mathrm{X} / \mathrm{H}=12$. This chapter continues with SPIV measurements for both fuel-air mixing and fuel-air combustion cases at $\mathrm{X} / \mathrm{H}=15$, followed by a comparison of SPIV measurements to CFD results obtained using a hybrid Large Eddy Simulation/Reynolds-averaged Navier-Stokes (LES/RANS) scheme.

\section{1 $\mathrm{X} / \mathrm{H}=12$ Measurement Plane Results}

SPIV measurement results for the $\mathrm{X} / \mathrm{H}=12$ measurement plane of Flowpath 2 are presented here. Figure 6.1 shows a schematic and photograph of Flowpath 2 with the $\mathrm{X} / \mathrm{H}=12$ measurement plane indicated. In the photograph of Fig. 6.1, the laser sheet at $\mathrm{X} / \mathrm{H}=12$ can be seen exiting one of the Test-section side-windows. A main concern for measurement planes in the test section is the presences of oblique shockwaves that negatively influence particle tracking. Figure 6.2 shows a shadowgraph of Flowpath 2 provided by Dr. Toshinori Kouchi. ${ }^{51}$ In the figure, oblique shockwaves can be seen near the $\mathrm{X} / \mathrm{H}=12$ and 15 measurement planes. A weak shockwave (wave angle $~ 33$-deg.) crosses the $\mathrm{X} / \mathrm{H}=12$ plane at a location very near the injector wall and passes through the $\mathrm{X} / \mathrm{H}=15$ plane near the center of the duct. It should also be noted that the curved shadow at the right of the picture is a window imperfection and not a structure of the flow. Only the fuel was seeded for these experiments and the fuel plume is located near the injector wall, therefore all velocity measurements at $\mathrm{X} / \mathrm{H}=12$ are in the area away from the shockwave. Furthermore, the majority of the measurements are outside the influence of the shockwave at $\mathrm{X} / \mathrm{H}=15$. At $\mathrm{X} / \mathrm{H}=15$, particle tracking error could 


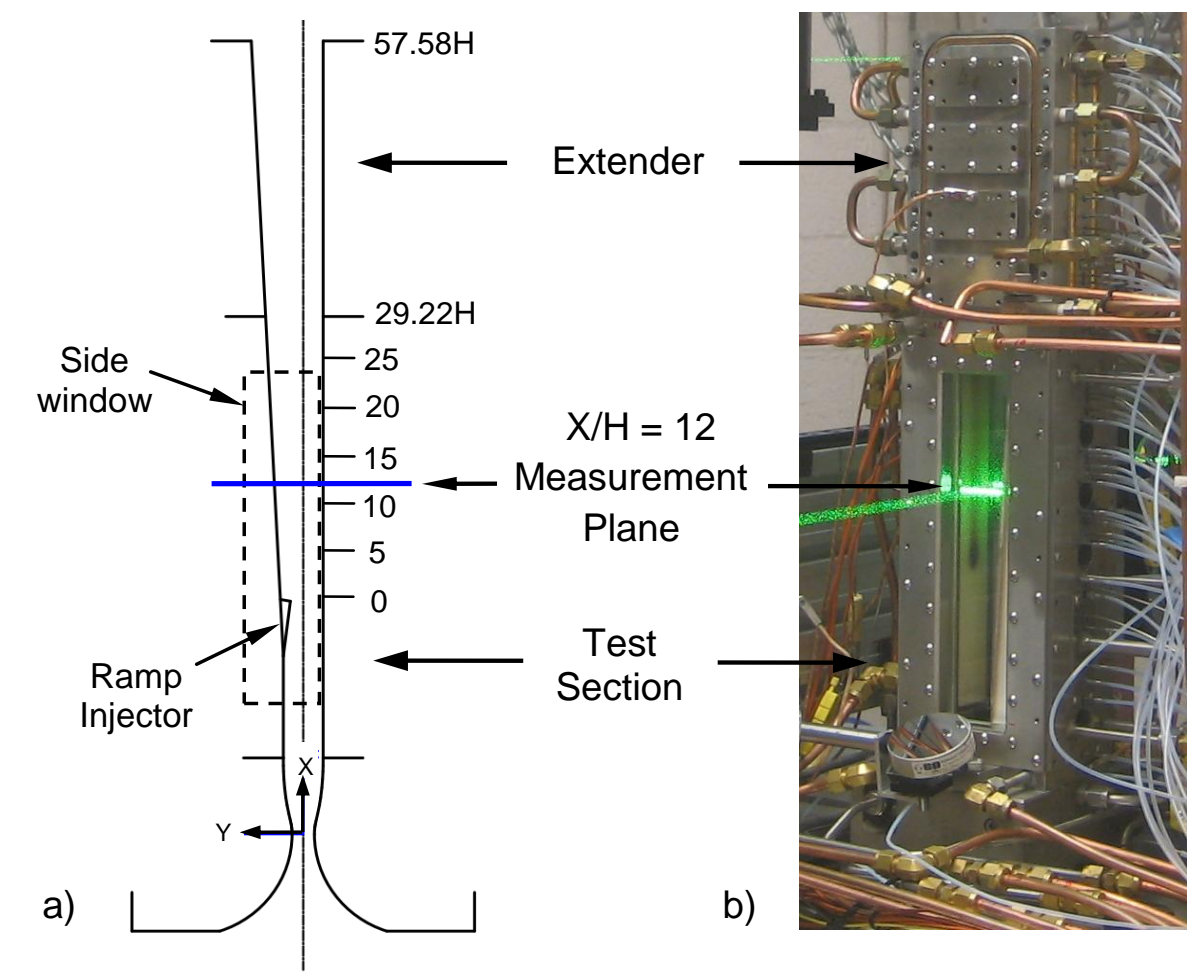

Figure 6.1: Flowpath 2 with $\mathrm{X} / \mathrm{H}=12$ measurement plane.

a) Schematic and b) Photograph with laser sheet exiting through tunnel side-window

occur at the edge of the plume (observation wall side) but the error is minimal because the weak shock only causes a small change in free-stream velocity.

Results from SPIV for the fuel-air mixing case at $\mathrm{X} / \mathrm{H}=12$ are presented briefly in section 6.1.1, with additional plots relegated to the appendices. Section 6.1.2 contains SPIV measurements for the more interesting case of fuel-air combustion at $\mathrm{X} / \mathrm{H}=12$ and those results are discussed in more detail than results of the fuel-air mixing case.

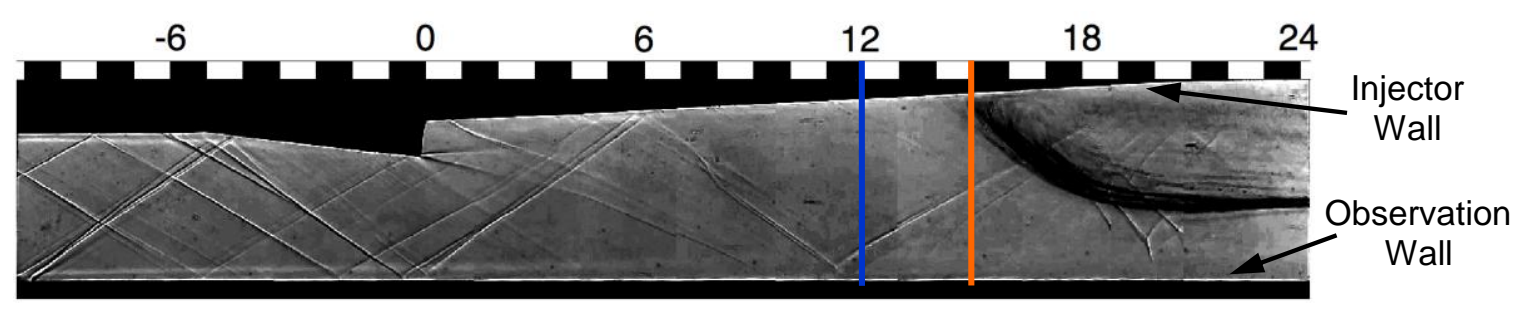

Figure 6.2: Shadowgraph of shockwaves in Flowpath 2 with $\mathrm{X} / \mathrm{H}=12$ and 15 planes indicated 


\subsubsection{Fuel-air Mixing Results, $\mathrm{X} / \mathrm{H}=12$}

Fuel-air mixing measurements are briefly presented here for the $\mathrm{X} / \mathrm{H}=12$ location and fuel equivalence ratio, $\Phi=0.17$. Figures which illustrate the most important characteristics of the fuel-air mixing case are included in this section, while supplementary figures can be found in Appendix D. Figure 6.3 shows $3 \mathrm{C}$ velocity contours and In-plane velocity vectors for the average velocity result from SPIV measurements at $\mathrm{X} / \mathrm{H}=12$. The $3 \mathrm{C}$ velocity magnitude range shown in Fig. 6.3 matches the velocity predicted by $1 \mathrm{D}$ isentropic calculations for flow through a Mach 2 nozzle at these conditions. The location of the injector wall is indicated in all figures of Chapter 6. The vector field of Fig. 6.3 shows two well-defined, counter-rotating vortices produced by the ramp fuel-injector. It should be noted that only every other vector is included in the vector field so that the vortices can clearly be seen. Once again, some degree of asymmetry of the vortices is present, just like in the fuel-air mixing case at $\mathrm{X} / \mathrm{H}$ $=10$ in Flowpath 1 . This time-average velocity result was created by averaging 1521 instantaneous vector fields with approximately $90 \%$ of the fields contributing to average vectors at the center of the measurement area. At least 100 instantaneous vectors contributed to the average at locations around the perimeter and Figure 6.4 shows the vector count distribution across the measurement area. Supplemental figures in Appendix D include examples of the instantaneous vector fields that contributed to the average fuel-air mixing velocity field.

Figure 6.5 shows the degree of rotation of the time-averaged result for fuel-air mixing at $\mathrm{X} / \mathrm{H}=12$ in Flowpath 2. The two, well-organized counter-rotating vortices are 
a)
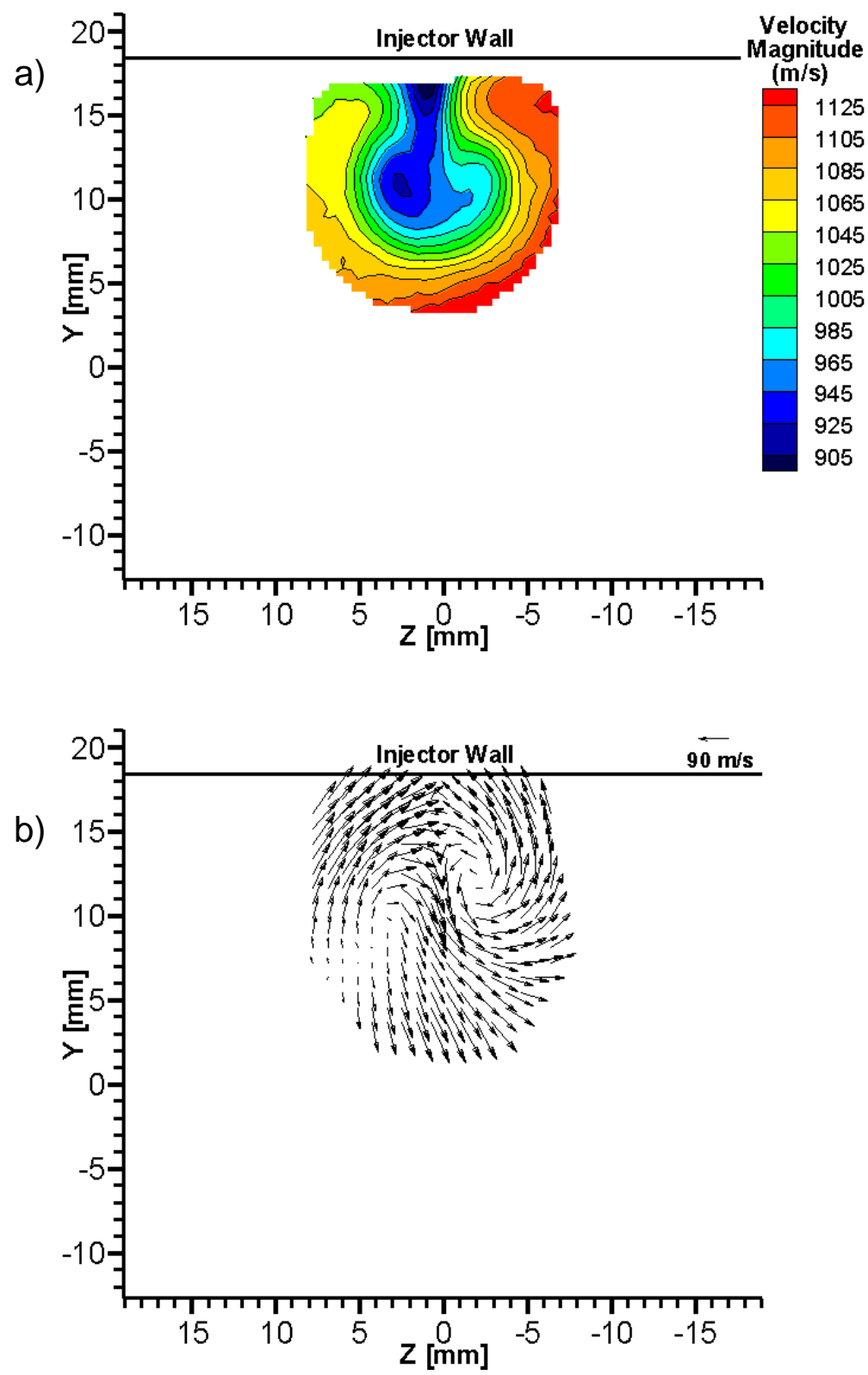

Figure 6.3: Average velocity field for Fuel-air Mixing case at $\mathrm{X} / \mathrm{H}=12$ a)Three-component (3C) velocity magnitude, and b) In-plane velocity vectors.

clearly visible and dominate the rotation plot. Each vortex has a very high degree of rotation, which is at a maximum at the vortex center. In fact, these vortices have higher absolute maximum degrees of rotation than maximums measured for the mixing vortices of Flowpath 1. Outside the area of the counter-rotating vortices, no other rotational features are present in the fuel plume for fuel-on without combustion. 


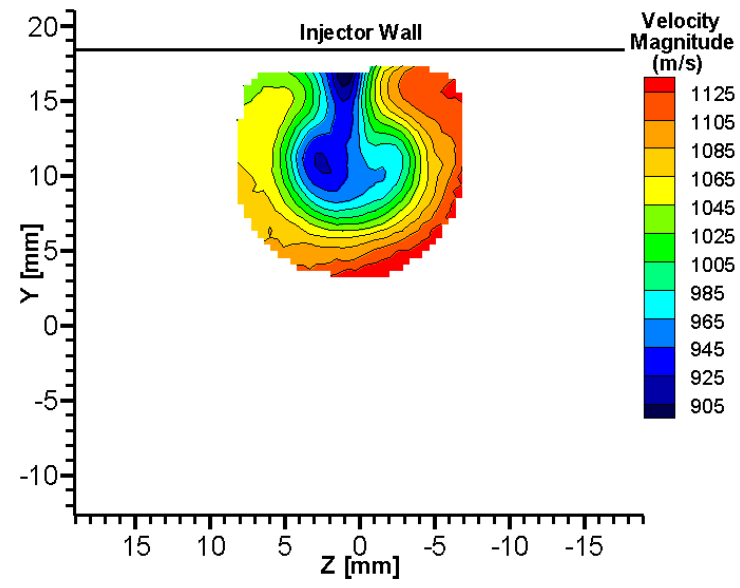

(a) Average of All (1521) Vector Fields [3C Velocity magnitude]

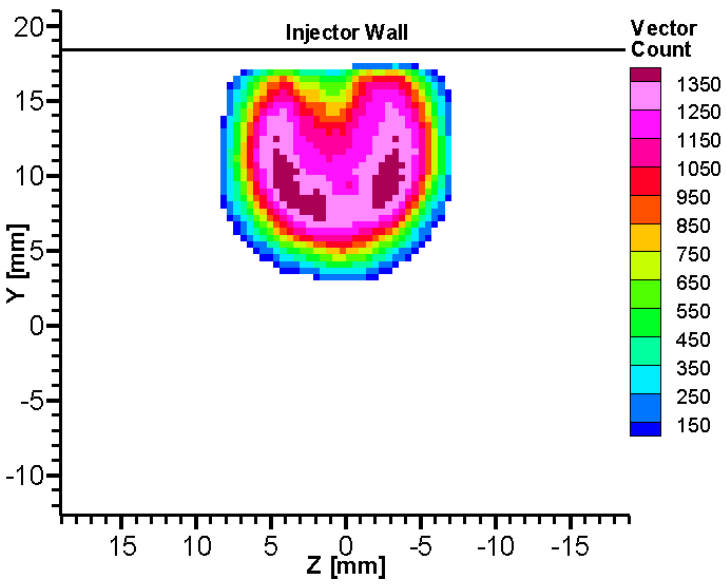

(b) Number of Vectors Averaged for Each Sub-region

Figure 6.4: $\Phi=0.17$, Fuel-air Mixing at $\mathrm{X} / \mathrm{H}=12$;

(a) Average $3 \mathrm{C}$ velocity field, and (b) Number of vectors averaged at each location

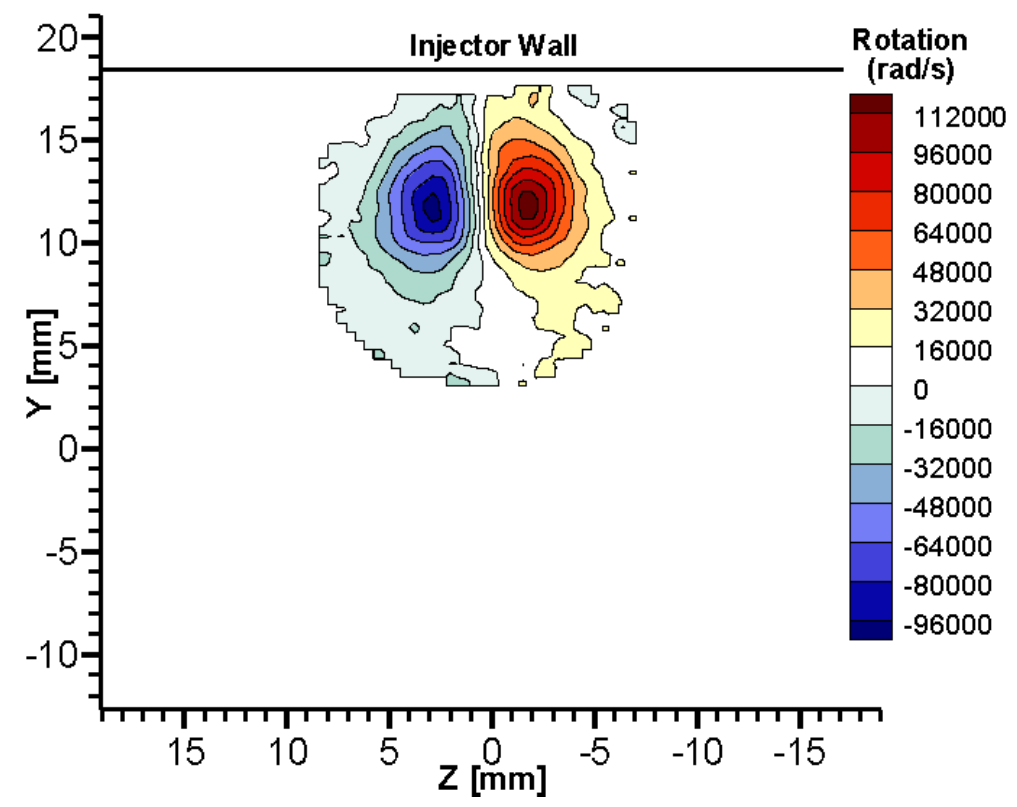

Figure 6.5: Degree of Fluid Rotation for Average SPIV result, $\Phi=0.17$, Fuel-air Mixing case at $\mathrm{X} / \mathrm{H}=12$.

Finally, a measure of the turbulence gives some insight into the fuel-air mixing case at $\mathrm{X} / \mathrm{H}=12$. Figure 6.6 shows a measure of turbulence intensity for fuel-air mixing case, 
calculated from velocity measurements at each location by normalizing the RMS of the 3C velocity magnitude by the average velocity. The plot shows relatively low levels of

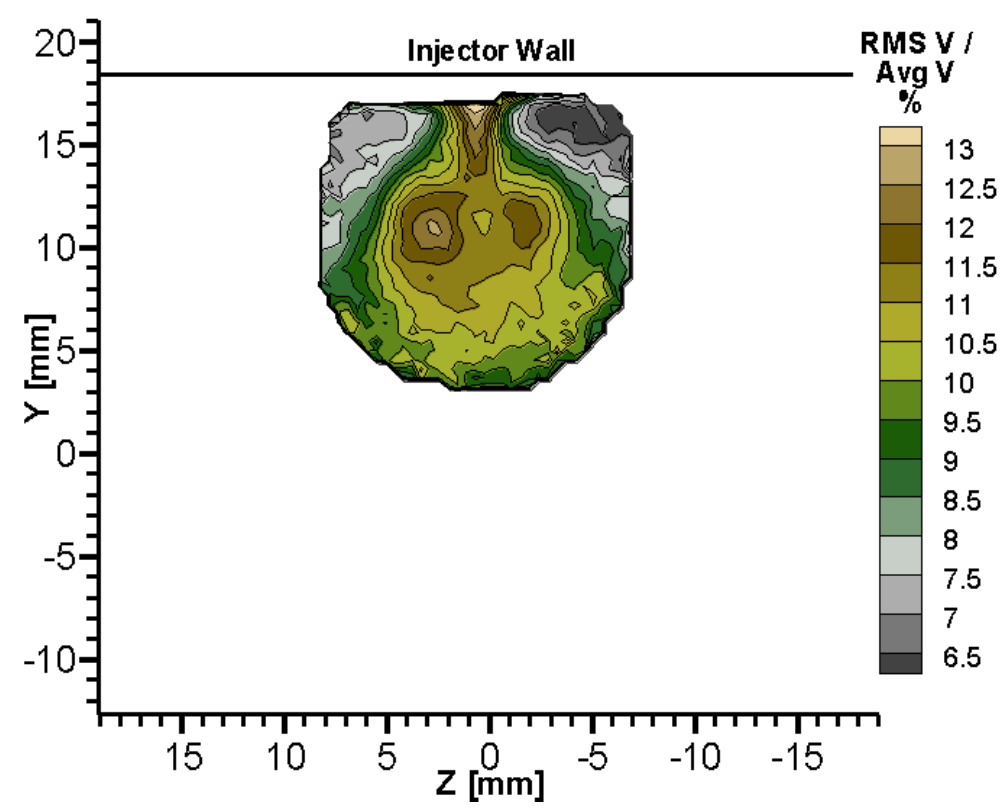

Figure 6.6: Turbulence Intensity (RMS of 3C Velocity normalized by Average velocity) of Fuel-air Mixing case at $\mathrm{X} / \mathrm{H}=12, \Phi=0.17$

turbulence ( $\sim 6 \%$ to $13.5 \%$ ) throughout the fuel plume, which indicates the absence of large velocity changes over time. The highest levels of turbulence are located near the ramp-induced vortices and this effect is mostly due to the movement (walk) of the vortices. Instantaneous measurements indicate the vortices move, relative to one another in both the $y$ and $\mathrm{z}$-directions, on the order of $1-2 \mathrm{~mm}$ from their average positions. The entire fuel plume also translates slightly between instantaneous measurements.

SPIV measurement for the fuel-air mixing case at $\mathrm{X} / \mathrm{H}=12$ in Flowpath 2 showed expected flow characteristics. However, the fuel-air mixing flowfield is not very complicated because of the lack of heat release from combustion. It was necessary to measure both fuel-air mixing and fuel-air combustion cases in order to understand the effects that combustion has on the flow. 


\subsubsection{Fuel-air Combustion Results, $\mathrm{X} / \mathrm{H}=12$}

Using the same experimental configuration as in the mixing case, SPIV measurements were acquired at the same plane during fuel-air combustion. Figure 6.7 shows the average velocity field for fuel-air combustion at $\mathrm{X} / \mathrm{H}=12$ with $\Phi=0.17$. Approximately 2500 SPIV measurements were acquired for this combustion case, however a number of measurements were not useful due to poor particle seeding, laser misfires, or images where the signal from particles was not separable from the background noise. The 1000 measurements acquired at the end of the run were useless due to poor seeding and high background noise in the images. For one measurement set, only approximately $1 / 3$ of 500 SPIV images could be used because flameout occurred near measurement \#165 of the set. After removal of useless SPIV images, 1162 instantaneous fields contributed to the average velocity result. The distribution of vector count was similar to that of the mixing case and a plot of vector count is included in Appendix D. A dashed line has been included in Fig. 6.7 (a) to show the extent of the fuel-plume for the combustion case. Incorrect vectors arose from correlations of particles with laser reflections and, as mentioned in Chapter 4, these vectors could not be removed with a filtering scheme. Instead, a mask was created to remove data in areas outside of approximately $\mathrm{z}=+/-8 \mathrm{~mm}$. Figure 6.8 shows the average vector field computed when the mask was not used to remove incorrect vectors and the large horizontal vectors in the figure are clearly non-physical results. For clarity only every other row of vectors is shown if Fig. 6.8. Returning to Figure 6.7, it is clear that the combustion flowfield is much more complicated than the fuel-air mixing case. The heat release from combustion 
a)

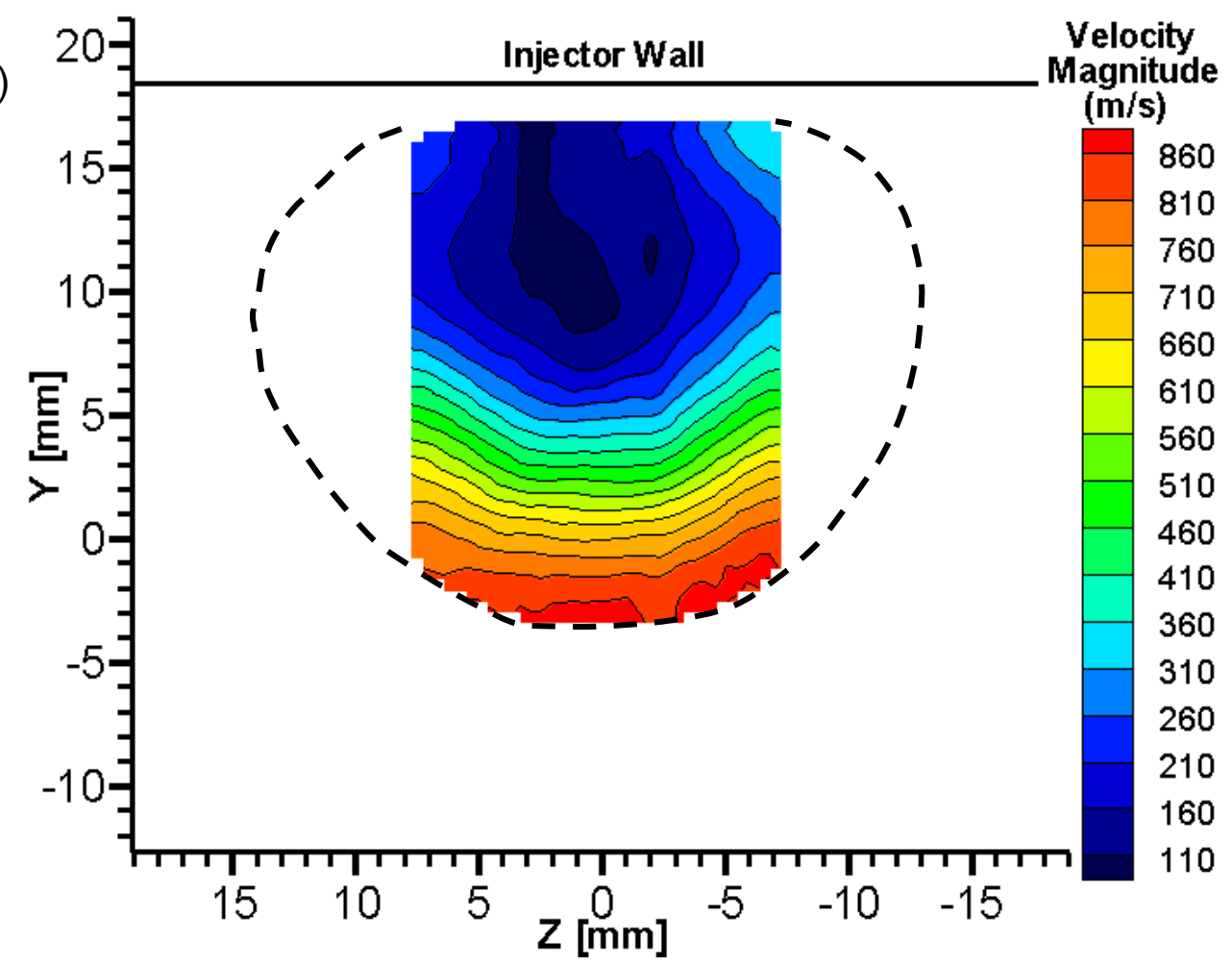

b)

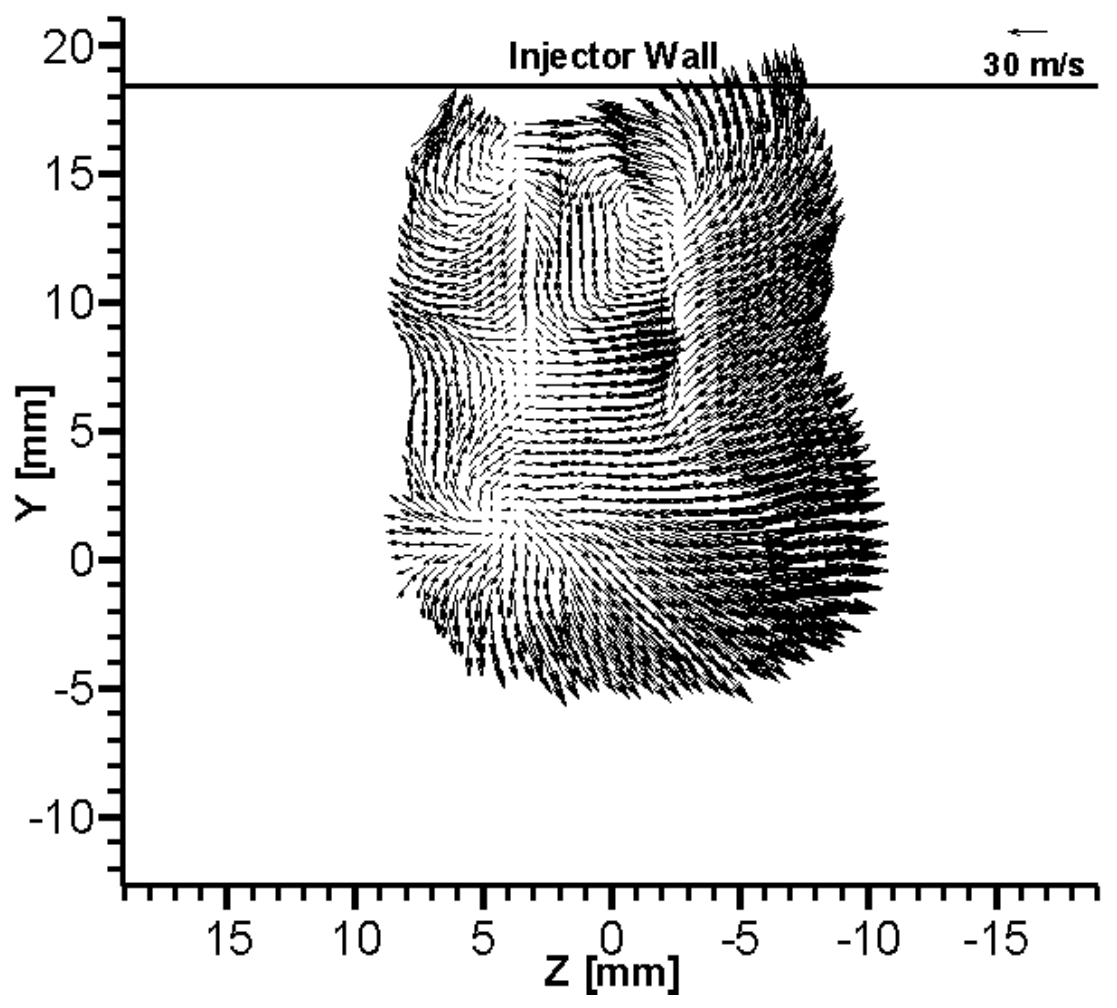

Figure 6.7: Average velocity field for Fuel-air Combustion case at $\mathrm{X} / \mathrm{H}=12$ a)Three-component (3C) velocity magnitude, and b) In-plane velocity vectors. 
causes a low-velocity region near the injector wall, while flow at the outer edged of the fuel plume (toward the observation wall) remains supersonic. In fact the $3 \mathrm{C}$ velocity

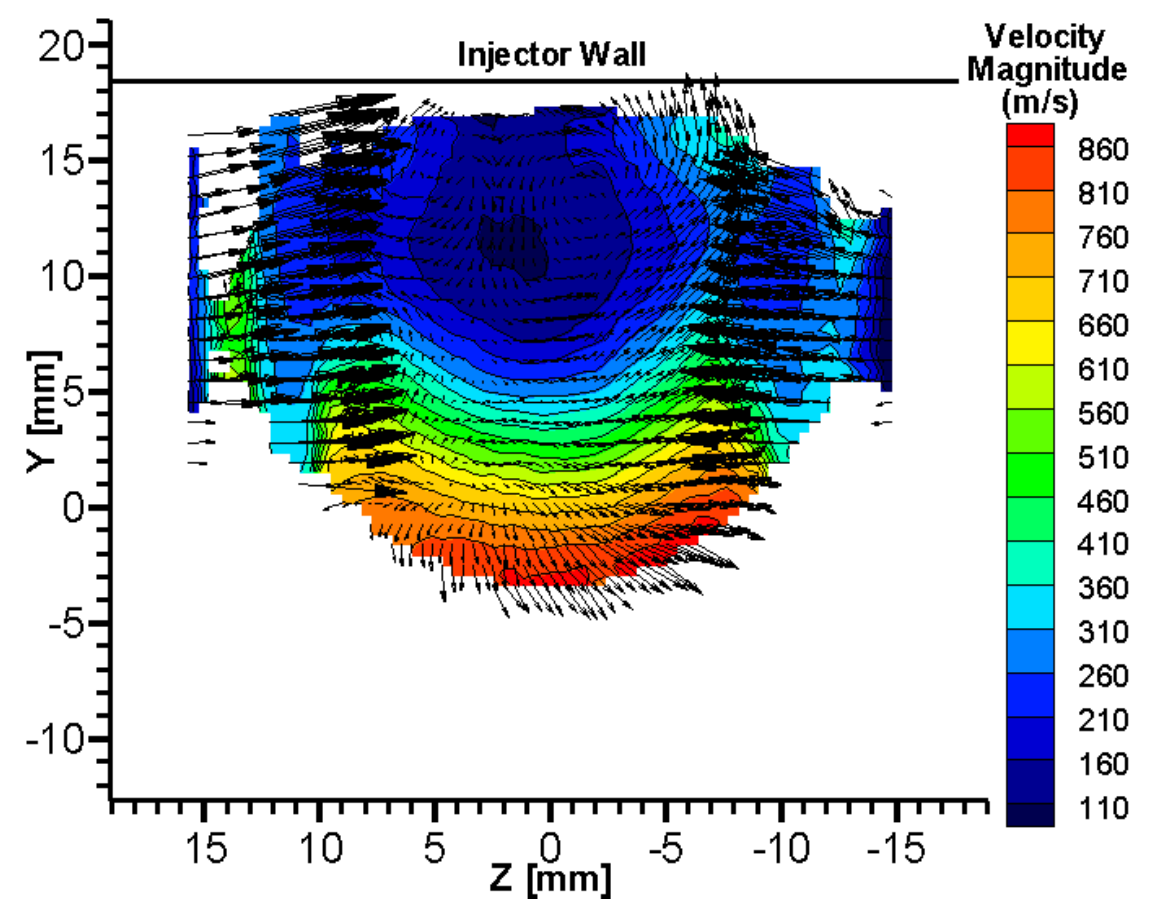

Figure 6.8: Average velocity field for Fuel-air Combustion case at $\mathrm{X} / \mathrm{H}=12$ without masking to remove velocity vectors arising from correlations with laser reflections.

range of this case is very large, from a minimum of approximately $90 \mathrm{~m} / \mathrm{s}$ close to the injector wall to a maximum near $900 \mathrm{~m} / \mathrm{s}$ at the out edge of the fuel-plume. This constitutes a large dynamic range for velocity measurements, but, the proper SPIV experimental configuration (presented in Chapter 4) allowed for determination of the velocity across the entire field. In-plane velocity vectors are shown in Fig. 6.7 (b) and indicate that the vortices do indeed remain intact during fuel-air combustion, albeit they appear weaker. The asymmetry of the vortices can again be viewed and it is interesting to note the average position of each vortex is reversed (z-direction) from the mixing case.

Figure 6.9 shows two examples of instantaneous velocity fields that contributed to the average result during combustion. From the instantaneous fields, the vortices can be 
Average In-plane Velocity Vectors

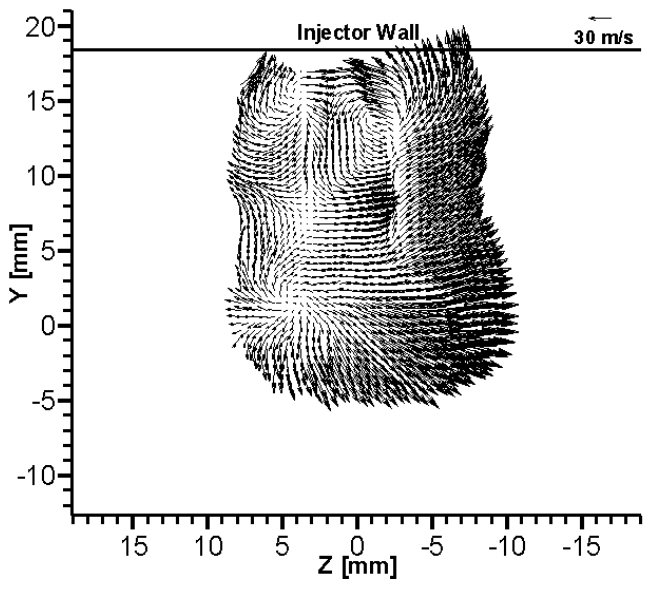

Instantaneous In-plane Velocity Vectors

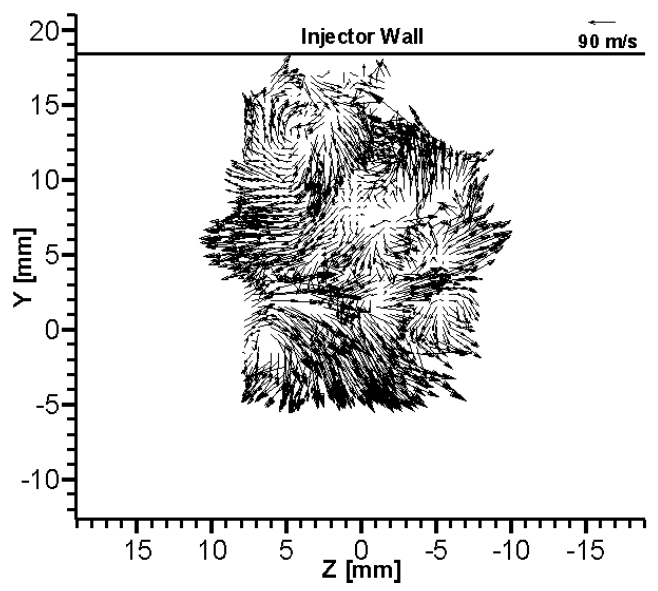

Instantaneous In-plane Velocity Vectors

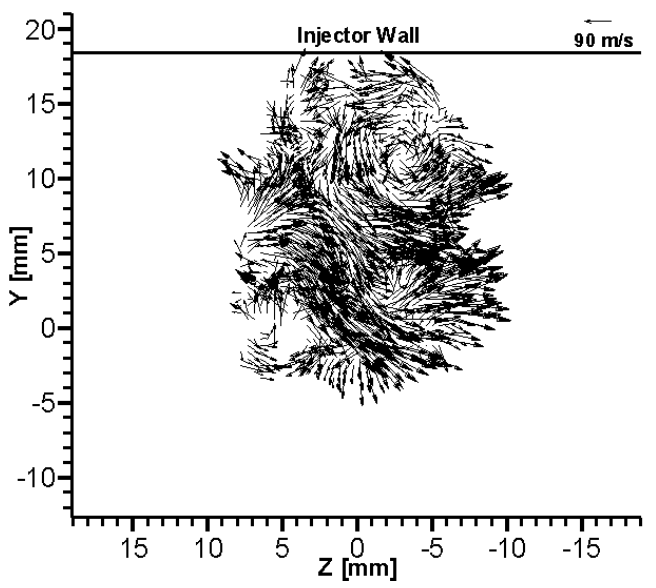

Average $3 C$ Velocity Field

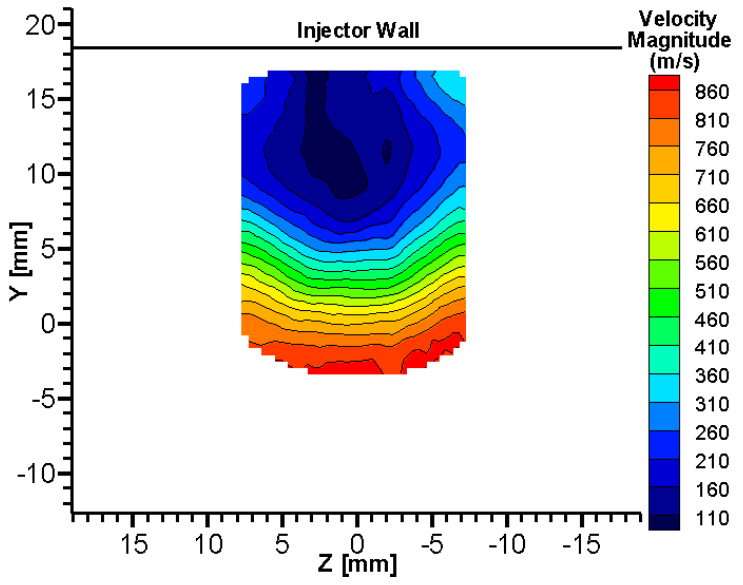

Instantaneous 3C Velocity

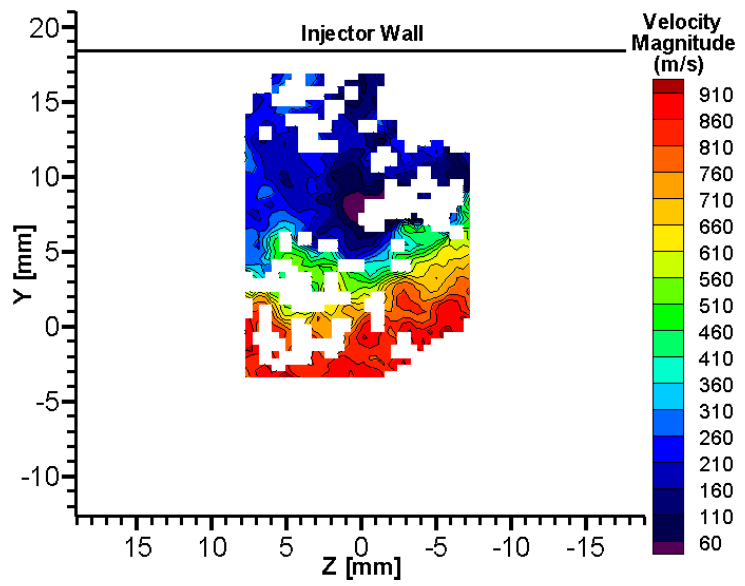

Instantaneous 3C Velocity

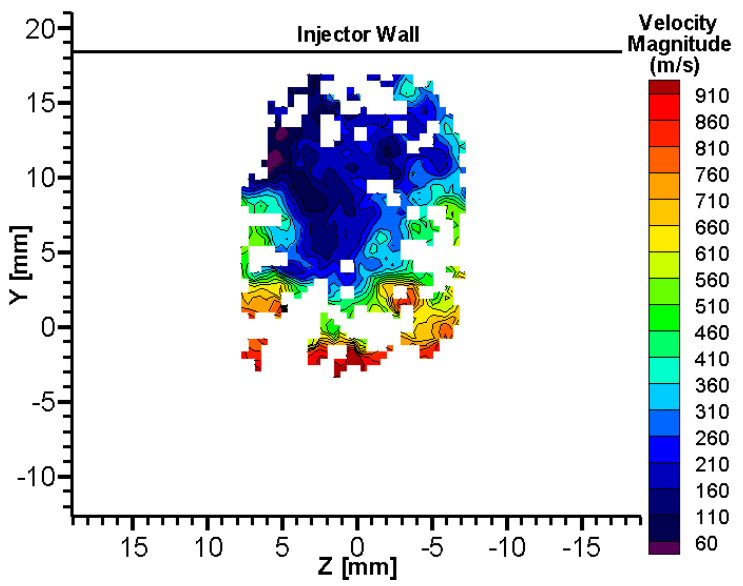

Figure 6.9: Average and Instantaneous velocity fields, Fuel-air Combustion at $\mathrm{X} / \mathrm{H}=12, \Phi=0.17$ 
seen to move across the plane more than in the mixing case. Computing the average field from instantaneous fields with vortices at different locations causes the average In-plane magnitude to be lower than the magnitude in each instant result. However, the location of the vortices is not completely random and the average field therefore shows the vortices at their mean locations. The instantaneous results also show there are missing vectors in some areas, but in the average the complete velocity field is calculated. The convergence of $3 \mathrm{C}$ velocity was again investigated by qualitatively comparing averages computed from increasing numbers of instantaneous fields. This comparison can be found in Appendix D and each average field of the figure has the same vector count distribution as the full average computed from 1162 instant fields. It was found that averaging between 300 and 400 instantaneous fields was sufficient for convergence, except for a small area of low-speed flow near the injector wall.

Figure 6.10 shows rotation of the combustion flowfield with areas in green indicating clockwise rotation and areas of orange for counter-clockwise. The counterrotating vortices can clearly be seen near the injector wall and have more spread and lower degree of rotation than the vortices of the mixing case. This effect is again due to the turbulence of the flow and the movement of the vortices over time. Rotation of each vortex in this combusting flowfield is approximately $20 \%$ of the magnitude of the corresponding vortices in the non-combusting field. It also appears that some secondary rotation exists in the area between the vortices and the far edge of the fuel-plume.

Figure 6.11 presents the turbulence intensity for this fuel-air combustion case. The plot shows that the minimum degree of turbulence occurs at the edge of the fuel plume, in the supersonic region, while maximum turbulence is in the low-speed region, between the 


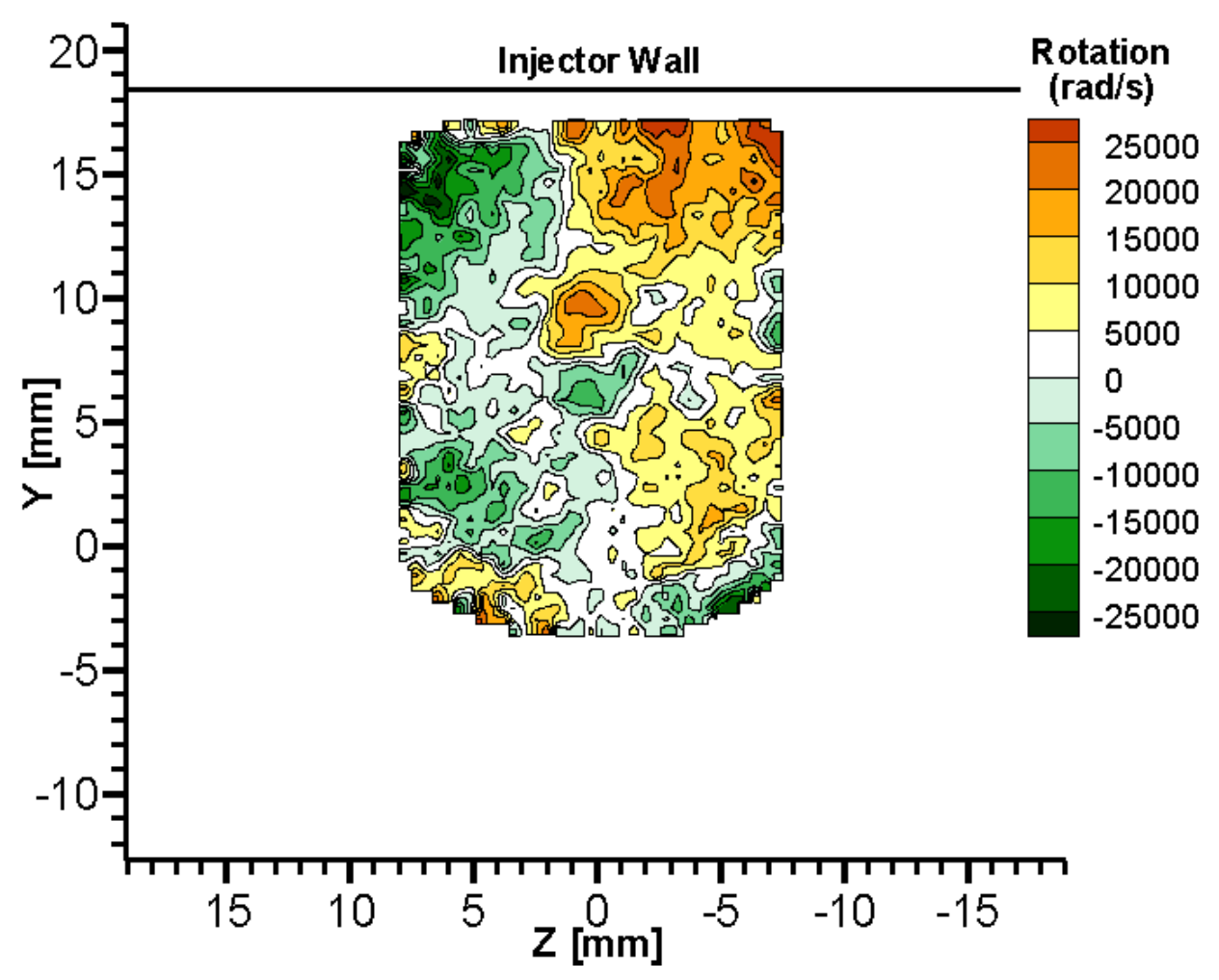

Figure 6.10: Degree of Fluid Rotation for Average SPIV result, $\Phi=0.17$, Fuel-air Combustion case at $\mathrm{X} / \mathrm{H}=12$.

vortices. Examining both the average $3 \mathrm{C}$ velocity distribution and the movement of vortices in the instantaneous fields shows that the turbulence distribution of Fig. 6.11 is not really a surprising result. Maximum turbulence is located in a small area around point $(y, z)=(10,1.5)$ in the plot and is near the interface between the two-counter rotating vortices. For sub-regions around $(\mathrm{y}, \mathrm{z})=(10,5)$ a vector in a given location comes from the left vortex for some instantaneous results, while for other instant measurements the vector at that location is part of the right vortex because of translation of the vortices across the line $\mathrm{z}=1.5$ between measurements. Therefore, the set of instantaneous measurements has In-plane vectors with approximately the same magnitude but opposite signs in the area between the vortices, and this leads to turbulence intensity near $100 \%$. Furthermore, high degrees of turbulence are possible in 
areas of low velocity where In-plane and axial velocity magnitudes are of the same order. In the supersonic region, $3 \mathrm{C}$ velocity fluctuations are small mostly due to the large and

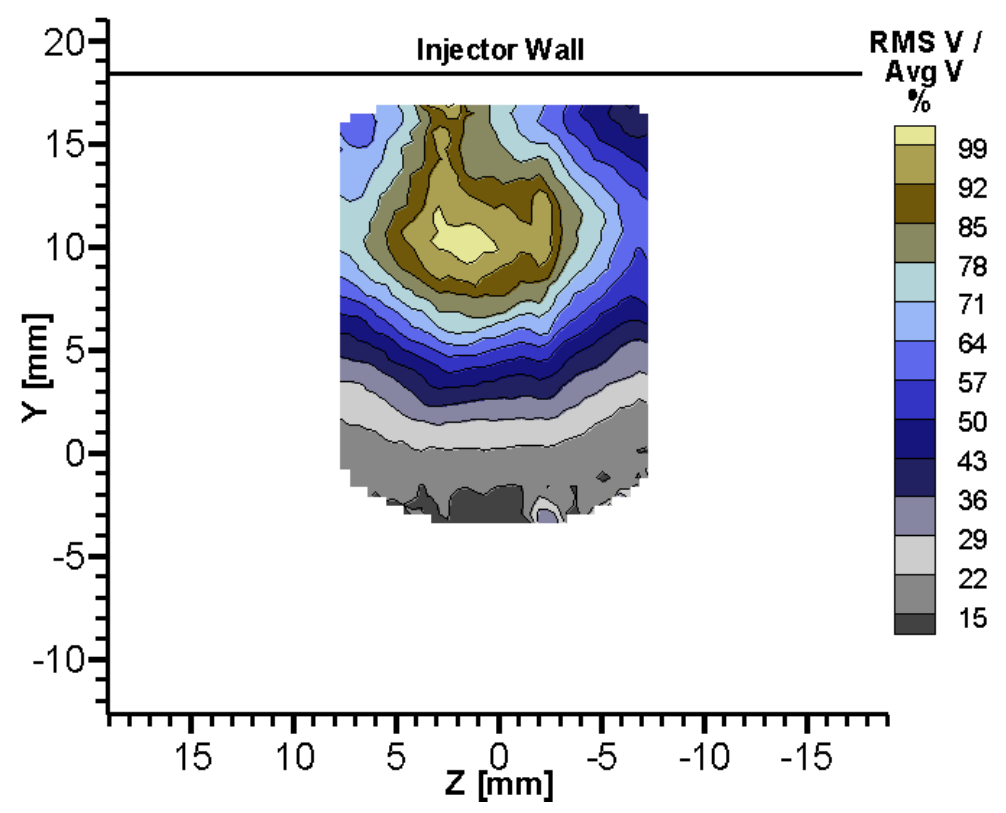

Figure 6.11: Turbulence Intensity (RMS of 3C Velocity normalized by Average velocity) of Fuel-air Combustion case at $\mathrm{X} / \mathrm{H}=12, \Phi=0.17$

consistent axial velocity component. Therefore turbulence is low in the supersonic region, as seen if Fig. 6.11.

\section{Turbulent length and time scales}

The SPIV measurements presented here can be used to determine other quantities of interest that give useful information about the flowfield. Two of these useful quantities are the turbulent mixing length scale and turbulent time scale. The turbulent mixing length scale can be found using a correlation function which shows the correlation of velocity values across the measurement area to the velocity at a point of interest. The correlation function shows a distance (from the point of interest) at which velocities are no longer appreciably correlated and this distance is the turbulent mixing length scale. ${ }^{52}$ 
Furthermore, this length scale is really a measure of the size of the largest turbulent eddies. Once the turbulent mixing length scale has been found, it can be used along with the magnitude of turbulent velocity fluctuations to find the turbulent time scale.

As an example, SPIV results at $\mathrm{X} / \mathrm{H}=12$ have been used to determine both the turbulent mixing length scale and turbulent time scale for one location of interest in the measurement plane at $\mathrm{X} / \mathrm{H}=12$. In the future, this method can be used to determine turbulent scales at other locations in the $\mathrm{X} / \mathrm{H}=12$ plane, or at locations in other planes. Figure 6.12 contains several plots which show the point of interest in the $\mathrm{X} / \mathrm{H}=12$ plane that was investigated, correlation maps for the $\mathrm{Y}$ and $\mathrm{Z}$ velocity components, and correlation profiles (correlation functions) determined from these correlation maps. The point of interest (shown as a black dot in the average velocity plot) lies in the shear layer region located between the low-speed region near the injector wall and the high-velocity free-stream. In the figure, a correlation map is shown for instantaneous Y-component velocities (v') at locations throughout the field correlated with the v' value at the point of interest. Likewise, a correlation map was determined for correlation of Z-component velocities.

The correlation function is determined by obtaining a profile of the correlation map, through the point of interest, in the direction of the correlated velocity component. For example, the appropriate correlation function for the $\mathrm{Y}$-component velocity would be a profile, in the Y-direction, of the Vy correlation map. For these measurements this correlation function is a vertical profile of the Vy correlation map starting from the point of interest at $(\mathrm{Z}, \mathrm{Y})=(0,0.8)$. In the Vy correlation map, the location of this profile is indicated by the pink line. The Y-component correlation function is plotted next to the 
Average $3 C$ Velocity Field with

investigation point at $(Z, Y)=(0,0.8)$

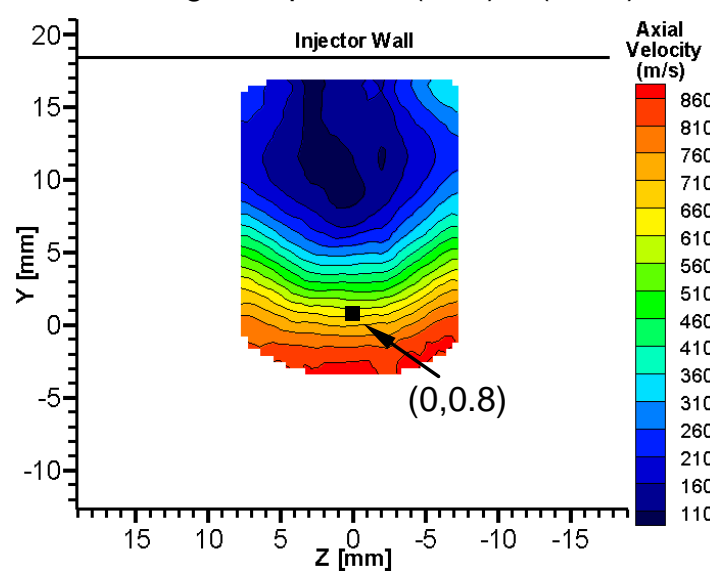

Vy Correlation Map for investigation at $(0,0.8)$

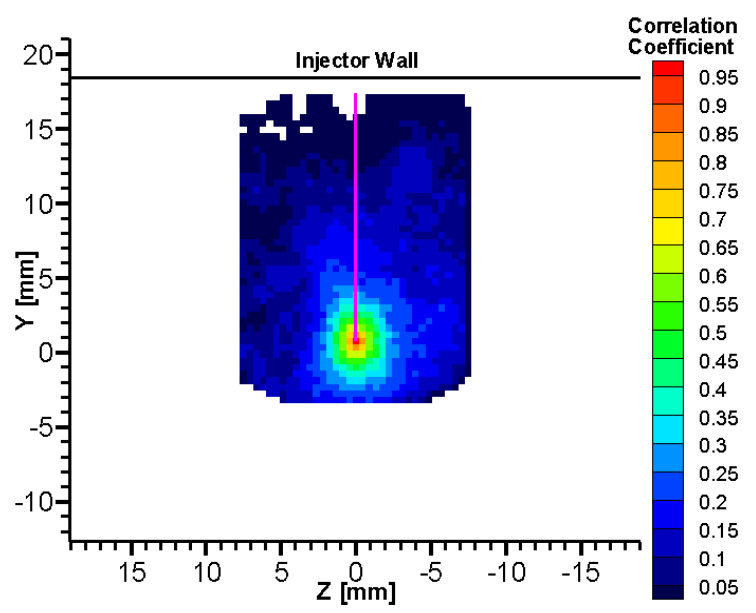

Vz Correlation Map for investigation at $(0,0.8)$

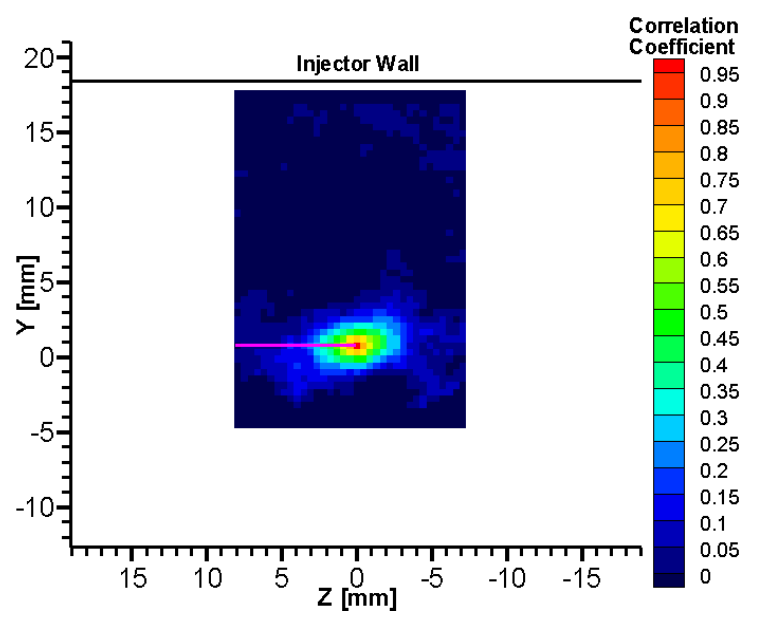

Measurement plane location, $\mathrm{X} / \mathrm{H}=12$

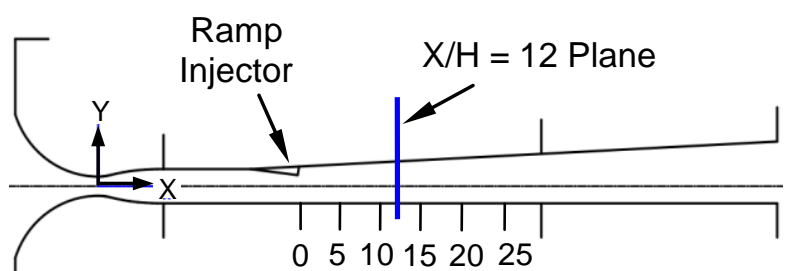

Vy Correlation Profile along line $Z=0$

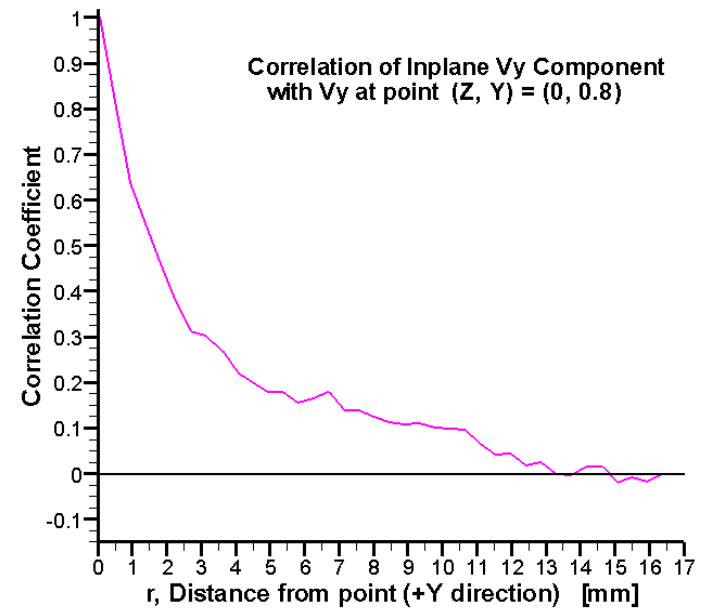

Vz Correlation Profile along line $Y=0.8$

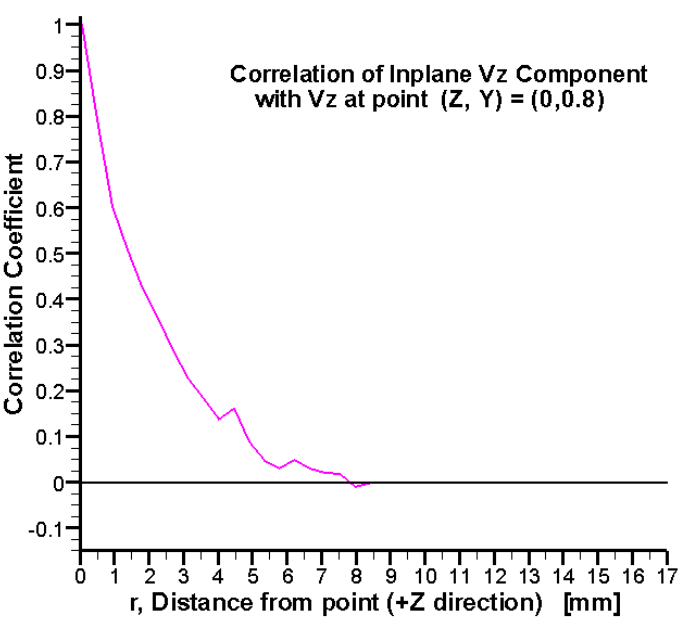

Figure 6.12: Correlation of In-plane velocity components (Vy \& Vz) with values at $(Z, Y)=(0,0.8)$ 
correlation map. The same procedure is used for the $\mathrm{Vz}$ component, with the correlation map and corresponding profile shown at the bottom of figure 6.12. The turbulent mixing length scale is determined by choosing the location in the correlation function (profile) where velocities have stopped being measurably correlated. Here, velocities are assumed to no longer be appreciably correlated for correlation coefficients of less than 0.15 . Using this cut-off value, the turbulent mixing length scale can be seen to be approximately 6-mm as determined from the correlation profile of the Y-component of velocity. The turbulent length scale is approximately $4-\mathrm{mm}$ when found using the Zcomponent velocity correlation function.

Dividing the turbulent length scales by the corresponding turbulent velocity fluctuations (v' or w') gives a measure of the turbulent time scale. From the SPIV data, the Y-component velocity fluctuation was found to be $\mathrm{v}^{\prime} \sim 60 \mathrm{~m} / \mathrm{s}$ and the Z-component fluctuation was found as $\mathrm{w}^{\prime} \sim 70 \mathrm{~m} / \mathrm{s}$. Using the Y-component values, the turbulent time scale was determined as $\left[6-\mathrm{mm}^{*}(1 \mathrm{~m} / 1000 \mathrm{~mm})\right] / 60 \mathrm{~m} / \mathrm{s}=1 \times 10^{-4} \mathrm{sec}$. Conducting the same calculation using the Z-component values shows the turbulent time scale as approximately $5.7 \times 10^{-5} \mathrm{sec}$. Therefore, the turbulent mixing length scale within the shear layer is on the order of $5-\mathrm{mm}$ and the turbulent time scale is of the order of $10^{-4}$ seconds. As mentioned, this method can be used to determine these turbulent length and time scales at other locations in the flow.

It is clear that the flowfield of the fuel-air combustion case in Flowpath 2 is quite complex and that $3 \mathrm{C}$ velocity measurements are necessary for understanding this flow. SPIV was also used to obtain $3 \mathrm{C}$ velocity measurements at a second plane of Flowpath 2 and results from those experiments are presented below. 


\section{2 $\mathrm{X} / \mathrm{H}=15$ Measurement Plane Results}

As mentioned above, it was desirable to investigate the flow at $\mathrm{X} / \mathrm{H}=18$, however, measurements could not be completed for the $\mathrm{X} / \mathrm{H}=18$ plane because of large background noise in the measurement area. Instead, measurements were conducted at $\mathrm{X} / \mathrm{H}=15$. Figure 6.13 shows a schematic of Flowpath 2 with the $\mathrm{X} / \mathrm{H}=15$ plane indicated and also a photograph of Flowpath 2 with a calibration target in place for calibration of the experiment at $\mathrm{X} / \mathrm{H}=15$. The SPIV configuration used for

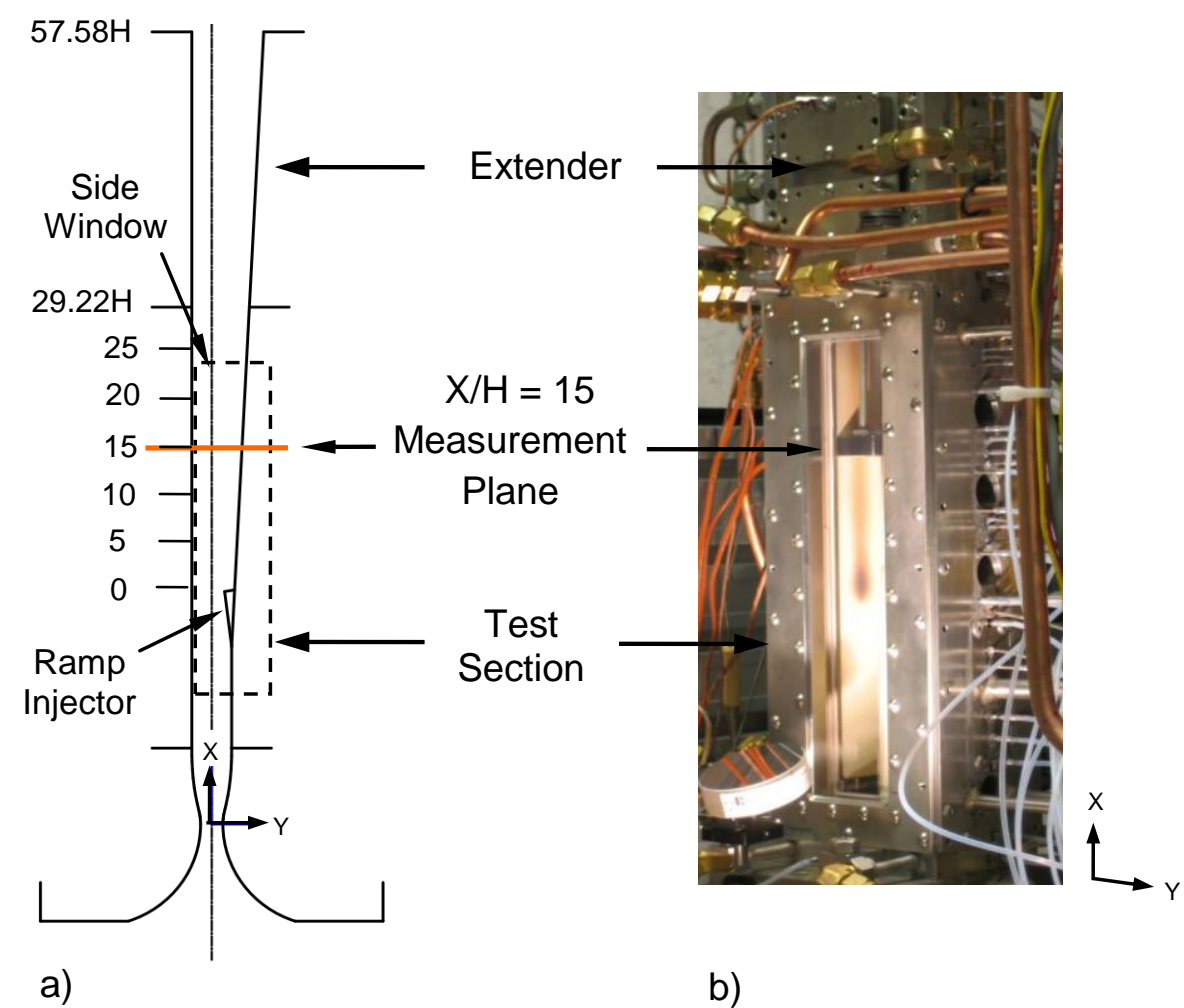

Figure 6.13: Flowpath 2 with $\mathrm{X} / \mathrm{H}=15$ measurement plane.

a) Schematic and b) Photograph with calibration target at $\mathrm{X} / \mathrm{H}=15$

measurements at $\mathrm{X} / \mathrm{H}=15$ was presented in Chapter 4. Results from the fuel-air mixing case are briefly presented in section 6.2.1 while other plots are located in Appendix E. 
The flowfield for fuel-air mixing is not a complex or interesting as the combustion case, so combustion results are discussed in more detail.

\subsubsection{Fuel-air Mixing Results, $\mathrm{X} / \mathrm{H}=15$}

Measurements of fuel-air mixing were completed at the $\mathrm{X} / \mathrm{H}=15$ plane and briefly presented here. Figure 6.14 shows average 3C velocity magnitude and In-plane vectors

a)

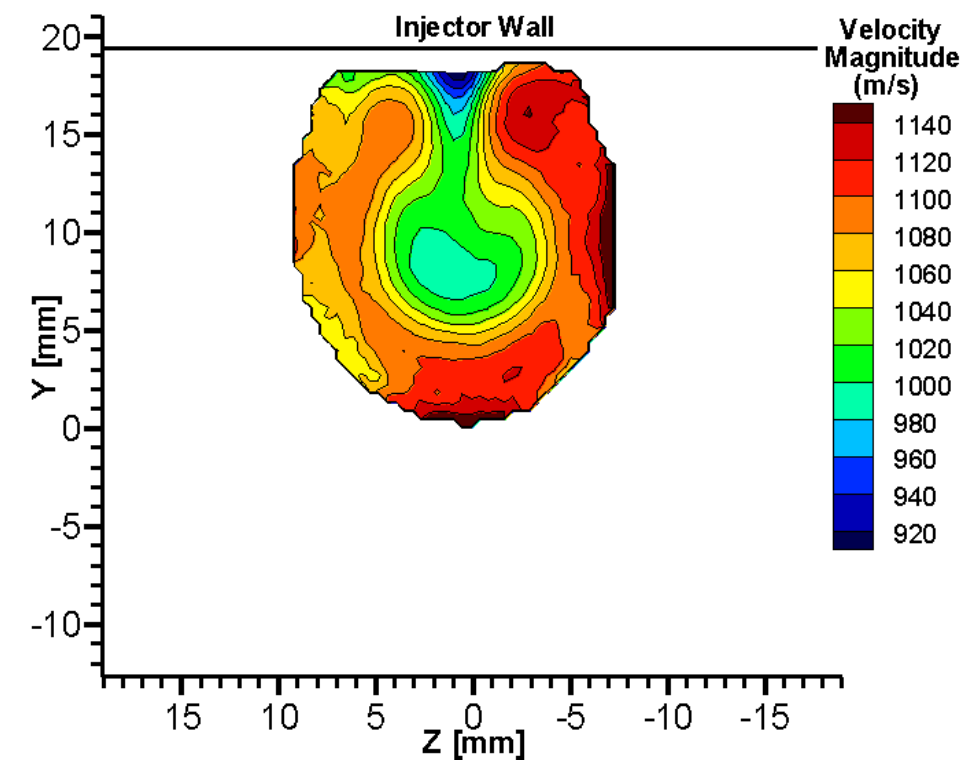

b)

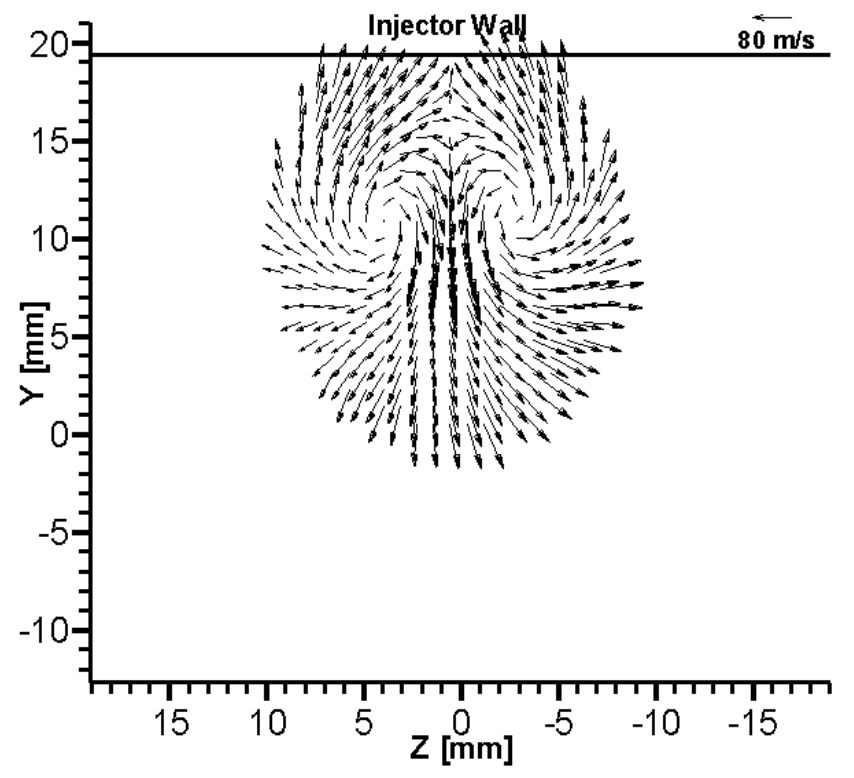

Figure 6.14: Average velocity field for Fuel-air Mixing case at $\mathrm{X} / \mathrm{H}=15$ a)Three-component (3C) velocity magnitude, and b) In-plane velocity vectors. 
for fuel-air mixing at $\mathrm{X} / \mathrm{H}=15$. This average field was computed using 1761 instantaneous velocity fields. The mixing results at $\mathrm{X} / \mathrm{H}=15$ are similar to those from $\mathrm{X} / \mathrm{H}=12-$ shape of the fuel plume, similar $3 \mathrm{C}$ velocity magnitude, and presence of the well-organized counter-rotating vortices. Just as in the previous mixing case, the $3 \mathrm{C}$ velocity field shows minimum velocity magnitude at the center, between the two vortices and maximum velocity at the edges of the plume. Note that the fuel plume at $\mathrm{X} / \mathrm{H}=15$ is larger than at $\mathrm{X} / \mathrm{H}=12$ because the plume expands as it propagates downstream. Some degree of asymmetry exists between vortices at $\mathrm{X} / \mathrm{H}=15$, but not to the same extent as at $\mathrm{X} / \mathrm{H}=12$. It is probable that the two vortices do not propagate axially downstream in perfect strait lines originating at the ramp injector, so viewing the flow at different downstream planes shows the vortices at different average positions in each plane.

Figure 6.15 shows average degree of rotation for fuel-air mixing at $\mathrm{X} / \mathrm{H}=15$, while Figure 6.16 presents the turbulence intensity. The distribution of rotation for mixing at

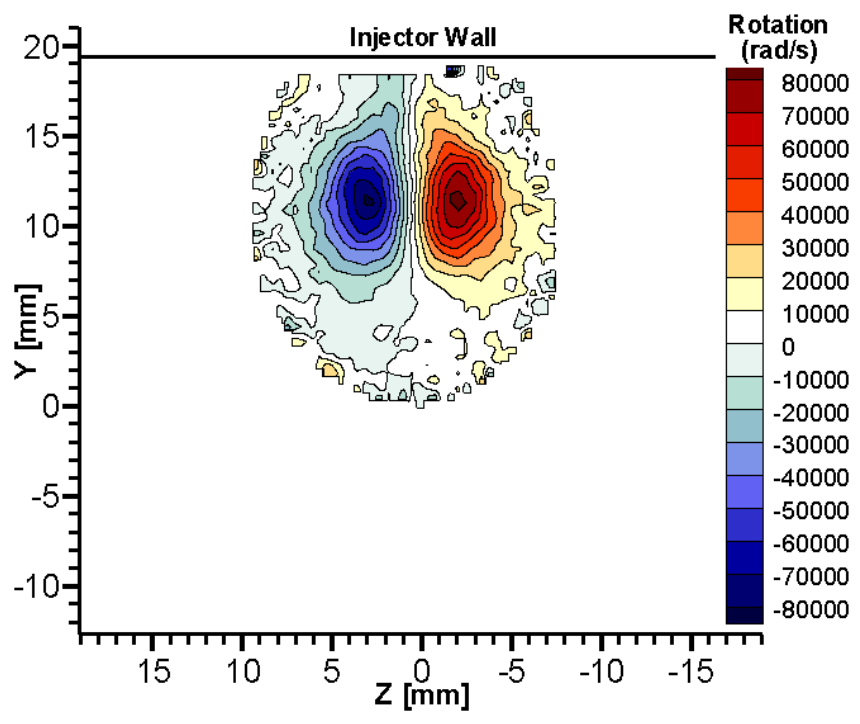

Figure 6.15: Degree of Fluid Rotation for Average SPIV result, $\Phi=0.17$, Fuel-air Mixing case at $\mathrm{X} / \mathrm{H}=15$. 
$\mathrm{X} / \mathrm{H}=15$ is similar to that from $\mathrm{X} / \mathrm{H}=12$, but with slightly lower absolute maximum rotation. The vortices are clearly still very well-organized and strong at this downstream plane. The turbulence intensity is similar in magnitude and distribution to that of mixing at $\mathrm{X} / \mathrm{H}=12$, with a high degree of turbulence between the vortices and lowest turbulence toward the injector wall, in areas parallel to the vortex centers. However, unlike mixing

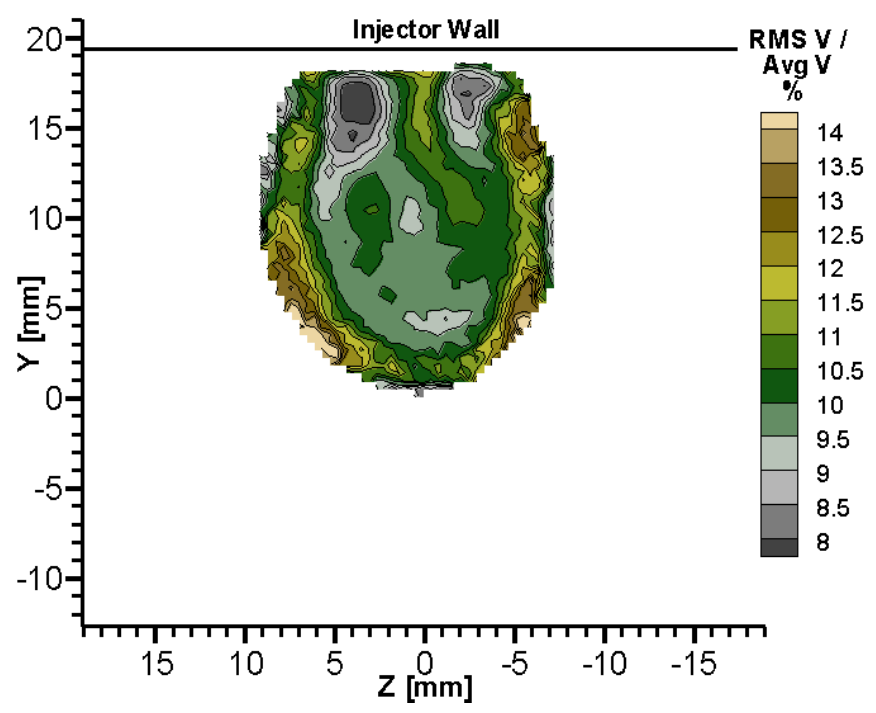

Figure 6.16: Turbulence Intensity (RMS of $3 \mathrm{C}$ Velocity normalized by Average velocity) of Fuel-air Mixing case at $\mathrm{X} / \mathrm{H}=15, \Phi=0.17$

at $\mathrm{X} / \mathrm{H}=12$, relatively high turbulence exits at the edge of the fuel plume in the mixing case at $\mathrm{X} / \mathrm{H}=15$.

\subsubsection{Fuel-air Combustion Results, $\mathrm{X} / \mathrm{H}=15$}

Stereoscopic Particle Image Velocimetry (SPIV) was also used to measure the three-component $(3 \mathrm{C})$ velocity field at $\mathrm{X} / \mathrm{H}=15$ for the case of fuel-air combustion. Unfortunately, SPIV images obtained during fuel-air combustion at X/H $=15$ contained large background noise which was not present in SPIV images at the $\mathrm{X} / \mathrm{H}=12$ plane, or in images for fuel-air mixing at $\mathrm{X} / \mathrm{H}=15$. The noise was not seen in images acquired for 
fuel-air mixing at $\mathrm{X} / \mathrm{H}=15$, and therefore, it seems that the combustion process itself caused the noise seen in the images, most likely due to particle depositing on windows. For these experiments, the laser sheet passed through the side-windows of the test section. Any particles that adhered to the side-windows during combustion, scattered

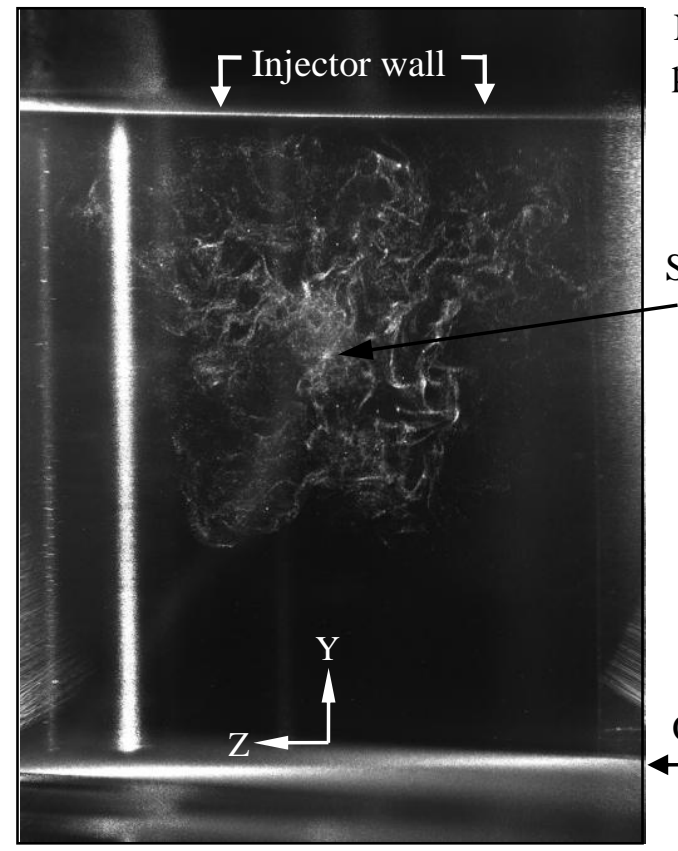

(a) Example SPIV image frame from Fuel-air combustion at $\mathrm{X} / \mathrm{H}=\mathbf{1 2}$

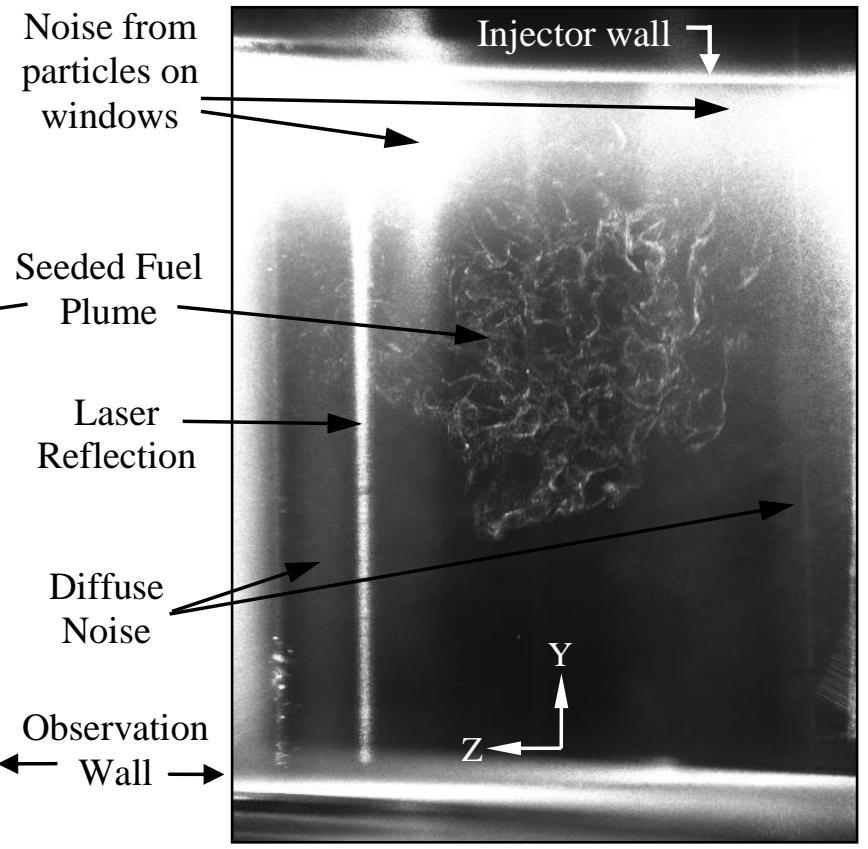

(b) Fuel-air combustion Image at $\mathrm{X} / \mathrm{H}=\mathbf{1 5}$ (High Background noise)

Figure 6.17: Comparison of noise in SPIV images during combustion at two planes in Flowpath 2. (a) Image from $\mathrm{X} / \mathrm{H}=12$ plane, and (b) Image from $\mathrm{X} / \mathrm{H}=15$

laser light which led to regions of high-intensity noise in the images. Figure 6.17 shows one image frame of a SPIV measurement (part a) for fuel-air combustion at $\mathrm{X} / \mathrm{H}=12$, and also an example image frame (part b) from the combustion case at $\mathrm{X} / \mathrm{H}=15$. Note that the images of Fig. 6.17 have been rotated by 90 -degrees to place the injector wall at the top. Figure 6.17 part (a) indicates that images acquired for combustion at $\mathrm{X} / \mathrm{H}=12$ contain some background noise due to laser reflections, which required masking to remove incorrect velocity vectors from correlation of particles with reflections, as discussed above. However, the image of Fig. 6.17(b) shows that SPIV images for 
combustion at $\mathrm{X} / \mathrm{H}=15$ contain high-intensity noise near the injector wall from laser light scattering off of particles coating side-windows, as well as laser reflections and areas of diffuse background noise. These problems required careful image processing and filtering of vector results in order to yield any velocity information from these SPIV images. However, areas near the injector wall did not yield any measurements, regardless of the processing used, because the noise completely obscured the seed particle in the fuel plume at that location. Despite these problems, SPIV measurements during combustion at $\mathrm{X} / \mathrm{H}=15$ did yield some useful results, albeit only for a relatively small area of the measurement plane.

Figure 6.18 presents average 3C velocity results and In-plane vectors for combustion at $\mathrm{X} / \mathrm{H}=15$. As mentioned above, velocity results are missing in the area near the injector wall due to noise in the images. Furthermore, a mask was used to remove incorrect vectors that resulted from correlations of particles with laser reflections. The final useful measurement area in Fig. 6.18 is relatively small, but does show some interesting results. The distribution and large range of $3 \mathrm{C}$ velocity magnitude (from approximately $40 \mathrm{~m} / \mathrm{s}$ to nearly $840 \mathrm{~m} / \mathrm{s}$ ) for combustion at $\mathrm{X} / \mathrm{H}=15$ is similar to the velocity range and distribution across the measurement area at $\mathrm{X} / \mathrm{H}=12$ during fuel-air combustion. Unfortunately, the area of useful measurements in Fig. 6.18 is too small to give an accurate idea of the size and shape of the region of low $3 \mathrm{C}$ velocity near the vortices. The In-plane vectors of Figure 6.18(b) show some expected characteristics. Vectors indicate two counter-rotating vortices in the upper portion of the measurement area, although the right vortex is incomplete because image noise obscured that area. The average vortex positions show asymmetry, which was also seen in previous 
a)
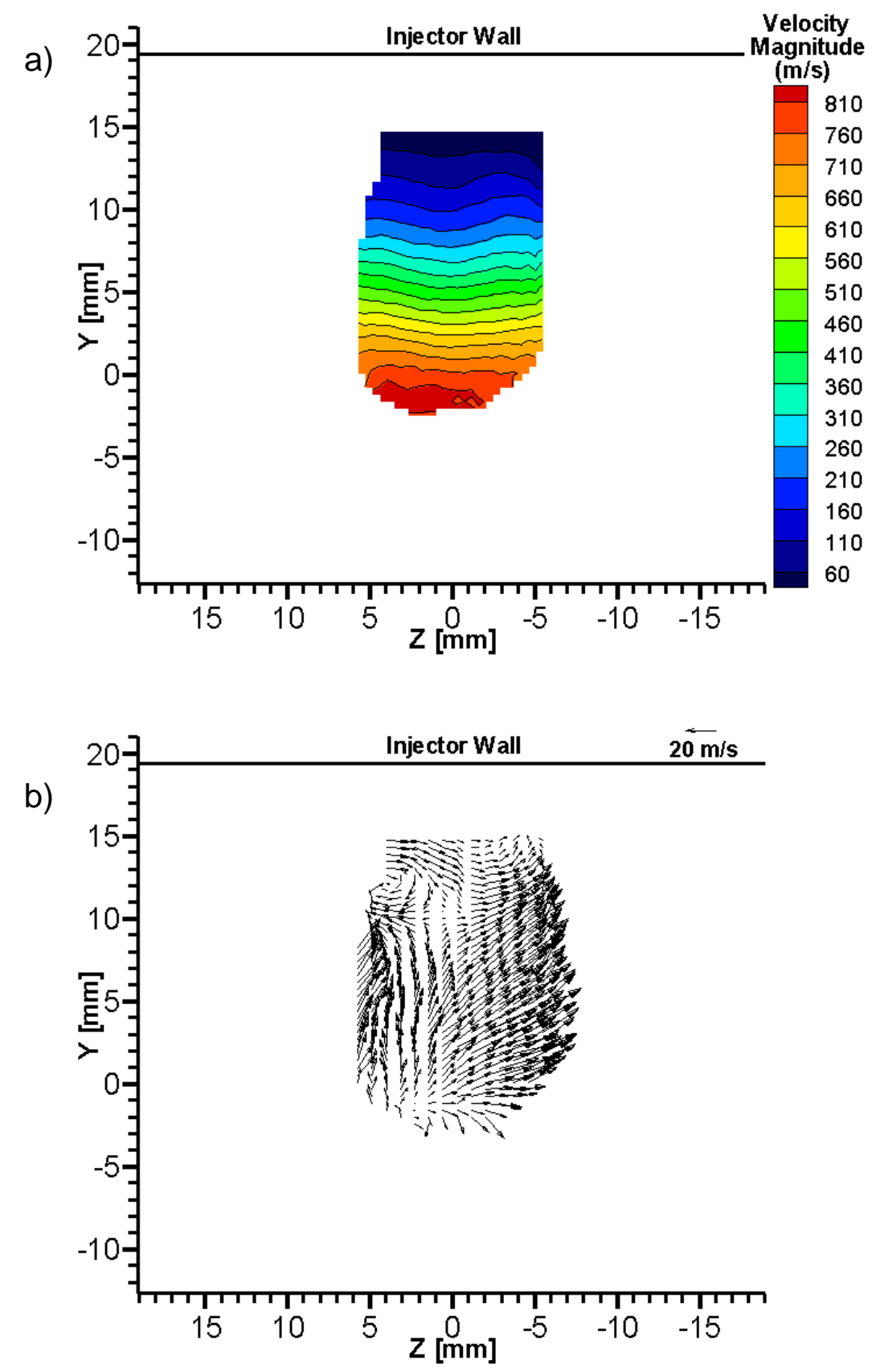

Figure 6.18: Average velocity field for Fuel-air Combustion case at $X / H=15$ a)Three-component (3C) velocity magnitude, and b) In-plane velocity vectors.

measurements. The average velocity field of Figure 6.18 was determined from 1083 instantaneous fields and the distribution of vector count can be viewed in Figure 6.19. However, many instantaneous velocity fields contained fewer numbers of velocity 
vectors than the instantaneous fields for combustion at $\mathrm{X} / \mathrm{H}=12$. Two of the instantaneous velocity fields that contributed to the average result can be viewed in Appendix E. A figure has also been included in Appendix E to summarize the average velocity field for combustion at $\mathrm{X} / \mathrm{H}=15$ and includes plots of: $3 \mathrm{C}$ velocity magnitude, In-plane magnitude, In-plane vectors; and $\mathrm{u}, \mathrm{v}$ and $\mathrm{w}$ velocity components.

Figure 6.20 shows rotation for this combustion case at $\mathrm{X} / \mathrm{H}=15$. The influence of the counter-rotating vortices can be seen in the upper portion of the figure. The magnitude of rotation is relatively low, although it is of the same order as the rotation for the combustion case at $\mathrm{X} / \mathrm{H}=12$. However, the highest absolute rotation in this case $(\mathrm{X} / \mathrm{H}=15)$ occurs over a smaller areas than in the combustion case at $\mathrm{X} / \mathrm{H}=12$, and this

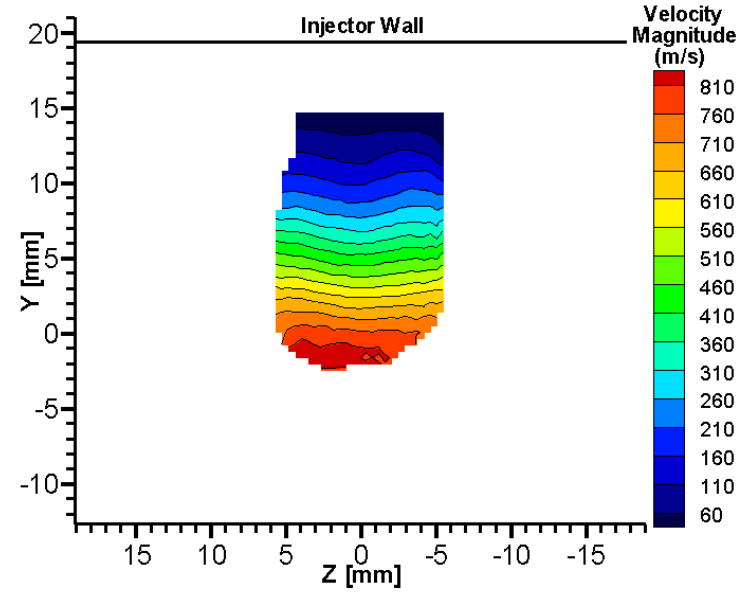

(a) Average of All (1083) Vector Fields [3C Velocity magnitude]

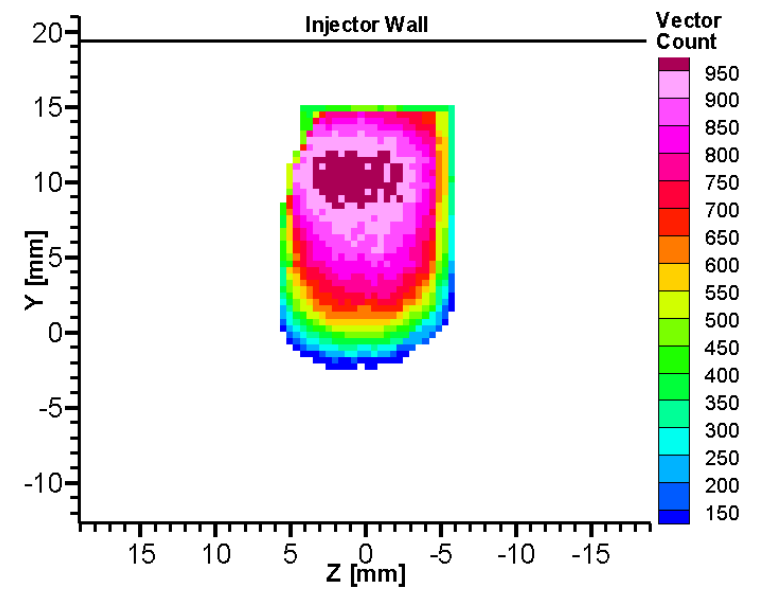

(b) Number of Vectors Averaged for Each Sub-region

Figure 6.19: $\Phi=0.17$, Fuel-air Combustion at $\mathrm{X} / \mathrm{H}=15$;

(a) Average 3C velocity field, and (b) Number of vectors averaged at each location

is most likely due to missing areas of the vortices in $\mathrm{X} / \mathrm{H}=15$ measurements due to background noise in the images. If velocity vectors could have been acquired in areas near the injector wall, the area of the counter-rotating vortices would likely be larger. No discernible pattern of rotation exists for the rest of the measurement area as seen if Figure 


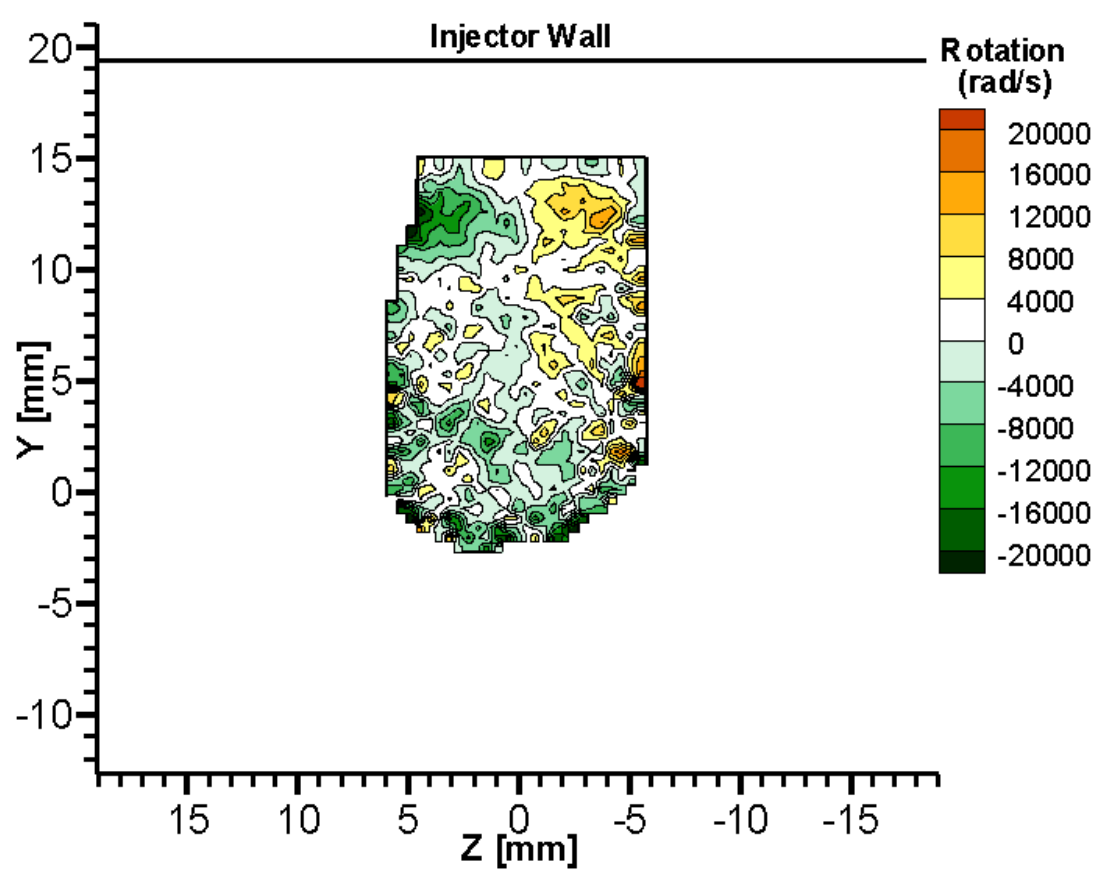

Figure 6.20: Degree of Fluid Rotation for Average SPIV result, $\Phi=0.17$, Fuel-air Combustion case at $\mathrm{X} / \mathrm{H}=15$.

6.20 Turbulence intensity was also calculated for this case and is presented in Figure 6.21. Once again the lowest turbulence occurs in the area of maximum velocity (supersonic flow) at the edge of the fuel plume, near $(y, z)=(0,1)$. The largest turbulence intensity is very high and is located in the low-speed region of the flowfield, between the vortices. Values similar to those of combustion at $\mathrm{X} / \mathrm{H}=12$ were expected for turbulence intensity at $\mathrm{X} / \mathrm{H}=15$ and indeed the pattern is similar. However, the magnitude of turbulence in the area of the vortices $(150-180 \%)$ is much higher for combustion at $\mathrm{X} / \mathrm{H}=15$, than at $\mathrm{X} / \mathrm{H}=12$. It is unclear whether this higher level of turbulence is a real characteristic of the flow, or if it is a results of missing data in the area of vortices that resulted from high background noise. Confidence in the turbulence intensity result for combustion at $\mathrm{X} / \mathrm{H}=15$ is not as high as for combustion at $\mathrm{X} / \mathrm{H}=12$ due to the large amount of background noise present in the SPIV images at $\mathrm{X} / \mathrm{H}=15$. 
However, the results at least give some useful indication of the degree and distribution of turbulence for the flowfield at $\mathrm{X} / \mathrm{H}=15$.

It is true that the measurements for combustion at $\mathrm{X} / \mathrm{H}=15$ only give information over a relatively small portion of the cross-plane and also that areas of interest are missing due

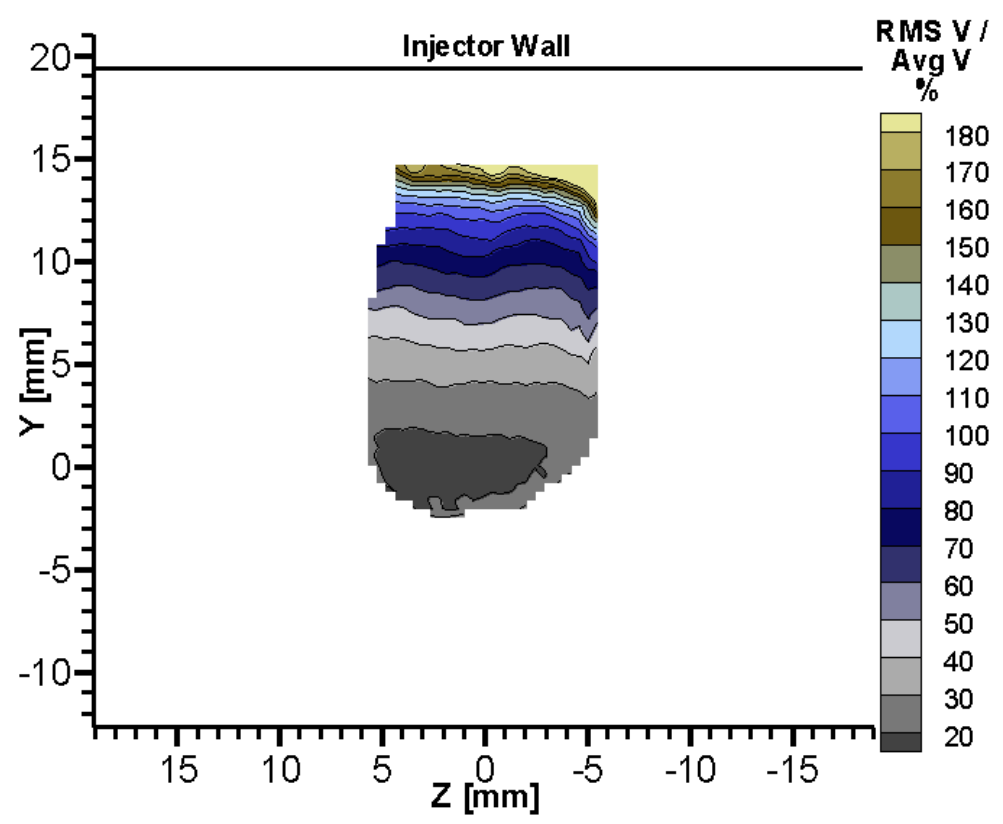

Figure 6.21: Turbulence Intensity (RMS of 3C Velocity normalized by Average velocity) of Fuel-air Combustion case at $\mathrm{X} / \mathrm{H}=15, \Phi=0.17$

to large noise in the images. However, considering the problem of particles adhering to windows (at $\mathrm{X} / \mathrm{H}=15$ ) which produced large noise in the images, any velocity results at this locations should be considered a success for the SPIV technique and for the experimental configuration. Furthermore, because no previous 3C velocity measurements have been obtained for this flowfield, even limited velocity measurements are useful for understanding the flow and for validating CFD simulations. 


\subsection{Qualitative Comparison to CFD results, Flowpath 2}

Computational Fluid Dynamics (CFD) simulations have been completed for Flowpath 2 of the direct-connect combustor in the University of Virginia supersonic combustion facility. This CFD work was completed by Dr. Jack Edwards and Mr. Jesse Fulton at North Carolina State University, using NC State's REACTMB flow solver. Information about this work is available in reference 53. These simulations used a hybrid Large Eddy Simulation/Reynolds-averaged Navier-Stokes (LES/RANS) approach. For LES/RANS simulations, largest scales of turbulence were resolved directly, as opposed purely RANS simulations where the turbulence effects are accounted for by including one of a number of available turbulence models where the dominant turbulence effects cannot be distinguished from secondary effects ${ }^{53}$. The entire cross-section of the flowpath was simulated. Asymmetric flow characteristics, that would be have missed had a symmetry plane at the centerline $(\mathrm{z}=0)$ been assumed, were captured in these simulations by modeling the entire flow cross-section. For the computational mesh, the grid in the area of the nozzle contained $\sim 17.85$ million cells, while the combustor section contained $\sim 33$ million cells ${ }^{54}$. A denser grid was used to simulate areas near the walls, with a coarser grid across the remainder of the flowpath. Boundary conditions were determined using information supplied by researchers at the University of Virginia. A uniform stagnation temperature profile of $1200 \mathrm{~K}$ was used in these simulations, while a fixed wall temperature of $600 \mathrm{~K}$ was used as the boundary condition on all walls, except near the ramp fuel injector where the temperature was set to $1000 \mathrm{~K}$. Further information about these LES/RANS simulations, including the numerical schemes, computational mesh, and reaction chemistry models used, can be found in reference 53. Results from 
these LES/RANS simulations were not examined prior to completion of SPIV measurements so that the CFD results would not influence processing of the SPIV data. As mentioned above, CFD modelers requested the Flowpath 2 configuration without an isolator section so that there would be a well-defined inflow condition for CFD models. All three components of velocity from LES/RANS results for combustion cases of Flowpath 2 are compared here to corresponding velocity components from SPIV measurements. LES/RANS simulations were also completed for fuel-air mixing cases of Flowpath 2 and comparison figures for mixing cases are included in the appendices.

Figure 6.22 shows plots for axial (u), v, and w velocity components from LES/RANS (CFD) and SPIV results for fuel-air combustion at the $\mathrm{X} / \mathrm{H}=12$ cross-plane for Flowpath 2, with fuel equivalence ratio of $\Phi=0.17$. Plots on the left side of the figure are averaged fields from SPIV measurements, while the CFD results are shown in the plots on the right side of the figure. For SPIV measurements, only the fuel was seeded so that velocity measurements occurred for only part of the plane. In order to facilitate comparison of SPIV and LES/RANS results, a solid outline is included in each LES/RANS plot to indicate the location of the corresponding SPIV measurements. Identical color scales have been used to plot SPIV measurements and CFD results for each velocity component.

From the plots of Figure 6.22 it is clear that the LES/RANS results match the SPIV measurements fairly well. The general shape of velocity contours matches particularly well for the Axial velocity component and velocity magnitudes are nearly identical over the area of the SPIV measurements. One difference is the size of the low-velocity region near the injector wall, where the SPIV results show the low-velocity area is larger in 
Experiment (SPIV) Axial (u) Velocity

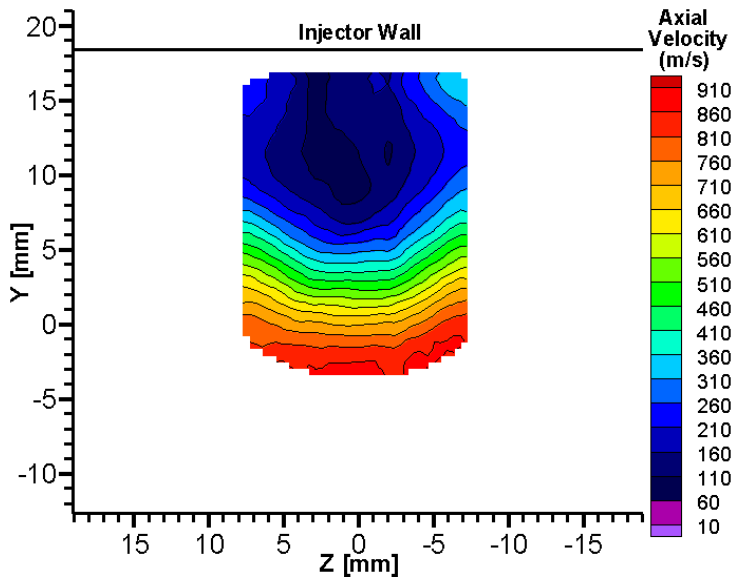

Experiment y-component (v) Velocity

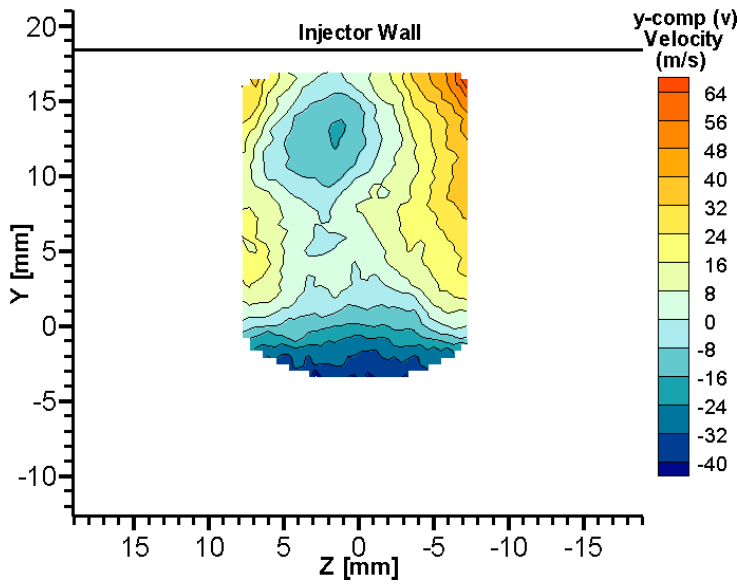

Experiment z-component (w) Velocity

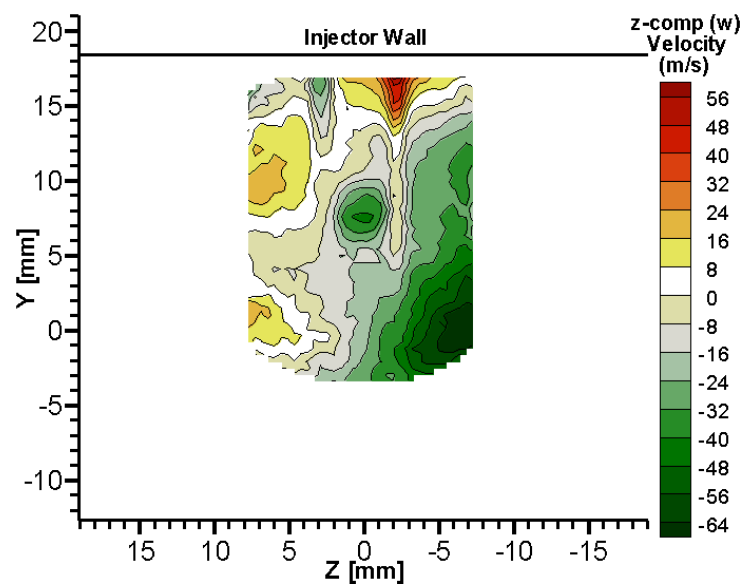

CFD Result, Axial (u) Velocity

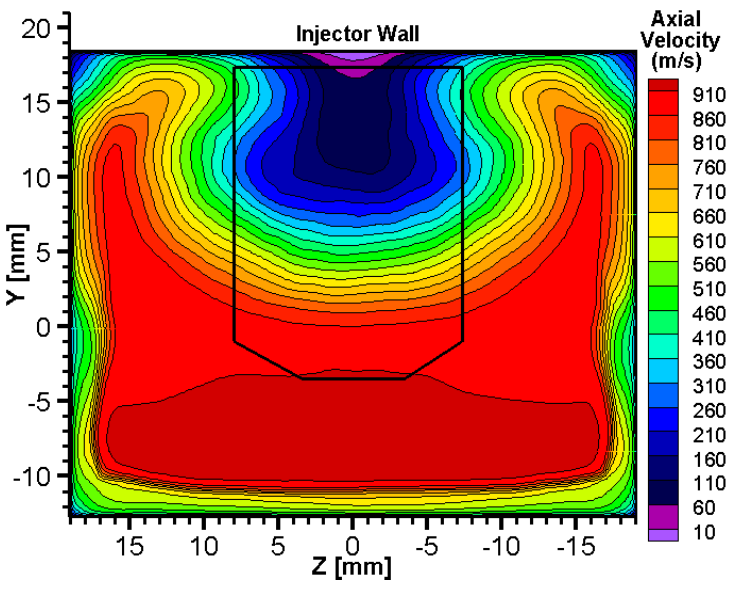

CFD y-component (v) Velocity

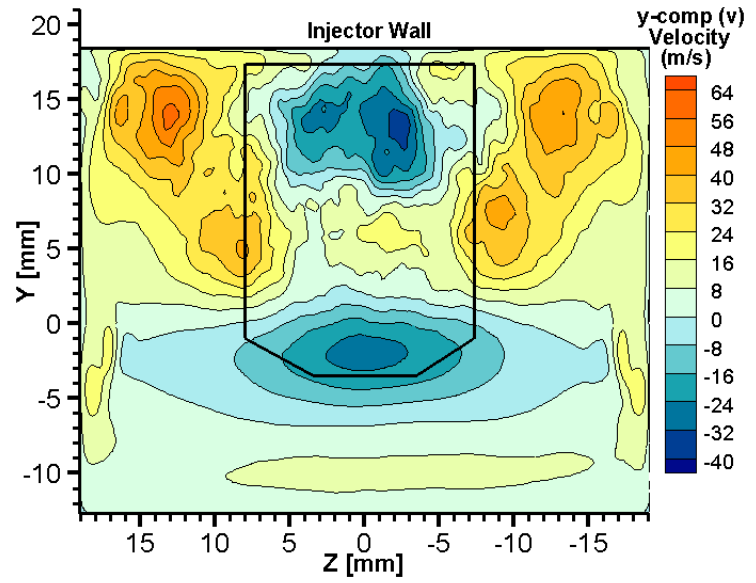

CFD z-component (w) Velocity

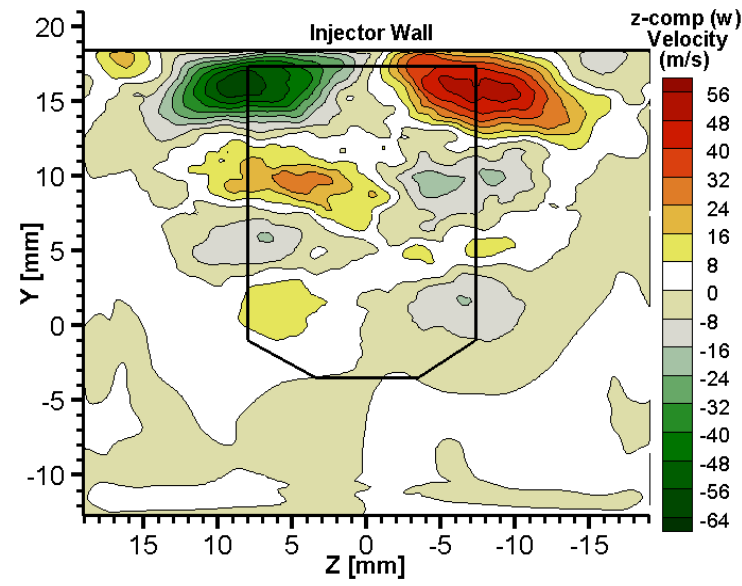

Figure 6.22: Experiment (SPIV) and CFD comparison, Fuel-air Combustion, $\mathrm{X} / \mathrm{H}=12, \Phi=0.17$ 
width (z-direction) than in the CFD simulation. Furthermore, the region of lowest axial velocity (shown as contours in shades of blue) extends slightly farther toward the center of the duct (approx. 2-mm further) in the average SPIV measurement than in the CFD simulation. The increase in velocity from the subsonic region to supersonic flow occurs over nearly the same (y-direction) distance in both the average SPIV result and the CFD simulation. The pattern of y-component velocity matches fairly well between CFD and SPIV results, but the magnitude of y-comp. velocity is slightly under-predicted by the CFD in areas at the edges of the fuel plume. The z-component velocity shows the least match between SPIV and CFD simulation, however, resolution of the In-plane components is a difficult task for any simulation. Figure 6.23 shows the In-plane vectors determined from SPIV measurements and LES/RANS simulations. The vortices are

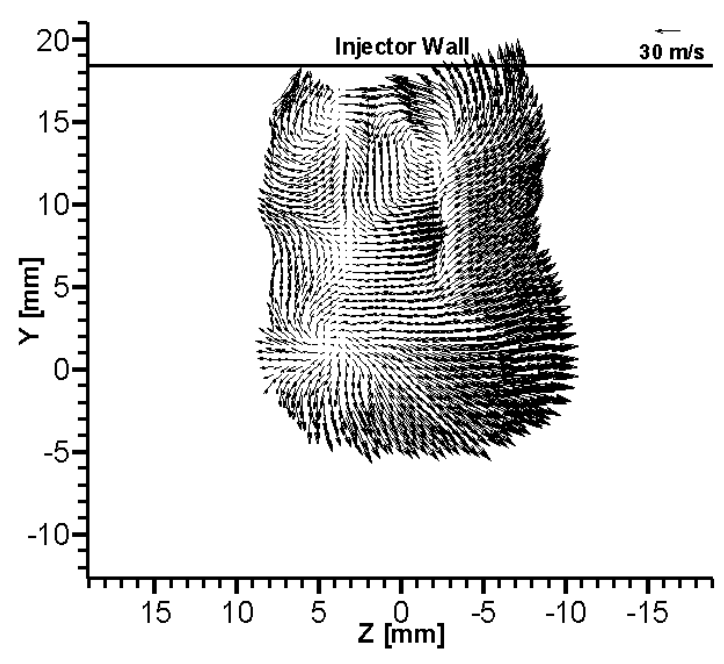

(a) Average Experimental (SPIV) Result

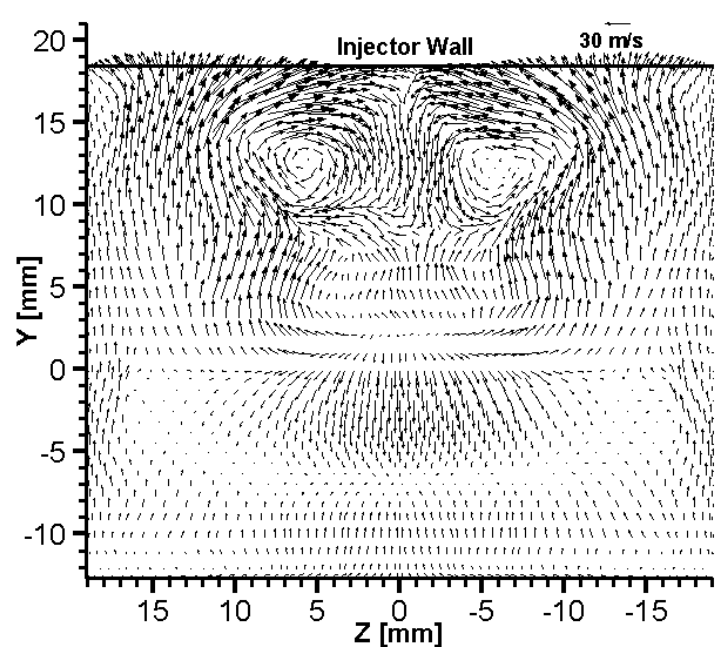

(b) CFD Result In-plane vectors

Figure 6.23: $\Phi=0.17$, Fuel-air Combustion at $\mathrm{X} / \mathrm{H}=12$

(a) Average SPIV velocity field showing In-plane vectors, and (b) CFD Result

smaller in the average SPIV measurement than in the CFD result and the LES/RANS simulation does not predict the asymmetry of the vortices seen in the SPIV results. However, the fact that some similarity exists between in-plane measurements and CFD 
predictions for such a difficult situation shows that modelers are making strides toward better CFD tools. Furthermore, this CFD model will likely be improved in the future after further comparison with experimental measurements.

The instantaneous SPIV measurements can also be used to obtain other turbulence quantities such as the turbulent kinetic energy. Turbulent kinetic energy (TKE) is defined as $1 / 2\left(V^{\prime}\right)^{2}$ where $V^{\prime}$ is the turbulent fluctuation of the three-component velocity, $\left(V^{\prime}\right)^{2}=$ $\left(\mathrm{u}^{\prime}\right)^{2}+\left(\mathrm{v}^{\prime}\right)^{2}+\left(\mathrm{w}^{\prime}\right)^{2}$. The hybrid LES/RANS CFD method can also be used to predict turbulent kinetic energy and it is useful to compare measured TKE to TKE found using CFD. Figure 6.24 shows plots of TKE from both SPIV measurements and CFD for the $\mathrm{X} / \mathrm{H}=12$ measurement plane. In the figure, it can be seen that both SPIV measurements

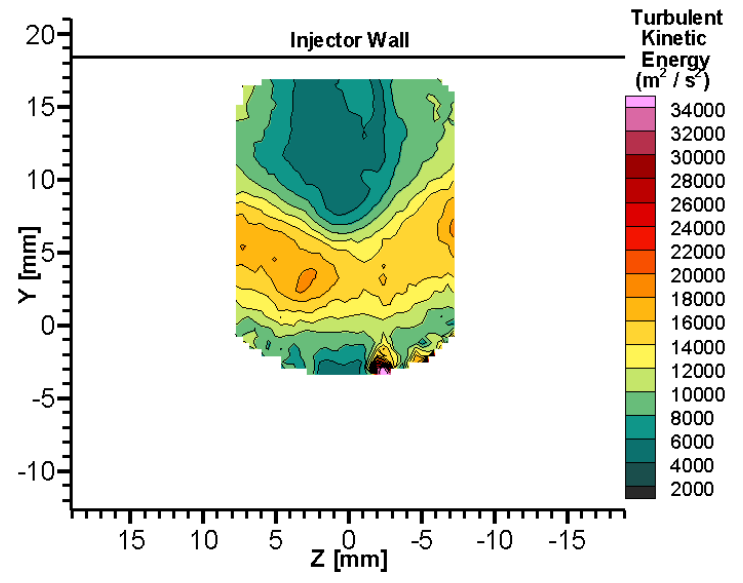

(a) Measured Turbulent Kinetic Energy from SPIV measurements

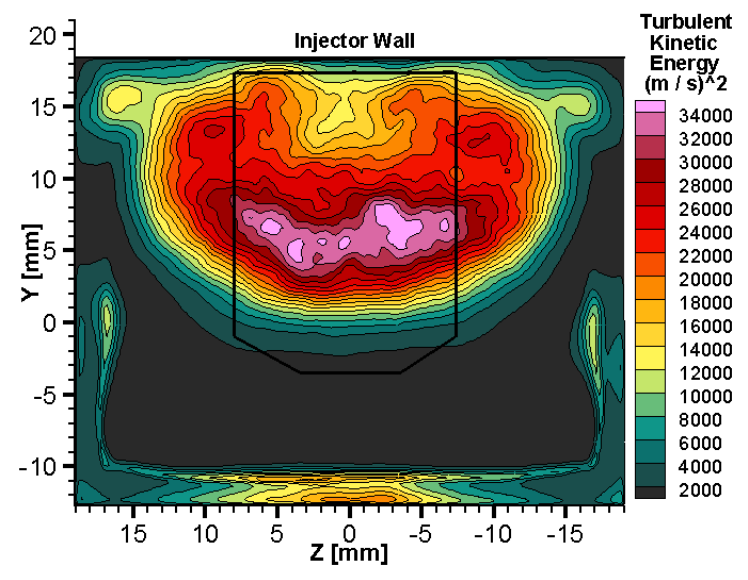

(b) Predicted Turbulent Kinetic Energy (CFD result)

Figure 6.24: Turbulent Kinetic Energy Comparison for $\Phi=0.17$, Fuel-air Combustion at $\mathrm{X} / \mathrm{H}=12$; (a) Measured TKE, and (b) Predicted TKE from CFD

and CFD results show that TKE is largest in the shear layer between the low-speed region and the supersonic free-stream. The relative shape of the TKE contours is the same between the CFD result and the result from SPIV measurements. Also, both CFD and SPIV measurements indicate that TKE in the shear layer is approximately 2.7 times the 
value of the low-speed region near the injector wall. However, the magnitude of TKE predicted by CFD does not match that measured using SPIV. The LES/RANS CFD overpredicts TKE by a factor of approximately $2-2.5$ throughout the measurement area. Furthermore, as was the case when comparing velocities from CFD and measurements, the CFD result underpredicts the size of the low-speed region near the injector wall. Clearly some improvement needs to be made in order to correct the disagreement in TKE magnitude between CFD predictions and the value found using SPIV measurements.

Comparison between SPIV measurements and LES/RANS simulations are presented in Figure 6.25 for fuel-air combustion at $\mathrm{X} / \mathrm{H}=15$. Again, a solid boundary has been included in the CFD results to show the corresponding location of the SPIV measurements. Comparison between CFD results and SPIV measurements is more difficult in this situation because of the relatively small area of SPIV measurements. General flow patterns appear similar between SPIV and CFD results for each of the velocity components, especially in the regions of high axial velocity. The magnitude of the axial velocity component in the subsonic region near the vortices does match well between SPIV and CFD results, however the shape of the subsonic region is very different. Because the subsonic region near areas of high background noise in the SPIV images, it is unclear whether the shape of this region in the measurements is correct or if it resulted because of missing velocity information due to image noise. However, the measurement area from approximately $\mathrm{z}=-2 \mathrm{~mm}$ to $\mathrm{z}=+9 \mathrm{~mm}$ was devoid of large image noise so high confidence exists for results in that area. That part of the measurement area shows the transition between the subsonic region and supersonic flow (near the center of the duct) and an almost perfect match exists between the average SPIV 
Experiment (SPIV) Axial (u) Velocity

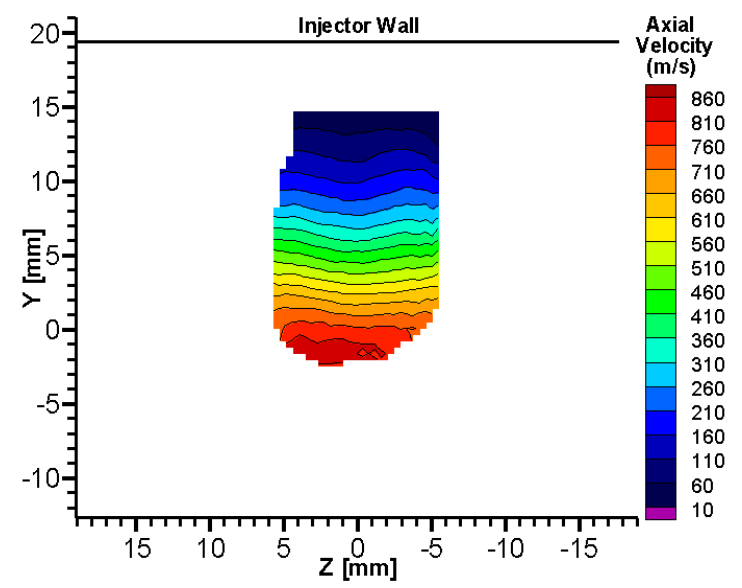

Experiment y-component (v) Velocity

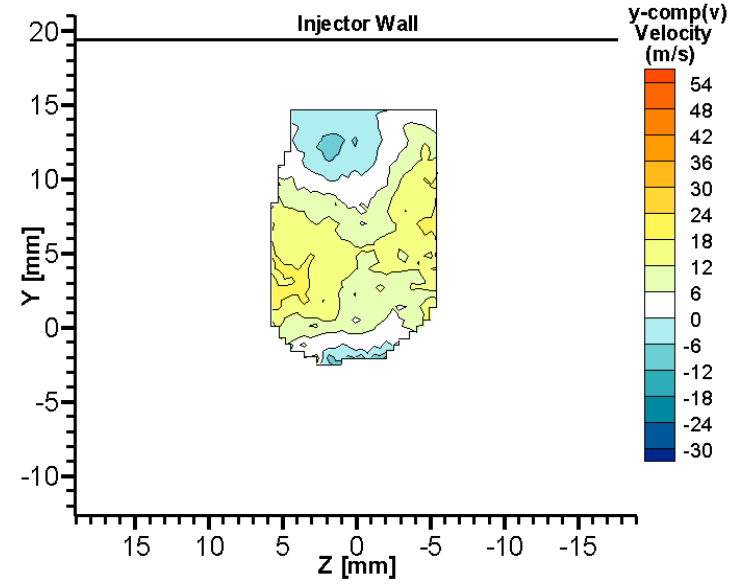

Experiment z-component (w) Velocity

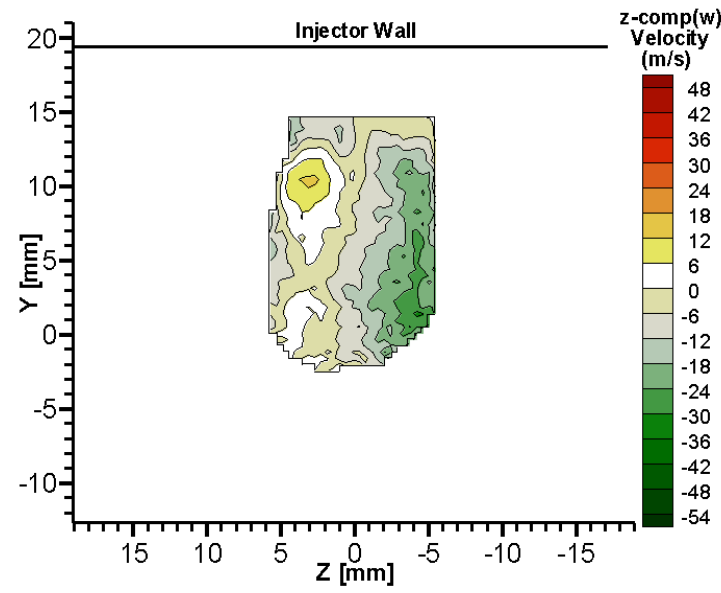

CFD Result, Axial (u) Velocity

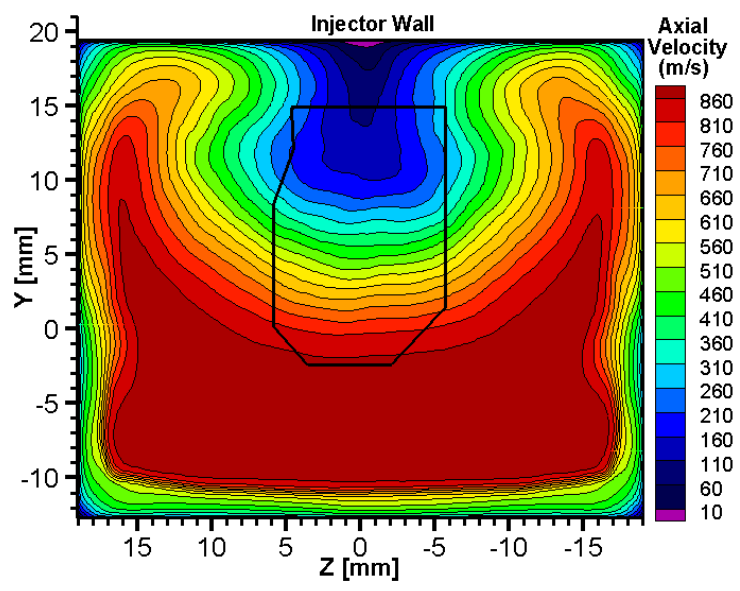

CFD y-component (v) Velocity

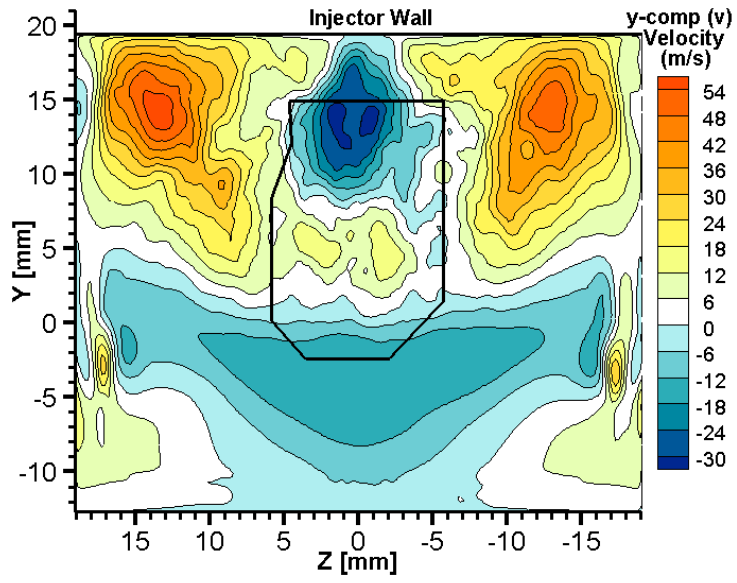

CFD z-component (w) Velocity

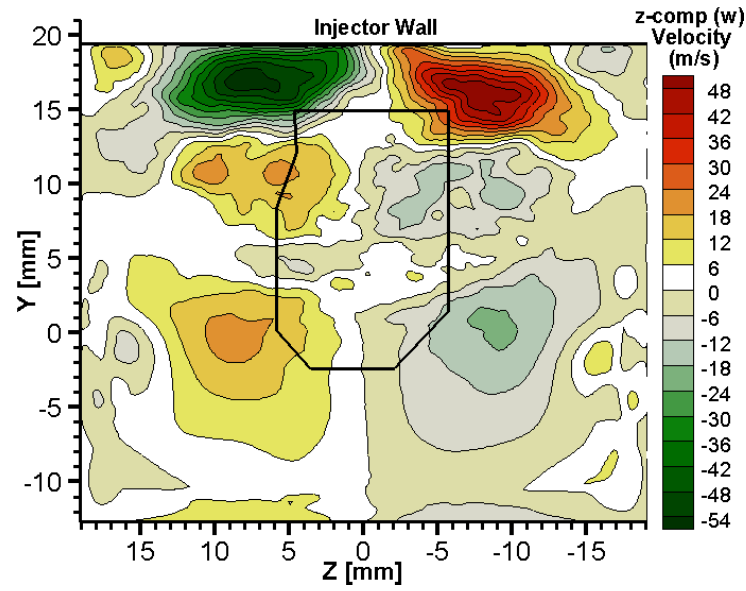

Figure 6.25: Experiment (SPIV) and CFD comparison, Fuel-air Combustion, $\mathrm{X} / \mathrm{H}=15, \Phi=0.17$ 
result and the CFD simulation for that region. For the $y$ and z-components of velocity, general agreement for the contour patterns exists between SPIV and CFD results, with some similarity in magnitude, but comparison is difficult without SPIV measurements over a larger area.

As mentioned above, LES/RANS simulations were also conducted for fuel-air mixing cases at $\mathrm{X} / \mathrm{H}=12$ and 15 , and comparison figures are included in Appendix D (for $\mathrm{X} / \mathrm{H}=12$ ) and for $\mathrm{X} / \mathrm{H}=15$ in Appendix E. Just as with the combustion cases, the LES/RANS CFD results for fuel-air mixing show fairly good agreement with SPIV measurements. The CFD results show similar velocity patterns when compared to the SPIV measurements, with only a slight under-prediction in the CFD results of the axial and y-component velocities in some areas at the edges of the fuel plume.

\section{Summary, Flowpath 2}

SPIV measurements at $\mathrm{X} / \mathrm{H}=12$ and 15 in Flowpath 2 showed a large range of velocity during fuel-air combustion with an area of low-speed flow near the injector wall and a high velocity stream in close proximity. A transition area with a large axial velocity gradient was noted between these two velocity extremes. Shearing between the two fluid streams caused this large velocity gradient. The vortices were noted for fuel-air mixing and combustion cases at $\mathrm{X} / \mathrm{H}=12$ and it was somewhat surprising that they remained largely organized during combustion. Again, that effect is useful for efficient fuel-air mixing and combustion. The same large velocity gradient was also observed at $\mathrm{X} / \mathrm{H}=15$. However, observation of the ramp vortices, as well as the extent of the fuel 
plume was not possible at $\mathrm{X} / \mathrm{H}=15$ due to large noise in the images which resulted due to particles depositing on portions of the windows.

Experiments were also undertaken at $\mathrm{X} / \mathrm{H}=23$ (not reported here) and the same noise problem was noted for the fuel-air combustion case, but actually to a much larger extent. At the $\mathrm{X} / \mathrm{H}=23$ plane, scattered laser light was of high enough intensity to cause damage to the cameras so measurements were not possible. Combustion experiments at $\mathrm{X} / \mathrm{H}=15$ and 23 indicate that the fuel plume is large enough to reach the tunnel side windows. The fuel plume carries seed particles to the boundary layer regions near the windows and the particles adhere to hot side-windows, especially in areas near the injector wall. The problem becomes more pronounced further downstream as more and more particles deposit on the windows. Therefore, future SPIV measurements may only be possible for planes upstream of $\mathrm{X} / \mathrm{H}=15$.

Comparison of LES/RANS simulations to SPIV shows fairly good agreement, especially for the axial velocity component. Simulation of the in-plane components can and should be improved, but the current CFD prediction of these velocity components does show the potential of the LES/RANS model. Future comparison of CFD results to experimental measurements will likely further improve the LES/RANS predictions. 


\section{Chapter 7: Summary and Recommendations}

Stereoscopic Particle Image Velocimetry (SPIV) was used to obtain velocity measurements of the complex flowfields of two DMSJ combustors at the University of Virginia. The objectives of the study were three-fold: 1) to develop an experimental apparatus for SPIV measurements of DMSJ combustor flowfields, 2) to use the velocity measurements obtained through SPIV to gain understanding of the DMSJ combustor flowfields, and 3) to qualitatively compare the SPIV velocity measurements to Computational Fluid Dynamics (CFD) simulations. CFD simulations for Flowpath 1 using a RANS based solver were completed by researchers at Embry-Riddle Aeronautical University, while a group at North Carolina State University completed simulations for Flowpath 2 using a hybrid LES/RANS scheme.

Objective 1 was met by creating an original design procedure for determining a suitable SPIV experimental configuration. This design procedure incorporated information from the literature into an iterative process that helped identify tradeoffs necessary for balancing opposing SPIV experimental constraints. The design procedure was presented in Chapter 3, while experimental configurations that were found using the design procedure were presented in Chapter 4. The SPIV technique was successfully implemented using these configurations and three-component velocity measurements of the DMSJ flowfields were obtained.

For Flowpath 1, further understanding of the DMSJ flowfield was gained by examining fuel-air combustion results as compared to fuel-air mixing at one plane in the test section $(\mathrm{X} / \mathrm{H}=10)$ and at a plane just downstream of the combustor exit. At $\mathrm{X} / \mathrm{H}=$ 10 in the test section, two strong, well-organized vortices were observed for the case of 
fuel-air mixing. These vortices resulted as flow "spilled" over the sides of the unswept ramp fuel injector due to a pressure gradient resulting from higher pressure on the face of ramp and lower pressure at the sides. The vortices are an expected characteristic of this geometry; however the measurements presented here were the first to quantify the rampinduced vortices of DMSJ combustor Flowpath 1 . The effect of heat release at $\mathrm{X} / \mathrm{H}=10$ during fuel-air combustion was seen as a drop in 3C velocity magnitude and this lower velocity is predicted by theory. Furthermore, combustion in the Test section also caused the vortices to spread in area and reduce in strength, while the degree of turbulence rose across the fuel plume, especially in areas near the vortices.

At the exit plane of Flowpath 1, two different fuel conditions were tested where one fuel condition $(\Phi=0.17)$ resulted in a predominantly supersonic combusting flowfield, whereas heat release from combustion for the $\Phi=0.34$ case yielded a flowfield which was mostly subsonic. These fuel conditions were chosen so the effect modetransition from a supersonic, to a subsonic, combusting flowfield could be examined. Fuel-air mixing measurements at the exit of Flowpath 1 did not yield very interesting or useful results, however, SPIV measurements of Fuel-air combustion were quite surprising. For the supersonic combustion case $(\Phi=0.17)$ well-organized vortices were observed in the exit cross-plane, which means the vortices remained intact to a distance of nearly 59 ramp heights $(\sim 37 \mathrm{~cm})$ downstream of their formation point. The influence of the vortices on the flowfield was also seen as a drop in axial velocity near the injector wall and extended into the core flow. For the subsonic combustion case, $(\Phi=0.34)$ a nearly uniform-velocity flowfield was observed across most of the plane, and the influence of the vortices was seen as a slight drop in axial velocity near the injector wall. 
For the higher fuelling case $(\Phi=0.34)$ the ramp induced vortices were not observed. This is most likely attributed to vortex breakdown in the complex flow downstream of the thermal throat that resulted from heat release during combustion. At the exit plane, turbulence intensity was again found to be higher near the vortices, with turbulence values between $15 \%$ and $20 \%$ in the area of the vortices as opposed to $9-12 \%$ in the remainder of the field.

Combustor Flowpath 2 has a very different geometry from that of Flowpath 1, in that divergence of the injector wall begins further upstream for Flowpath 2 and the ramp injector is located on the wall downstream of the start of divergence. Comparison of the velocity fields in the Test-sections of the two flowpaths is interesting and useful for understanding the effect of flow geometry, however, it should be kept in mind that an Isolator section was present in Flowpath 1, but absent for Flowpath 2. Furthermore, experiments were conducted with different equivalence ratios, $\Phi=0.26$ versus 0.17 . Well-organized vortices were again noted for fuel-air mixing cases in Flowpath 2, with degree of fluid rotation that was nearly identical to that observed for mixing in Flowpath 1. While fuel-air mixing results were very similar in the Test-sections of both flowpaths, the difference in flowpath geometry produced substantially different velocity fields during fuel-air combustion. SPIV measurements for the fuel-air combustion case $(\Phi=$ 0.17 ) at both the $\mathrm{X} / \mathrm{H}=12$ and $\mathrm{X} / \mathrm{H}=15$ planes of Flowpath 2 showed average fields containing an extremely large range of velocity. Measured 3C velocity was very low $(\sim 50 \mathrm{~m} / \mathrm{s}$ to $80 \mathrm{~m} / \mathrm{s})$ in the area of the vortices near the injector wall, while a maximum velocity of $\sim 900 \mathrm{~m} / \mathrm{s}$ was measured at the edge of the fuel plume. Transition between the subsonic and supersonic regions was found to occur over a short distance and is attributed 
to a strong shearing between the two extreme velocities. The ramp-induced vortices were also observed for combustion at $\mathrm{X} / \mathrm{H}=12$, along with characteristic asymmetry. Unfortunately, areas of large background noise occurred in SPIV images for the combustion case at $\mathrm{X} / \mathrm{H}=15$ and this prevented velocity measurements where the rampinduced vortices reside. Despite this problem, useful velocity measurements were obtained for the region of supersonic flow in the plane at $\mathrm{X} / \mathrm{H}=15$, as well as in the transitional region from subsonic to supersonic speeds.

The area relief in Flowpath 2, due to the divergence of the injector wall attributed to the largely different combusting velocity fields observed in Flowpath 1 and Flowpath 2. Much larger degrees of turbulence were noted in the area of the vortices during combustion $\mathrm{X} / \mathrm{H}=12$ in Flowpath 2 than at $\mathrm{X} / \mathrm{H}=10$ in Flowpath 1. High turbulence in this low-velocity region is expected due to heat release from combustion and also due to movement of the vortices across the plane. Higher turbulence intensity was noted for combustion at $\mathrm{X} / \mathrm{H}=15$ than at $\mathrm{X} / \mathrm{H}=12$, but it is unclear if this is a physical effect or if background noise contributed to that result.

In all cases, the decrease in velocity in the area of the vortices during combustion indicates heat release from efficient fuel-air combustion. This efficient combustion was accomplished due to the enhancement of fuel-air mixing provided by the ramp-induced vortices. The observation of these general flow characteristics provided further understanding of the complex flow of a DMSJ combustor and, therefore, Objective 2 of this study was met.

For objective 3 of this study, SPIV measurements from both flowpaths were compared to CFD results. SPIV measurements in Flowpath 1 were compared to RANS 
simulations for fuel-air combustion cases and it was found that the simulations underpredicted the degree of turbulent mixing in the Test-section. This lead to an overprediction of axial velocity in the core of the fuel plume because, at the $\mathrm{X} / \mathrm{H}=10$ plane, CFD predicted a core in the fuel plume that was pure hydrogen fuel. The CFD results showed that this pure hydrogen core had a velocity closer in magnitude to the velocity at the exit of the fuel injector nozzle. In reality, the entire hydrogen fuel plume mixes with free-stream air and combustion occurs by the time fuel reaches the $\mathrm{X} / \mathrm{H}=10$ plane, thus causing a lower velocity than the velocity of a pure hydrogen core predicted by RANS CFD simulations. The problem of under-predicting turbulent mixing was not as noticeable at the exit because fuel had time to properly mix with free-stream air before reaching the exit plane. RANS simulations did predict the drop in axial velocity near the injector wall for $\Phi=0.17$. However, In-plane velocity component magnitudes were under-predicted for all cases.

For Flowpath 2, SPIV measurements were compared to hybrid LES/RANS results and the combustion case is the most interesting. Flow patterns and predicted velocity magnitudes matched the measurements fairly well for each velocity component, but especially well for the axial component. The LES/RANS simulations were also able to predict the in-plane vortices, but did not correctly predict the size of these vortices and did not predict the asymmetry between the vortices that was observed in the SPIV measurements. The main area of disagreement was in the size and shape of the low speed region of the combusting flow. Simulations predicted a slightly smaller area of the low-speed region at $\mathrm{X} / \mathrm{H}=12$ than was measured using SPIV. Simulations and SPIV measurements match well for the supersonic and transition regions at $\mathrm{X} / \mathrm{H}=15$. 
However, measurements in the low-speed region at $\mathrm{X} / \mathrm{H}=15$ are questionable due to image noise, as mentioned above, so no comparison could be made between SPIV and LES/RANS results in that area.

Several original contributions resulted from this study. First, this work constitutes the first application of SPIV to the supersonic combusting flow of a DMSJ combustor and three-component, instantaneous velocity measurements were obtained for the first time. Second, an original design procedure, that combines information from the literature, was created to aid in design of SPIV experiments for complex flows. Third, velocity measurements for a DMSJ combustor were compared for the first time to RANS CFD simulations. The first comparison of SPIV to hybrid LES/RANS simulations was also conducted as part of this study. Lastly, comparison of the flowfields for cases on each side of mode-transition (supersonic combustion and subsonic combustion) were conducted as part of the work presented here.

\section{Recommendations}

Several recommendations are made here for continuing work with the current data, as well as for future experiments. First, turbulence intensity of each velocity component, as well as Reynolds stresses could be determined from the current data, however, values would likely only be estimates because a larger data set is required for statistical convergence for quantities involving velocity fluctuations. Second, the turbulent mixing length scale and turbulent time scale were found, as an example, at one interesting location in the $\mathrm{X} / \mathrm{H}=12$ measurement plane for the fuel-air combustion case. Turbulent scales can be found for other locations of interest in the $\mathrm{X} / \mathrm{H}=12$ plane, as well as at 
locations in future measurement planes. Third, direct measurement of the uncertainty would likely be more accurate than assuming 0.1 pixel displacement uncertainty that is reported in the literature for well-posed SPIV experiments. One method for direct measurement of uncertainty is to simulate SPIV data by copying and shifting a single measured SPIV image by a known amount. Processing for vectors and comparing to the known displacement would give a measure of the uncertainty for the SPIV configuration.

It is recommended that future measurements be conduct for a different geometry of Flowpath 2 and at different fuel equivalence ratios. It would be interesting to see what flowfield is produced with an isolator and investigation of greater heat release from combustion would likely produce further understand of the complex DMSJ flowfield. The large windows of Flowpath 2 make it well suited for such experiments, especially for planes upstream of $\mathrm{X} / \mathrm{H}=12$. In the current work, it was found that SPIV measurements were severely impacted by particles adhering the windows during combustion at planes downstream of $\mathrm{X} / \mathrm{H}=12$. Measurements at planes downstream of $\mathrm{X} / \mathrm{H}=15$ will likely not be possible without substantial changes to the SPIV configuration. Scattering of the laser sheet as it passes though particle-coated windows is the major problem and could possibly be mitigated if the cameras did not view through the same windows that pass the laser sheet. Directing the laser sheet through a window on the Observation wall would accomplish this, however, such a window may not be possible due to high heat loads on that wall. The best solution would be to keep particles from adhering to the windows altogether, or to obtain SPIV measurements in the short time before particles coat the windows. The problem of window fouling seems to be universal for particle-based measurement techniques and no solution has yet been reported in the literature. 


\section{References:}

[1] Ferlemann, S. M., McClinton, C. R., Rock, K. E., and Voland, R. T., "Hyper-X Mach 7 Scramjet Design, Ground Test and Flight Results," AIAA Paper 2005-3322, 2005

[2] Heiser, W. H., and Pratt, D. T., with Daley, D. H., and Mehta, U. B. Hypersonic Airbreathing Propulsion, American Institute of Aeronautics and Astronautics, Inc., Washington, DC, 1994.

[3] NASA Langley Research Center (LaRC), "Propulsion-Airframe Integrated Scramjet," Schematic, 2006. Retrieved on 8/21/2011 from http://www.nasa.gov/centers/langley/images/content/142861main_x43a_intscramjet_550.gif

[4] Billig, F.S., "Research on Supersonic Combustion," Journal of Propulsion and Power, Vol. 6, No.4, 1993, pp. 499-514.

[5] Cockrell, C.E., Auslender, A.H., Guy, R.W., McClinton, C.R., and Welch, S.S., "Technology Roadmap for Dual-Mode Scramjet Propulsion to Support Space-Access Vision Vehicle Development," AIAA Paper 2002-5288, 2002.

[6] Gauba, G., Klavuhn, K.G., McDaniel, J.C., Victor, K.G., Krauss, R.H., and Whitehurst, R.B., "OH Planar Laser-Induced Fluorescence Velocity Measurements in a Supersonic Combustor," AIAA Journal, Vol. 35, No. 4, 1997, pp. 678-686.

[7] Miles, R.B., and Lempert, W.R., "Quantitative Flow Visualization in Unseeded Flows," Аnnи. Rev. Fluid Mech., 29, pp.285-326, 1997.

[8] Smith, M.W., "Application of a Planar Doppler Velocimetry System to a High Reynolds Number Compressible Jet," AIAA Paper 98-0428, Jan. 1998.

[9] Meyers, J.F., and Komine, H., "Doppler Global Velocimetry: A New Way to Look at Velocity," Laser Anemometry, Vol. 1, 1991, pp.289-296.

[10] Raffel, M., Willert, C., and Kompenhans, J., Particle Image Velocimetry - A Practical Guide, Springer-Verlag, Berlin, 1998.

[11] Goyne, C.P., McDaniel, J.C., Krauss, R.H., and Day, S.W., "Velocity Measurement in a Dualmode Supersonic Combustor using Particle Image Velocimetry," AIAA Paper 2001-1761, April 2001.

[12] McIntyre, T. J., Houwing, A. F. P., Palma, P. C., Rabbath, P. A. B., and Fox, J. S., "Optical and Pressure Measurements in Shock Tunnel Testing of a Model Scramjet Combustor," Journal of Propulsion and Power, Vol. 13, No. 3, 1997, pp. 388 - 394.

[13] Parker, T. E., Allen, M. G., Reinecke, W. G., Legner, H. H., Foutter, R. R., and Rawlins, W. T., "High-Temperature Supersonic Combustion Testing with Optical Diagnostics," Journal of Propulsion and Power, Vol. 9, No. 3, 1993, pp. 486 - 492. 
[14] Donbar, J. M., Gruber, M. R., Jackson, T. A., and Carter, C. D., "OH Planar Laser-Induced Fluorescence Imaging in a Hydrocarbon-Fueled Scramjet Combustor," Proceedings of the Combustion Institute, Vol. 28, 2000, pp. $679-687$.

[15] Gauba, G., "OH PLIF Velocity Measurement and Computational Fluid Dynamics Modeling for a Combusting High Speed Flow," Ph.D. Dissertation, Dept. of Mechanical and Aerospace Engineering, University of Virginia, Charlottesville, VA, 1995.

[16] Samimy, M., and Wernet, M.P., "Review of Multiple-Component Velocimetry in High-Speed Flows," AIAA Journal, Vol. 38, No. 4, 2000, pp.553-574.

[17] Willert, C.E., and Gharib, M., "Digital Particle Image Velocimetry," Experiments in Fluids, Vol. 10, 1991, pp. 181 - 193.

[18] Prasad, A. K., "Stereoscopic Particle Image Velocimetry," Experiments in Fluids, Vol. 29, 2000, pp. $103-116$.

[19] Keane, R.D., and Adrian, R.J., "Optimization of Particle Image Velocimeters. Part I: Double Pulsed Systems," Measurement, Science and Technology, Vol.1 No. 11, Nov. 1990, pp 1202 1215 .

[20] Gupta, A. K., Lourenco, L., Linck, M., and Archer, S., "New Particle-Image-Velocimetry Method to Measure Flowfields in Luminous Spray Flames," Journal of Propulsion and Power, Vol. 20, No.2, March-April 2004, pp. 369 - 372.

[21] Tanahashi, M., Shinichirou, M., Choi, G-M., Fukuchi, Y., and Miyauchi, T., "Simultaneous CHOH PLIF and Stereoscopic PIV Measurements of Turbulent Premixed Flames," Proceedings of the Combustion Institute, Vol. 30, 2005, pp.1665 - 1672.

[22] Ikeda, Y., Kuratani, N., and Nakajima, T., "M2.5 Supersonic PIV Measurement in Step-back Flow with the Normal Injection,” AIAA Paper 2002-0232, January 2002.

[23] Scarano, F., and van Oudheusden B.W., "Planar Velocity Measurements of a Two-dimensional Compressible Wake," Experiments in Fluids, Vol. 34, 2003, pp 430 - 441.

[24] Arai, T., Sakaue, S., Morisaki, T., Kondo, A., Hiejima, T., and Nishioka, M., "Supersonic Streamwise Vortices Breakdown in Scramjet Combustor," AIAA Paper 2006-8025.

[25] Alkislar, M. B., Krothapalli, A., and Lourenco, L. M., "Structure of a Screeching Rectangular Jet: A Stereoscopic Particle Image Velocimetry Study," Journal of Fluid Mechanics, Vol. 489, 2003, pp. 121-154.

[26] Beresh, S. J., Henfling, J. F., Erven, R. J., and Spillers, R. W., "Vortex Structure Produced by a Laterally Inclined Supersonic Jet in Transonic Crossflow," Journal of Propulsion and Power, Vol. 23, No.2, March-April 2007, pp. 353 - 363.

[27] Weisgerber, H., Marinuzzi, R., Brummund, U., and Magre, Ph., "PIV Measurements in a Mach 2 Hydrogen-air Supersonic Combustion,” AIAA Paper 2001-1757, April, 2001.

[28] Goyne, C.P., Rodriguez, C.G., Krauss, R.H., McDaniel, J.C., and McClinton, C.R., "Experimental and Numerical Study of a Dual-Mode Scramjet Combustor," Journal of Propulsion and Power, Vol. 22, No. 3, 2006. pp. 481-489

[29] Scheel, F., "PIV Measurement of a 3-dimensional Reacting Flow in a Scramjet Combustion Chamber," AIAA Paper 2004-1038, January 2004. 
[30] Krauss, R.H., McDaniel, J.C., Scott J.E., Whitehurst, R.B., Segal, C., Mahoney, G.T., and Childers, J.M., "Unique, clean-air, continuous-flow, high-stagnation-temperature facility for supersonic combustion research,” AIAA Paper 88-3059, July, 1988.

[31] Krauss, R.H., and McDaniel, J.C., "A Clean Air Continuous Flow Propulsion Facility,” AIAA Paper 92-3912, July, 1992.

[32] Lawson, N. J., and Wu, J., "Three-dimensional Particle Image Velocimetry: Error Analysis of Stereoscopic Techniques," Meas. Science and Technology, Vol. 8, 1997, pp. 894 - 900.

[33] Donohue, J. M. and McDaniel, J. C. 1996 Complete Three-Dimensional Multiparameter Mapping of a Supersonic Ramp Fuel Injector Flowfield AIAA Journal, Vol. 34, 1996, pp. 455462

[34] Donohue, J. M. "Experimental and Numerical Study of Ramp Injectors for Supersonic Fuel/Air Mixing" Ph.D. Dissertation, Dept. of Mechanical and Aerospace Engineering, University of Virginia, Charlottesville, VA, 1995.

[35] Howison, J. C. and Goyne, C. P., "Assessment of Seeder Performance for Particle Velocimetry in a Scramjet Combustor," Journal of Propulsion and Power, Vol. 26, No.3, 2010, pp. 514 523. doi: $10.2514 / 1.44469$

[36] Melling, A., "Tracer Particles and Seeding for Particle Image Velocimetry," Measurement, Science and Technology, Vol.8 No.12, Dec. 1997, pp 1406 - 1416

[37] Hinsch, K. D., "Three-dimensional Particle Velocimetry," Meas., Science and Technology, Vol. 6,1995 , pp. $742-753$.

[38] Meehan, J. The Art of Close-up Photography, Fountain Press, Kingston-Upon-Thames, Surry, 1994, p 9

[39] Giuni, M., Green, R. B., and Benard, E., "Investigation of a Trailing Vortex Near Field by Stereoscopic Particle Image Velocimetry,” AIAA Paper 2011-992, January 2011

[40] Son, S. Y., Kihm, K. D., and Han, J.-C., "PIV Flow Measurements for Heat Transfer Characterization in Two-pass Square Channels with Smooth and $90^{\circ}$ Ribbed Walls," International Journal of Heat and Mass Transfer, Vol. 45, 2002, pp. 4809 - 4822.

[41] Goyne, C. P., McDaniel, J.C., Quagliaroli, T. M., Krauss, R. H., and Day, S. W., "Dual-Mode Combustion of Hydrogen in a Mach 5, Continuous-Flow Facility," Journal of Propulsion and Power, Vol. 17, No.6, 2001, pp. 1313 - 1318.

[42] Scarano, F., David, L., Bsibsi, M., and Calluaud, D., "S-PIV Comparative Assessment: Image Dewarping Misalignment Correction and Pinhole Geometric Back Projection," Experiments in Fluids, Vol. 39, 2005, pp. $257-266$.

[43] Wieneke, B., "Stereo-PIV Using Self-Calibration on Particle Images," Experiments in Fluids, Vol. 39, 2005, pp. $267-280$.

[44] Durst, F., Melling, A., and Whitelaw, J. H., Principles and Practice of Laser-Doppler Anemometry, $2^{\text {nd }}$ Edition, Academic Press, London, 1981.

[45] Guezennec, Y. G., and Kiritsis, N., "Statistical Investigation of Errors in Particle Image Velocimetry," Experiments in Fluids, Vol. 10, 1990, pp. 138 - 146. 
[46] Samimy, M., and Wernet, M. P., "Review of Planar Multiple-Component Velocimetry in HighSpeed Flows," AIAA Journal, Vol. 38, No. 4, 2000, pp. 553 - 574.

[47] Anderson, J. D. Jr, Modern Compressible Flow with Historical Perspective, $2^{\text {nd }}$ Edition, McGraw Hill, New York 1990, p. 83-84.

[48] Towne, C.E., “Wind-US Users Guide, Version 2.0,” NASA/TM - 2009-215804, 2009.

[49] Lankford, D.W., and Mani, M., "Wind Thermochemical Models and Recent Improvements," AIAA Paper 2003-545, 2003.

[50] Vyas, M.A., Engblom, W. A., Georgiadis, N. J., Trefny, C. J., and Bhagwandin, V. A., "Numerical Simulation of Vitiated Effects on a Hydrogen-fueld Dual-Mode Scramjet," AIAA Paper 2010-1127, January 2010.

[51] Kouchi, T., Personal communication, September 2011.

[52] Davidson, P. A., Turbulence - An Introduction for Scientists and Engineers, Oxford Universtiy Press, Oxford, New York, 2004, pp 88-90.

[53] Fulton, J. A., Edwards, J. R., Hassan, H. A., Rockwell, R., Goyne, C., McDaniel, J., Smith, C., Cutler, A., Johansen, C., Danehy, P. M., and Kouchi, T., "Large-Eddy/Reynolds-Averaged Navier-Stokes Simulations of a Dual-Mode Scramjet Combustor," AIAA Paper AIAA-2012115, January 2012.

[54] Fulton, J. A., Personal communication, January 5, 2012. 


\section{Appendix A}

\section{Supplemental Figures for Flowpath 1, Plane at X/H = 10}

\section{List of figures}

$3 D$ Velocity Plots

Figure A.1: $3 \mathrm{D}$ plot of Average 3C velocity for Fuel-air Mixing at $\mathrm{X} / \mathrm{H}=10$; 3D surface representation of velocity

Figure A.2: $3 \mathrm{D}$ plot of Average 3C velocity for Fuel-air Combustion case at $\mathrm{X} / \mathrm{H}=10$; $3 \mathrm{D}$ surface representation of velocity

Summary Figures

Figure A.3: Summary of Average velocity fields for Fuel-air Mixing at $\mathrm{X} / \mathrm{H}=10$; $3 \mathrm{C}$ magnitude, In-plane magnitude, In-plane vectors; and $\mathrm{u}, \mathrm{v}$ and $\mathrm{w}$ velocity components (Average of 708 instantaneous fields)

Figure A.4: Summary of Average velocity fields for Fuel-air Combustion at $\mathrm{X} / \mathrm{H}=10$; 3C magnitude, In-plane magnitude, In-plane vectors; and $\mathrm{u}, \mathrm{v}$ and $\mathrm{w}$ velocity components (Average of 775 instantaneous fields) 


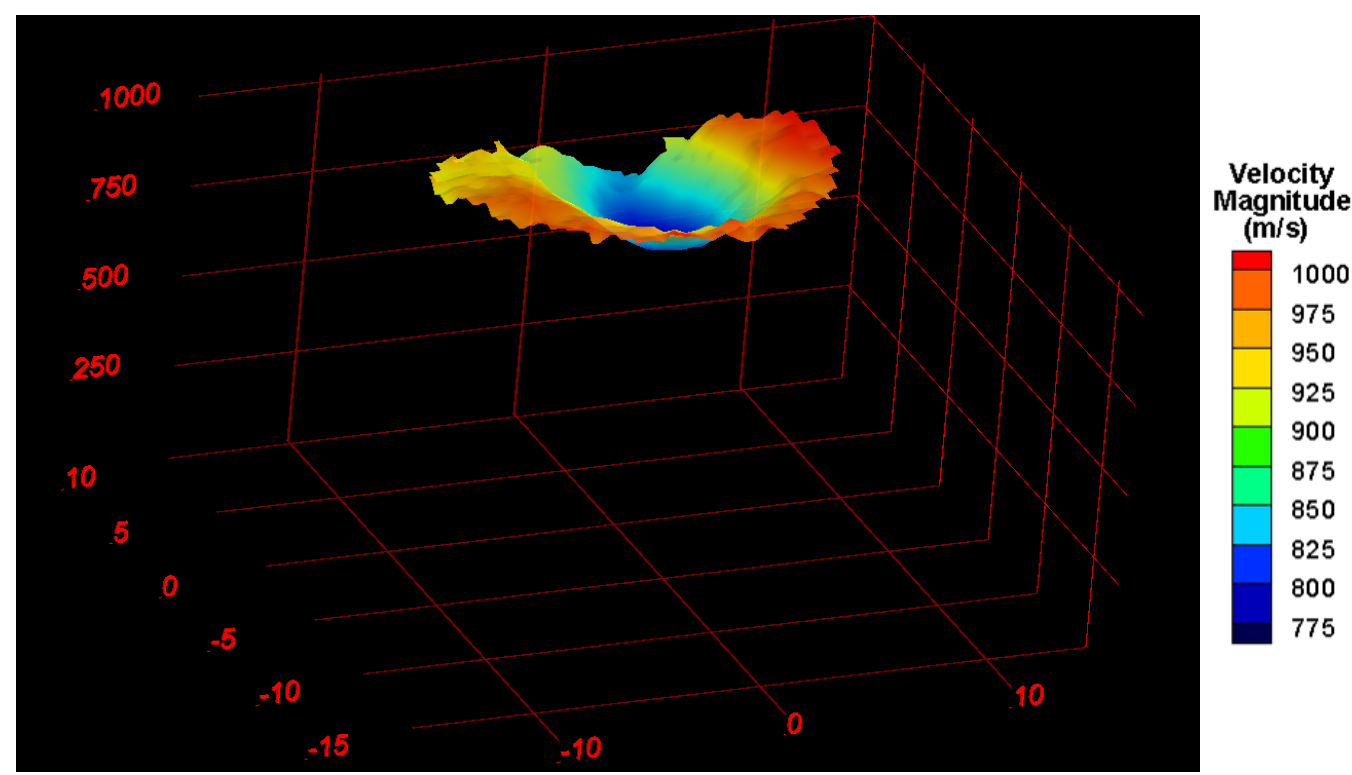

Figure A.1: Average three-component (3C) velocity result from SPIV measurements for Fuel-air Mixing case at $\mathrm{X} / \mathrm{H}=10 ; 3 \mathrm{C}$ velocity represented as a 3D Surface.
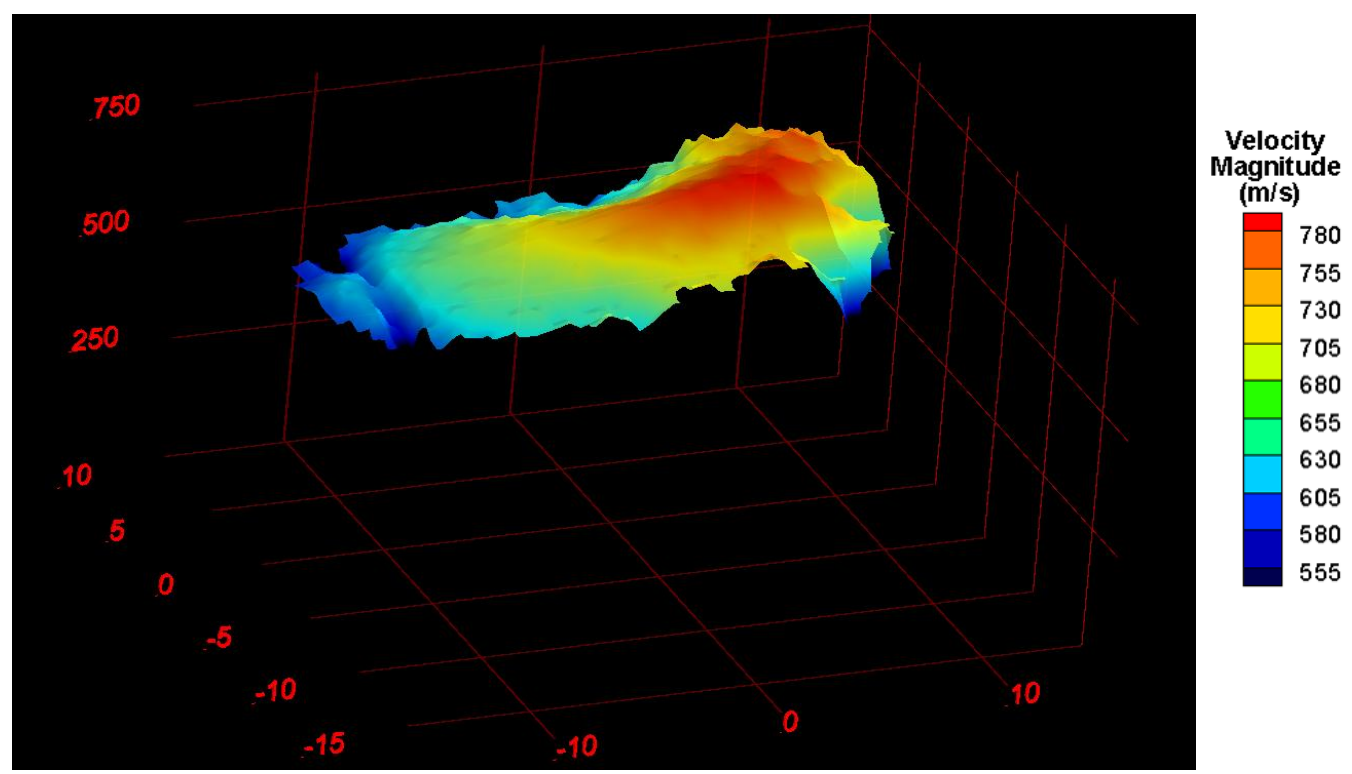

Figure A.2: Average three-component (3C) velocity result from SPIV measurements for Fuel-air Combustion case at $\mathrm{X} / \mathrm{H}=10 ; 3 \mathrm{C}$ velocity represented as a 3D Surface. 
3C Velocity Magnitude

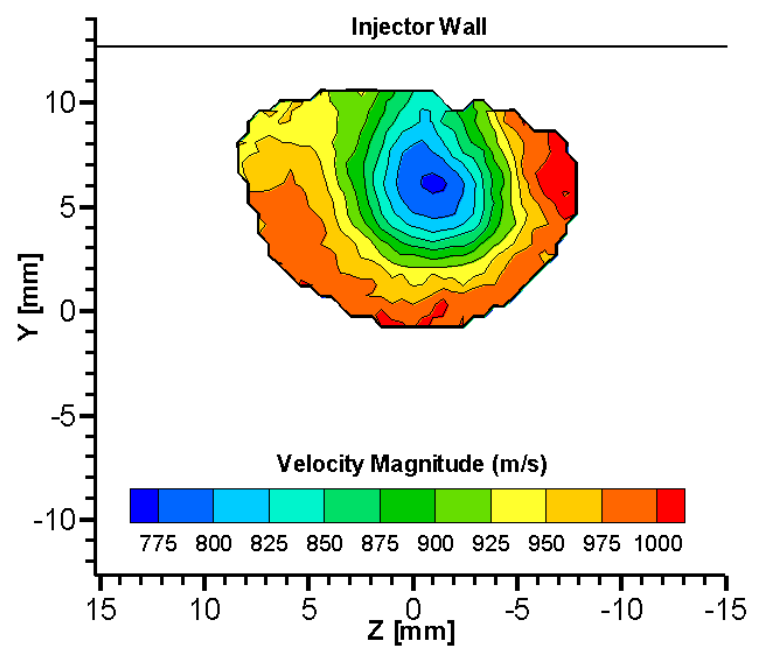

In-plane Velocity

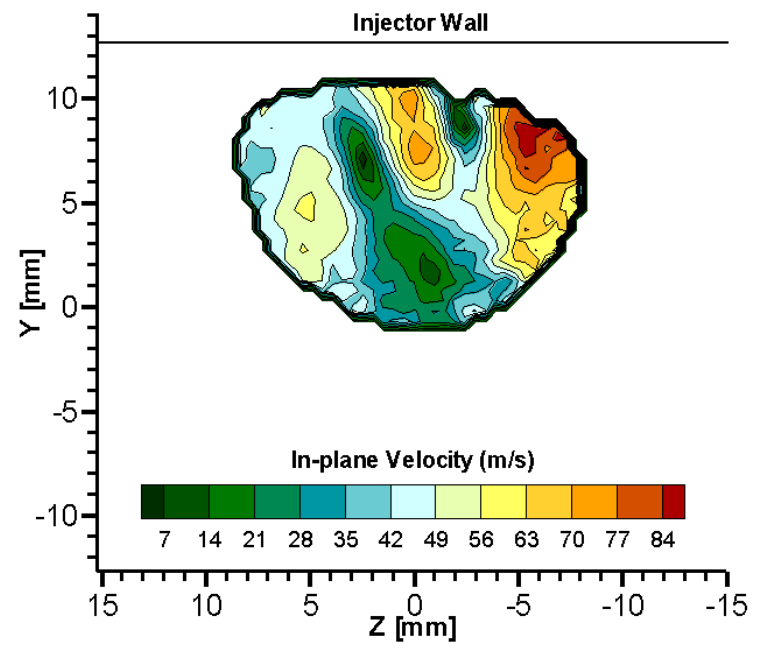

In-plane Velocity Vectors

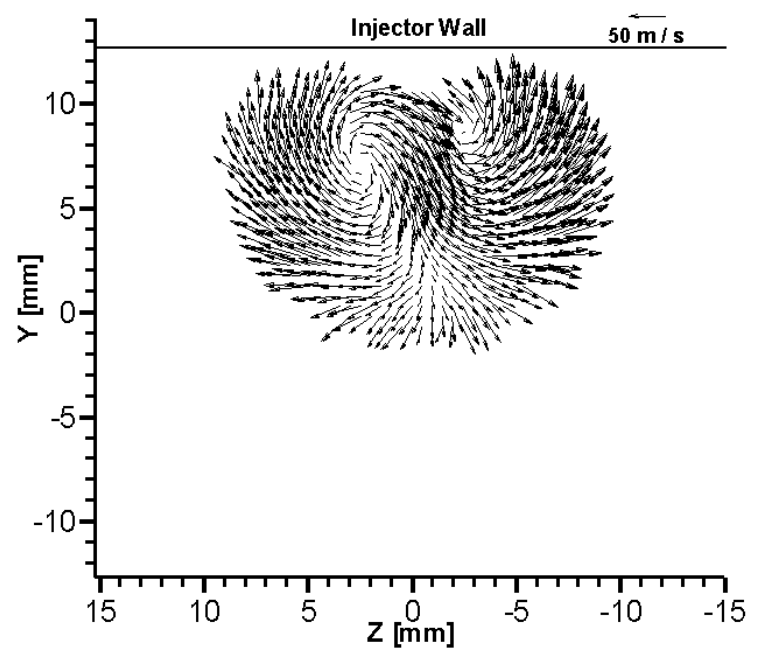

Axial (u) Velocity

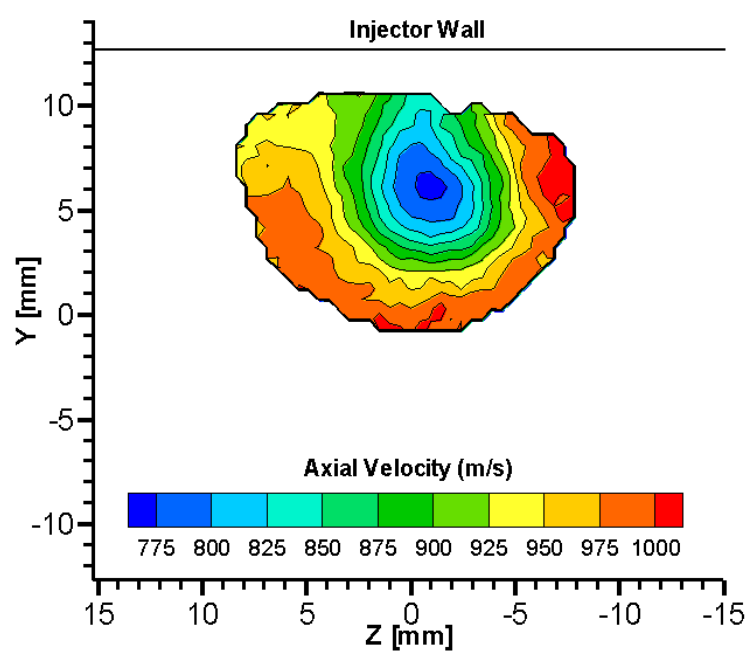

y-component (v) Velocity

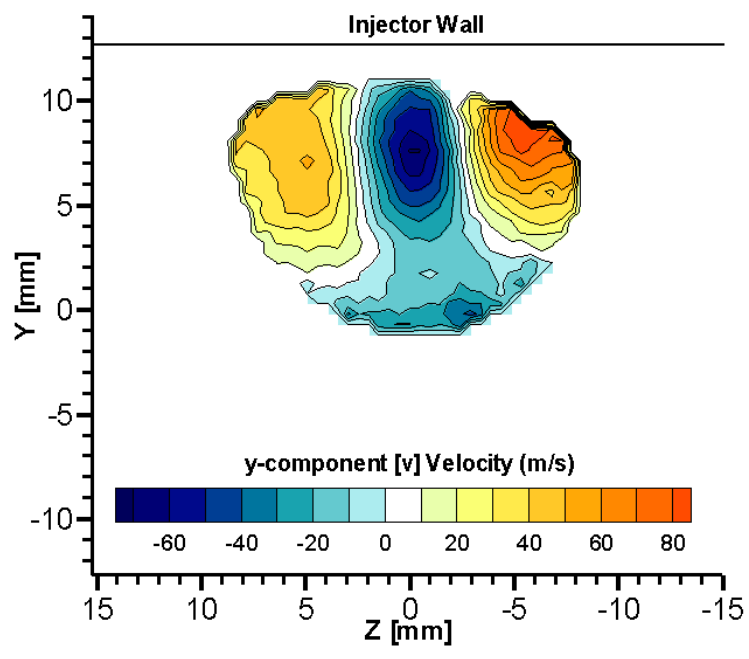

z-component (w) Velocity

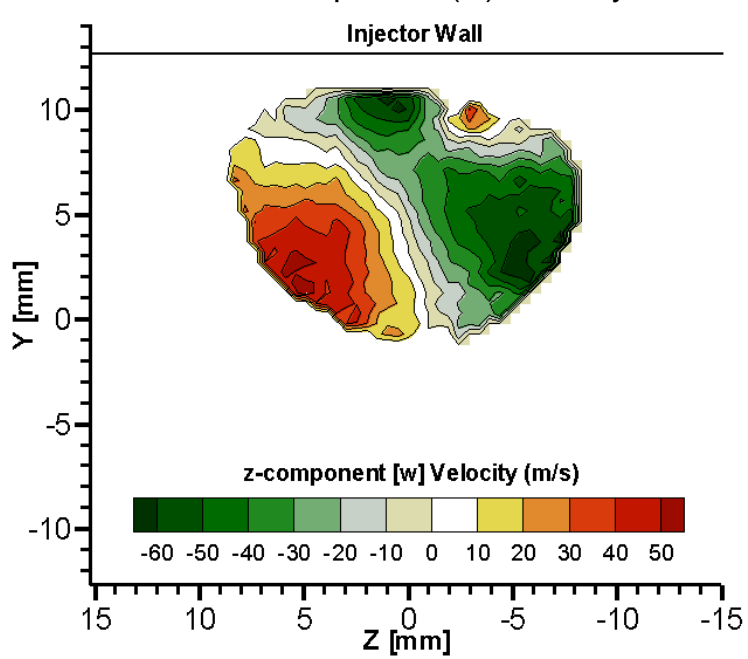

Figure A.3: Average velocity fields for Fuel-air Mixing at $\mathrm{X} / \mathrm{H}=10$ (Average of 708 results) 
3C Velocity Magnitude

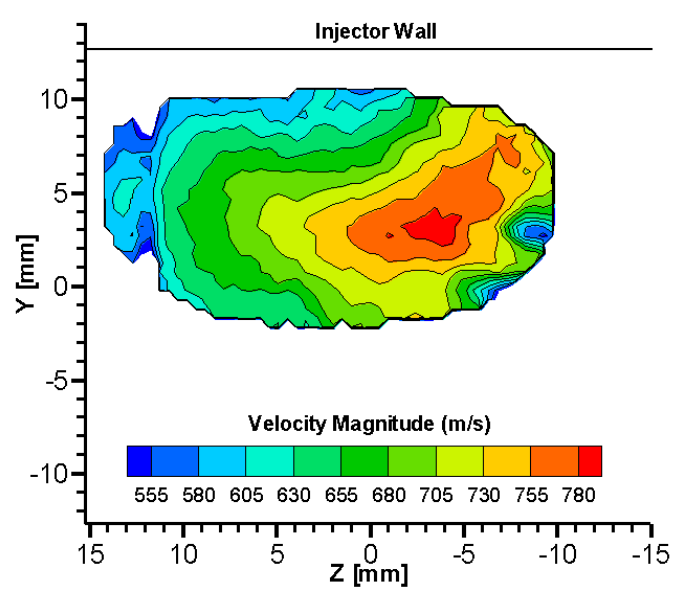

In-plane Velocity

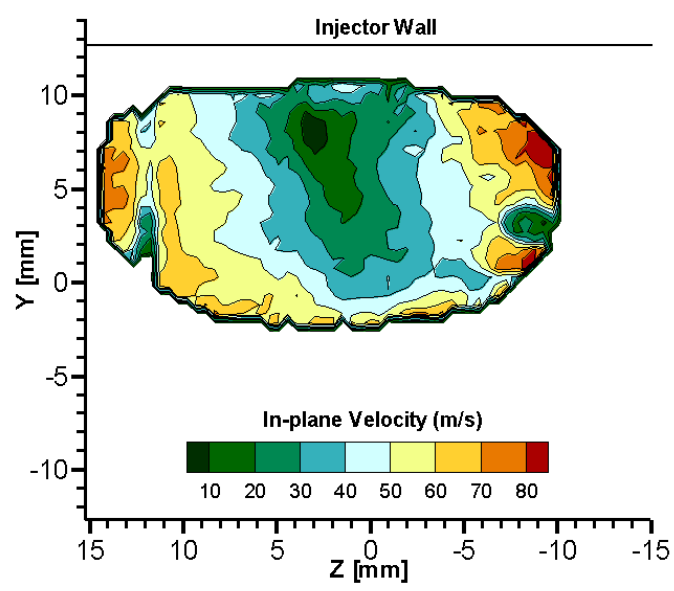

In-plane Velocity Vectors

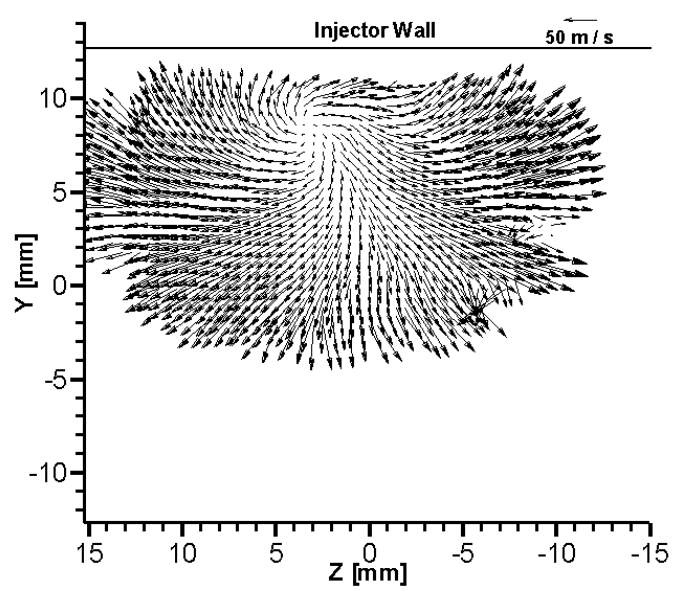

Axial (u) Velocity

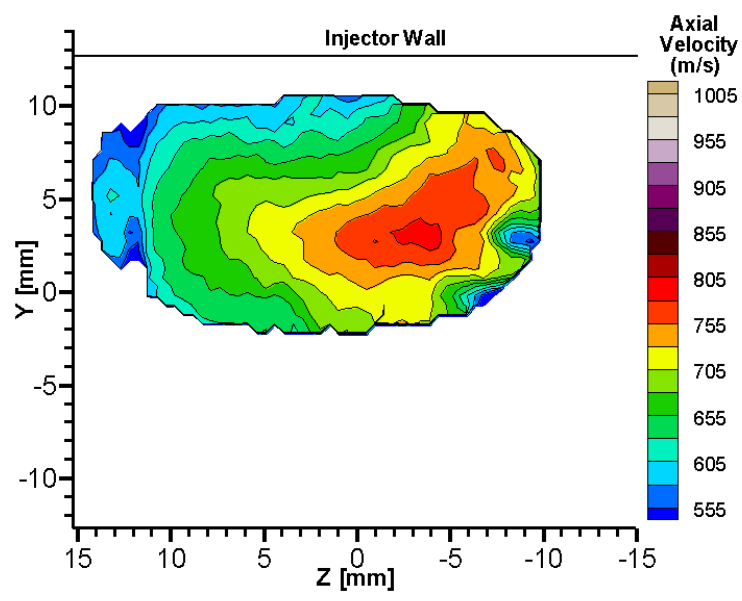

y-component (v) Velocity

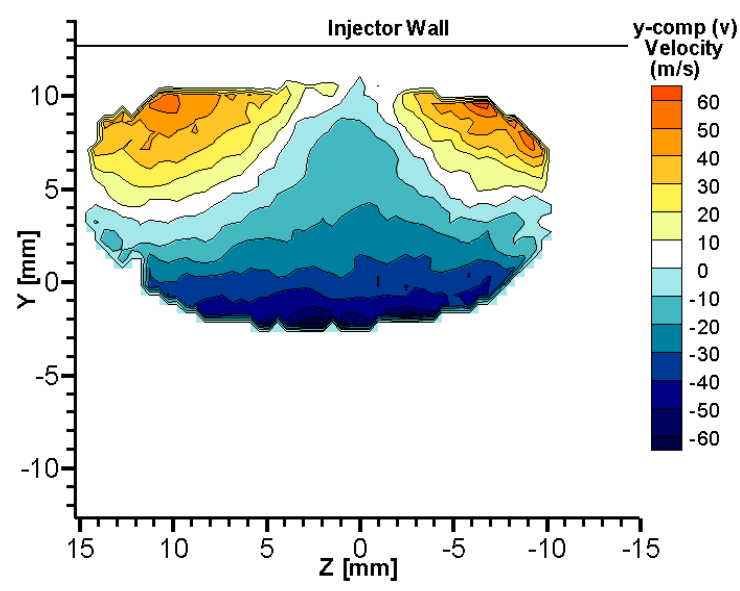

z-component (w) Velocity

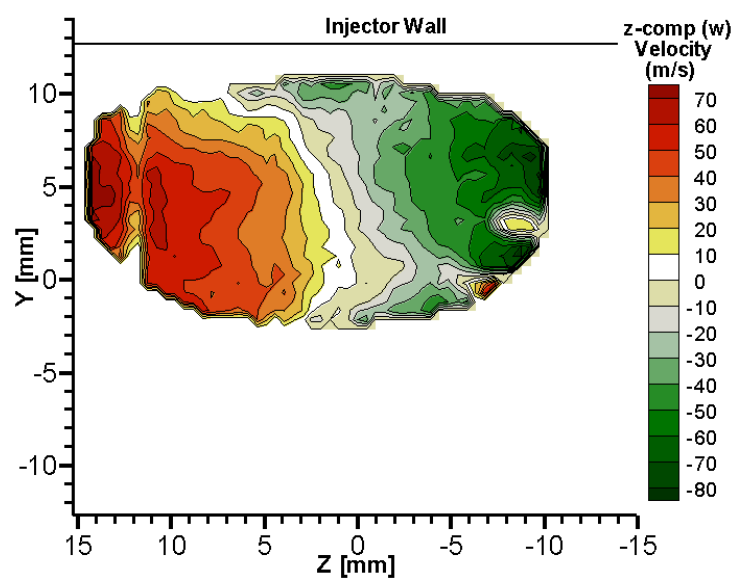

Figure A.4: Average velocity fields for Fuel-air Combustion at $\mathrm{X} / \mathrm{H}=10$ (Average of 775 results) 


\section{Appendix B \\ Supplemental Figures for Flowpath 1, Plane at $\mathrm{X} / \mathrm{H}=\mathbf{5 8 . 6}($ Exit plane $)$ for $\Phi=0.17$}

\section{List of figures}

Fuel-air Mixing

Figure B.1: $3 \mathrm{D}$ plot of Average 3C velocity for Fuel-air Mixing at $\mathrm{X} / \mathrm{H}=58.6$;

$3 \mathrm{D}$ view of velocity vectors and $3 \mathrm{D}$ surface representation of velocity

Figure B.2: Average $3 \mathrm{C}$ velocity and vector count for Fuel-air Mixing case at $\mathrm{X} / \mathrm{H}=58.6$ (Exit Plane) for $\Phi=0.17$ (Average of 992 instantaneous fields)

Figure B.3: Average and instantaneous velocity fields: Fuel-air Mixing case at $\mathrm{X} / \mathrm{H}=58.6$ (Exit Plane) for $\Phi=0.17$

Figure B.4: Statistical convergence of 3C velocity: Fuel-air Mixing case at $\mathrm{X} / \mathrm{H}=58.6$ (Exit Plane) for $\Phi=0.17$

Figure B.5: Summary of Average fields for Fuel-air Mixing at $\mathrm{X} / \mathrm{H}=58.6, \Phi=0.17$; $3 \mathrm{C}$ magnitude, In-plane magnitude, In-plane vectors; and $\mathrm{u}, \mathrm{v}$ and $\mathrm{w}$ velocity components (Average of 992 instantaneous fields)

\section{Fuel-air Combustion}

Figure B.6: Statistical convergence of 3C velocity: Fuel-air Combustion case at $\mathrm{X} / \mathrm{H}=58.6$ (Exit Plane) for $\Phi=0.17$

Figure B.7: Summary of Average fields, Fuel-air Combustion at $\mathrm{X} / \mathrm{H}=58.6, \Phi=0.17$; $3 \mathrm{C}$ magnitude, In-plane magnitude, In-plane vectors; and $\mathrm{u}, \mathrm{v}$ and $\mathrm{w}$ velocity components (Average of 1000 instantaneous fields) 
a)

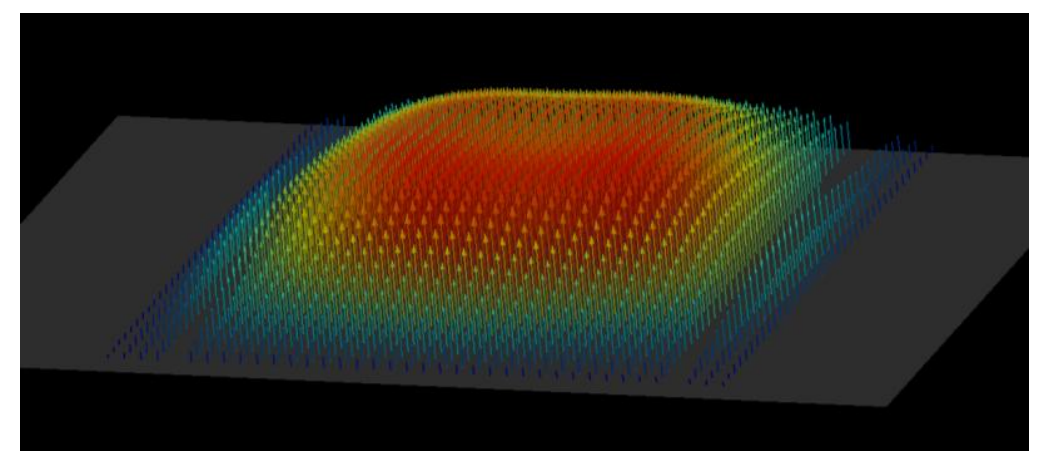
Velocity
Magnitude $(\mathrm{m} / \mathrm{s})$

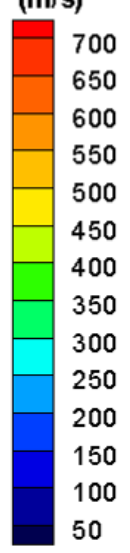

b)

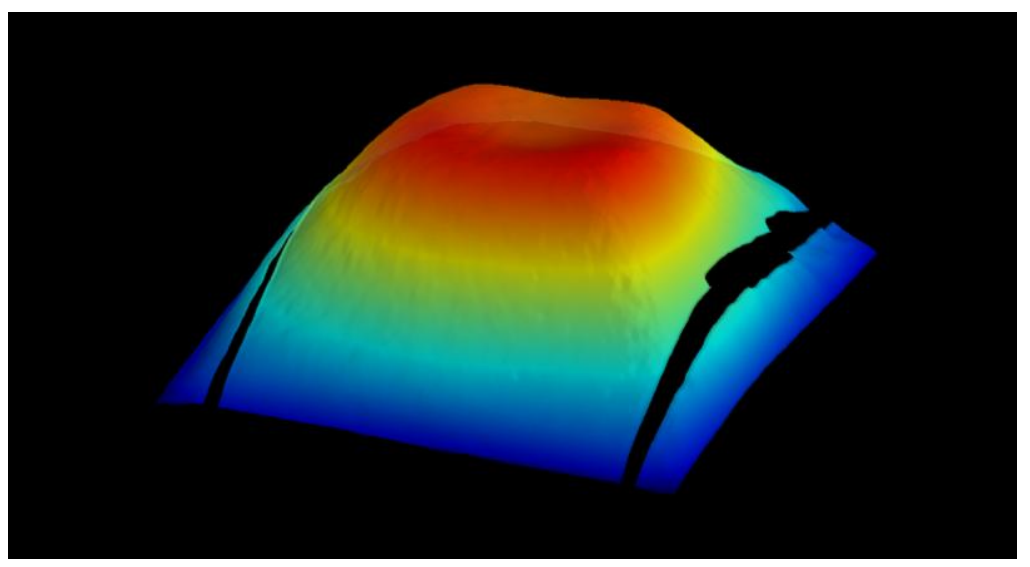

Figure B.1: Average three-component (3C) velocity result from SPIV measurements for Fuel-air Mixing case $(\Phi=0.17)$ at $\mathrm{X} / \mathrm{H}=58.6$ a) Vector representation, and b) Surface representation.

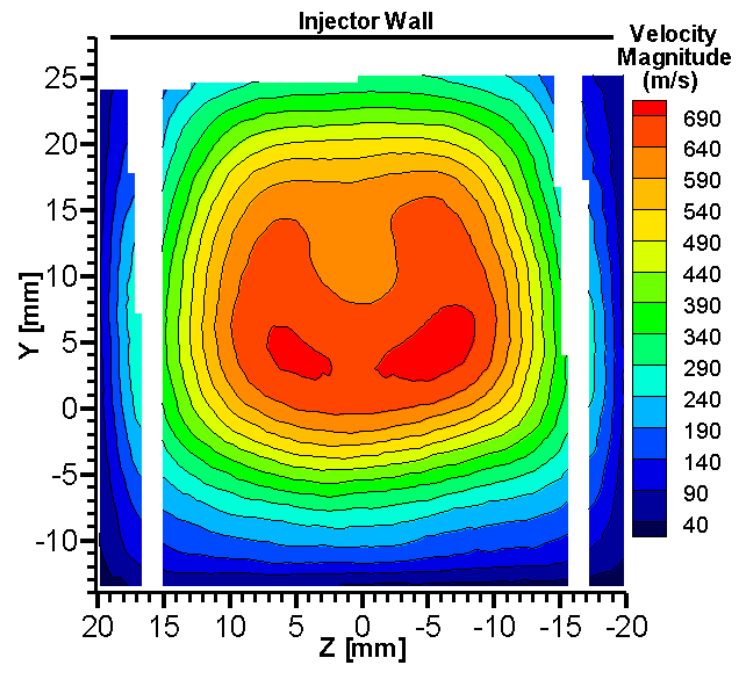

(a) Average of All (992) Vector Fields [3C Velocity magnitude]

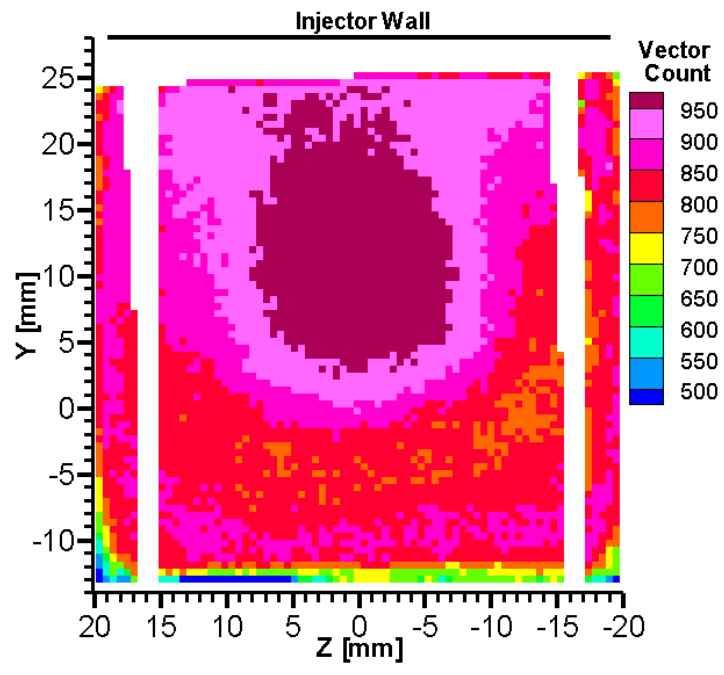

(b) Number of Vectors Averaged for Each Sub-region

Figure B.2: $\Phi=0.17$, Fuel-air Mixing case at $\mathrm{X} / \mathrm{H}=58.6$ (Exit plane);

(a) Average $3 \mathrm{C}$ velocity field, and (b) Number of vectors averaged at each location 
Average In-plane Velocity Vectors

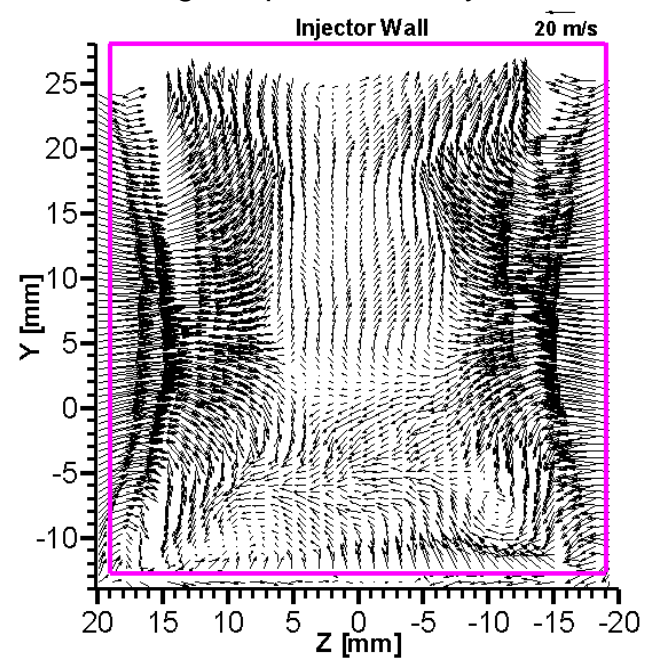

Instantaneous In-plane Velocity Vectors

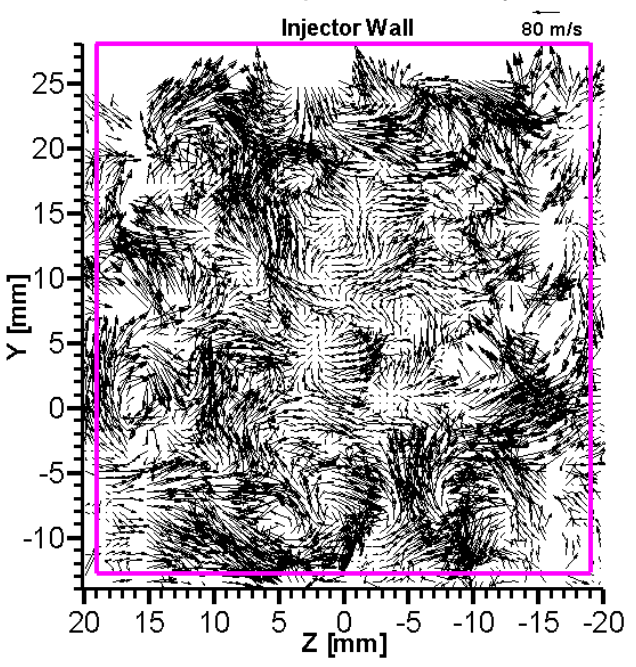

Instantaneous In-plane Velocity Vectors

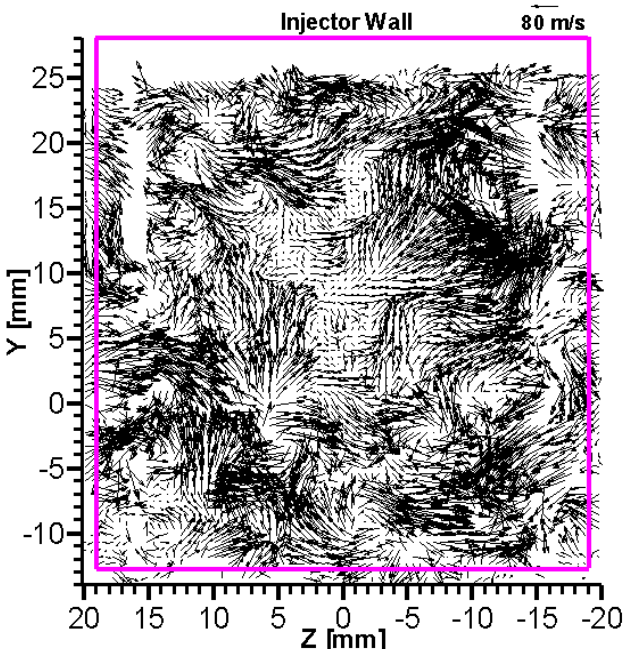

Average $3 C$ Velocity Field

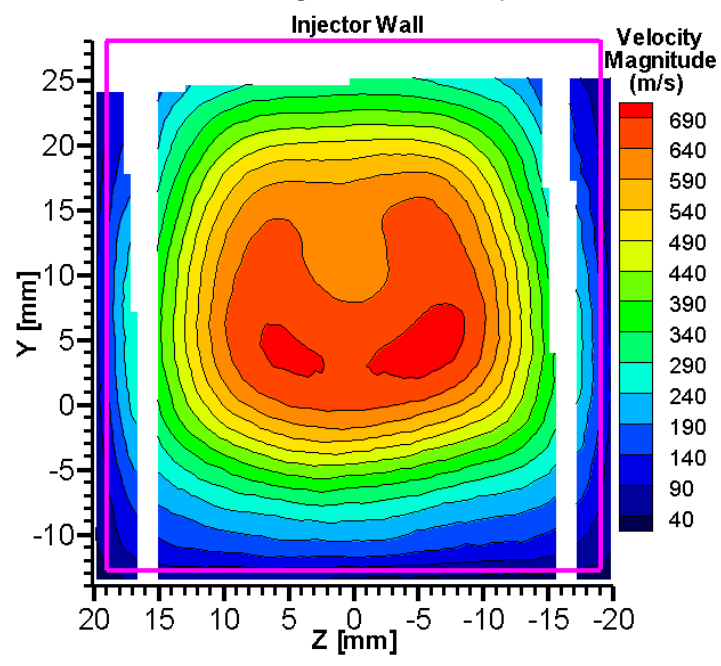

Instantaneous 3C Velocity

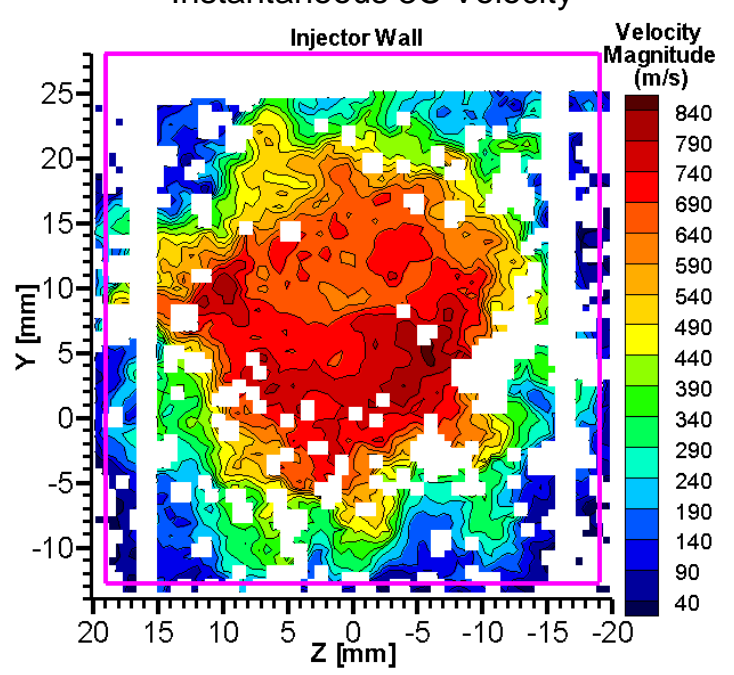

Instantaneous 3C Velocity

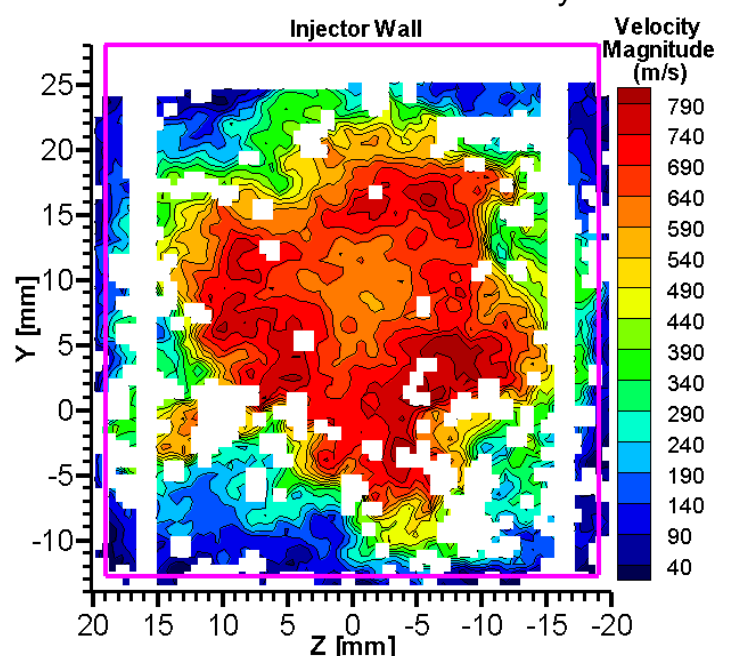

Figure B.3: Average and Instantaneous velocity fields, Fuel-air Mixing case at $\mathrm{X} / \mathrm{H}=58.6, \Phi=0.17$ 

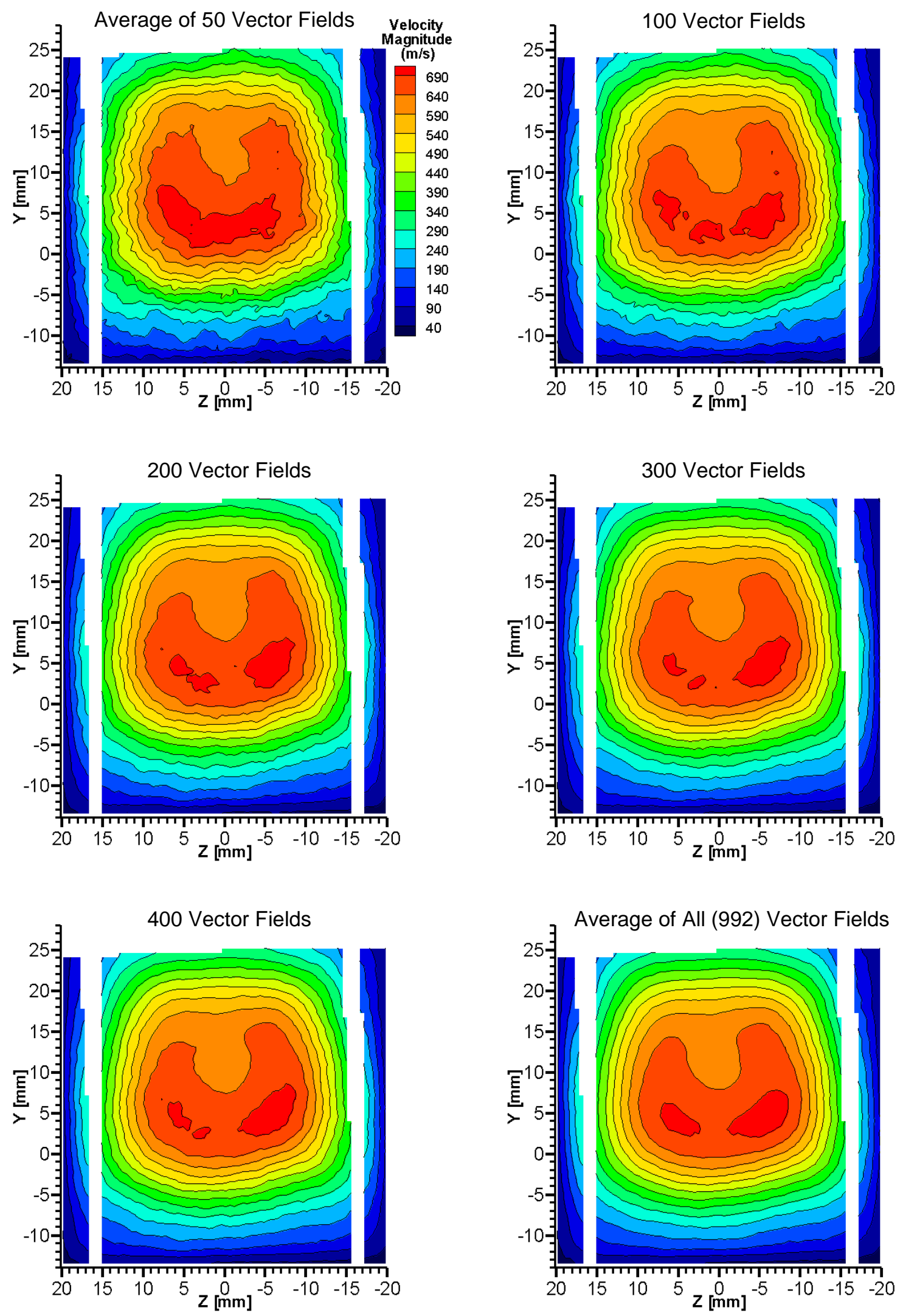

Figure B.4: Statistical convergence of $3 \mathrm{C}$ velocity, Fuel-air $\underline{\text { Mixing, }} \mathrm{X} / \mathrm{H}=58.6, \Phi=0.17$ 

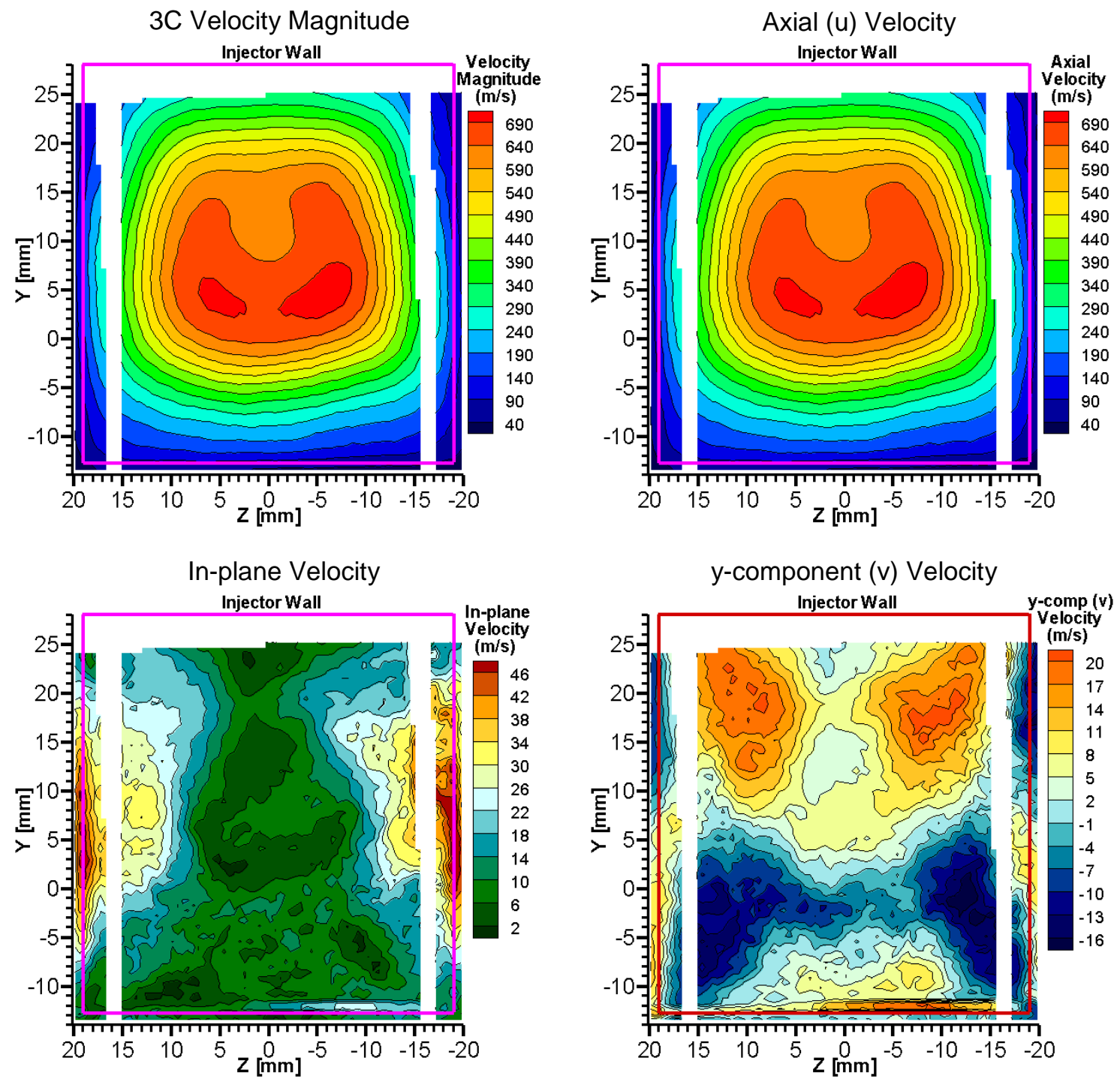

In-plane Velocity Vectors
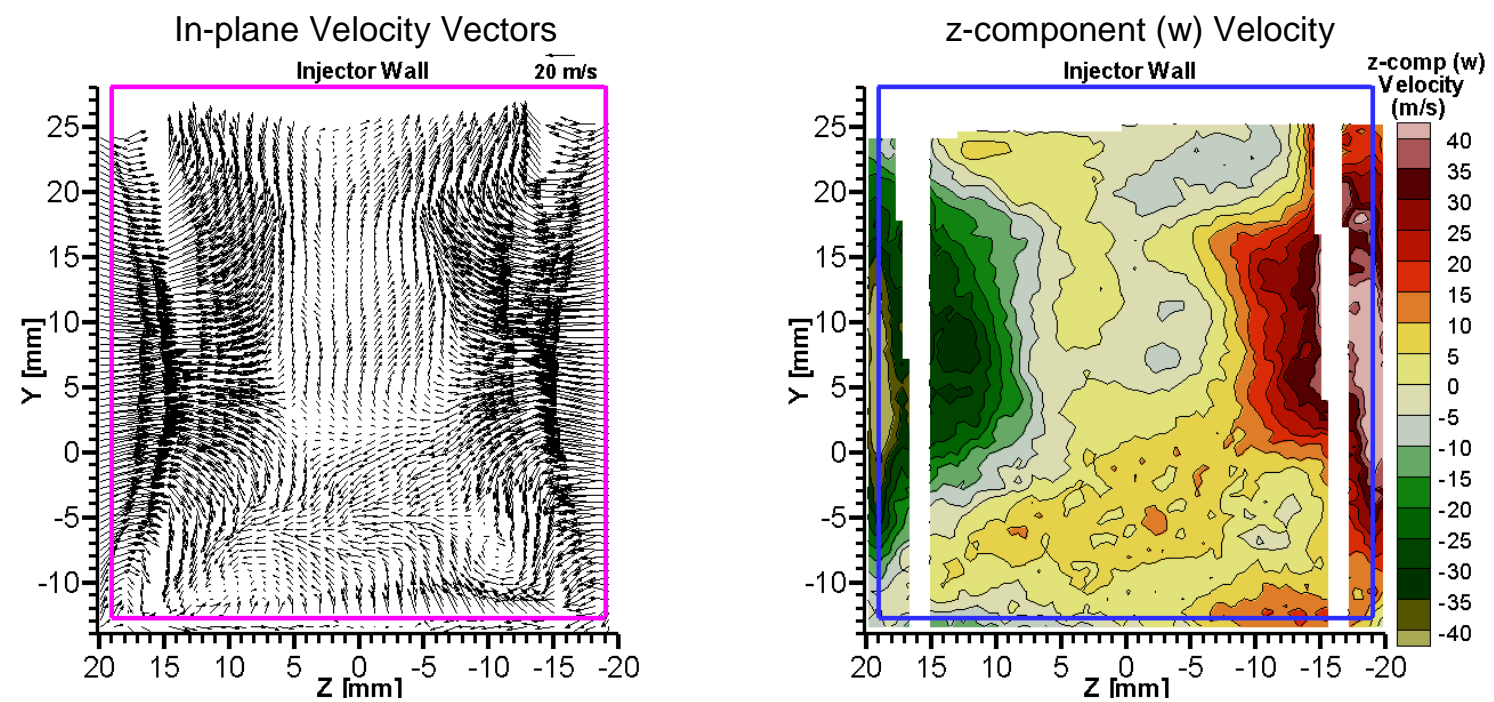

Figure B.5: Average velocity fields for $\Phi=0.17$ Fuel-air Mixing at $\mathrm{X} / \mathrm{H}=58.6$ (Avg. of 992 results) 

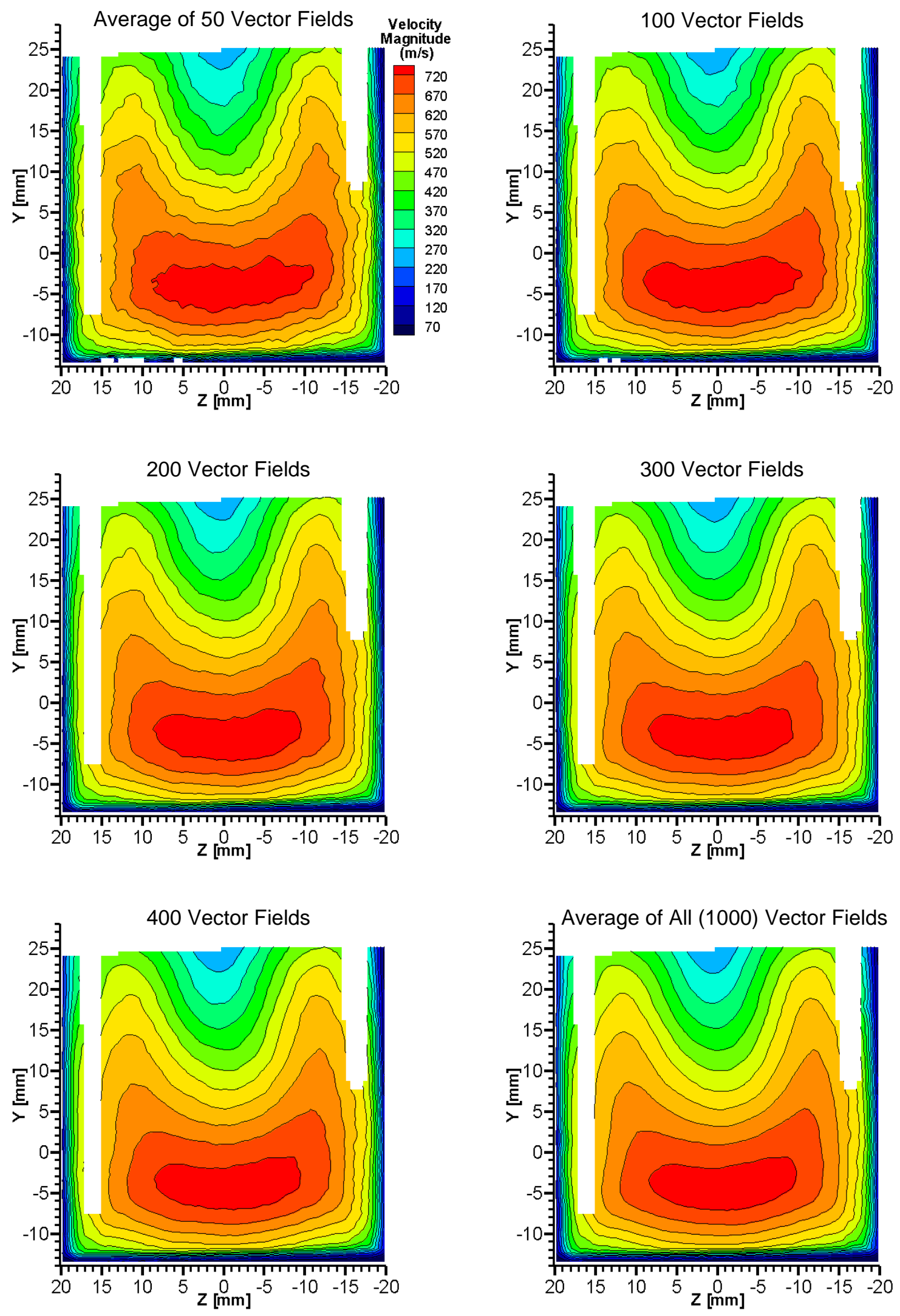

Figure B.6: Statistical convergence of $3 \mathrm{C}$ velocity, Fuel-air $\underline{\text { Combustion, }} \mathrm{X} / \mathrm{H}=58.6, \Phi=0.17$ 

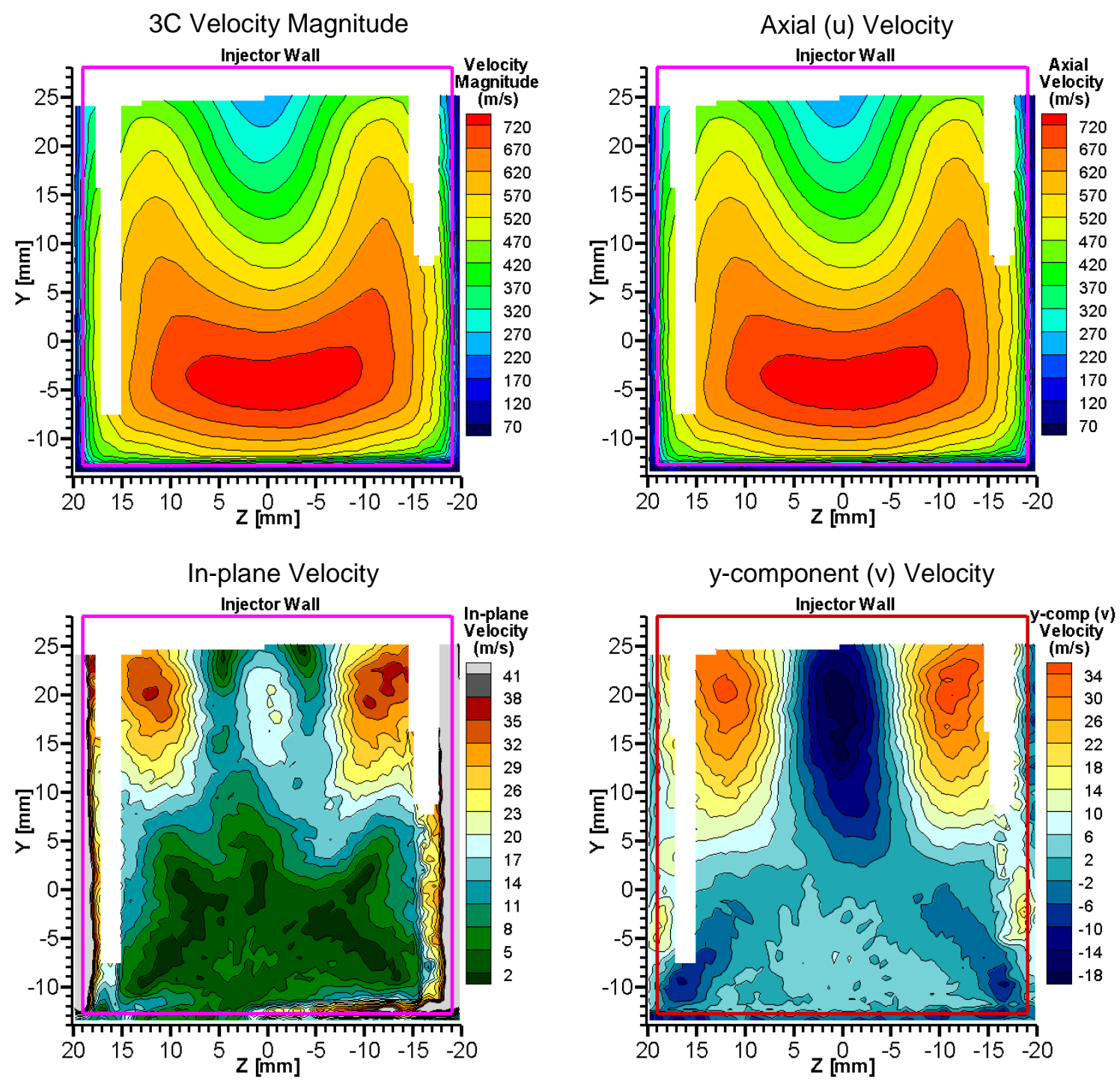

In-plane Velocity Vectors
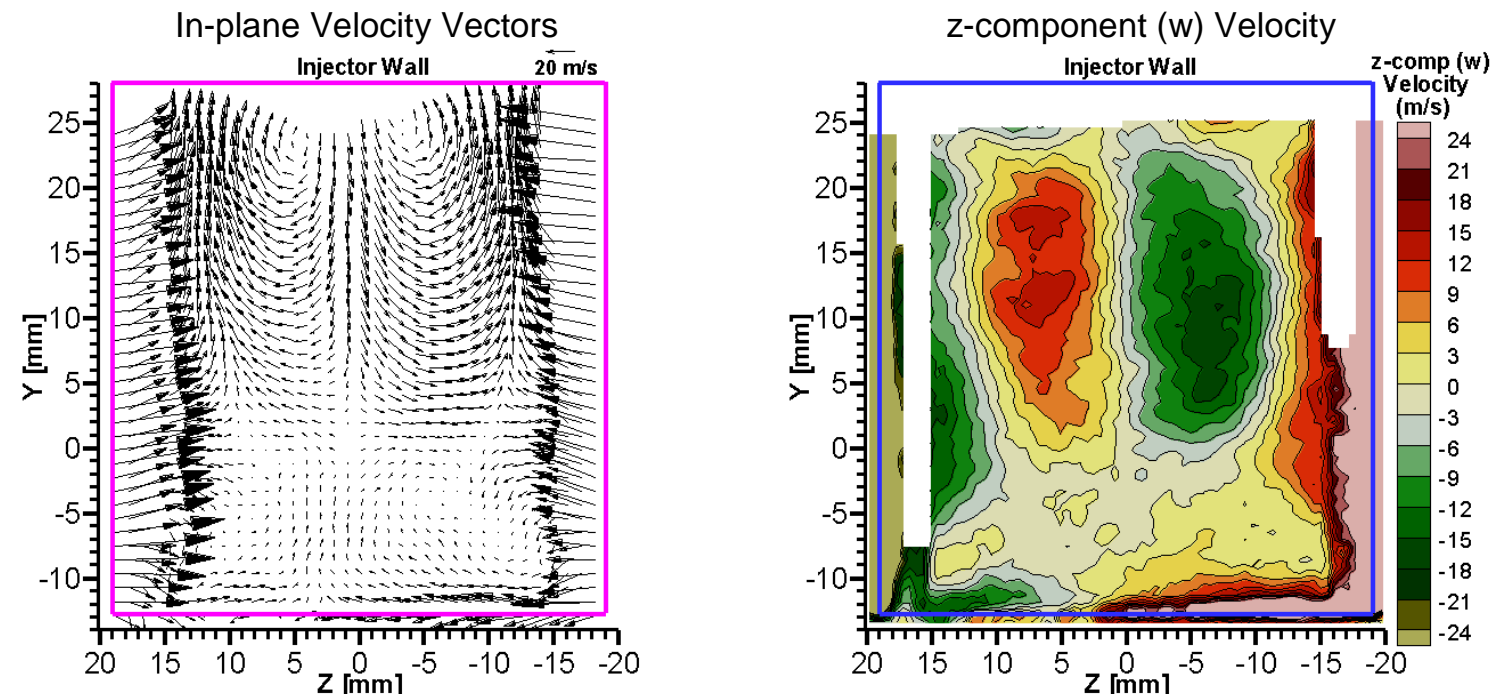

Figure B.7: Average velocity for $\Phi=0.17$ Fuel-air Combustion at $\mathrm{X} / \mathrm{H}=58.6$ (Avg. of 1000 results) 


\section{Appendix C \\ Supplemental Figures for Flowpath 1, \\ Plane at $\mathrm{X} / \mathrm{H}=\mathbf{5 8 . 6}($ Exit plane) for $\Phi=\mathbf{0 . 3 4}$}

\section{List of figures}

Fuel-air Mixing

Figure C.1: $3 \mathrm{D}$ plot of Average 3C velocity for Fuel-air Mixing at $\mathrm{X} / \mathrm{H}=58.6, \Phi=0.34$ $3 \mathrm{D}$ view of velocity vectors and $3 \mathrm{D}$ surface representation of velocity

Figure C.2: Average $3 \mathrm{C}$ velocity and vector count for Fuel-air Mixing case at $\mathrm{X} / \mathrm{H}=58.6$ (Exit Plane) for $\Phi=0.34$ (Average of 988 instantaneous fields)

Figure C.3: Average and instantaneous velocity fields: Fuel-air Mixing case at $\mathrm{X} / \mathrm{H}=58.6$ (Exit Plane) for $\Phi=0.34$

Figure C.4: Statistical convergence of 3C velocity: Fuel-air Mixing case at $\mathrm{X} / \mathrm{H}=58.6$ (Exit Plane) for $\Phi=0.34$

Figure C.5: Summary of Average fields for Fuel-air Mixing at $\mathrm{X} / \mathrm{H}=58.6, \Phi=0.34$; $3 \mathrm{C}$ magnitude, In-plane magnitude, In-plane vectors; and $\mathrm{u}, \mathrm{v}$ and $\mathrm{w}$ velocity components (Average of 988 instantaneous fields)

\section{Fuel-air Combustion}

Figure C.6: Statistical convergence of 3C velocity: Fuel-air Combustion case at $\mathrm{X} / \mathrm{H}=58.6$ (Exit Plane) for $\Phi=0.34$

Figure C.7: Summary of Average fields, Fuel-air Combustion at $\mathrm{X} / \mathrm{H}=58.6, \Phi=0.34$; $3 \mathrm{C}$ magnitude, In-plane magnitude, In-plane vectors; and $\mathrm{u}, \mathrm{v}$ and $\mathrm{w}$ velocity components (Average of 979 instantaneous fields) 
a)
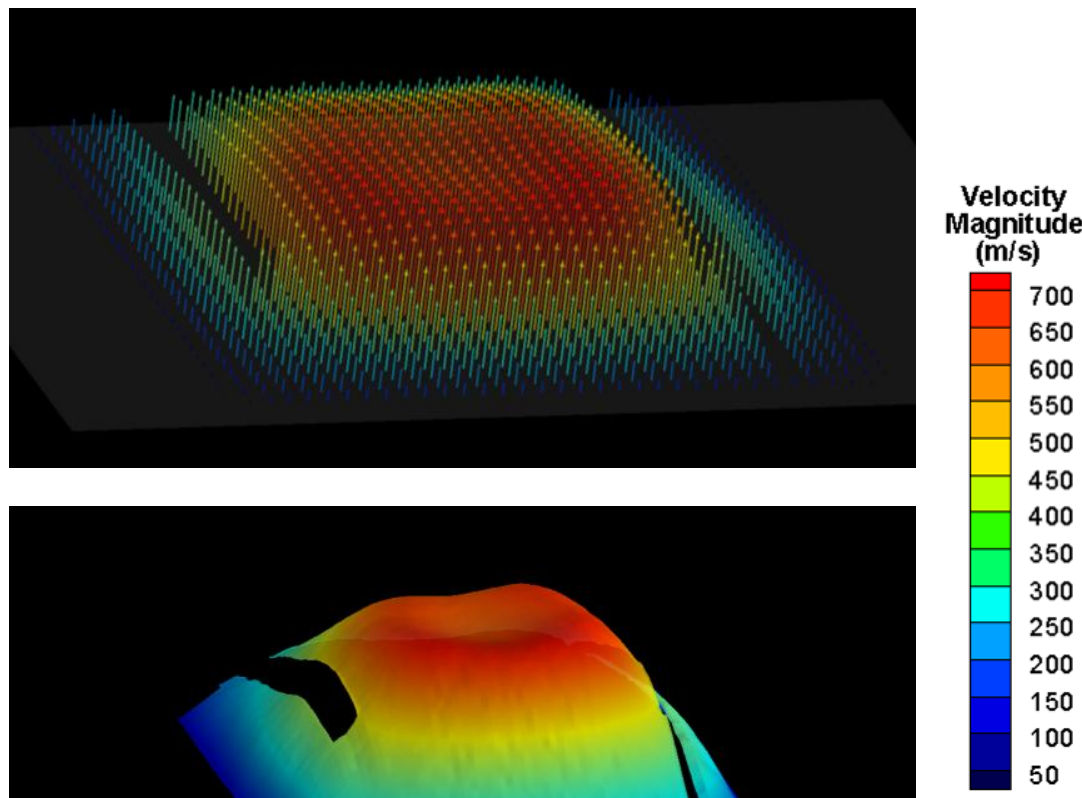

b)

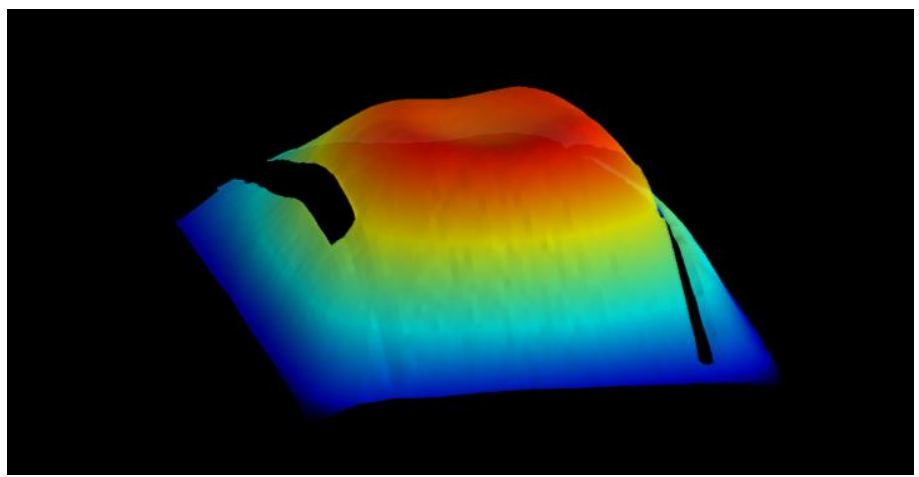

Figure C.1: Average three-component (3C) velocity result from SPIV measurements for Fuel-air Mixing case $(\Phi=0.34)$ at $\mathrm{X} / \mathrm{H}=58.6$ a) Vector representation, and b) Surface representation.

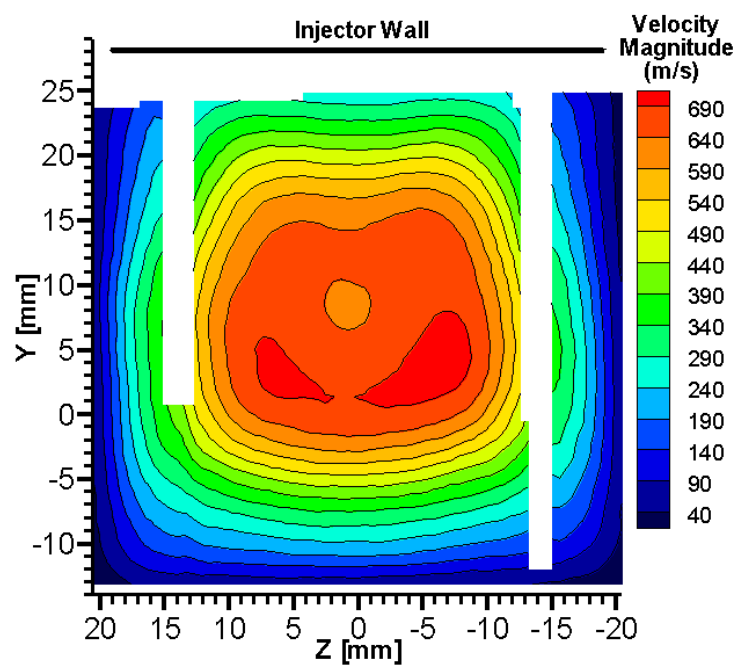

(a) Average of All (988) Vector Fields [3C Velocity magnitude]

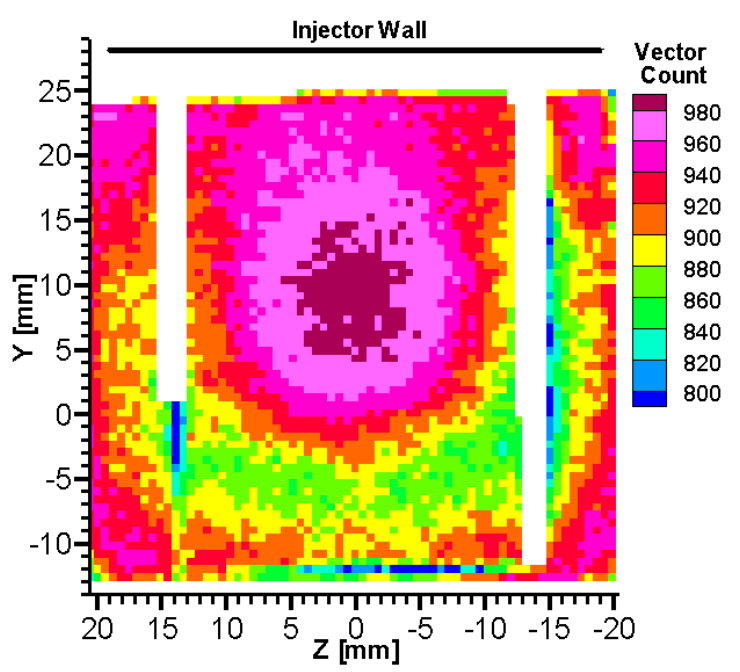

(b) Number of Vectors Averaged for Each Sub-region

Figure C.2: $\Phi=0.34$, Fuel-air Mixing case at $\mathrm{X} / \mathrm{H}=58.6$ (Exit plane);

(a) Average $3 \mathrm{C}$ velocity field, and (b) Number of vectors averaged at each location 
Average In-plane Velocity Vectors

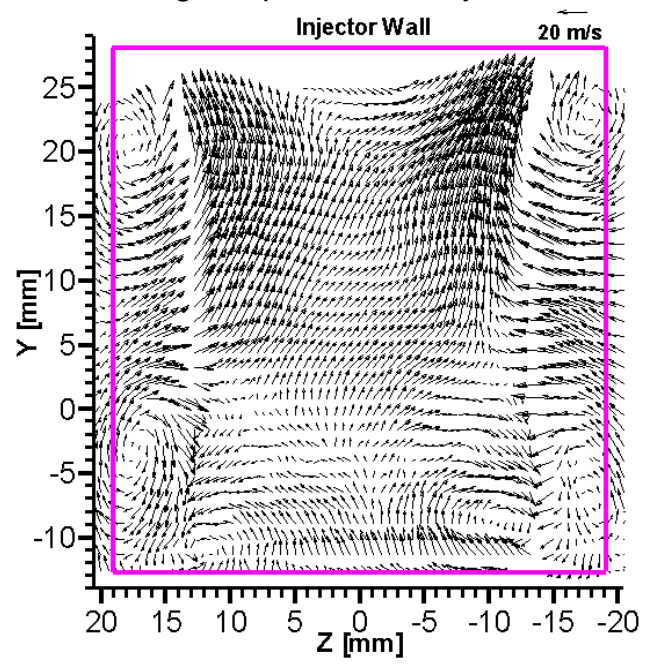

Instantaneous In-plane Velocity Vectors

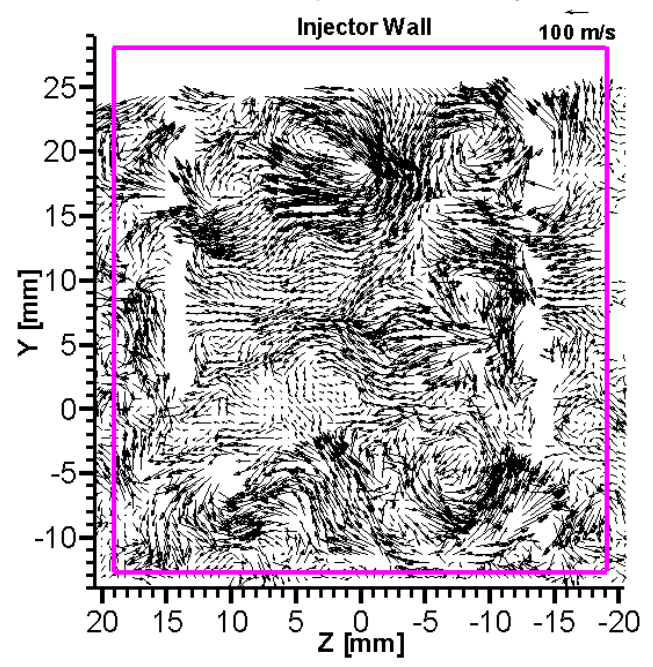

Instantaneous In-plane Velocity Vectors

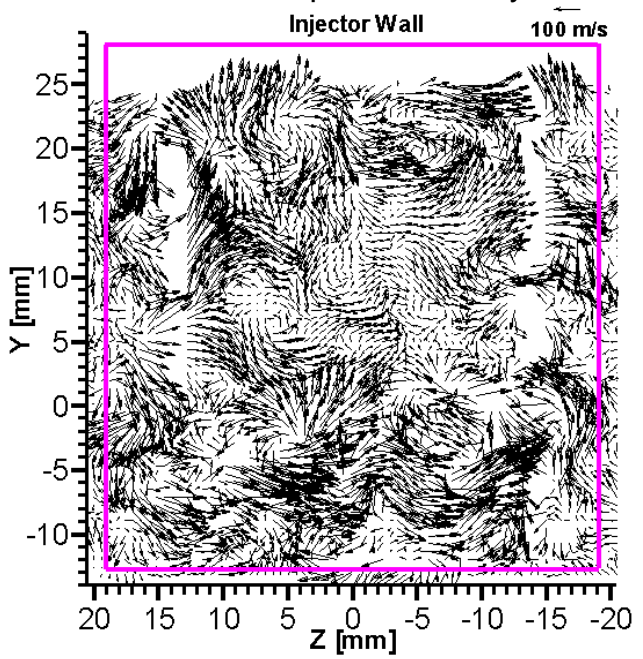

Average $3 \mathrm{C}$ Velocity Field

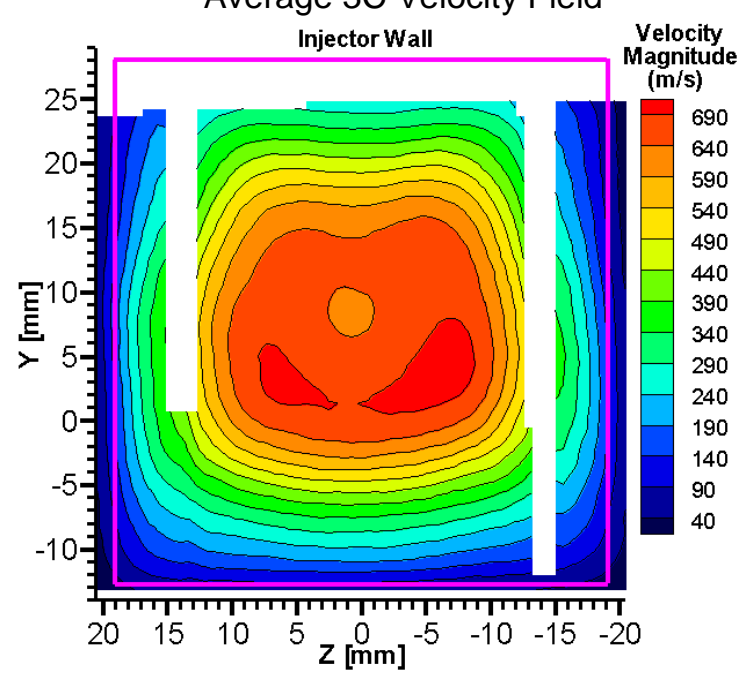

Instantaneous 3C Velocity

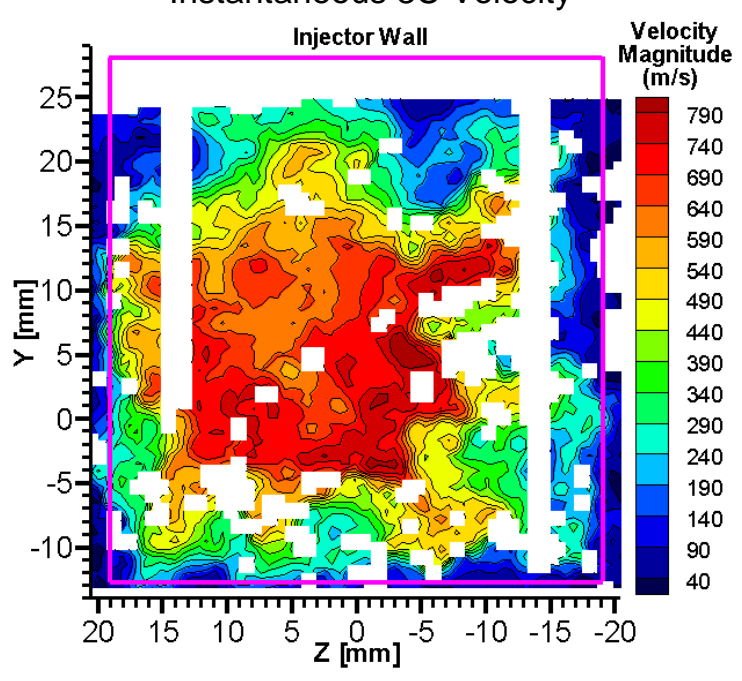

Instantaneous 3C Velocity

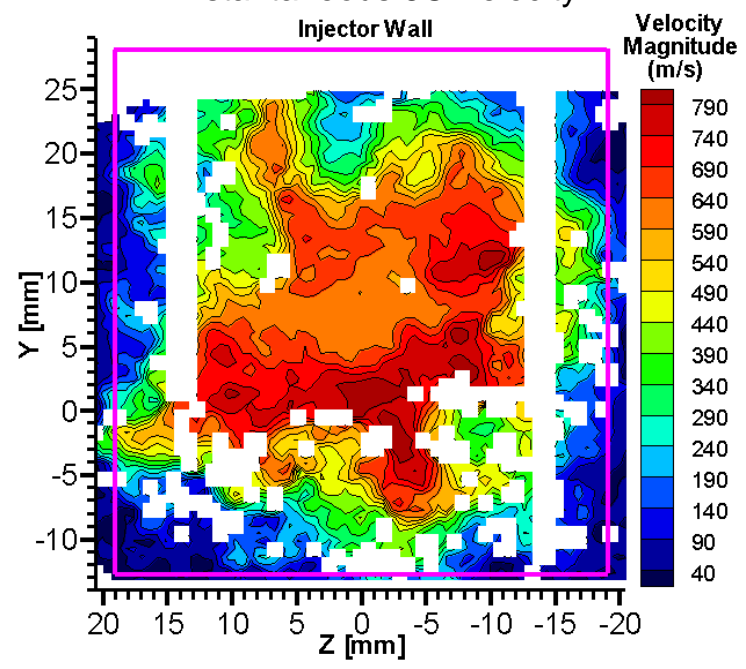

Figure C.3: Average and Instantaneous velocity fields, Fuel-air Mixing at $\mathrm{X} / \mathrm{H}=58.6, \Phi=0.34$ 

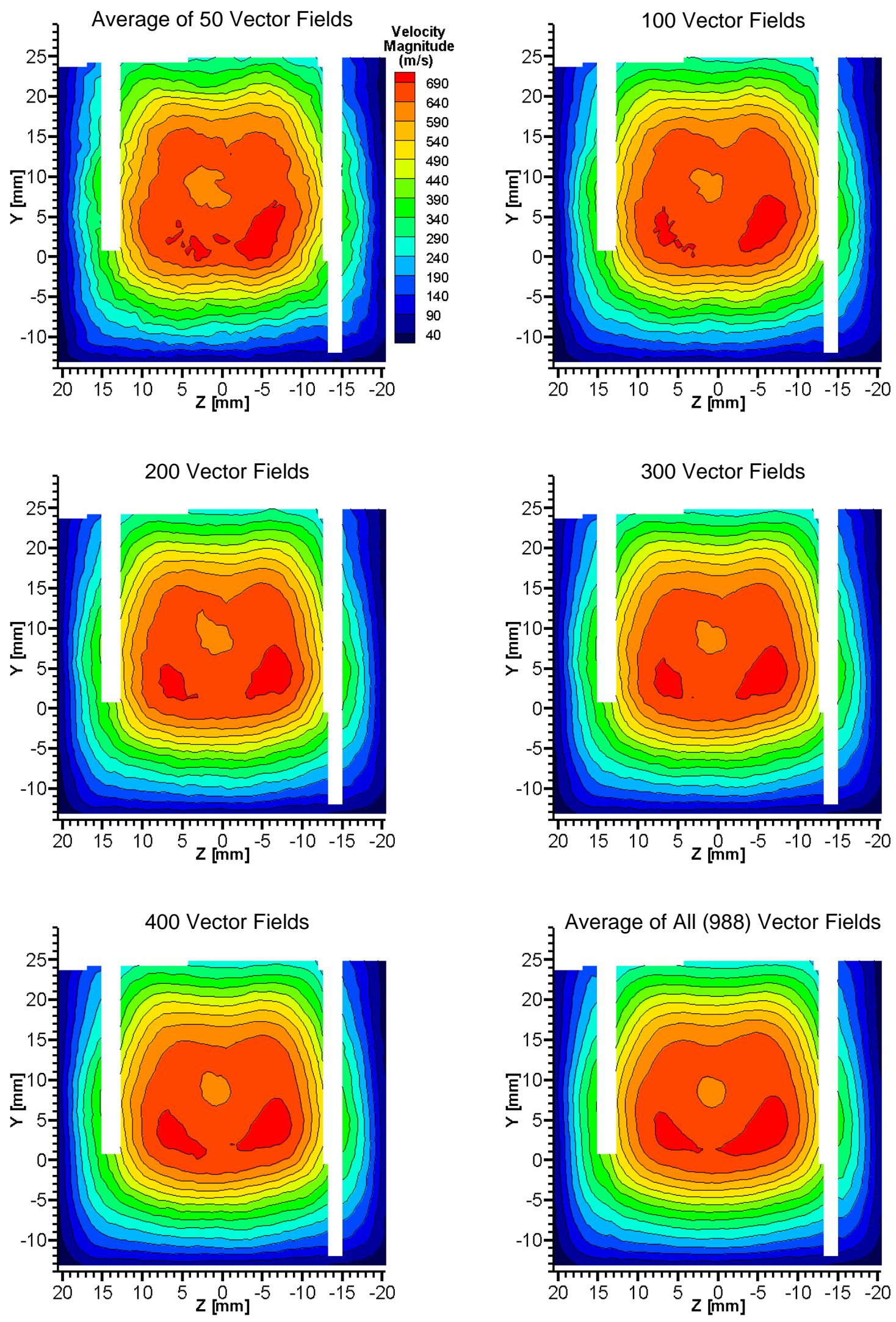

Figure C.4: Statistical convergence of $3 \mathrm{C}$ velocity, Fuel-air Mixing, $\mathrm{X} / \mathrm{H}=58.6, \Phi=0.34$ 

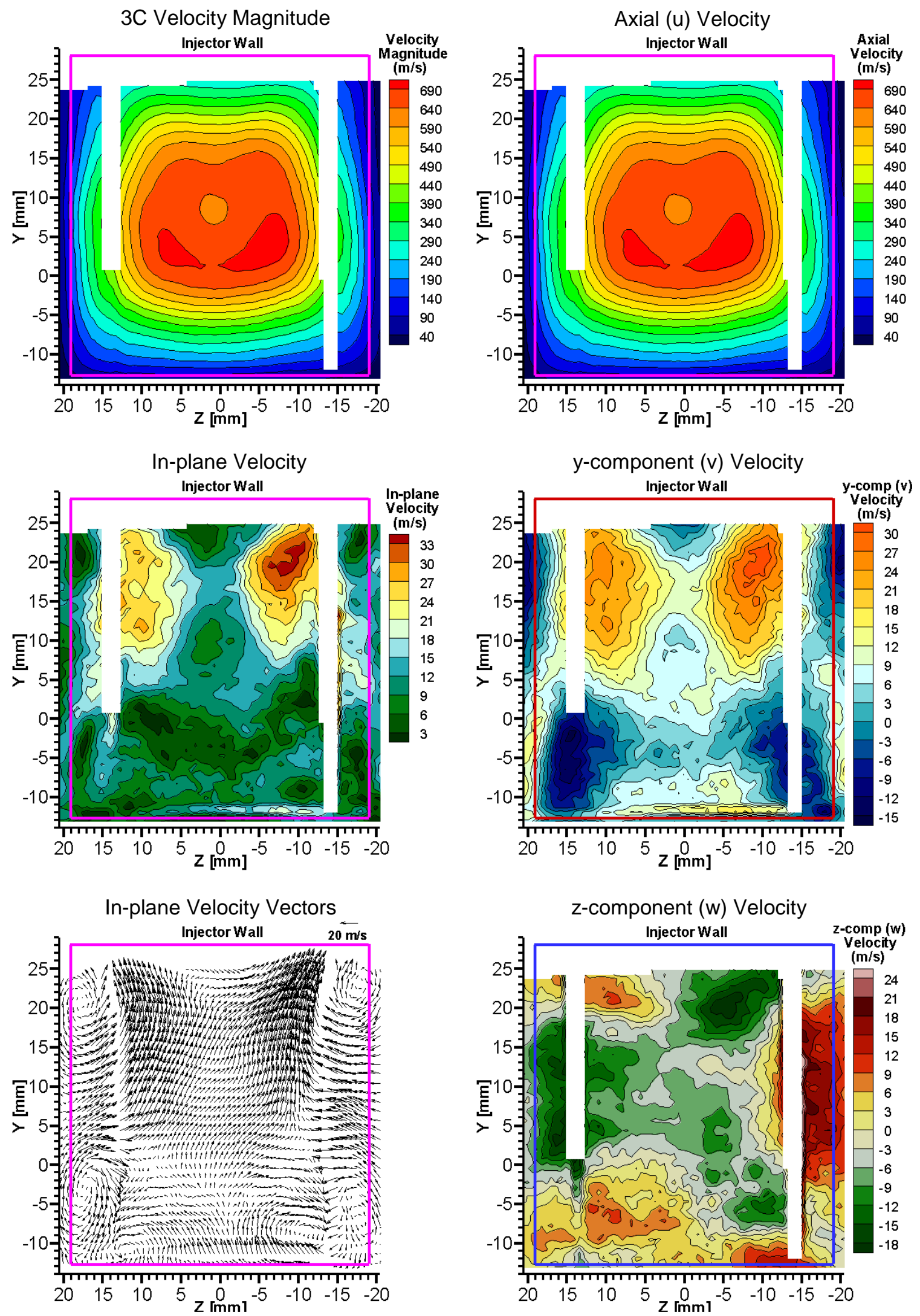

Figure C.5: Average velocity for $\Phi=0.34$ Fuel-air Mixing case at $\mathrm{X} / \mathrm{H}=58.6$ (Avg. of 988 results) 

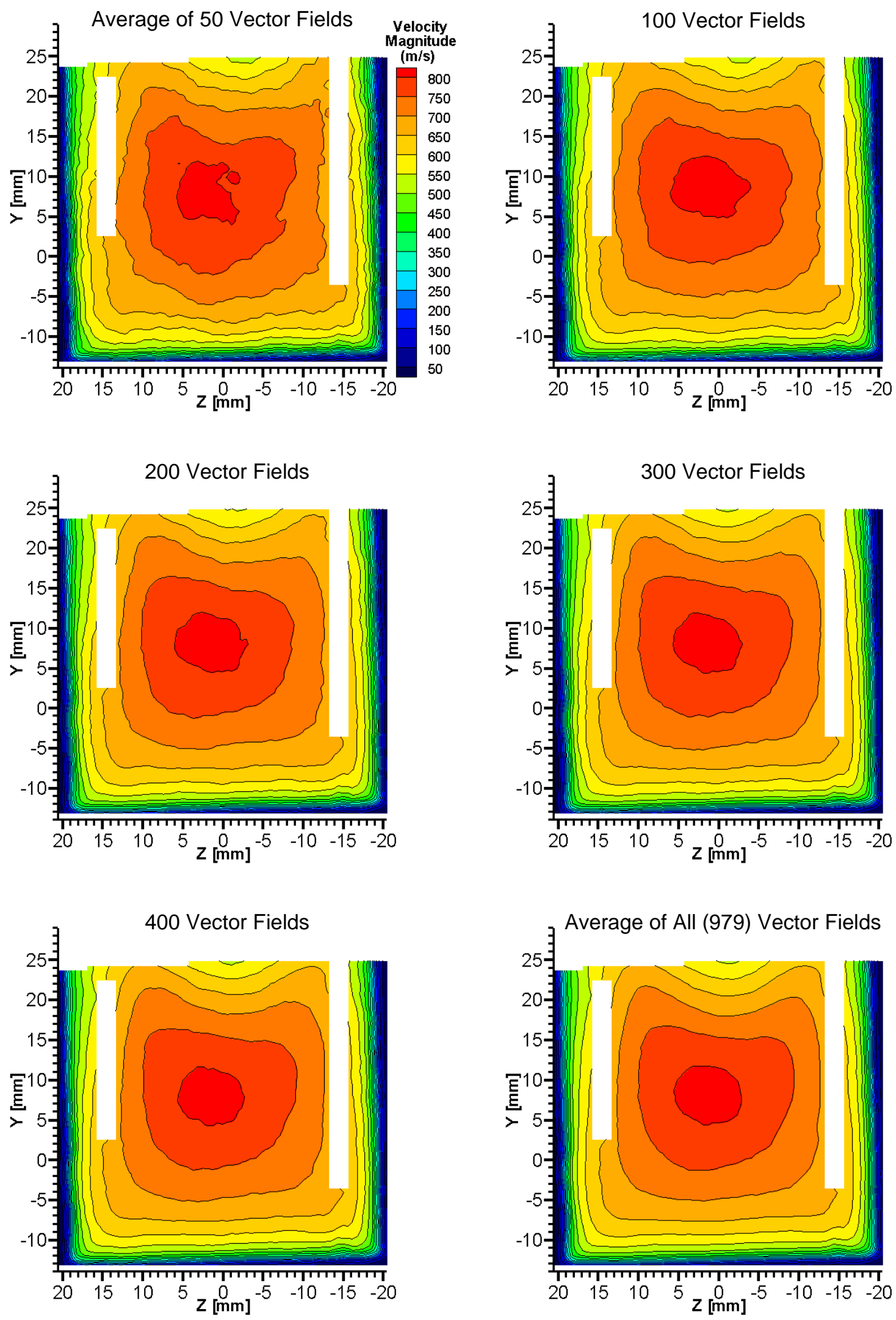

Figure C.6: Statistical convergence of $3 \mathrm{C}$ velocity, Fuel-air $\underline{\text { Combustion, }} \mathrm{X} / \mathrm{H}=58.6, \Phi=0.34$ 

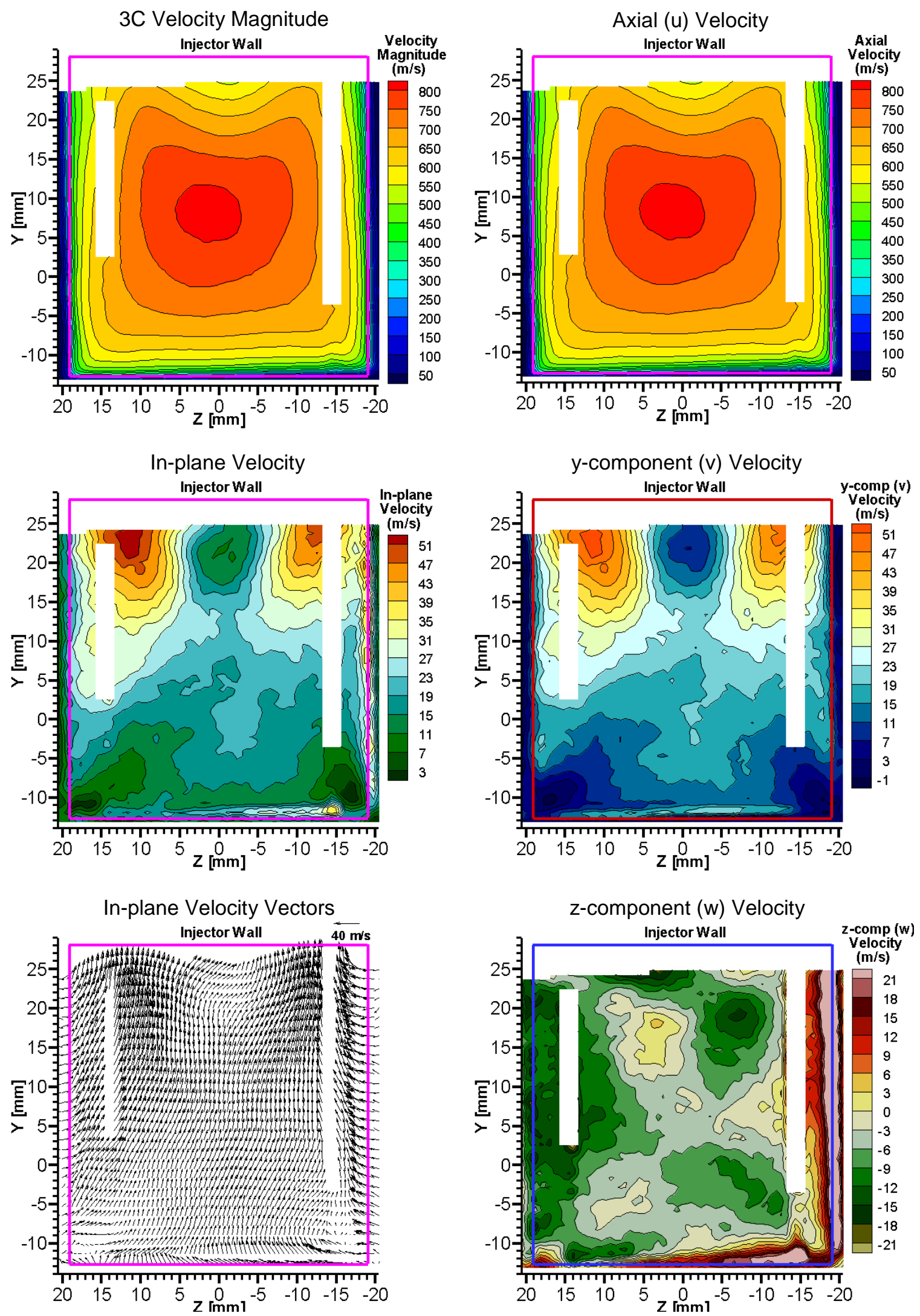

Figure C.7: Average velocity for $\Phi=0.34$ Fuel-air Combustion at $\mathrm{X} / \mathrm{H}=58.6$ (Avg. of 979 results) 


\section{Appendix D \\ Supplemental Figures for Flowpath 2, Plane at X/H = 12}

\section{List of figures}

Fuel-air Mixing

Figure D.1: Average and instantaneous velocity fields: Fuel-air Mixing case at $\mathrm{X} / \mathrm{H}=12$, Flowpath 2, $\Phi=0.17$

Figure D.2: Statistical convergence of 3C velocity: Fuel-air Mixing case at $\mathrm{X} / \mathrm{H}=12$, Flowpath 2, $\Phi=0.17$

Figure D.3: Summary of Average fields for Fuel-air Mixing at $\mathrm{X} / \mathrm{H}=12, \Phi=0.17$;

$3 \mathrm{C}$ magnitude, In-plane magnitude, In-plane vectors; and $\mathrm{u}, \mathrm{v}$ and $\mathrm{w}$ velocity components (Average of 1521 instantaneous fields)

Figure D.4: Comparison of experimental (SPIV) measurements to CFD results (LES/RANS) for Fuel-air Mixing; X/H = 12, Flowpath 2

Fuel-air Combustion

Figure D.5: $3 \mathrm{D}$ plot of Average $3 \mathrm{C}$ velocity for Fuel-air Combustion at $\mathrm{X} / \mathrm{H}=12$, Flowpath 2, $\Phi=0.17$; $3 \mathrm{D}$ view of velocity vectors

Figure D.6: Average 3C velocity and vector count for Fuel-air Combustion case at $\mathrm{X} / \mathrm{H}=12$, Flowpath 2, $\Phi=0.17$ (Average of 1162 instantaneous fields)

Figure D.7: Statistical convergence of 3C velocity: Fuel-air Combustion case at $\mathrm{X} / \mathrm{H}=12$, Flowpath 2, $\Phi=0.17$

Figure D.8: Summary of Average fields for Fuel-air Combustion at $\mathrm{X} / \mathrm{H}=12$; 3C magnitude, In-plane magnitude, In-plane vectors; and $\mathrm{u}, \mathrm{v}$ and $\mathrm{w}$ velocity components (Average of 1162 instantaneous fields) 
Average In-plane Velocity Vectors

Average 3C Velocity Field
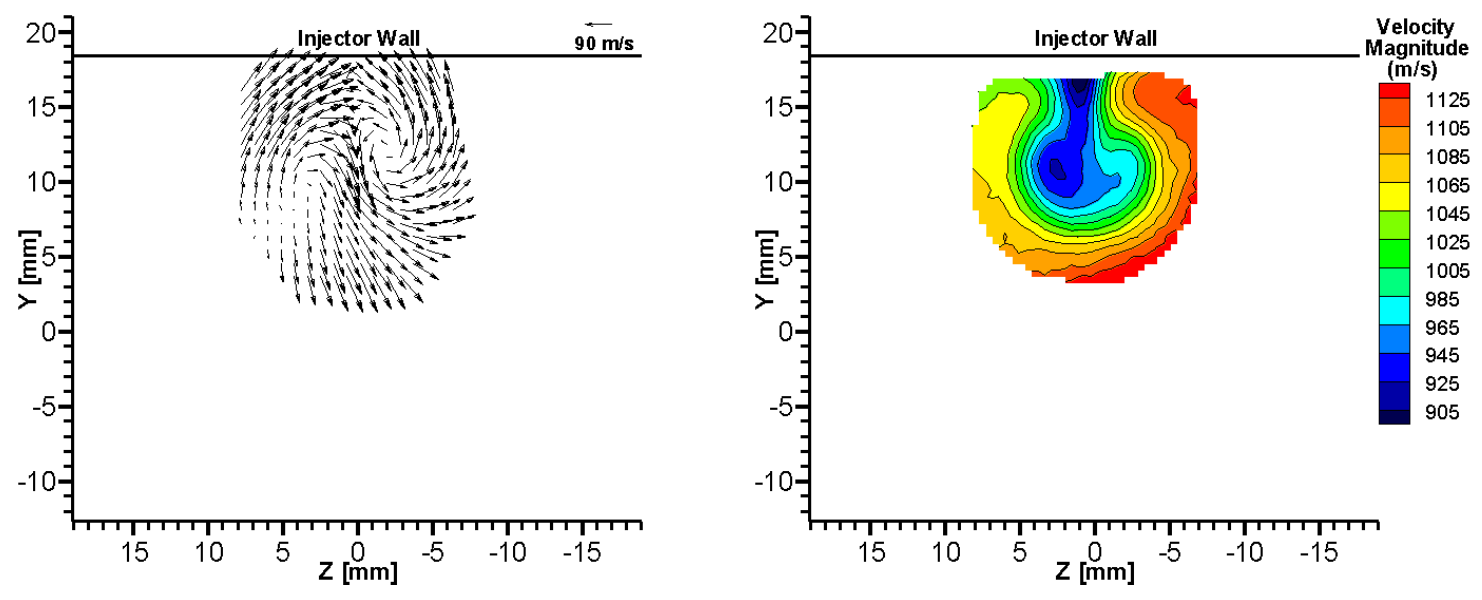

Instantaneous In-plane Velocity Vectors

Instantaneous 3C Velocity
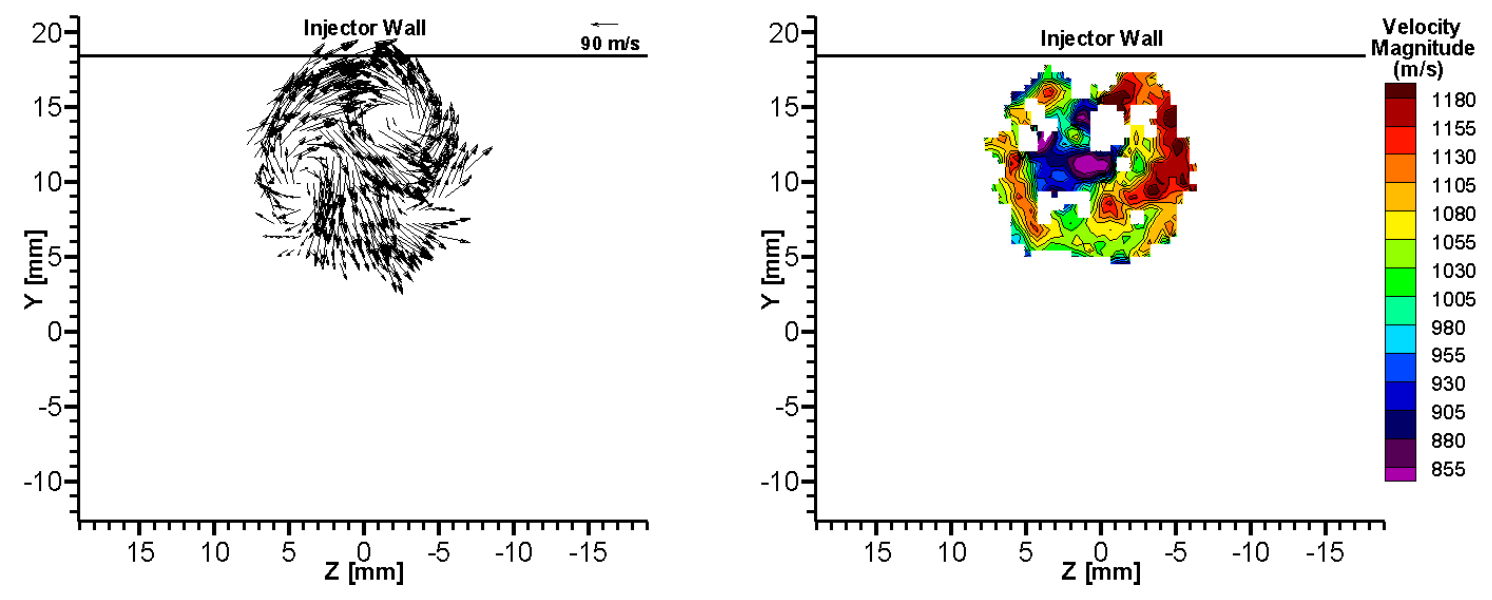

Instantaneous In-plane Velocity Vectors

Instantaneous 3C Velocity
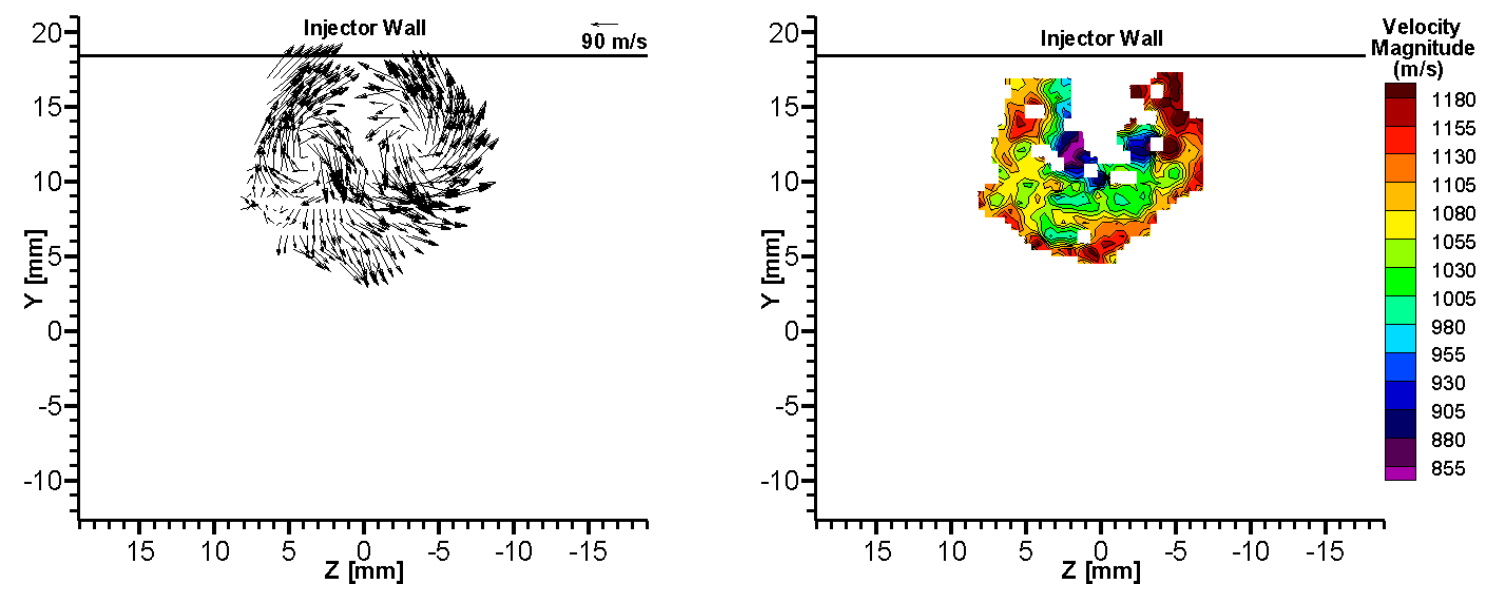

Figure D.1: Average and Instantaneous velocity fields, Fuel-air Mixing case at $\mathrm{X} / \mathrm{H}=12, \Phi=0.17$ 
Average of 100 Vector Fields

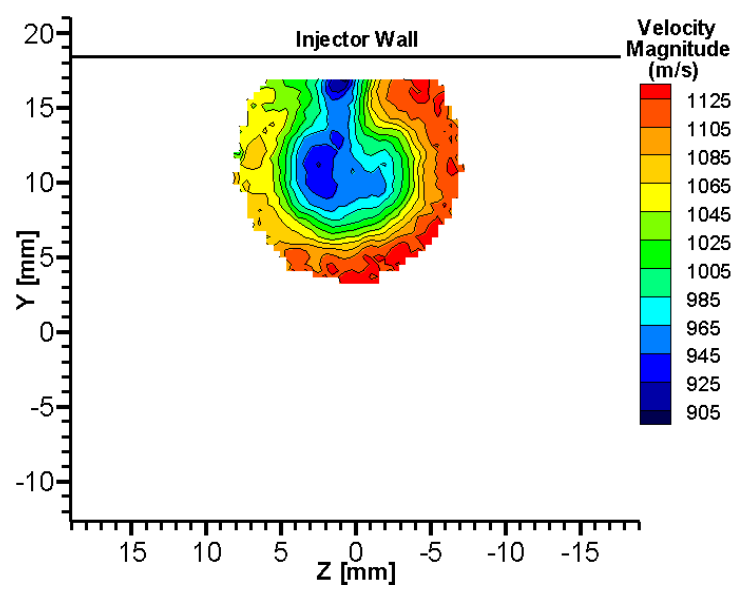

300 Vector Fields

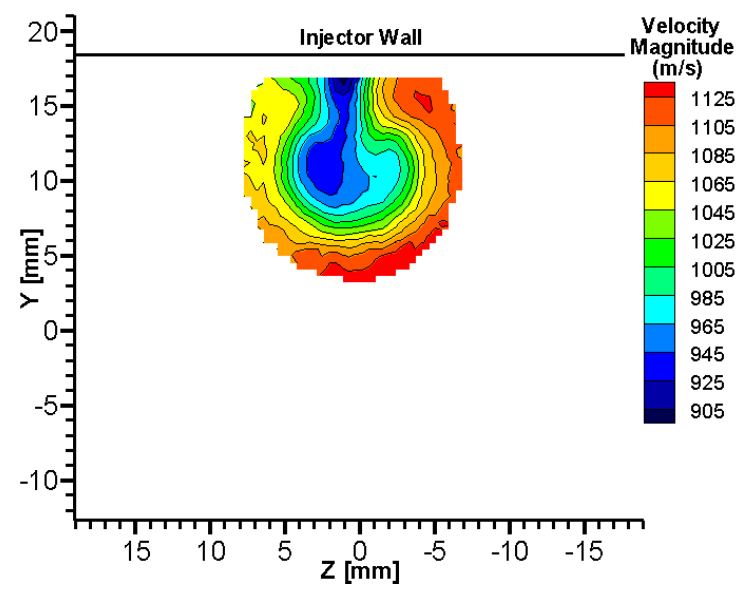

500 Vector Fields

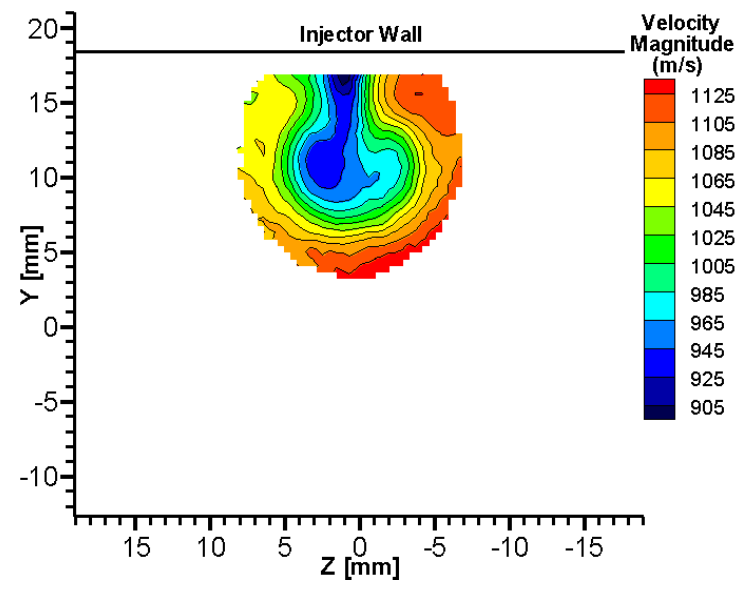

200 Vector Fields

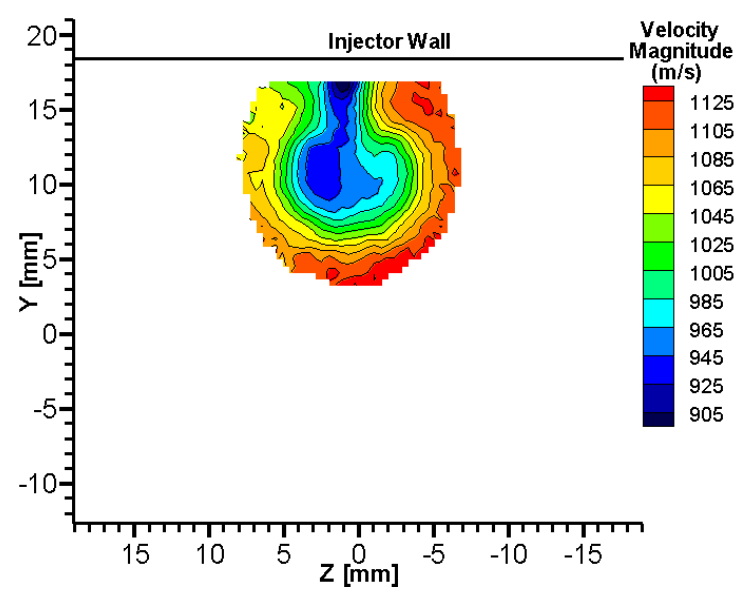

400 Vector Fields

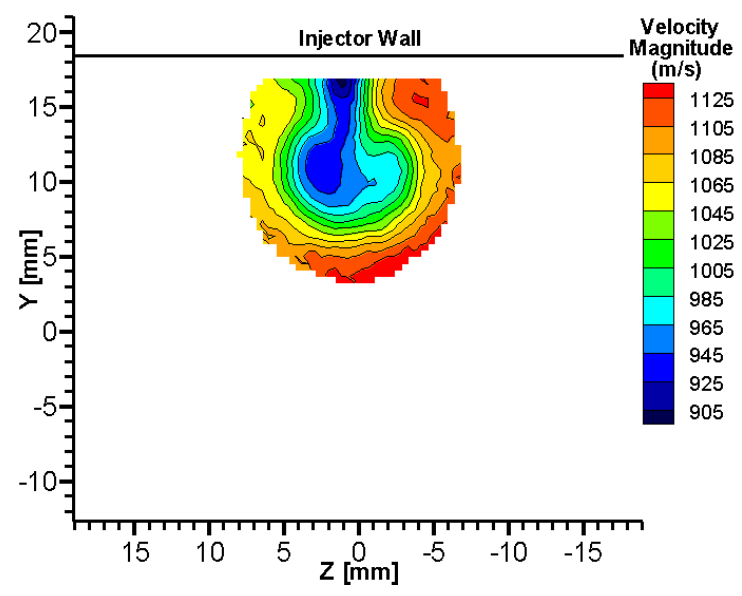

Average of All (1521) Vector Fields

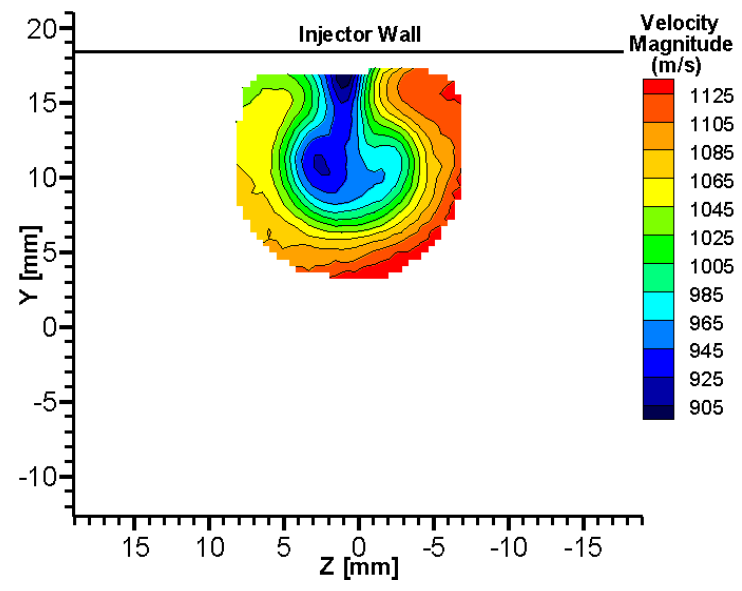

Figure D.2: Statistical convergence of $3 \mathrm{C}$ velocity, Fuel-air Mixing case at $\mathrm{X} / \mathrm{H}=12, \Phi=0.17$ 
3C Velocity Magnitude

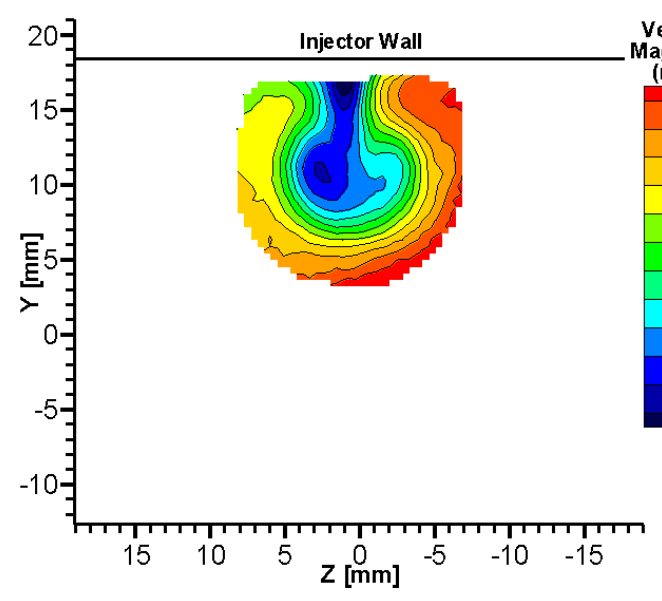

In-plane Velocity

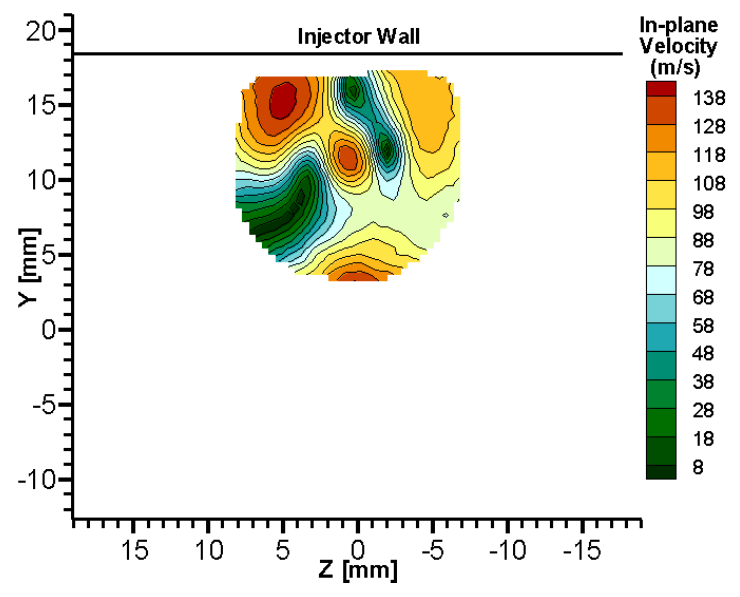

In-plane Velocity Vectors

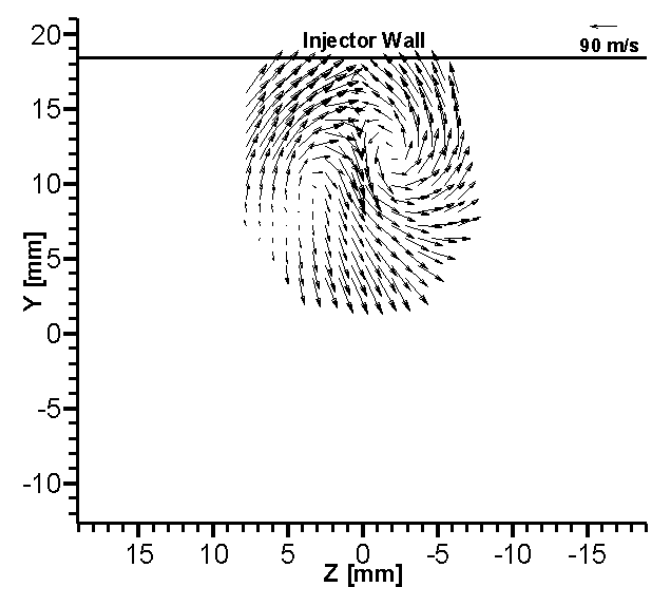

Axial (u) Velocity

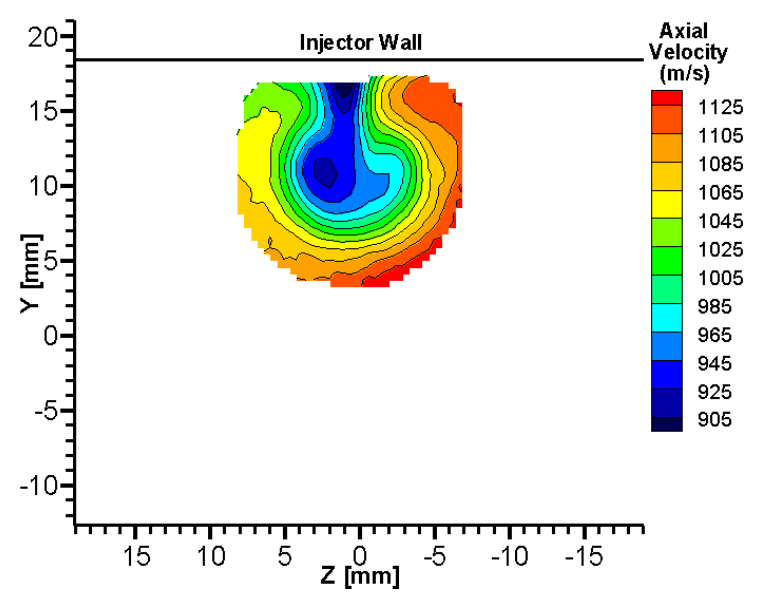

y-component (v) Velocity

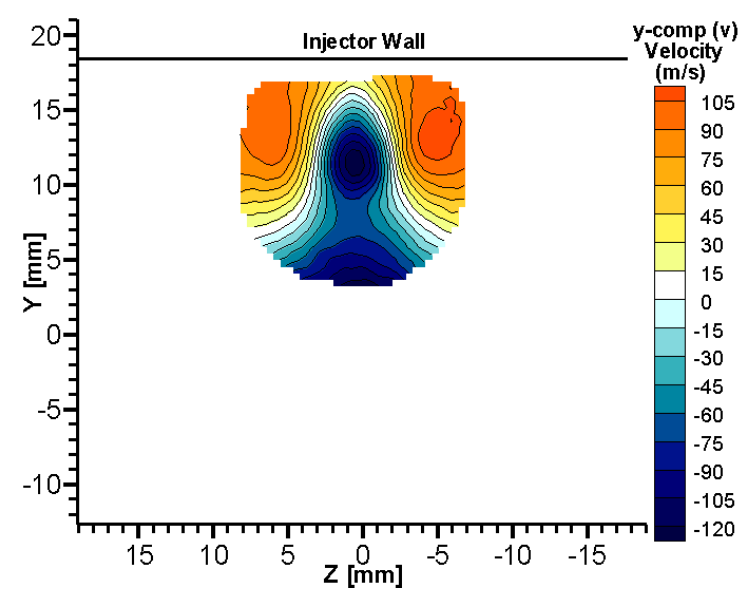

z-component (w) Velocity

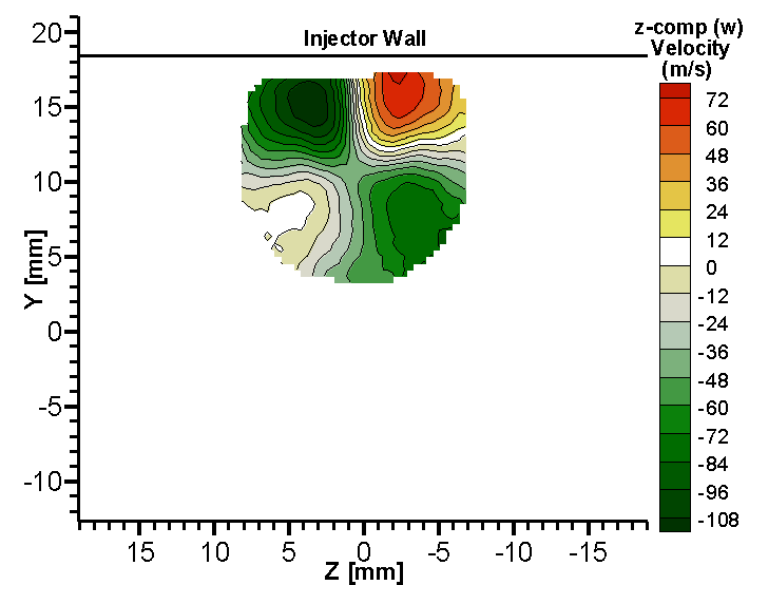

Figure D.3: Average velocity fields for Fuel-air Mixing case at $\mathrm{X} / \mathrm{H}=12$ (Avg. of 1521 results) 
Experiment (SPIV) Axial (u) Velocity

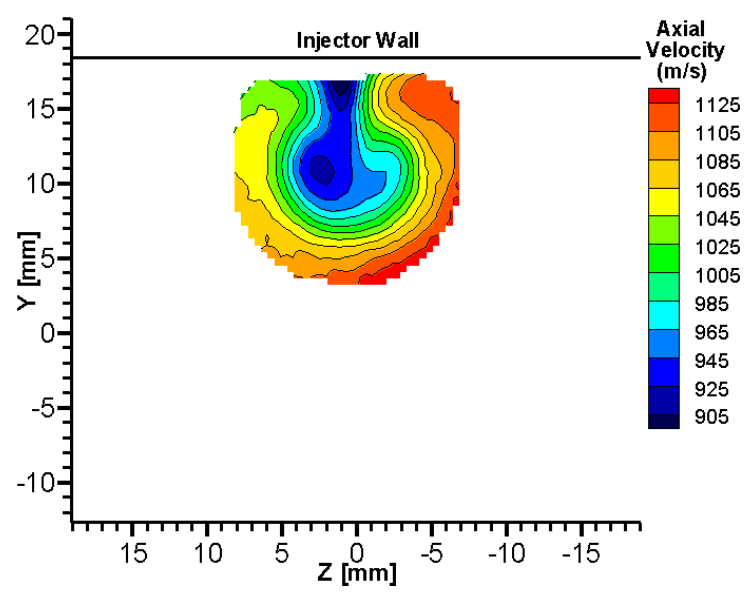

Experiment y-component (v) Velocity

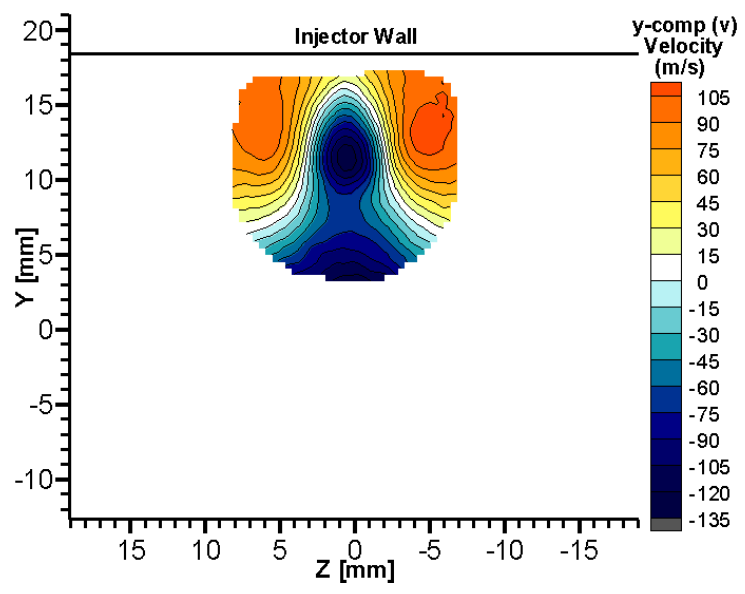

Experiment z-component (w) Velocity

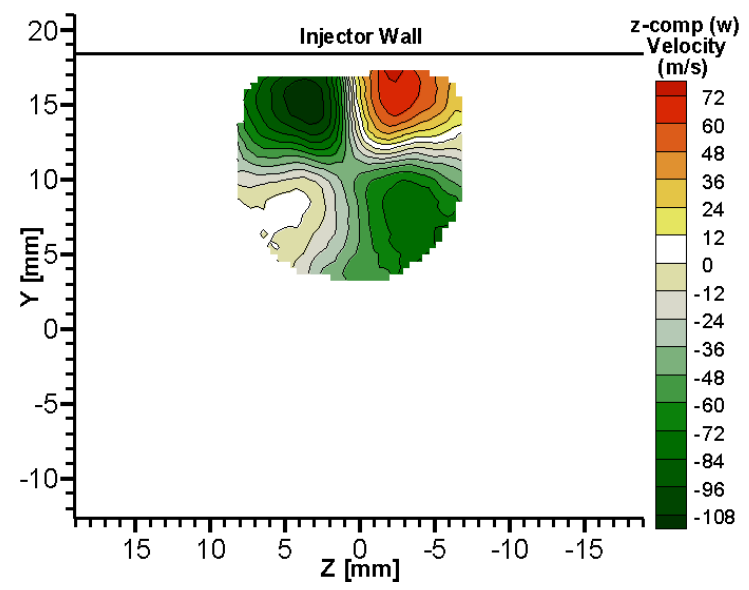

CFD Result, Axial (u) Velocity

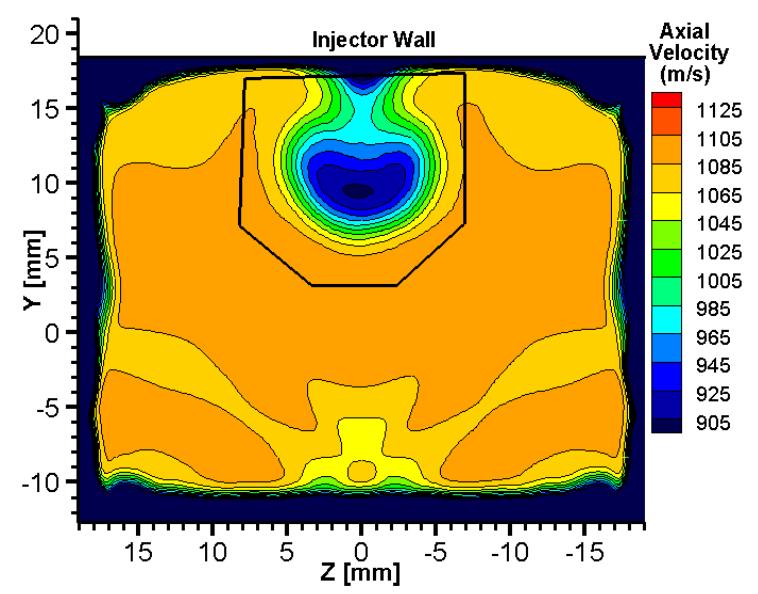

CFD y-component (v) Velocity

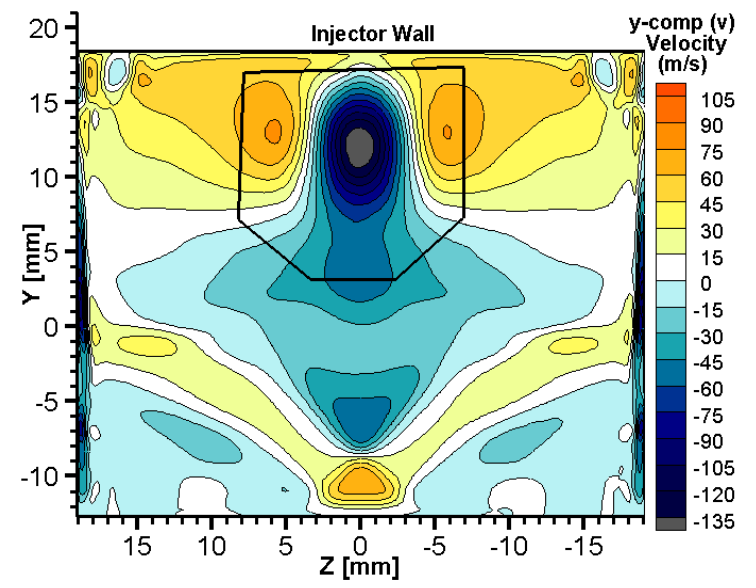

CFD z-component (w) Velocity

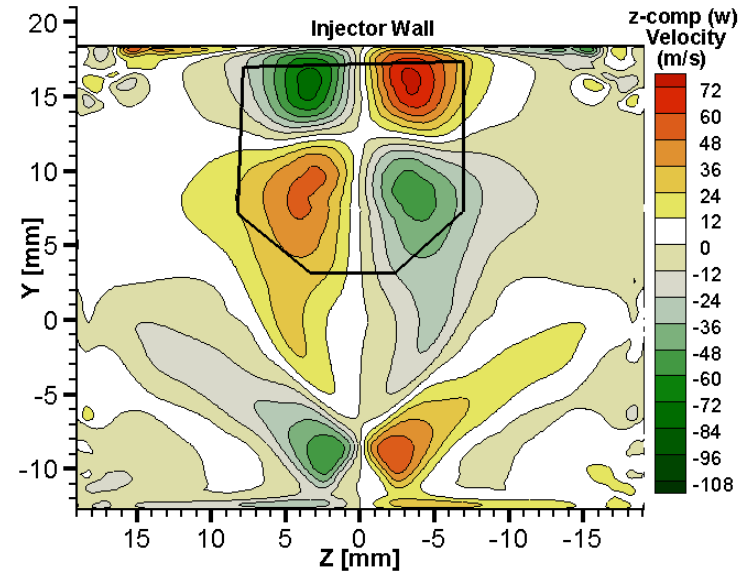

Figure D.4: Experiment (SPIV) and CFD comparison, Fuel-air Mixing case, $\mathrm{X} / \mathrm{H}=12, \Phi=0.17$ 


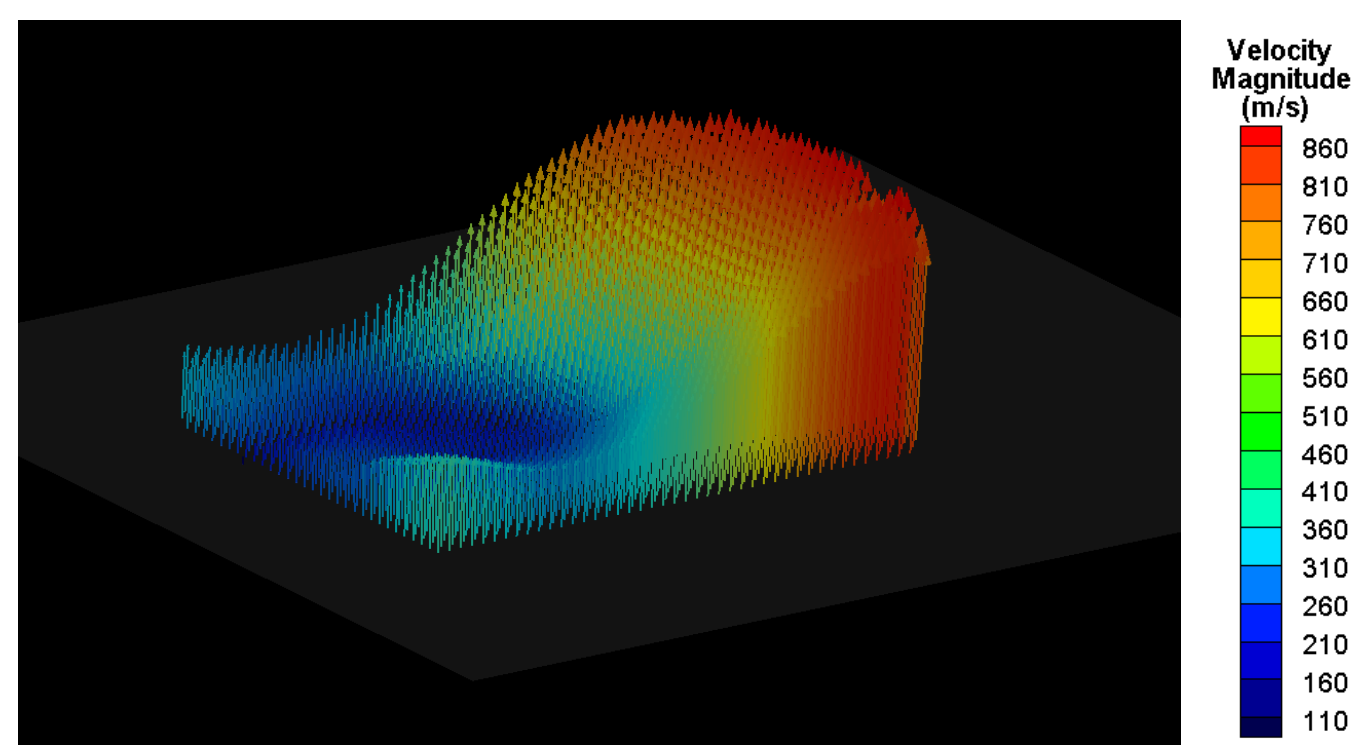

Figure D.5: Average three-component (3C) velocity result from SPIV measurements for Fuel-air Combustion case at $\mathrm{X} / \mathrm{H}=12$, Flowpath $2 ; 3 \mathrm{D}$ view of three-component velocity vectors

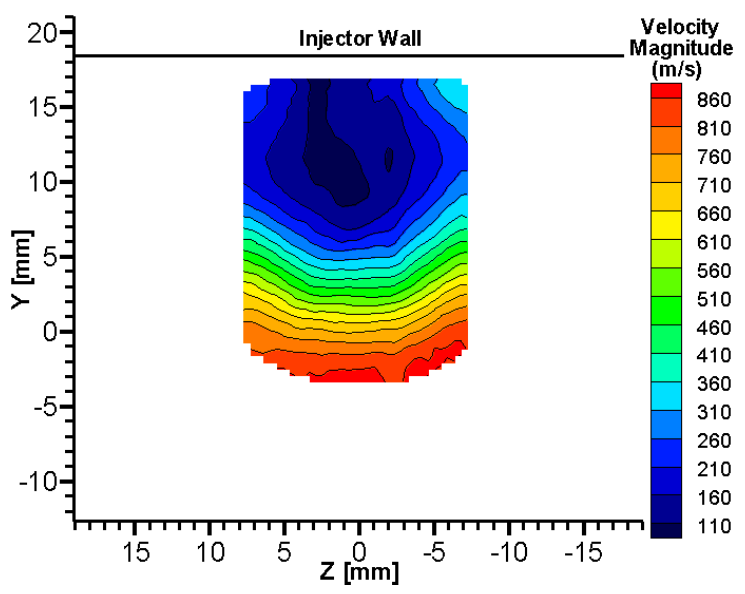

(a) Average of All (1162) Vector Fields [3C Velocity magnitude]

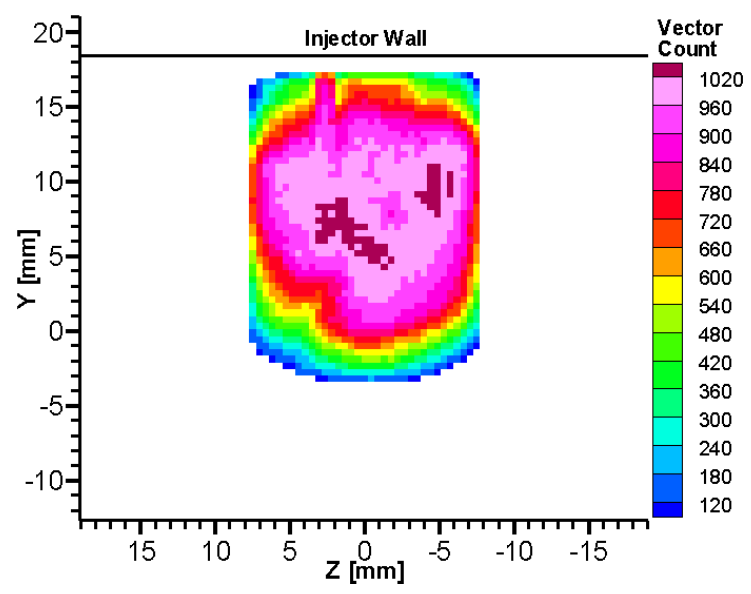

(b) Number of Vectors Averaged for Each Sub-region

Figure D.6: $\Phi=0.17$, Fuel-air Combustion at $\mathrm{X} / \mathrm{H}=12$;

(a) Average $3 \mathrm{C}$ velocity field, and (b) Number of vectors averaged at each location 
Average of 50 Vector Fields

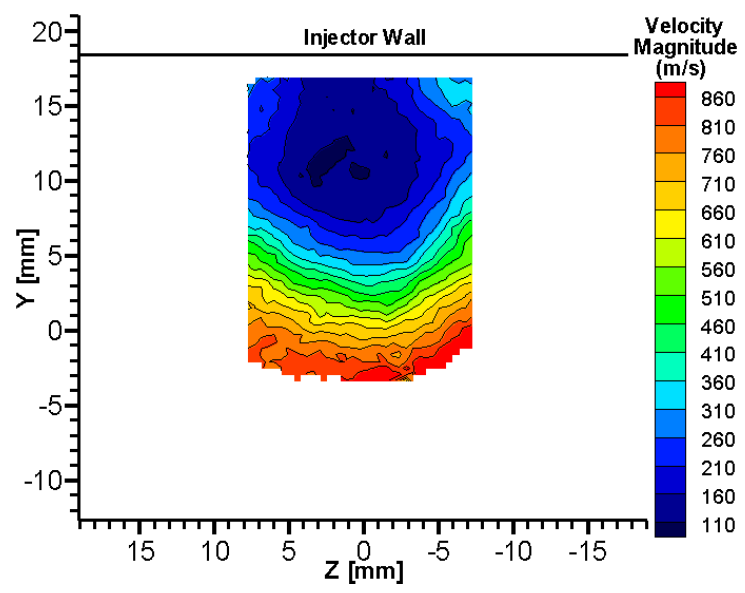

200 Vector Fields

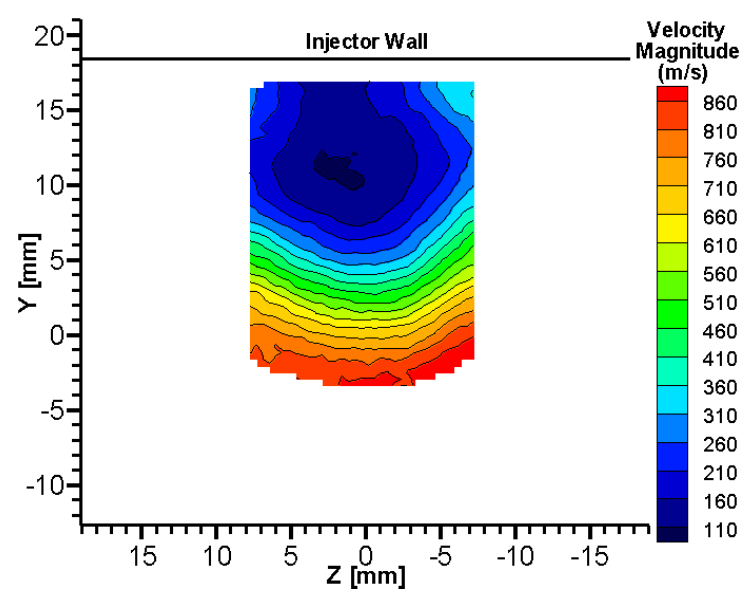

400 Vector Fields

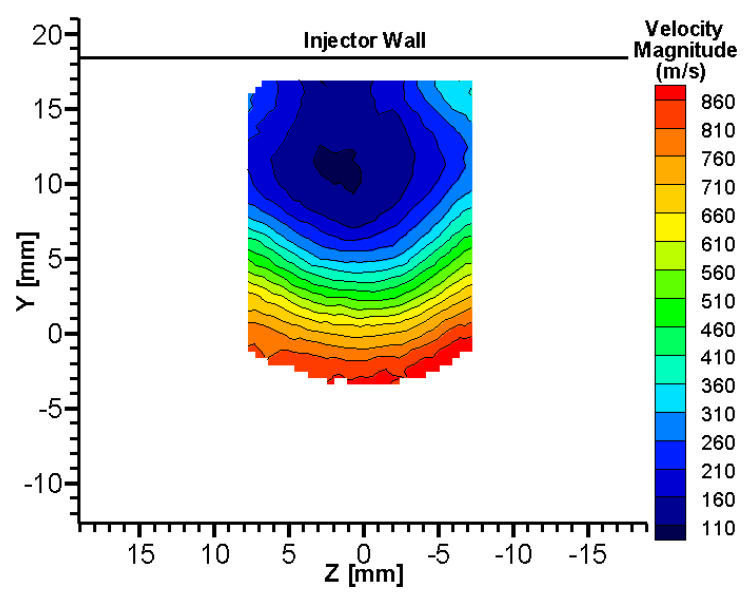

100 Vector Fields

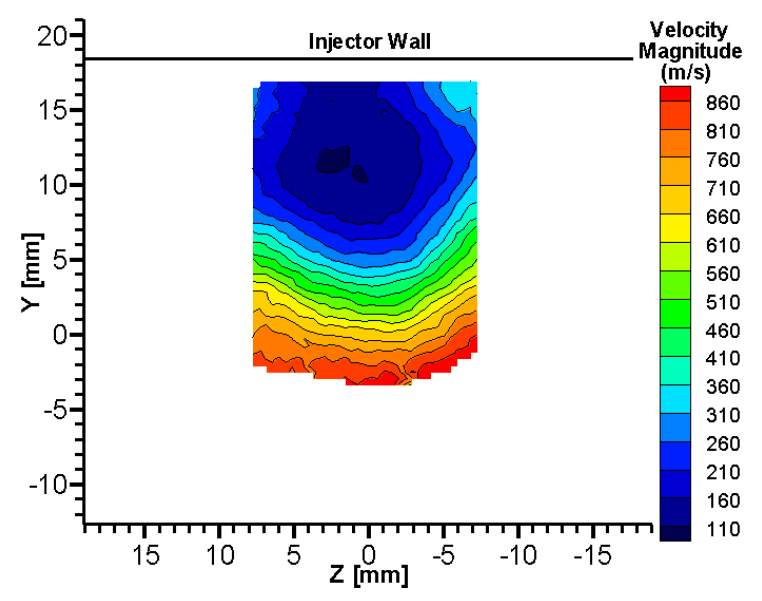

300 Vector Fields

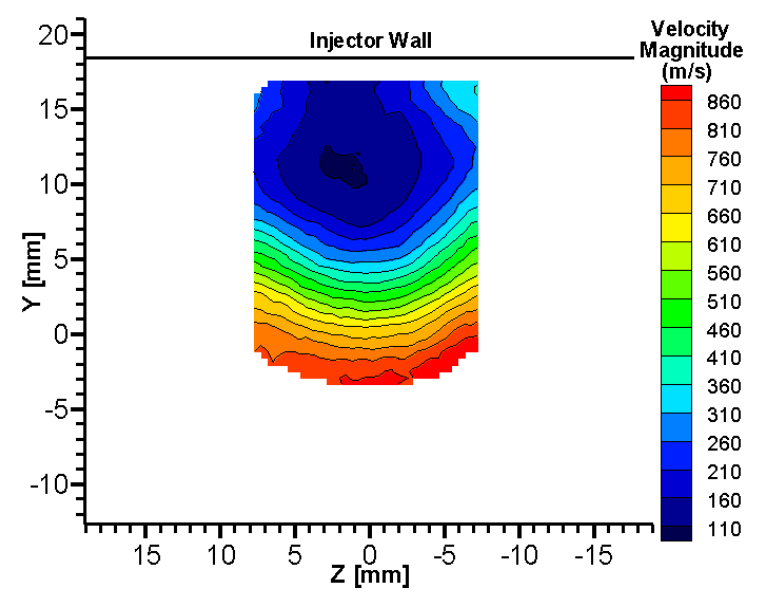

Average of All (1162) Vector Fields

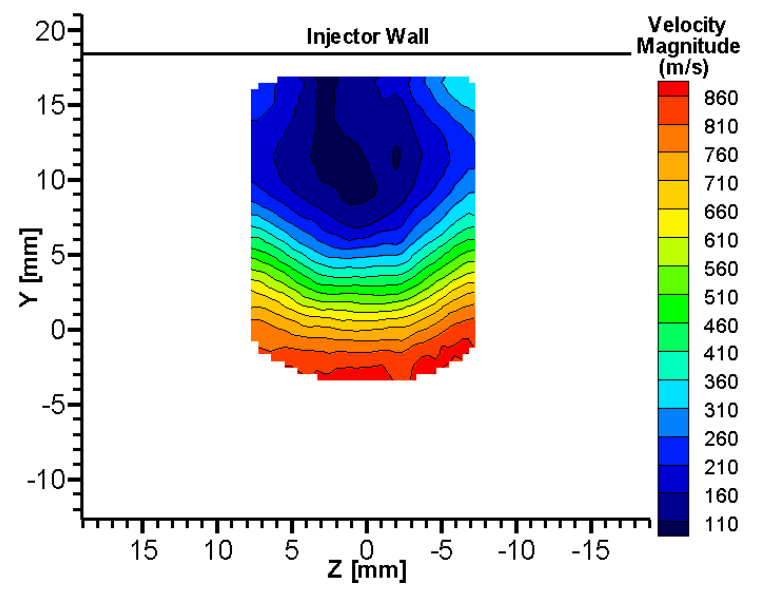

Figure D.7: Statistical convergence of $3 \mathrm{C}$ velocity, Fuel-air $\underline{\text { Combustion }}$ at $\mathrm{X} / \mathrm{H}=12, \Phi=0.17$ 
3C Velocity Magnitude

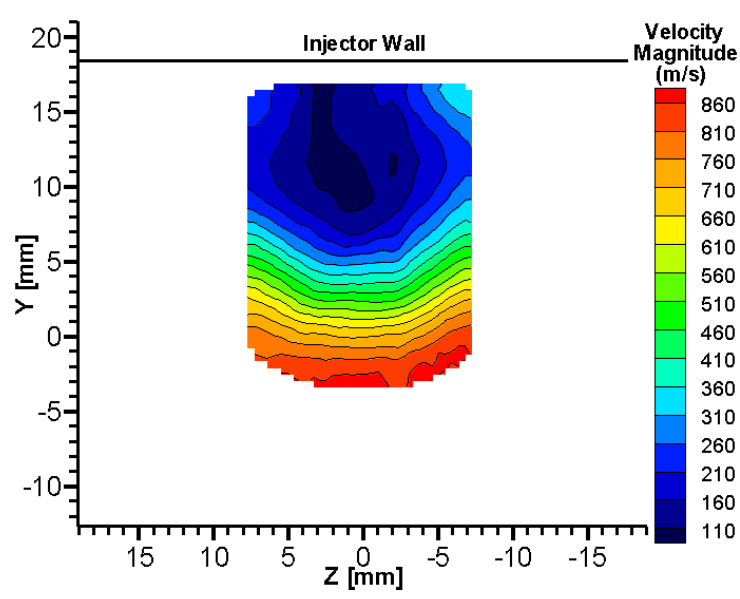

In-plane Velocity

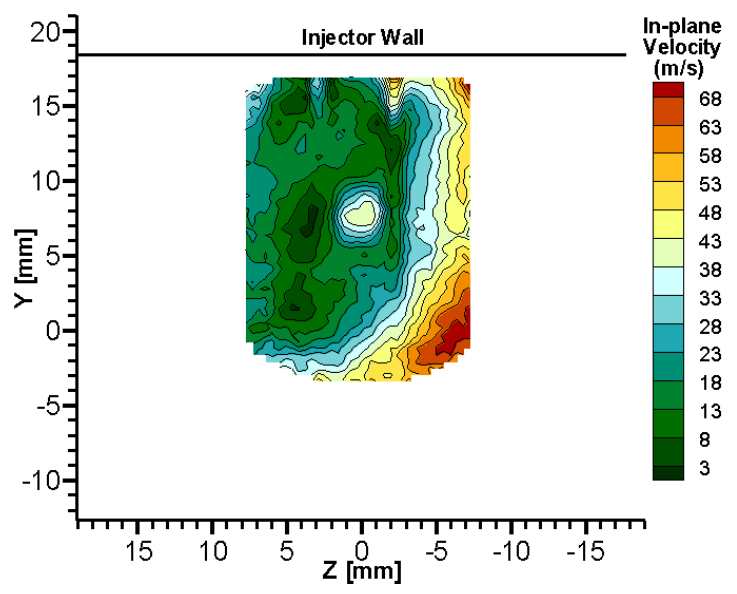

In-plane Velocity Vectors

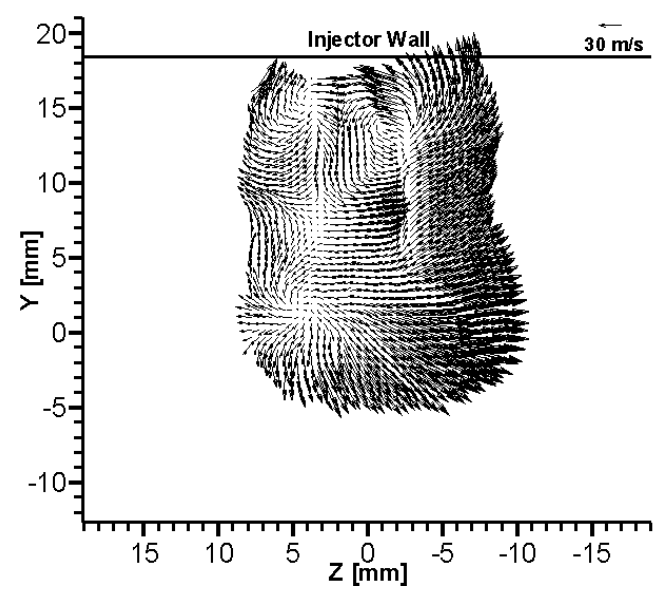

Axial (u) Velocity

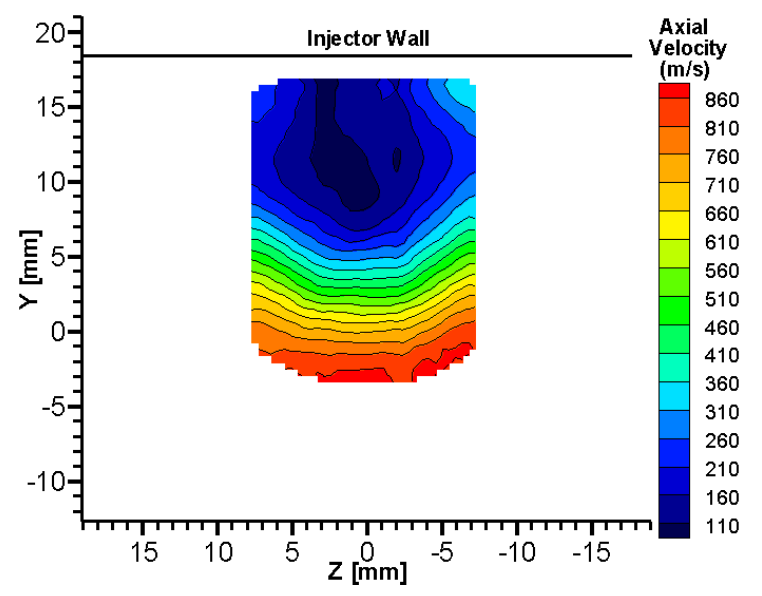

y-component (v) Velocity

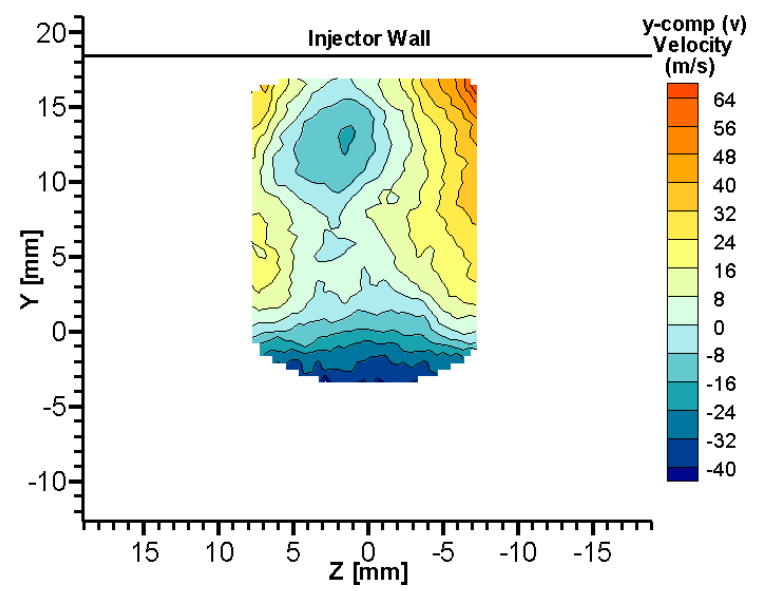

z-component (w) Velocity

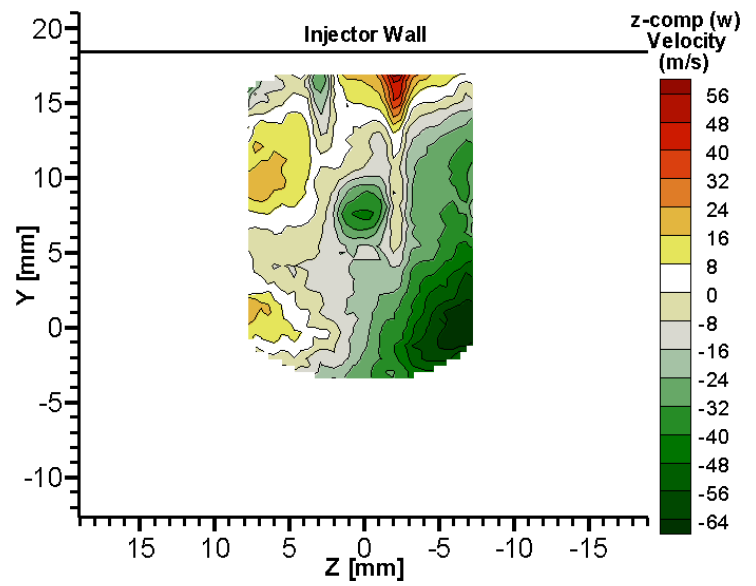

Figure D.8: Average velocity fields for Fuel-air Combustion at $\mathrm{X} / \mathrm{H}=12$ (Avg. of 1162 results) 


\section{Appendix E}

\section{Supplemental Figures for Flowpath 2, Plane at X/H = 15}

\section{List of figures}

Fuel-air Mixing

Figure E.1: Average and instantaneous velocity fields: Fuel-air Mixing case at $\mathrm{X} / \mathrm{H}=15$, Flowpath 2, $\Phi=0.17$

Figure E.2: Summary of Average fields for Fuel-air Mixing at $\mathrm{X} / \mathrm{H}=15, \Phi=0.17$;

3C magnitude, In-plane magnitude, In-plane vectors; and $\mathrm{u}, \mathrm{v}$ and $\mathrm{w}$ velocity components (Average of 1761 instantaneous fields)

Figure E.3: Comparison of experimental (SPIV) measurements to CFD results (LES/RANS) for Fuel-air Mixing; X/H = 15, Flowpath 2

\section{Fuel-air Combustion}

Figure E.4: Average and instantaneous velocity fields: Fuel-air Combustion case at $\mathrm{X} / \mathrm{H}=15$, Flowpath $2, \Phi=0.17$

Figure E.5: Summary of Average fields for Fuel-air Combustion at $\mathrm{X} / \mathrm{H}=15$;

3C magnitude, In-plane magnitude, In-plane vectors; and $\mathrm{u}, \mathrm{v}$ and $\mathrm{w}$ velocity components (Average of 1083 instantaneous fields) 
Average In-plane Velocity Vectors

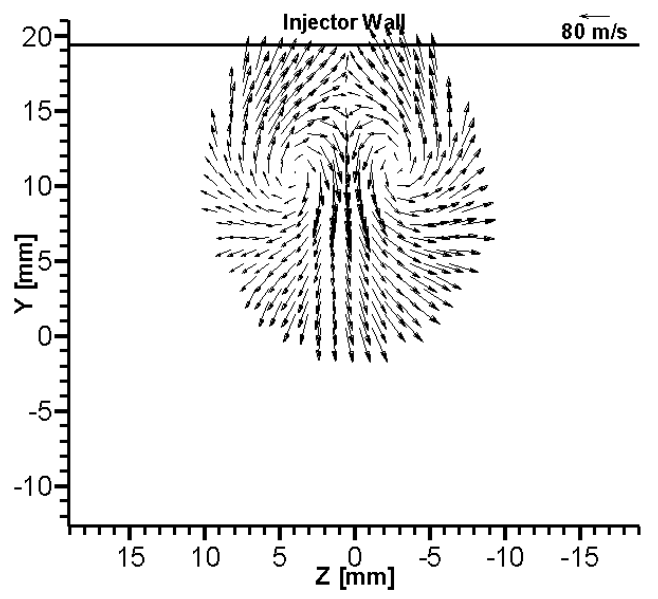

Instantaneous In-plane Velocity Vectors

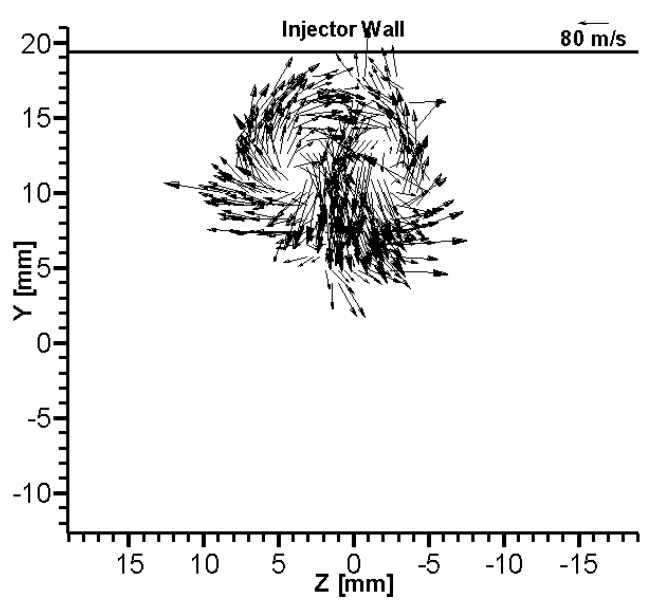

Instantaneous In-plane Velocity Vectors

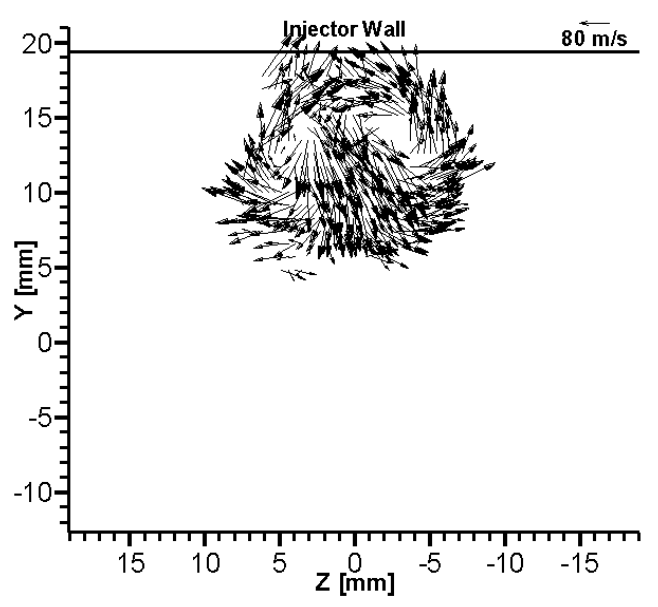

Average $3 C$ Velocity Field

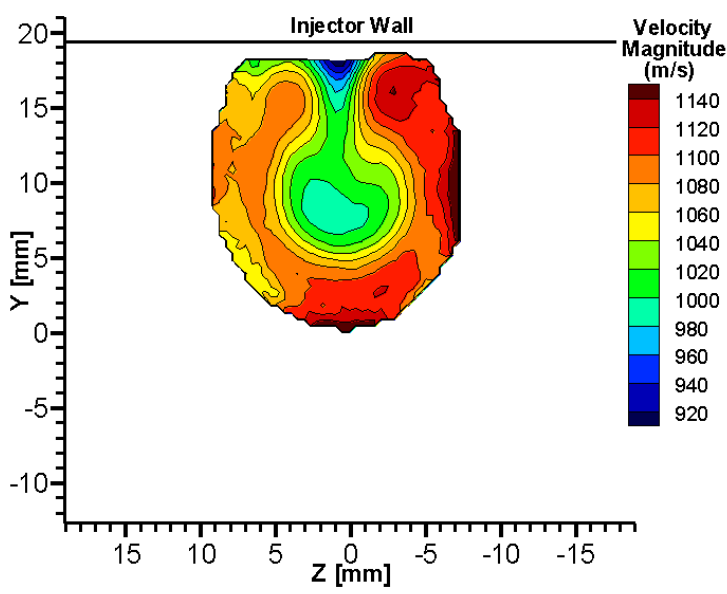

Instantaneous 3C Velocity

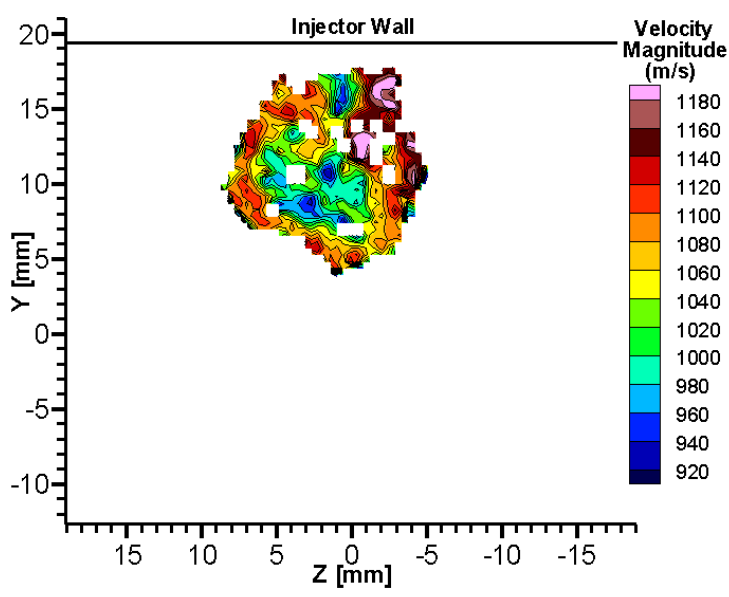

Instantaneous 3C Velocity

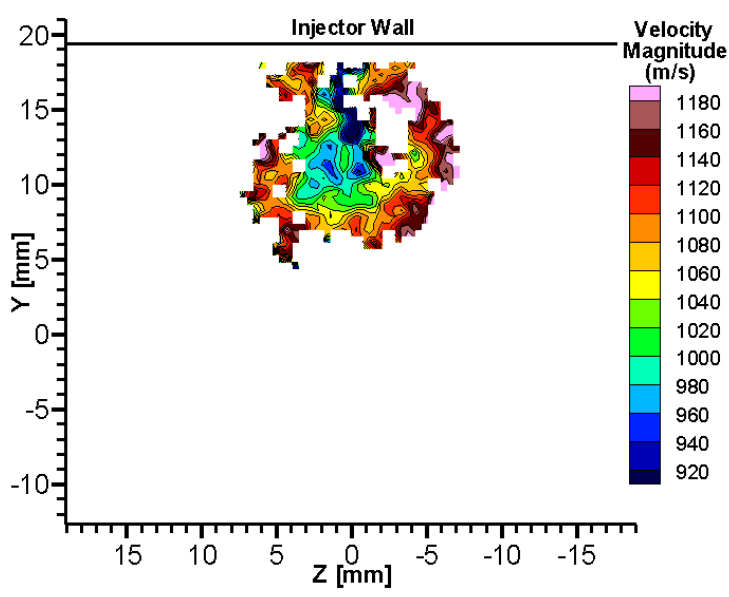

Figure E.1: Average and Instantaneous velocity fields, Fuel-air Mixing case at $\mathrm{X} / \mathrm{H}=15, \Phi=0.17$ 
3C Velocity Magnitude

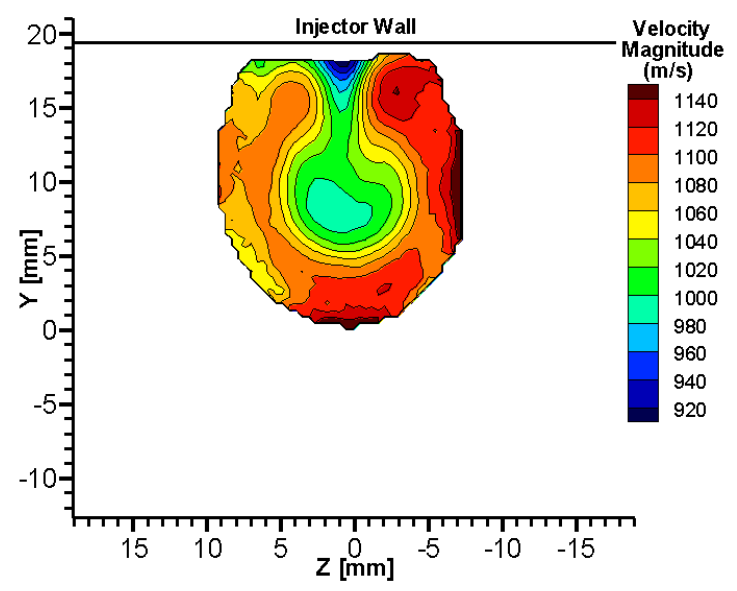

In-plane Velocity

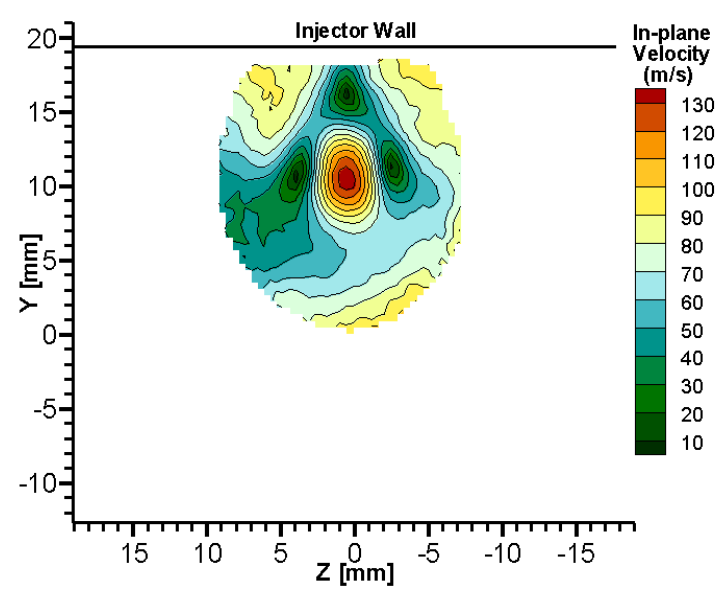

In-plane Velocity Vectors

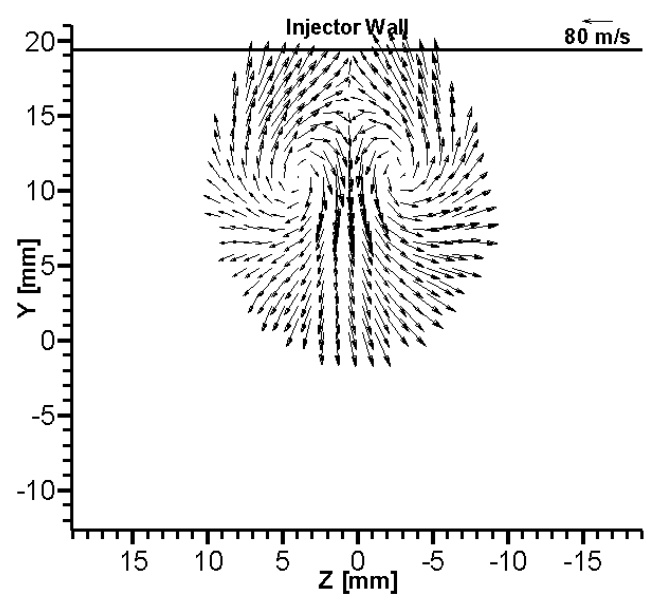

Axial (u) Velocity

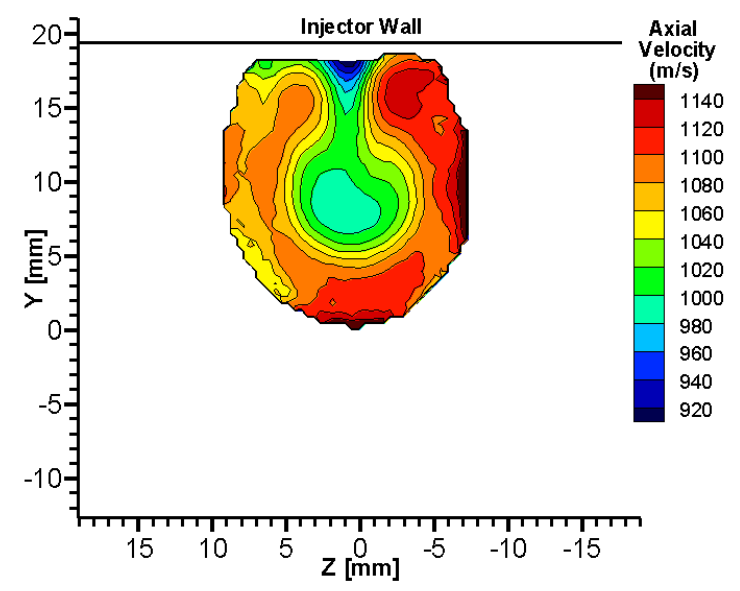

y-component (v) Velocity

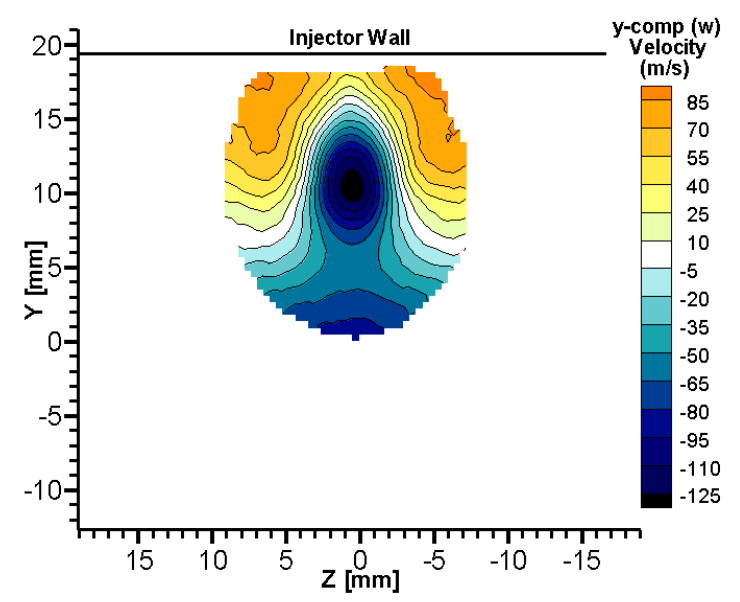

z-component (w) Velocity

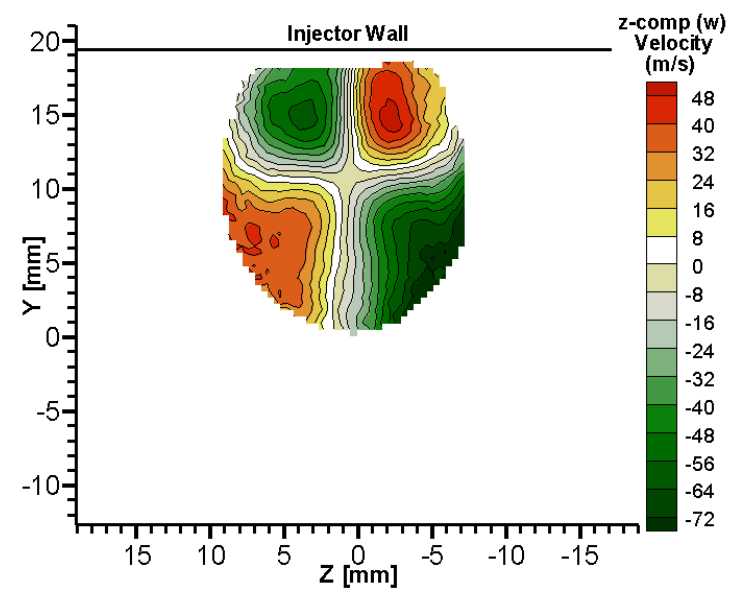

Figure E.2: Average velocity fields for Fuel-air Mixing case at $\mathrm{X} / \mathrm{H}=15$ (Avg. of 1761 results) 
Experiment (SPIV) Axial (u) Velocity

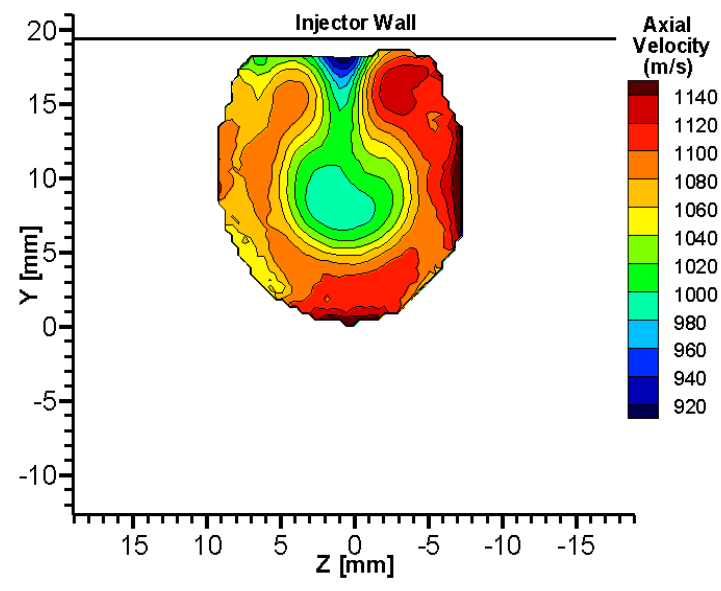

Experiment y-component (v) Velocity

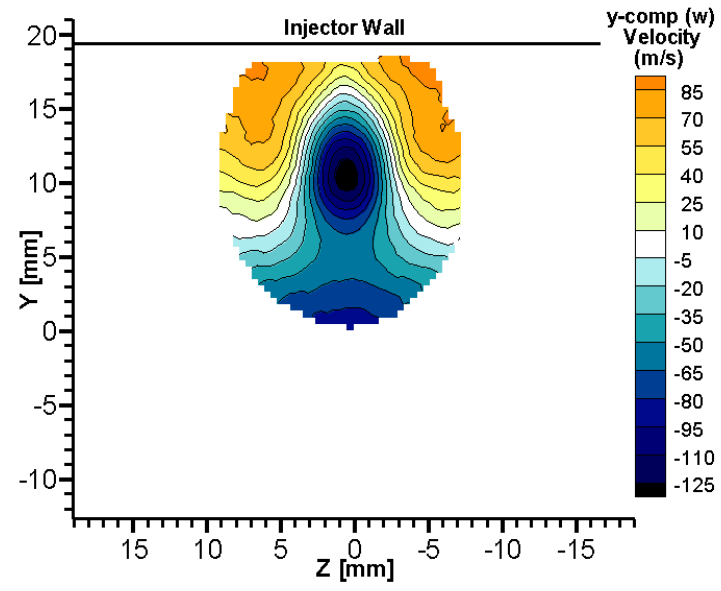

Experiment z-component (w) Velocity

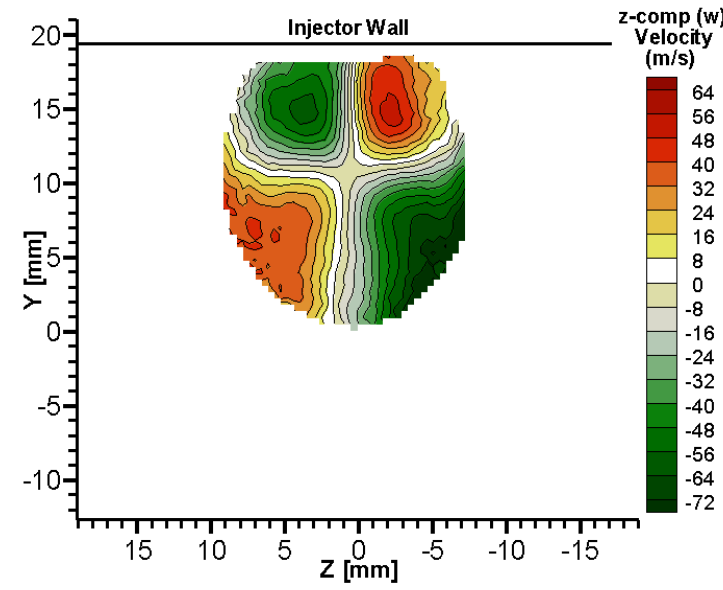

CFD Result, Axial (u) Velocity

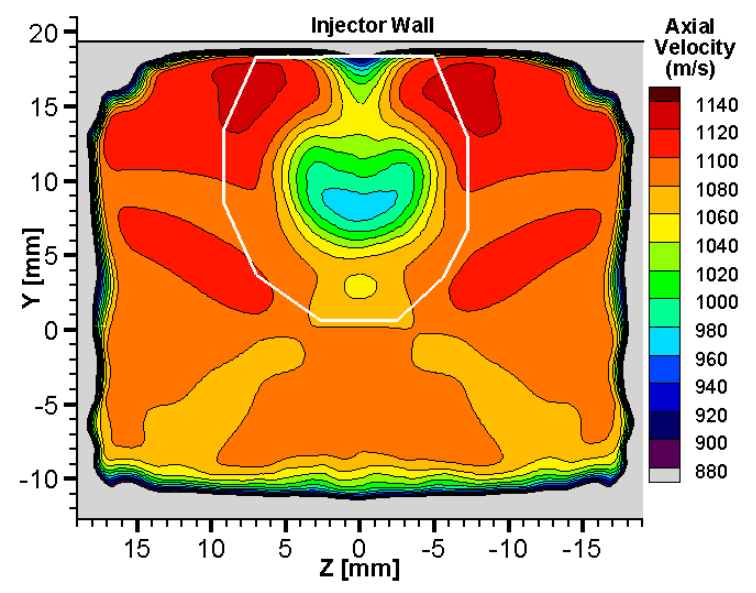

CFD y-component (v) Velocity

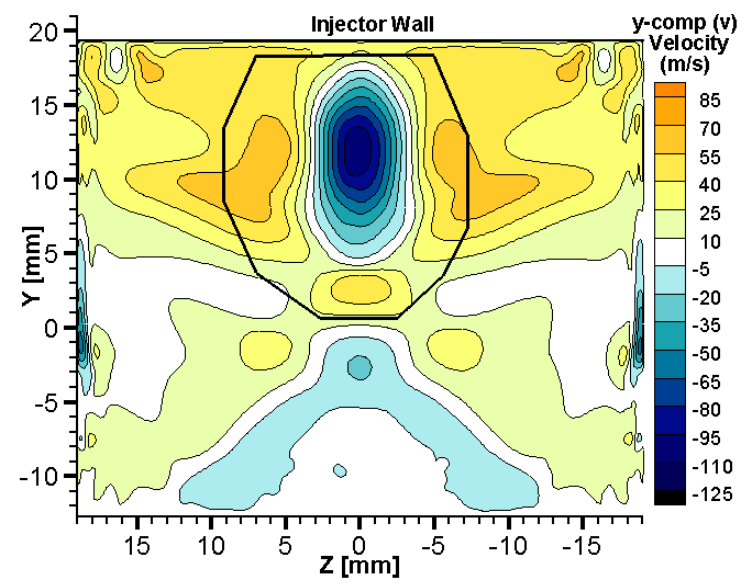

CFD z-component (w) Velocity

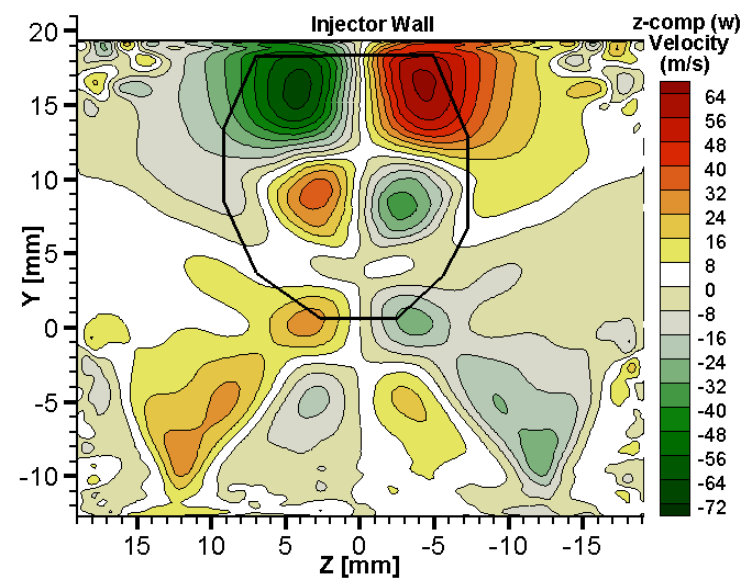

Figure E.3: Experiment (SPIV) and CFD comparison, Fuel-air Mixing case, $\mathrm{X} / \mathrm{H}=15, \Phi=0.17$ 
Average In-plane Velocity Vectors

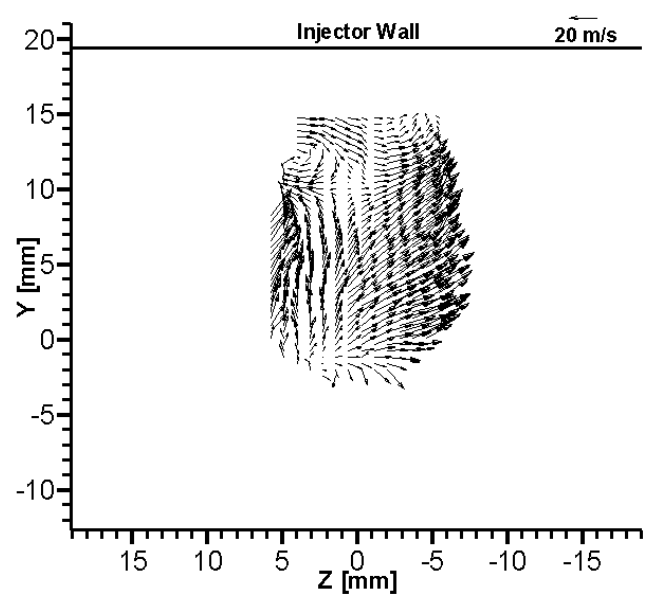

Instantaneous In-plane Velocity Vectors

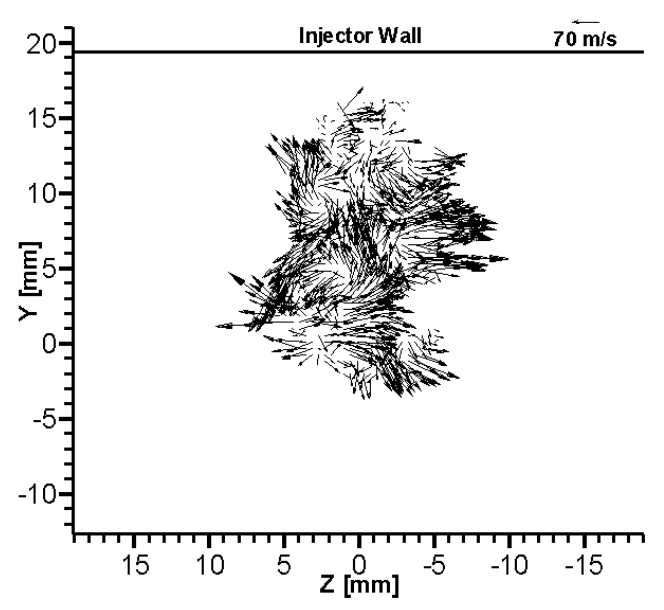

Instantaneous In-plane Velocity Vectors

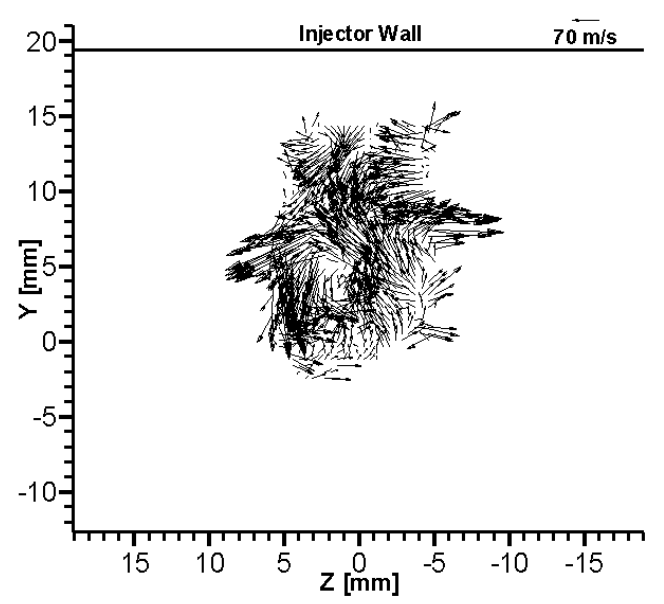

Average 3C Velocity Field

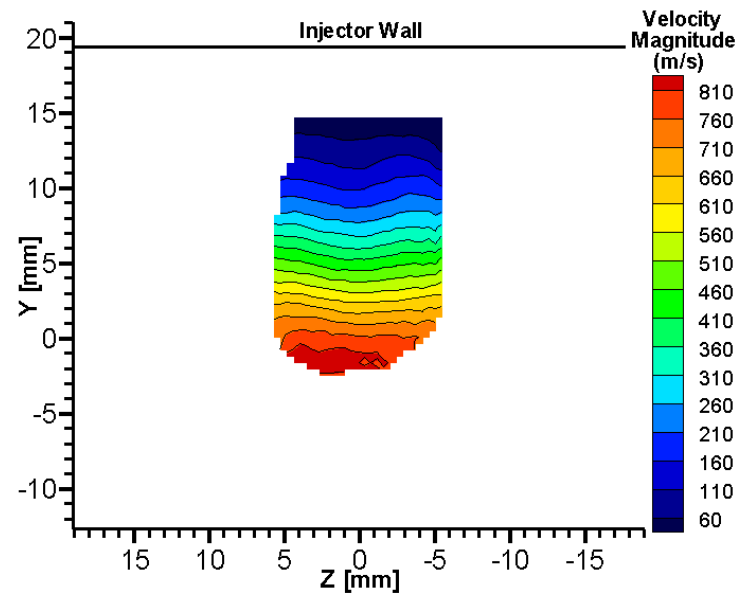

Instantaneous 3C Velocity

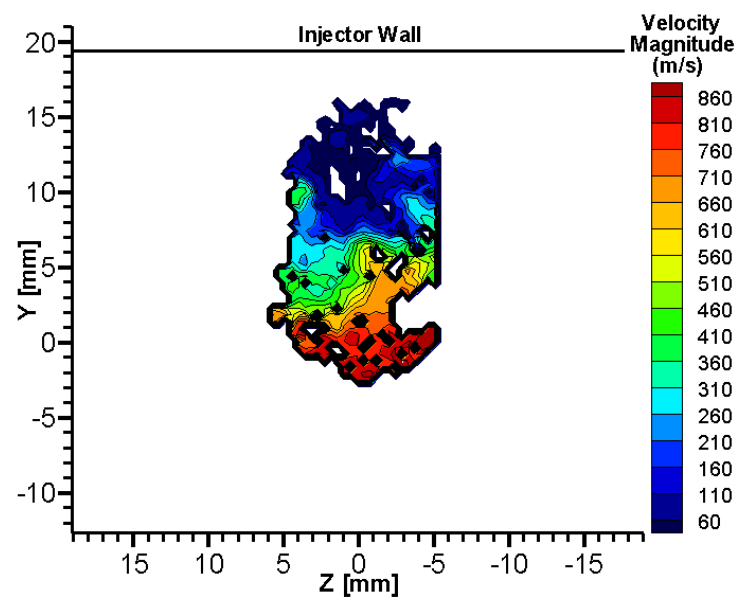

Instantaneous 3C Velocity

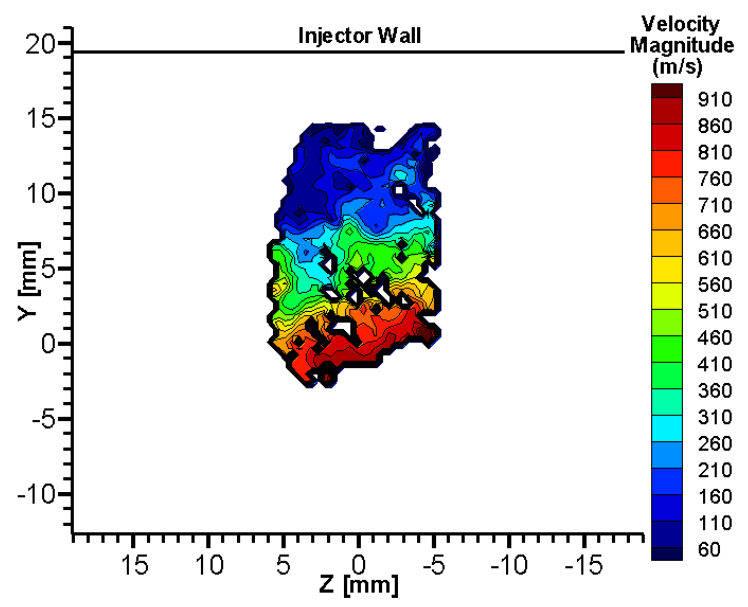

Figure 6.4C: Average and Instantaneous velocity fields, Fuel-air Combustion at $\mathrm{X} / \mathrm{H}=15, \Phi=0.17$ 

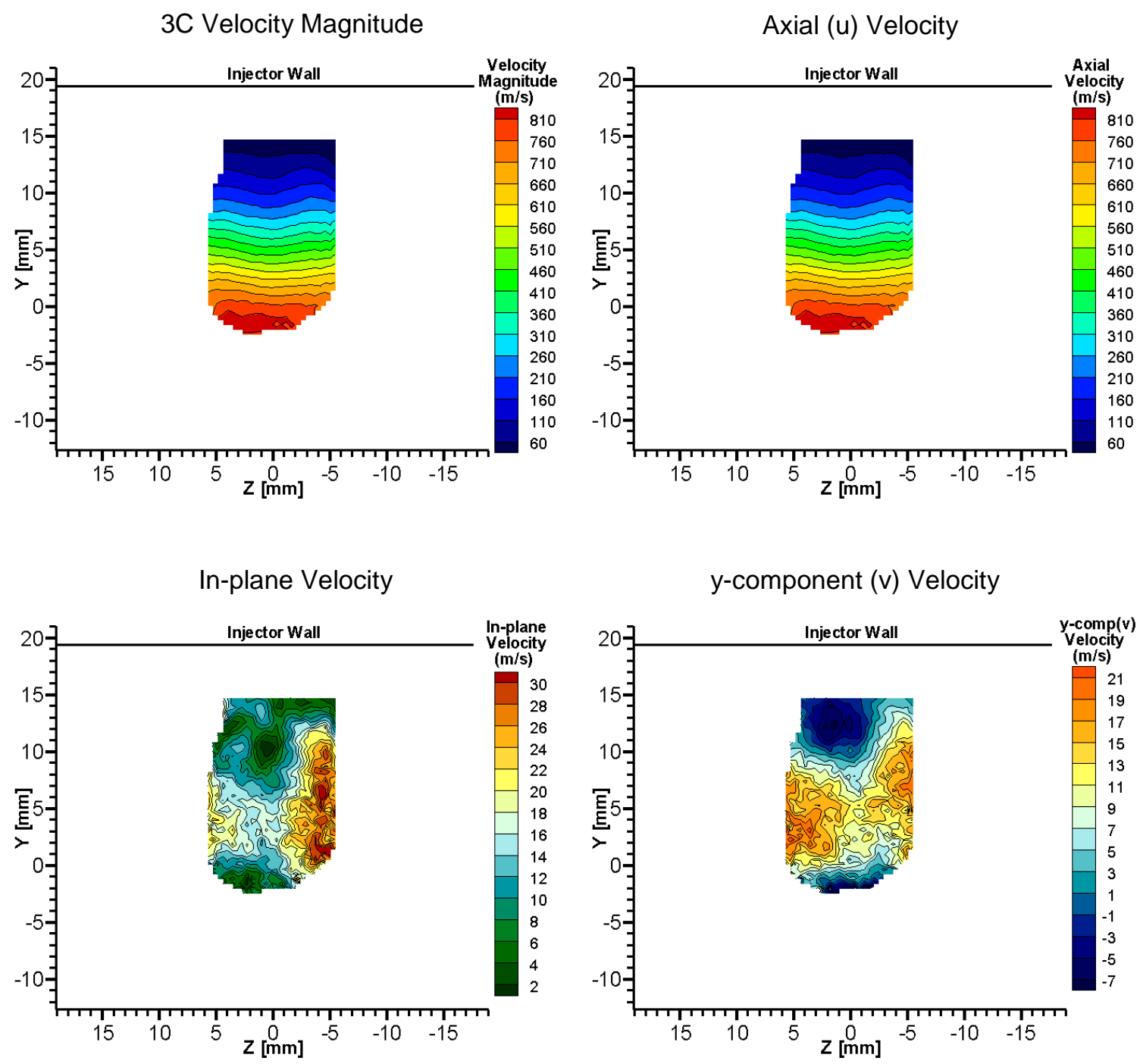

In-plane Velocity Vectors

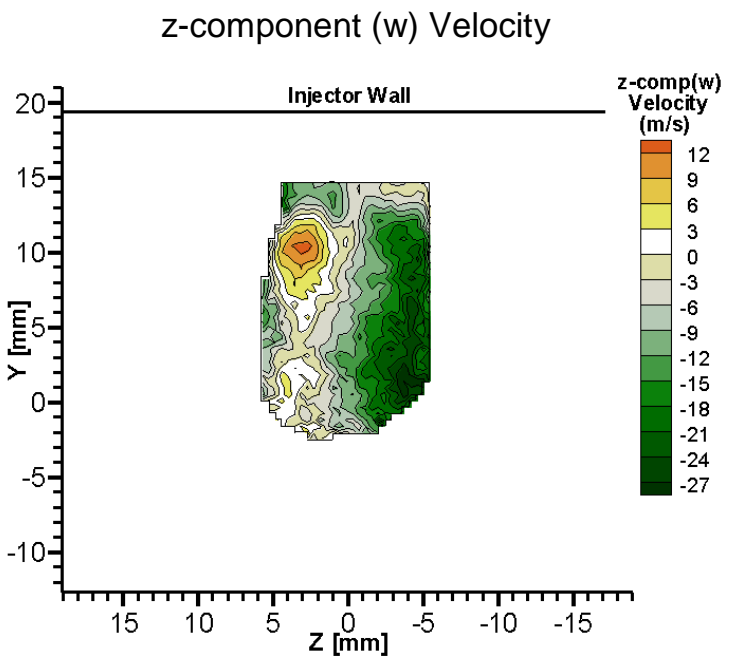

Figure E.5: Average velocity fields for Fuel-air Combustion at $\mathrm{X} / \mathrm{H}=15$ (Avg. of 1083 results) 


\section{Appendix F}

\section{Uncertainty Estimation Details for $\mathrm{X} / \mathrm{H}=10$ plane results}

Uncertainty estimation for the $\mathrm{X} / \mathrm{H}=10$ plane of Flowpath 1 is discussed in detail here. The experimental uncertainty of a stereoscopic PIV system is due to four major sources of error: 1) registration error, or when a $2 \mathrm{C}$ vector from one camera is not combined with the correct corresponding $2 \mathrm{C}$ vector viewed by the other camera, 2) particle flow tracking error in regions of high flow acceleration or deceleration, 3) uncertainty of measuring the displacement of the particles in the image plane of each camera (uncertainty in determining the two-component (2C) vectors), 4) uncertainty of the time between laser pulses. Each of these types of error is discussed below.

Registration error (error type 1) is introduced if there is misalignment of the calibration target with the laser sheet. When a misalignment of the target is present, corresponding $2 \mathrm{C}$ vectors from each camera back-project to different areas of the image plane during image-to-world mapping. Therefore, different $2 \mathrm{C}$ vectors combine to

produce incorrect $3 \mathrm{C}$ vectors. This type of error is discussed in the literature. ${ }^{18,42}$ Scarano et al. ${ }^{42}$ investigated registration error and found that even small angular or translational misalignment (less than 3 degrees, or $1 \mathrm{~mm}$, respectively) of the calibration target caused particle images to be mapped to locations that were 10 or more pixels away from the correct image plane locations. The LaVision DaVis 7.1 software package contains a "self-calibration" feature where particle images themselves are used to correct the imageto-world mapping functions to a form for no misalignment between calibration target and 
laser sheet. This "self-calibration" process is fully described in Reference 43. After applying the "self-calibration," the disparity distance between the two (dewarped, backprojected) images was less than half the length of a sub-region. Therefore, corresponding sub-regions from each camera overlapped and correct $3 \mathrm{C}$ vectors were generated. Thus, registration error was neglected.

Particle tracking (error 2) in regions near shocks or expansion fans can be a significant source of error when applying PIV to a supersonic flow. However, previous CFD and wall pressures ${ }^{28}$ indicate that there are no shocks or expansion fans near the $\mathrm{X} / \mathrm{H}=10$ measurement plane and therefore particle lag in the measurement region is assumed to be negligible.

Another potential source of particle tracking error is radial acceleration and a calculation was completed to examine the measurement error that could be introduced by the vortices of this flowfield. The radial movement of a particle in a rotating field of constant angular velocity $\omega$ can be predicted using a relation by Durst et al. ${ }^{44}$ with viscosity from the Sutherland relation. A particle starts at an initial radius $\left(\mathrm{r}_{1}\right)$ and moves to a larger radius $\left(\mathrm{r}_{2}\right)$ during axial motion downstream. Previous $\mathrm{CFD}^{15}$ predicts that $\sim 6 \mathrm{~mm}$ diameter vortices are fully formed by the $\mathrm{X} / \mathrm{H}=4$ plane, with a tangential velocity of approximately $100 \mathrm{~m} / \mathrm{sec}$ expected at $\mathrm{r}_{1} \approx 1 \mathrm{~mm}$. Using the equation of Durst et al. ${ }^{44}$ and an expected axial velocity of $1000 \mathrm{~m} / \mathrm{s}$, a particle starting at $\mathrm{r}_{1}$ is expected to move from $r_{1}=1 \mathrm{~mm}$ to $r_{2}=1.25 \mathrm{~mm}$ during axial motion from $\mathrm{X} / \mathrm{H}=4$ to $\mathrm{X} / \mathrm{H}=10$. A value of $\Delta \mathrm{r}=0.25 \mathrm{~mm}$ indicates the particle experiences an average radial velocity component of $6.5 \mathrm{~m} / \mathrm{sec}$. The cross-plane velocity measured using PIV would be the vector sum of the tangential velocity $(100 \mathrm{~m} / \mathrm{sec})$ and the radial velocity $(6.5 \mathrm{~m} / \mathrm{sec})$ due to one of the 
ramp vortices, yielding a measurement of $100.2 \mathrm{~m} / \mathrm{sec}$ at 3.7 degrees from the true tangential velocity vector. This is an acceptably small particle tracking error of $0.21 \%$ in the magnitude of the measured cross-plane velocity.

The next major uncertainty of a SPIV experiment is the accuracy of the particle displacement that can be measured using particle images. Cross-correlation algorithms can, in general, achieve an accuracy of 0.1 pixel in the image plane. ${ }^{45}$ For the camera configuration of these SPIV experiments, the average spatial resolution was 28.8 $\mu \mathrm{m} /$ pixel so 0.1 pixel corresponds to a displacement across the image plane of $2.88 \mu \mathrm{m}$. Furthermore, in the flow under investigation, particle motion is due in large part to the dominant axial velocity component. In an effort to determine the maximum reasonable error, it is assumed here that the uncertainty of 0.1 pixels is associated entirely with the axial component. This assumption and a time separation of $\mathrm{D}_{t}=400 \mathrm{nsec}$ results in an uncertainty of $7.2 \mathrm{~m} / \mathrm{sec}$ associated with the projection of the axial velocity component onto the image plane. This error is the uncertainty for the axial velocity projected onto the image plane due to camera angle $\theta$. Therefore, geometrically resolving the error back to the physical world (for $\theta=30^{\circ}$ ) results in an error for the axial velocity component of $\pm 14.4 \mathrm{~m} / \mathrm{sec}$. Instantaneous vector fields from the SPIV measurements show that the mean axial velocity component is approximately $690 \mathrm{~m} / \mathrm{sec}$ for the fuel-air combustion case, so the uncertainty for particle displacement $(14.4 \mathrm{~m} / \mathrm{sec})$ corresponds to $2.1 \%$ uncertainty for the axial velocity component.

For an angular SPIV configuration with a camera angle of $\theta=30^{\circ}$, Lawson and $\mathrm{Wu}^{32}$ report that the error associated with the out-of-plane velocity component (here the axial component) is approximately 1.7 times the in-plane error and this ratio is nearly constant 
for locations across the camera field of view. Therefore, an axial velocity component displacement error estimate of $\pm 14.4 \mathrm{~m} / \mathrm{sec}$ corresponds to an in-plane error of \pm 8.5 $\mathrm{m} / \mathrm{sec}$ for $\theta=30^{\circ}$. Examining instantaneous velocity fields for the fuel-air combustion case showed that the mean in-plane velocity component magnitude was approximately 72 $\mathrm{m} / \mathrm{sec}$, so the particle displacement uncertainty for the in-plane velocity component is roughly $12 \%$.

Uncertainty in the time separation between laser pulses $\left(D_{t}\right)$ has been previously estimated to be $1 \%$ and that uncertainty is mostly attributed to laser pulse jitter. ${ }^{11}$ Assuming the uncertainties in displacement measurement and time separation $\left(D_{t}\right)$ are normally distributed and independent, they can be combined as a standard root sum square to yield total uncertainties near $2.3 \%$ and $12 \%$ for the axial and in-plane velocity components, respectively. 